\title{
Maths and science education: the supply of high achievers at A level
}

Education Standards Analysis and Research Division 
This research report was commissioned before the new UK Government took office on 11 May 2010. As a result the content may not reflect current Government policy and may make reference to the Department for Children, Schools and Families (DCSF) which has now been replaced by the Department for Education (DFE). 


\section{Summary of Findings}

\section{Headline Maths \& Science Trends}

- Provisional 2010 data show a continued increase in the number of entries to $\mathrm{A}$ level maths since 2003, now reaching 70 thousand per year; the rise on the previous year was $8 \%$, a little lower than the previous year's increase, when growth in entries achieved a record $12 \%$. These increases mark a successful recovery since maths entries fell by 18\% following curriculum changes in 2002.

- The number of $A$ level maths grades $A^{*}$-B increased to 47 thousand in 2010, also continuing the trend of the previous 7 years. The percentage of entries awarded these grades fell fractionally from 2009, standing at 67\%. 2010 was the first year in which grades of $A^{*}$ were awarded; they were achieved by $17 \%$ of maths entrants.

- The number of A level biology entries reached 53 thousand in 2010, surpassing their previous peak of 49 thousand in 1998. This increase by $10 \%$ on the previous year follows a $1 \%$ drop in 2009 , preceded by small but steady annual increases since 2004.

- The number of A level biology grades $A^{*}$-B increased to 27 thousand in 2010, continuing the trend of the previous 7 years. The percentage of entries awarded these grades rose fractionally from 2009 , standing at $51 \%$. $A^{*}$ grades were achieved by $8 \%$ of biology entrants in 2010 .

- Chemistry A level entries declined between 1998 (37 thousand) and 2003 (31 thousand), but then recovered gradually regaining their 1998 level by 2009, and reaching a new peak of 40 thousand in 2010; this latest represented a $9 \%$ increase on the previous year.

- The number of A level chemistry grades $A^{*}-B$ increased to 24 thousand in 2010, also continuing the trend of the previous 7 years. The percentage of entries awarded these grades fell fractionally from 2009 , standing at $59 \%$. A* grades were achieved by $9 \%$ of chemistry entrants.

- Physics A level entries are the weakest of the three traditional sciences, starting from the lowest base and falling continuously between 1999 and 2006.

Provisional 2010 data show that the slow recovery since 2006 has continued, with the number of entries now standing at 28 thousand, which is 2 thousand fewer than in 1999. Encouragingly, the $8 \%$ rise in entries in 2010 was the largest over the period, and double the size of the previous year's increase.

- The number of A level physics grades $A *-B$ increased to 15 thousand in 2010, continuing the trend of the previous 3 years. The percentage of entries awarded these grades rose by 1 percentage point from 2009 , standing at $55 \%$. A* grades were achieved by $11 \%$ of physics entrants.

- The average grades achieved for all maths and science A levels have risen over the period from 1996 to 2010. The main increases have been in the proportions of A grades, and to a lesser extent, B grades. The proportions of C-D grades have remained fairly stable, with Ungraded results and, to a lesser extent, grade Es shrinking as a proportion of entries.

- AS level entries in all four maths and science subjects increased in 2010, suggesting that further increases in A level entries are likely in 2011 . These likely increases look robust in maths and biology, but smaller in chemistry, and less certain in physics due to its slightly higher and increasing proportion of nonconverted AS levels, where the candidates have not continued to full A level. 
- The pass rate for GCSE maths in 2010 was 97\%, having remained stable at around this level since 2005 . The percentage of entrants achieving the highest maths grades ( $A^{*}$ or $A$ ) has increased to $16 \%$, up by 1 percentage point since 2005.

- Entries in the separate sciences at GCSE have more than doubled between 2007 and 2010; in 2007, they stood at between 51 and 54 thousand for each of biology, chemistry and physics, but by 2010, the entries had reached between 112 and 116 thousand. These entries were climbing much more gradually prior to 2007 .

- In 2008, double science GCSEs were replaced by core and additional sciences. Entries in these qualifications have continued to decline, corresponding to increases in both separate science GCSEs, and in other science qualifications, notably BTEC and OCR nationals.

- The pass rate for GCSE biology has remained stable at 99\%, whereas the percentage of entries awarded grades $A^{*}$ or A has risen from $46 \%$ in 2005 to $48 \%$ in 2010. By contrast, just $15 \%$ of GCSE additional science entries attracted these grades in 2010, reflecting the selection of higher ability pupils into the separate sciences.

- The percentage of GCSE chemistry entrants achieving grades $A^{*}$ or A has risen slightly from $48 \%$ in 2005 to $49 \%$ by 2010 . For physics, the percentage of entrants achieving these grades stands at $49 \%$ having risen from this level since 2005, but then dropped back again in 2010.

- By 2010, separate science GCSEs supplied more pupils with grades $A^{*}$ or $A$ in each of biology, chemistry and physics than the number of $A^{*}$ or $A$ grades in core science or additional science, despite separate science entrants being outnumbered two-and-a-half to one by core and additional entrants.

- Achievement in the maths and science subjects has continued to increase despite the widening of access to separate science GCSEs during the last few years.

- National GCSE and A level maths and science participation rates mask considerable variation between Local Authorities. In 2010, the percentage of maintained mainstream pupils who took separate sciences at GCSE ranged from as low as $4 \%$ in one LA up to almost ten times this rate, at 39\%, in another. The range of $A$ level uptake rates across LAs was $1 \%-16 \%$ for maths, $2 \%-21 \%$ for biology, 1\%-13\% for chemistry, and $<1 \%-11 \%$ for physics.

\section{Prior Attainment, Continuation and Achievement}

- Pupils who have achieved at above the expected level within subject prior to Key Stage 5 are more likely to take A levels in maths and science. Comparing between Key Stages, the odds of entering A level maths for pupils with high prior attainment at Key Stage1 are 9 times higher than the odds for pupils who attained at the expected level or below; this increases to 88 times the odds for pupils with GCSE maths at grades $A^{*}$ or A compared with other pupils.

- For biology, the odds of entering A level are 3 times higher for pupils with high prior attainment in science at Key Stage 1, than for pupils with KS1 attainment at the expected level or below; this rises to 23 times the odds for pupils with GCSE biology or double science at grades $A^{*}$ or $A$ compared with other pupils.

- For chemistry A level, the odds of entry are 4 times higher for pupils with KS1 science attainment at the expected level or below; this rises to 40 times the odds for pupils with GCSE chemistry or double science at grades $A^{*}$ or A compared with other pupils. 
- The odds of entering A level physics for pupils with high prior attainment in science at Key Stage 1 are 4 times higher than those for pupils with KS1 attainment at the expected level or below; this rises to 40 times the odds for pupils with GCSE physics or double science at grades $A^{*}$ or A compared with other pupils.

- Only 3\% of A level maths entrants manage to progress from grades of B or lower at GCSE to achieve an A or B at A level. For biology, this is slightly higher at $7 \%$, falling to $6 \%$ for chemistry, and $5 \%$ for physics. This suggests that $\mathrm{B}$ grades at GCSE are not enough to secure a good chance of achieving high A level grades for maths and science subjects.

- Pupils who have achieved at above the expected level within subject prior to Key Stage 5 are also more likely to achieve high grades at A level. The odds of achieving grades $A$ or $B$ in maths for pupils with high prior attainment at Key Stage1 are twice the size of the odds for pupils who attained at the expected level or below; this increases to 11 times the odds for pupils with GCSE maths at grades $A^{*}$ or $A$ compared with other pupils. Similarly, the odds effects for achievement in A level biology, chemistry and physics are in the order of two at Key Stage 1, rising to 10-12 times the odds by GCSE.

- The importance of path dependence for GCSE attainment is stronger for maths than for science, but for both subjects, more pupils fall out of the high attaining groups between Key Stages 1 and 4 than join the high attaining groups by GCSE having not attained at above the expected level consistently through the earlier Key Stages.

\section{Pupil Characteristics and A level Outcomes}

- Female entrants had higher odds than boys of achieving grades A or B at A level in all four subjects. However, a given girl at Key Stage 4 was only more likely to end up with these grades in biology; and less likely to end up with them in maths, physics or chemistry, because she had lower odds of taking on these A levels (but higher odds of entering biology). The gender effects on A level entry were most pronounced for physics.

- Pupils eligible for free school meals were less likely to achieve grades A or B at A level maths and science subjects; this reflected both a shortage of pupils with free school meals entering these A levels, and an achievement gap for those who did. Again, physics was the most affected subject.

- Chinese and Indian pupils have the highest odds of entry and achievement for maths and science A levels, followed by Pakistani, Black African and Bangladeshi pupils, with Black Caribbean pupils having the lowest odds for these outcomes.

- For A level entry effects, all minority groups apart from Black Caribbean have stronger odds of taking A level maths and sciences than White British pupils, except in the case of physics, where only Chinese pupils are clearly ahead of the majority.

- For the smaller effects on achievement of high A level grades, White British pupils fit in between Indian and Pakistani pupils (as the third ranked ethnic group), except in the case of physics, where they fit in after Chinese, but ahead of all other ethnic groups.

\section{Provision: Education Settings and Pathways}

- Subsequent A level maths and science outcomes vary according to which type of 
Key Stage 4 school the pupil attended. Pupils from grammar schools and independent schools have the highest rates of continuation to $A$ levels in these subjects; they also have the highest achievement of A level grades A or B.

- However, maths and computing / science specialist schools, along with grammars, have the highest rates of continuation for those pupils with GCSE grades $A^{*}$ or $A$ within subject. Other maintained mainstream schools contribute the largest overall share of maths and science A level entrants despite having lower continuation rates.

- Taking separate science GCSEs increases the chances of taking A level science qualifications compared with core and additional science GCSEs (as was historically the case with double science). The association between separate sciences and A level entry was strongest for physics, at almost 3 times the odds of pupils who took additional science.

- A level entry and achievement rates for the different GCSE science routes are partly driven by selection of higher attaining pupils into the separate science route; however, the separate science advantage persists within the subset of pupils who achieved grades $A^{*}$ or A at GCSE, with odds of achieving grades A or $B$ at A level 2-3 times higher for separate science takers. The link with A level achievement was strongest for biology.

- Nevertheless, pupils who took combined science GCSEs (whether double science previously or additional science for the latest A level outturn) still accounted for the majority of $A$ level science entries, and a smaller majority of grades A or B.

- But the shift away from combined sciences and towards separate sciences at GCSE continues. Across all schools (including independents) in England, the offer of separate sciences at GCSE has expanded from fewer than half of schools in 2008 , to $52 \%$ in 2009 and $70 \%$ by 2010 .

- Amongst Key Stage 5 institution types, independent and grammar schools again had the highest rates of achieving grades A or B in A level maths and sciences; in third position were science specialist schools for maths and physics, and sixth form colleges for biology and chemistry. FE colleges had the lowest achievement of the institutions offering A levels. The variation between school types was largest for chemistry.

- Maintained mainstream schools still make up the largest share of A levels in maths and science at between $32 \%$ and $36 \%$ of the total. Independent schools account for the next largest share at between $14 \%$ and $17 \%$. Sixth form colleges and grammar schools follow next, each contributing 13\%-17\% of the total entries. Specialist schools with either maths and computing or science specialisms make up the final $9 \%-10 \%$ of entries.

\section{Choice and Continuation}

- Increases in maths, biology and chemistry A level entries since 1996 have been below the overall level of growth for all A levels, and physics has seen no growth over the period. The fastest growing subjects since 1996 (religious studies, media or communication studies, and physical education), have grown at twice the rate or more compared with maths and the sciences. However, looking at a shorter timescale, since 2002, places maths and further maths amongst the fastest growing in more recent years.

- The growth in A level entries has mainly taken place amongst candidates taking three subjects at A level, with the numbers of entrants taking 1-2 or more than 3 
subjects remaining fairly stable since 2003. Entrants taking 3 subjects have slightly higher than average entry rates for maths and biology, but the same as the average for chemistry, and lower than average for physics (where almost half of entrants take 4 or more A levels).

- Of the 75 thousand A level entrants who entered at least one science subject, $57 \%$ (42 thousand) took one science; 38\% (29 thousand) entered two sciences, and just $5 \%$ took three sciences. The proportion of these groups who also took $\mathrm{A}$ level maths in combination with sciences, was $48 \%$ of the one science group, $59 \%$ of the two sciences group, and $48 \%$ of the three sciences group.

- The subjects that were most commonly combined with maths and/or science at A level were general studies, further maths, history and geography.

- Controlling for prior attainment, entrants in A level maths were less likely to achieve grades A or B than entrants in English, but they were more likely to gain these grades than entrants in history or modern foreign languages. Physics and biology entrants had even smaller chances of achieving grades A or B than any of these subjects, with chemistry appearing in the middle of this estimated scale of subject difficulty.

- Pupils with a relative strength in the subject (achieving a higher grade in it than their own average) outnumber those with a relative weakness for maths GCSE, and separate biology, chemistry and physics GCSEs. This is also the case for English, but the opposite is true for history and modern foreign languages. There is some coincidence between a subject's distribution being skewed towards relative strength at GCSE, and increases in entries in that subject at A level, but the pattern is imperfect.

\section{Modelling Maths and Science Uptake and Attainment}

- The influence on A level entry of achieving a grade A at GCSE is largest for maths, followed by chemistry, then physics, then biology; the odds effects range from 400 down to 50 times the odds of a pupil with grade $C$ or below, from amongst the Key Stage 4 cohort.

- For achievement (grade A or B) at A level, the link with GCSE prior attainment at grade $A$ is also strongest for maths, followed by biology, then chemistry, then physics; the odds effects range from 200 down to 60 times the odds of a pupil with grade $\mathrm{C}$ or below, from amongst the $\mathrm{A}$ level cohort.

- In addition to prior attainment at GCSE, pupil level factors that are statistically significantly associated with both A level entry and achievement of grades A or B for maths, biology, chemistry and physics are Key Stage 3 prior attainment (Level $7+$ ), relative strength in the subject at GCSE, taking separate science GCSEs (for the three science A levels), pupil gender and ethnicity.

- There were some subject-specific differences in A level outcomes for pupils with special educational needs and those eligible for free school meals. At school level, the type of maintained Key Stage 4 school attended did not have any sizeable impact on subsequent uptake and achievement after prior attainment was controlled for; however attending a school with a sixth form did predict greater A level continuation generally.

- Smaller but significant increases in uptake and achievement for maths and science A levels exist at Key Stage 5 when an individual has taken a larger number of A level subjects, when the school or institution offers fewer subject options, and when the school or institution has a larger cohort taking that particular subject. 
- The most important wider factors in A level maths and science uptake and achievement, measured by survey data, are enjoyment of the subject, belief in one's ability at the subject, working hard at school and having an internal locus of control. Subject specific effects existed for family factors such as attendance at school parent's evenings, eating together as a family, living with married or cohabiting parents, and receiving private tuition.

- Highest parental qualification, parental employment status, household income, pupil aspirations to continue in full-time education, and the perceived reputation of the Key Stage 4 school attended were amongst factors which were not found to be important in predicting uptake and achievement in A level maths and science subjects. 


\section{Contents}

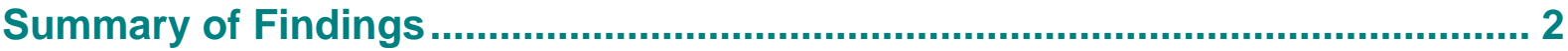

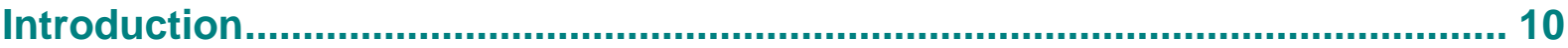

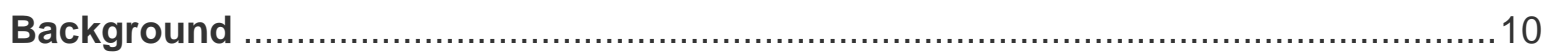

Aims

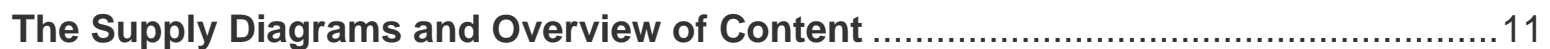

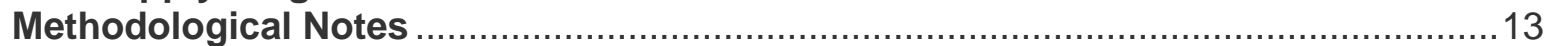

Chapter 1. Headline Maths \& Science Trends................................................ 14

1.1 Number of A Level Entries, Passes, and Grades A / B .................................... 14

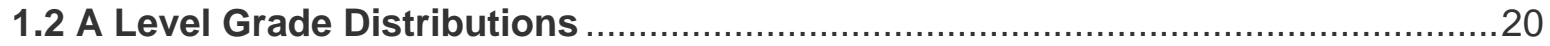

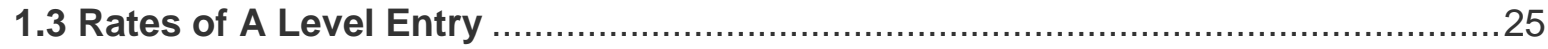

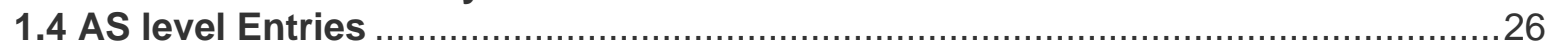

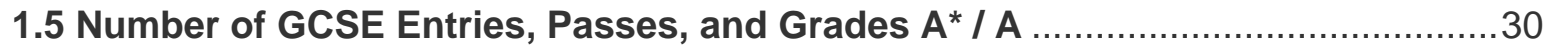

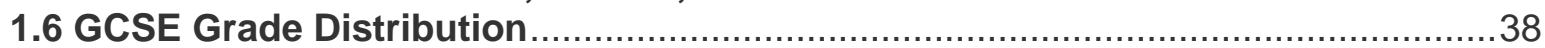

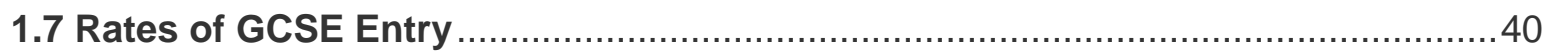

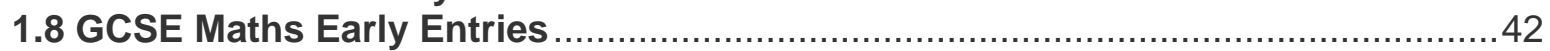

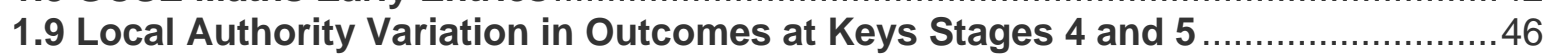

Chapter 2. Prior Attainment, Continuation \& Progression................................... 49

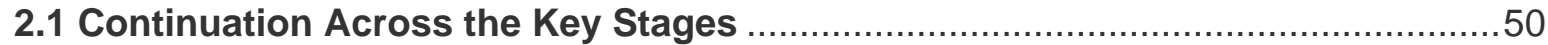

2.2 GCSE Attainment and Progression to A level ................................................. 60

2.3 Key Stage 3 Attainment and A level Progression ............................................ 72

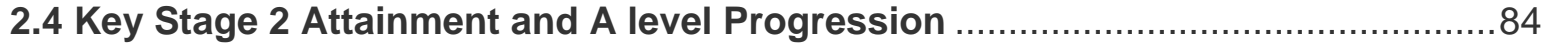

2.5 Key Stage 1 Attainment and A level Progression ..........................................96

2.6 A level Progression Comparisons Between Subjects and Key Stages ..............109

2.7 Path Dependence: Pulling Ahead and Slipping Back in Maths and Science .....110

Chapter 3. Pupil Characteristics and A Level Outcomes ................................. 114

3.1 Gender and A level Maths and Sciences ....................................................... 114

3.2 Free School Meals and A level Maths and Sciences.....................................123

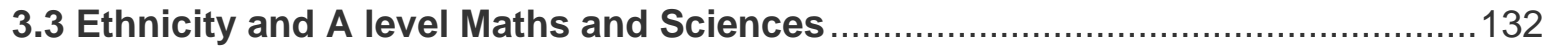

Chapter 4. Provision: Education Settings \& Pathways .................................. 143

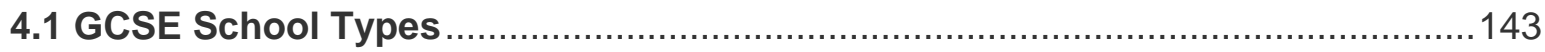

4.2 GCSE Qualification Routes: The Separate Sciences Advantage .......................155

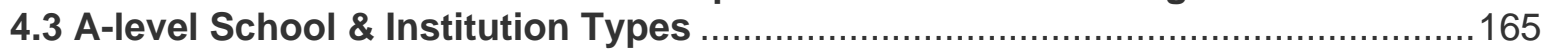

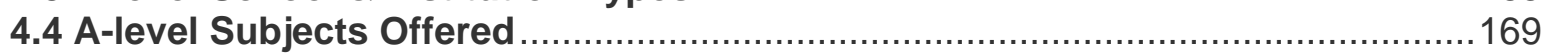

Chapter 5: Choice \& Continuation ................................................................. 170

5.1 Competing for Entrants - Relative Subject Popularity Trends .........................170

5.2 Trend in the Number of Entries per Pupil .........................................................173

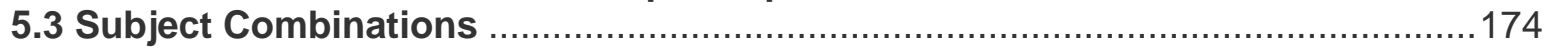

5.4 How Hard is it to Achieve a Top Grade in Maths and Science? ........................177

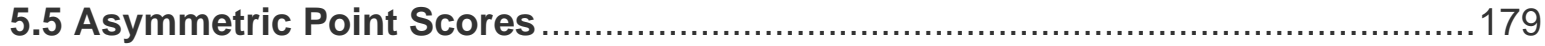


Chapter 6. Modelling Maths \& Science Uptake \& Attainment

6.1 Introduction to the Models

6.2 A level Entry for KS4 MM Pupils

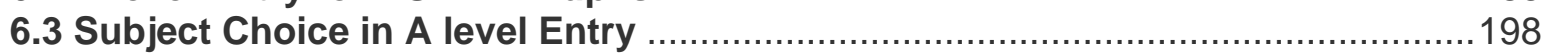

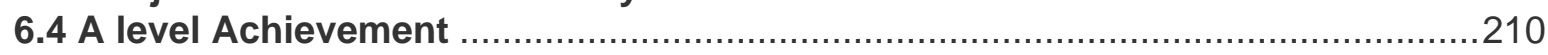

6.5 Comparing and Summarising the Model Findings ..................................... 221

Annex A: Numbers of Pupils Progressing to A level

Annex B: School Type Pathways and A level Achievement

Annex C: Model Effects Translated to Probabilities for Maths, Biology \& Chemistry 243

Annex D: Local Authority Maths Science Indicators 246 


\section{Introduction}

\section{Background}

In 2002, DfE $^{1}$ began collecting individual pupil-level data on the characteristics of pupils in maintained schools through the Schools Census. This information has been linked to attainment data from Key Stage tests to create the National Pupil Database, which has become a resource enabling detailed longitudinal analysis of pupil, school and national performance and the factors which are associated with it. The 2009 Key Stage 5 results report on the first cohort for which DfE holds complete Key Stage records for KS1 - KS5, enabling analysis of progress from age 7 through to A level.

An area of considerable policy interest at Key Stage 5 has been the supply of young people gaining good $\mathrm{A}$ level grades in maths and science subjects, such that they would be eligible to continue on to university to study these subjects and subsequently to take up careers in these subject fields. The Royal Society (2008) characterise these policy concerns as viewing science education as "... a means to increase the number of scientists in order, first and foremost, to grow the amount of research, development and innovation in the UK. This is presented as an almost direct path to maintaining and improving a high-value economy and international competitiveness (DfES 2006a; HMTreasury 2006; DIUS 2007; Sainsbury 2007)."

\section{Aims}

The principle aim of this paper is to present a comprehensive set of statistical analyses building up a picture of the production function for the supply of young people in England with good A levels in maths and science. In this way, the questions addressed are focused towards high attainers in these subjects, with the desired output being grades $A$ and $B$ at $A$ level. The focus is on traditional A level qualifications in maths, biological sciences (hereafter referred to as biology), chemistry and physics, which make up the majority of maths and science Level 3 qualifications. This paper is not intended to provide figures which will be regularly updated. It aims to provide in-depth insight into the statistical evidence available at this point in time.

Key questions considered in the following chapters include: How have participation and attainment in maths and science $A$ levels changed over time? Who takes $A$ levels in maths and science? Who achieves grades A or B at these subjects? What are the prior attainment pre-requisites for reaching this stage in maths or science education and succeeding in gaining good grades? How do aspects of provision of maths and science education during compulsory schooling influence Key Stage 5 outcomes? What factors in individual choices to study maths and science at KS5 can be identified? And finally, how does the range of factors examined combine in influencing participation and attainment for $A$ level maths and science?

The approach of the paper is to present a diagrammatic summary of the evidence on participation and attainment in A level maths and science that can be drawn from DfE's data sources, and to examine systematically the various components in the proposed production function through detailed analyses, culminating in logistic

\footnotetext{
${ }^{1}$ DfE = Department for Education, which was then the Department for Education and Skills (DfES).
} 
regression modelling of the key A level outcomes in order to draw together the relationships described throughout the paper.

All analyses in this paper are limited to data for England only. The scope varies according to the type of question at hand; the majority of the analysis is limited to pupils who attended maintained mainstream schools, for whom prior attainment information at Key Stages 1-4 is available; this limitation also applies to all analysis including pupil characteristics data. Exceptions to this scope are made where only KS5 data are required for the analysis, and entries for maintained schools, independent schools and FE sector institutions for all pupils aged 16-18 are included. These selected "complete KS5" sections appear in chapter 1 (looking at trends in participation and attainment at KS5), Chapter 4 (covering aspects of KS5 provision), and chapter 5 (comparisons of KS5 subject options).

\section{The Supply Diagrams and Overview of Content}

The following diagrams summarise the areas of evidence on participation and attainment in A level maths and science that can be drawn from DfE's data sources. Diagram D1 presents the components of the supply of young people achieving grades A or B in A level maths that will be examined in the following chapters. Central to the supply is the chain of progression at above expected levels at each Key Stage, driven by each previous level of prior attainment, school standards, and individual pupil characteristics. Additional factors related to pupil choice are introduced at Key Stage 5, where participation becomes optional.

\section{Diagram D1: Supply of Maths A levels at Grades A and B}

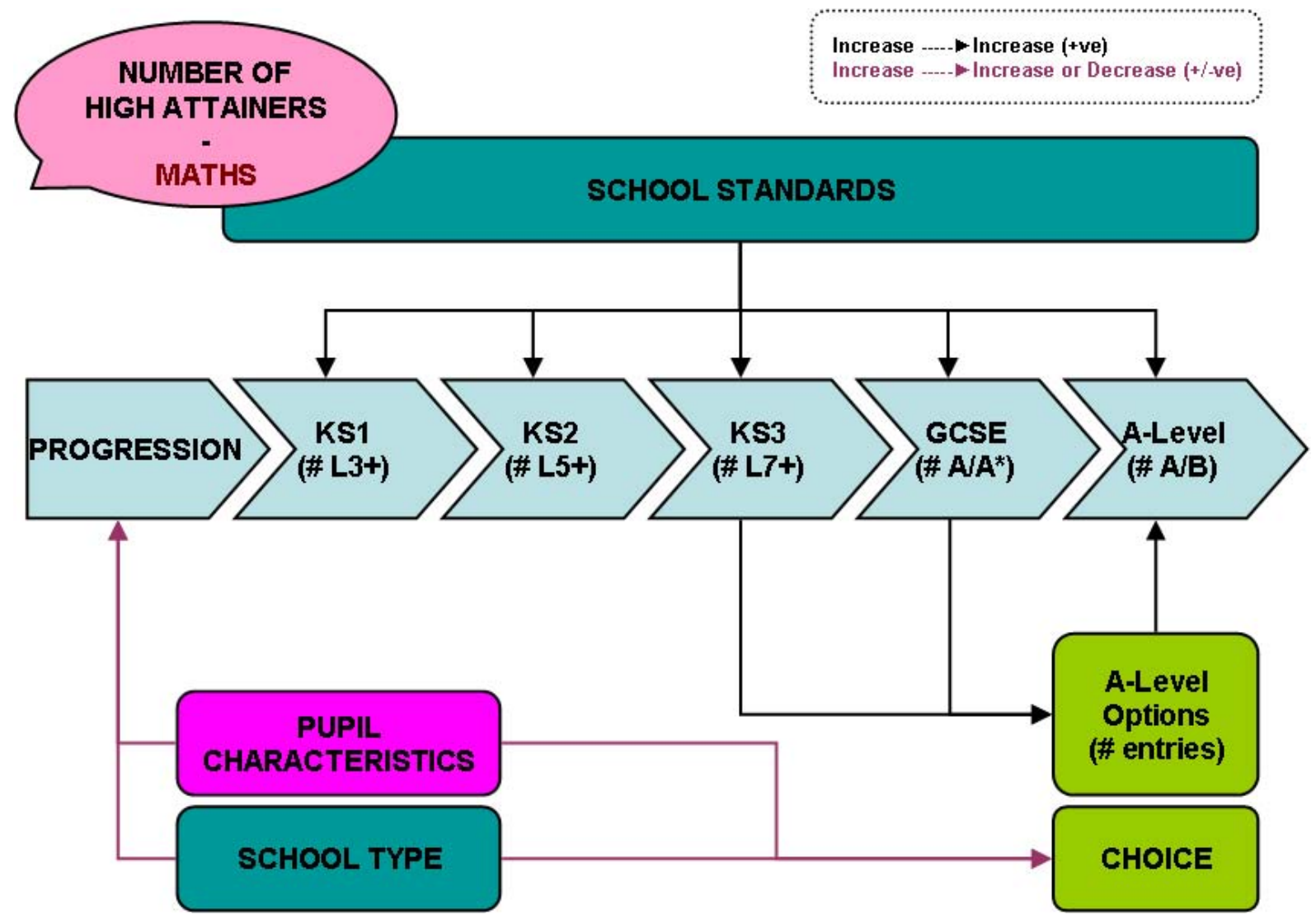


Diagram D2 presents the equivalent components of the supply of young people achieving grades A or B in the A level traditional sciences, biology, chemistry and physics. The main differences pertain to factors related to choice, which begins to exert an earlier influence than for maths, with different GCSE science routes associated with differing rates of $A$ level continuation. Although science in some form is compulsory at KS4, the distinction between double science GCSE awards and the separate science GCSE route has historically been linked to differential outcomes at Key Stage 5; from 2010, the KS5 cohorts have fed through from GCSE cohorts eligible for Core and Additional science GCSEs, as the current alternative route to separate sciences.

\section{Diagram D2: Supply of Science A levels at Grades A and B}

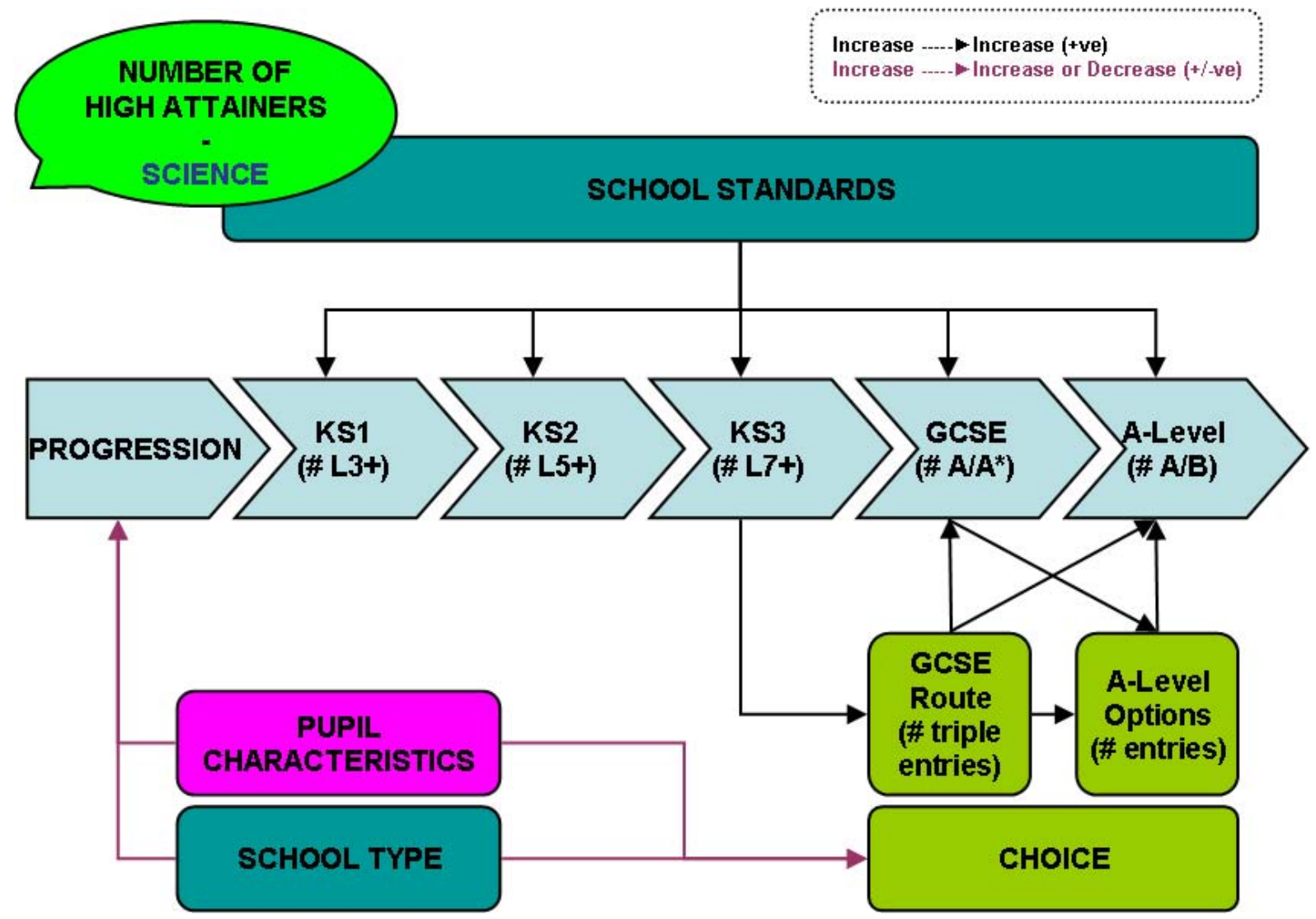

Chapter 1 begins by introducing the trends over time in A level maths and science entries and grade distributions. Chapter $\mathbf{2}$ then examines the progression chain back through the Key Stages tracking the influence of prior subject attainment on $\mathrm{A}$ level continuation and achievement, stretching back to Key Stage 1. Chapter 3 focuses on the key pupil characteristics of gender, deprivation (as measured by eligibility for Free School Meals) and ethnicity, and how maths and science outcomes vary between different groups of pupils. Chapter 4 considers school provision and how the types of school and institution attended and the GCSE science route taken influence A level participation and achievement. Chapter $\mathbf{5}$ reviews what can be learned about A level option choices, situating maths and the sciences as alternatives within the full range of available A level subjects. Finally, chapter $\mathbf{6}$ 
draws together factors from across chapters 2-5 into a suite of logistic regression models to answer the questions: "who takes A level maths [/science]?", and "who achieves grades A or B at A level maths [/science]?".

\section{Methodological Notes}

\section{Data}

Provisional data for A levels taken in 2010 became available at a late stage in the production of this paper; 2010 results have been added to the sections covering headline trends (Chapter 1), and to section 4.2 on GCSE science routes. The remainder of the analysis in the paper takes 2009 as the latest year covered.

Continuation I A level Entry Throughout the paper these terms are used to describe entry to full $A$ level in a given subject, where the candidate has received a result of grade A-G or Unclassified. Students who have begun a course of study at Key Stage 5, e.g. studying for one year and entering for an AS level award, but not entering to convert this to A level are not included in the counts of $A$ level entries / continuation used in the analysis.

High Prior Attainers For the purpose of analysing progression between Key Stages, the following categories have been deemed to be high attaining groups: grades $A$ star and A at GCSE / KS4; level 7 and above at KS3 (where the expected level is 6); level 5 and above at KS2 (where the expected level is 4); and level 3 and above at KS1 (where the expected level is 2). For Key Stages 1-3, historical results are available for maths and science. At KS4 a variety of science routes are possible depending on the cohort in question; grades in double certification science are treated as equivalent to the same letter grades for separate sciences. For example, in some analyses, GCSE high prior attainers at GCSE will be a mixture of those gained $A^{*}$ or $A$ grade at biology, chemistry or physics and those who gained ${ }^{* \star}$ or $A A$ grades at double science. Separate analysis of the different GCSE science routes appears in section 4.2

Charting Conventions For ease of reference, throughout the paper, pink designates maths and green designates science subjects; these colours are also used to distinguish the most recent year's data. Darker or brighter tones (as opposed to paler ones) designate high attainment, attainment as opposed to participation, or the most recent year's data. Blue tones are used throughout in both maths and science charts to provide extra categories as needed. 


\section{Chapter 1. Headline Maths \& Science Trends}

This chapter reviews changes over time in the numbers of entries, passes and top grades at A level, AS level and GCSE, in absolute numbers and rates of incidence. Passes and individual grades are expressed as a percentage of entrants in sections 1.1 and 1.2; entries and grades A or B are then presented as a percentage of the estimated age 17 population in section 1.3. Section 1.4 compares AS level entry numbers with the number of full A level entries in the following year, hinting at what may happen to A level numbers in 2011. Moving down the age range to GCSEs, passes and individual grades are reported as percentages of GCSE entrants in sections 1.5 and 1.6. GCSE science and maths entries, and grades $A^{*}$ or $A$ in these subjects, are presented as population rates in section 1.7. Section 1.8 then examines early GCSE maths entries, and subsequent retakes by early entrants. Concluding the chapter, section 1.9 briefly considers variation between Local Authorities in maths and science outcomes.

Coverage Analyses in this chapter include all A level entrants aged 16-18, including those at independent schools, FE sector institutions, and special schools.

\subsection{Number of A Level Entries, Passes, and Grades A / B}

Figure 1.1a depicts the trend in A level maths entries, and in the rate of achievement by entrants, from 1996 to 2010 (provisional data). Entries were relatively stable from 1996 to 2001, at between 54 and 57 thousand 16-18 year olds per year. There is then a dramatic decrease, with 10 thousand fewer entrants in 2002, which coincided with the introduction of curriculum changes in all subjects, meaning that all entrants entered AS level qualifications during their first year of Key Stage 5, and decided whether to continue and convert to A level in the following year after receiving their AS level results. The pass rate for AS level maths fell in 2001 under this new curriculum, feeding through to lower numbers continuing into their second (A2) year in maths and entering for a full A level.

It is believed that the new curriculum acted as a filter for lower achieving A level candidates, resulting in the observed fall in entries and simultaneous rise in the pass rate, and in the percentage of entrants achieving grades A or $\mathrm{B}$. The proportion of entrants gaining grade A or B rose by 10 percentage points from 2001 to 2002, from stable levels of $45-46 \%$ to $56 \%$; the rate then rose steadily thereafter reaching $67 \%$ in 2009 and 2010, despite the gradual but accelerating recovery of entry numbers widening the field of entrants again. The pass rate (grades A-E) also increased in 2002 , from stable previous levels of $88-89 \%$, to $94 \%$ in 2002 , rising further to $98 \%$ by 2010. 
The dark blue bars in figure 1.1a represent the numbers of grades $A$ and $B$ achieved each year (including the new $A^{*}$ grades in 2010), and demonstrate that the drop in entries in 2002 came from the lower achieving end of the distribution, as there was no fall in the total numbers of As and Bs corresponding to the slump in entries. A steady recovery in the number of maths entries is apparent, reaching pre-2002 levels again by 2008, and exceeding these levels (at almost 70 thousand) by 2010 .

\section{Fig. 1.1a}

Mathematics A-level Entries and Passes Trends for 1996-2010

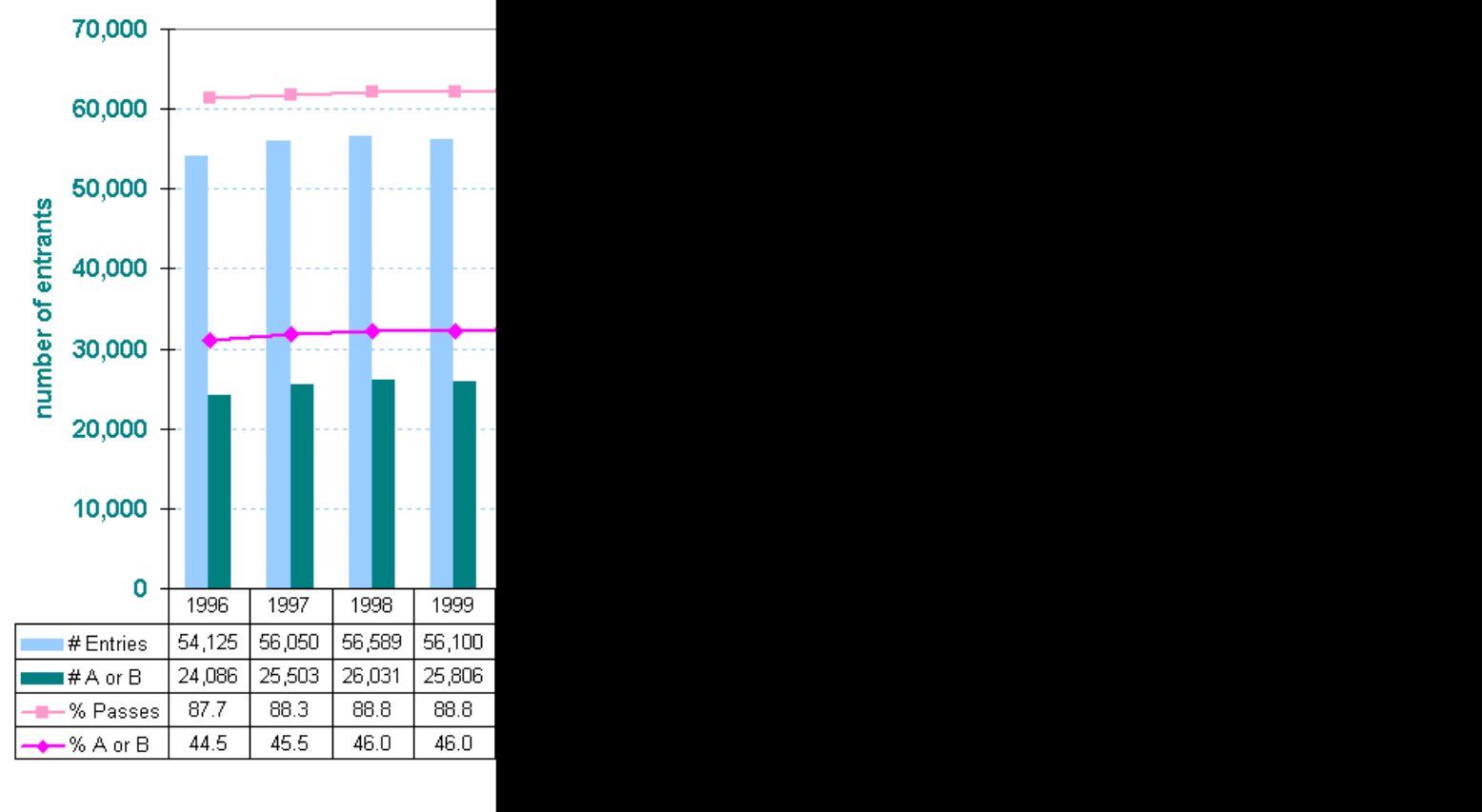


Turning to A level biology, figure $1.1 \mathrm{~b}$ shows a steady increase in the pass rate and percentage of entrants achieving grades A or B throughout the period of 1996-2010. The proportion of entrants passing (grades A-E) rose from 85\% to $98 \%$; that for grades A or B increased from $33 \%$ to $51 \%$ (including $A^{*}$ s) in 2010 . Entries in biology declined gradually from 49 thousand per year to 44 thousand between 1998 and 2004, but did not suffer any sharp decrease (as with maths entries) following the curriculum changes in 2002. Biology entries recovered to 53 thousand by 2010, exceeding their previous 1998 peak for the first year since. Numbers of entrants achieving grades $A$ or $B$ in biology have been increasing from 17 thousand in each of the years 1998 to 2001, up to 27 thousand by 2010 .

\section{Fig. 1.1b}

Biological Sciences A-level Entries and Passes Trends for 1996-2010

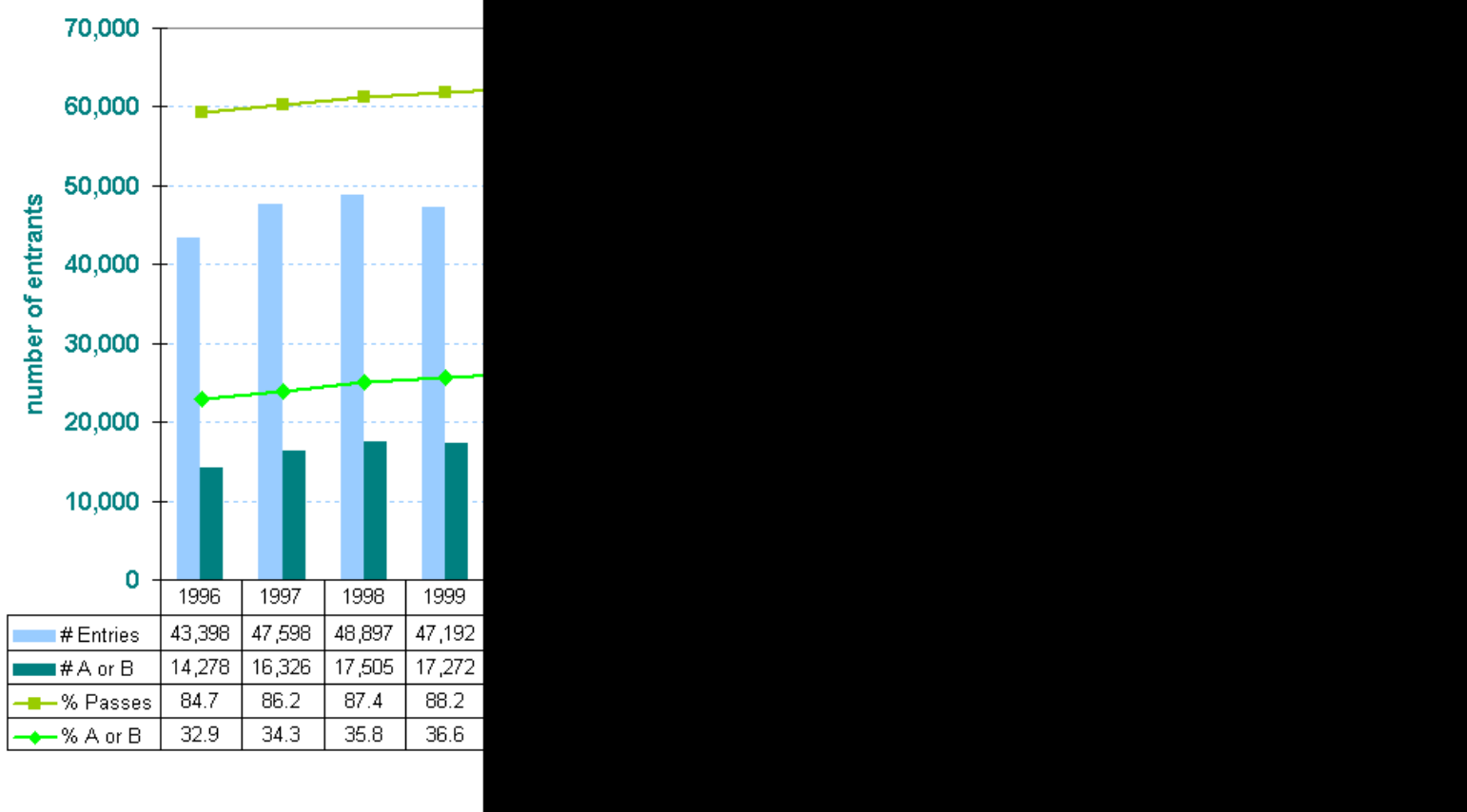


A level chemistry entry and pass trends for 1996 to 2010 are depicted in figure 1.1c. As for biology, the pass rate and percentage of entrants gaining grades $A$ or $B$ have increased steadily over the period, from $86 \%$ to $98 \%$ for grade A-E passes. The A-B rate began from a higher base than biology, but lower than that of maths, rising from $41 \%$ in 1998 to 59\% in each of 2008-2010. Chemistry A level entries declined between 1998 (37 thousand) and 2003 (31 thousand), but then recovered more gradually regaining their 1998 level by 2009, and reaching a new peak of 40 thousand in 2010. As with biology, numbers of grades A or B in chemistry were steady at 17 thousand from 1998 to 2003, with no decline in 2002, then began to grow reaching 24 thousand by 2010.

\section{Fig. 1.1c}

\section{Chemistry A-level Entries and Passes Trends for 1996-2010}

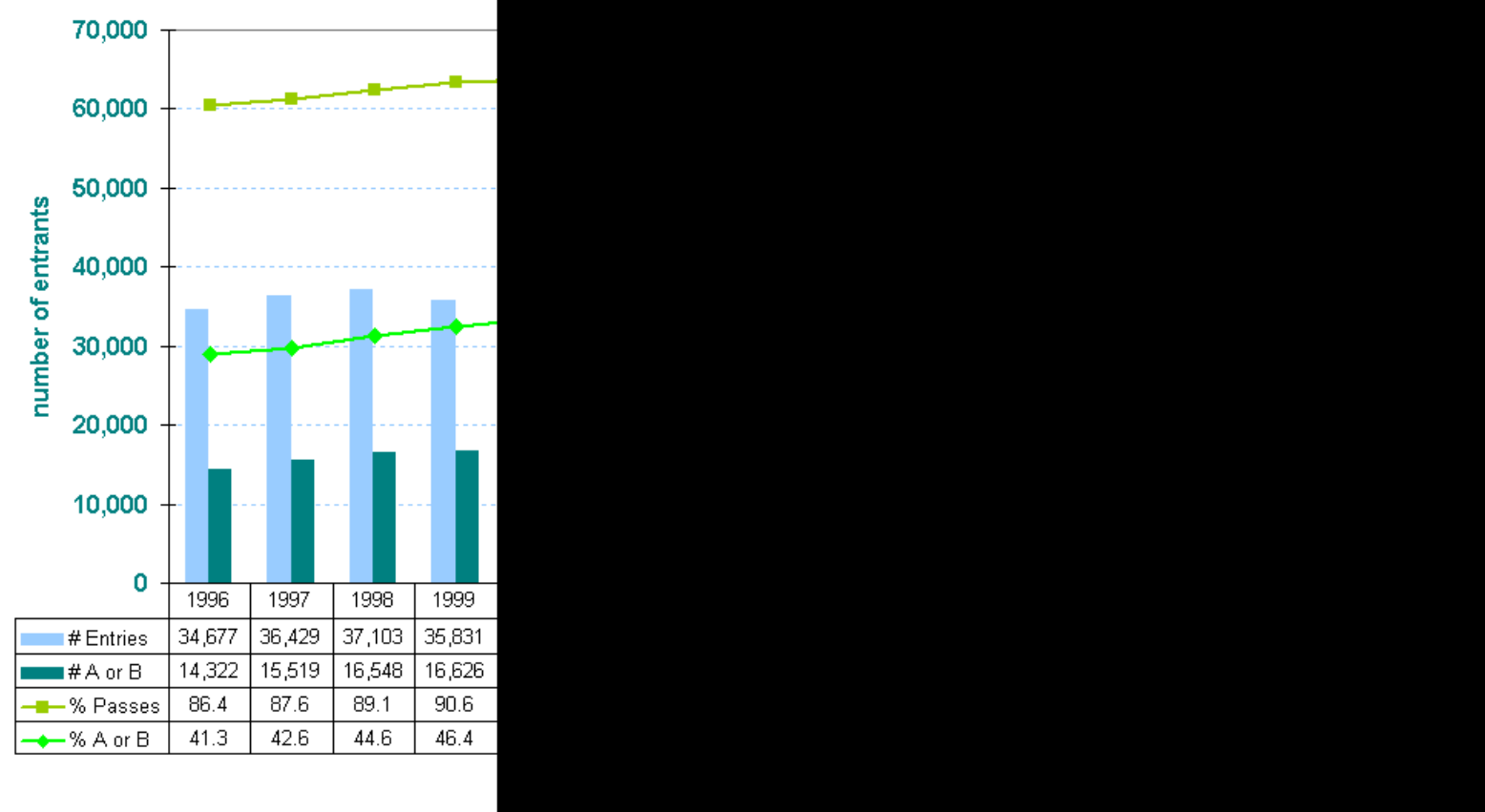


Turning to physics, figure 1.1d shows a longer decline in A level entries than for biology or chemistry, falling from 30 thousand in 1998 to 24 thousand in 2005. A recovery in entries is underway, reaching 28 thousand by 2010 , but with physics entries remaining the weakest of the three traditional sciences. There is no clear trend in the number of entrants achieving grades A or B in physics until around 2007, when the numbers began to climb gradually from their previous level of between 11 and 13 thousand per year, reaching 15 thousand in 2010. The decrease in physics entries combined with a modest increase in the numbers of $A$ and $B$ grades results in rising rates of achievement. The percentage of entrants passing $A$ level physics has risen from $86 \%$ in 1996 to $97 \%$ in 2010; the percentage gaining grades A or B has increased from $40 \%$ to $55 \%$ over the same period.

\section{Fig. 1.1d}

\section{Physics A-level Entries and Passes Trends for 1996-2010}

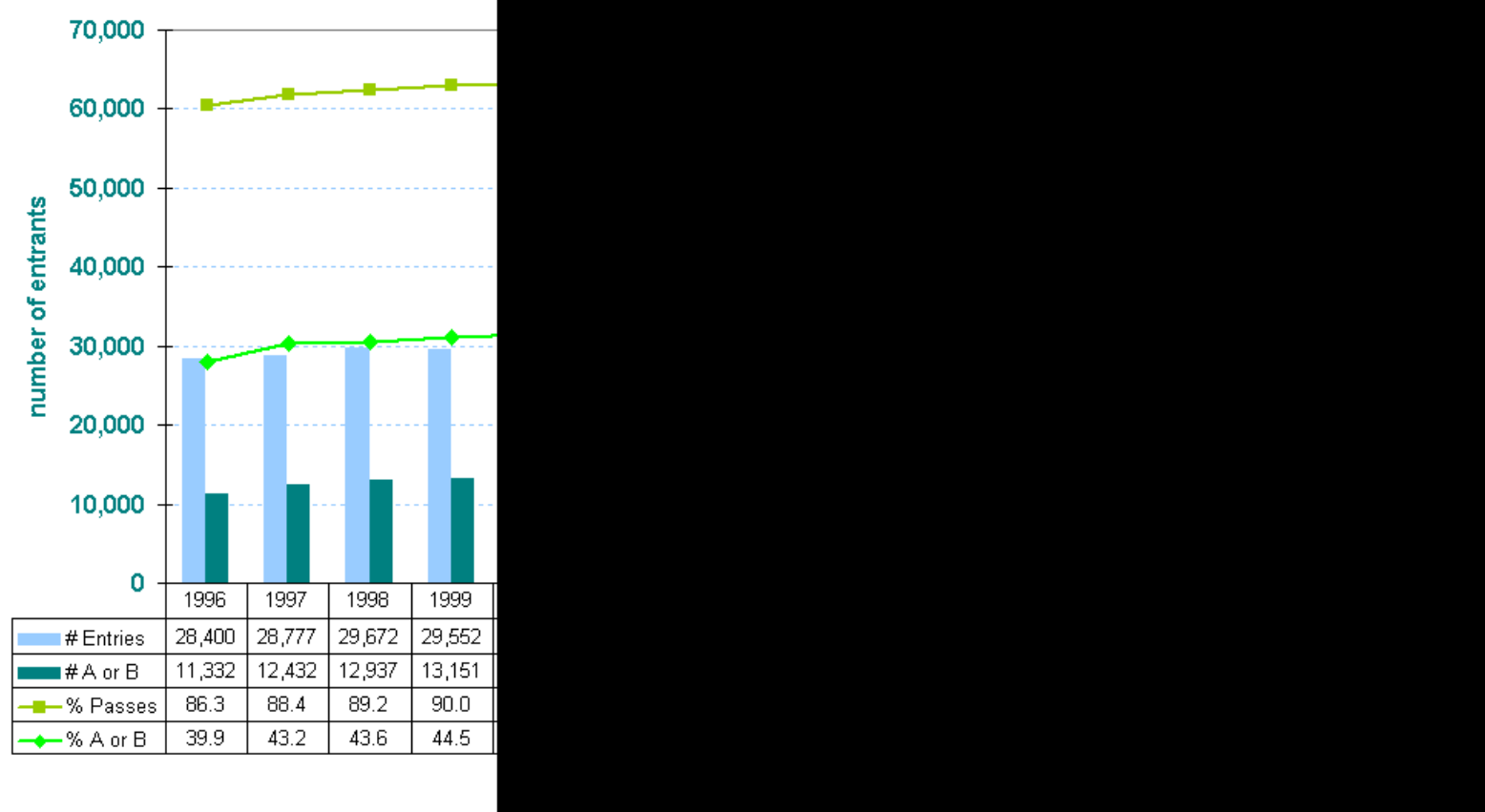


Figure 1.1e shows the number of other science A level entries stable at approximately 4 thousand per year from 1996 to 2010 . As with the traditional sciences, the number and rates of achieving grades $A$ or $B$ have increased over the period, from around 1,000 or $25 \%$ to 2,100 or $48 \%$. The pass rate has also increased from $83 \%$ in 1996 to $97 \%$ in 2010 . The "other science" group of A level subjects includes single award science, electronics, environmental science, geology, psychology and science for public understanding qualifications. This group of $A$ levels will not be subject to further analysis in subsequent chapters due to the small numbers of entries per year, which contributed only $3 \%$ of the science A levels awarded to 16-18 year olds in 2010 .

\section{Fig 1.1e}

\section{Other Science A-level Entries and Passes Trends for 1996-2010}

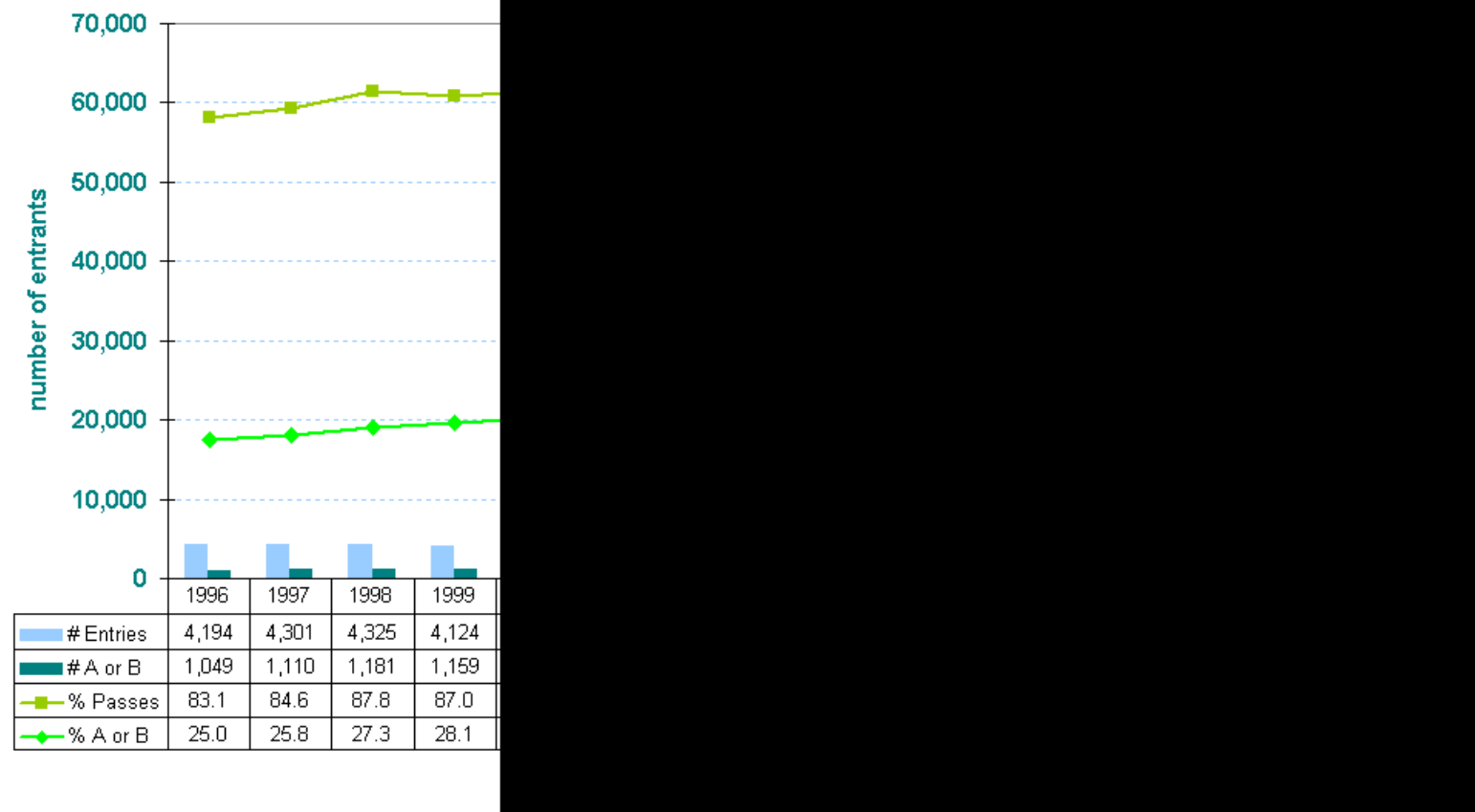


Figure $1.1 \mathrm{f}$ summarises the entries and passes for the three traditional sciences and maths at A level. Maths was the only one of the four subjects to be significantly affected by the curriculum changes in 2002, although slightly above average increases in the pass rates also took place for the three sciences, but without any corresponding loss of $A$ level entries. Maths has also seen the steepest increases in entries and achievement of grades A or B since 2002, with chemistry entries climbing fastest of the three science subjects, all of which had been declining between 1998 and 2002. In contrast, physics entry numbers have been slow to recover and are still below their 1998 level. The proportions of entrants gaining grades A or B have increased for all four subjects, with the rate of such successes in maths boosted significantly by the drop in lower achieving entrants in 2002, in addition to the longer term upward trend.

\section{Fig. 1.1f}

Mathematics \& Science A-level Entries and Passes Trends for 1996-2010

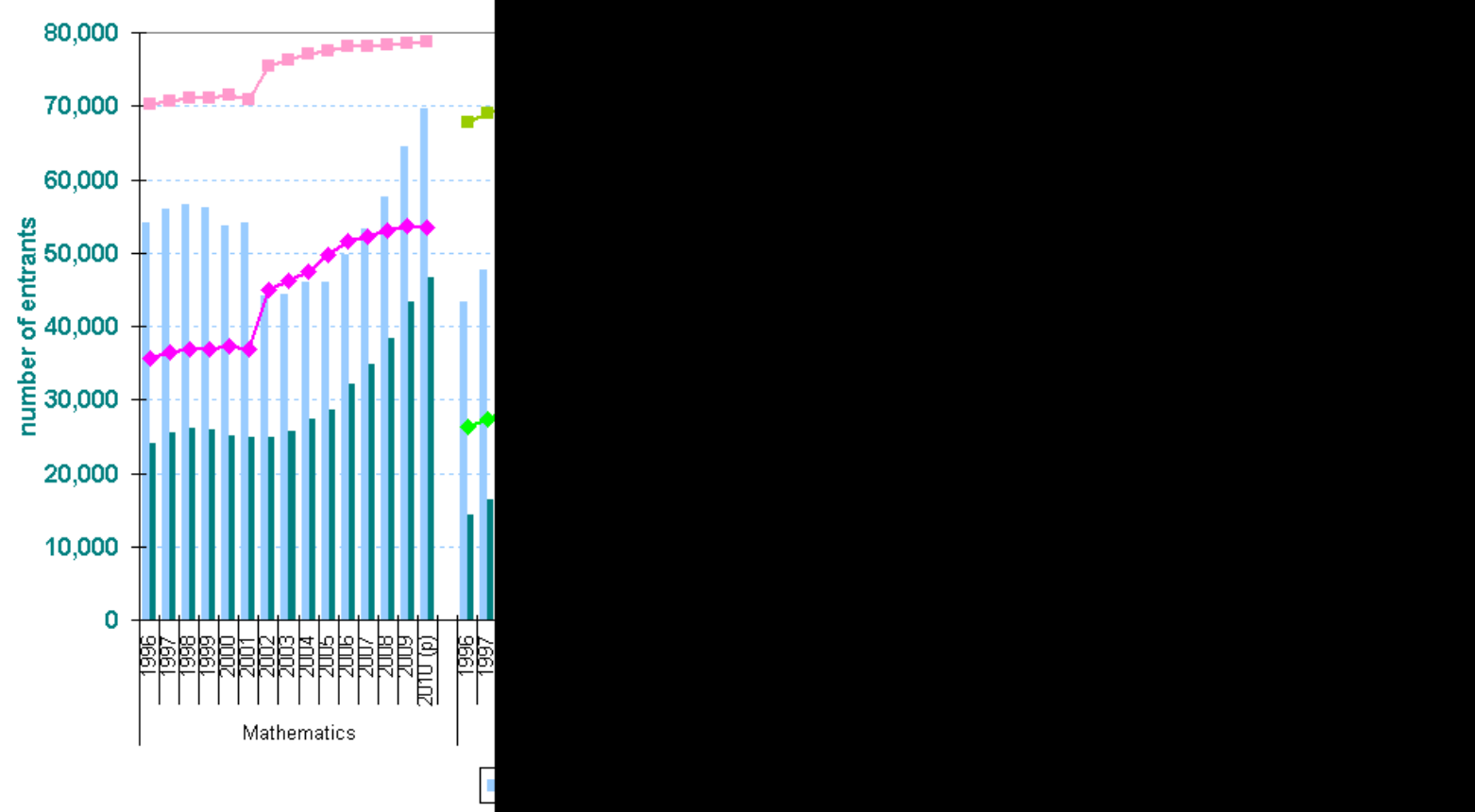

\subsection{A Level Grade Distributions ${ }^{2}$}

The following charts in figures $1.2 \mathrm{a}-1.2 \mathrm{e}$ present the grade distributions at A level for maths, biology, chemistry, physics, and other science subjects. Figure $1.2 f$ then shows the main subjects side-by-side for ease of comparison. The average grades achieved for all maths and science A levels have risen over the period from 1996 to 2010. The main increases have been in the proportions of $A$ grades, and to a lesser extent, B grades. The proportions of C-D grades have remained fairly stable, with Ungraded results and, to a lesser extent, grade Es shrinking as a proportion of entries.

\footnotetext{
${ }^{2}$ Analysis in this section includes entrants aged 16-18.
} 
Fig. 1.2a

Mathematics A-level Grade Distribution Trend for 1996-2010

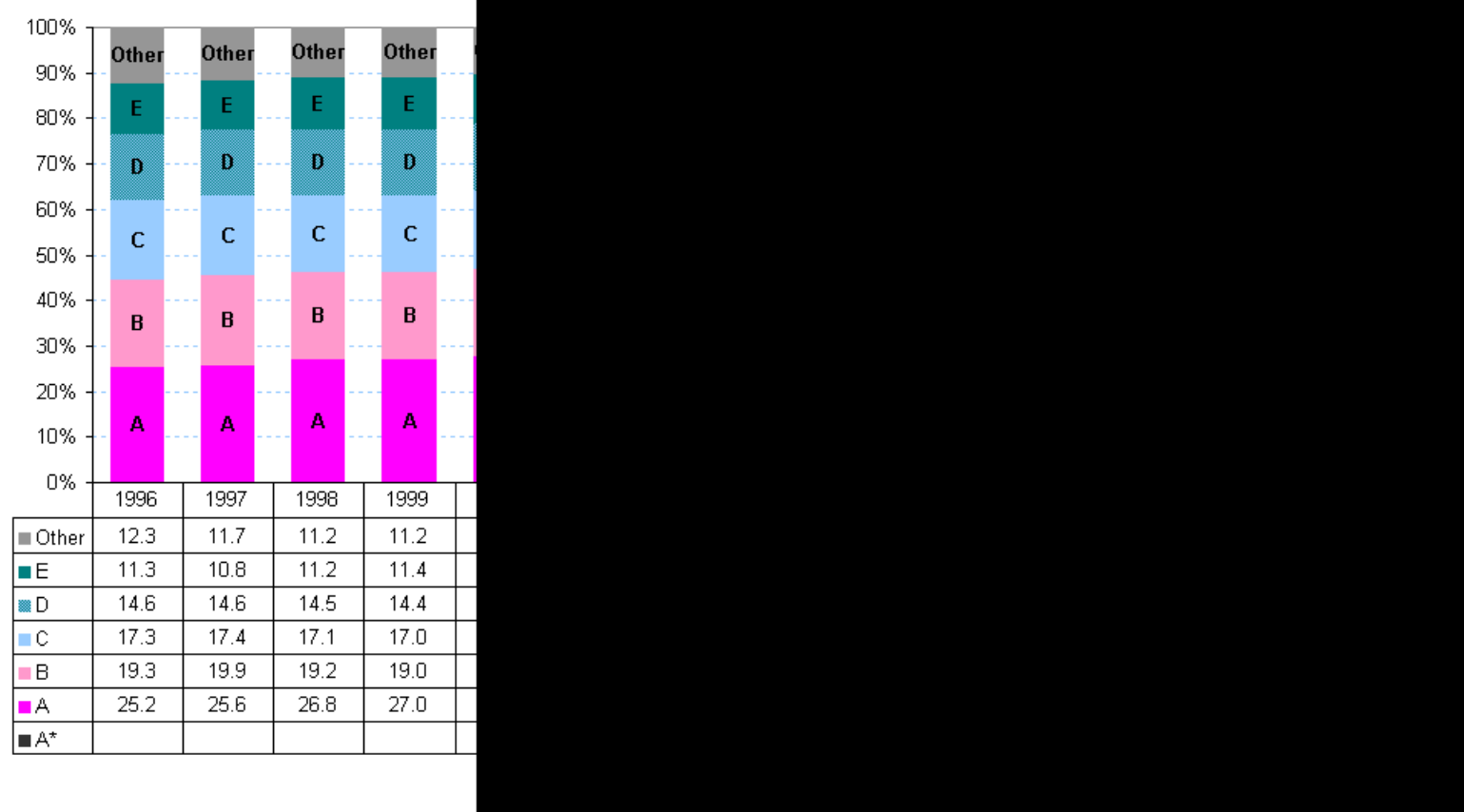

Fig. 1.2b

Biological Sciences A-level Grade Distribution Trend for 1996-2010

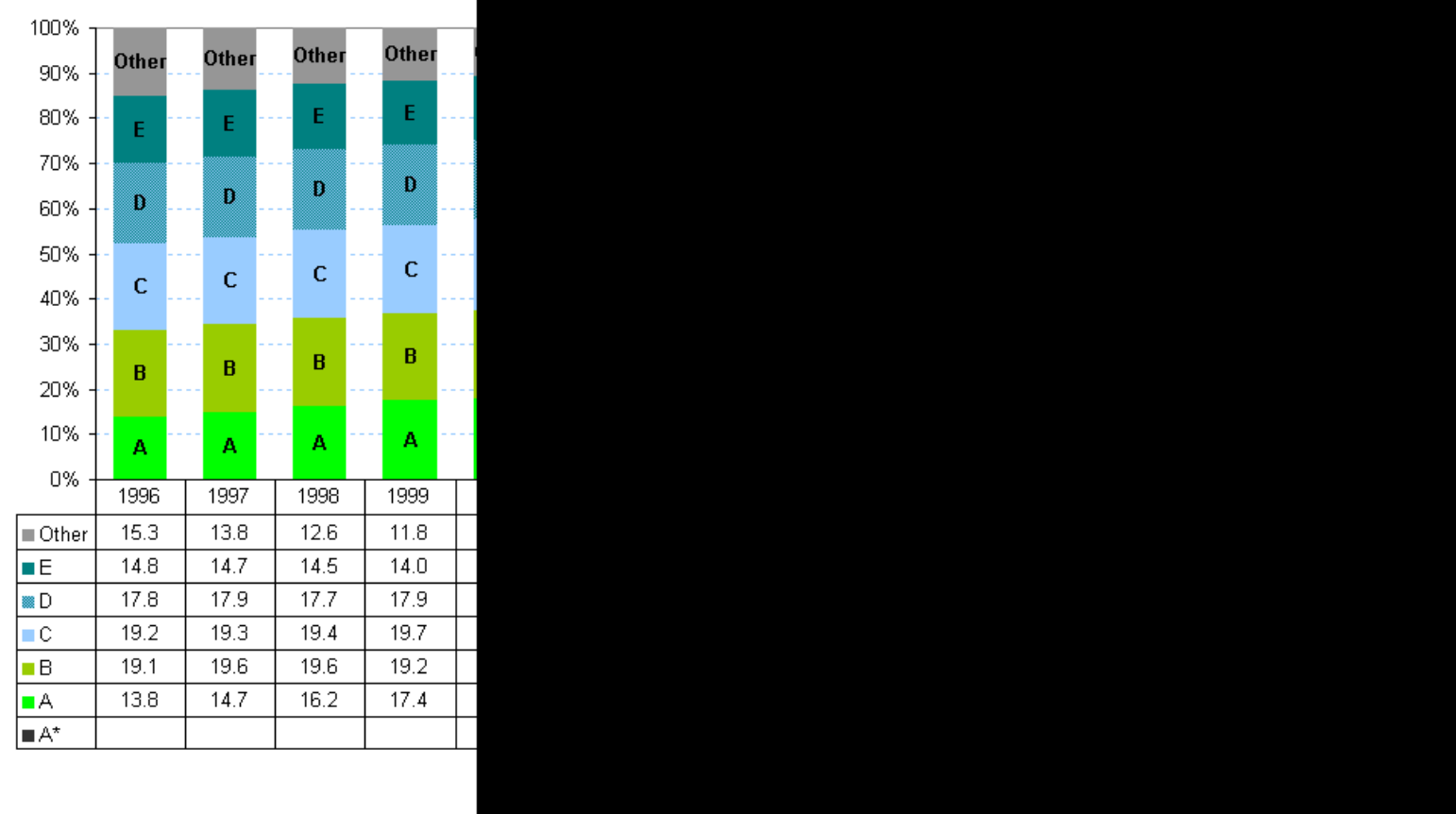


Fig. 1.2C

\section{Chemistry A-level Grade Distribution Trend for 1996-2010}

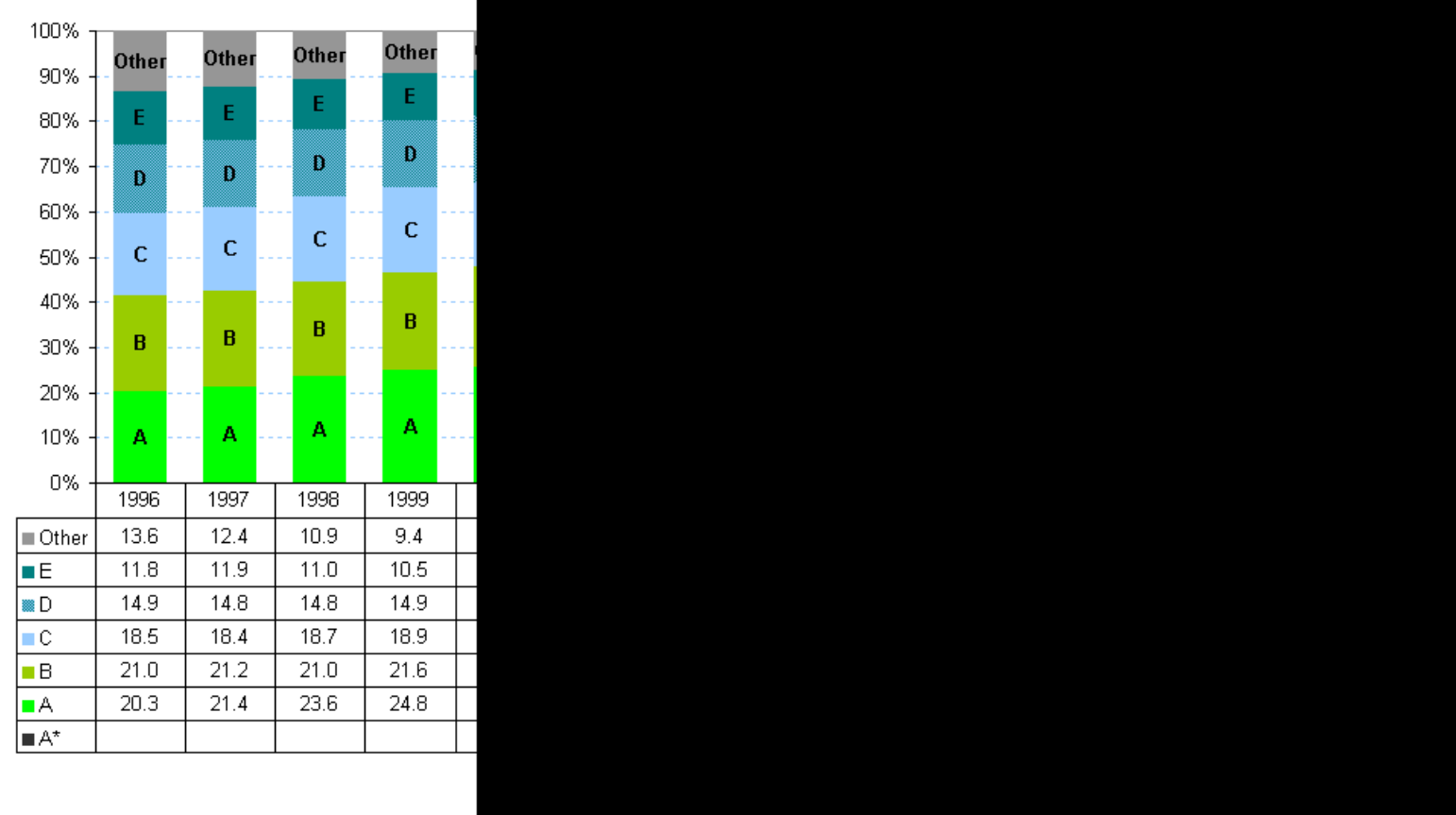

Fig. 1.2d

Physics A-level Grade Distribution Trend for 1996-2010

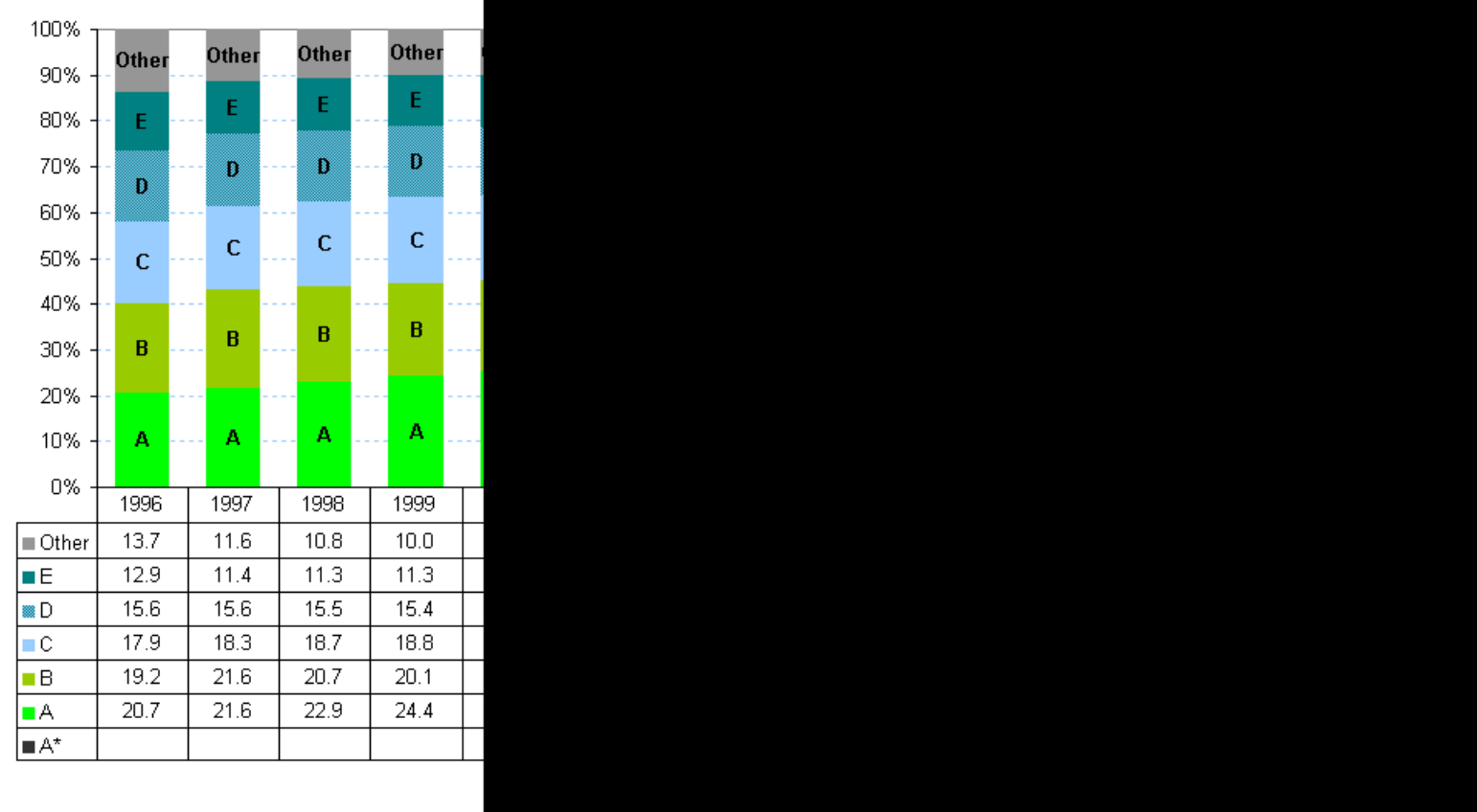


Fig. 1.2e

Other Science A-level Grade Distribution Trend for 1996-2010

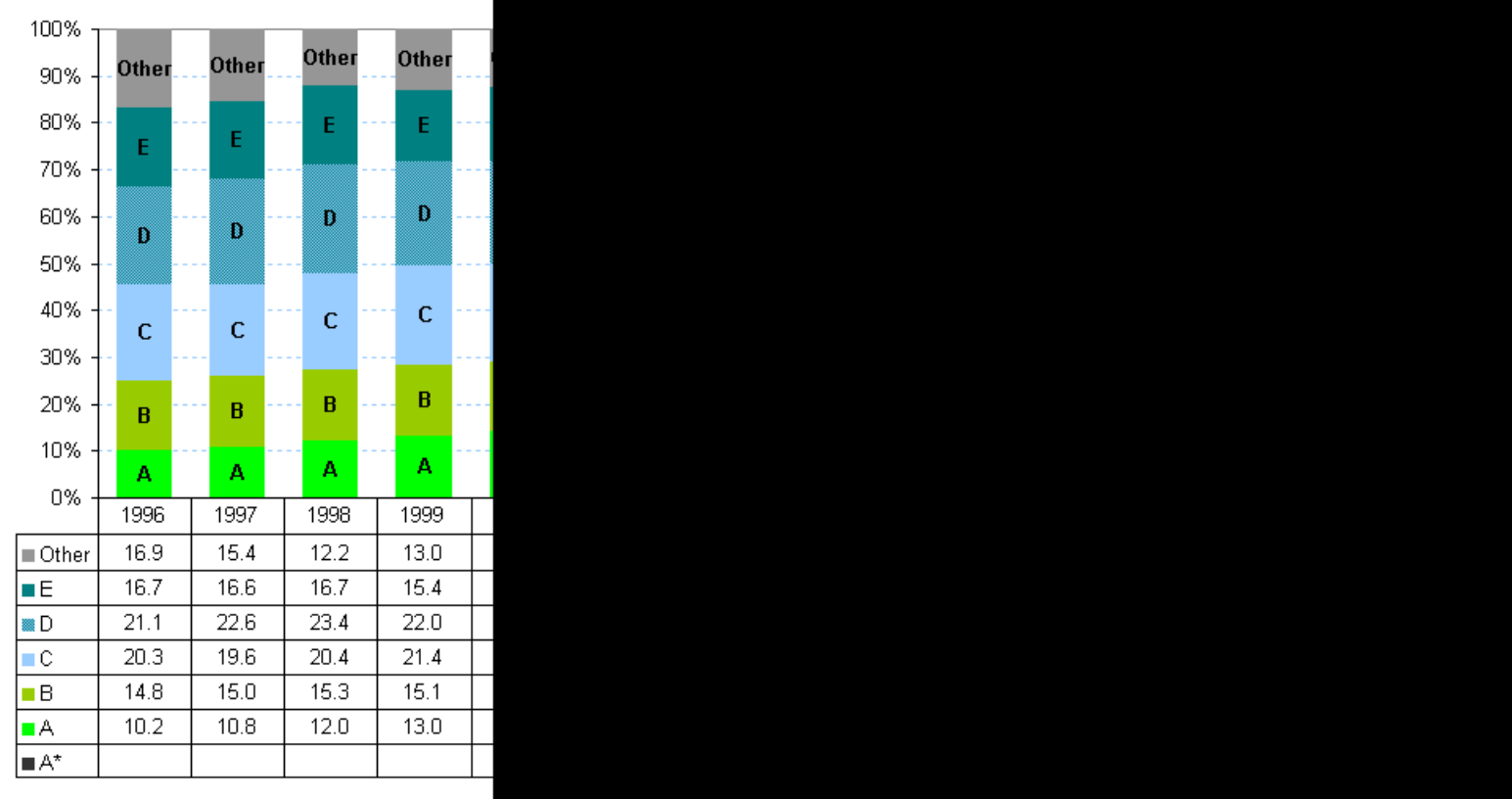

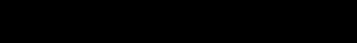


The comparison of grade distributions between the maths and traditional science subjects in figure $1.2 \mathrm{f}$ shows the higher percentage of entries gaining A grades in maths (45\% in 2009) than in the science subjects. Biology has the lowest proportion of $A$ grades at $28 \%$ in 2009 , despite this figure having grown fastest since 1996 , when entrants were only half as likely to achieve an A grade. The proportion of physics entrants gaining A grades was $33 \%$ on 2009 , compared with $35 \%$ of chemistry entrants. Provisional data for 2010, the year in which A star grades were introduced, shows a fractional decrease in the total proportion of A grades (including starred As) for maths, biology and chemistry, but a fractional increase in these grades for physics. $A^{*}$ grades accounted for $16.9 \%$ of entries in maths, $7.7 \%$ of biology entries, $8.9 \%$ of chemistry entries, and $10.5 \%$ of physics entries.

\section{Fig. 1.2f}

Mathematics \& Science A-level Grade Distribution Trend for 1996-2010

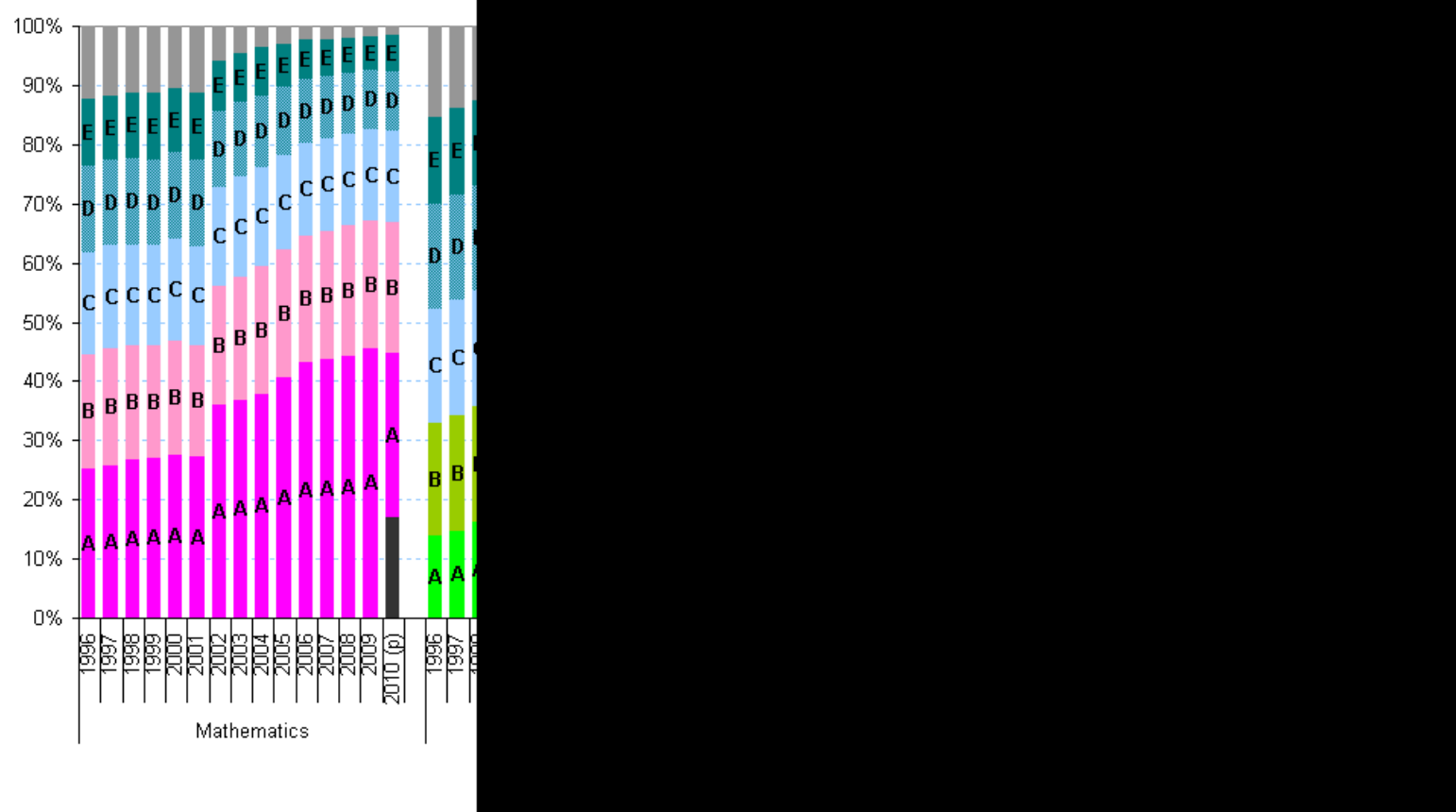




\subsection{Rates of A Level Entry ${ }^{3}$}

Although numbers of A level entries are higher than in 1996 for maths, biology and chemistry, as a proportion of the total estimated cohort aged $17^{3}$, the rate of entry has fallen for biology, chemistry and physics over the years 1998-2009 (see figure 1.3a). The increases in entries have not kept pace with the increase in the population over the same period, resulting in falling rates of participation. However, provisional data for 2010 show marked increases in the rates of participation for all three sciences, bringing chemistry back to 1998 levels, with biology just below the 1998 peak and physics beginning to recover. Proportional maths entries fell until 2006 , including a large drop coinciding with the curriculum changes in 2002 , but have increased sharply since 2006 , reaching almost $11 \%$ of the age group by 2010 . The 2010 rates of participation for the sciences were $8 \%$ for biology, $6 \%$ for chemistry and $4 \%$ for physics.

As a proportion of the total estimated age group, achievement of grades A or B has increased since 1998 for maths, biology and chemistry, remaining fairly stable for physics. As seen in sections 1.1 and 1.2 above, the rates of entrants achieving these top grades have increased significantly over the period, with the fall in the percentage of the population entering the sciences at A level responsible for dampening these increases when expressed as a rate of the estimated age 17 population. Maths has seen a sharper increase in the proportion of the age group achieving grades A or B since 2003 , coinciding with the increase in population entry rates.

\section{Fig. 1.3a}

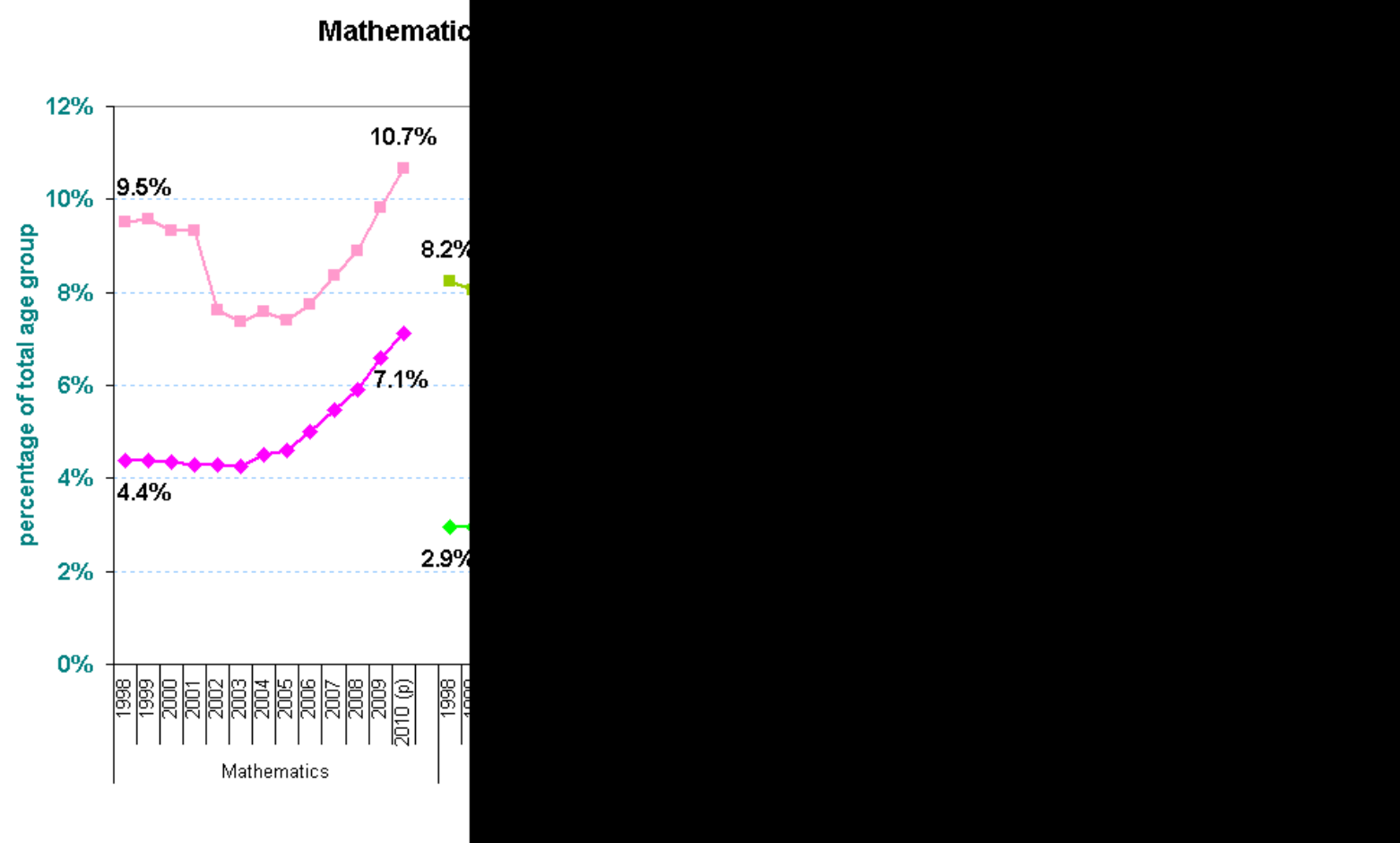

\footnotetext{
${ }^{3}$ Analysis in this section is based on Key Stage 4 cohorts; the cohort at the end of KS4 two years prior to the A-level year stated form the denominators for each year. This approximates to the age 17 population, but does not account for migration or deaths between KS4 and KS5.
} 


\subsection{AS level Entries}

The following charts present the number of AS level entries in maths and the traditional sciences. The AS entries have been aligned against the year in which most students would go on to convert them to full A levels (year $Y$, appearing along the horizontal chart axes), rather than the year in which the AS papers were sat (year $\mathrm{Y}-1$ ); this is to align them with the numbers of full A level entries for the same cohort so that the difference between the two can be seen.

In each year, a number of candidates sit AS level exams but do not continue to A level; this may be because the candidate wished to accumulate AS levels across a wider range of subjects rather than specialising in a smaller number in depth; alternatively, in some cases it may be that the candidate has found the subject challenging at AS level and decided it would not be a good use of study time to attempt the full A level in that subject. However, the majority of entrants in maths and science subjects do continue to A level, as demonstrated in figures $1.4 \mathrm{a}-1.4 \mathrm{~d}$.

The number of AS level maths entries for 16-18 year olds in 2010, pre-figuring the potential number of A level entries in 2011, rose to 79 thousand, up by around 5 thousand on the previous year. The rising trend in the number of AS maths entries has been followed by the increase in full $A$ level entries reported in section 1.1, with fewer non-continuing AS entrants over the last 4-5 years as the curriculum changes of 2002 bedded in. A further increase in A level maths entries in 2011 is suggested by the continued upward trend in AS entries in 2010.

\section{Fig. 1.4a}

Mathematics AS level Entries and Conversions to A level

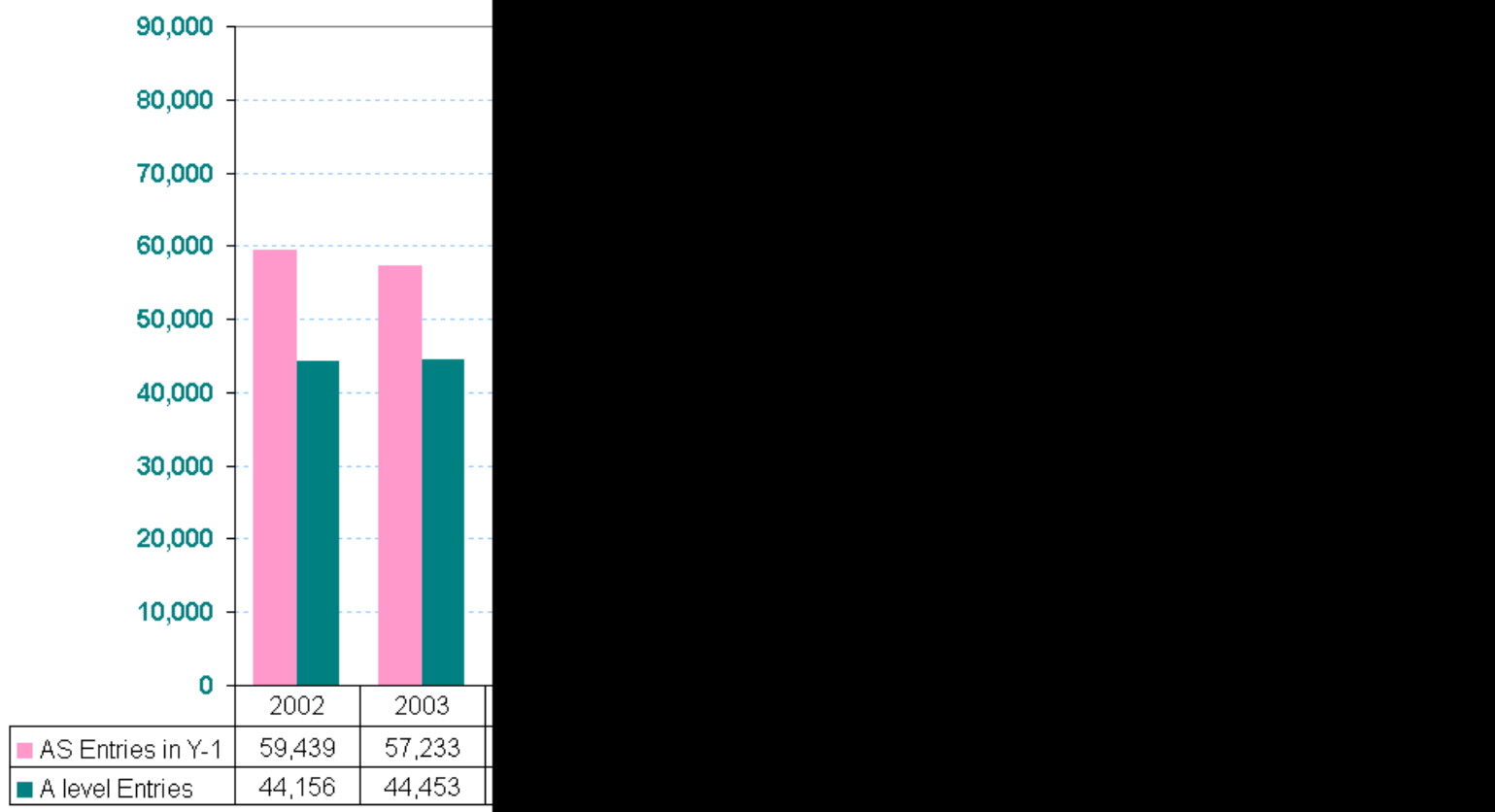

For biology, the number of non-converted AS levels has varied a little from year to year, but does not form a clear pattern of decline as with maths. AS numbers fell 
slightly to 58 thousand in 2008 (A level $Y=2009$ ), but recovered and continued their upward trend in 2009 (A level 2010). AS levels in biology increased again to over 66 thousand in 2010, suggesting that the A level numbers are likely to continue to increase in 2011.

Fig. 1.4b

Biology AS level Entries and Conversions to A level

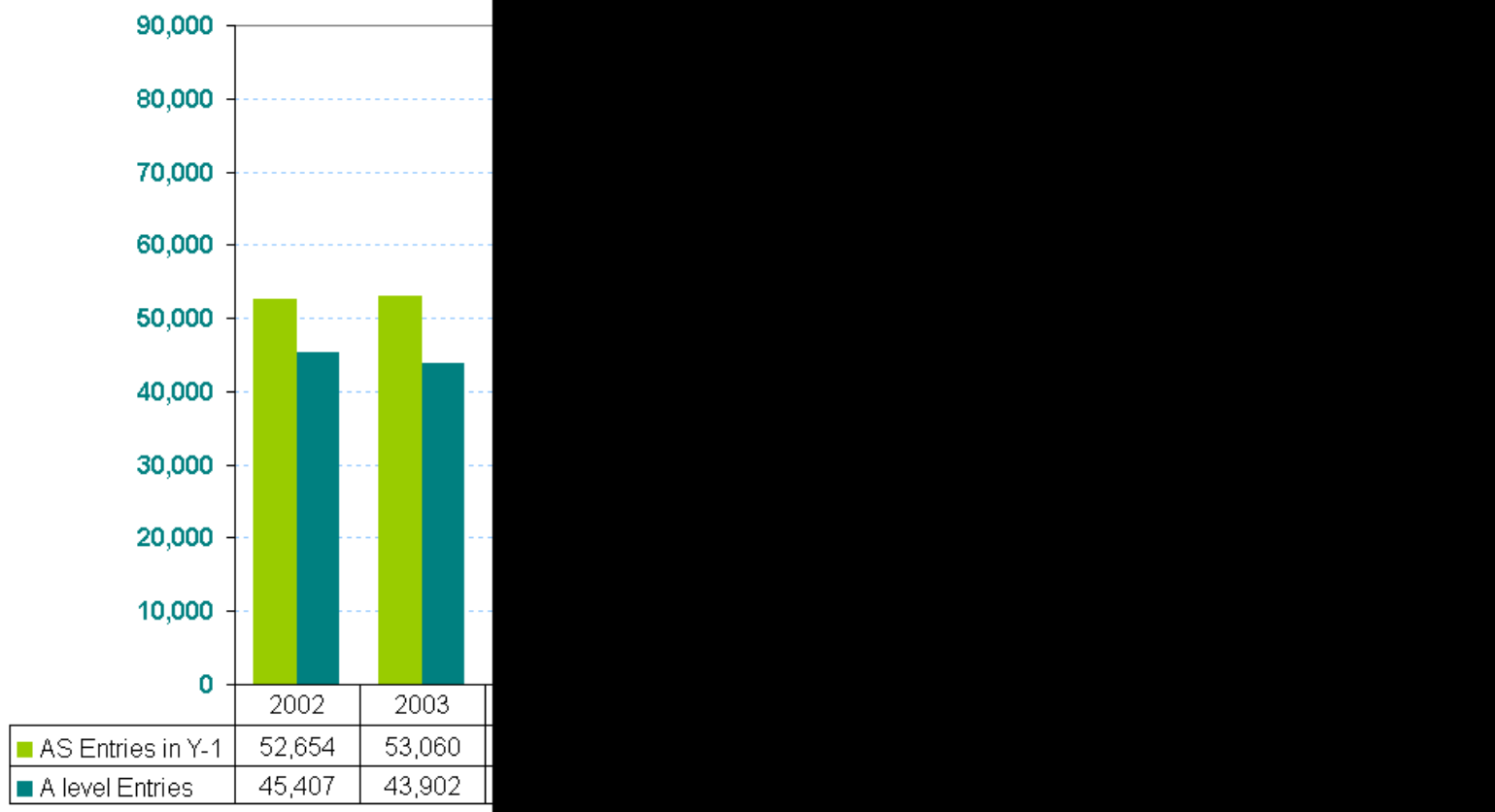


For chemistry, numbers of AS levels not followed up with full A levels have increased a little since 2002, standing at 6 thousand in 2009 (A level $Y=2010$ ). The number of AS entries has increased from 36 thousand to 49 thousand in 2010, followed by slightly smaller increases in full A levels in each following year to date (figure 1.4c). A further small increase in the number of chemistry A level entries looks likely for 2011 based on the continued rise in AS numbers.

\section{Fig. 1.4c}

\section{Chemistry AS level Entries and Conversions to A level}

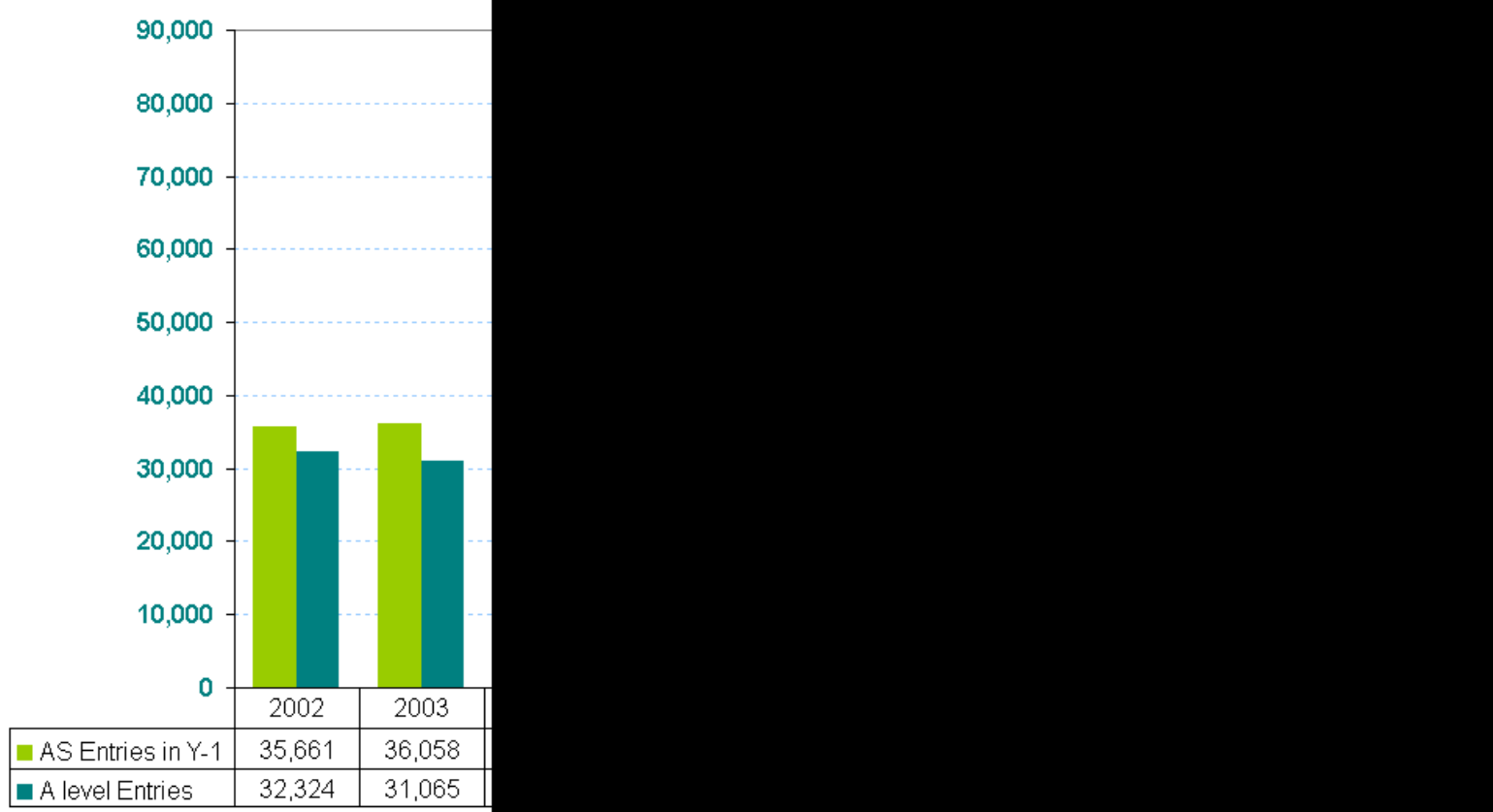


The pattern of physics A level entries witnessed in section1.1, declining then beginning to recover, is mirrored at slightly higher numbers in the prior AS level entries depicted in figure 1.4d. The numbers of AS levels not followed up as A levels has generally increased in the years since 2002. Although AS numbers reached 37 thousand in 2010 (6 thousand more than in 2001), the size of any likely increase in A level numbers for 2011 looks uncertain because of this trend in non-converted AS levels, making the recovery in physics A levels entries appear more fragile than those in biology or chemistry.

\section{Fig. 1.4d}

Physics AS level Entries and Conversions to A level

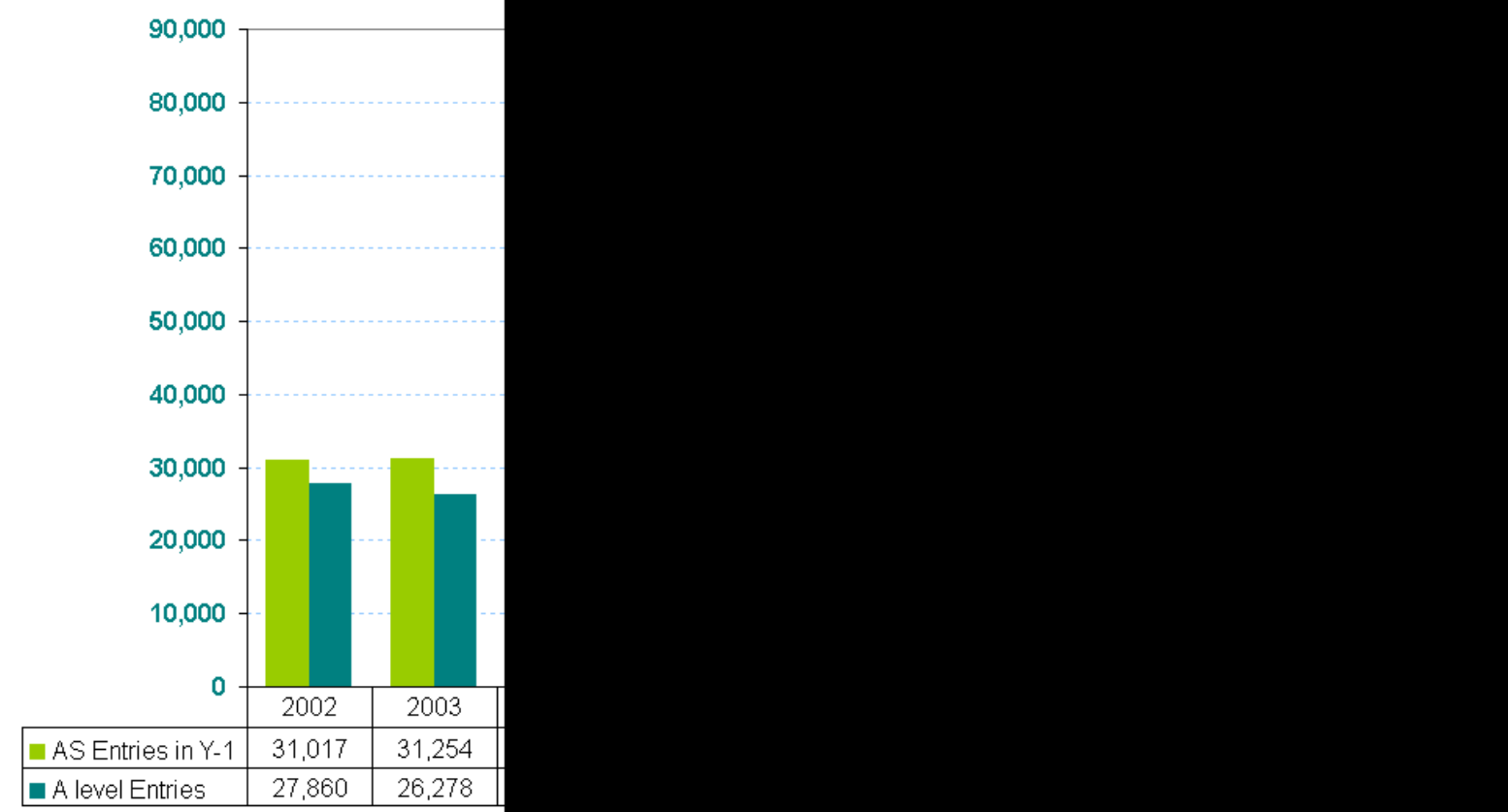


Figure 1.4e presents the AS level trends for the three sciences and maths side by side for comparison. With the exception of maths in the three years following the curriculum changes, biology has the largest numbers of non-converted AS levels, at 7-11 thousand per year, compared with 3-7 thousand for chemistry and 3-6 thousand for physics. When considered as a percentage of the number of AS entries, however, the levels of non-conversion are similar for biology and physics, averaging $18 \%$ over the time series, but a little lower for chemistry, averaging $14 \%$.

\section{Fig. 1.4e}

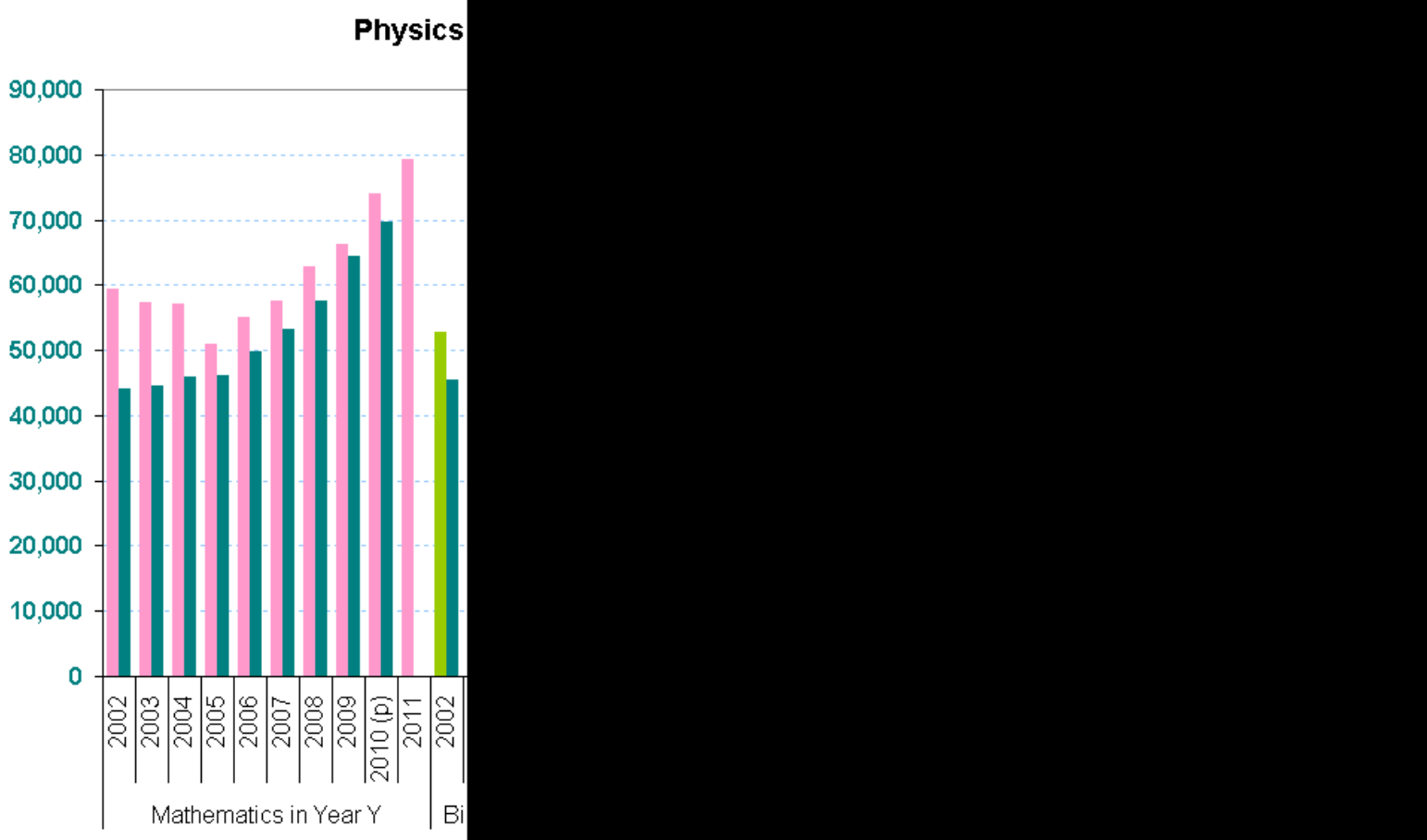

\subsection{Number of GCSE Entries, Passes, and Grades A* I A}

The following charts shift the focus down a Key Stage to GCSEs, in order to assess the trends in maths and science performance at the end of compulsory education. The figures reported in this section cover all schools in England including independent and special schools. In 2005, there was a change in the basis of GCSE reporting, from achievements expressed as a percentage of 15 year olds, to achievements expressed as a percentage of pupils at the end of Key Stage 4 in their studies (i.e. having completed year 11). The break in the achievement rate lines in the following charts represents this shift in methodology.

At GCSE, changes in the numbers of maths entries are largely driven by population changes, with small additional scopes for variation deriving from alternative types of qualification, and post-2005, from very small proportions of pupils sitting GCSEs at age 14 or 16 rather than 15 . As the study of maths is largely compulsory, the main features of interest in figure 1.5a are the rates of achievement. The pass rate has remained essentially stable at around $97 \%$ in most years, on both the age 15 and end of KS4 bases. The rate of achievement of grades $A^{*}$ or $A$ has increased from $12 \%$ to $13 \%$ of entries by 15 year olds between 2001 and 2004 , then from $15-16 \%$ of 
entries by pupils at the end of KS4 from 2005-2010. This increase in the percentage of high grades awarded is small compared with those seen at A level in sections 1.1 and 1.2 .

Fig. 1.5a

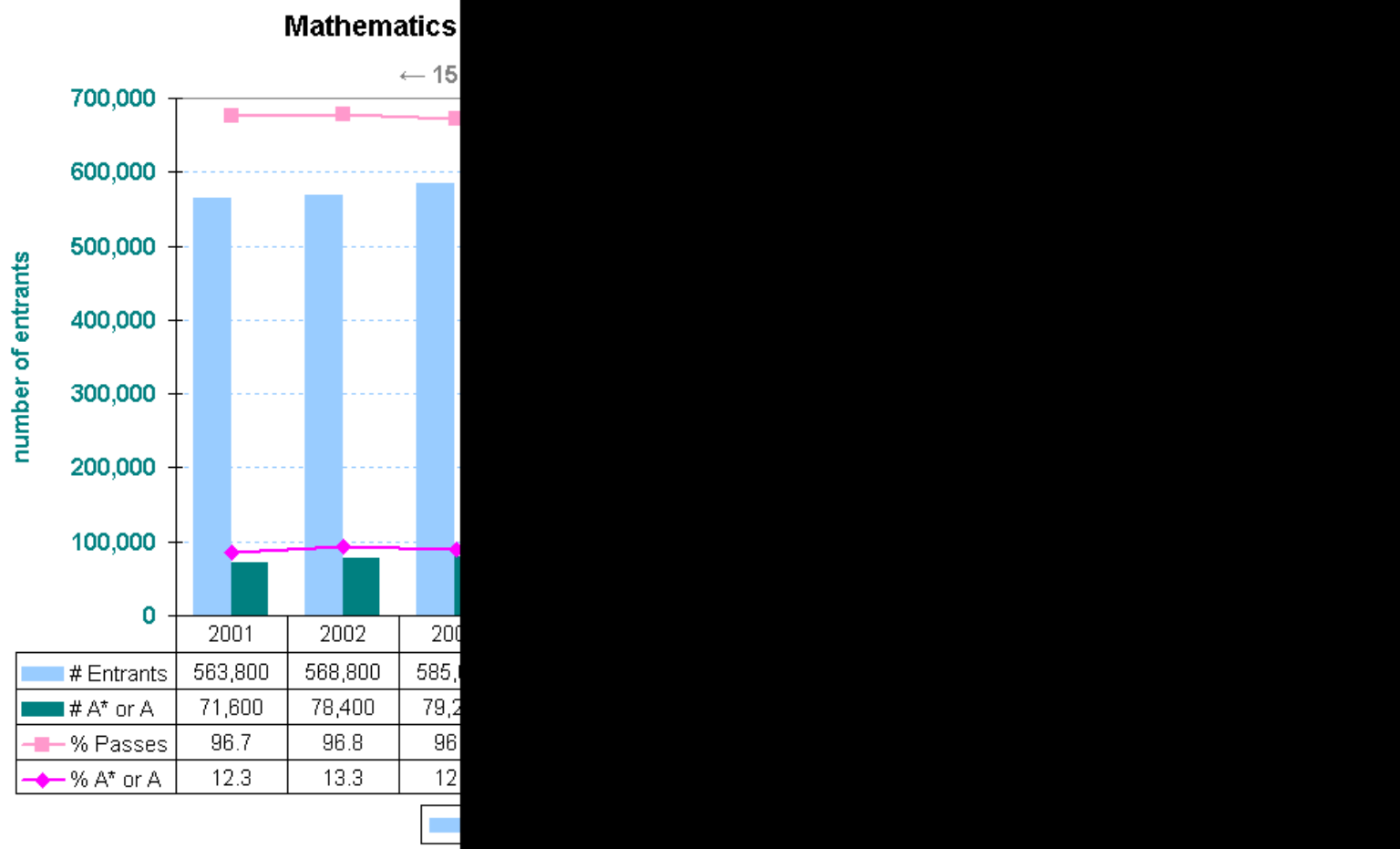

Science is compulsory at Key Stage 4, but there are alternative qualification routes capable of fulfilling this requirement. Up to 2007, the majority of pupils sat double science GCSEs as their science curriculum, and from 2008, these were replaced with core and additional science GCSEs. Most pupils still study this GCSE science route, worth 2 GCSEs, although a shift is taking place away from double / core and additional science, and towards separate GCSEs in biology, chemistry and physics. 
Figure 1.5b charts the rise in the numbers of pupils entering GCSE biology from 2001 to 2010 . There were just over 40 thousand entrants in 2001, rising gently to 54 thousand by 2007 , then sharply to 116 thousand by 2010 in response to government policies promoting the provision of separate sciences at GCSE. The pass rate for GCSE biology has remained at over $99 \%$ of entries throughout this period, whereas the percentage of entries attracting grades $A^{*}$ or $A$ has floated up from $43 \%$ in 2001 to almost $50 \%$ in 2008 , then falling back slightly to $48 \%$ in 2010 .

\section{Fig. 1.5b}

Biology GCSE Entries and Passes Trends for 2001-2010

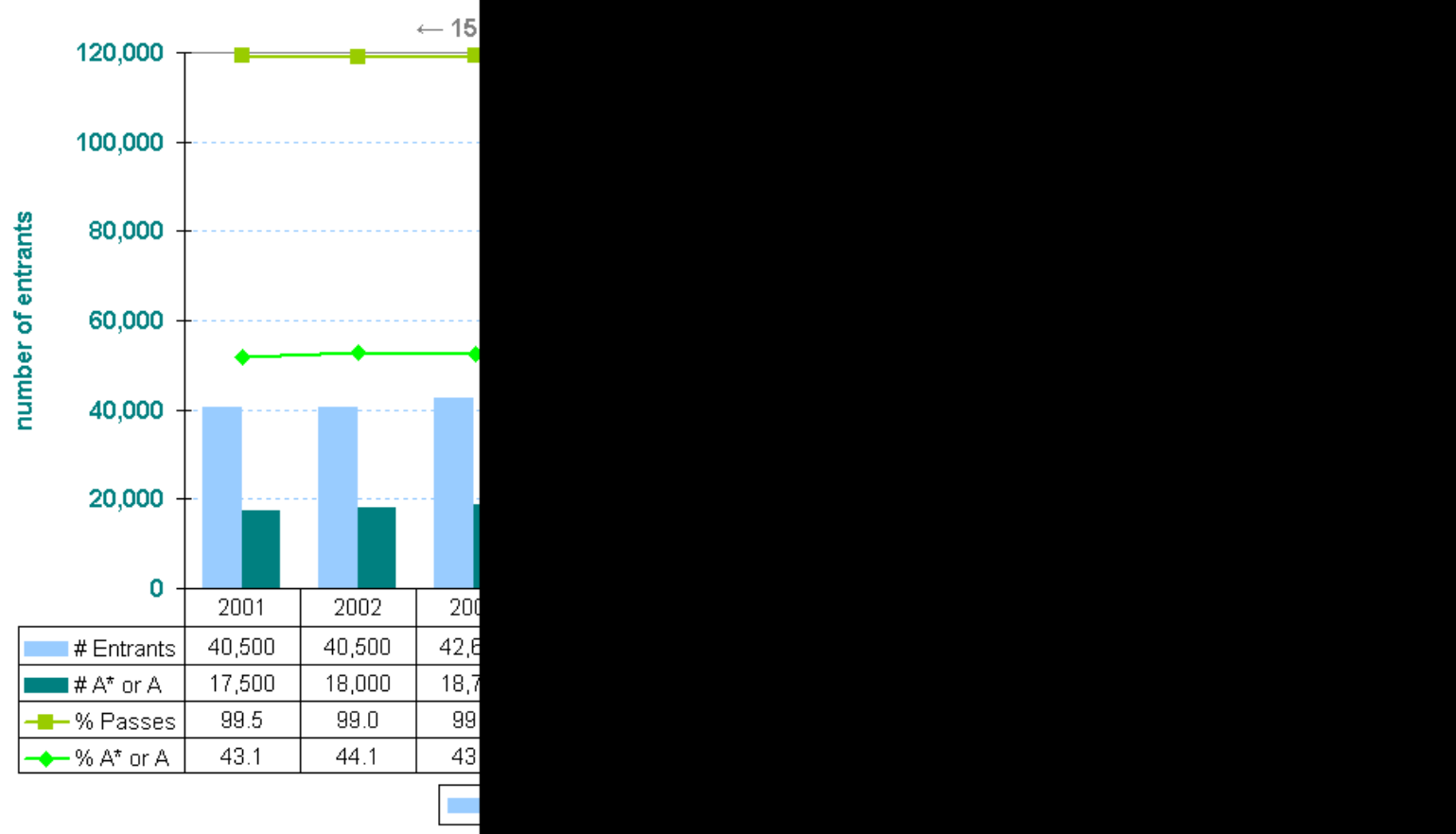


Figure $1.5 \mathrm{c}$ shows a similar pattern of increased entries in separate chemistry GCSEs, although the numbers are marginally lower than biology throughout the period at 39 thousand in 2001, 51 thousand in 2007, and 113 thousand in 2010. As with biology, around half of entries result in grades $A^{*}$ or A in GCSE chemistry, increasing from $45 \%$ in 2001 , to $54 \%$ by 2008 , then dropping back to $49 \%$ by 2010 . The pass rate has remained between $99 \%$ and $100 \%$ over the last decade.

\section{Fig. 1.5c}

\section{Chemistry GCSE Entries and Passes Trends for 2001-2010}

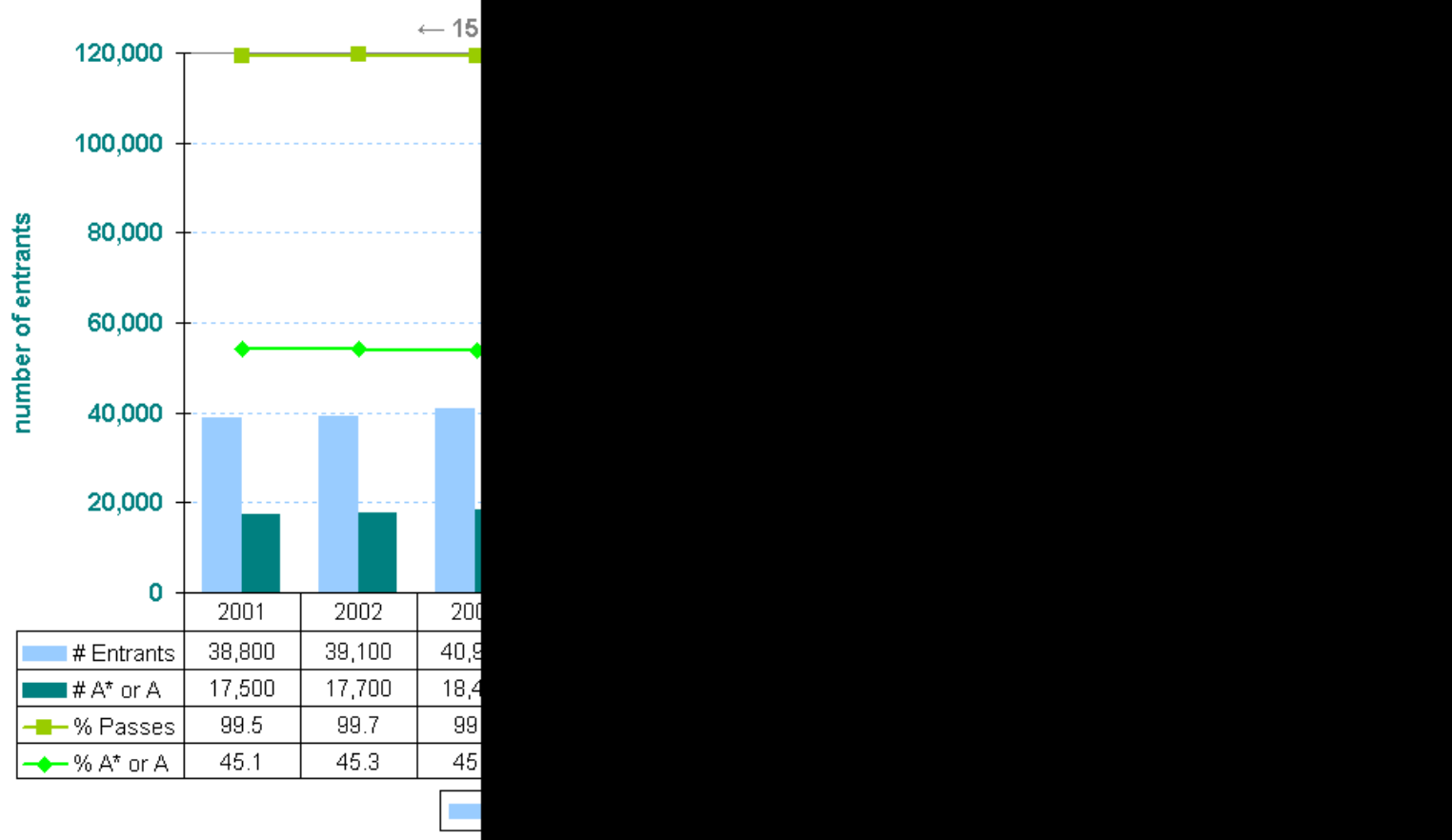


Figure 1.5d repeats the trend of increased separate science GCSE entries for physics, again with numbers a little lower than biology at 39 thousand in 2001, 51 thousand by 2007, and 112 thousand by 2010 . Similarly, the pass rate and percentage of entries awarded grades $A^{*}$ or $A$ also mirror the pattern established for biology and chemistry GCSEs. Pass grades are near-universal and top grades have grown from $48 \%$ to over 50\%, then fallen back to $49 \%$ in 2010 .

\section{Fig. 1.5d}

Physics GCSE Entries and Passes Trends for 2001-2010

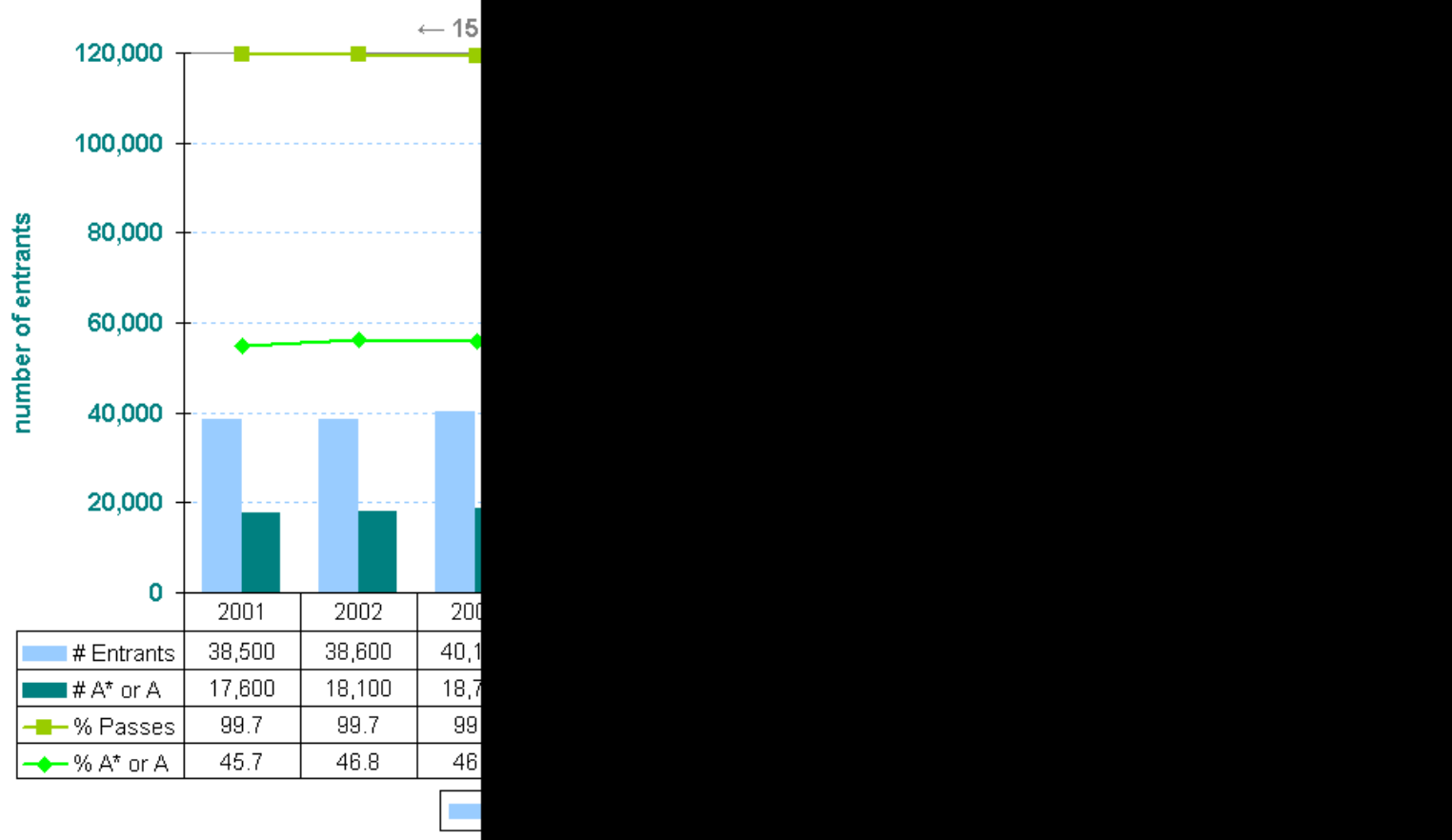


Figure 1.5e compares the above GCSE data for biology, chemistry and physics data, and adds results for the available years in double science, single science, core science and additional science GCSEs. The historical dominance of double science, with over 400,000 entrants per year can be seen, along with its drastically lower percentage of entries attracting grades $A^{*}$ or $A$, standing at $15 \%$ in 2007 , and dropping back to the same level two years after the introduction of the equivalent additional science GCSE in 2008. Single science GCSEs were entered by between 50 thousand and 71 thousand pupils per year until they were replaced by core and additional science in 2008.

\section{Fig. 1.5e}

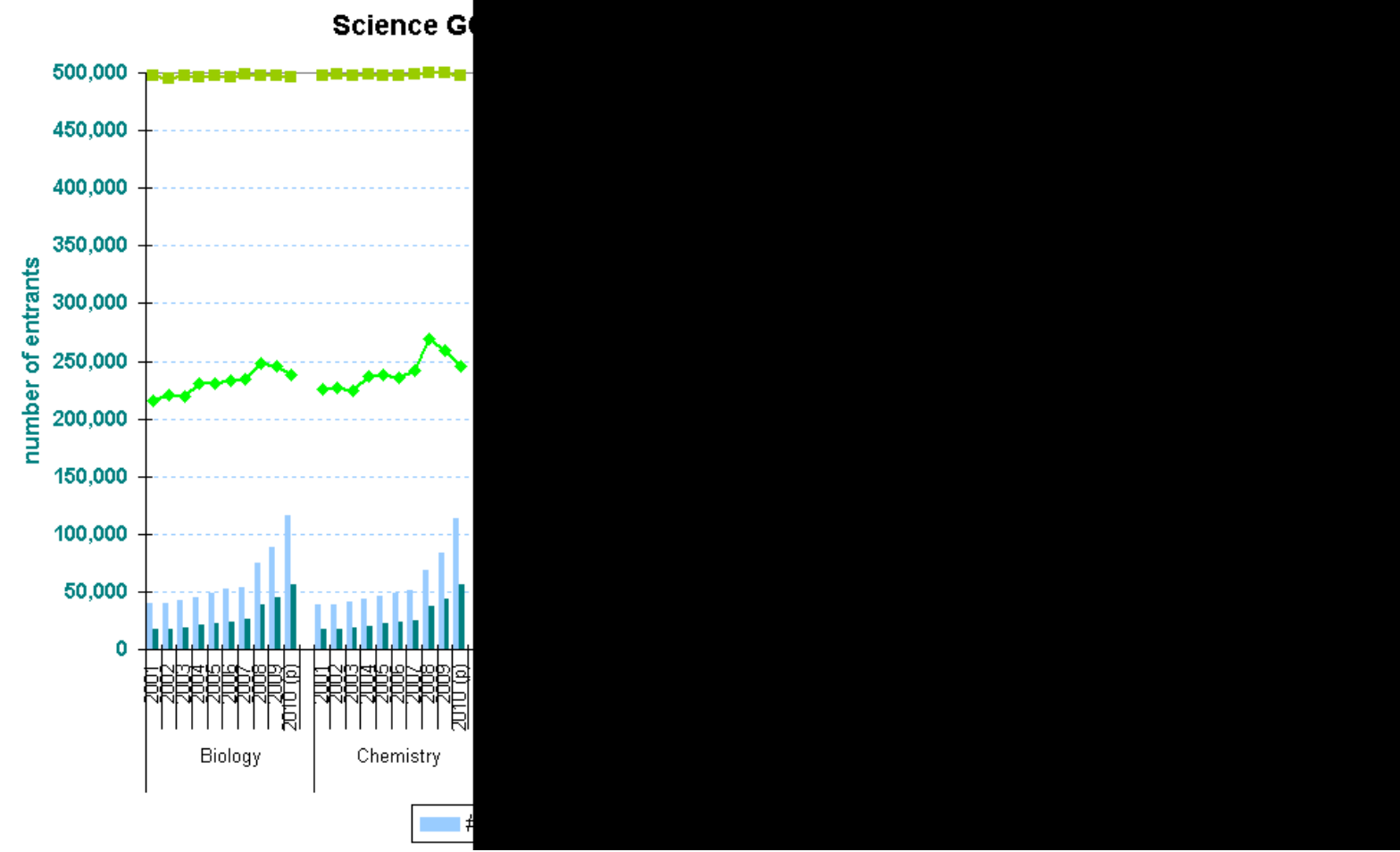


Figure $1.5 f$ takes a more detailed view of science GCSE entry numbers over the same period of 2001 to 2010 . The numbers of entrants to combined science GCSEs began to fall in 2005, with double science falling from 480 thousand entrants the previous year to 451 thousand; this decline continued in subsequent years, reaching 435 thousand by the final year for double science in 2007. Core science GCSEs (equivalent to the old single science qualifications) began at 492 thousand in 2008, 355 thousand of which were topped up with additional science (equivalent to the old double science GCSE). By 2010, this had fallen to 405 thousand core science entrants, 289 thousand of whom went on to enter additional science.

\section{Fig. $1.5 f$}

Science GCSE Entry Trends for 2001-2010

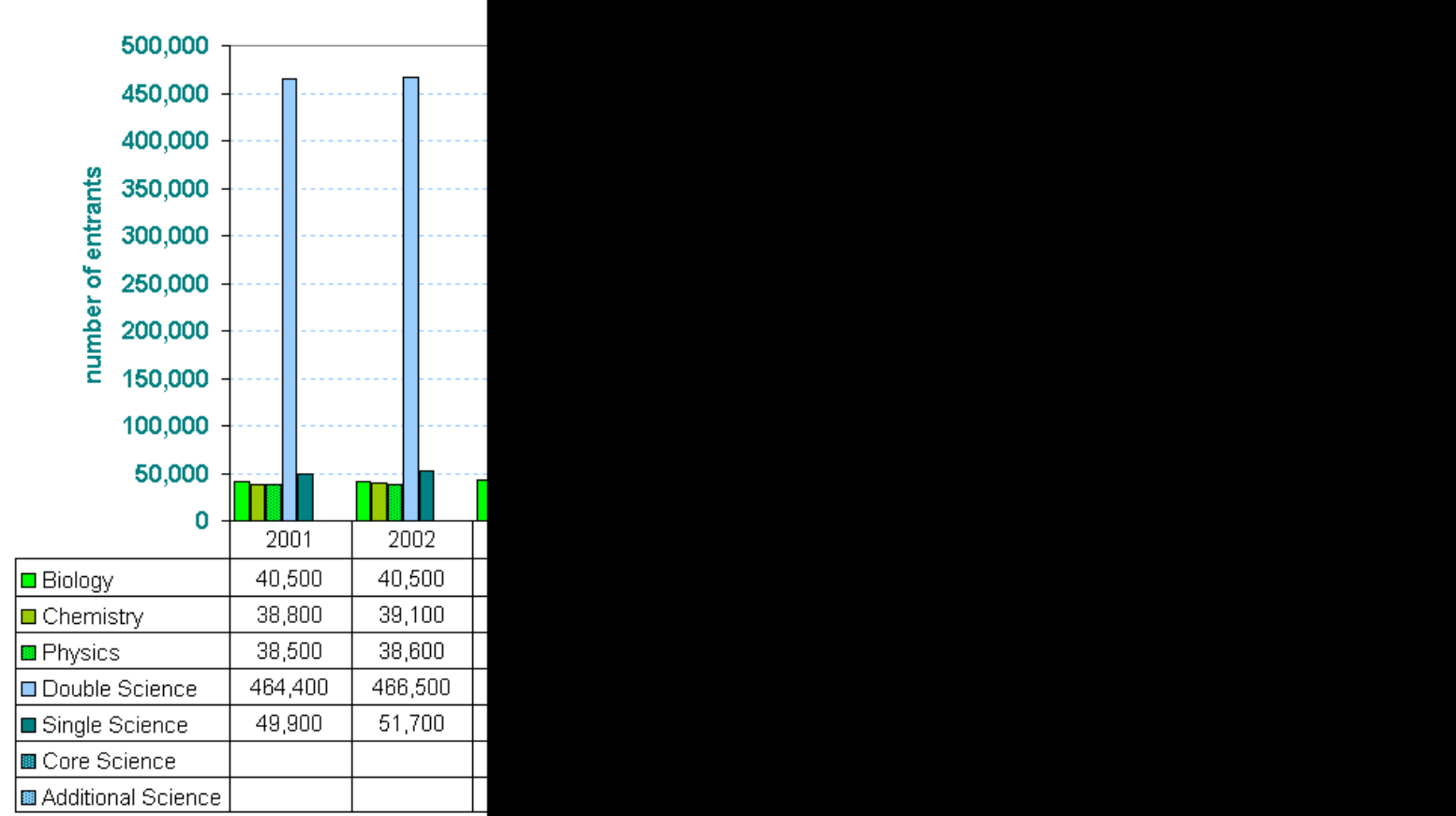


The decreases in the numbers of core science and additional science entries seen since 2008 (by 87 thousand and 66 thousand respectively) are only partially accounted for by increases in separate science GCSEs (of $41-45$ thousand entries). The shortfall is made up by increases in alternative Key Stage 4 science qualifications; BTEC and OCR applied science entries have increased by 56 thousand over the same period (see figure $1.5 \mathrm{~g}$ ).

\section{Fig. 1.5g}

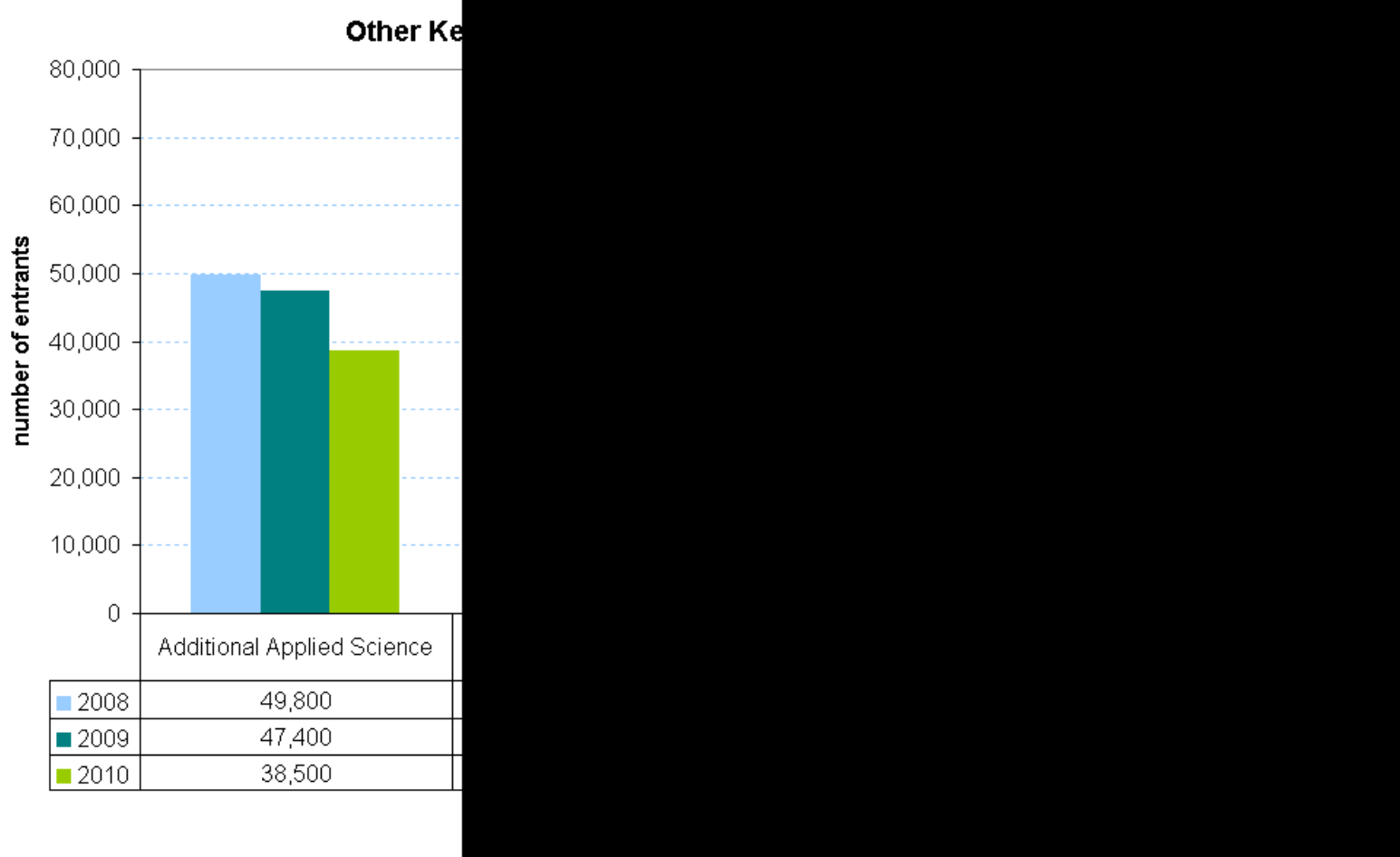


Figure 1.5h presents an equivalent analysis of achievements at grade $A^{*}$ or $A$ in the science GCSEs. Although separate science GCSEs are still outnumbered by threeand-a-half to one by core science, and by two-and-a-half to one by additional science, the number of top grades is now higher for separate sciences. In 2010, there were 57 thousand grades $A^{*}$ or A in biology, 56 thousand in chemistry, and 55 thousand in physics. This exceeded the 51 thousand $A^{*}$ / A grades awarded in core science, and the 45 thousand for additional science. The drop in top grades in the combined science GCSEs is partly driven by the selection of the most able candidates who would have previously sat this GCSE science route into the extra entry numbers for separate sciences. This selection effect compounds the raw transfer of entries from combined sciences to separate sciences, and the difference in success rates between the routes, resulting in the new numerical dominance of separate sciences in accounting for top grades.

Fig. 1.5h

Science GCSE Grade $A^{*}$ or A Trends for 2001-2010

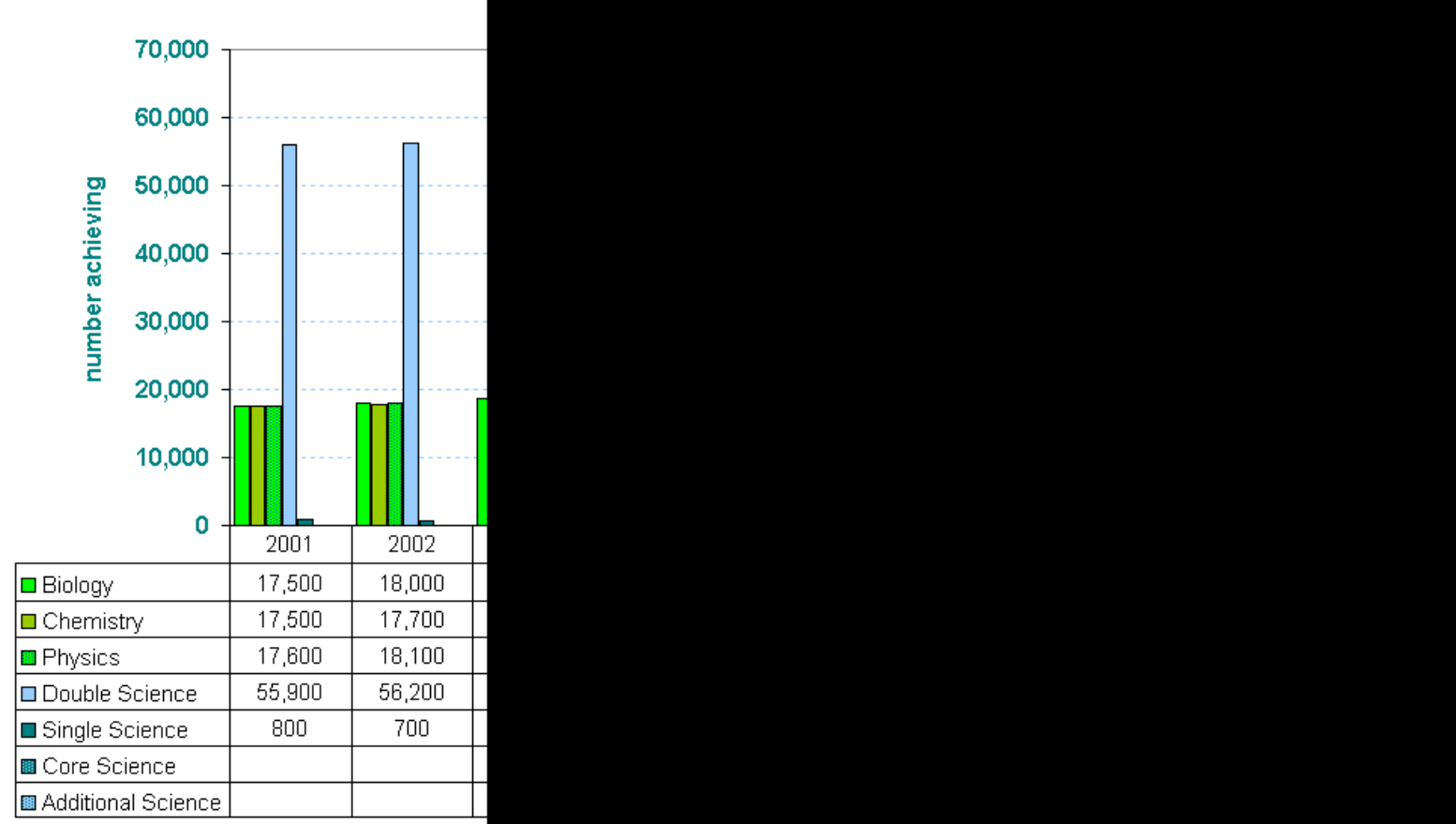

\subsection{GCSE Grade Distribution}

The charts in figure 1.6a depict the detailed grade distributions for GCSEs in maths, biology, chemistry and physics, from 2001 to 2010. Achievement in the maths and science subjects has continued to increase despite the widening of access to separate science GCSEs during the last few years. There is a small but noticeable additional jump in the grade distributions in 2008, when the double and single science alternatives were replaced by core and additional science. There appears to have been some correction of this in 2009 and 2010, with the very highest grades ( $A^{*}$ and A) stabilising, while passes at grades A-C continue to increase for biology, but also tail off slightly for maths, chemistry and physics. The grade distribution is much more evenly spread for maths than for the separate science GCSEs, with top grades $A^{*}$ or $A$ accounting for around half of entrants in the sciences, but less than one fifth 
of entrants for maths. This is the reverse of the pattern at A level, where grades $A^{*}$ $B$ are significantly more common in maths than in the sciences.

Fig. 1.6a

Mathematics GCSE Grade Distribution Trend 2001-2010

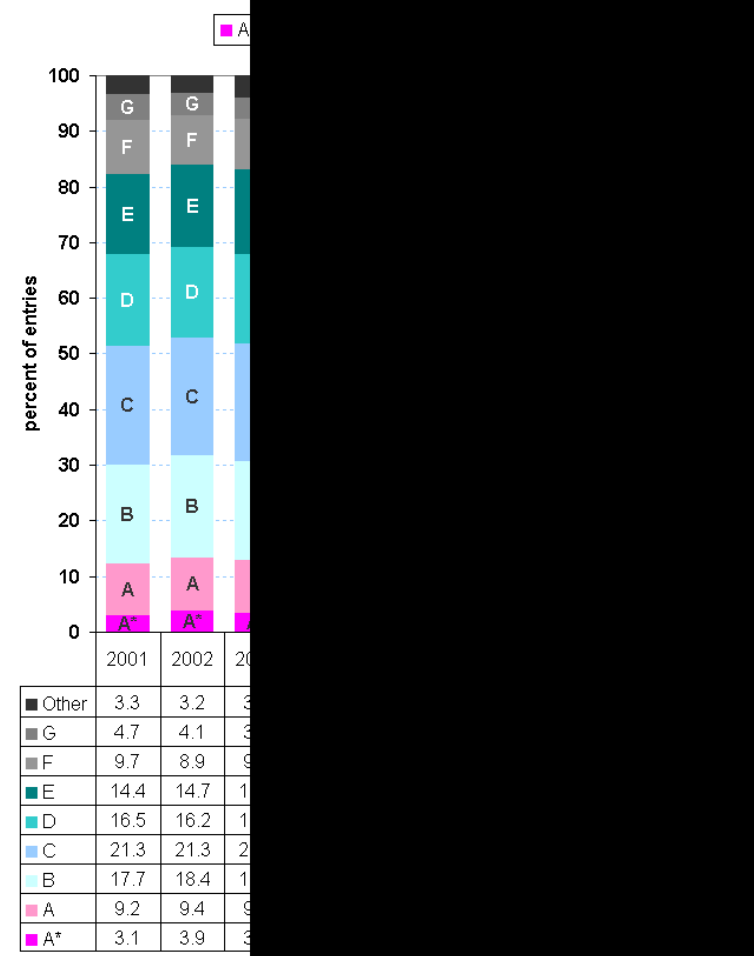

Chemistry GCSE Grade Distribution Trend 2001-2010

$\square \mathrm{A}^{*} \backsim \mathrm{A} \quad \mathrm{B} \backsim \mathrm{C}\|\mathrm{D} \backsim \mathrm{E}\| \mathrm{F} \| \mathrm{G} \backsim$ Other

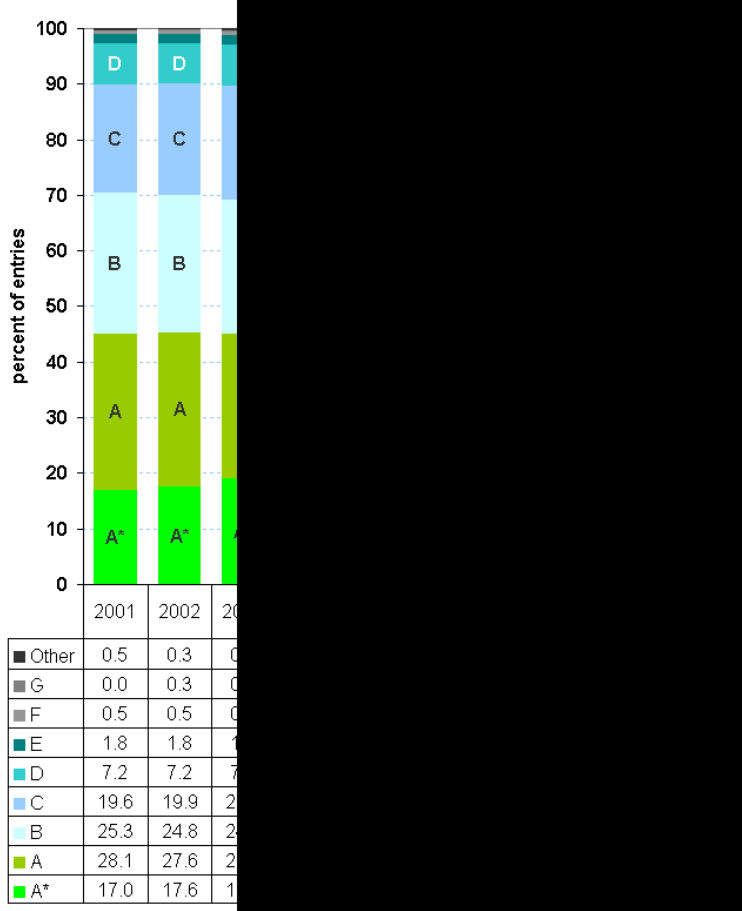

Biology GCSE Grade Distribution Trend 2001-2010

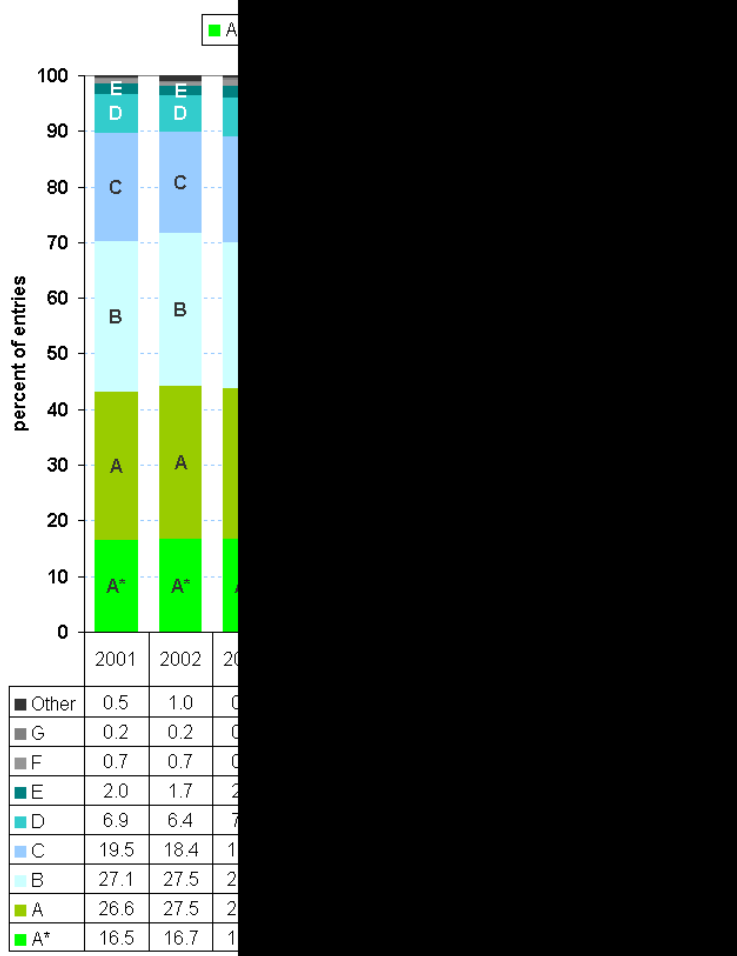

Physics GCSE Grade Distribution Trend 2001-2010

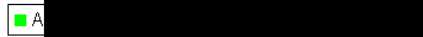

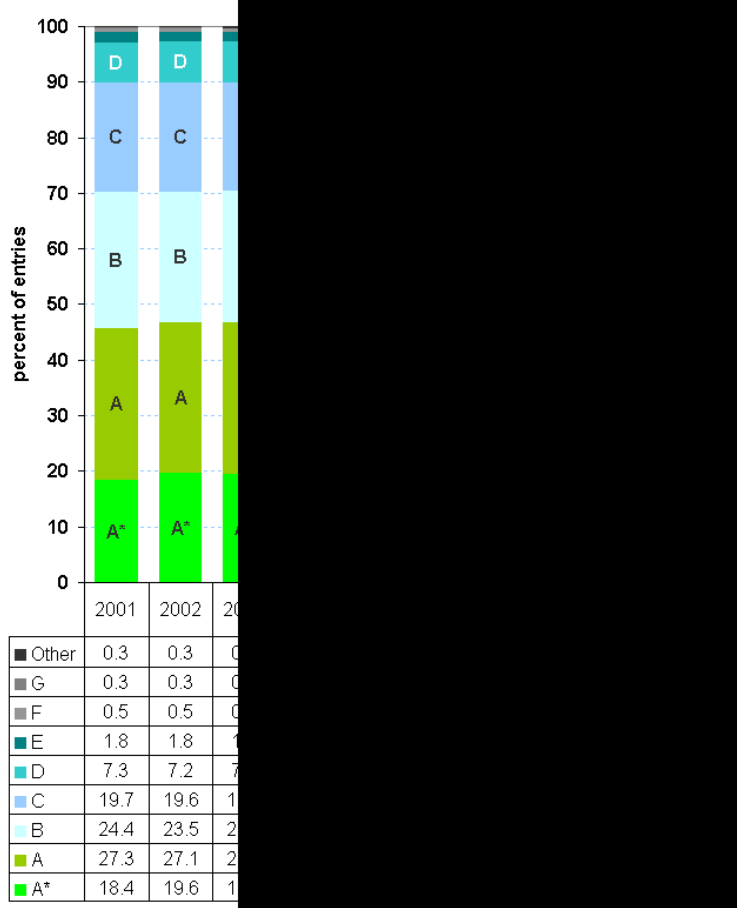


Figure 1.6b summarises the science GCSE grade distributions, adding the combined science GCSEs to the detailed picture of the separate sciences presented above. Less than one fifth of entries results in grades $A^{*}$ or $A$ across the combined science GCSEs, both previous and current. Single science had the lowest achievement of all science GCSEs, with only around a fifth of entries awarded grades $A^{*}-C$. The core science GCSE which has replaced it has better rates of achievement, a fraction lower than those for additional science, where an element of selection takes place.

\section{Fig. 1.6b}

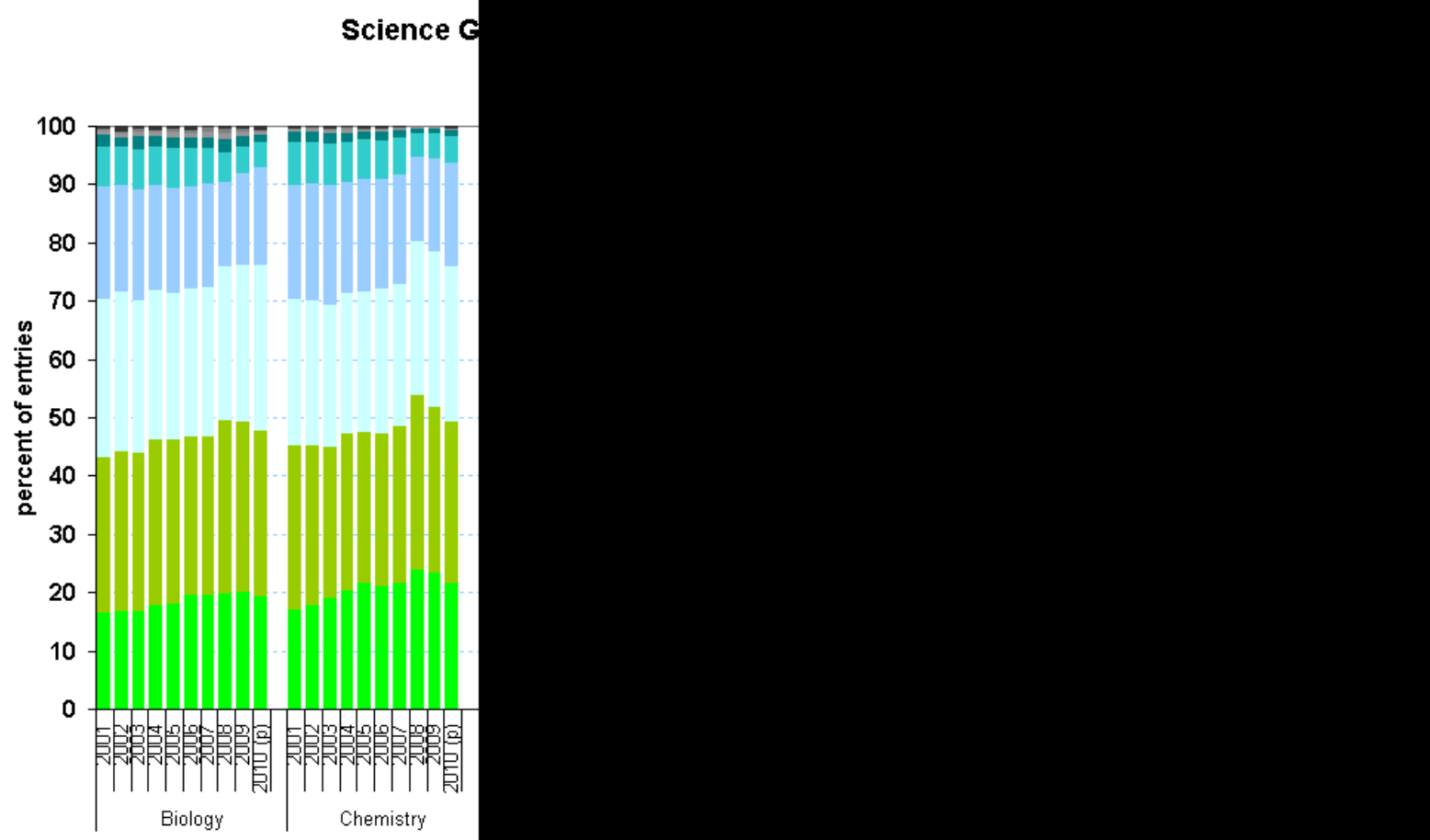

\subsection{Rates of GCSE Entry}

The following analyses present the participation rates for maths and science GCSES, expressed as percentages of the relevant cohort. For years 2001-2004, the cohort is counted as pupils aged 15; from 2005, the basis for reporting changed to pupils at the end of Key Stage 4 in their studies, allowing a more flexible approach to the timing of an individual pupil's qualifications to be adopted, with a fraction of pupils sitting GCSE exams at age 14 or 16 instead of 15.

For GCSE maths, participation is over $90 \%$ with this qualification being the main vehicle for delivering maths as a compulsory element of the curriculum. The increases in the numbers of top grades in GCSE maths described in the previous section are also reflected in the proportion of the cohort achieving grades $A^{*}$ or $A$. This demonstrates that the numerical increases in these grades are not just an artefact of changes in the population size.

In fact, the existence of multiple attempts at GCSE maths by some pupils means that a higher percentage of the cohort (18.9\%) achieves grade $A^{*}$ or $A$, than the percentage of maths entries resulting in the same grades $(16.1 \%)$. The rates of entry and achievement were slightly higher following the change in cohort basis to the end 
of Key Stage 4, as achievements by pupils sitting GCSEs early or late began to be included. The percentage of the cohort achieving grades $A^{*}$ or $A$ in GCSE maths has risen from $15 \%$ in 2005 to $19 \%$ in 2010.

Fig. 1.7a

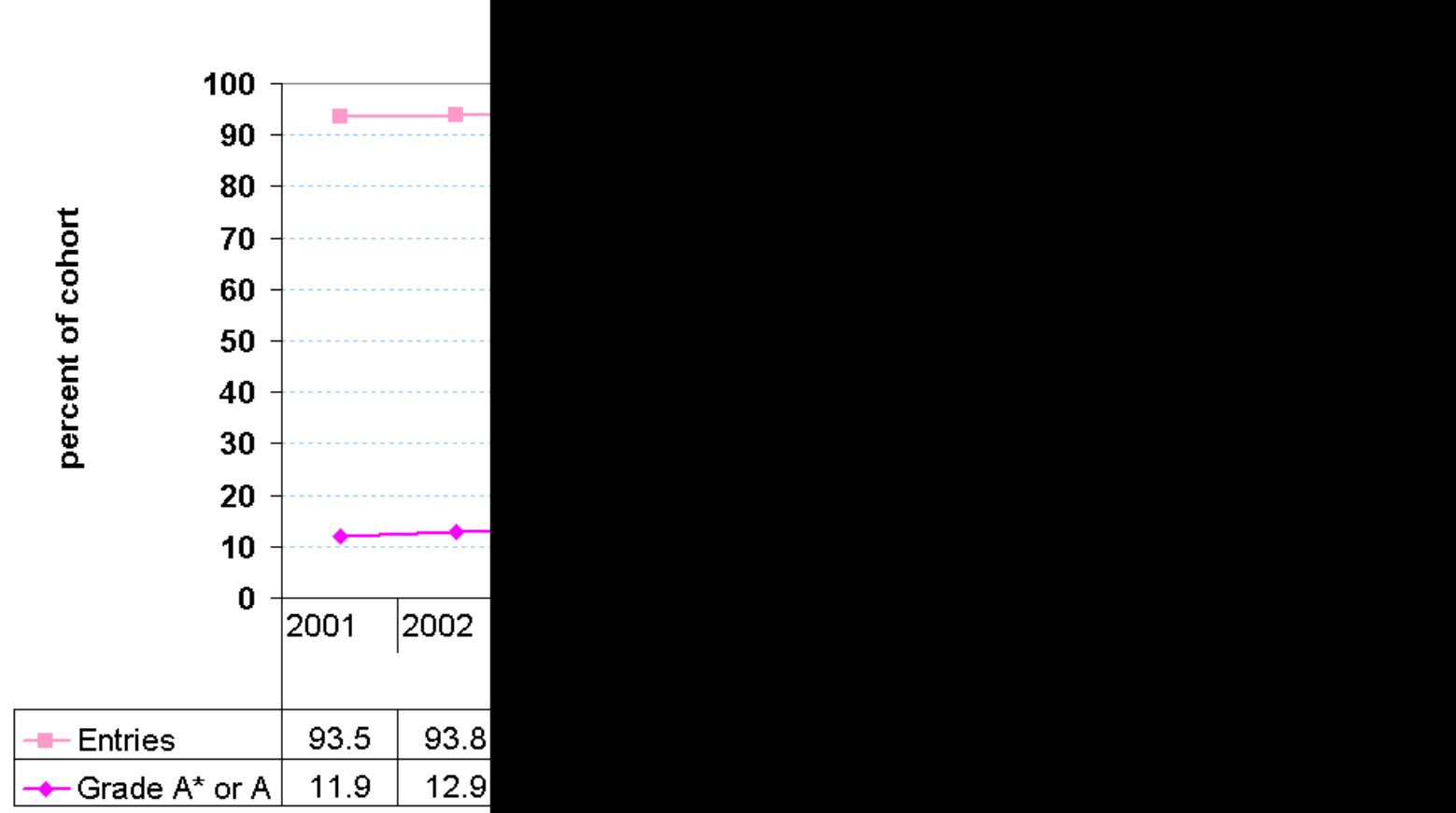


The charts in figure 1.7b depict the cohort rates of participation and achievement for the various science GCSEs. The pattern of transferring popularity from combined science GCSEs to separate sciences is confirmed by these analyses, with separate science participation now reaching $18 \%$ of the cohort and core science entry declining to $63 \%$ of the cohort (with $45 \%$ continuing to additional science). In 2010 , achievement of grades $A^{*}$ or $A$ has increased to $9 \%$ of the cohort for the separate sciences, and fallen to $8 \%$ for core science and $7 \%$ for additional science.

\section{Fig. 1.7b}
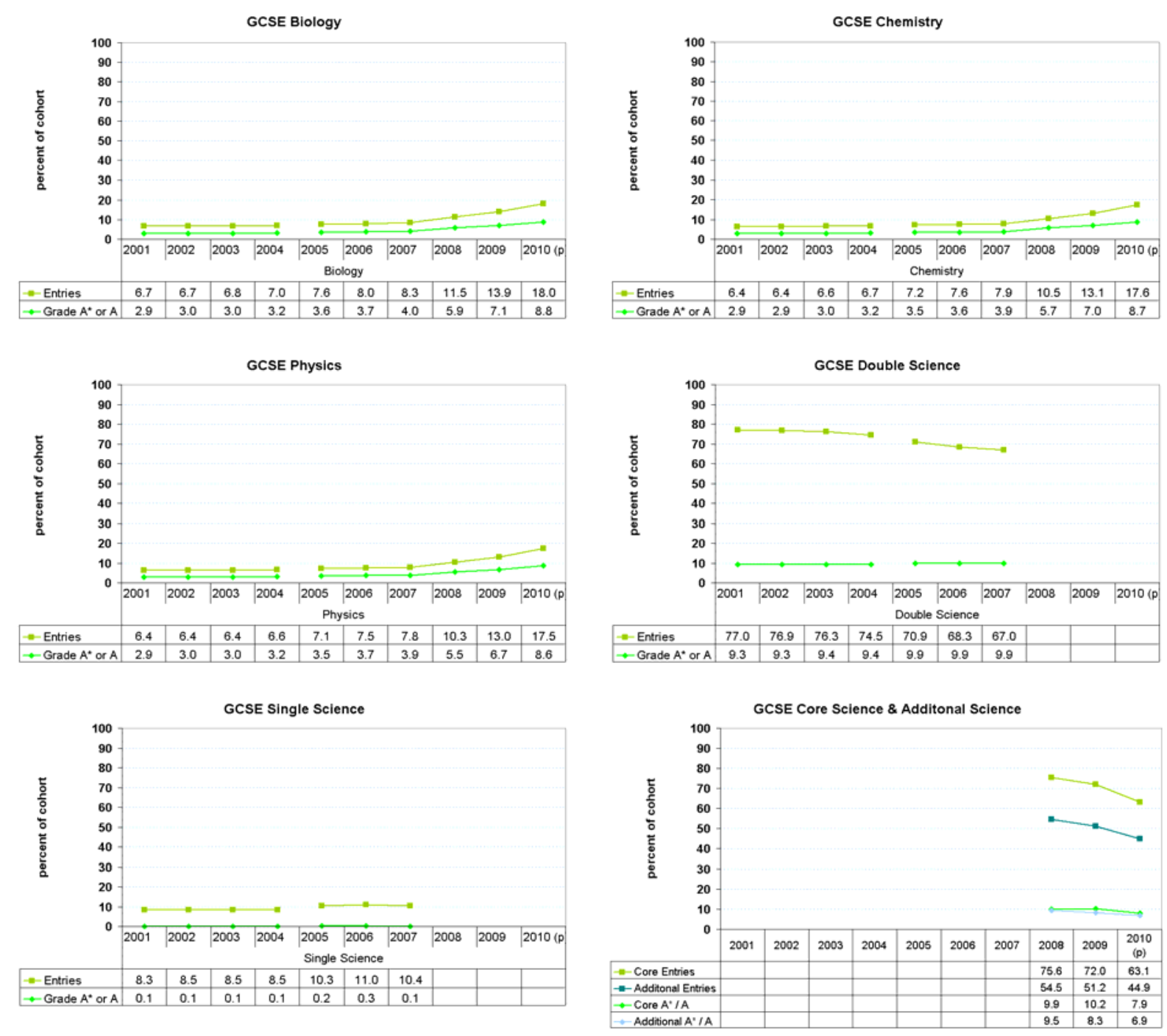

\subsection{GCSE Maths Early Entries}

This section describes analysis of early entries to GCSE maths by pupils who have not yet reached the end of year 11 . The number of early entries in GCSE maths has been increasing annually over the last few years, as has the subset of these pupils who then retake the qualification by the time they reach the end of their Key Stage 4 studies.

Of the pupils at the end of Key Stage 4 in 2009, 554 thousand (94\%) took GCSE maths only once; 23 thousand of these were early entries. Including this $4 \%$ of pupils 
who took GCSE maths early and only once, 59 thousand (10\%) in total took maths early; the remaining 36 thousand (6\%) from the early entrants group took the GCSE more than once. This multiple entry group breaks down into 32 thousand with one retake, 4 thousand with two retakes, 160 with three retakes and 14 with four retakes.

Figure 1.8a depicts the rise in early entries, subsequent retakes, and the number of those retakes which resulted in an improved grade. Of the 59 thousand early maths entries in the 2009 Key Stage 4 cohort, over half retook by the end of year 11, and one quarter eventually achieved a better grade than their first attempt.

\section{Fig. 1.8a}

\section{Early Entries to GCSE Maths, 2005-2009}

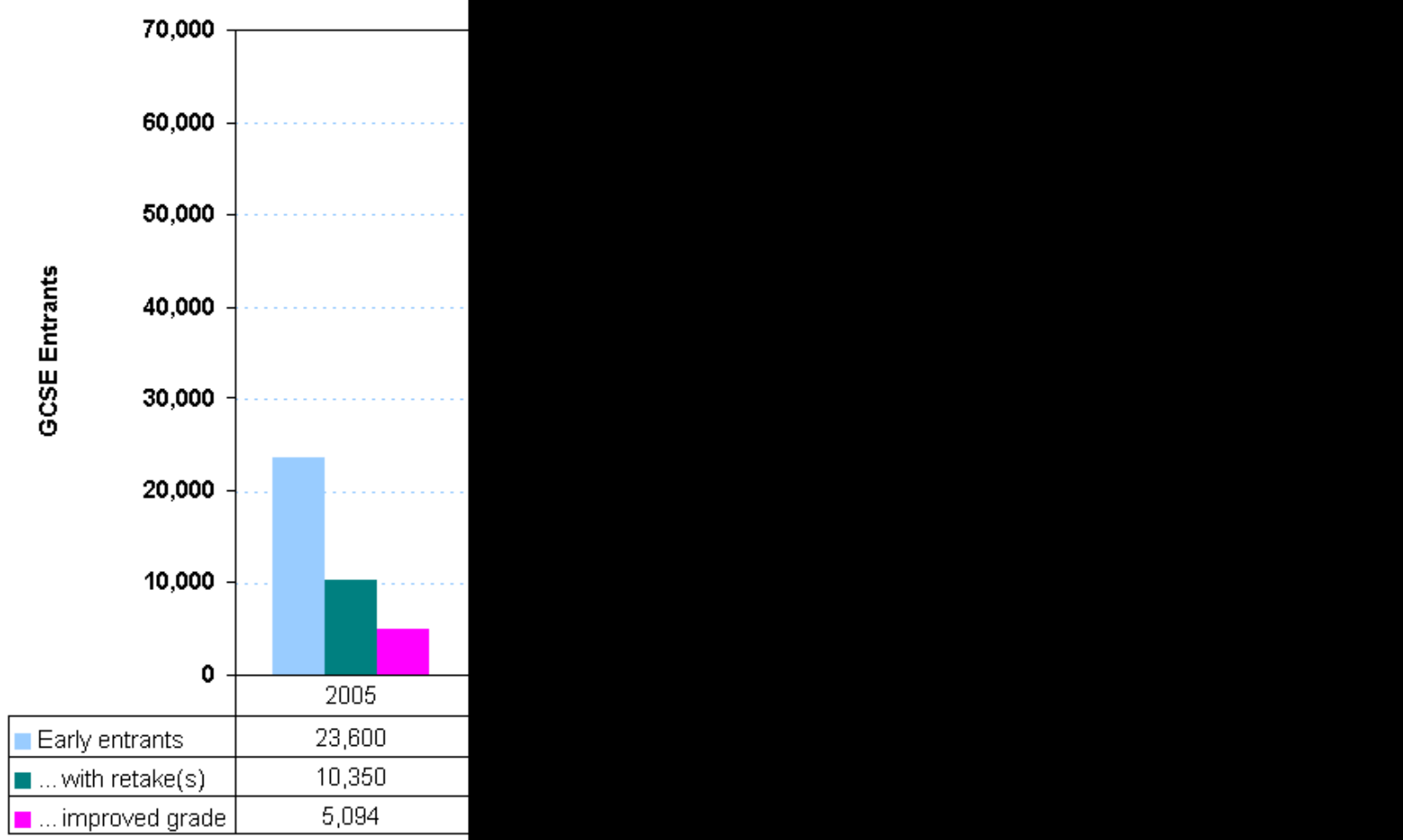


The best eventual grades achieved in GCSE maths by the 2009 Key Stage 4 cohort are plotted in figure $1.8 \mathrm{~b}$. Compared with all pupils, those who had early entries were slightly more likely to achieve an A* star grade, but less likely to achieve grades A-C, with the biggest difference for grade $B$. This reflects the fact that early entry is not only used for high achievers, but also in other instances to allow the possibility of retakes where pupils may struggle to achieve a good grade at the first attempt.

\section{Fig. 1.8b}

Highest Grades Achieved in GCSE Maths, 2009

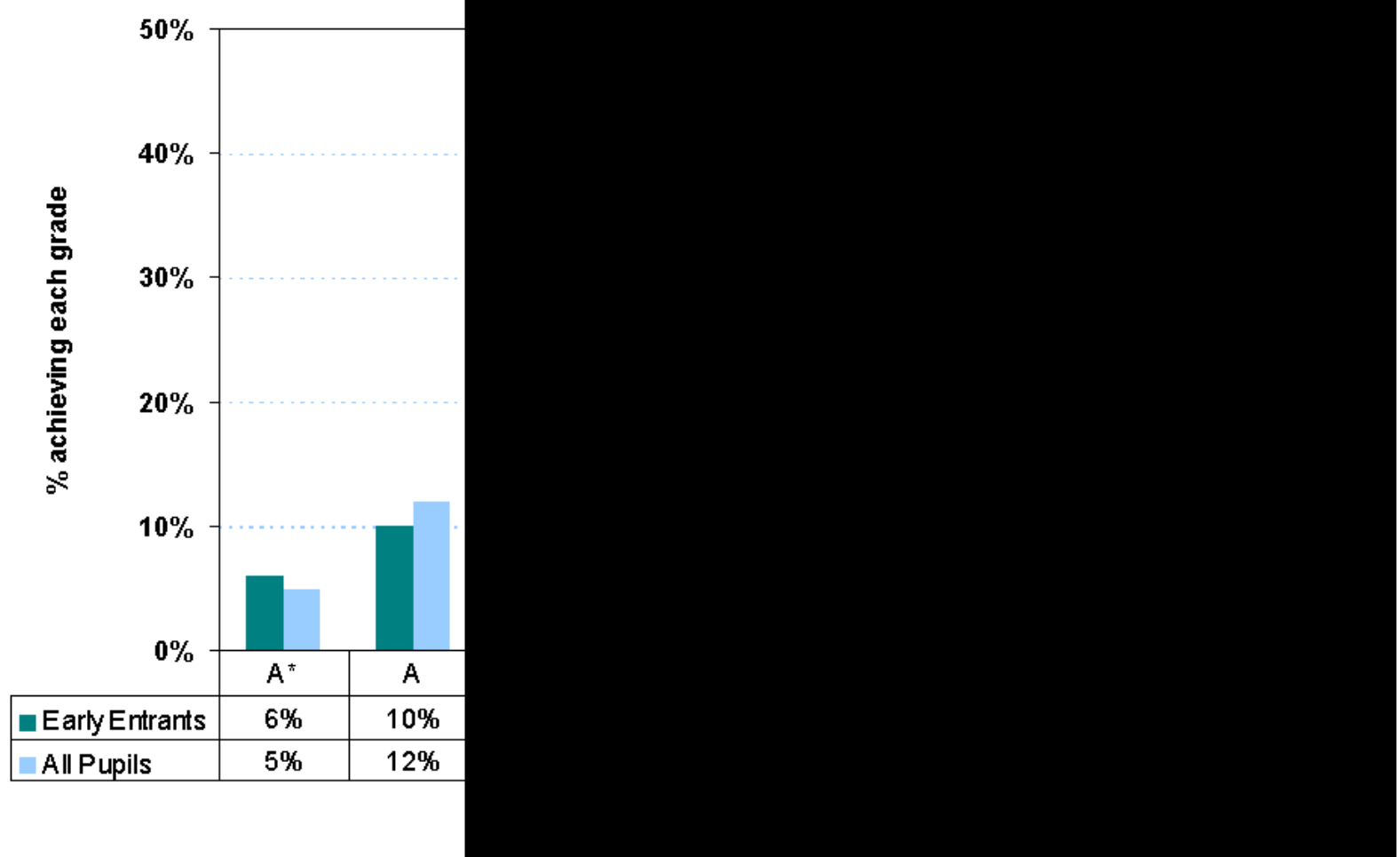


The earliest entrants, sitting GCSE maths for the first time at the end of year 10, are most likely to achieve $A^{*}$ or $A$ as their best grade in maths; on the other hand they also run the greatest risk of ending up with grades $F, G$ and $U$ (see figure 1.8c). Entrants in mid year 11 are least likely to achieve grades $A^{*}-B$ as their best grade, but are the most likely group to receive grades $\mathrm{C}$-E. Traditional end of year 11 entrants are the most likely group to achieve a grade $B$ and the least likely to receive grades F-U.

\section{Fig. 1.8c}

Highest Grades Achieved in GCSE Maths, 2009

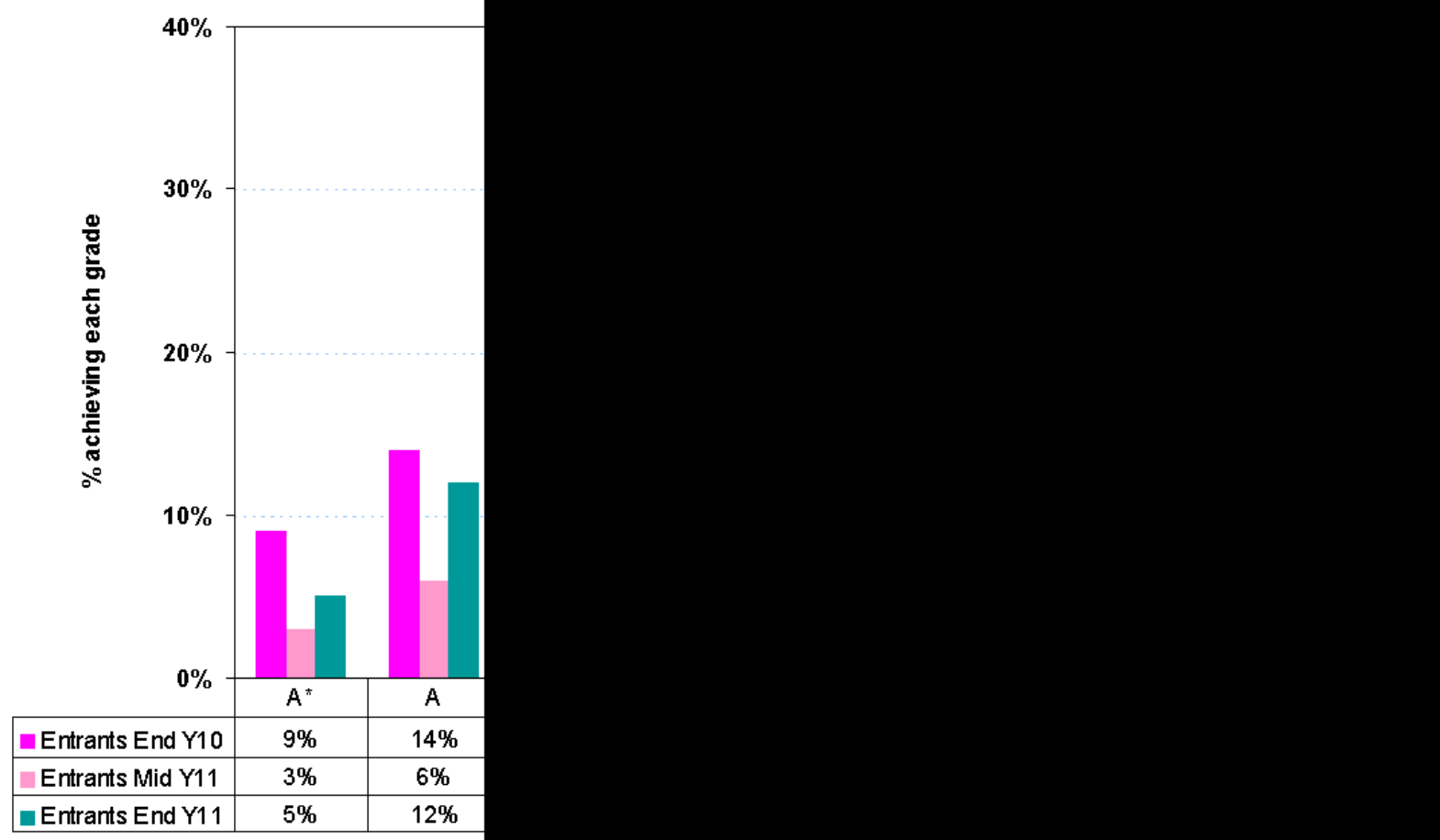


Year 10 early entrants who initially achieved grade D were the most likely of this group to retake GCSE maths by the end of year 11 (see figure 1.8d). Meanwhile, over half of pupils whose initial year 10 grade was B or C did not attempt to better this grade by retaking. This apparent level of satisfaction with a grade $C$ or better may reflect the responses of schools or pupils to the use of threshold measures of attainment in previous government targets, and more generally to define socially acceptable levels of success at GCSE.

\section{Fig. 1.8d}

Number of GCSE Maths Retakes by Early Takers, 2009

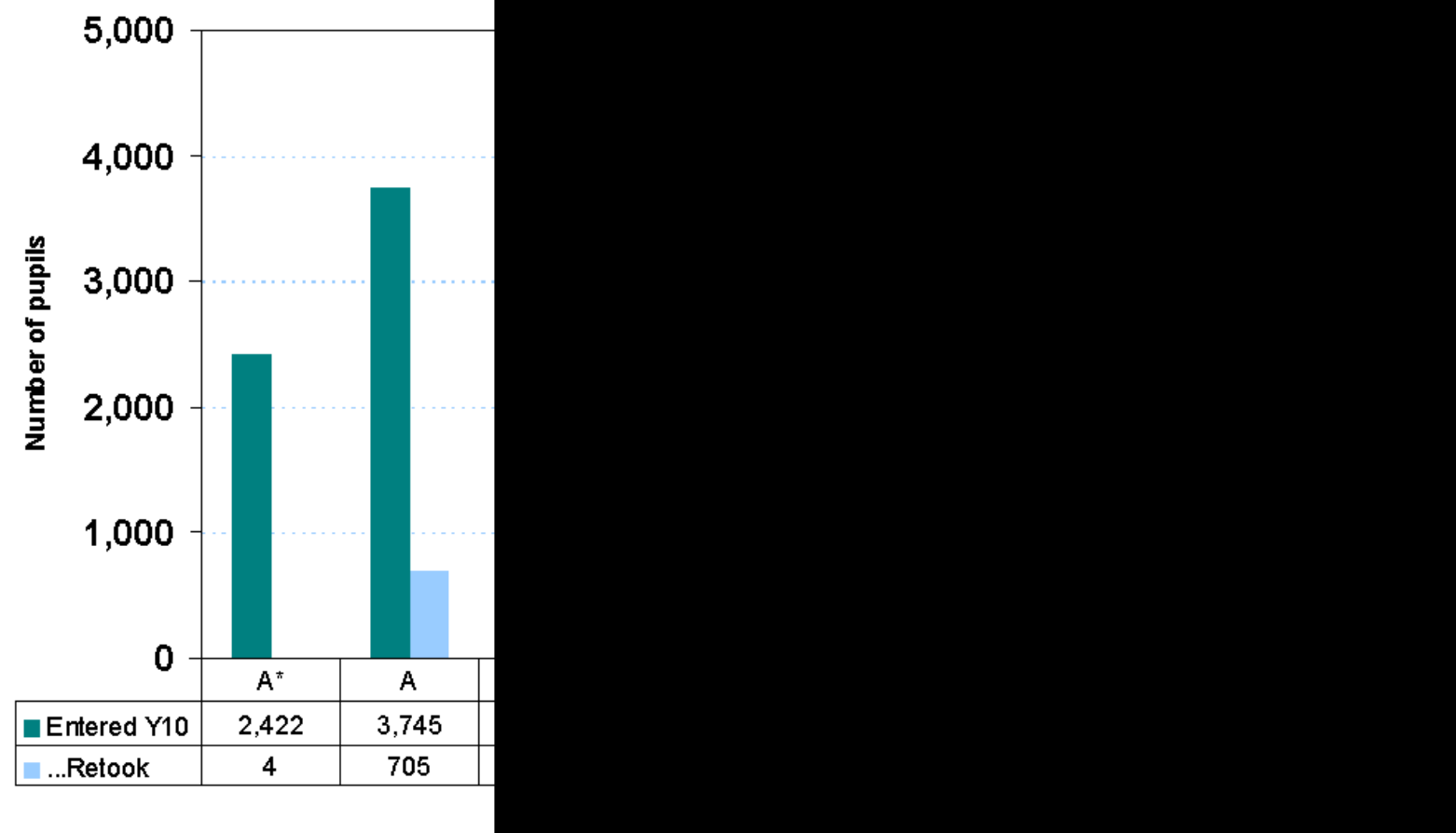

\subsection{Local Authority Variation in Outcomes at Keys Stages 4 and 5}

The national GCSE and A level maths and science participation figures reported earlier in this chapter mask considerable variation between Local Authorities. For example, in 2010 , the percentage of maintained mainstream pupils who took triple science (all three of biology, chemistry and physics) at GCSE ranged from as low as $4 \%$ up to almost ten times this rate, at 39\%. Uptake of A level maths in 2010 by the 2008 GCSE cohort varied from $1 \%$ up to $16 \%$; for A level biology this was $2 \%$ to $21 \%{ }^{4}$, for chemistry $1 \%$ to $13 \%$, and for physics $<1 \%$ to $11 \%$. Annex D contains underlying data for the following charts as well as additional context variables for each LA.

Figure 1.9a maps the relationship between triple science GCSE and A level science entries at LA level. The association between GCSE triple science and A level uptake

\footnotetext{
${ }^{4}$ City of London had an even higher rate of biology continuation at 33\%, but this is excluded from the reported range as this LA had fewer than 200 KS4 pupils, all of whom attended independent school.
} 
is strongest for physics $\left(r^{2}=0.26\right)$, followed by chemistry $\left(r^{2}=0.19\right)$, then biology $\left(r^{2}\right.$ $=0.15$ ); these are relatively small correlations, but nevertheless there is a clearly visible pattern of higher A level entry in Local Authorities where triple science is more prevalent.

Fig. 1.9a

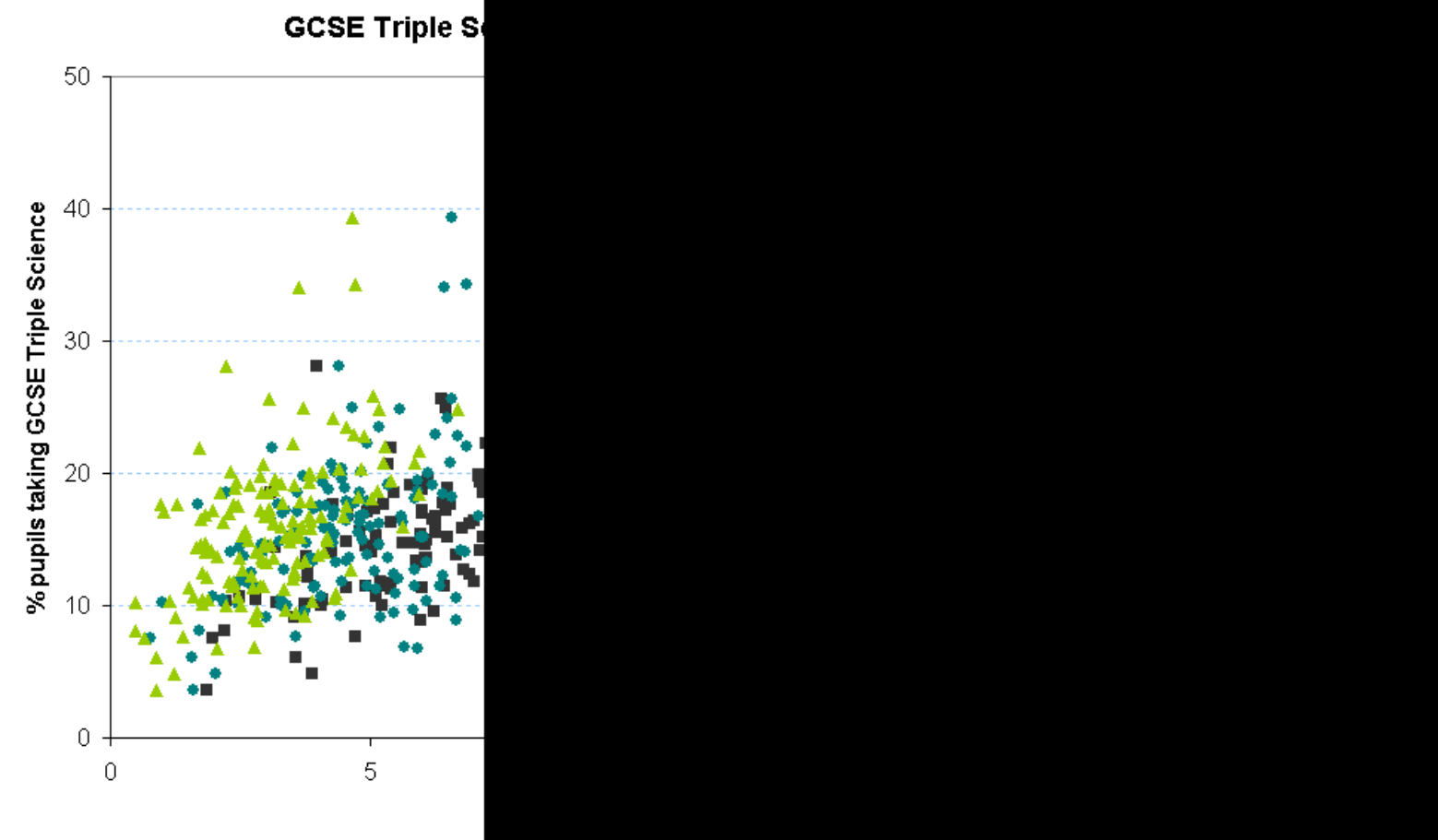


Figure 1.9b charts the relationship between the levels of deprivation in the areas in which key Stage 4 pupils live, and their rates of maths and science entry at A level. There is no correlation between IDACI (Income Deprivation Affecting Children Index) scores and uptake of biology or chemistry at A level $\left(r^{2}<0.02\right)$. For physics and maths however, there is a small relationship between deprivation and uptake, with Local Authorities characterised by higher levels of deprivation exhibiting smaller proportions of pupils continuing to these A level subjects (physics $r^{2}=0.14$; maths $r^{2}$ $=0.07)$.

\section{Fig.1.9b}

Area Deprivation and A level Science Entry at LA level (2010)

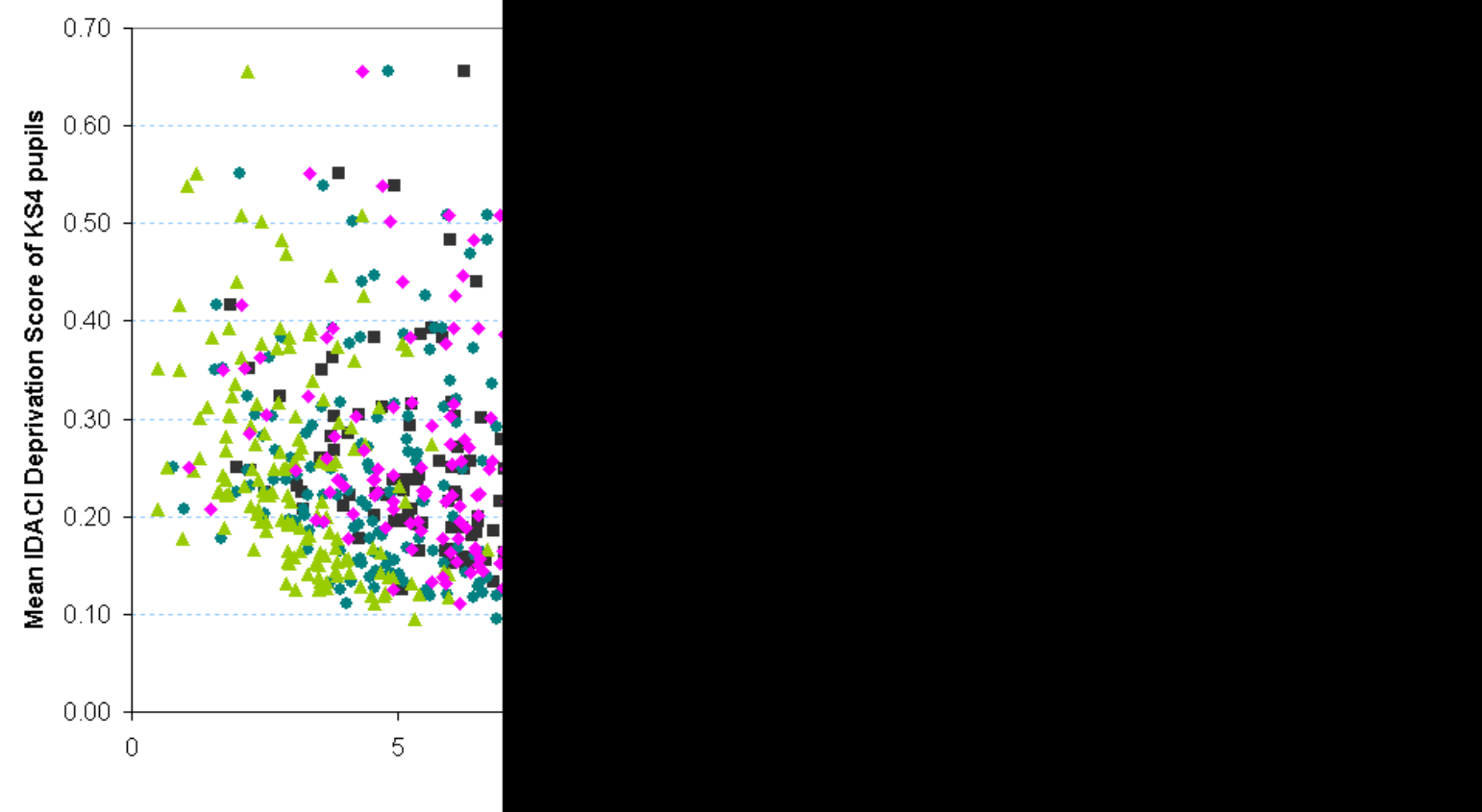




\section{Chapter 2. Prior Attainment, Continuation \& Progression}

This chapter examines the importance of prior attainment in influencing the likelihood of continuing to A level and of subsequently achieving grades A or B in maths and science subjects. Section 2.1 presents the rates of participation in maths, biology, chemistry and physics A levels as percentages of the cohort as a whole, contrasted with continuation for high attainers (those achieving above the expected level) at each prior Key Stage.

Sections 2.2 to 2.5 shift the focus from A level entries to progression to top grades at A level in each of the subjects, and from each prior Key Stage, comparing the incidences of A level achievement for high prior attainers with achievement of the cohort as a whole. The effects of choice and selection can be seen through the contrasting of cohort incidence rates, and rates of incidence amongst those who actually went on to enter the relevant A level subject. Grade A and B achievements are then split to give placements by prior attainment at each Key Stage; this analysis reveals the attainment histories of the largest groups of pupils who go on to achieve high grades at A level.

Finally, section 2.6 draws together progression across Key Stages 1-4, and section 2.7 assesses the extent of path dependence in reaching GCSE as a high attainer in maths or science.

Coverage All analyses in this chapter cover maintained mainstream schools cohorts at the relevant Key Stage. Complete prior attainment data are available for this group in most cases. Special schools and independent schools are not included in the analysis. Years reported are the KS5 A level exam year for the cohort; where a 2009 result is reported, this refers to percentages of the 2007 GCSE cohort, i.e. the scope is pupils continuing directly to KS5 after GCSE and completing A levels in two years. 


\subsection{Continuation Across the Key Stages}

Chart 2.1a presents the percentages of maintained mainstream pupils who went on to enter a full A level in maths in 2007-2009. The strongest Key Stage influence on continuation by a significant margin is prior attainment at Key Stage 4 (GCSE). In $2009,47 \%$ of high attainers at GCSE maths continued to A level maths, compared with $26 \%$ who achieved above the expected level at Key Stage 3, 22\% who exceeded the expected level at Key Stage 2, and 21\% who did so at Key Stage 1. This compares with a continuation rate of $7 \%$ for the entire cohort; hence high prior attainers at KS4 were nearly seven times as likely as average to enter A level maths, and those who exceeded the expected level at Key Stages 1-4 were three to four times as likely to continue to A level maths.

Fig. 2.1a

Continuation to A Level Maths, All Pupils Compared with High Prior Attainers

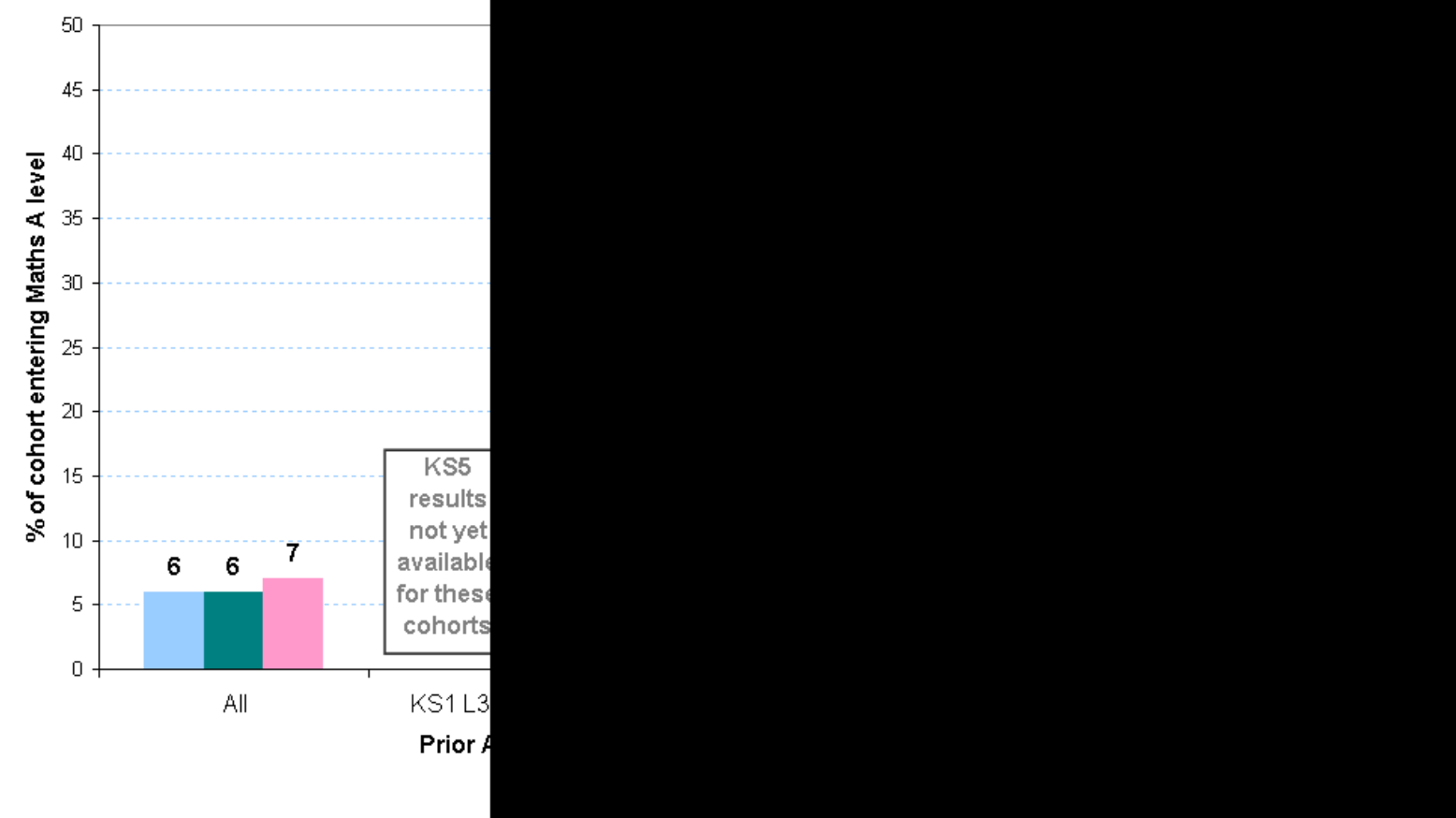


Turning to the numbers of A level maths entries contributed by the high prior attaining groups, figure 2.1b illustrates the dominance of the high attaining groups at Key Stages 2-4. As there are a larger number of high attainers in maths at KS3 than at GCSE, pupils with level 7+ at KS3 outnumber those with grades $A^{*}$ or A at GCSE despite the much stronger progression rate for the GCSE high attainers. The vast majority of A level maths entrants have a history of high attainment in the subject that stretches back at least as far as the end of primary education.

\section{Fig.2.1b}

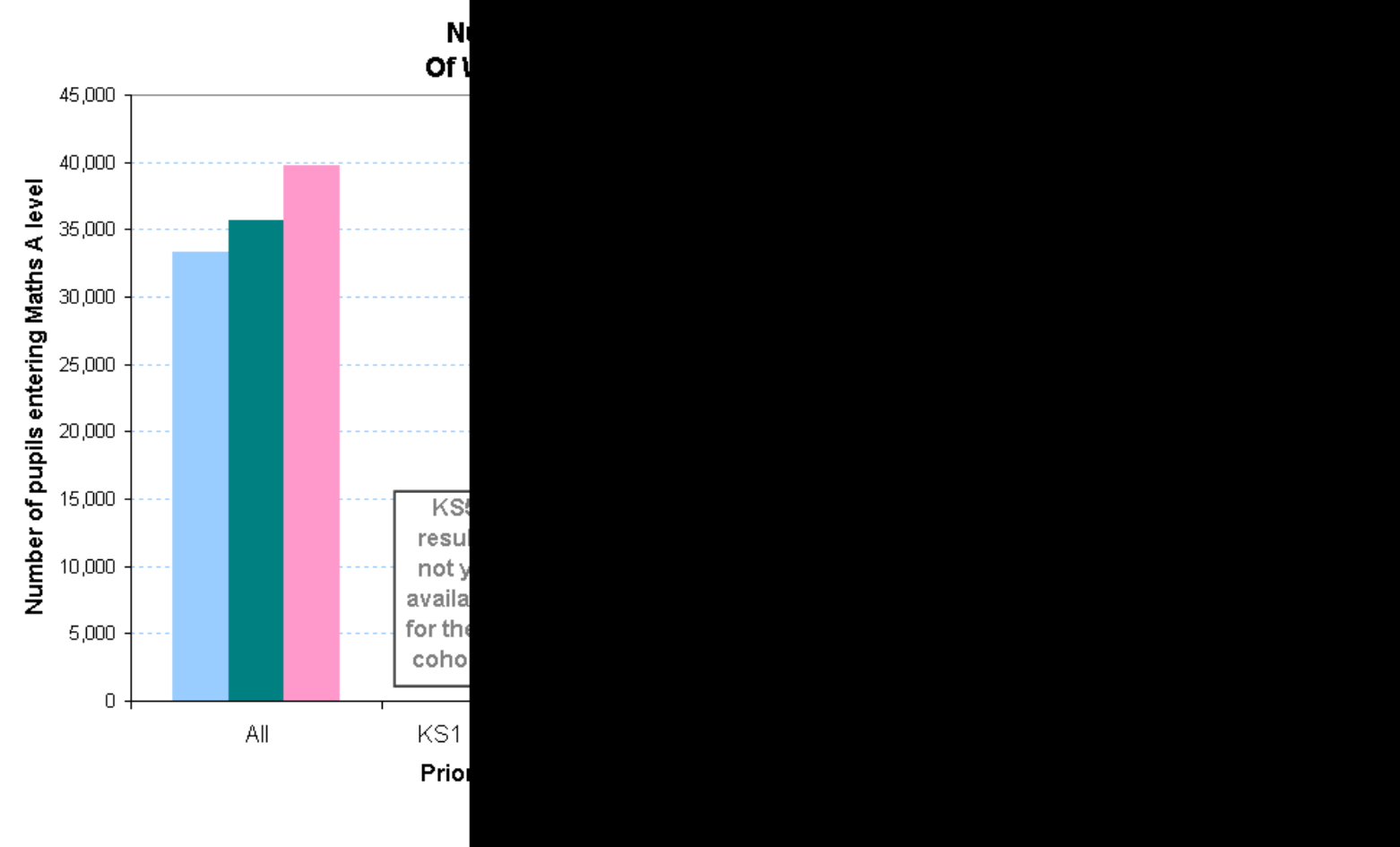


Moving on to biology A level continuation, figure 2.1c compares the rates of entry for high prior attainers in science and the cohort as a whole. Again, Key Stage 4 provides the strongest influence on continuation, although the margin between KS3 and GCSE prior attainment is much smaller than was the case for maths, mainly due to weaker continuation of the GCSE high attainers in biology than maths; KS2 science attainment is noticeably less influential than was the case for maths.

For A level biology entrants in $2009,32 \%$ of pupils who had achieved grade $A^{*}$ or $A$ at GCSE science or biology continued to A level. For KS3 high attainers (with level $7+$ in science), the continuation rate was $26 \% ; 12 \%$ of KS2 high attainers continued, as did the same percentage of KS1 high attainers. These rates of continuation mean that GCSE high attainers were six times as likely to enter A level biology as the cohort average of $5 \%$; KS3 high attainers were 5 times as likely to continue to A level, and KS2 and KS1 high attainers were both twice as likely as the average.

Fig. 2.1c

Continuation to A Level Biology, All Pupils Compared with High Prior Attainers

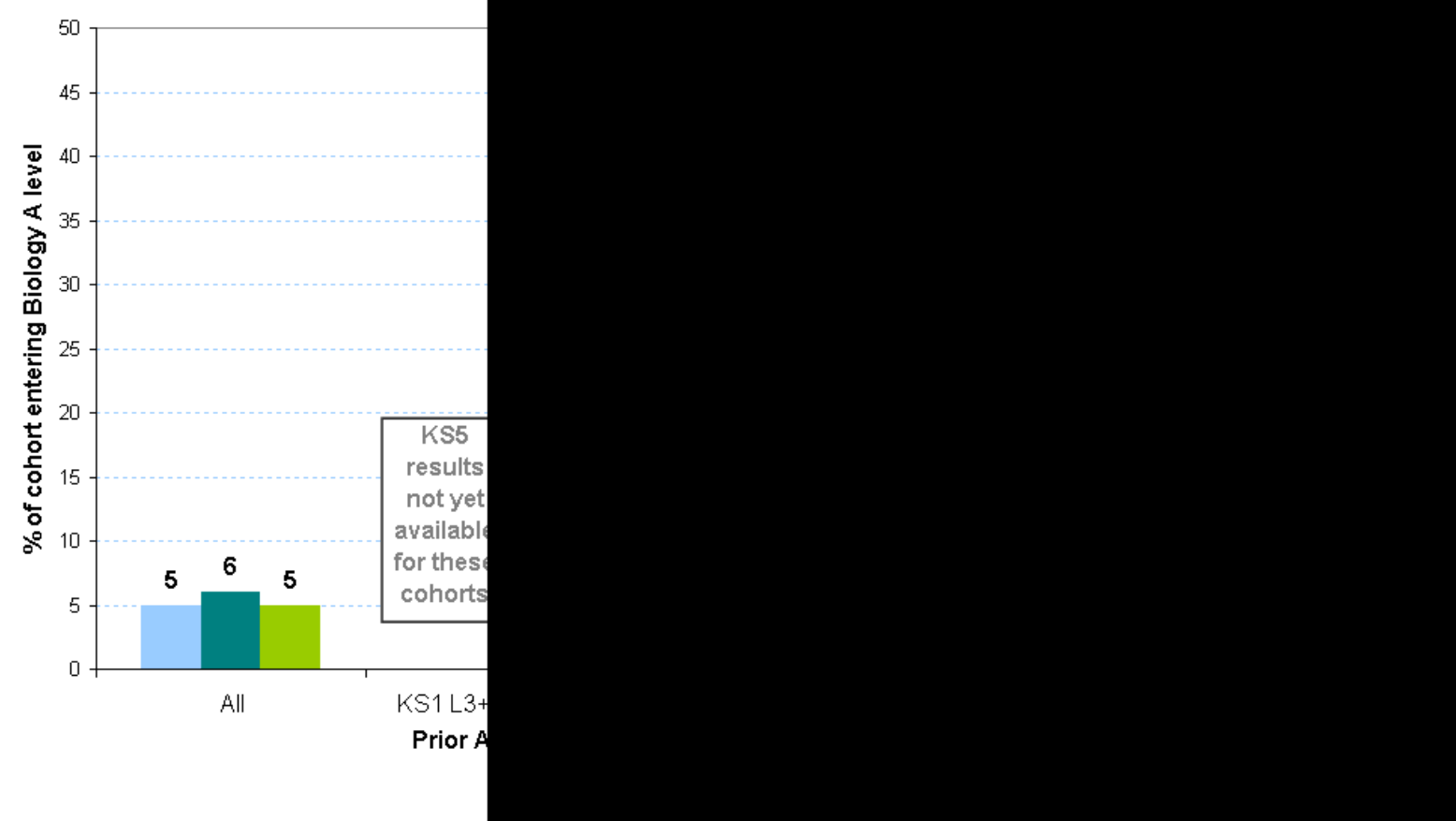


It can be seen from figure 2.1d that the total number of continuations to A level biology contains a lower share of high prior attainers from Key Stages 3 and 4 than was the case for maths. Pupils who had attained above the expected level in science at Key Stage 2 contribute a larger number of A level entries than the higher attainers at the later Key Stages despite having a lower rate of continuation as seen in figure 2.1c. These effects reflect the differing numbers of high attainers at each prior Key Stage forming smaller or larger pools from which the high attainers continuing to A level are drawn. The large number of pupils who attained level $5+$ at KS2 in science results in a significant contribution to the total A level entrants number despite this group only having a continuation rate of $12 \%$.

Fig. 2.1d

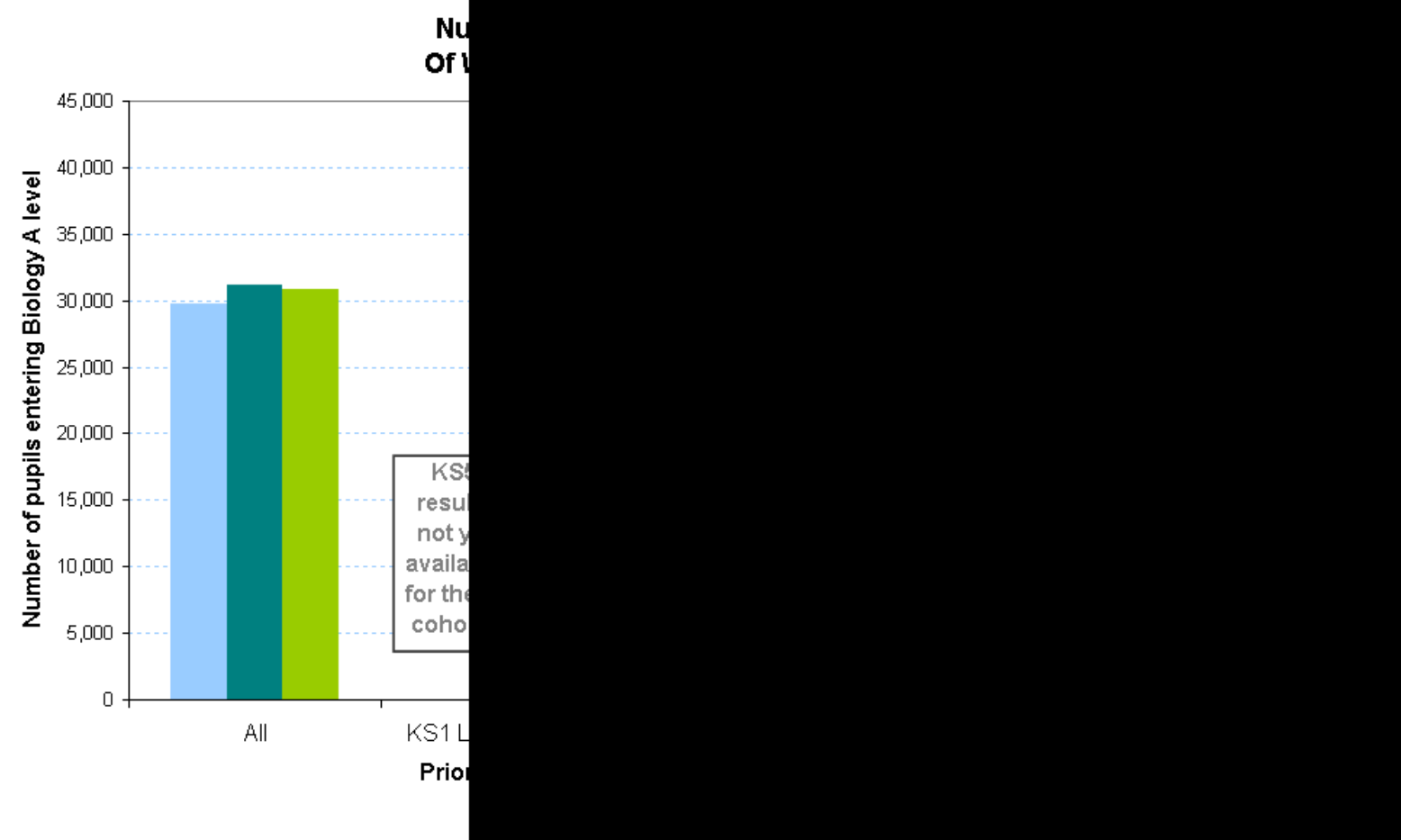


Continuation to A level chemistry for high prior attaining groups is presented in figure 2.1e. The pattern of lower A level entry than for maths is evident here, and the increased importance to the chances of A level entry of Key Stage 3 attainment, relative to GCSE attainment, can be seen (as was the case for biology continuation). Of the KS4 cohort expected to reach A level in 2009, 29\% of those who attained grades $A^{*}$ or $A$ in GCSE chemistry or science went on to take A level chemistry. The rate of A level continuation for KS3 high attainers was 23\%, compared with $9 \%$ for KS2 high attainers, and 10\% for those who exceeded the expected level at KS1. The chances of continuation are thus seven times as high as the cohort average of $4 \%$ for GCSE high attainers, six times as high for KS3 high attainers and 2-3 times as high for KS2 and KS1 high attainers.

\section{Fig. 2.1e}

Continuation to A Level Chemistry, All Pupils Compared with High Prior Attainers

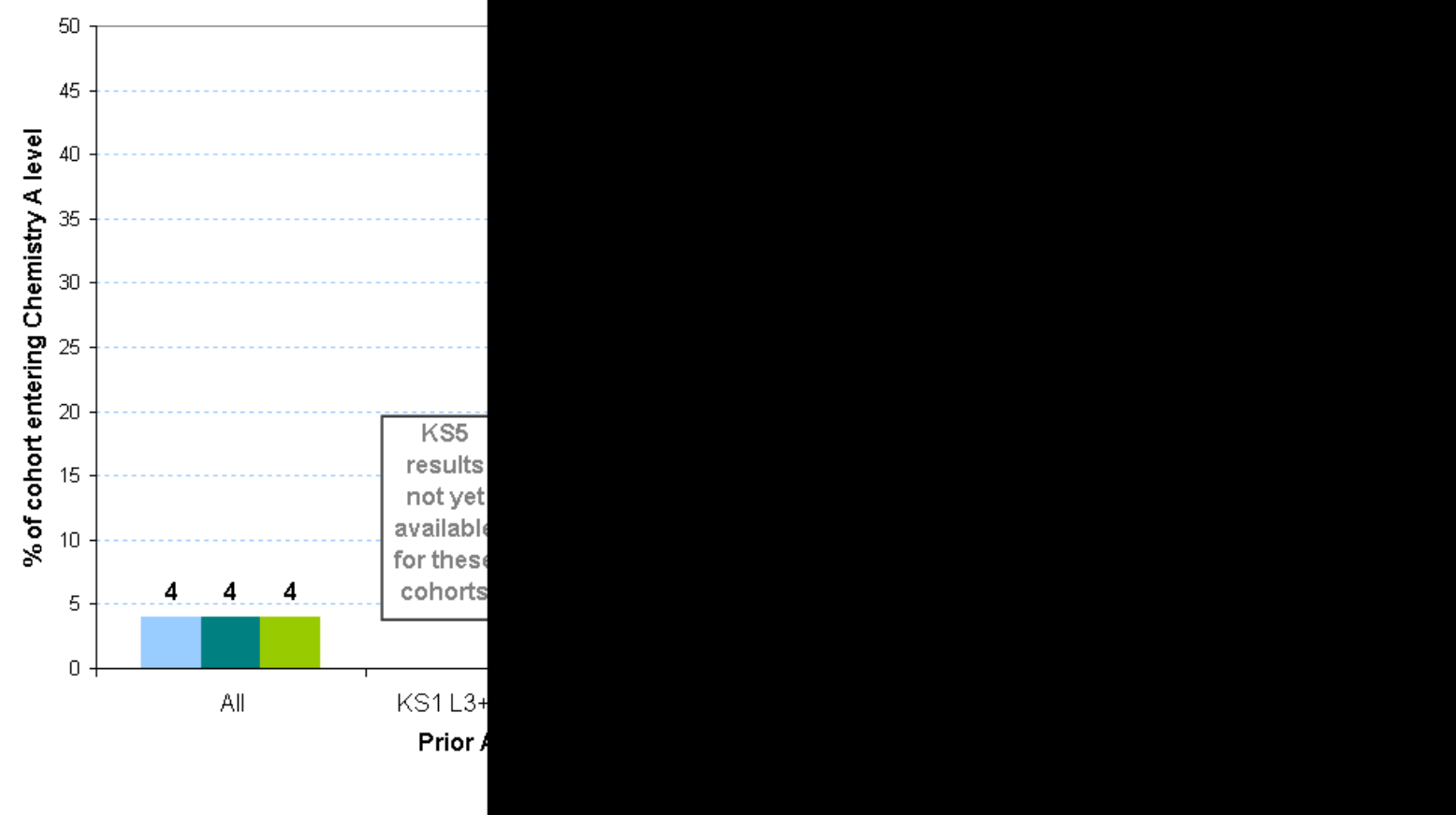


As with maths and biology, a high share of A level Chemistry entrants from the maintained mainstream schools cohorts were high prior attainers at Key Stages 2-4. Echoing the pattern in biology, Key Stage 2 high attainers contribute the largest share of the cohort's entries to A level chemistry, reflecting a larger pool of high attainers in science at this level rather than the rate of continuation for the group. Pupils achieving grades $A^{*}$ or A at GCSE chemistry or science contribute the next largest number of the A level chemistry entrants, followed by those who exceeded the expected level of attainment at Key Stage 3. Less than half of A level continuers had achieved level 3+ in science at KS1, this key stage being the least important contributor as with biology.

\section{Fig. 2.1f}

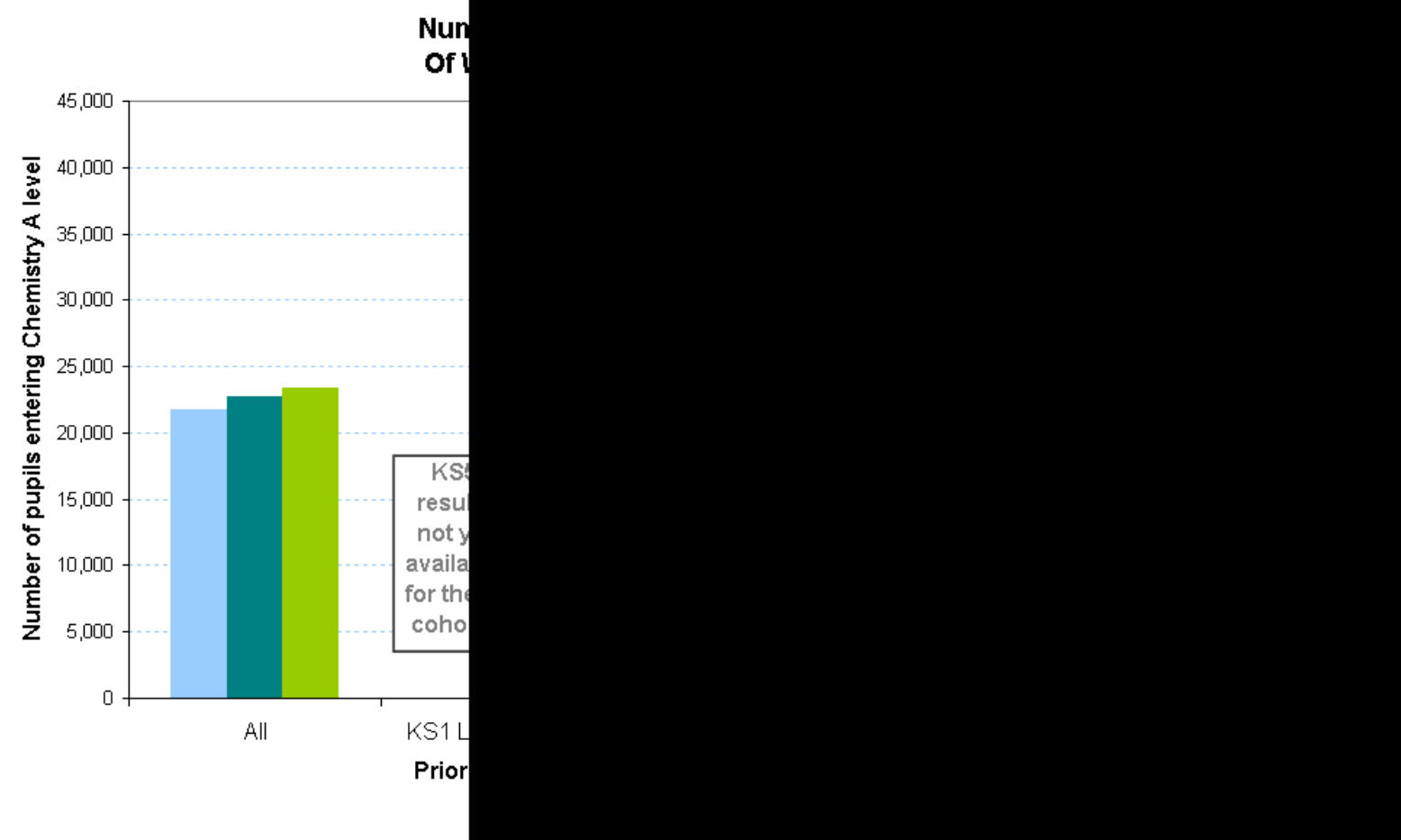


The likelihood of continuing to A level physics for high attainers in science at Key Stage 3 is very similar to that of GCSE high attainers, making KS3 more important for physics $A$ level entries than for entries in biology or chemistry. Of pupils gaining grades $A *$ or $A$ at GCSE physics or science, $19 \%$ went on to enter for physics $A$ level, closely followed with $18 \%$ continuation by those with level $7+$ in KS3 science; and $7-8 \%$ continuation by high attainers at KS1 and 2. Compared with the cohort average continuation rate of 3\%, the GCSE and KS3 high attainers were both six times as likely to enter A level physics; KS1 and 2 high attainers were 2-3 times as likely as the average to continue to physics $A$ level.

\section{Fig. 2.1g}

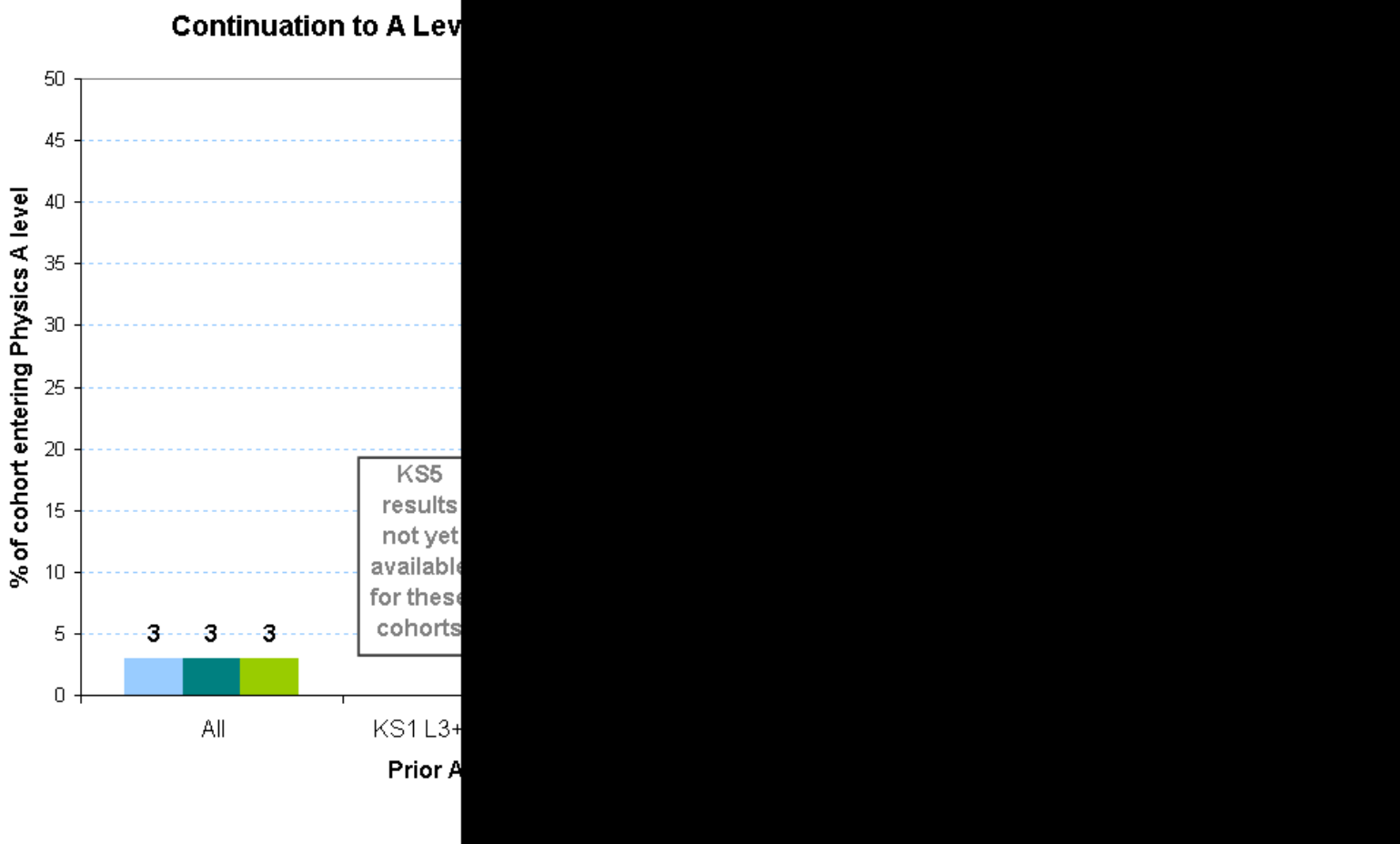


As with biology and chemistry, Key Stage 2 high attainers contribute the largest share of the cohort's entries to A level physics, reflecting the larger pool of high attainers in science at that Key Stage. Pupils who achieved grades $A^{*}$ or A at GCSE chemistry or science and those with level $7+$ at KS3 made up similar number of the $A$ level chemistry entrants, with KS1 high attainers contributing less than half of A level chemistry entries.

\section{Fig. 2.1h}

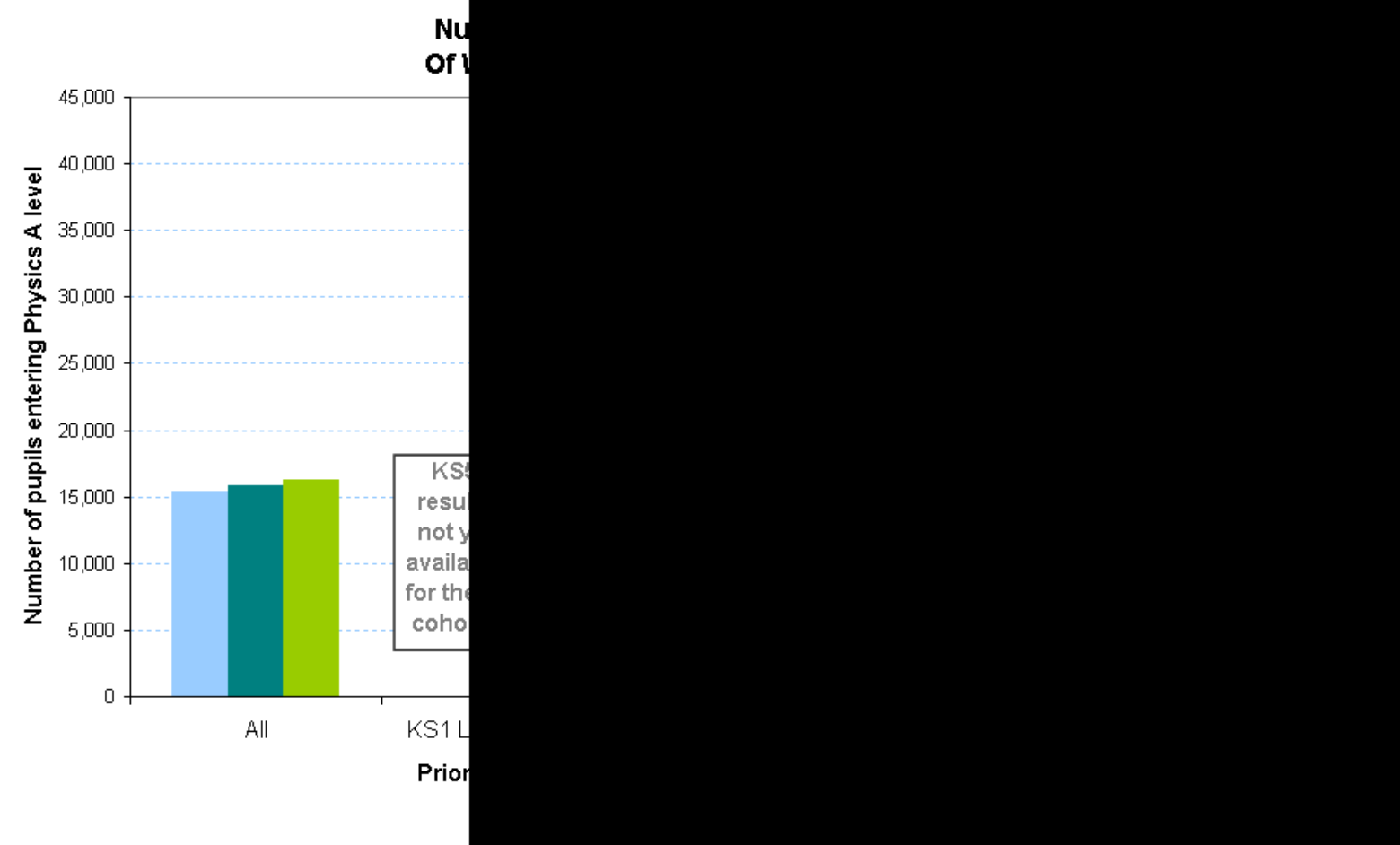




\section{Comparisons Across Key Stages and Between Subjects}

\section{Threshold Attainment Measures}

The Key Stage measures (\% of pupils achieving various levels) have been used to capture different groups of pupils in the preceding analyses of $A$ level continuation. However, certain characteristics of these measures mean that they should be interpreted with care when making comparisons across Key Stages or between different subjects; this is because the current average attainment for these different subjects / Key Stages varies, meaning that smaller or larger continuation percentages may be expected due to the choice of measures rather than the size of the prior attainment effects.

\section{Odds Ratios}

Odds Ratios are used below as a way of quantifying the differences between pupils with high prior attainment within the maths and science subjects, and those with lower prior attainment - they provide a like-for-like comparison of groups with different underlying levels of attainment, and for different subjects or Key Stages where the national attainment level is currently at a higher or lower percentage.

An odds ratio of greater than 1, for high attainers compared with non-high attainers, would suggest that high prior attainment is positively associated with A level entry; similarly an odds ratio of less than 1 would imply a lesser association with continuation.

Figure 2.1i presents an odds ratio comparison of the association between prior attainment and continuation to A level for maths, biology, chemistry and physics. From the pattern of odds ratios plotted, it is clear that the importance of high prior attainment increases with age and as the pupils get closer to the end of compulsory education. This is not surprising as pupils are likely to have more regard to their most recent attainment performance when considering post-16 options, and their GCSE attainment is likely to act as a filter for the sorts of further study or employment options available to them.

The odds of continuing to study at A level for high attainers at KS1 are 3-8 times as high as those for pupils achieving at the expected level or below. This rises to between 7 and 28 times the odds by KS2, and an order of influence emerges between maths and the sciences, with maths continuation being the most dependent on prior attainment, followed by physics, and with chemistry and biology being the least dependent. There is a smaller increase in the importance of prior attainment between Key Stages 2 and 3, with the odds of A level entry for high prior attainers rising to between 11 and 35 times those of pupils with the expected level of achievement or below.

By GCSE, the odds of A level progression for the high attainers have reached between 23 and 88 times those of the other pupils, with the chemistry ratios overtaking those of physics and biology, but the maths odds ratio now being significantly larger than those for the science subjects. From this, it can be concluded that maths GCSE attainment has an exceptionally strong influence on whether a pupil goes on to enter A level maths, with GCSE chemistry attainment also having a very strong influence on continuation in that subject. Attainment at all Key 
Stages is important for A level participation, in all four maths and science subjects, but especially from Key Stage 2 onwards.

\section{Fig. 2.1i}

Continuation to A level Entry - Comparing Across Subjects and Key Stages

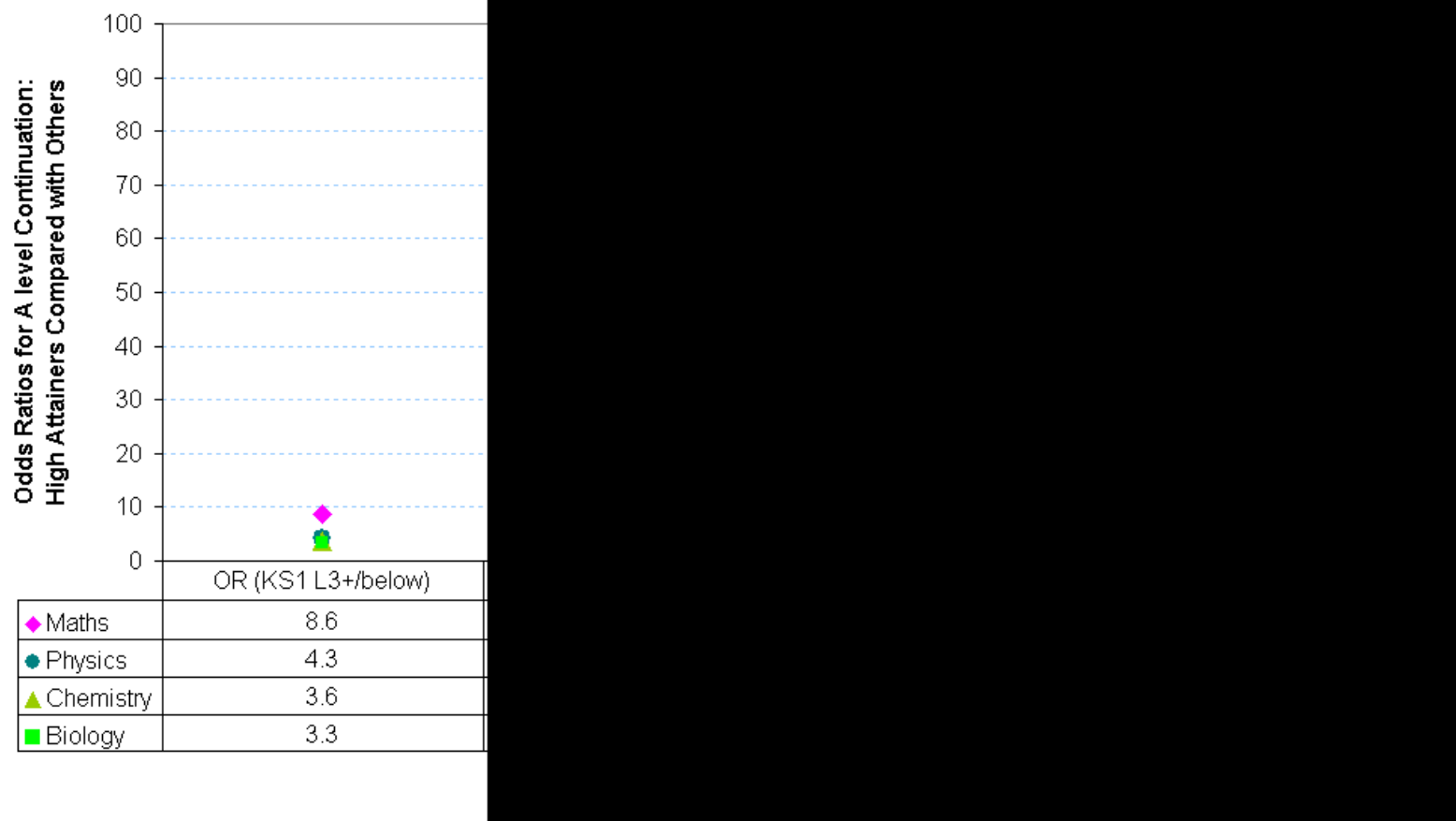




\subsection{GCSE Attainment and Progression to A level}

A level maths and science achievements can also be broken down according to prior attainment groups to analyse the progression from each prior Key Stage. The first chart displayed for each maths and science subject answers the question: what was the A level grade achieved by entrants with a given level of prior attainment at GCSE?

Figure 2.2a shows the percentage of A level maths entrants, split by GCSE maths attainment, who went on to achieve grade A, grade B, and grades below B. In 2009, of entrants from the maintained mainstream cohort with GCSE grades B or below, just $7 \%$ achieved grade $A$ at $A$ level, $17 \%$ achieved grade $B$ and the remaining $76 \%$ had results below grade $B$ (including ungraded). The rate of achievement of $A$ grades was almost seven times as high for the grade $A^{\star} / A$ GCSE maths attainers at $46 \%$, with $24 \%$ achieving grade B and $30 \%$ awarded grades C or below.

\section{Fig. 2.2a}

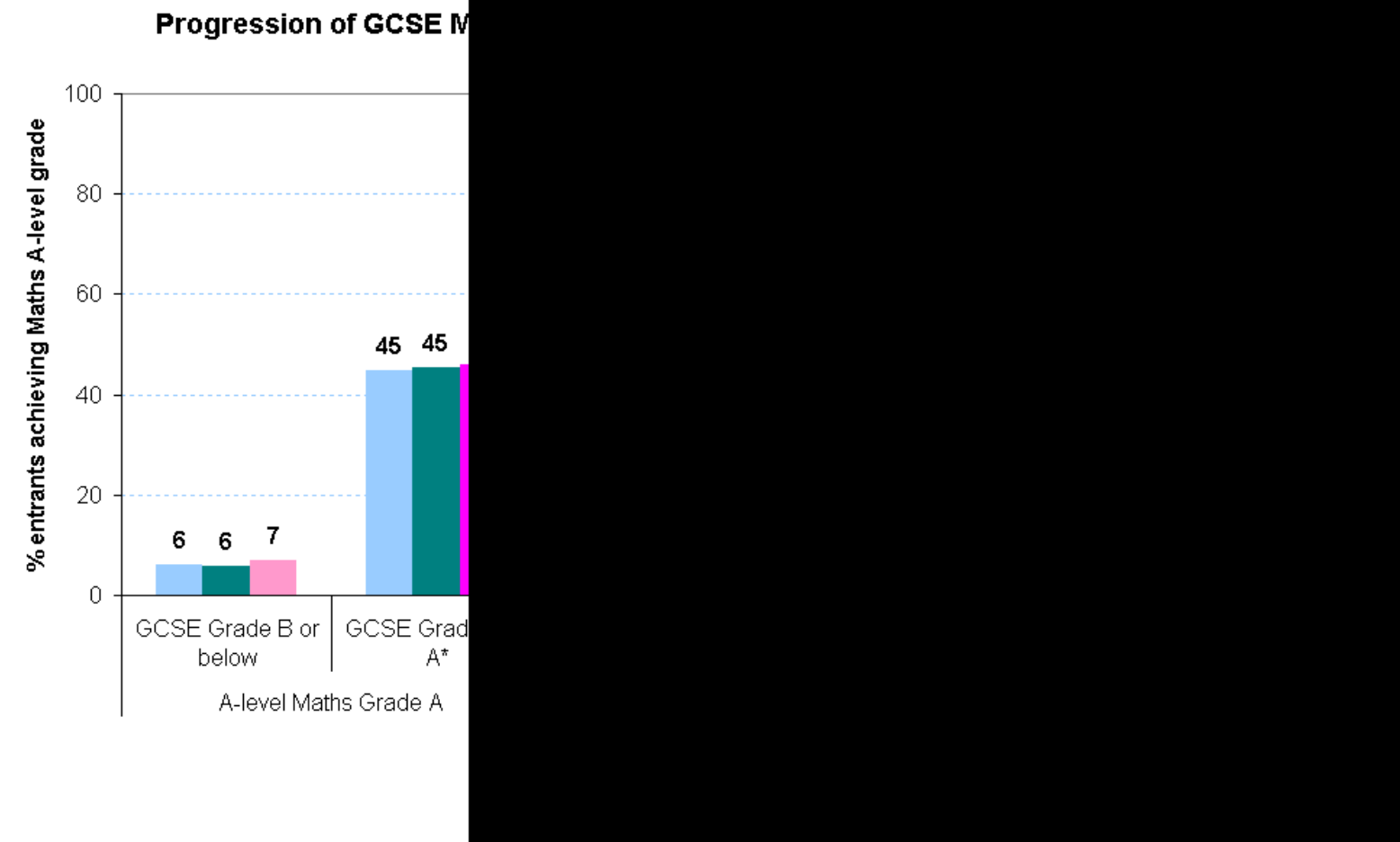

Charts showing the numbers of pupils progressing to A level, corresponding with the percentages in figure 2.2a, and its equivalents throughout the chapter, can be found in Annex A.

Figure $2.2 \mathrm{~b}$ takes the same data and expresses the numbers of entrants who were high attainers at GCSE maths, as a percentage of the group with a given subsequent outcome at $A$ level maths. In other words, we ask: what was the prior attainment of those who went on to achieve a high grade at A level maths?

The large (blue) pie on the left splits all cohort entries to A level maths into those achieving grades $A$ or $B(64 \%)$ and those with results below grade $B-9 \%$ with GCSE grades other than $A^{*}$ or $A$, and a further $27 \%$ who achieved $A^{\star} / A$ at GCSE but 
slipped to below grade B at A level. The smaller (pink) pie on the right breaks down the $64 \%$ into As and Bs, revealing that $40 \%$ achieved the highest possible $(A)$ grade at $A$ level after achieving $A^{*}$ or $A$ at GCSE; $1 \%$ came from grade $B$ or lower at GCSE to achieve an $A$ at $A$ level; $21 \%$ slipped to grade $B$ at $A$ level after achieving $A^{*}$ or $A$ at GCSE; the final $2 \%$ achieved grade $B$ at A level coming from grade B or lower at GCSE.

\section{Fig. 2.2b}

\section{A Level Maths Entries by Grade and GCSE Prior Attainment, 2009}

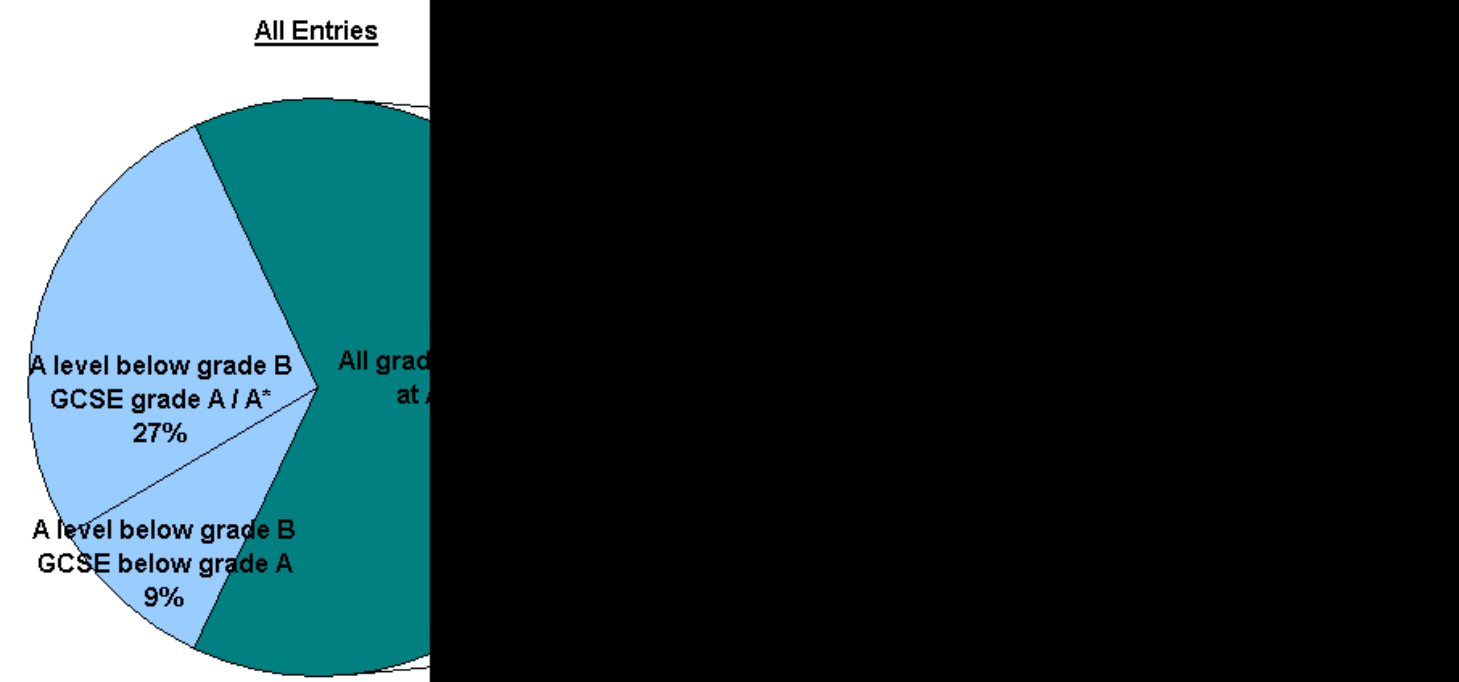

Figure 2.2c presents the percentages of the complete maintained mainstream GCSE cohort who went on to achieve high grades at $A$ level maths. This takes into account the proportions of pupils who selected themselves out of maths at A level, either because they didn't continue to Key Stage 5, because they took qualifications other than A levels, or because they chose to study other subjects.

Of the complete GCSE cohort, $3 \%$ went on to achieve a top A grade at A level maths in 2009, with high maths attainers at GCSE seven times as likely to achieve this, at $21 \%$. A further $2 \%$ of the cohort achieved grade B at A level maths, compared with $11 \%$ of the high attainers at GCSE, or five times the overall percentage. The remainder of $A$ level maths entries with results at grade $C$ or lower made up 3\% of the GCSE cohort, or $14 \%$ of the high attaining group at GCSE maths. 


\section{Fig. 2.2c}

Progression of GCSE Maths Cohorts to A-level Maths for 2007-2009, \% of Cohort

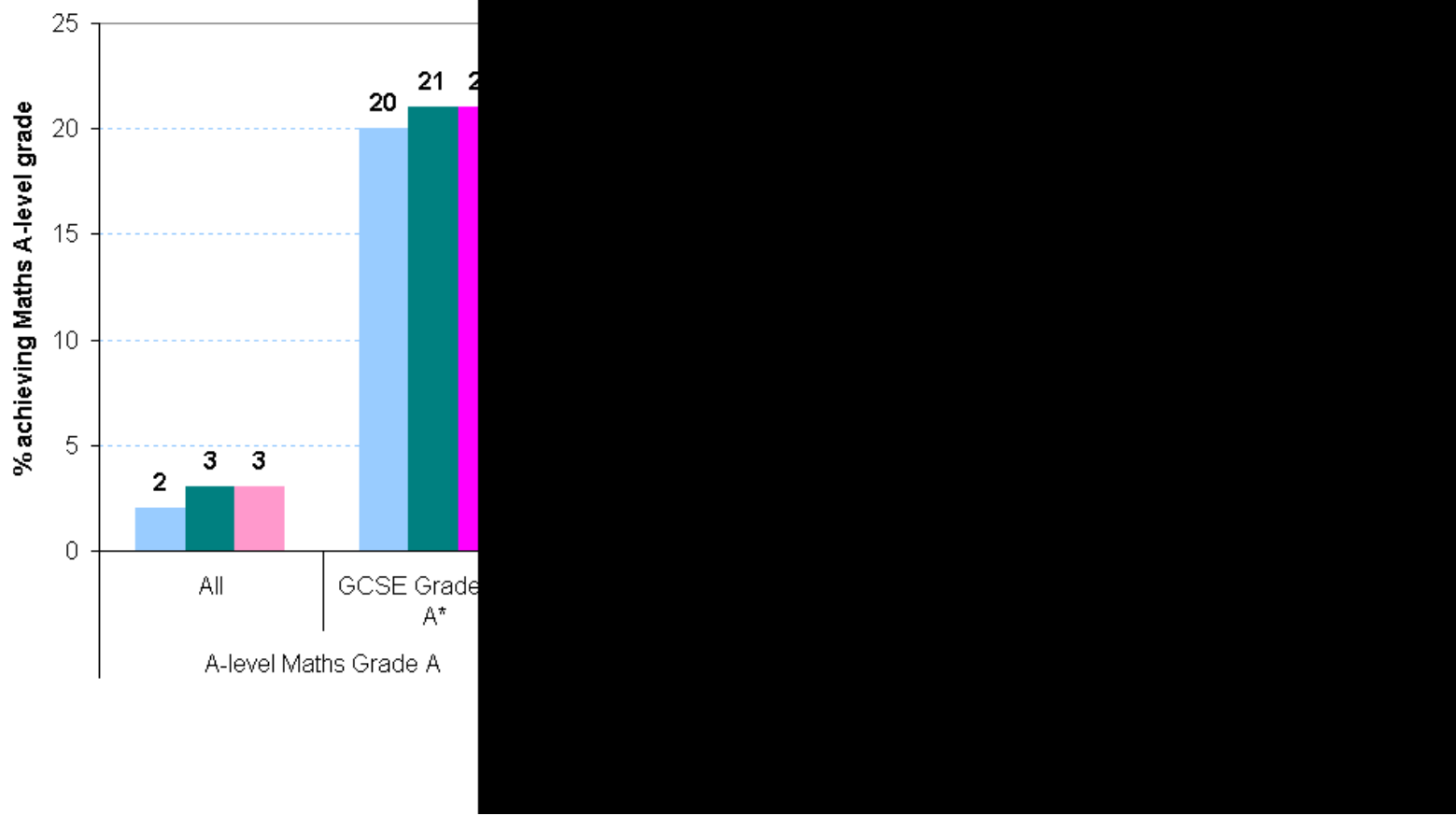


Turning to A level biology progression analyses, figure $2.2 \mathrm{~d}$ shows the percentage of biology entrants, split by GCSE science or biology attainment, who went on to achieve grade $A$, grade $B$, and grades below $B$. In 2009, of entrants from the maintained mainstream cohort with GCSE grades B or below, just $5 \%$ achieved grade $A$ at $A$ level, $12 \%$ achieved grade $B$ and the remaining $84 \%$ had results below grade $B$ (including ungraded). The rate of achievement of $A$ grades was nearly eight times as high for the grade A*/A GCSE science attainers at $38 \%$, with $28 \%$ achieving grade $\mathrm{B}$ and $34 \%$ awarded grades $\mathrm{C}$ or below.

\section{Fig. 2.2d}

Progression of GCSE Science Cohorts to A-level Biology for 2007-2009, $\%$ of Entrants

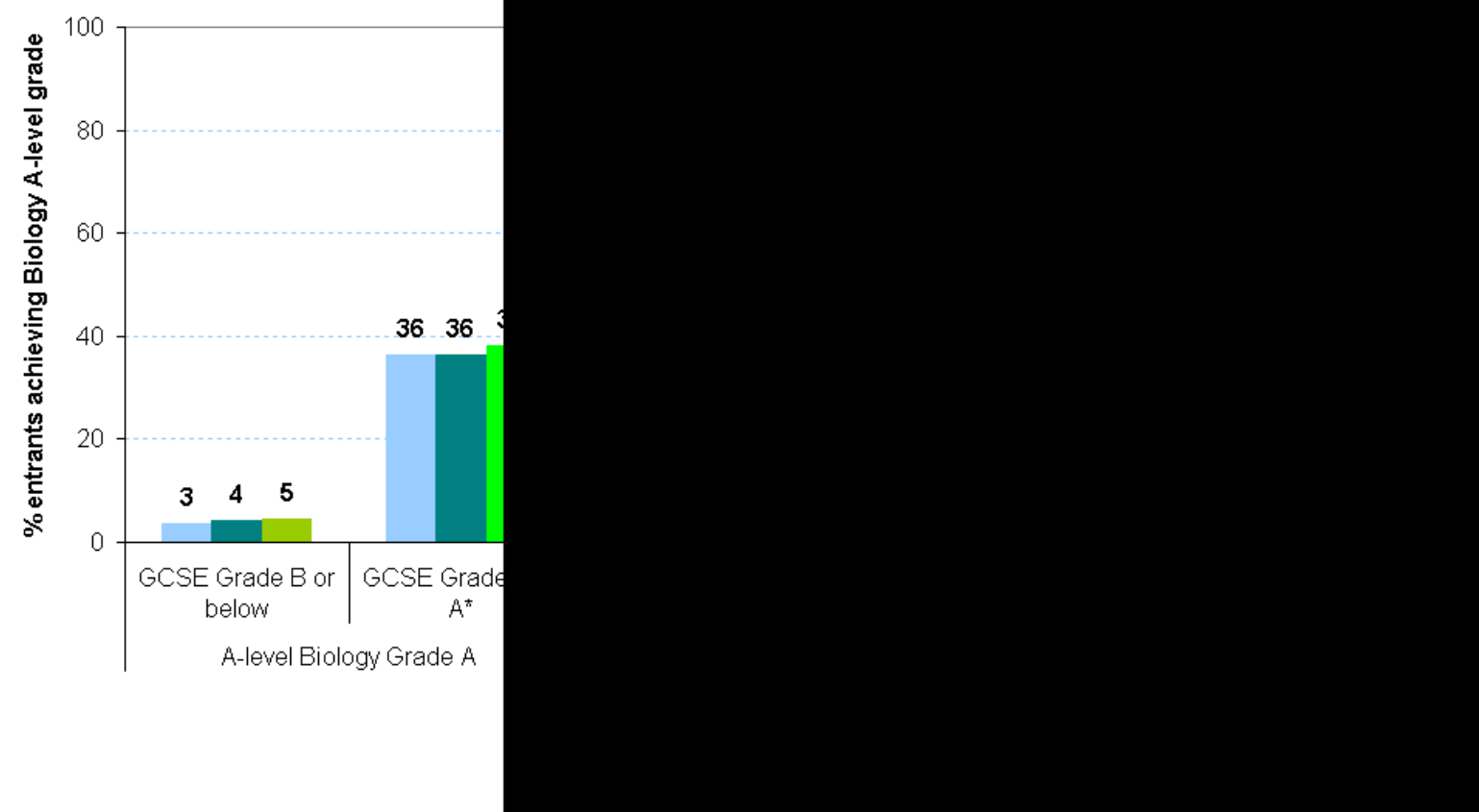


Figure 2.2e examines the prior attainment of those who went on to achieve highly at A level biology; where $49 \%$ of entrants went on to achieve grades A or B. Focusing on those with $A$ level results below grade $B, 22 \%$ of entrants had slipped from grades $A^{*}$ or $A$ at GCSE, and $28 \%$ had prior GCSE grades of B or lower. Breaking down further the biology $A$ level results at grades $A$ and $B$ in the right-hand pie, $25 \%$ achieved the highest possible (A) grade at A level after achieving $A^{*}$ or $A$ at GCSE; $2 \%$ came from grade $B$ or lower at GCSE to achieve an A at A level; $18 \%$ slipped to grade $B$ at $A$ level after achieving $A^{*}$ or A at GCSE; the final $5 \%$ achieved grade $B$ at A level coming from grade $B$ or lower at GCSE.

\section{Fig. 2.2e}

\section{A Level Biology Entries by Grade and GCSE Prior Attainment, 2009}

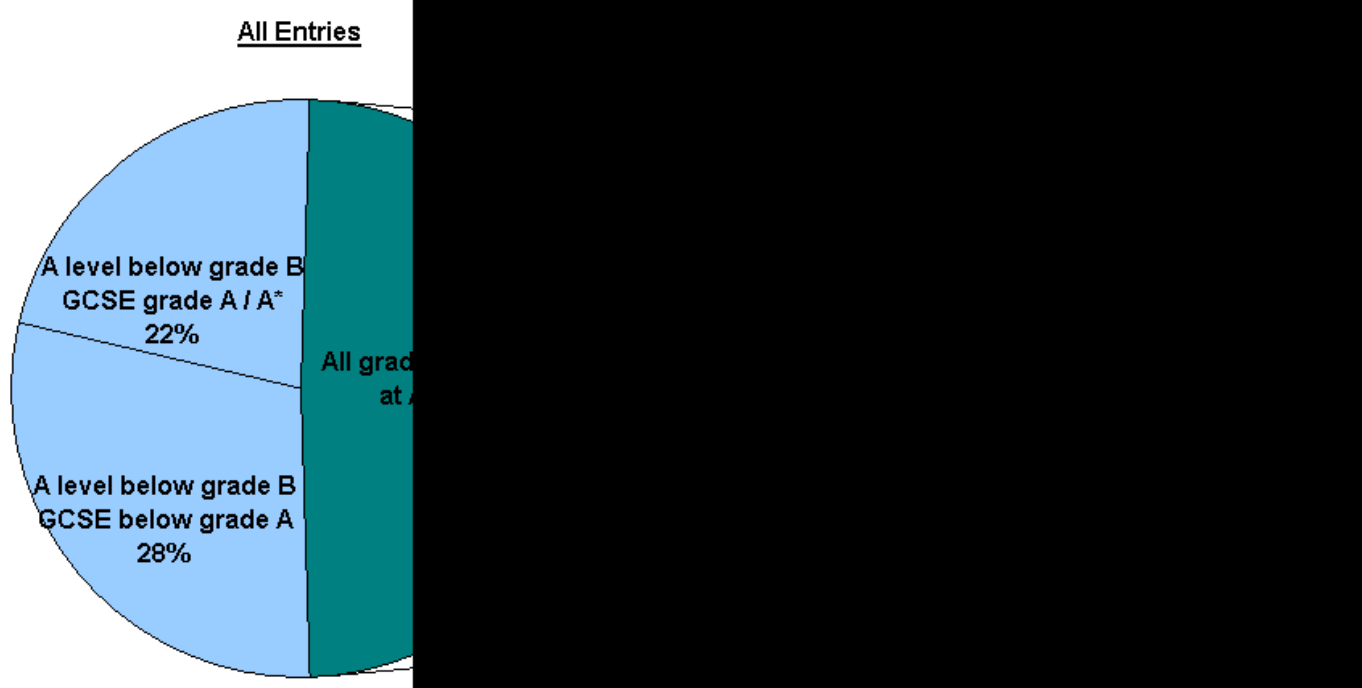


Covering all pupils from the GCSE cohort, including non-entrants to A level biology, figure $2.2 \mathrm{f}$ presents the percentages who went on to achieve high grades. Of the cohort, just $1 \%$ went on to achieve a top A grade at A level biology in 2009, with high science attainers at GCSE over ten times as likely to achieve this, at $12 \%$. A further $1 \%$ of the cohort achieved grade B at A level biology, compared with $9 \%$ of the high attainers at GCSE. The remainder of $A$ level biology entries with results at grade $C$ or lower made up 3\% of the GCSE cohort, or $11 \%$ of the high attaining group at GCSE science.

\section{Fig. 2.2f}

Progression of GCSE Science Cohorts to A-level Biology for 2007-2009, $\%$ of Cohort

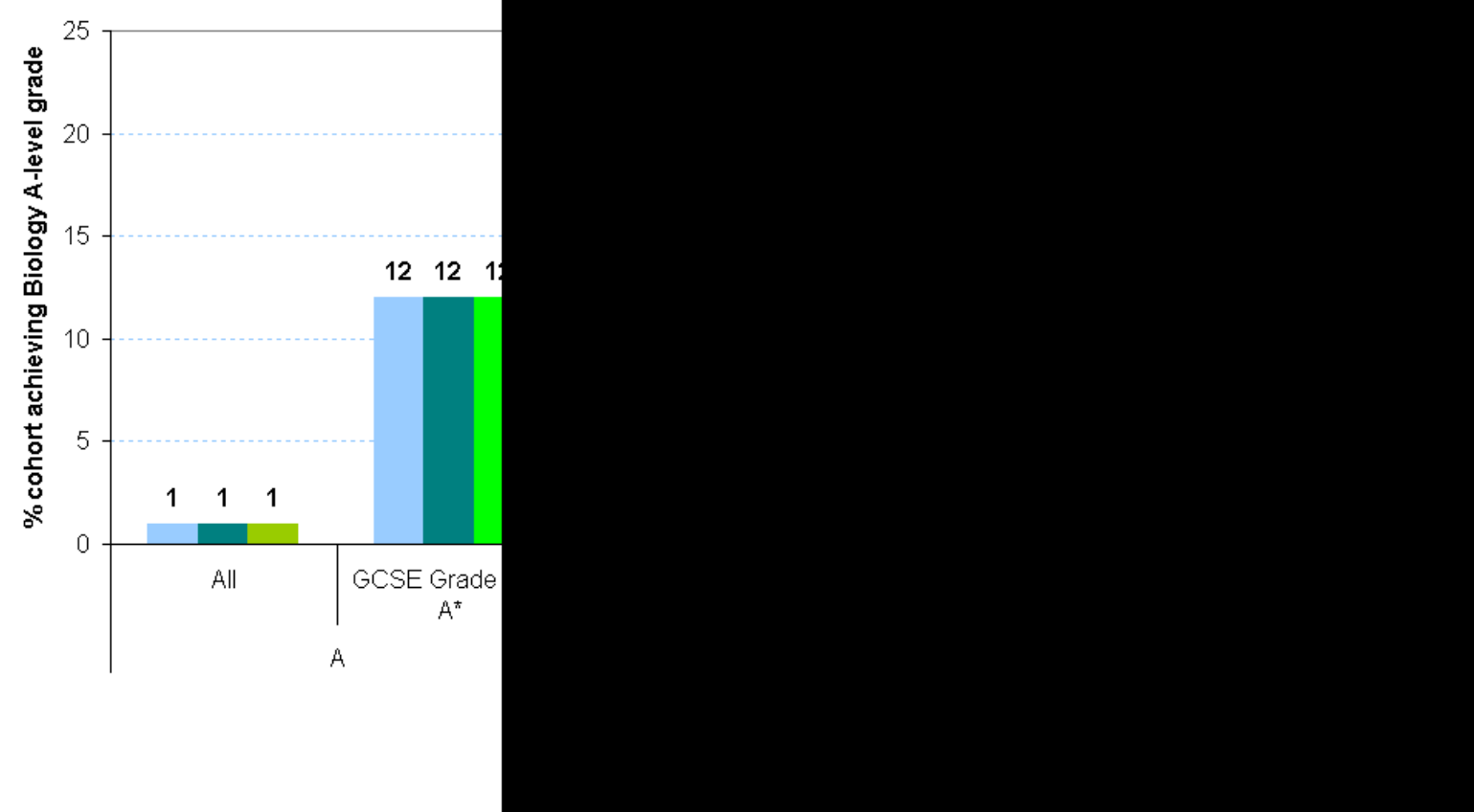


Turning next to A level chemistry progression analyses, figure $2.2 \mathrm{~g}$ shows the percentage of chemistry entrants, split by GCSE science or chemistry attainment, who went on to achieve grade A, grade B, and grades below B. In 2009, of entrants from the maintained mainstream cohort with GCSE grades B or below, only $6 \%$ achieved grade $A$ at $A$ level, $17 \%$ achieved grade $B$ and the remaining $77 \%$ had results below grade $B$ (including ungraded). The rate of achievement of $A$ grades was almost seven times as high for the grade A*/A GCSE science attainers at $40 \%$, with $27 \%$ achieving grade $B$ and $33 \%$ awarded grades $C$ or below.

\section{Fig. 2.2g}

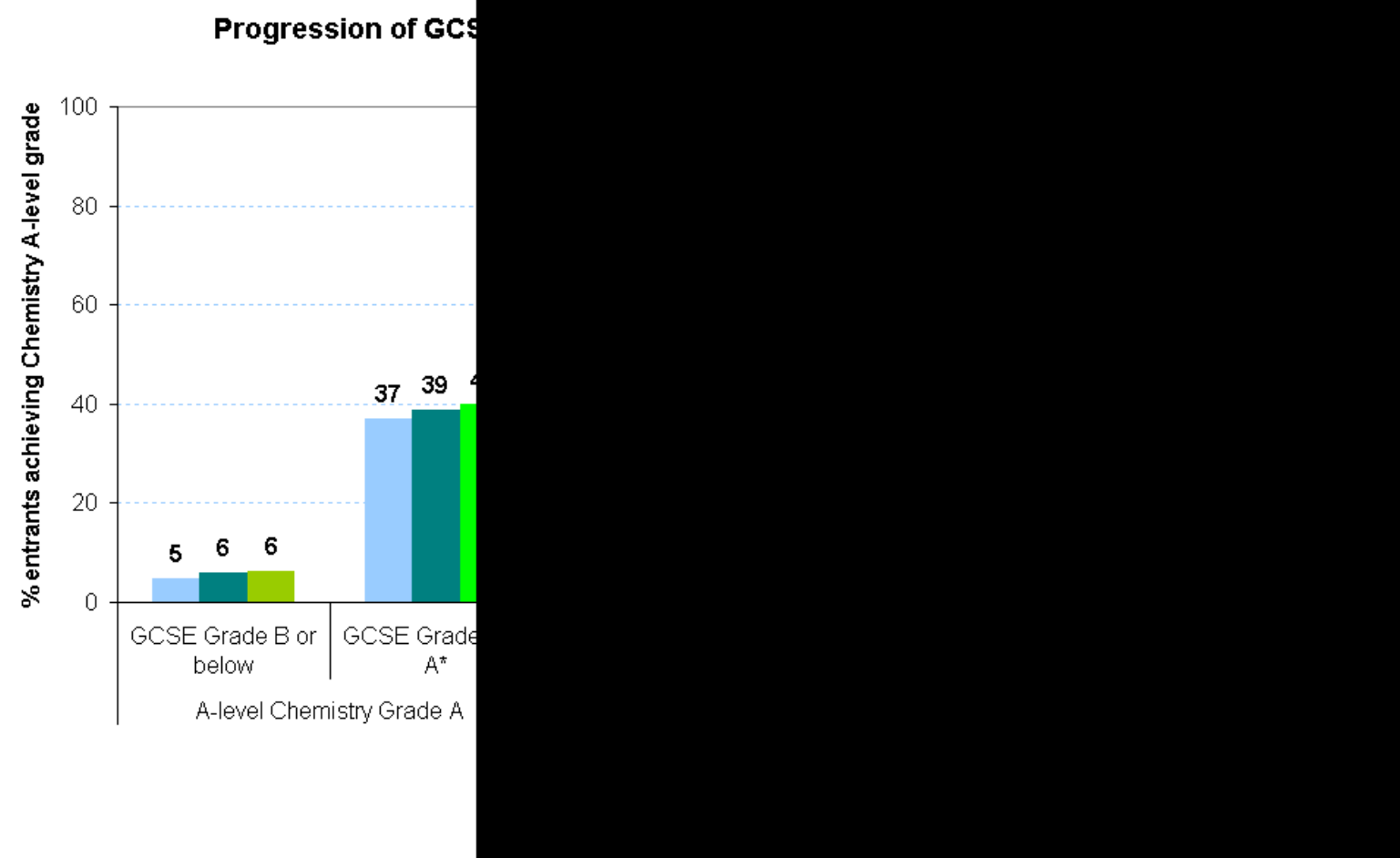


Figure 2.2h examines the prior attainment of those who went on to achieve highly at A level chemistry; where $55 \%$ of cohort entrants went on to achieve grades A or B. Focusing on those with $A$ level results below grade $B, 25 \%$ of entrants had slipped from grades $A^{*}$ or A at GCSE, and $19 \%$ had prior GCSE grades of B or lower.

Breaking down further the chemistry $A$ level results at grades $A$ and $B$ in the righthand pie, 29\% achieved the highest possible (A) grade at $A$ level after achieving $A^{*}$ or A at GCSE; $2 \%$ came from grade B or lower at GCSE to achieve an A at A level; $21 \%$ slipped to grade B at A level after achieving A* or A at GCSE; the final $4 \%$ achieved grade $B$ at $A$ level coming from grade $B$ or lower at GCSE.

\section{Fig. 2.2h}

\section{A Level Chemistry Entries by Grade and GCSE Prior Attainment, 2009}

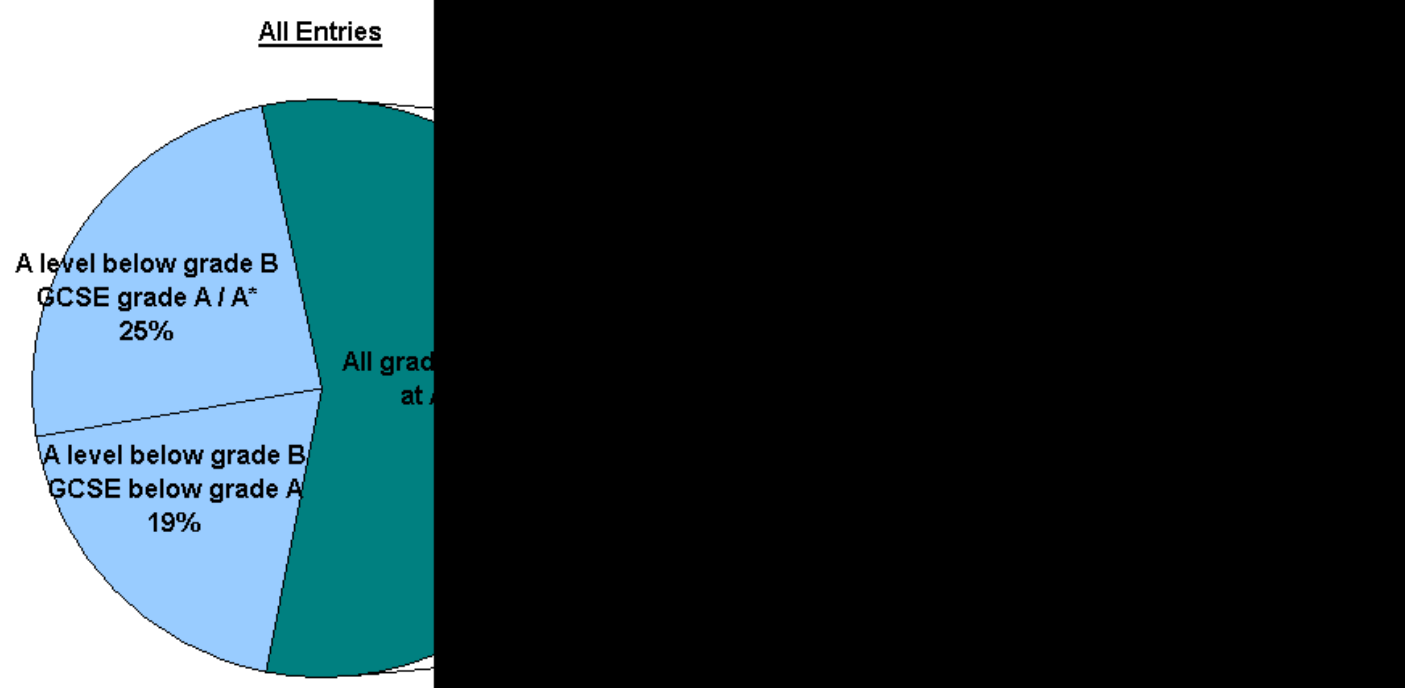


Covering all pupils from the GCSE cohort, including those who did not enter A level chemistry, figure $2.2 \mathrm{i}$ presents the percentages who went on to achieve high grades. Of the cohort, just 1\% went on to achieve a top A grade at A level chemistry in 2009, with high science attainers at GCSE over ten times as likely to achieve this, at $12 \%$. A further $1 \%$ of the cohort achieved grade B at A level chemistry, compared with $8 \%$ of the high attainers at GCSE. The remainder of A level chemistry entries with results at grade C or lower made up $2 \%$ of the GCSE cohort, or $9 \%$ of the high attaining group at GCSE science.

\section{Fig. 2.2i}

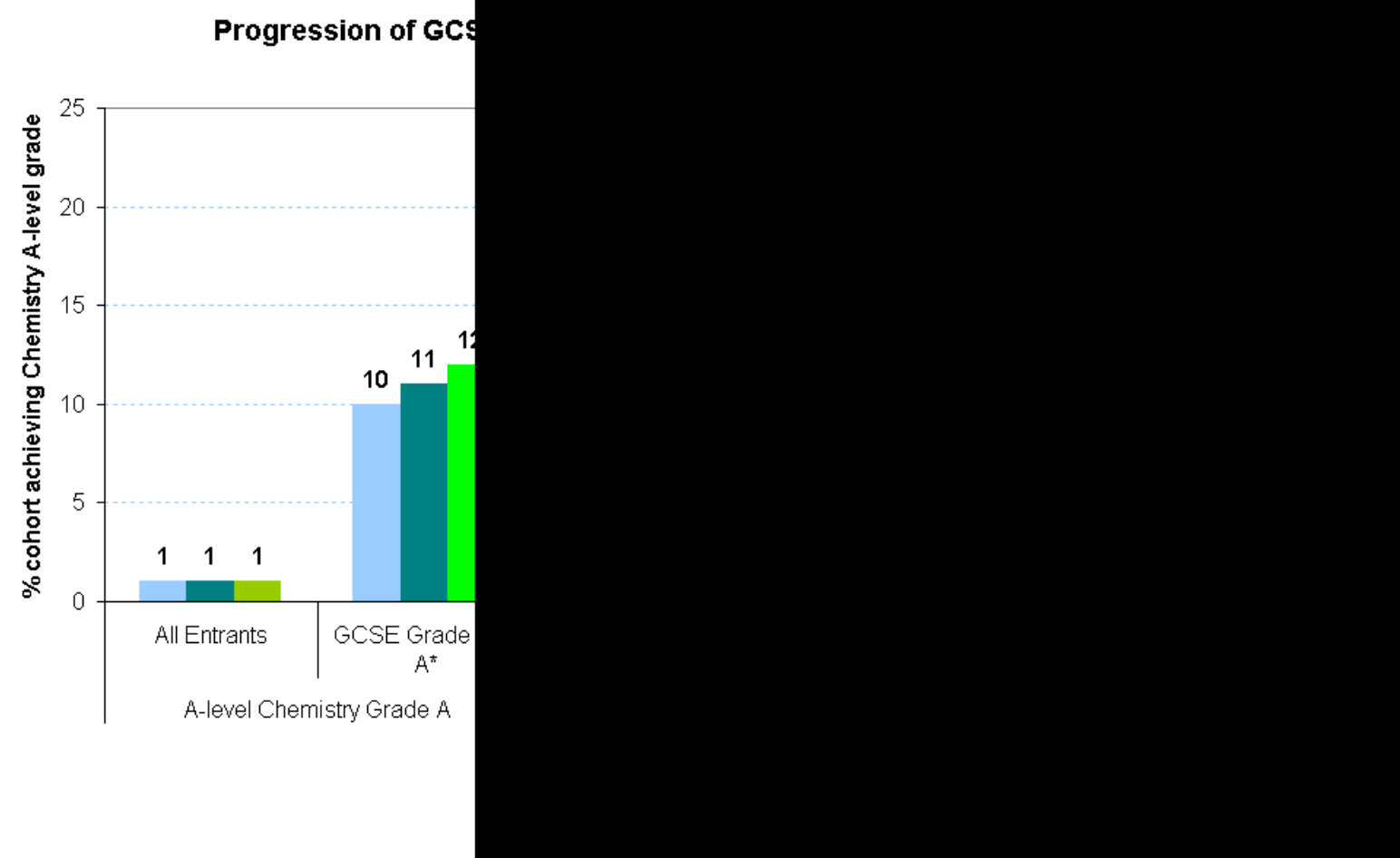


Concluding the GCSE cohorts progression section with A level physics analyses, figure 2.2j shows the percentage of physics entrants, split by GCSE science or physics attainment, who went on to achieve grade $A$, grade $B$, and grades below $B$. In 2009, of entrants from the maintained mainstream cohort with GCSE grades $B$ or below, just $5 \%$ achieved grade $A$ at $A$ level, $13 \%$ achieved grade $B$ and the remaining $82 \%$ had results below grade $B$ (including ungraded). The rate of achievement of $A$ grades was almost eight times as high for the grade $A * / A$ GCSE science attainers at 39\%, with $24 \%$ achieving grade $B$ and $37 \%$ awarded grades C or below.

\section{Fig. 2.2j}

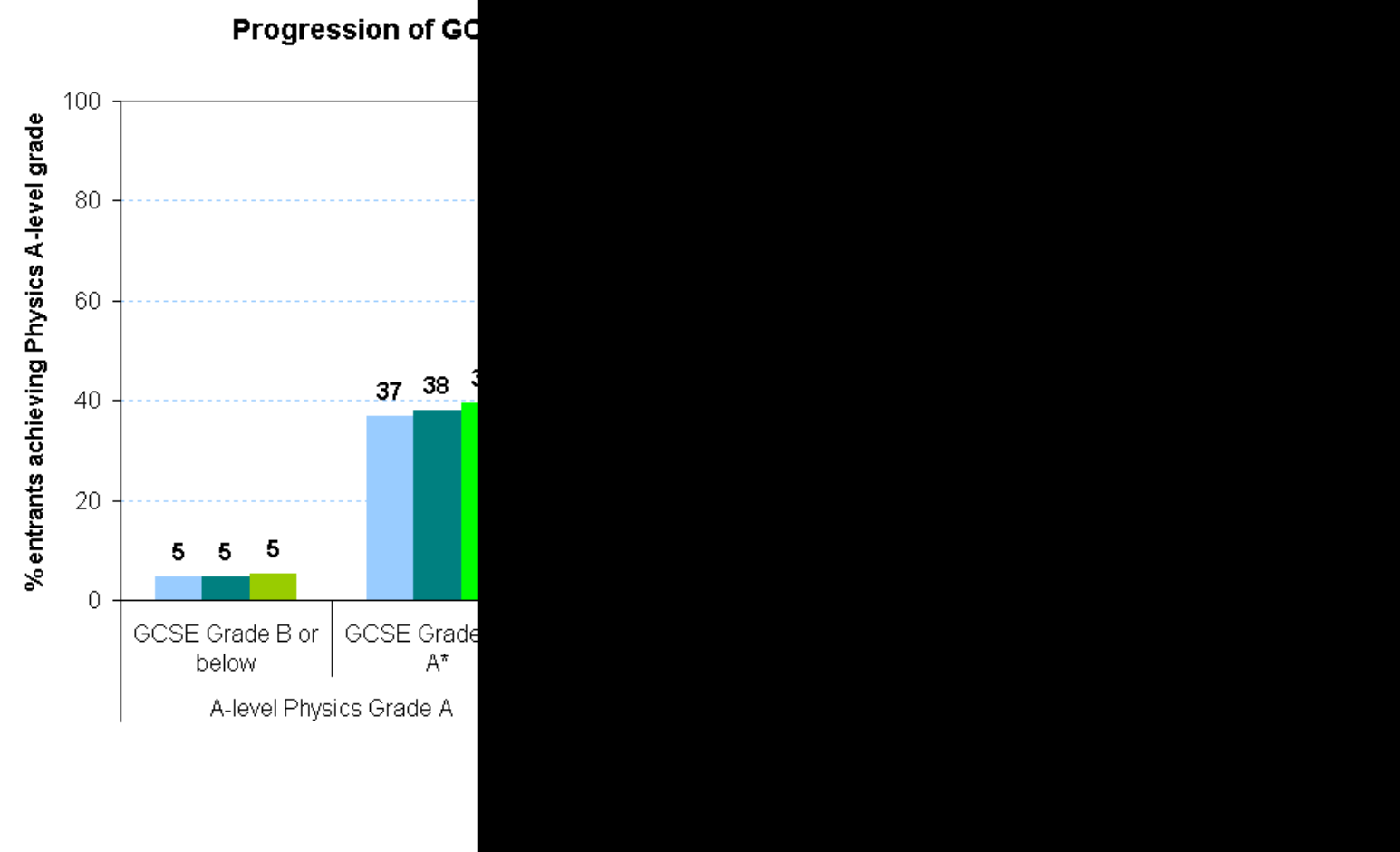


Figure 2.2k examines the prior attainment of those who went on to achieve highly at A level physics; where $51 \%$ of cohort entrants went on to achieve grades A or B. Focusing on those with $A$ level results below grade $B, 27 \%$ of entrants had slipped from grades $A^{*}$ or $A$ at GCSE, and $23 \%$ had prior GCSE grades of $B$ or lower.

Breaking down further the physics $A$ level results at grades $A$ and $B$ in the right-hand pie, $28 \%$ achieved the highest possible $(A)$ grade at $A$ level after achieving $A^{*}$ or $A$ at GCSE; 1\% came from grade B or lower at GCSE to achieve an A at A level; $17 \%$ slipped to grade $B$ at A level after achieving $A^{*}$ or A at GCSE; the final $4 \%$ achieved grade $B$ at $A$ level coming from grade $B$ or lower at GCSE.

\section{Fig. 2.2k}

\section{A Level Physics Entries by Grade and GCSE Prior Attainment, 2009}

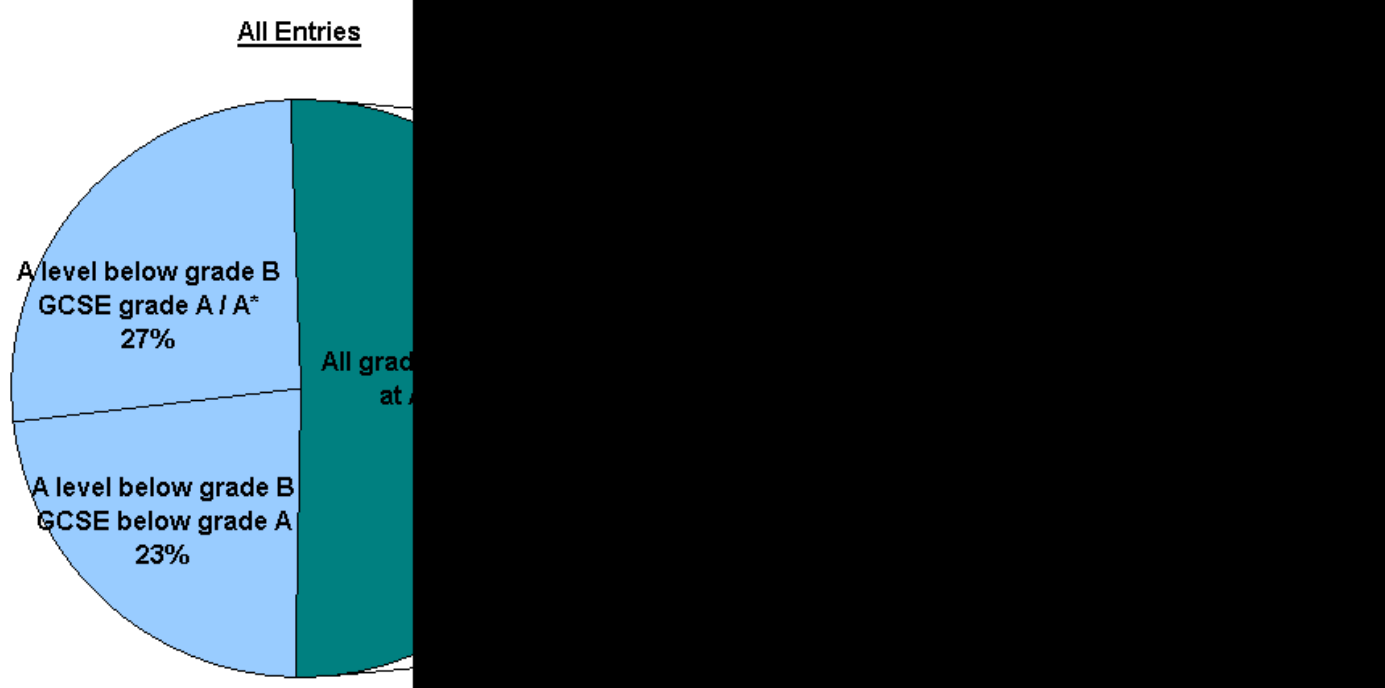


Covering all pupils from the GCSE cohort, including those who did not enter A level physics, figure 2.21 presents the percentages who went on to achieve high grades. Of the cohort, just 1\% went on to achieve a top A grade at A level physics in 2009, compared with $8 \%$ of the high science attainers at GCSE. A further $1 \%$ of the cohort achieved grade $B$ at A level physics, as did $5 \%$ of the high attainers at GCSE. The remainder of $A$ level physics entries with results at grade $C$ or lower made up only $1 \%$ of the GCSE cohort, but $7 \%$ of the high attaining group at GCSE science.

\section{Fig. 2.21}

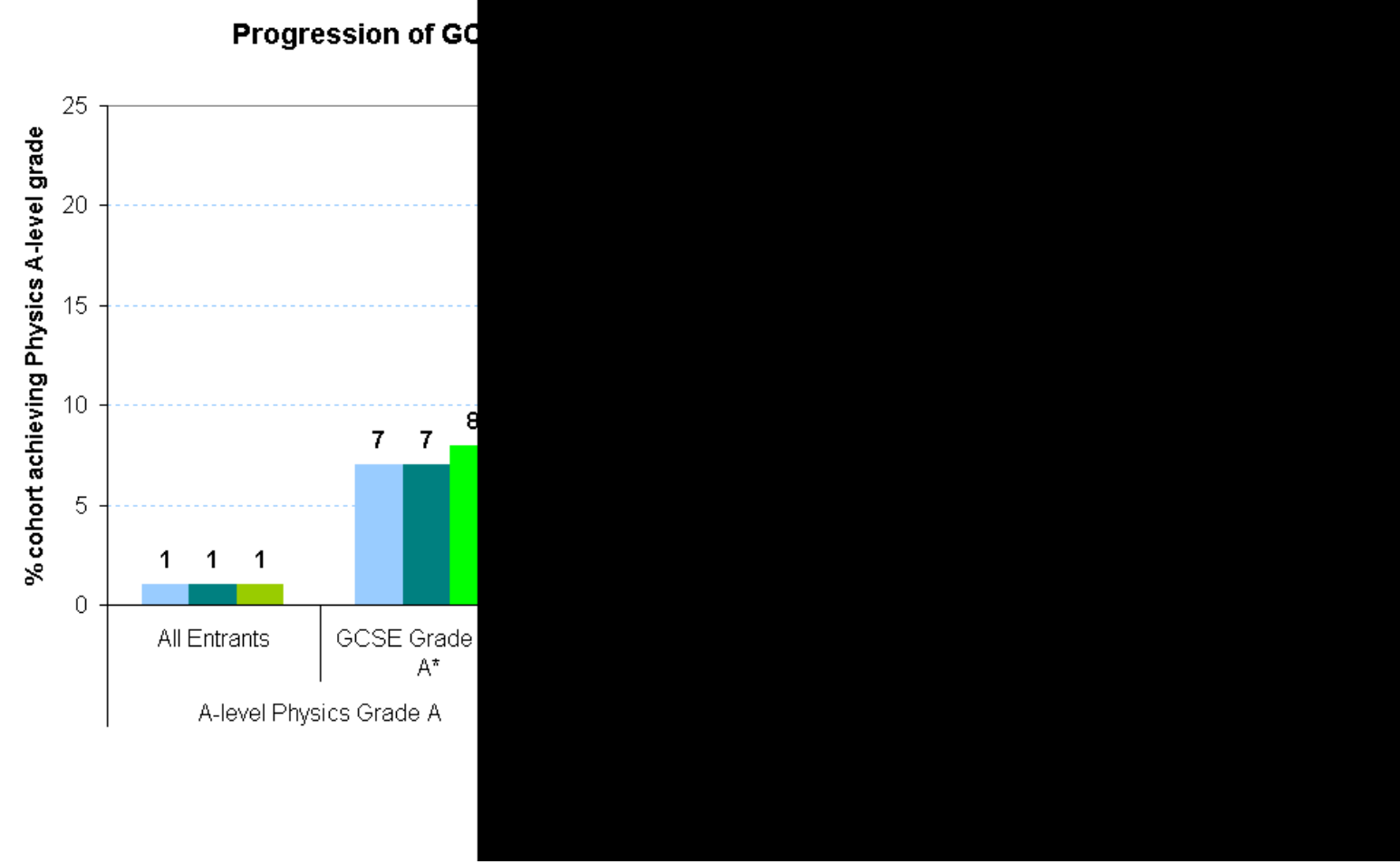

\section{Section Summary}

Section 2.2 has examined achievement of high grades in A level maths and science subjects. The analyses have revealed a consistent pattern of high dependence on strong prior attainment within subject. Only $3 \%$ of $A$ level maths entrants manage to progress from grades of B or lower at GCSE to achieve an A or a B at A level. For biology, this figure is slightly higher at $7 \%$, falling to $6 \%$ for chemistry, and $5 \%$ for physics. Across the four subjects, the chances of achieving grade $A$ at $A$ level are seven to eight times higher for pupils who gained $A^{*}$ or A grades at GCSE than for those with grade $B$ or lower. From this it may be concluded that B grades at GCSE are not enough to secure a good chance of progressing to high A level grades for maths and science subjects.

See section 2.6 for odds ratio comparisons of progression between maths and the three science subjects and with other Key Stages. 


\subsection{Key Stage 3 Attainment and A level Progression}

Following from the analysis of GCSE to A level progression in section 2.2 above, this section looks back to the next previous Key Stage to assess how far back the influence of prior attainment on A level achievement stretches. A level maths and science achievements are broken down according to prior attainment at Key Stage 3 to answer the questions: what was the A level grade achieved by entrants with a given level of achievement at KS3? And, what was the KS3 prior attainment of those who went on to achieve a high grade at A level? At Key Stage 3, the expected level of achievement is level 6 , hence we define high prior attainers as those achieving level 7 or above.

Figure 2.3a shows the percentage of A level maths entrants, split by KS3 maths attainment, who went on to achieve grade A, grade B, and grades below B. In 2009, of entrants from the maintained mainstream cohort with maths achievement at KS3 of below level 7, 14\% achieved grade A at A level, 21\% achieved grade B and the remaining $65 \%$ had results below grade $B$ (including ungraded). The rate of achievement of $A$ grades was three times as high for the level 7+ maths attainers at $43 \%$, with $23 \%$ achieving grade B and 34\% awarded grades C or below.

\section{Fig. 2.3a}

Progression of KS3 Maths Cohorts to A-level Maths for 2007-2009, \% of Entrants

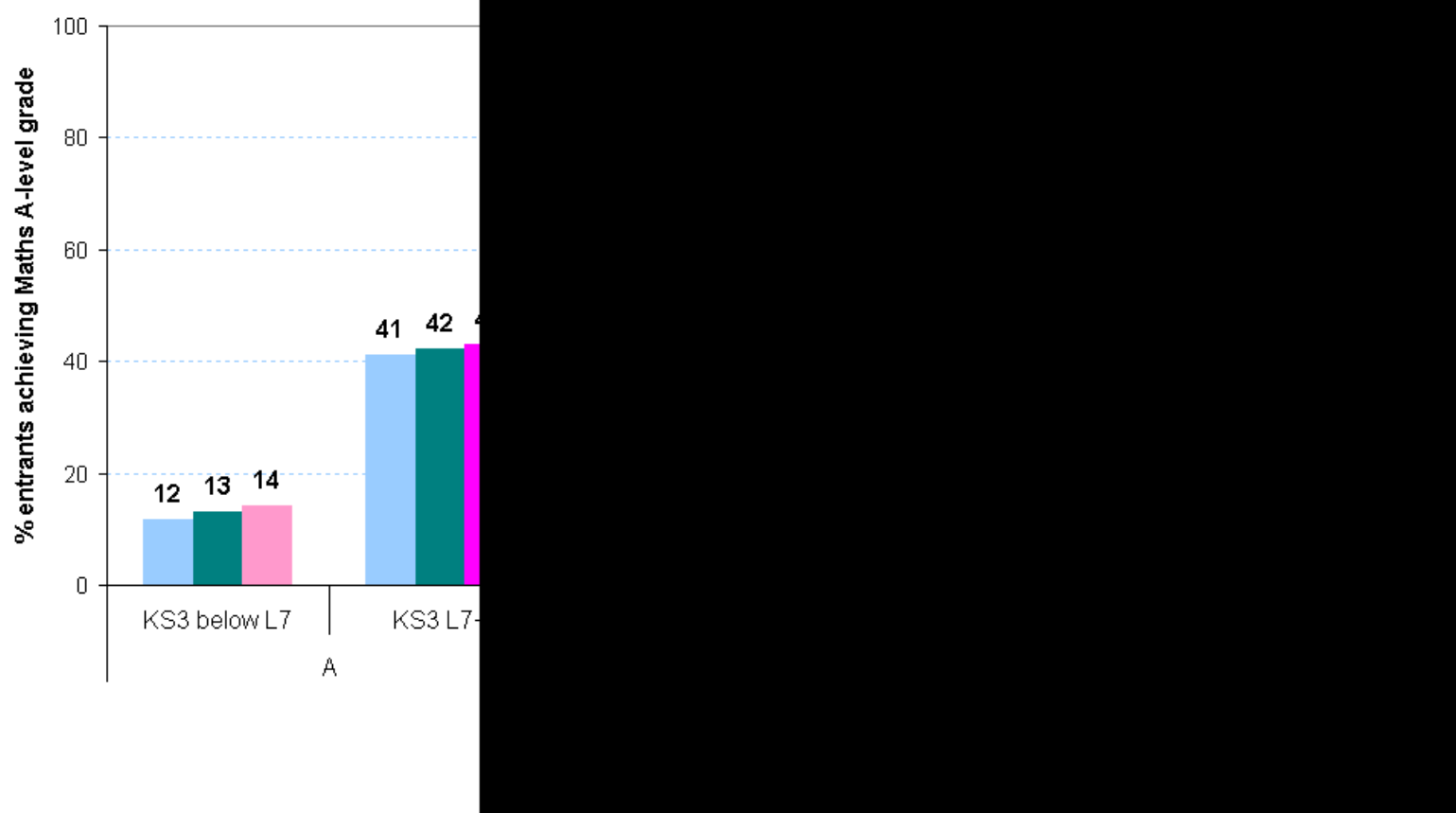

Figure 2.3b takes the same data and expresses the numbers of entrants who were high attainers at Key Stage 3 maths, as a percentage of the group with a given subsequent outcome at A level maths. The large pie on the left splits all cohort entries to A level maths into those achieving level 7 and above at KS3 (65\%) and those with lower results - 4\% with KS3 levels of below level 7 , and a further $32 \%$ who did achieve level 7+ at KS3 but slipped to below grade B at A level. The smaller pie on the right breaks down the $65 \%$ into As and Bs, revealing that $41 \%$ achieved the highest possible (A) grade at A level after achieving level 7+ at KS3 maths; $1 \%$ 
came from below level 7 to achieve an A at A level; 21\% managed to achieve a grade $B$ at $A$ level having secured level $7+$ at $K S 3$; the final $2 \%$ achieved grade $B$ at A level coming from below level 7 at KS3.

\section{Fig. 2.3b}

A Level Maths Entries by Grade and KS3 Prior Attainment, 2009

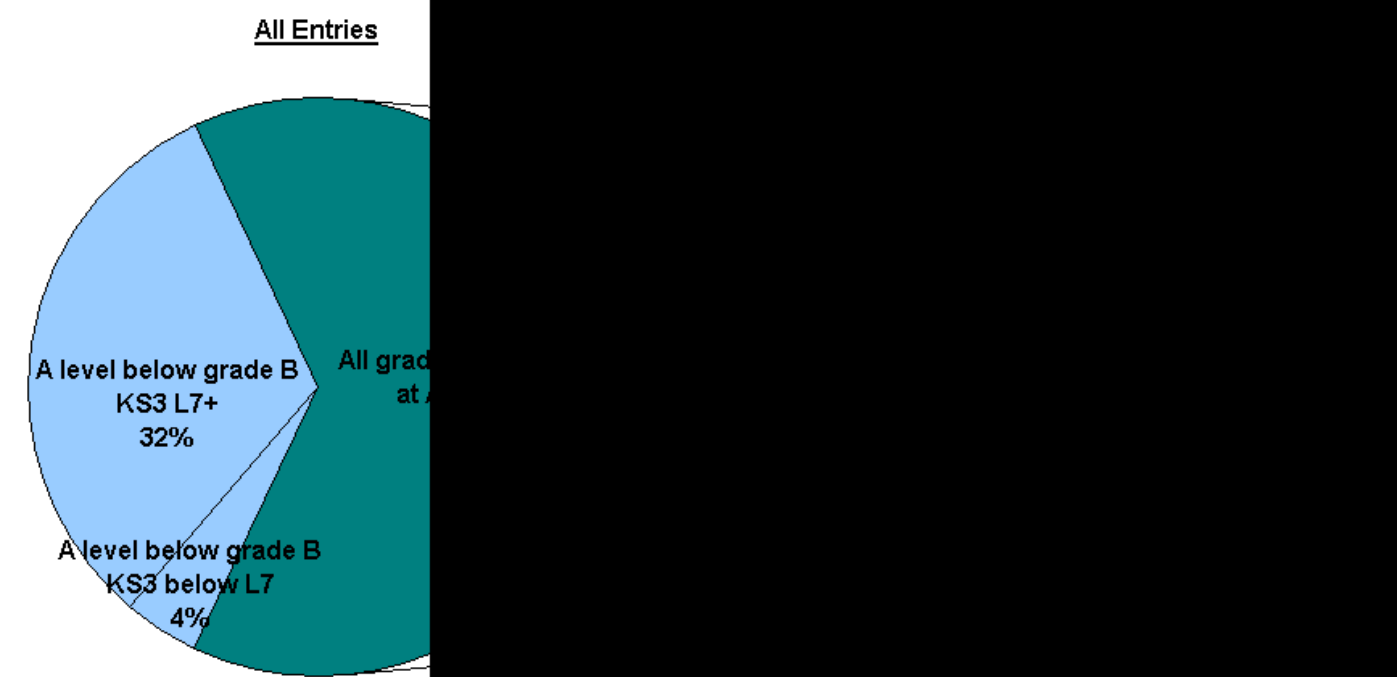


Figure 2.3c presents the percentages of the complete maintained mainstream Key Stage 3 cohort who went on to achieve high grades at A level maths. This takes into account the proportions of pupils who selected themselves out of maths at A level, either because they didn't continue to Key Stage 5, because they took qualifications other than A levels, or because they chose to study other subjects.

Of the complete KS3 cohort, 3\% went on to achieve an A grade at A level maths in 2009, with high maths attainers at KS3 just under 4 times as likely to achieve this, at $11 \%$. A further $2 \%$ of the cohort achieved grade B at A level maths, compared with $6 \%$ of the high attainers at KS3. The remainder of A level maths entries with results at grade $\mathrm{C}$ or lower made up 3\% of the KS3 cohort, or $9 \%$ of the high attaining group at KS3 maths.

Fig. 2.3c

Progression of KS3 Maths Cohorts to A-level Maths for 2007-2009, \% of Cohort

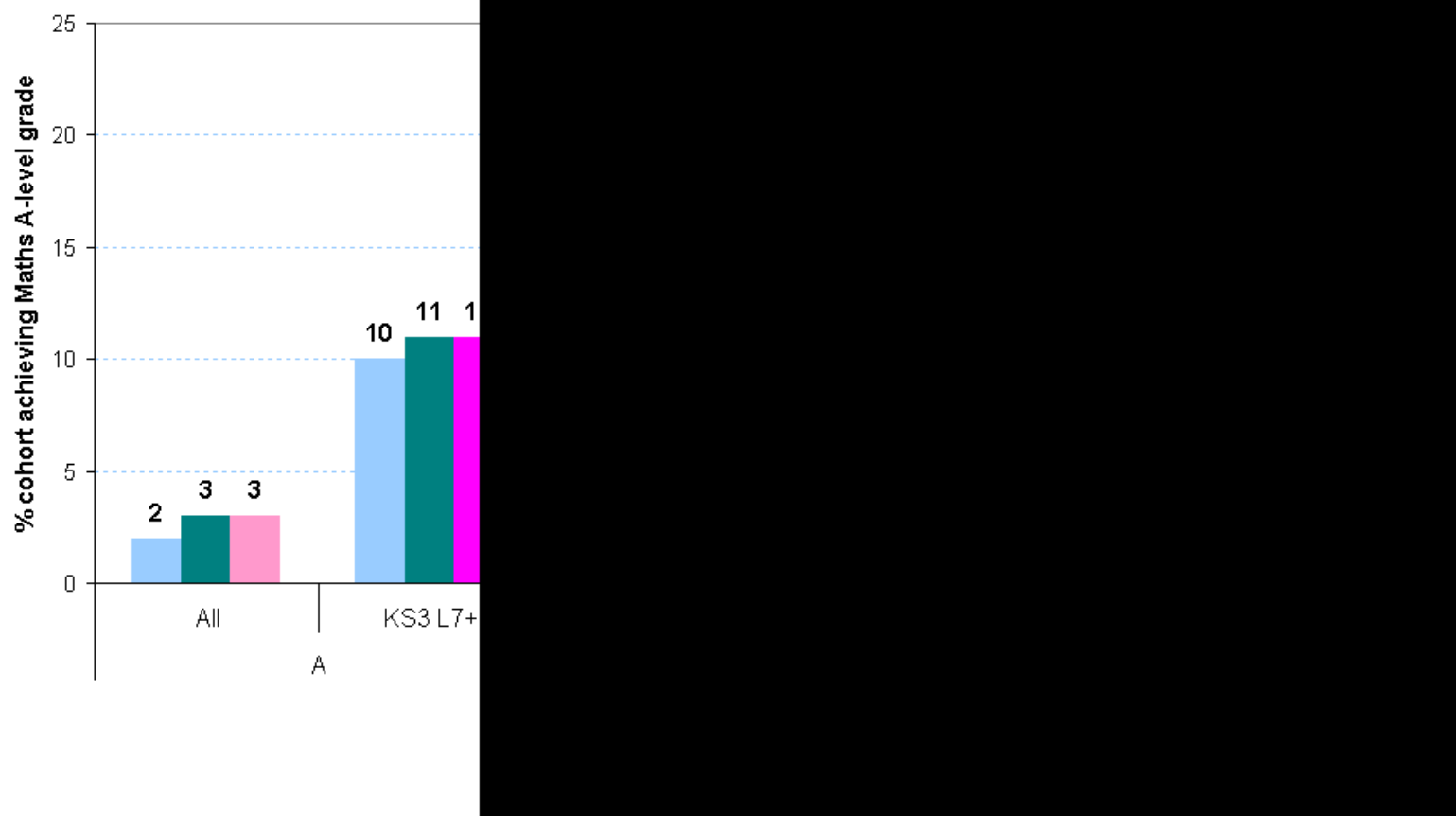


Moving to the science subjects, figure $2.3 \mathrm{~d}$ shows the percentage of $A$ level biology entrants, split by KS3 science attainment, who went on to achieve grade A, grade B, and grades below B. In 2009, of entrants from the maintained mainstream cohort with science achievement at KS3 of below level 7, 10\% achieved grade A at A level, $19 \%$ achieved grade $B$ and the remaining $71 \%$ had results below grade $B$ (including ungraded). The rate of achievement of $A$ grades was almost 4 times as high for the level $7+$ science attainers at 39\%, with $26 \%$ achieving grade B and $35 \%$ awarded grades $\mathrm{C}$ or below.

\section{Fig. 2.3d}

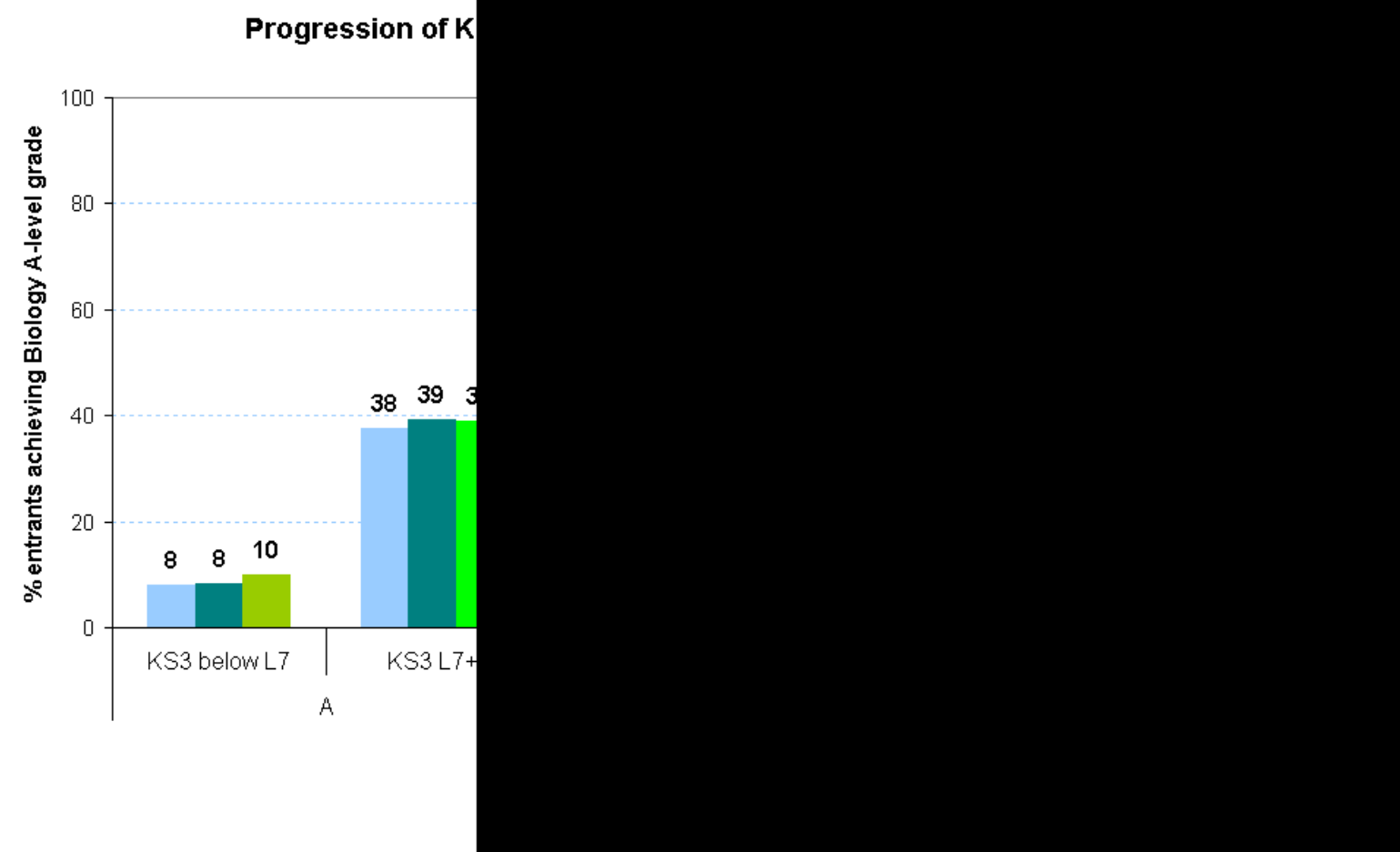


Figure 2.3e takes the same data and expresses the numbers of entrants who were high attainers at Key Stage 3 science, as a percentage of the group with a given subsequent outcome at $A$ level biology. The large pie on the left splits all cohort entries to A level biology into those achieving level 7 and above at KS3 (49\%) and those with lower results - 31\% with KS3 levels of below level 7, and a further $20 \%$ who did achieve level 7+ at KS3 but slipped to below grade B at A level. The smaller pie on the right breaks down the $49 \%$ into As and Bs, revealing that $22 \%$ achieved the highest possible (A) grade at A level after achieving level 7+ at KS3 science; 4\% came from below level 7 to achieve an $A$ at $A$ level; $14 \%$ managed to achieve a grade $B$ at $A$ level having secured level $7+$ at $K S 3$; the final $9 \%$ achieved grade $B$ at A level coming from below level 7 at KS3.

\section{Fig. 2.3e}

A Level Biology Entries by Grade and KS3 Prior Attainment, 2009

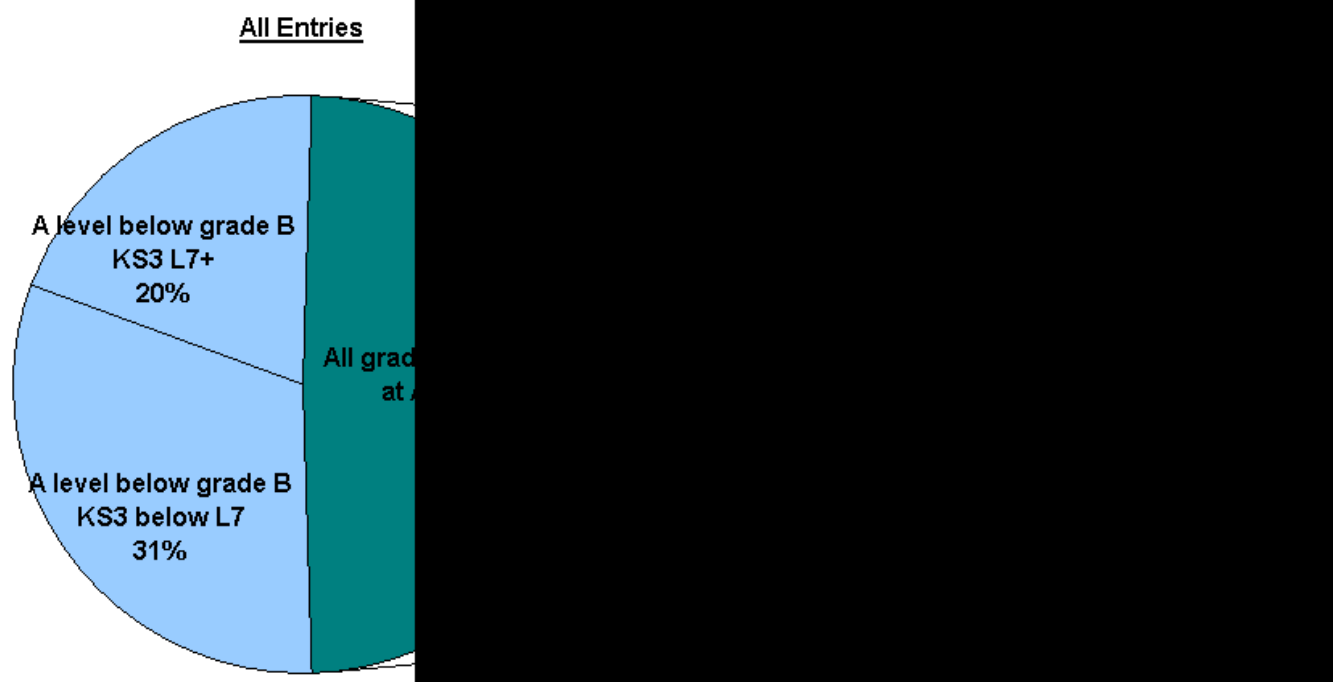


Figure 2.3f presents the percentages of the complete maintained mainstream Key Stage 3 cohort who went on to achieve high grades at A level biology. This takes into account the proportions of pupils who selected themselves out of biology at $A$ level, either because they didn't continue to Key Stage 5, because they took qualifications other than A levels, or because they chose to study other subjects.

Of the complete KS3 cohort, 1\% went on to achieve a top A grade at A level biology in 2009 , with high science attainers at KS3 ten times as likely to achieve this, at $10 \%$. A further $1 \%$ of the cohort achieved grade B at A level biology, compared with $7 \%$ of the high attainers at KS3 science. The remainder of A level biology entries with results at grade C or lower made up 3\% of the KS3 cohort, or $9 \%$ of the high attaining group at KS3 science.

\section{Fig. $2.3 f$}

Progression of KS3 Science Cohorts to A-level Biology for 2007-2009, \% of Cohort

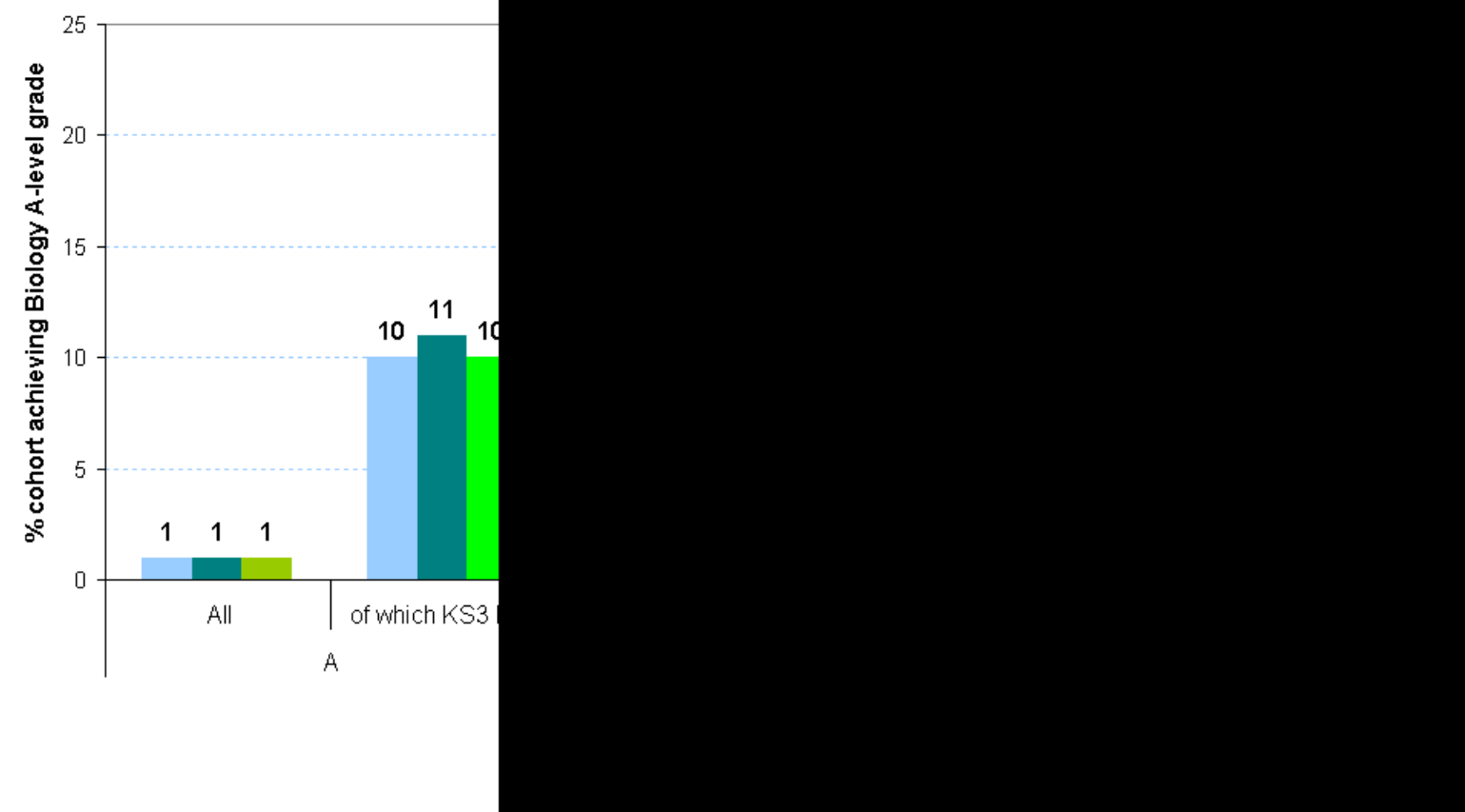


Turning to the next science subject, figure $2.3 \mathrm{~g}$ shows the percentage of A level chemistry entrants, split by KS3 science attainment, who went on to achieve grade A, grade $B$, and grades below $B$. In 2009 , of entrants from the maintained mainstream cohort with science achievement at KS3 of below level 7, 14\% achieved grade A at A level, $23 \%$ achieved grade $B$ and the remaining $63 \%$ had results below grade $B$ (including ungraded). The rate of achievement of $A$ grades was almost three times as high for the level $7+$ science attainers at $41 \%$, with $26 \%$ achieving grade $B$ and $33 \%$ awarded grades C or below.

\section{Fig. $2.3 \mathrm{~g}$}

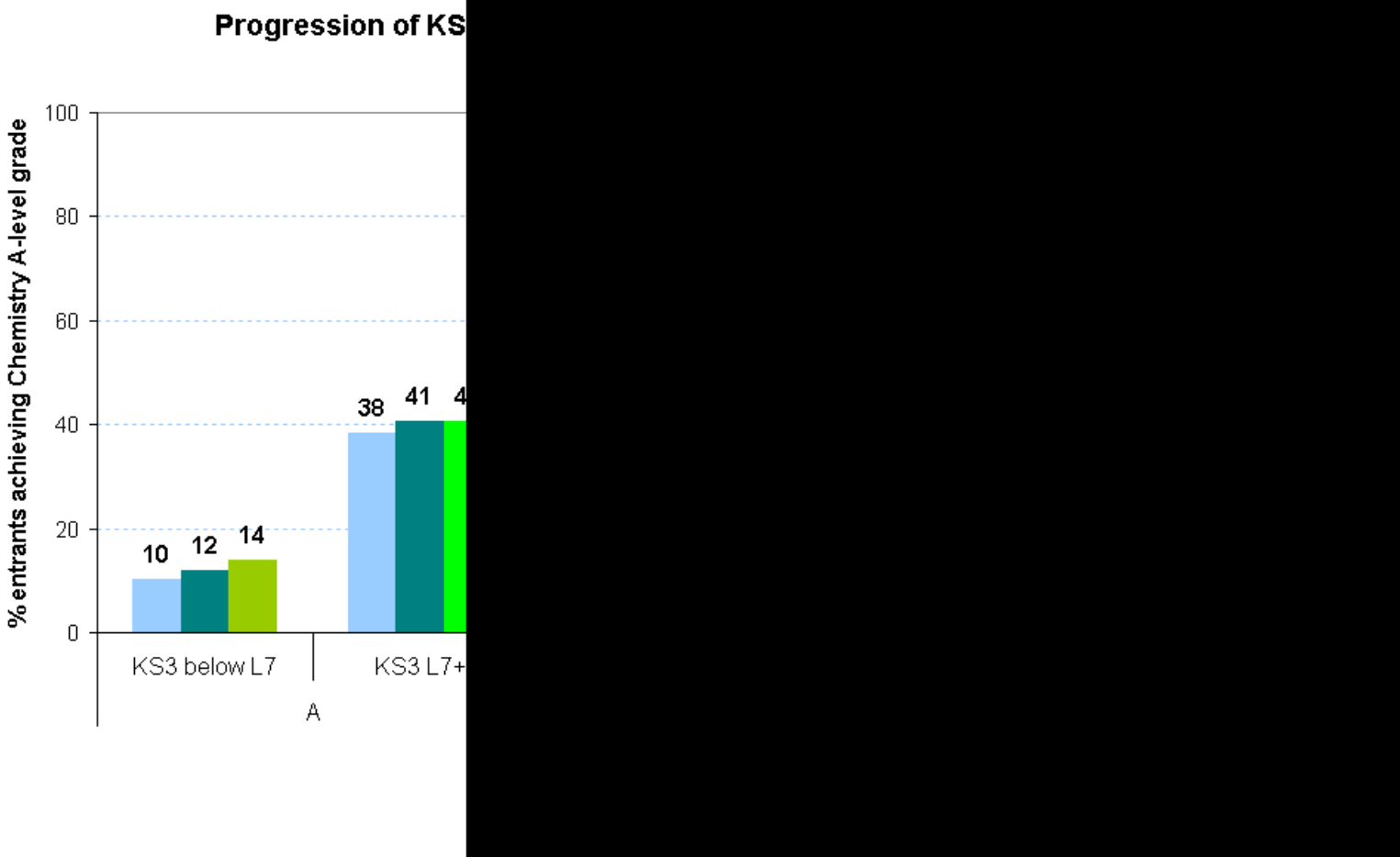


Figure 2.3h takes the same data and expresses the numbers of entrants who were high attainers at Key Stage 3 science, as a percentage of the group with a given subsequent outcome at A level chemistry. The large pie on the left splits all cohort entries to A level chemistry into those achieving level 7 and above at KS3 (56\%) and those with lower results - $21 \%$ with KS3 levels of below level 7 , and a further $22 \%$ who did achieve level 7+ at KS3 but slipped to below grade B at A level. The smaller pie on the right breaks down the $56 \%$ into As and Bs, revealing that $27 \%$ achieved the highest possible (A) grade at A level after achieving level $7+$ at KS3 science; $5 \%$ came from below level 7 to achieve an $A$ at $A$ level; $17 \%$ managed to achieve a grade $B$ at $A$ level having secured level $7+$ at $K S 3$; the final $8 \%$ achieved grade $B$ at A level coming from below level 7 at KS3.

\section{Fig. 2.3h}

\section{A Level Chemistry Entries by Grade and KS3 Prior Attainment, 2009}

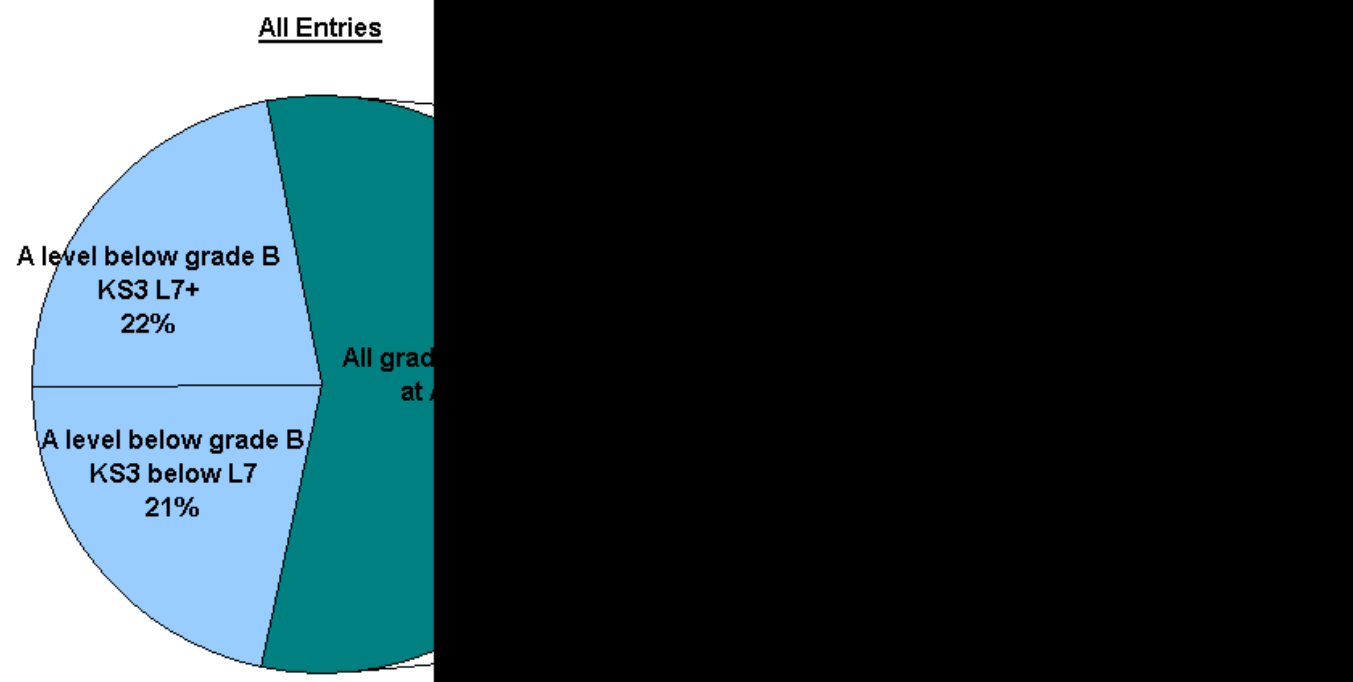


Figure 2.3i presents the percentages of the complete maintained mainstream Key Stage 3 cohort who went on to achieve high grades at A level chemistry. This takes into account the proportions of pupils who selected themselves out of chemistry at A level, either because they didn't continue to Key Stage 5, because they took qualifications other than A levels, or because they chose to study other subjects.

Of the complete KS3 cohort, $1 \%$ went on to achieve an A grade at A level chemistry in 2009, with high science attainers at KS3 nine times as likely to achieve this, at $9 \%$. A further $1 \%$ of the cohort achieved grade B at A level chemistry, compared with $6 \%$ of the high science attainers at KS3. The remainder of A level chemistry entries with results at grade C or lower made up $2 \%$ of the KS3 cohort, or $8 \%$ of the high attaining group at KS3 science.

Fig. 2.3i

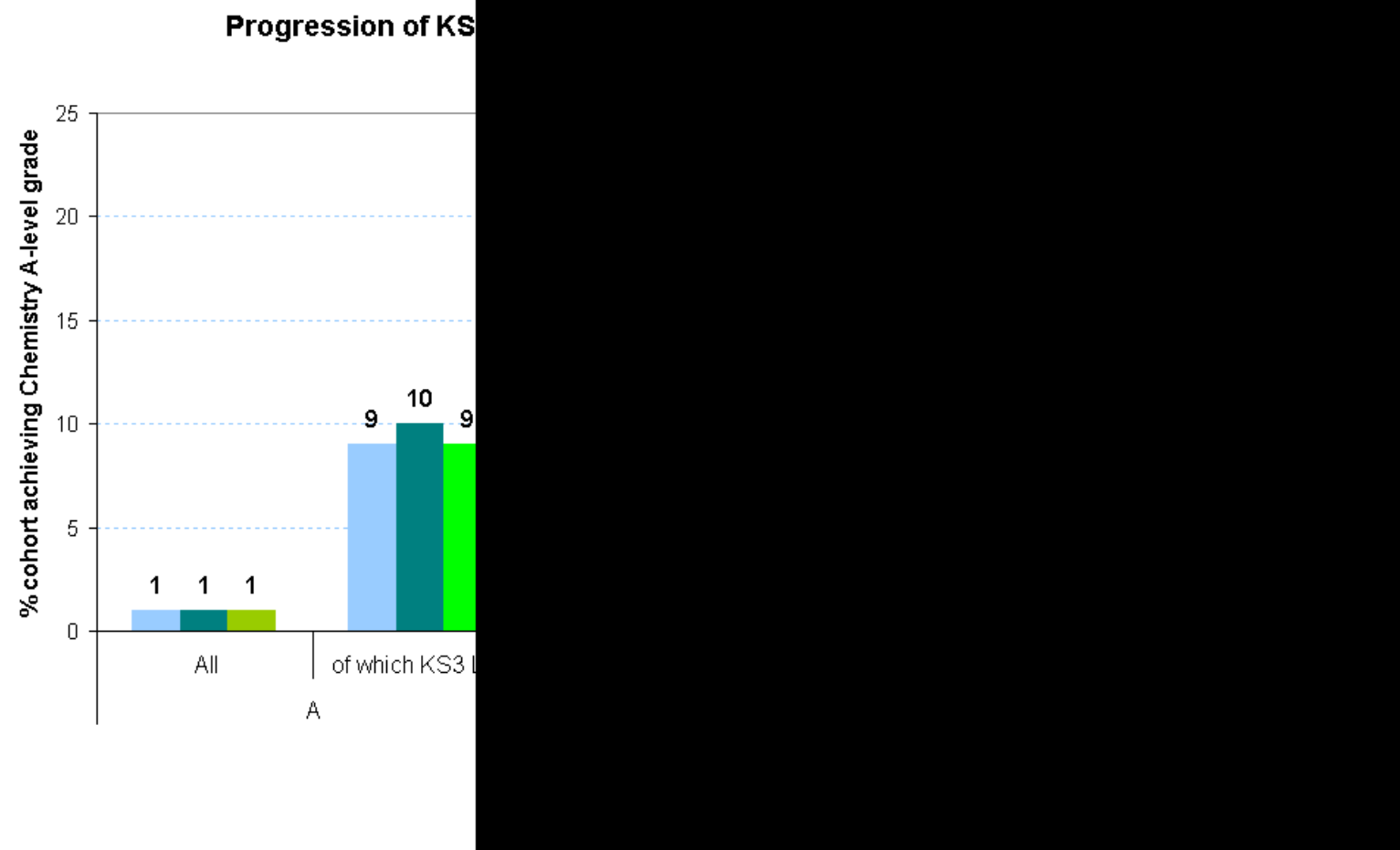


Moving to the third and final traditional science, figure 2.3j shows the percentage of $A$ level physics entrants, split by KS3 science attainment, who went on to achieve grade $A$, grade $B$, and grades below $B$. In 2009, of entrants from the maintained mainstream cohort with science achievement at KS3 of below level 7, 8\% achieved grade $A$ at $A$ level, 15\% achieved grade $B$ and the remaining $77 \%$ had results below grade $B$ (including ungraded). The rate of achievement of $A$ grades was almost five times as high for the level $7+$ science attainers at $38 \%$, with $23 \%$ achieving grade $B$ and $40 \%$ awarded grades $\mathrm{C}$ or below.

\section{Fig. 2.3i}

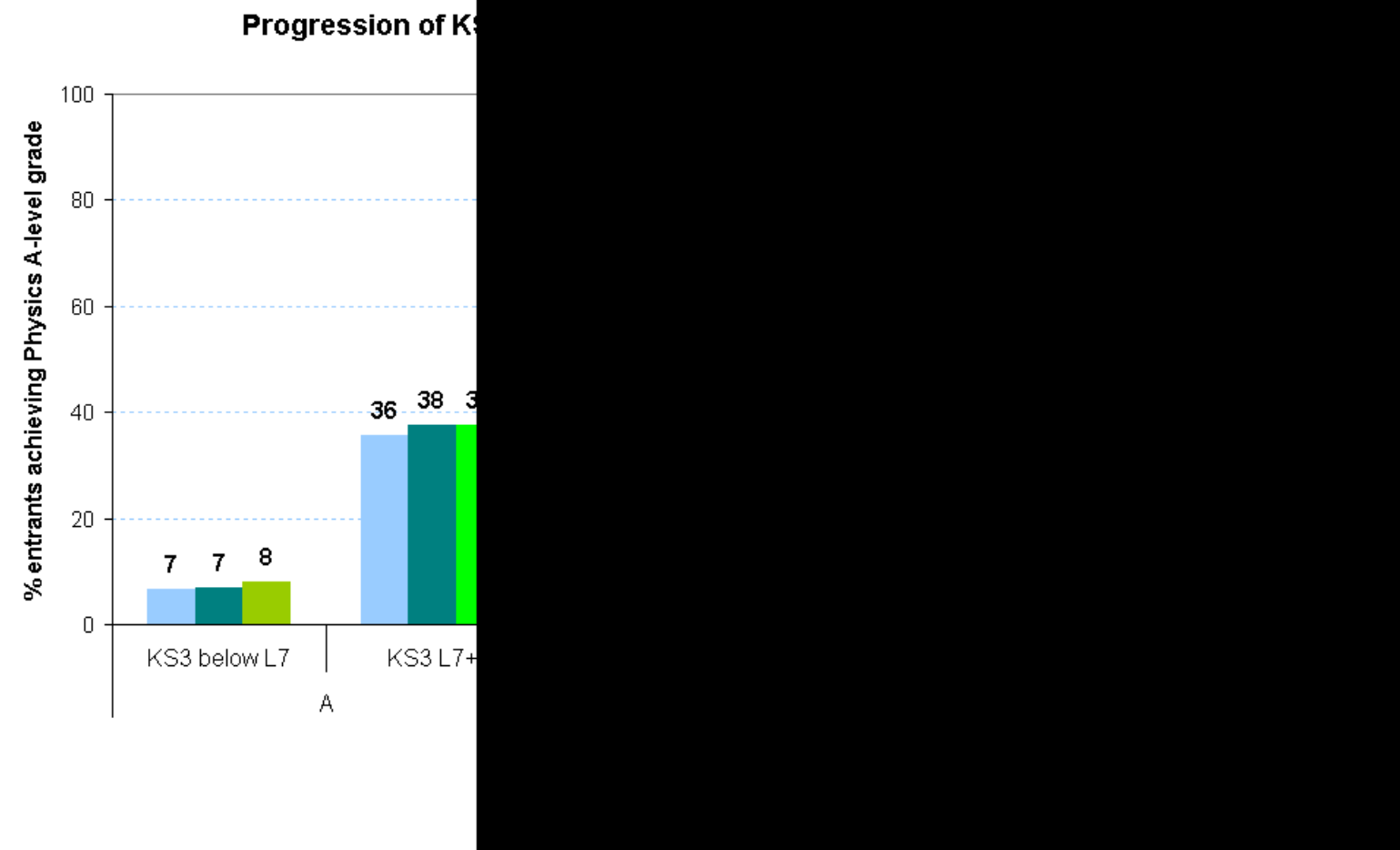


Figure 2.3k takes the same data and expresses the numbers of entrants who were high attainers at Key Stage 3 science, as a percentage of the group with a given subsequent outcome at $A$ level physics. The large pie on the left splits all cohort entries to A level physics into those achieving level 7 and above at KS3 (51\%) and those with lower results - $20 \%$ with KS3 levels of below level 7 , and a further $29 \%$ who did achieve level 7+ at KS3 but slipped to below grade B at A level. The smaller pie on the right breaks down the $51 \%$ into As and Bs, revealing that $28 \%$ achieved the highest possible (A) grade at A level after achieving level 7+ at KS3 science; $2 \%$ came from below level 7 to achieve an $A$ at $A$ level; $17 \%$ managed to achieve a grade $B$ at $A$ level having secured level $7+$ at $K S 3$; the final $2 \%$ achieved grade $B$ at A level coming from below level 7 at KS3.

\section{Fig. 2.3k}

\section{A Level Physics Entries by Grade and KS3 Prior Attainment, 2009}

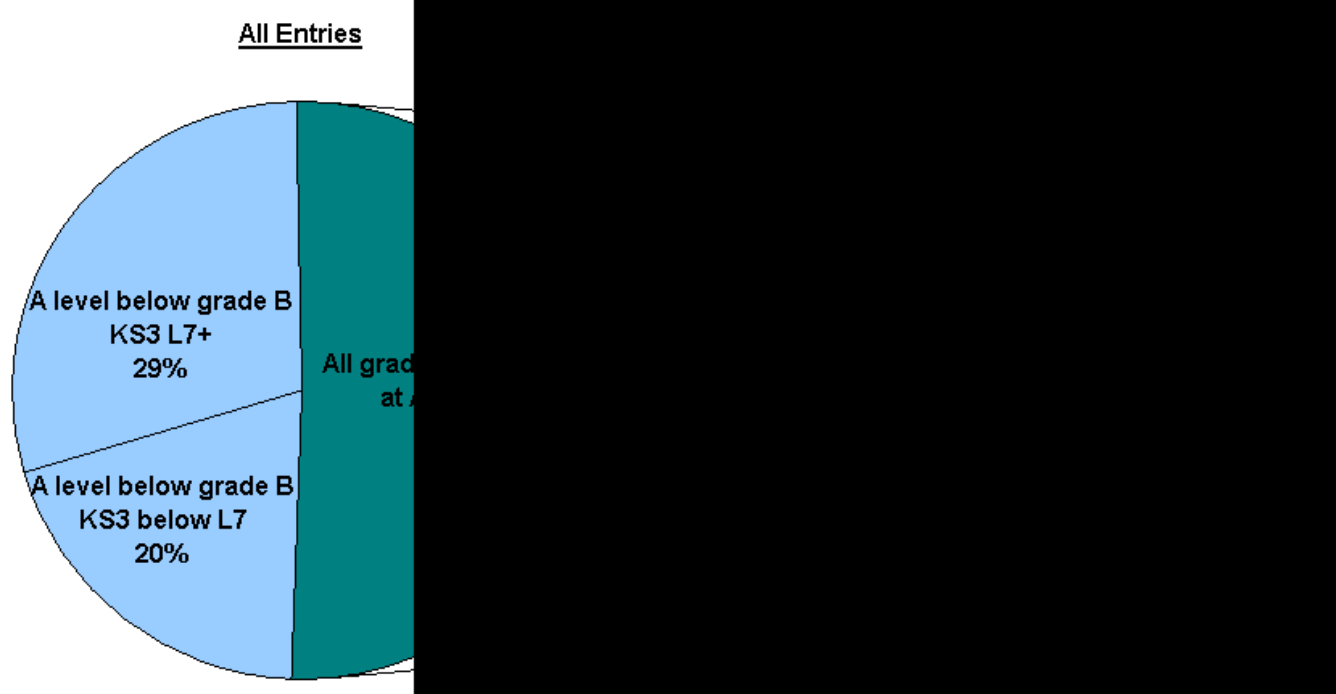

Figure 2.3l presents the percentages of the complete maintained mainstream Key Stage 3 cohort who went on to achieve high grades at A level physics. This takes into account the proportions of pupils who selected themselves out of physics at $A$ level, either because they didn't continue to Key Stage 5, because they took qualifications other than A levels, or because they chose to study other subjects. 
Of the complete KS3 cohort, 1\% went on to achieve a top A grade at A level physics in 2009, with high science attainers at KS3 seven times as likely to achieve this, at $7 \%$. A further $1 \%$ of the cohort achieved grade B at A level physics, compared with $4 \%$ of the high science attainers at KS3. The remainder of A level physics entries with results at grade $\mathrm{C}$ or lower made up 1\% of the KS3 cohort, or $7 \%$ of the high attaining group at KS3 science.

\section{Fig. 2.31}

Progression of KS3 Science Cohorts to A-level Physics for 2007-2009, \% of Cohort

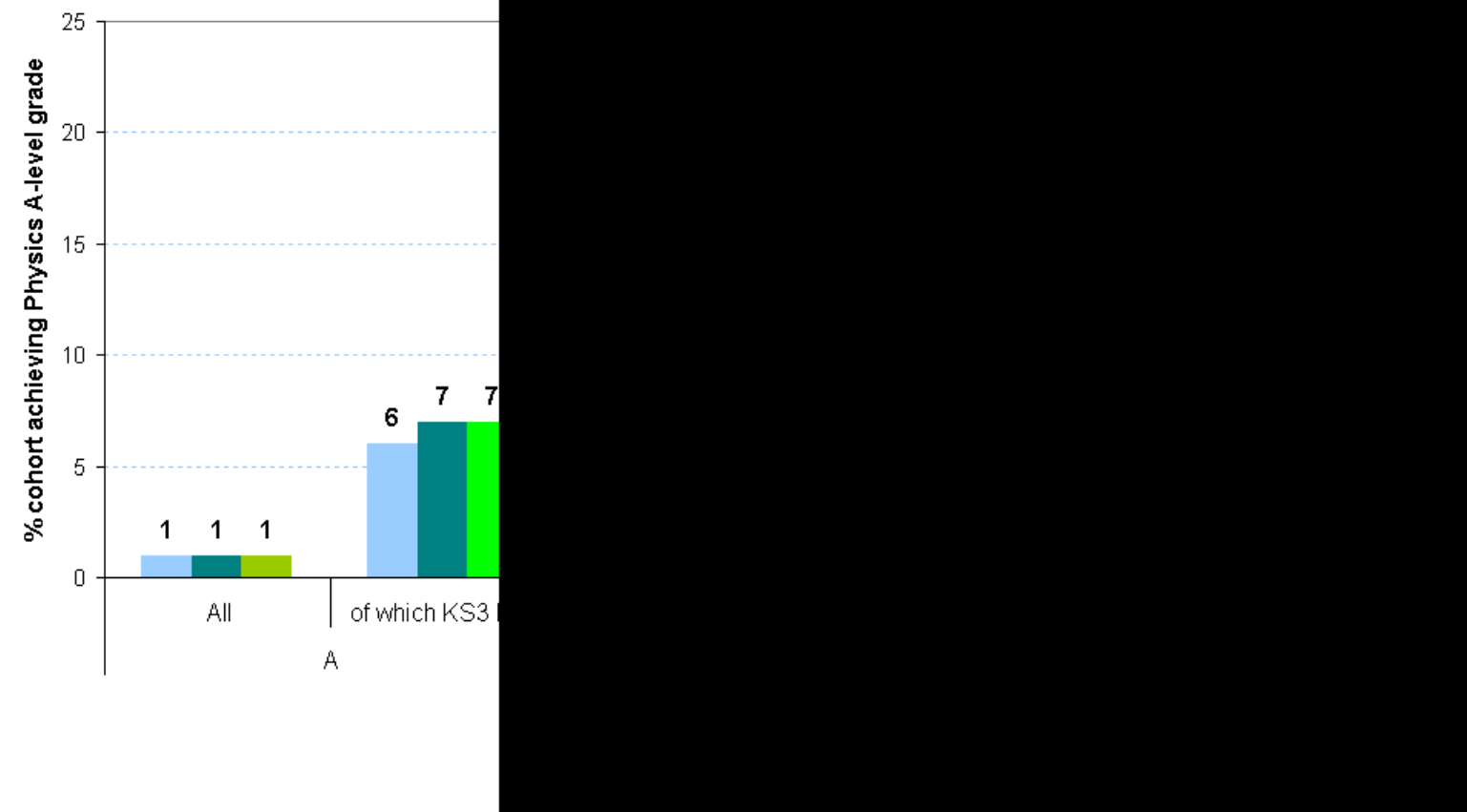

\section{Section Summary}

Section 2.3 has examined progression to high grades in A level maths and science subjects from Key Stage 3. The analyses have revealed a pattern of dependence on strong prior attainment within subject. Only $2 \%$ of A level maths entrants manage to progress from below level 7 at KS3 to achieve an A or a B at A level. For biology and chemistry, this figure is higher at $13 \%$, falling to $6 \%$ for physics. Across the four subjects, the chances of achieving grade $A$ at $A$ level are three to five times higher for pupils who gained level 7+ at KS3 than for those with below level 7 .

Effects of prior attainment across subjects and Key Stages will be compared with one another in section 2.6; for this purpose, odds ratios will be used to remove the effects of differing numbers of pupils falling into the high attaining groups at different Key Stages, and of different achievement rates between the subjects. 


\subsection{Key Stage 2 Attainment and A level Progression}

Moving back one further Key Stage from the analysis of Key Stage 3 to A level progression in section 2.3 above, A level maths and science achievements are broken down according to prior attainment at Key Stage 2 to answer the questions: what was the A level grade achieved by entrants with a given level of achievement at the end of primary education? And, what was the KS2 prior attainment of those who went on to achieve a high grade at A level? At Key Stage 2, the expected level of achievement is level 4 , hence we define high prior attainers as those achieving level 5 or above.

Figure 2.4a shows the percentage of A level maths entrants, split by KS2 maths attainment, who went on to achieve grade A, grade B, and grades below B. In 2009, of entrants from the maintained mainstream cohort with maths achievement at KS2 of below level 5, 20\% achieved grade A at A level, 24\% achieved grade B and the remaining $56 \%$ had results below grade $B$ (including ungraded). The rate of achievement of $A$ grades was just over twice as high for the level 5+ maths attainers at 44\%, with 23\% achieving grade B and 33\% awarded grades C or below.

Fig. 2.4a

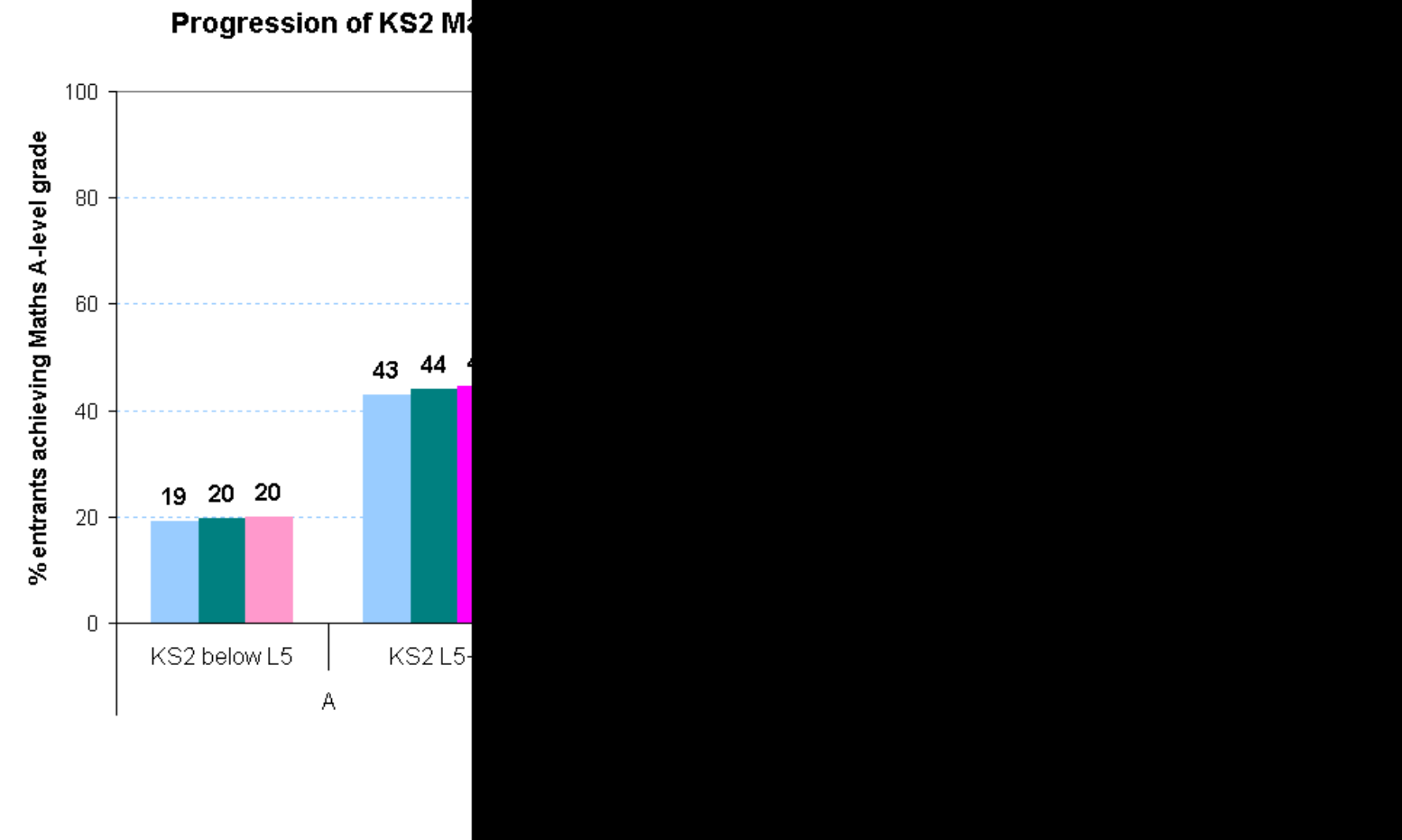

Figure 2.4b takes the same data and expresses the numbers of entrants who were high attainers at Key Stage 2 maths, as a percentage of the group with a given subsequent outcome at A level maths. The large pie on the left splits all cohort entries to A level maths into those achieving level 5 and above at KS2 (64\%) and those with lower results - 8\% with KS2 levels of below level 5 , and a further $29 \%$ who did achieve level $5+$ at KS2 but slipped to below grade B at A level. The smaller pie on the right breaks down the $64 \%$ into As and Bs, revealing that $38 \%$ achieved the highest possible (A) grade at A level after achieving level 5+ at KS2 maths; 3\% came from below level 5 to achieve an A at A level; 19\% managed to achieve a 
grade $B$ at $A$ level having secured level $5+$ at $\mathrm{KS} 2$; the final $3 \%$ achieved grade $\mathrm{B}$ at A level coming from below level 5 at KS2.

Fig. 2.4b

A Level Maths Entries by Grade and KS2 Prior Attainment, 2009

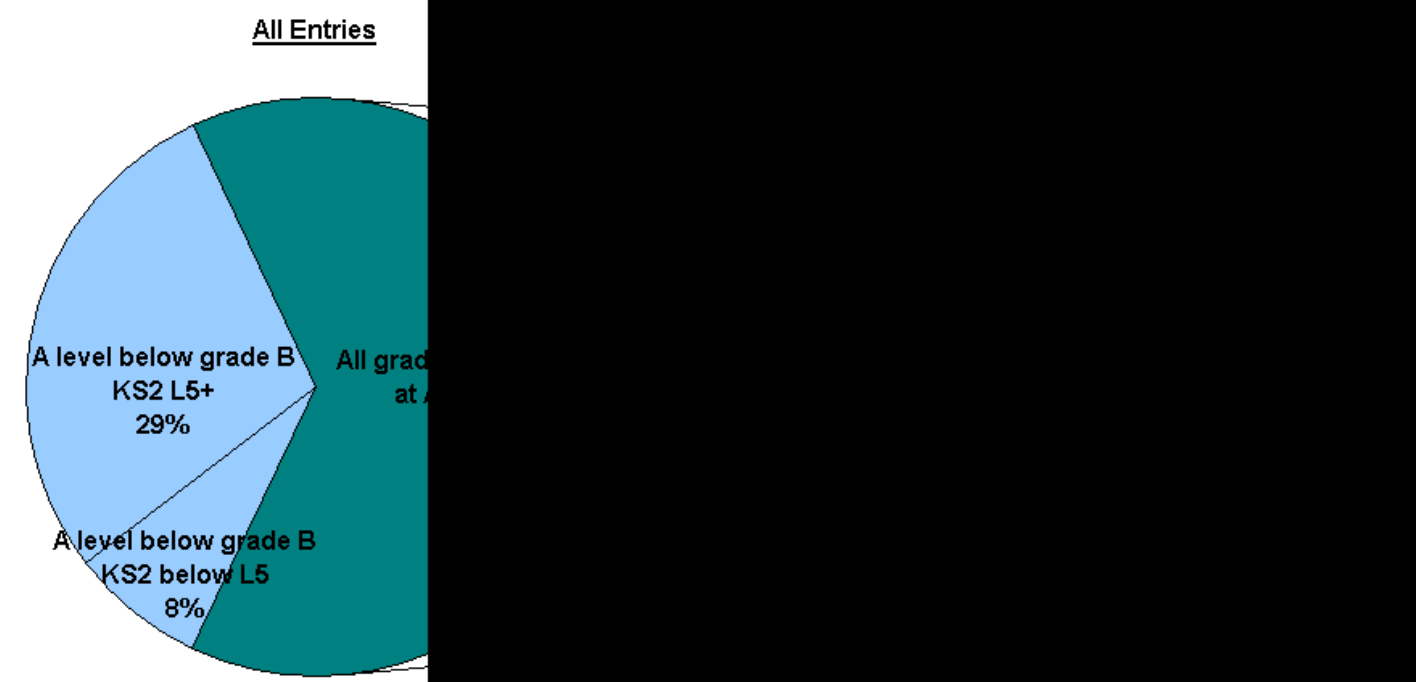

Figure 2.4c presents the percentages of the complete maintained mainstream Key Stage 2 cohort who went on to achieve high grades at A level maths. This takes into account the proportions of pupils who selected themselves out of maths at A level, either because they didn't continue to Key Stage 5, because they took qualifications other than A levels, or because they chose to study other subjects. 
Of the complete KS2 cohort, 3\% went on to achieve a top A grade at A level maths in 2009 , with high maths attainers at KS2 three times as likely to achieve this, at $10 \%$. A further $2 \%$ of the cohort achieved grade B at A level maths, compared with $5 \%$ of the high attainers at KS2. The remainder of A level maths entries with results at grade $\mathrm{C}$ or lower made up 3\% of the $\mathrm{KS} 2$ cohort, or $7 \%$ of the high attaining group at KS2 maths.

\section{Fig. 2.4c}

Progression of KS2 Maths Cohorts to A-level Maths for 2007-2009, \% of Cohort

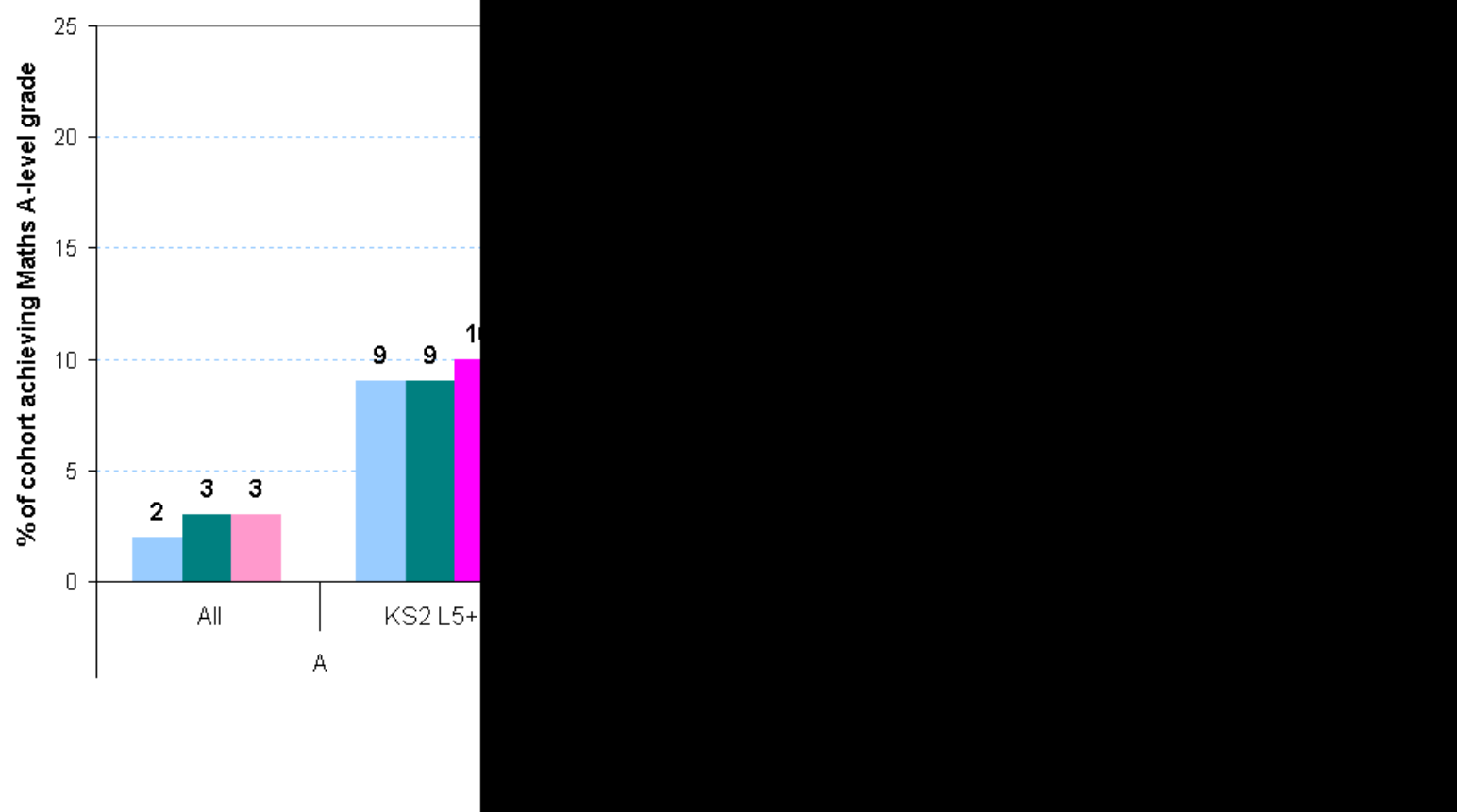


Moving to the science subjects, figure 2.4d shows the percentage of $A$ level biology entrants, split by KS2 science attainment, who went on to achieve grade A, grade $B$, and grades below B. In 2009, of entrants from the maintained mainstream cohort with science achievement at KS2 of below level 5, 12\% achieved grade A at A level, $20 \%$ achieved grade $B$ and the remaining $68 \%$ had results below grade $B$ (including ungraded). The rate of achievement of $A$ grades was over twice as high for the level $5+$ science attainers at 29\%, with 23\% achieving grade B and $47 \%$ awarded grades C or below.

\section{Fig. 2.4d}

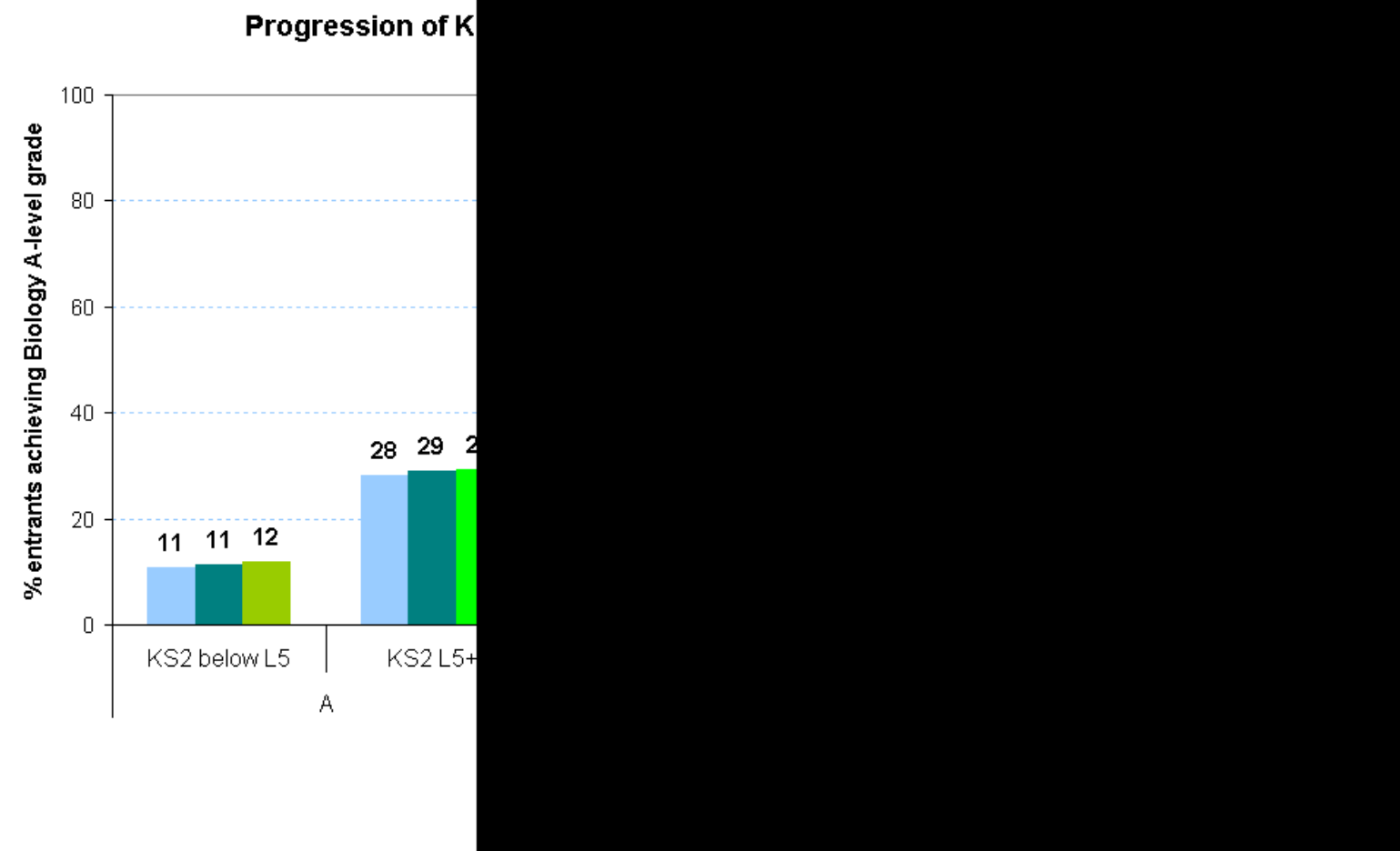


Figure 2.4e takes the same data and expresses the numbers of entrants who were high attainers at Key Stage 2 science, as a percentage of the group with a given subsequent outcome at $A$ level biology. The large pie on the left splits all cohort entries to A level biology into those achieving level 5 and above at KS2 (49\%) and those with lower results - 12\% with KS2 levels of below level 5 , and a further $39 \%$ who did achieve level 5+ at KS2 but slipped to below grade B at A level. The smaller pie on the right breaks down the $49 \%$ into As and Bs, revealing that $24 \%$ achieved the highest possible $(A)$ grade at A level after achieving level $5+$ at KS2 science; $2 \%$ came from below level 5 to achieve an A at A level; 19\% managed to achieve a grade $B$ at A level having secured level $5+$ at $K S 2$; the final $4 \%$ achieved grade $B$ at A level coming from below level 5 at KS2.

\section{Fig. 2.4e}

A Level Biology Entries by Grade and KS2 Prior Attainment, 2009

All Entries

Grades A and B

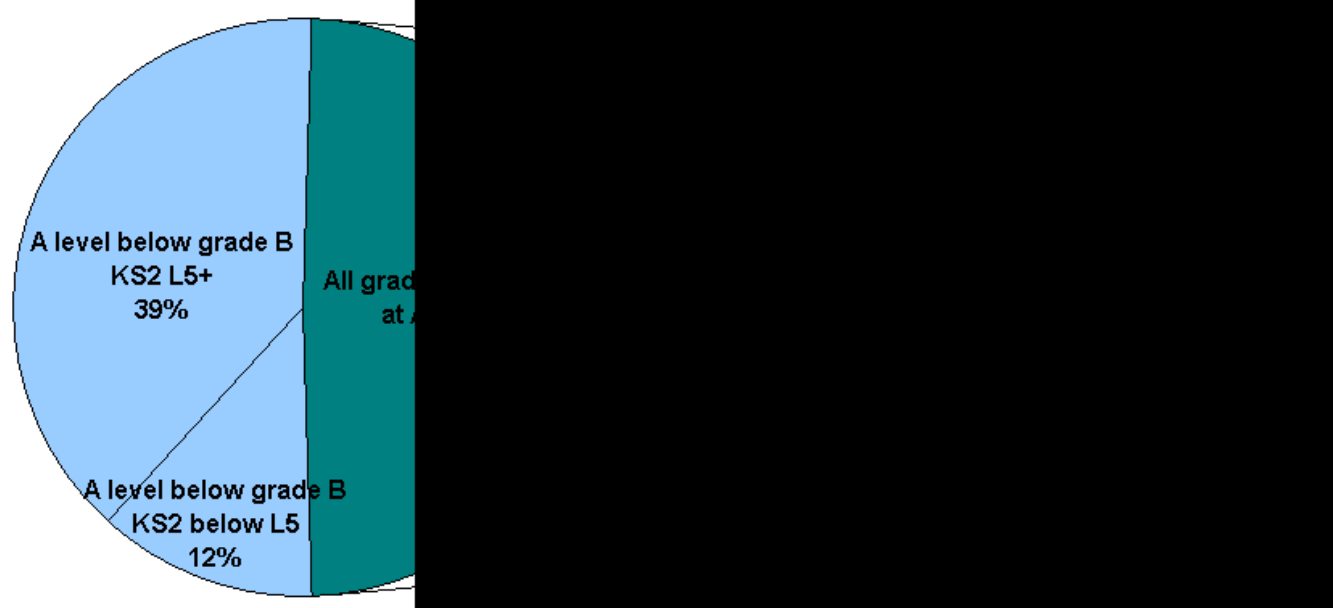


Figure 2.4f presents the percentages of the complete maintained mainstream Key Stage 2 cohort who went on to achieve high grades at A level biology. This takes into account the proportions of pupils who selected themselves out of biology at $A$ level, either because they didn't continue to Key Stage 5, because they took qualifications other than A levels, or because they chose to study other subjects.

Of the complete KS2 cohort, 1\% went on to achieve a top A grade at A level biology in 2009 , with high science attainers at KS2 three times as likely to achieve this, at $3 \%$. A further $1 \%$ of the cohort achieved grade B at A level biology, compared with $3 \%$ of the high attainers at KS2. The remainder of A level biology entries with results at grade $\mathrm{C}$ or lower made up 3\% of the KS2 cohort, or 6\% of the high attaining group at KS2 science.

\section{Fig. $2.4 f$}

Progression of KS2 Science Cohorts to A-level Biology for 2007-2009, \% of Cohort

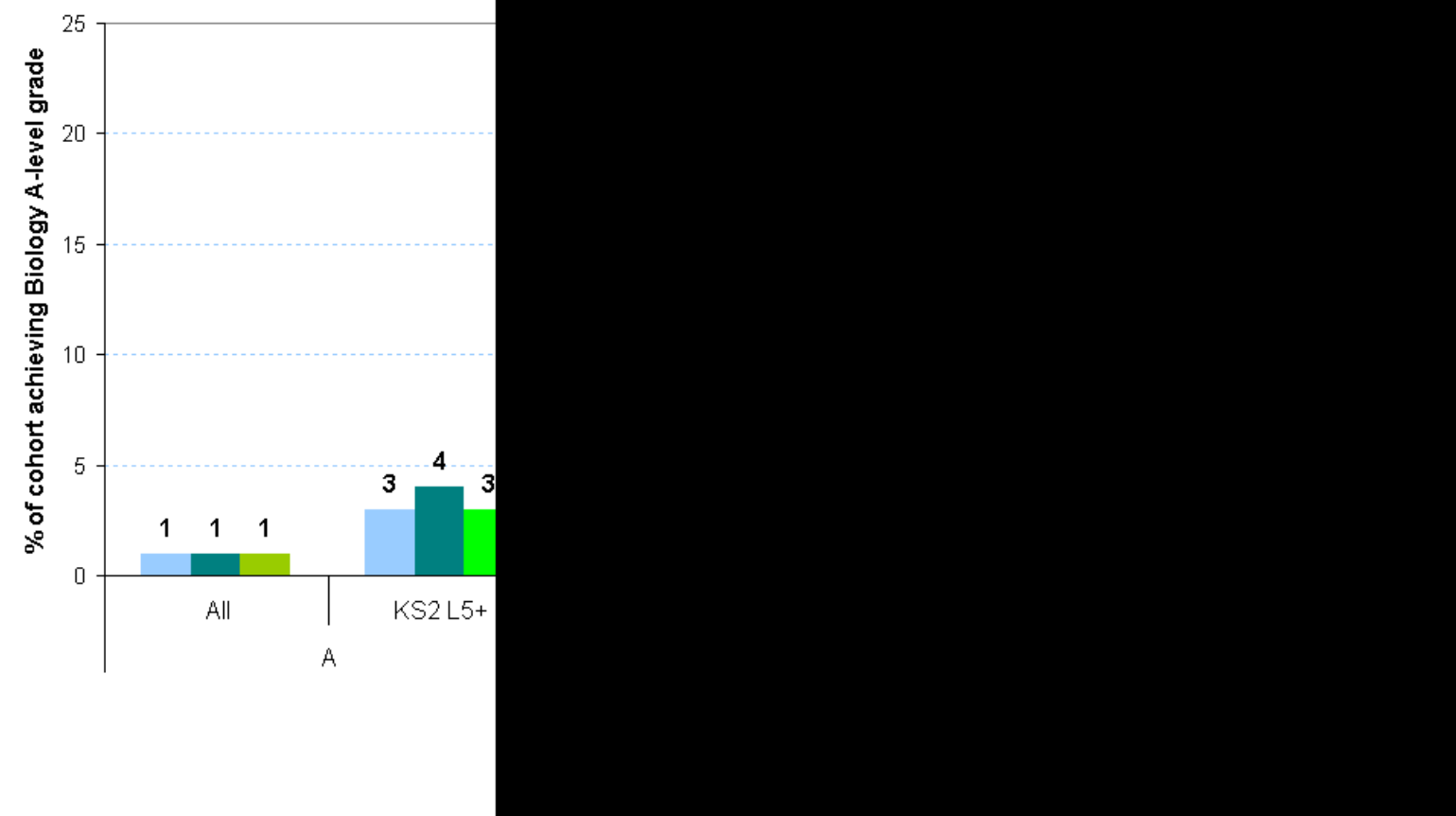


Turning to the next science subject, figure $2.4 \mathrm{~g}$ shows the percentage of A level chemistry entrants, split by KS2 science attainment, who went on to achieve grade A, grade $B$, and grades below $B$. In 2009 , of entrants from the maintained mainstream cohort with science achievement at KS2 of below level 5, 16\% achieved grade $A$ at $A$ level, $23 \%$ achieved grade $B$ and the remaining $61 \%$ had results below grade $B$ (including ungraded). The rate of achievement of $A$ grades was twice as high for the level 5+ science attainers at 34\%, with 25\% achieving grade B and $41 \%$ awarded grades $\mathrm{C}$ or below.

\section{Fig. $2.4 \mathrm{~g}$}

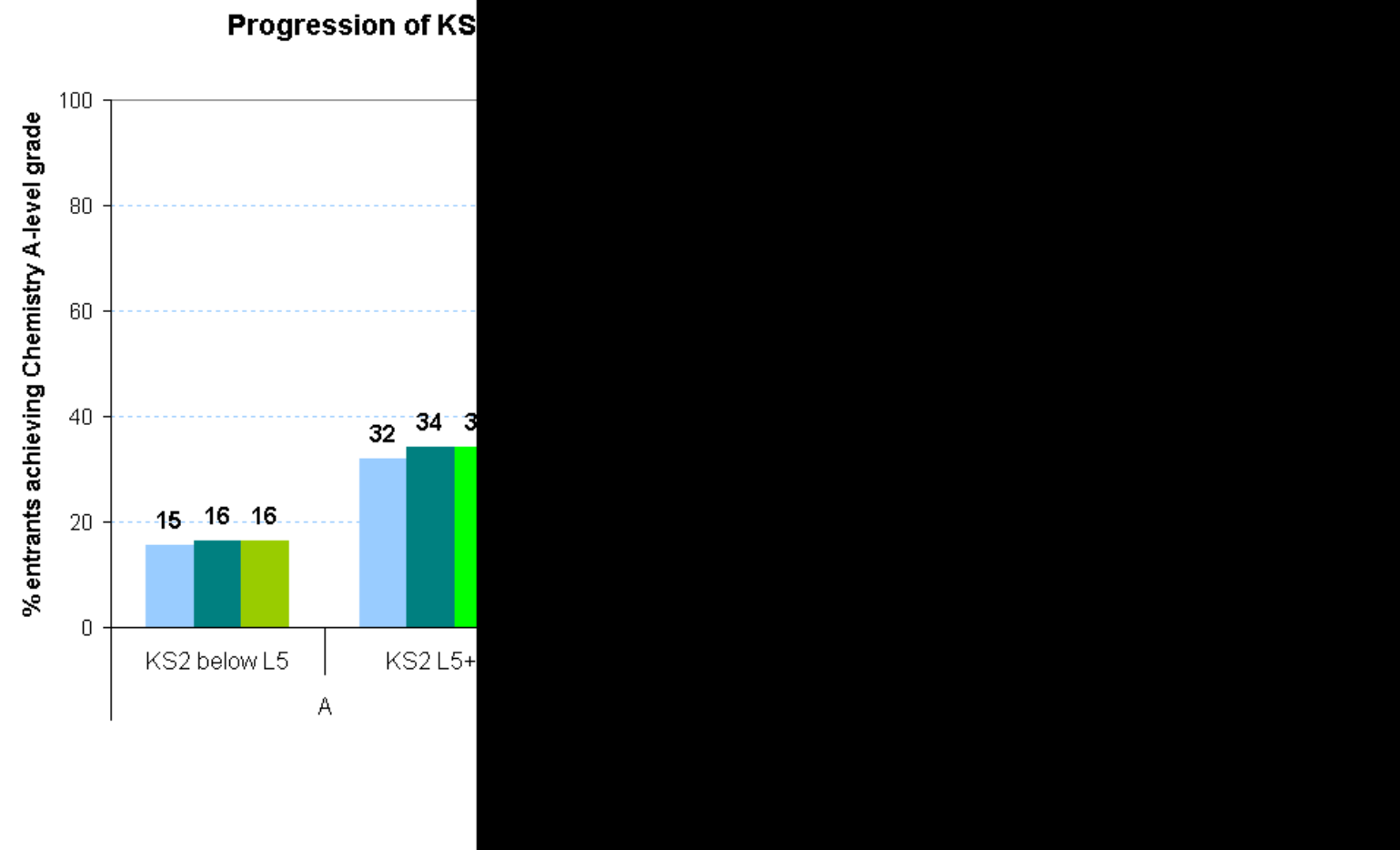


Figure 2.4h takes the same data and expresses the numbers of entrants who were high attainers at Key Stage 2 science, as a percentage of the group with a given subsequent outcome at A level chemistry. The large pie on the left splits all cohort entries to A level chemistry into those achieving level 5 and above at KS2 (56\%) and those with lower results - 9\% with KS2 levels of below level 5 , and a further $36 \%$ who did achieve level $5+$ at KS2 but slipped to below grade B at A level. The smaller pie on the right breaks down the $56 \%$ into As and Bs, revealing that $29 \%$ achieved the highest possible (A) grade at A level after achieving level 5+ at KS2 science; 2\% came from below level 5 to achieve an $A$ at $A$ level; $21 \%$ managed to achieve a grade $B$ at $A$ level having secured level $5+$ at $\mathrm{KS} 2$; the final 3\% achieved grade $B$ at A level coming from below level 5 at KS2.

Fig. 2.4h

A Level Chemistry Entries by Grade and KS2 Prior Attainment, 2009

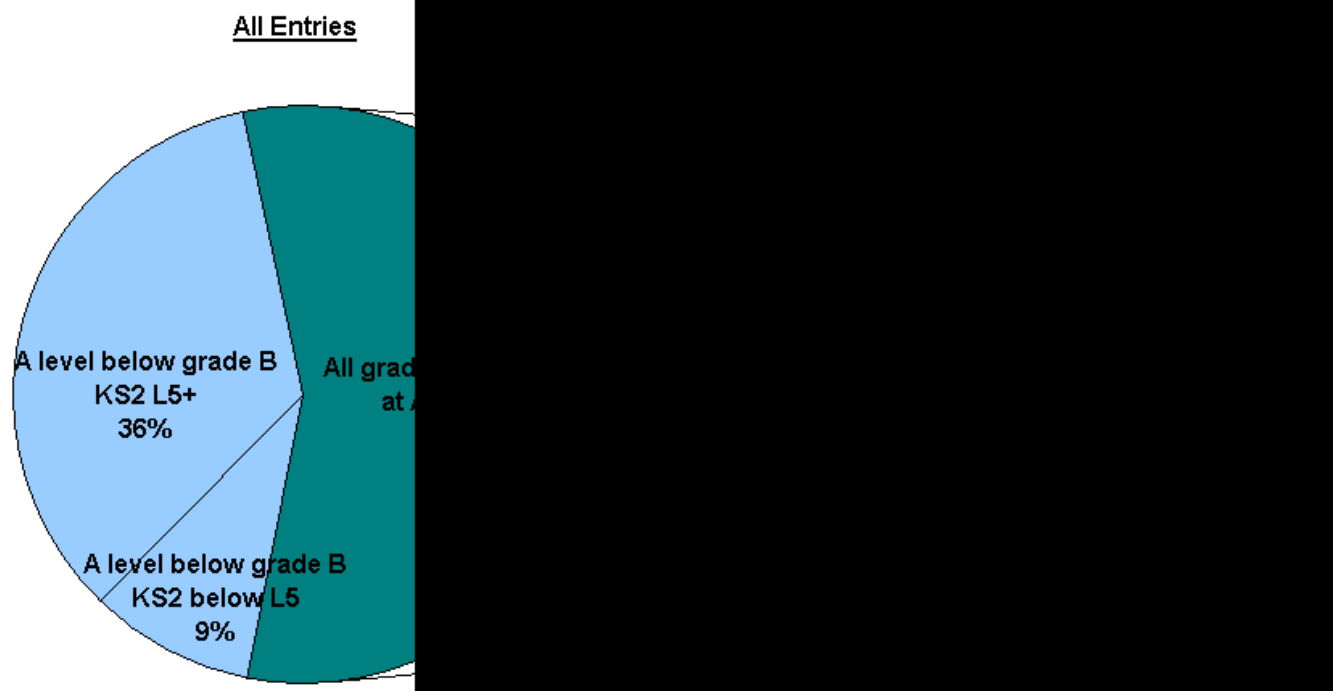


Figure 2.4i presents the percentages of the complete maintained mainstream Key Stage 2 cohort who went on to achieve high grades at A level chemistry. This takes into account the proportions of pupils who selected themselves out of chemistry at A level, either because they didn't continue to Key Stage 5, because they took qualifications other than A levels, or because they chose to study other subjects.

Of the complete KS2 cohort, $1 \%$ went on to achieve a top A grade at A level chemistry in 2009, with high science attainers at KS2 three times as likely to achieve this, at $3 \%$. A further $1 \%$ of the cohort achieved grade B at A level chemistry, compared with $2 \%$ of the high attainers at KS2. The remainder of A level chemistry entries with results at grade C or lower made up $2 \%$ of the KS2 cohort, or $4 \%$ of the high attaining group at KS2 science.

Fig. 2.4i

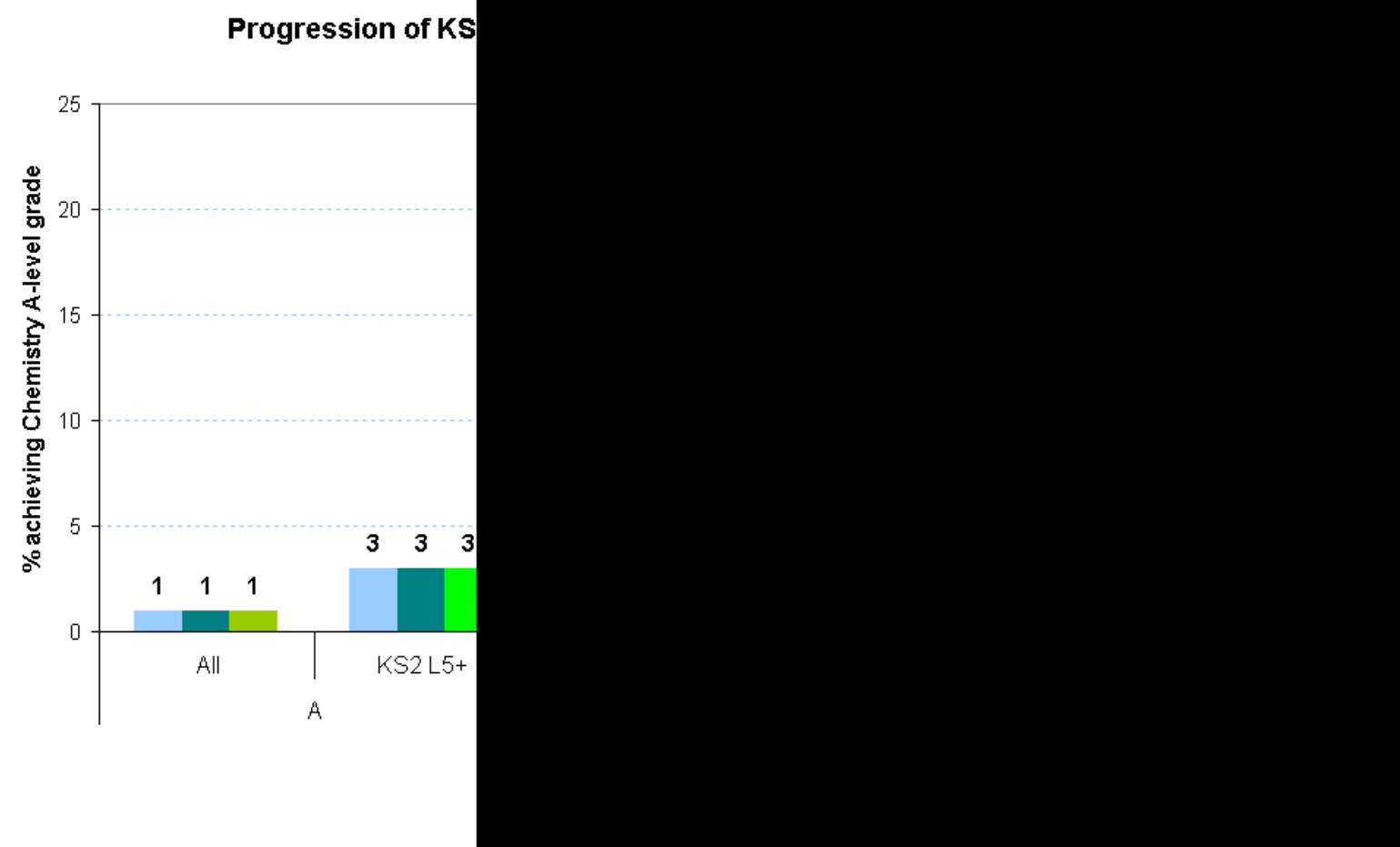


Moving to the third and final traditional science, figure 2.4j shows the percentage of $A$ level physics entrants, split by KS2 science attainment, who went on to achieve grade A, grade B, and grades below B. In 2009, of entrants from the maintained mainstream cohort with science achievement at KS2 of below level 5, 11\% achieved grade $A$ at $A$ level, $15 \%$ achieved grade $B$ and the remaining $74 \%$ had results below grade $B$ (including ungraded). The rate of achievement of $A$ grades was almost three times as high for the level 5+ science attainers at 32\%, with 22\% achieving grade B and $47 \%$ awarded grades $\mathrm{C}$ or below.

\section{Fig. 2.4j}

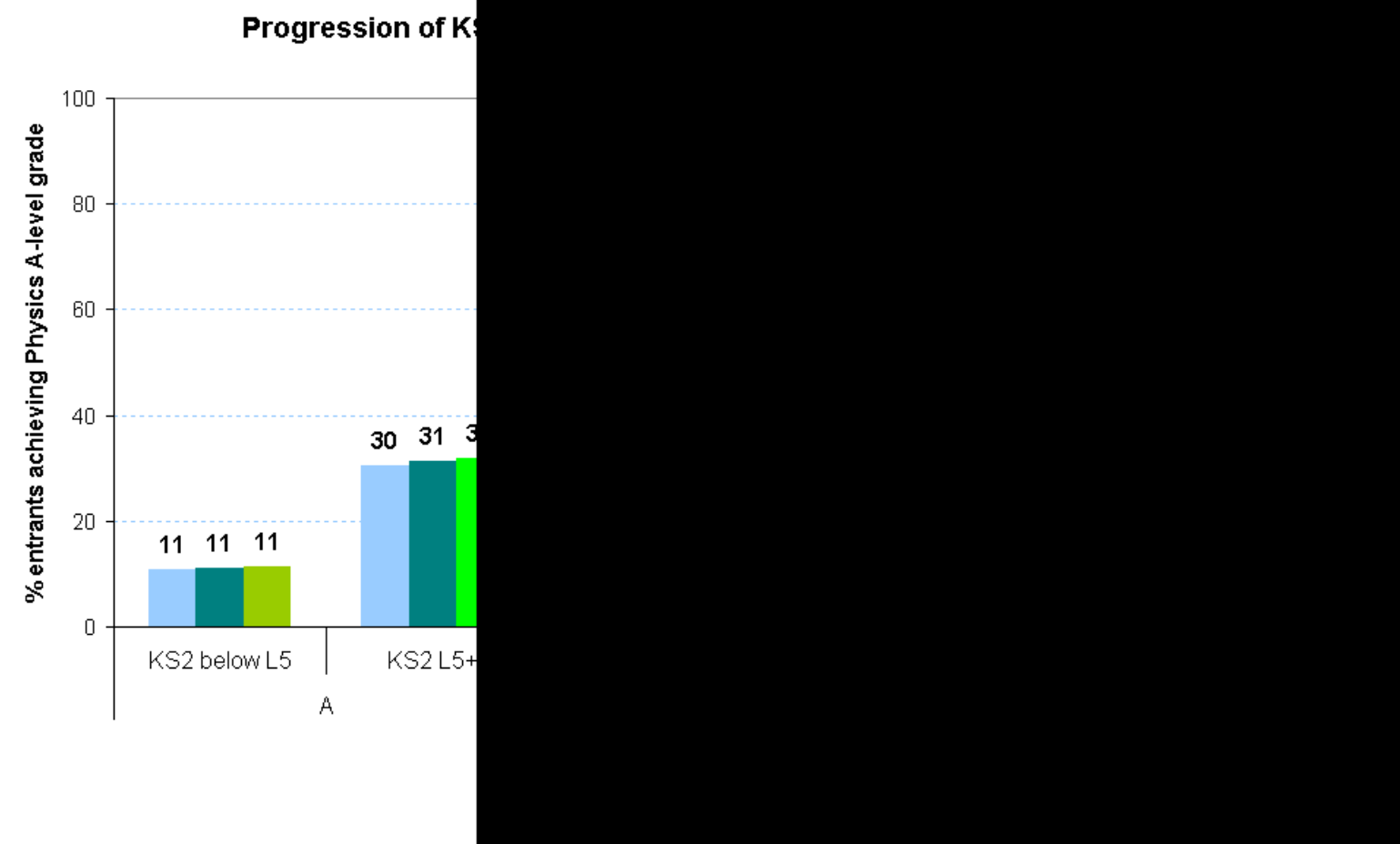


Figure 2.4k takes the same data and expresses the numbers of entrants who were high attainers at Key Stage 2 science, as a percentage of the group with a given subsequent outcome at $A$ level physics. The large pie on the left splits all cohort entries to A level physics into those achieving level 5 and above at KS2 (61\%) and those with lower results - 7\% with KS2 levels of below level 5 , and a further $43 \%$ who did achieve level $5+$ at KS2 but slipped to below grade B at A level. The smaller pie on the right breaks down the $61 \%$ into As and Bs, revealing that $29 \%$ achieved the highest possible (A) grade at A level after achieving level $5+$ at $\mathrm{KS} 2$ science; $1 \%$ came from below level 5 to achieve an $A$ at $A$ level; $19 \%$ managed to achieve a grade $B$ at $A$ level having secured level $5+$ at $\mathrm{KS} 2$; the final $1 \%$ achieved grade $B$ at A level coming from below level 5 at KS2.

\section{Fig. 2.4k}

\section{A Level Physics Entries by Grade and KS2 Prior Attainment, 2009}

All Entries

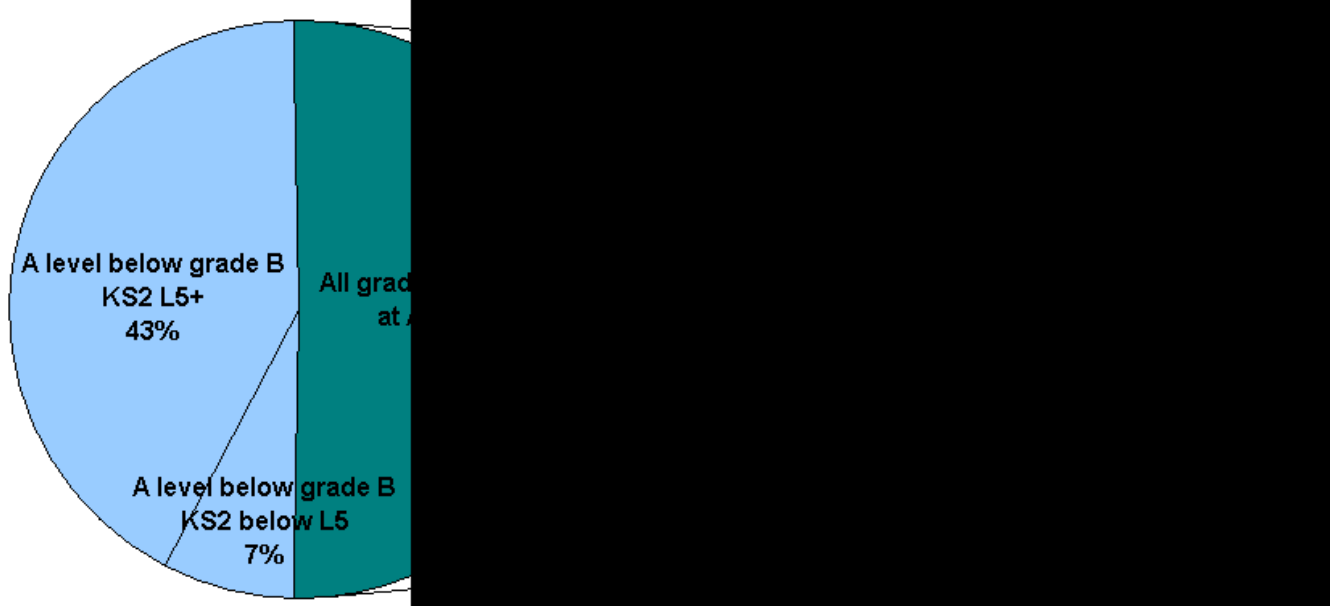


Figure 2.4I presents the percentages of the complete maintained mainstream Key Stage 2 cohort who went on to achieve high grades at A level physics. This takes into account the proportions of pupils who selected themselves out of physics at $A$ level, either because they didn't continue to Key Stage 5, because they took qualifications other than A levels, or because they chose to study other subjects.

Of the complete KS2 cohort, 1\% went on to achieve a top A grade at A level physics in 2009 , with high science attainers at KS2 at $2 \%$. A further $1 \%$ of the cohort achieved grade $B$ at A level physics, compared with the same percentage of the high attainers at KS2. The remainder of A level chemistry entries with results at grade $\mathrm{C}$ or lower made up 1\% of the KS2 cohort, or 3\% of the high attaining group at KS2 science.

\section{Fig. 2.41}

Progression of KS2 Science Cohorts to A-level Physics for 2007-2009, \% of Cohort

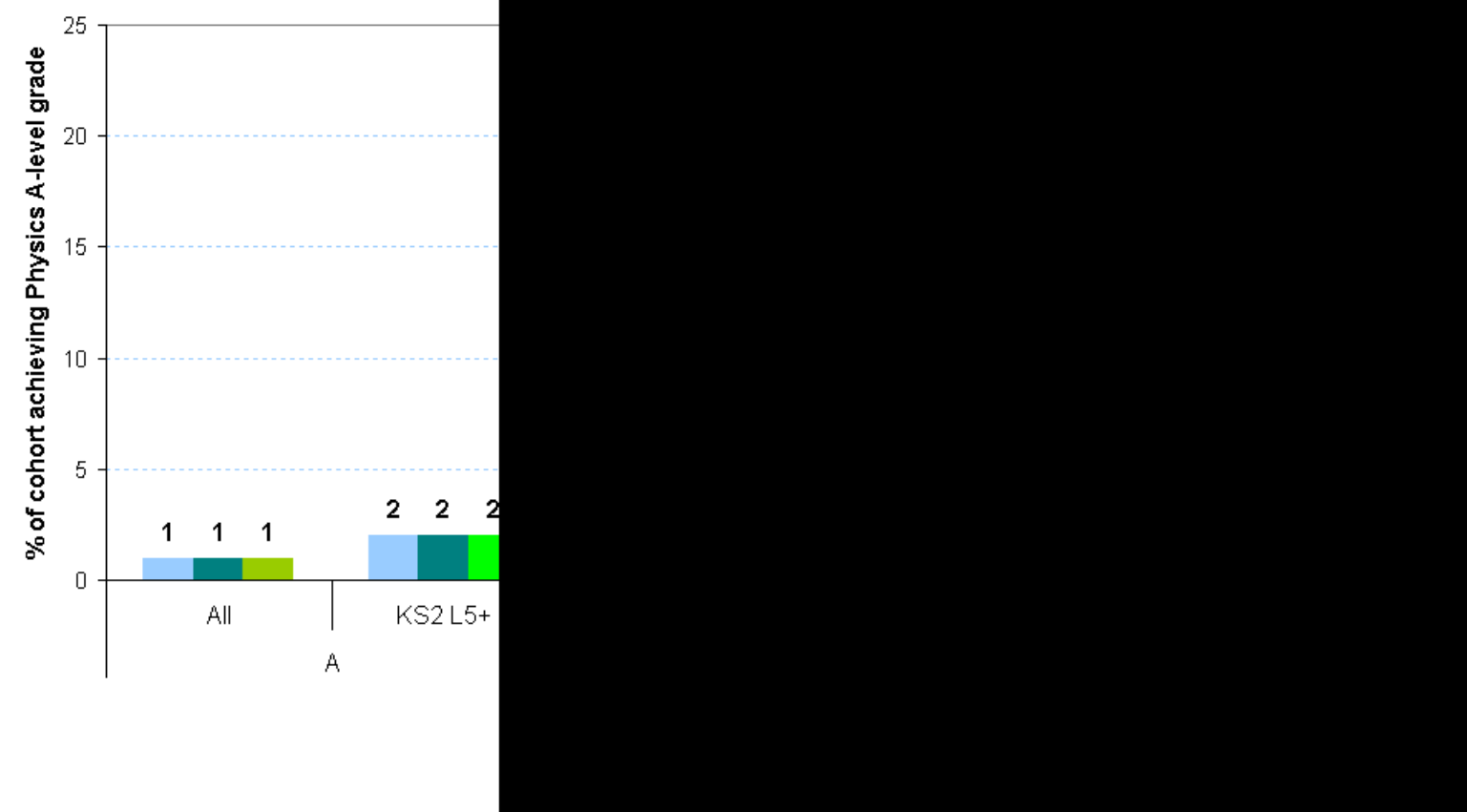

\section{Section Summary}

Section 2.4 has examined progression to high grades in A level maths and science subjects from Key Stage 2. The analyses have revealed a pattern of persisting dependence on strong prior attainment within subject from the end of primary education. Six percent of $A$ level maths entrants manage to progress from below level 5 at KS2 to achieve an A or a B at A level. For biology, this figure is also 6\%, falling to $5 \%$ for chemistry and just $2 \%$ for physics. Across the four subjects, the chances of achieving grade $A$ at $A$ level are two to three times higher for pupils who gained level 5+ at KS2 than for those with below level 5.

Effects of prior attainment across subjects and Key Stages will be compared with one another in section 2.6; for this purpose, odds ratios will be used to remove the effects of differing numbers of pupils falling into the high attaining groups at different Key Stages, and of different achievement rates between the subjects. 


\subsection{Key Stage 1 Attainment and A level Progression}

Only one cohort of pupils is shown in the analyses below as cohorts with Key Stage 1 data held by DfE have only recently begun to reach the end of Key Stage 5. A level maths and science achievements are broken down according to prior attainment at Key Stage 1 to answer the questions: what was the A level grade achieved by entrants with a given level of achievement at KS1? And, what was the KS1 prior attainment of those who went on to achieve a high grade at A level? At Key Stage 1, the expected level of achievement is level 2 , hence we define high prior attainers as those achieving level 3 or above.

Figure 2.5a shows the percentage of $A$ level maths entrants, split by KS1 maths attainment, who went on to achieve grade A, grade B, and grades below B. In 2009, of entrants from the maintained mainstream cohort with maths achievement at KS1 of below level 3, 30\% achieved grade A at A level, 24\% achieved grade B and the remaining $46 \%$ had results below grade $B$ (including ungraded). The rate of achievement of A grades was $48 \%$ for the level 3+ maths attainers, with $22 \%$ achieving grade $\mathrm{B}$ and $30 \%$ awarded grades $\mathrm{C}$ or below.

\section{Fig. 2.5a}

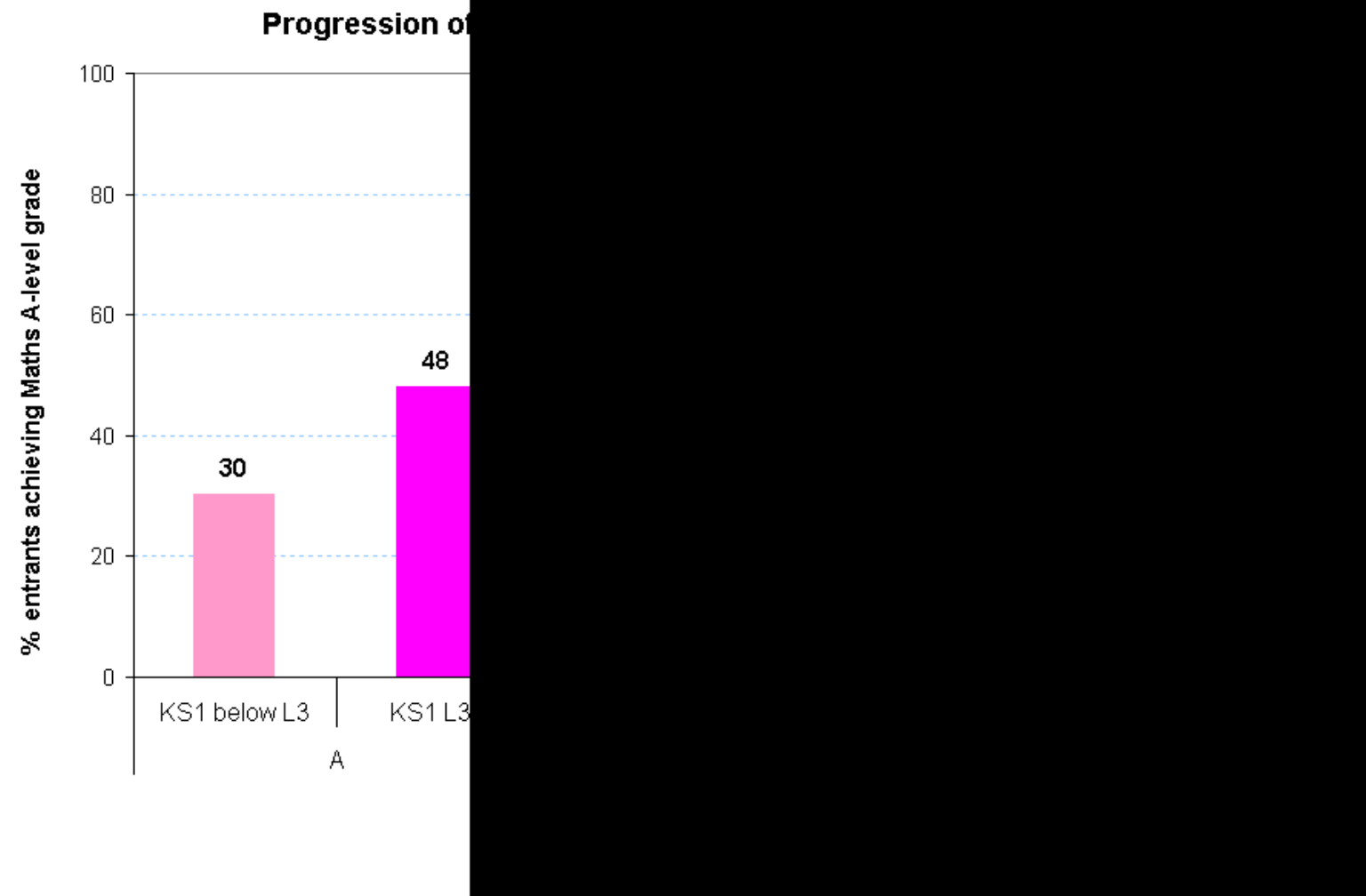

Figure 2.5b takes the same data and expresses the numbers of entrants who were high attainers at Key Stage 1 maths, as a percentage of the group with a given subsequent outcome at $A$ level maths. The large pie on the left splits all cohort entries to A level maths into those achieving level 3 and above at KS1 (64\%) and those with lower results - 19\% with KS2 levels of below level 3, and a further $18 \%$ who did achieve level 3+ at KS1 but slipped to below grade B at A level. The smaller pie on the right breaks down the $64 \%$ into As and Bs, revealing that $28 \%$ achieved 
the highest possible (A) grade at A level after achieving level 3+ at KS1 maths; $12 \%$ came from below level 3 to achieve an $A$ at $A$ level; $13 \%$ managed to achieve a grade B at A level having secured level 3+ at KS1; the final 10\% achieved grade B at A level coming from below level 3 at $\mathrm{KS} 1$.

\section{Fig. 2.5b}

A Level Maths Entries by Grade and KS1 Prior Attainment, 2009

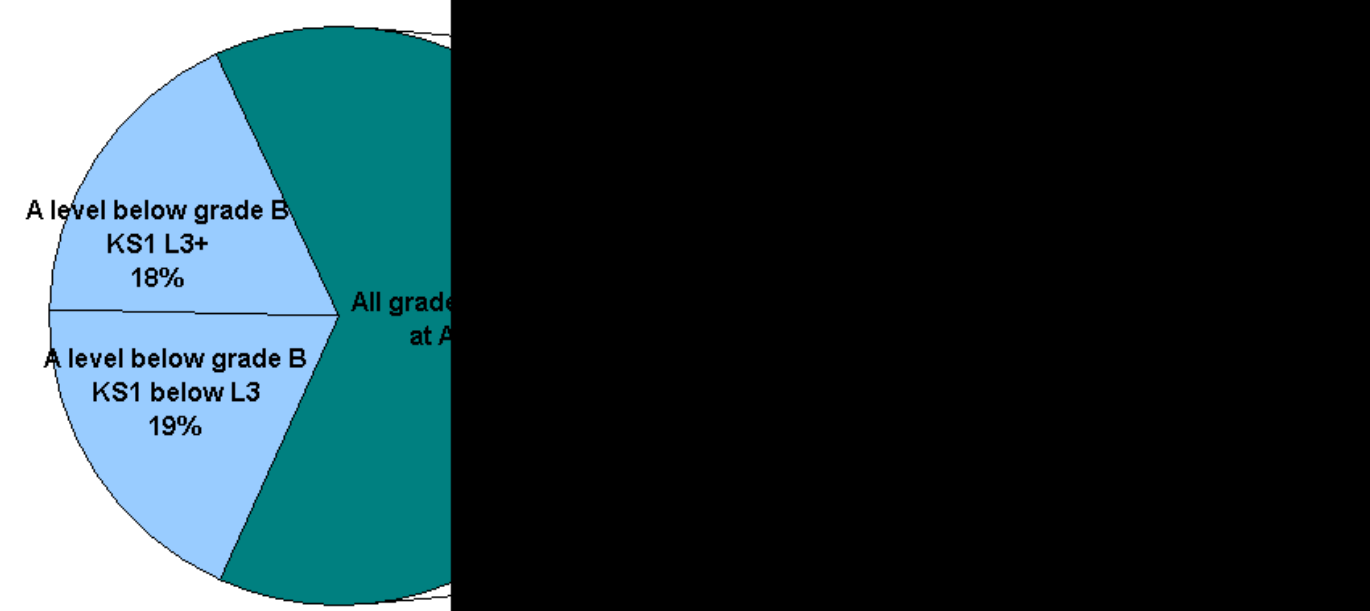


Figure 2.5c presents the percentages of the complete maintained mainstream Key Stage 1 cohort who went on to achieve high grades at A level maths. This takes into account the proportions of pupils who selected themselves out of maths at A level, either because they didn't continue to Key Stage 5, because they took qualifications other than A levels, or because they chose to study other subjects.

Of the complete KS1 cohort, 3\% went on to achieve a top A grade at A level maths in 2009 , with high maths attainers at KS1 three times as likely to achieve this, at $10 \%$. A further $2 \%$ of the cohort achieved grade B at A level maths, compared with $4 \%$ of the high attainers at KS1. The remainder of A level maths entries with results at grade $\mathrm{C}$ or lower made up $2 \%$ of the $\mathrm{KS} 1$ cohort, or $6 \%$ of the high attaining group at KS1 maths.

\section{Fig.2.5c}

Progression of KS1 Cohort to A-level Maths for in 2009, \% of Cohort

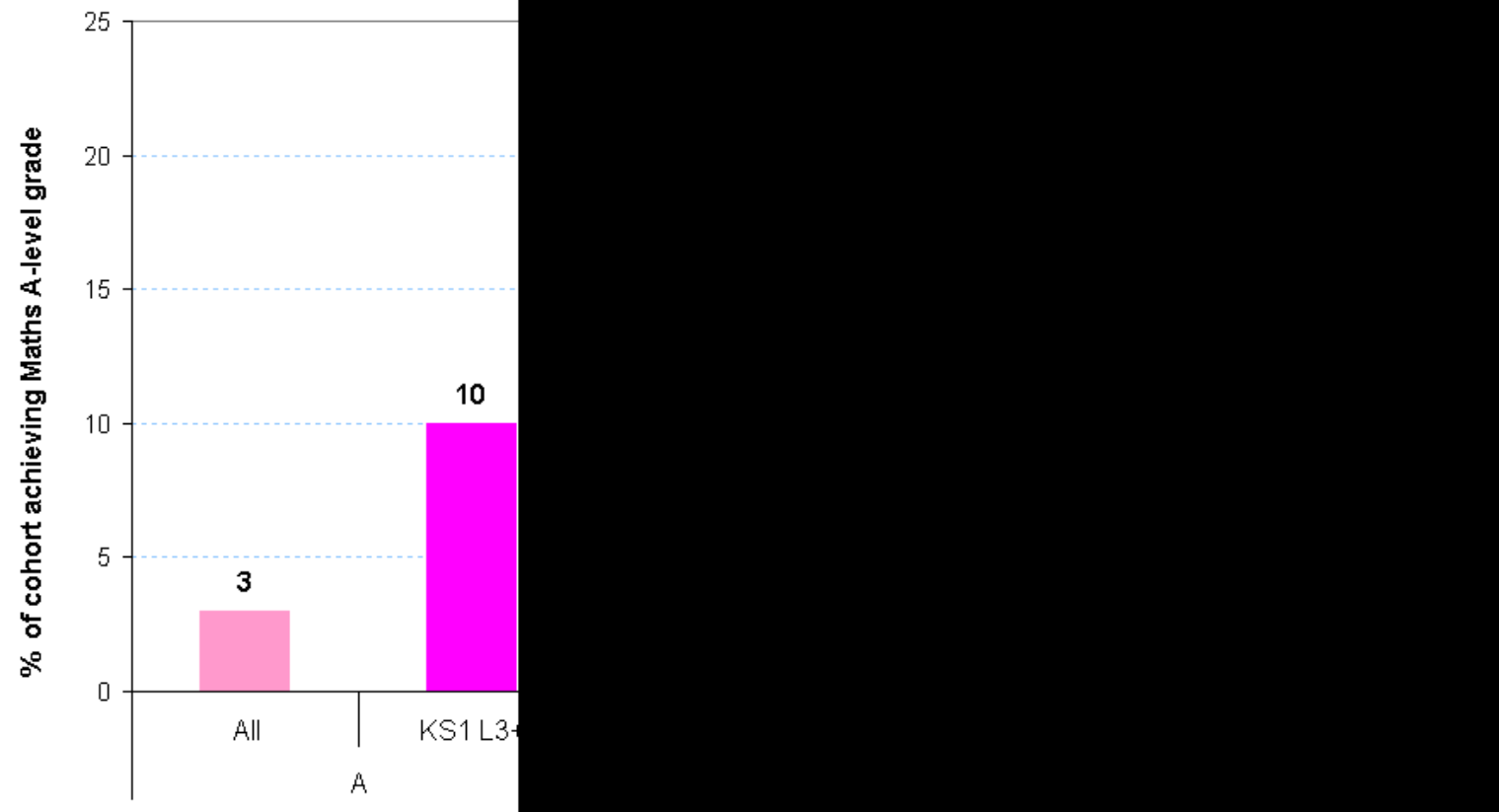

KS1 Prior Attainment

A-level Maths Grade 
Turning to KS1 to A level biology progression, figure $2.5 \mathrm{a}$ shows the percentage of $\mathrm{A}$ level biology entrants, split by KS1 science attainment, who went on to achieve grade A, grade B, and grades below B. In 2009, of entrants from the maintained mainstream cohort with science achievement at KS1 of below level 3, 30\% achieved grade $A$ at A level, $24 \%$ achieved grade B and the remaining $46 \%$ had results below grade $B$ (including ungraded). The rate of achievement of $A$ grades was $48 \%$ for the level $3+$ maths attainers, with $22 \%$ achieving grade B and $30 \%$ awarded grades C or below.

\section{Fig. 2.5d}

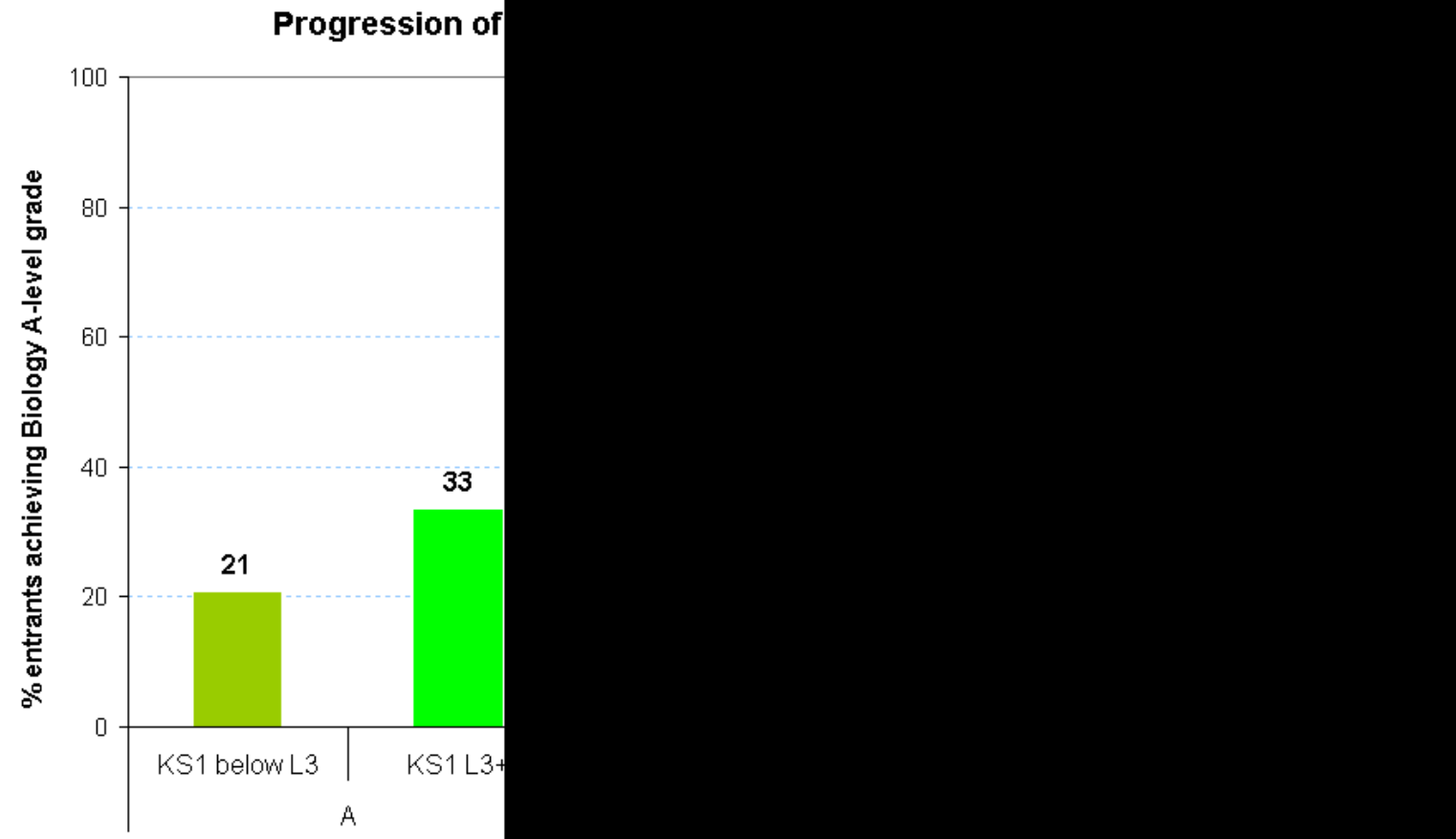

KS1 Prior Attainment A-level Biology Grade 
Figure 2.5e takes the same data and expresses the numbers of entrants who were high attainers at Key Stage 1 science, as a percentage of the group with a given subsequent outcome at $A$ level biology. The large pie on the left splits all cohort entries to A level biology into those achieving level 3 and above at KS1 (49\%) and those with lower results - 33\% with KS2 levels of below level 3, and a further $18 \%$ who did achieve level 3+ at KS1 but slipped to below grade B at A level. The smaller pie on the right breaks down the $49 \%$ into As and Bs, revealing that $14 \%$ achieved the highest possible $(A)$ grade at $A$ level after achieving level $3+$ at $\mathrm{KS} 1$ science; $12 \%$ came from below level 3 to achieve an $A$ at $A$ level; $10 \%$ managed to achieve a grade $B$ at $A$ level having secured level $3+$ at $\mathrm{KS} 1$; the final 13\% achieved grade $B$ at A level coming from below level 3 at KS1.

\section{Figure 2.5e}

A Level Biology Entries by Grade and KS1 Prior Attainment, 2009

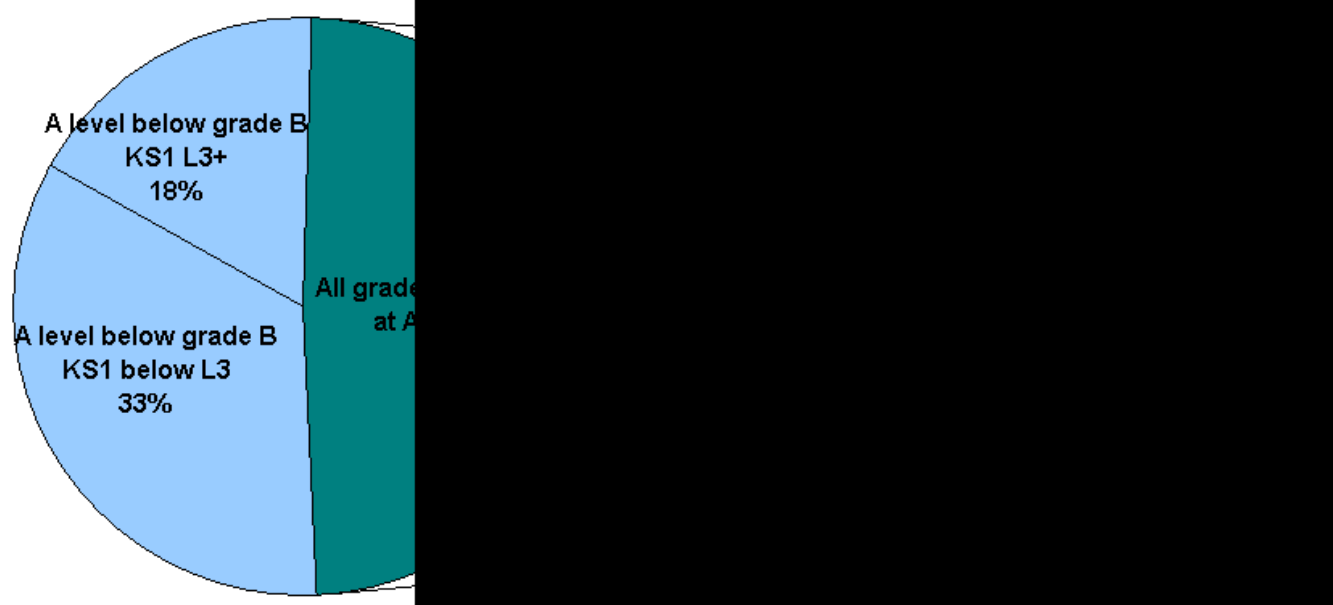

Figure $2.5 f$ presents the percentages of the complete maintained mainstream Key Stage 1 cohort who went on to achieve high grades at A level biology. This takes into account the proportions of pupils who selected themselves out of biology at $A$ level, either because they didn't continue to Key Stage 5, because they took qualifications other than A levels, or because they chose to study other subjects. 
Of the complete KS1 cohort, 1\% went on to achieve a top A grade at A level biology in 2009 , compared with $4 \%$ of the high science attainers at KS1. A further $1 \%$ of the cohort achieved grade $B$ at $A$ level biology, compared with $3 \%$ of the high attainers at $\mathrm{KS} 1$. The remainder of $A$ level biology entries with results at grade $\mathrm{C}$ or lower made up 3\% of the KS1 cohort, or 5\% of the high attaining group at KS1 science.

\section{Fig. 2.5f}

Progression of KS1 Cohorts to A-level Biology for 2007 in 2009, \% of Cohort

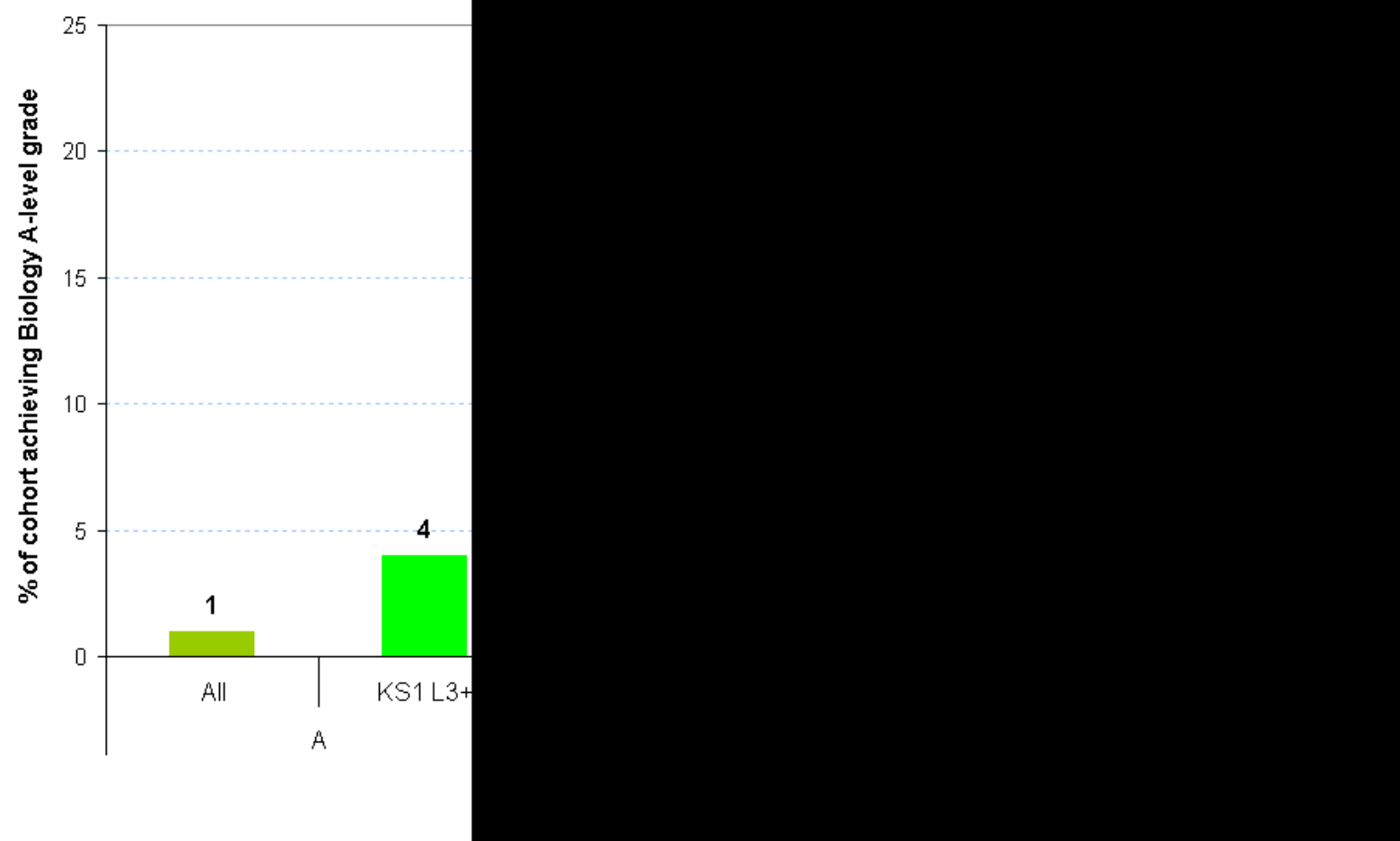


Turning to A level chemistry progression, figure $2.5 \mathrm{~g}$ shows the percentage of A level entrants, split by KS1 science attainment, who went on to achieve grade A, grade B, and grades below B. In 2009, of entrants from the maintained mainstream cohort with science achievement at KS1 of below level 3, 25\% achieved grade A at A level, $24 \%$ achieved grade $B$ and the remaining $51 \%$ had results below grade $B$ (including ungraded). The rate of achievement of $A$ grades was $39 \%$ for the level 3+ science attainers, with $26 \%$ achieving grade $\mathrm{B}$ and $36 \%$ awarded grades $\mathrm{C}$ or below.

\section{Fig. $2.5 \mathrm{~g}$}

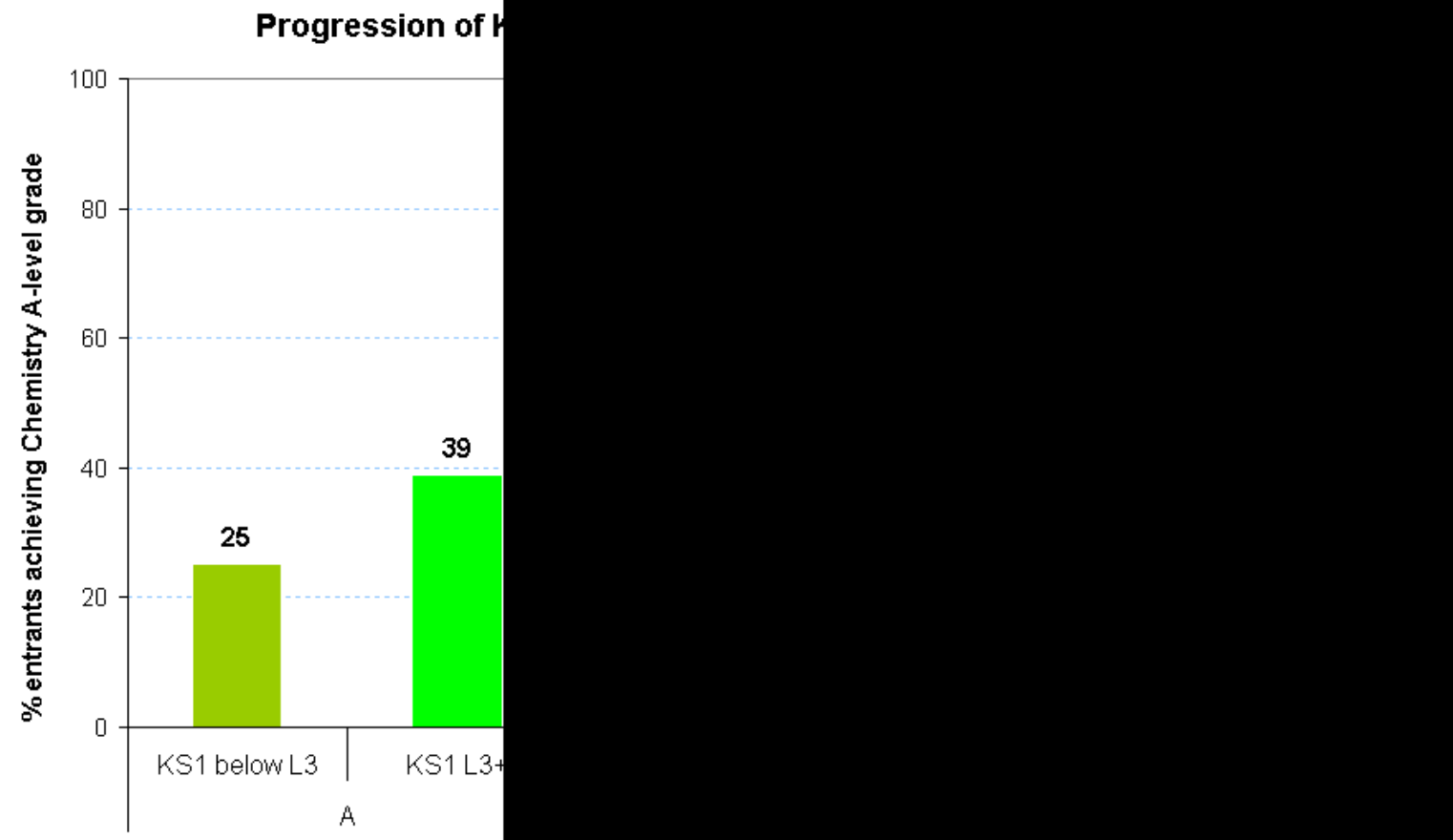

KS1 Prior Attainment A-level Chemistry Grade 
Figure 2.5h takes the same data and expresses the numbers of entrants who were high attainers at Key Stage 1 science, as a percentage of the group with a given subsequent outcome at A level chemistry. The large pie on the left splits all cohort entries to A level chemistry into those achieving level 3 and above at KS1 (56\%) and those with lower results - $27 \%$ with KS1 levels of below level 3, and a further $16 \%$ who did achieve level 3+ at KS1 but slipped to below grade B at A level. The smaller pie on the right breaks down the $56 \%$ into As and Bs, revealing that $18 \%$ achieved the highest possible (A) grade at A level after achieving level 3+ at KS1 science; $14 \%$ came from below level 3 to achieve an $A$ at $A$ level; $12 \%$ managed to achieve a grade $B$ at $A$ level having secured level $3+$ at $\mathrm{KS} 1$; the final $13 \%$ achieved grade $B$ at A level coming from below level 3 at KS1.

Fig. 2.5h

A Level Chemistry Entries by Grade and KS1 Prior Attainment, 2009

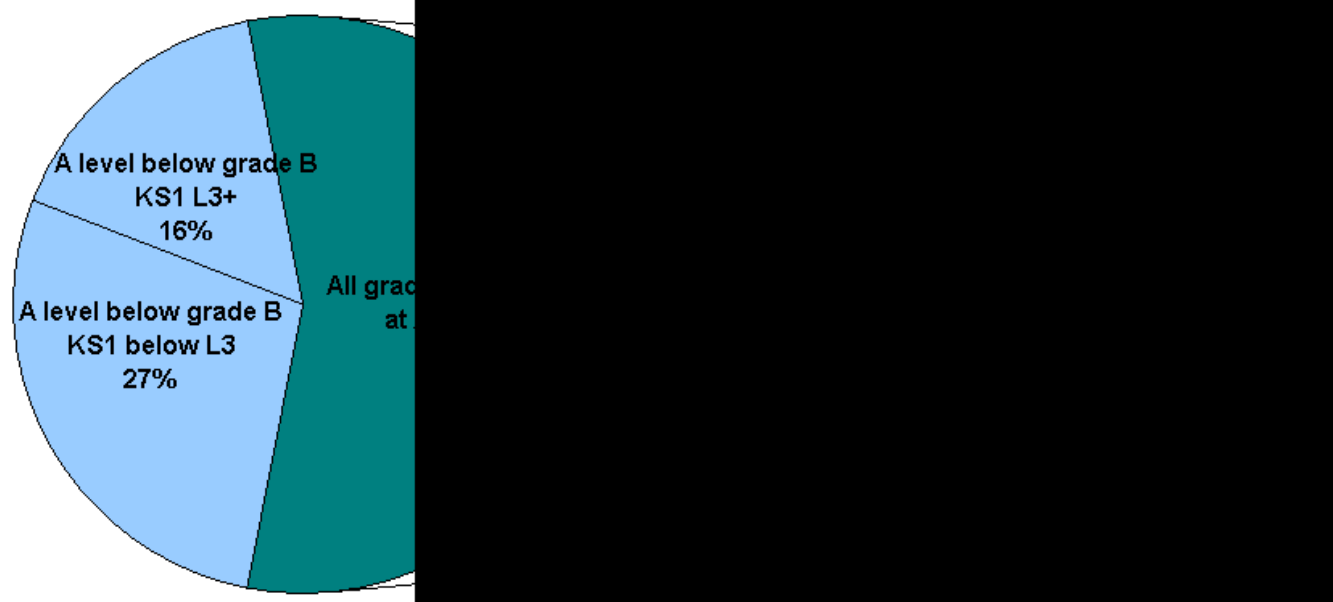


Figure 2.5i presents the percentages of the complete maintained mainstream Key Stage 1 cohort who went on to achieve high grades at A level chemistry. This takes into account the proportions of pupils who selected themselves out of chemistry at A level, either because they didn't continue to Key Stage 5, because they took qualifications other than A levels, or because they chose to study other subjects.

Of the complete KS1 cohort, $1 \%$ went on to achieve a top A grade at A level chemistry in 2009 , compared with $4 \%$ of the high science attainers at KS1. A further $1 \%$ of the cohort achieved grade B at A level chemistry, compared with $2 \%$ of the high attainers at KS1. The remainder of A level chemistry entries with results at grade C or lower made up $2 \%$ of the KS1 cohort, or $3 \%$ of the high attaining group at KS1 science.

\section{Fig. 2.5i}

Progression of KS1 Cohorts to A-level Chemistry in 2009, \% of Cohort

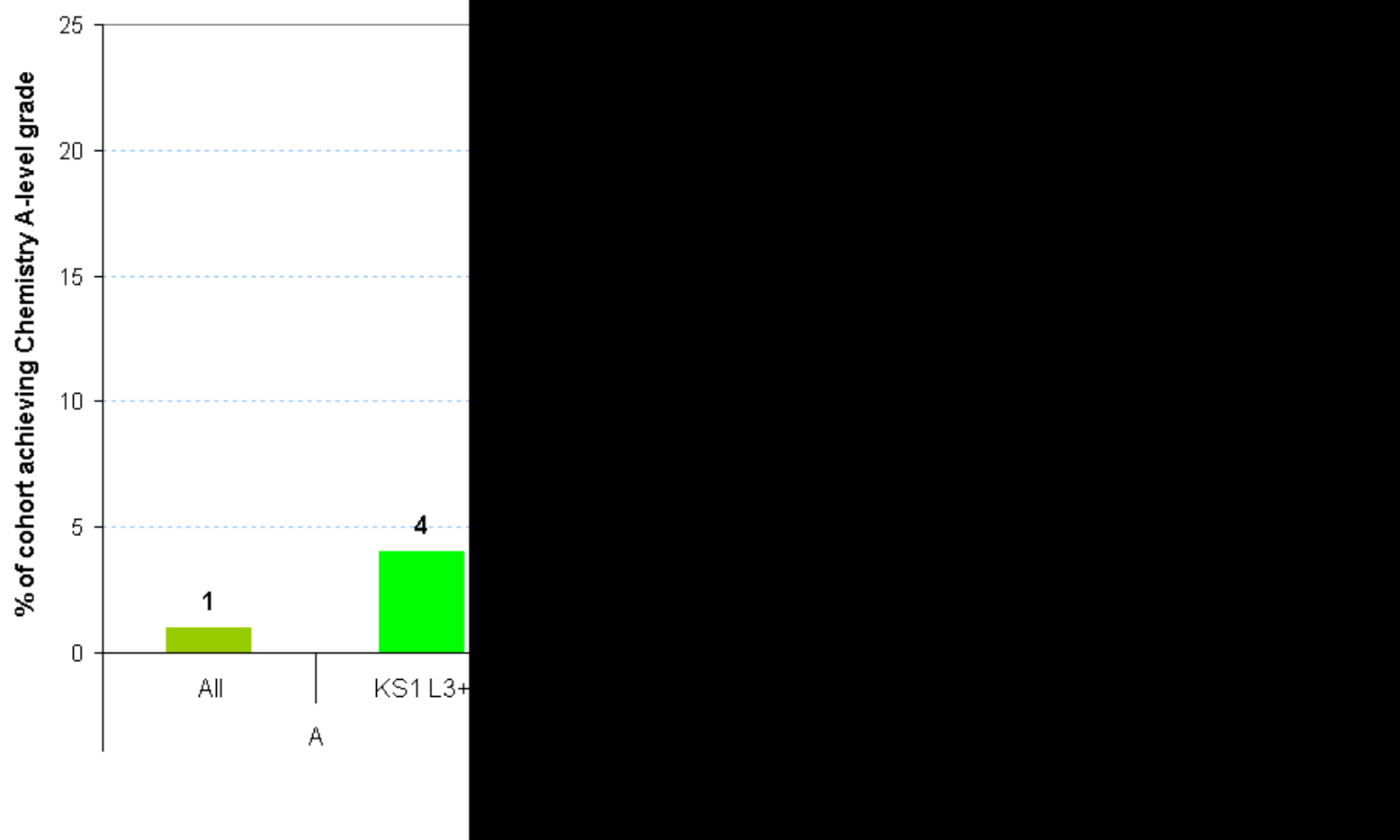


Moving to the third and final traditional science, figure 2.5j shows the percentage of $A$ level physics entrants, split by KS1 science attainment, who went on to achieve grade A, grade B, and grades below B. In 2009, of entrants from the maintained mainstream cohort with science achievement at KS1 of below level 3, 22\% achieved grade $A$ at A level, 20\% achieved grade B and the remaining 59\% had results below grade $B$ (including ungraded). The rate of achievement of $A$ grades for the level 3+ science attainers was $37 \%$, with $22 \%$ achieving grade $B$ and $41 \%$ awarded grades $C$ or below.

\section{Fig. 2.5j}

Progression of KS1 Cohorts to A-level Physics in 2009, \% of Entrants

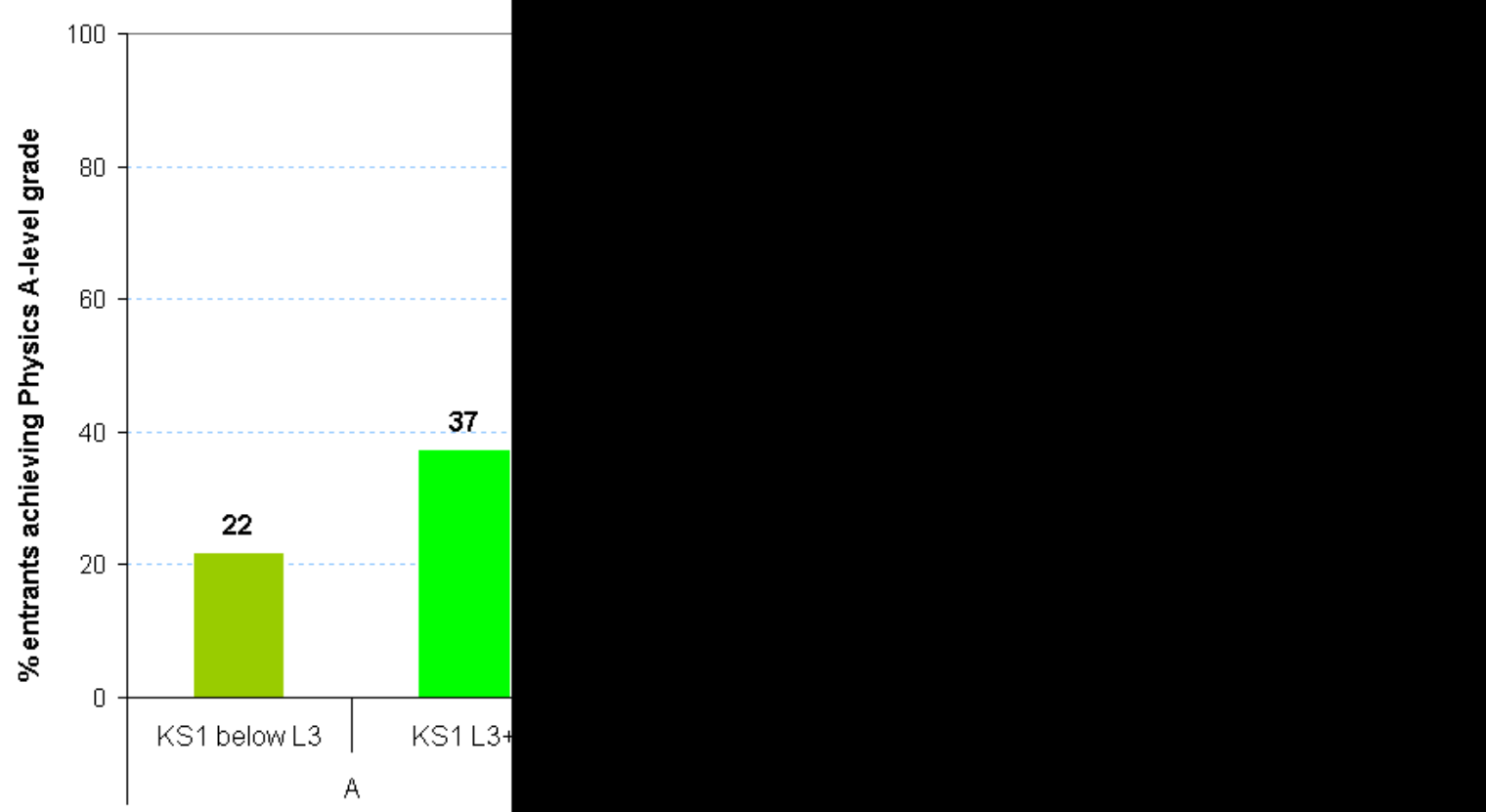

KS1 Prior Attainment

A-level Physics Grade 
Figure 2.5k takes the same data and expresses the numbers of entrants who were high attainers at Key Stage 1 science, as a percentage of the group with a given subsequent outcome at A level chemistry. The large pie on the left splits all cohort entries to A level chemistry into those achieving level 3 and above at KS1 (56\%) and those with lower results - $27 \%$ with KS1 levels of below level 3, and a further $16 \%$ who did achieve level 3+ at KS1 but slipped to below grade B at A level. The smaller pie on the right breaks down the $56 \%$ into As and Bs, revealing that $18 \%$ achieved the highest possible (A) grade at A level after achieving level 3+ at KS1 science; $14 \%$ came from below level 3 to achieve an $A$ at $A$ level; $12 \%$ managed to achieve a grade $B$ at $A$ level having secured level $3+$ at $\mathrm{KS} 1$; the final $13 \%$ achieved grade $B$ at A level coming from below level 3 at KS1.

\section{Fig. 2.5k}

A Level Physics Entries by Grade and KS1 Prior Attainment, 2009

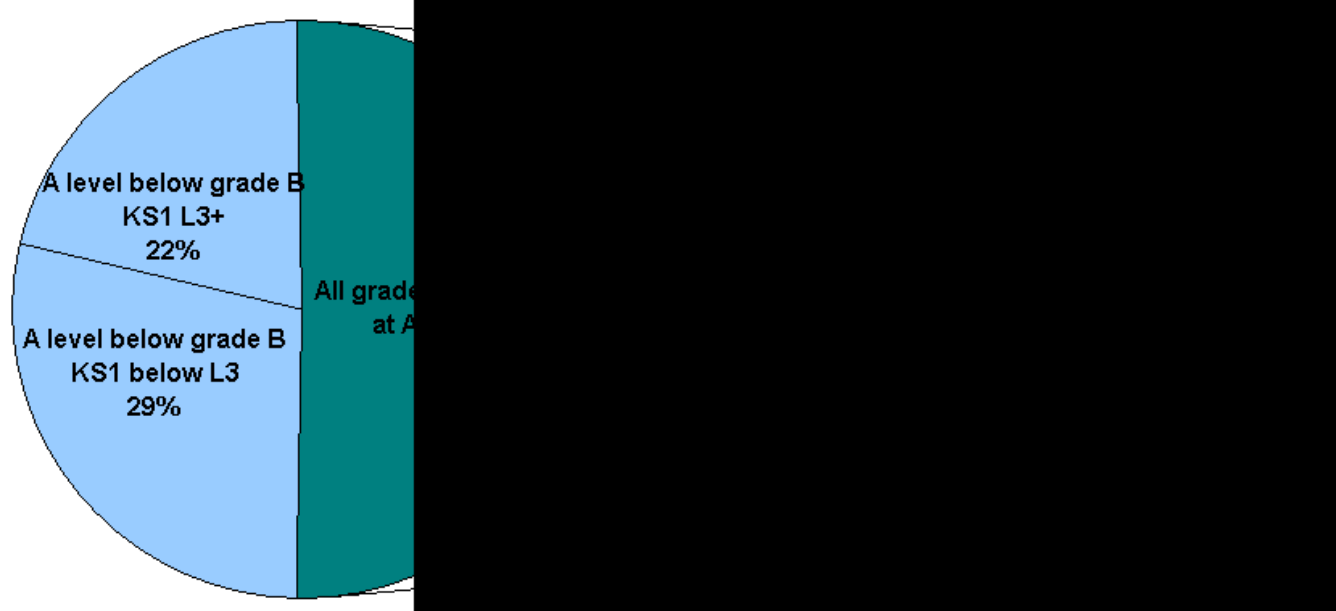


Figure 2.5I presents the percentages of the complete maintained mainstream Key Stage 1 cohort who went on to achieve high grades at A level physics. This takes into account the proportions of pupils who selected themselves out of physics at $A$ level, either because they didn't continue to Key Stage 5, because they took qualifications other than A levels, or because they chose to study other subjects.

Of the complete KS1 cohort, 1\% went on to achieve a top A grade at A level physics in 2009 , compared with $3 \%$ of the high science attainers at KS1. A further $1 \%$ of the cohort achieved grade B at A level physics, compared with $2 \%$ of the high attainers at KS1. The remainder of A level physics entries with results at grade $\mathrm{C}$ or lower made up $1 \%$ of the $\mathrm{KS} 1$ cohort, or $3 \%$ of the high attaining group at KS1 science.

\section{Fig. 2.5I}

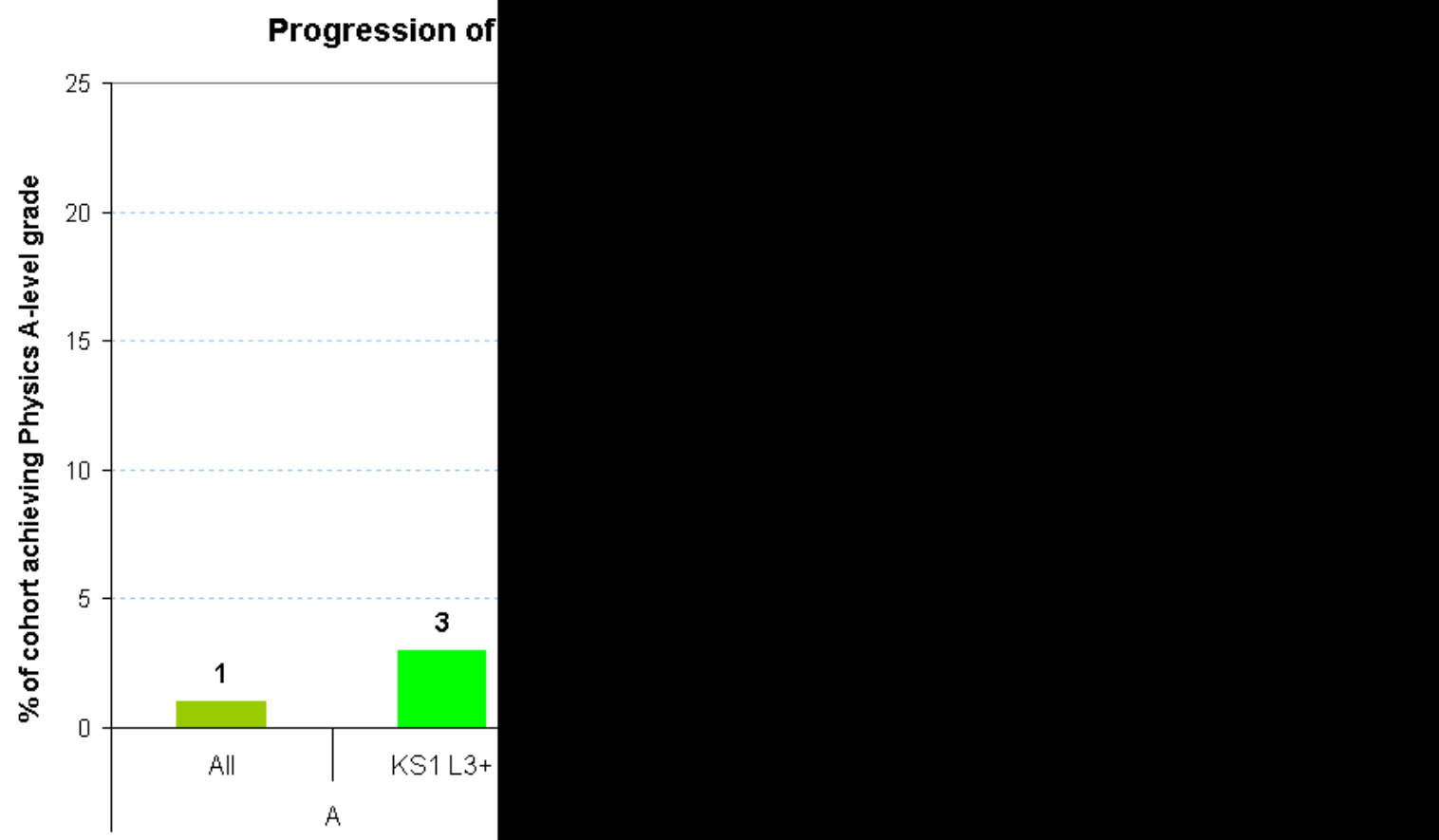

KS1 Prior Attainment

A-level Physics Grade

\section{Section Summary}

Section 2.5 has examined progression to high grades in A level maths and science subjects from Key Stage 1. The analyses have revealed a diminished pattern of dependence on strong prior attainment within subject, showing a markedly smaller association than at Key Stages 2-4. Twenty-two percent of A level maths entrants managed to progress from below level 3 at KS1 to achieve an A or a B at A level. For biology, this figure is also $25 \%$, rising to $27 \%$ for chemistry, and a little lower at $19 \%$ for physics. Across the four subjects, the chances of achieving grade $A$ at $A$ level are less than twice as high for pupils who gained level 3+ at KS1 as for those with below level 3.

Effects of prior attainment across subjects and Key Stages will be compared with one another in the following section; for this purpose, odds ratios will be used to remove 
the effects of differing numbers of pupils falling into the high attaining groups at different Key Stages, and of different achievement rates between the subjects. 


\subsection{A level Progression Comparisons Between Subjects and Key Stages}

As discussed at the end of section 2.1, an odds ratio of greater than 1, for high attainers compared with non-high attainers, would suggest that high prior attainment is positively associated with progression to A level; similarly an odds ratio of less than 1 would imply a lesser association with the progression outcome.

Figure 2.6a presents an odds ratio comparison of the association between prior attainment and later A level achievement for maths, biology, chemistry and physics. From the pattern of odds ratios plotted, it is clear that the importance of high prior attainment increases with age and as the pupils get closer to the A level attainment measured as the progression output. This is not surprising as pupils have less remaining time to catch up or pull ahead of the expected attainment standard by the time they reach the later Key Stages.

The odds of achieving grade A at A level for high attainers at KS1 are around twice as high as those for pupils achieving at the expected level or below. This rises to between two and four times the odds by KS2, and there begins to be a distinction between the subjects, with physics progression being the most dependent on prior attainment, and chemistry the least. High prior attainment has become noticeably more important to A level achievement by Key Stage 3, with between four and seven times the odds of achieving grade A for those achieving above the expected levels; biology progression has now become more dependent than maths progression, between the high point of physics and the low point of chemistry. By GCSE, the odds of A level progression for the high attainers have reached around ten to twelve times those of the other pupils, with the four maths and science subjects moving closer together again, but retaining the same order in terms of the importance of prior attainment to progression.

\section{Fig. 2.6a}

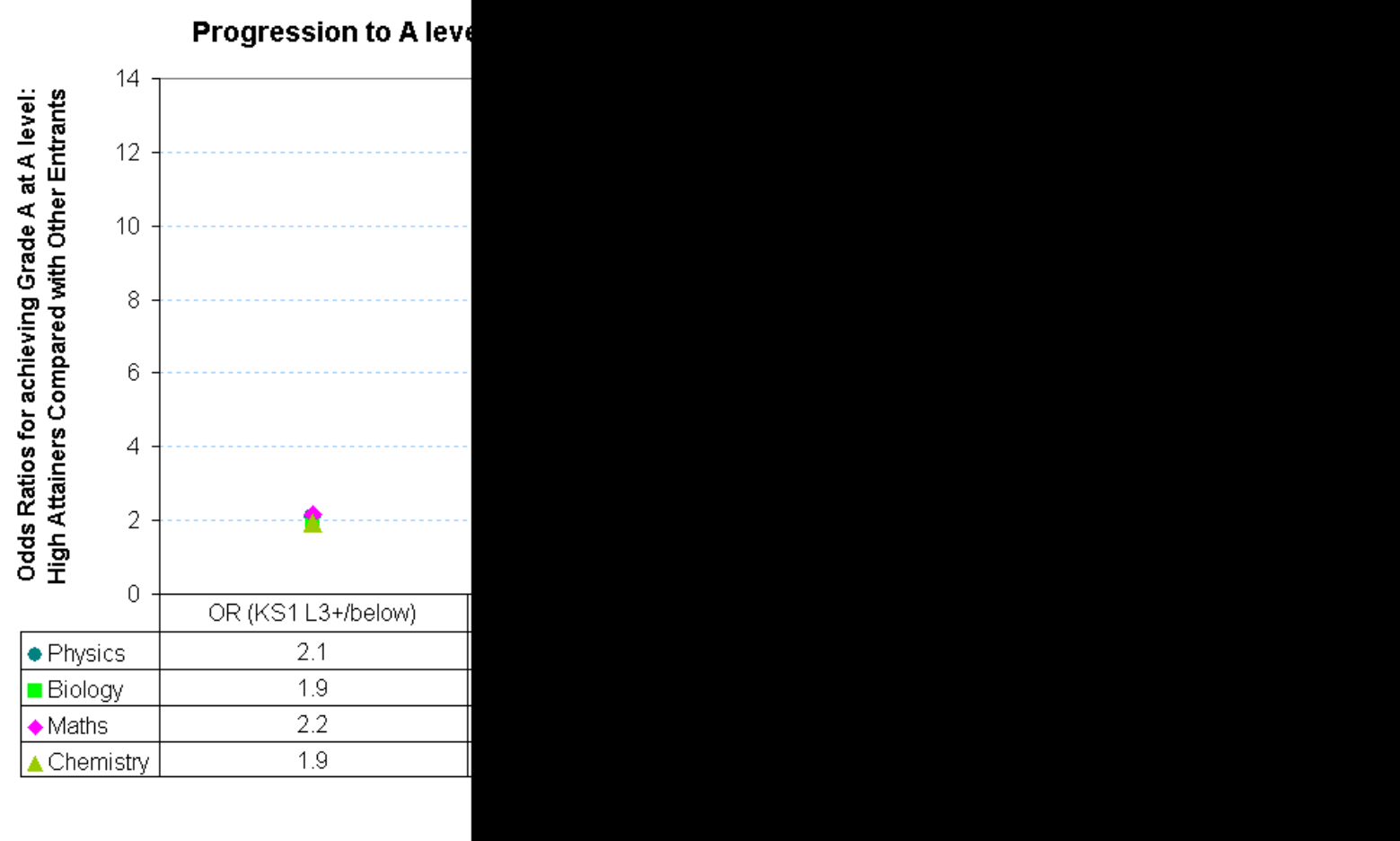




\subsection{Path Dependence: Pulling Ahead and Slipping Back in Maths and Science}

The importance of high prior attainment for participation in A level maths and science subjects, increasing with each step closer to A level, triggers a new set of questions about the stability of subject attainment across the Key Stages. This section investigates to what extent it is the same group of pupils who attain at above the expected levels in each Key Stage. This can be described as an analysis of path dependence for high maths and science attainment through Key Stages 1-4, to address the following questions:

- How likely is it that a pupil who has not previously been high attaining in that subject can accelerate into the high attaining group by Key Stage 4?

- How many pupils begin their education as high maths or science attainers, but fall below this level by GCSE?

- Do those who have dropped out of the high attaining group frequently manage to catch up again and rejoin it?

Figure 2.7a begins with the 79,000 high attainers at GCSE maths (top left Venn diagram), defined as those achieving grades $A^{*}$ or $A$, and looks back at the previous Key Stages to identify how dominant the overlap is between attainers at above the expected level for each Key Stage. The top right Venn figure looks back at the prior maths attainment of the remaining 515,000 pupils, whose GCSE maths grades were B or below, i.e. those not in the high attaining GCSE group. The bottom two Venn figures repeat this analysis for GCSE science entrants (using the highest science grade achieved, whether in double science or individual biology, chemistry or physics GCSEs).

From the maths diagrams, the group of high attainers in GCSE maths is dominated by pupils with a record of earlier high attainment at one or more Key Stages; only 3\% of the high attaining GCSE group had not had results at above the expected level in any of Key Stages 1-3. Almost half (49\%) of the high GCSE maths attainers had been high attaining at all three previous Key Stages, and a further third (32\%) were high attaining at KS2 and KS3, having joined the group after KS1. Only $2 \%$ of high attaining GCSE pupils had rejoined the high attaining group, having been in it at KS1 or KS2, but dropped behind at KS3. Key Stage 3 had the strongest transference of high maths performance to GCSE, with 95\% of all GCSE high attainers having carried forward this status for at least one Key Stage.

In the larger non-high attaining group at GCSE maths, the majority (74\%) had not attained at above the expected level in maths at any of the prior Key Stages. Of this group, $5 \%$ had slipped below grades $A^{*}$ or $A$ at GCSE after having previously had high attainment consistently from $\mathrm{KS1}-3$. A further $5 \%$ had high prior maths attainment at both KS2 and KS3, but not at GCSE. Of those with grades B or below at GCSE, a total of $14 \%$ were high attaining at KS3, in contrast to the $95 \%$ of high GCSE attainers. In number terms, the total $26 \%$ of GCSE non-high attainers who dropped out of the group having had high maths attainment in at least one previous Key Stage is much larger, at 134,000 , than the $51 \%$ of high GCSE attainers who had pulled ahead having not had high maths attainment at all prior Key Stages (numbering 40,000). 
Turning to the GCSE science high attainers, a slightly higher percentage of this group (10\%) had joined having not previously attained at above the expected level in any of the three prior Key Stages. However, one third (33\%) of the high GCSE attainers were consistently high attaining throughout $\mathrm{KS} 1-3$, and a further $28 \%$ were high attaining at KS 2 and KS3, having joined the high attaining group subsequent to KS1. Key Stage 3 high attainers were less dominant for science subjects than maths, with Key Stage 2 high attainers forming a larger proportion of those with grades $A^{*}$ or $A$ at GCSE science subjects. A total of $66 \%$ of the GCSE high attainers continued this status from KS3, compared with $84 \%$ who had achieved at above the expected level in KS2 science. Correspondingly, it was more common for pupils to drop out of the high attaining group at KS3, having been in it at KS1 or KS2, but rejoin it at GCSE, with $25 \%$ of the GCSE high attainers following this trajectory. 
In the larger group of pupils who did not have high attainment (grades $A^{*}$ or $A$ ) in one of the main GCSE science subjects, over half (65\%) had not attained at above the expected level in maths at any of the prior Key Stages. Of the group, $2 \%$ had slipped below grades $A^{*}$ or $A$ at GCSE after having previously had high attainment consistently from KS1-3. A further $2 \%$ had high prior maths attainment at both $\mathrm{KS} 2$ and KS3, but not at GCSE. Of those with grades B or below at GCSE, just under one third (30\%) had high attainment in science at KS2, compared with $84 \%$ of the high GCSE attainers. The total 35\% of GCSE non-high attainers who dropped out of the group having had high science attainment in at least one previous Key Stage is much larger, at 183,000 , than the $67 \%(48,000)$ of high GCSE attainers who had pulled ahead having not had high science attainment at all of KS1-3.

\section{Fig. 2.7a}
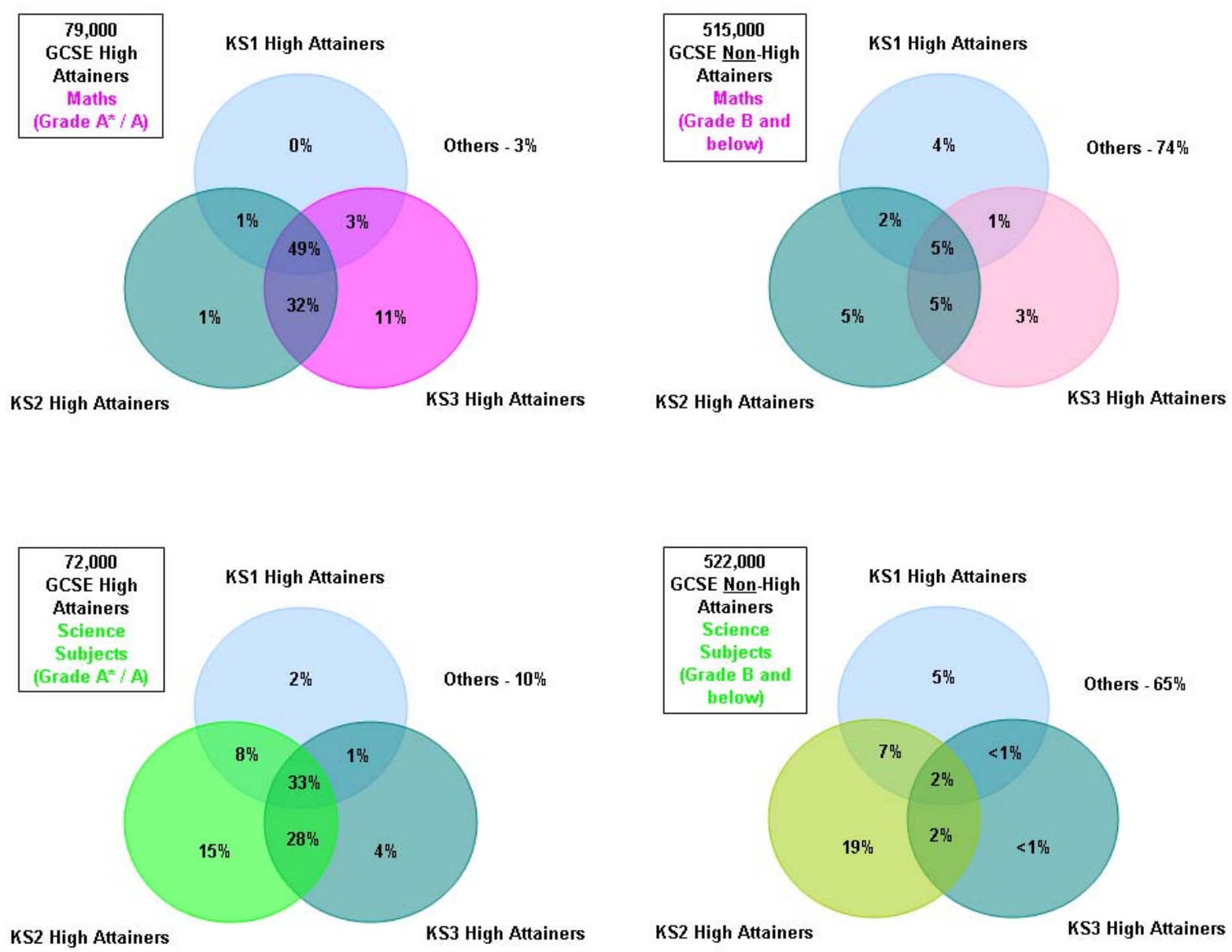

In figure 2.7b, the perspective of the analysis is reversed to look forwards from high attaining status at the earlier Key Stages, presenting the associated chances of high attainment at GCSE for each pathway of prior maths and science attainment. The points plotted on the chart represent the odds ratios for achievement of grade $A^{*}$ or $A$ at GCSE, of pupils with high prior attainment at the given Key Stages, compared with those who had not attained at above the expected level in any of Key Stages 1-3 (the base case with $\mathrm{OR}=1$ ). 
All attainment patterns including at least one prior Key Stage with high subject attainment have odds ratios of greater than one, indicating that pupils in these groups are more likely to be high attaining at GCSE than those with no prior history of high attainment within the subject. Pupils who achieve at above the expected level in maths or science at all of KS1-3 have odds of high GCSE attainment for these subjects in the order of 100 times higher than pupils without any previous high attainment. The odds for pupils in the high attaining group at Key Stages 2 and 3 (but not KS1) are of approximately the same order, although with a larger drop in the GCSE advantage for maths than for science.

Having only KS3, or KS1 and KS3 (but not KS2), with high subject attainment again has broadly similar odds ratios for science, falling further for maths, indicating a greater importance of having a consistent record of high attainment stretching back to primary school for maths than for science subjects. The largest drop in the odds ratios for high GCSE attainment, for both maths and science, is associated with not having high prior attainment at KS3. In both subjects the odds ratios decline to in the order of 10 times that for pupils with no high prior attainment, then steadily decline towards twice the odds when only KS2 or only KS1 high attainment is analysed.

\section{Fig. 2.7b}

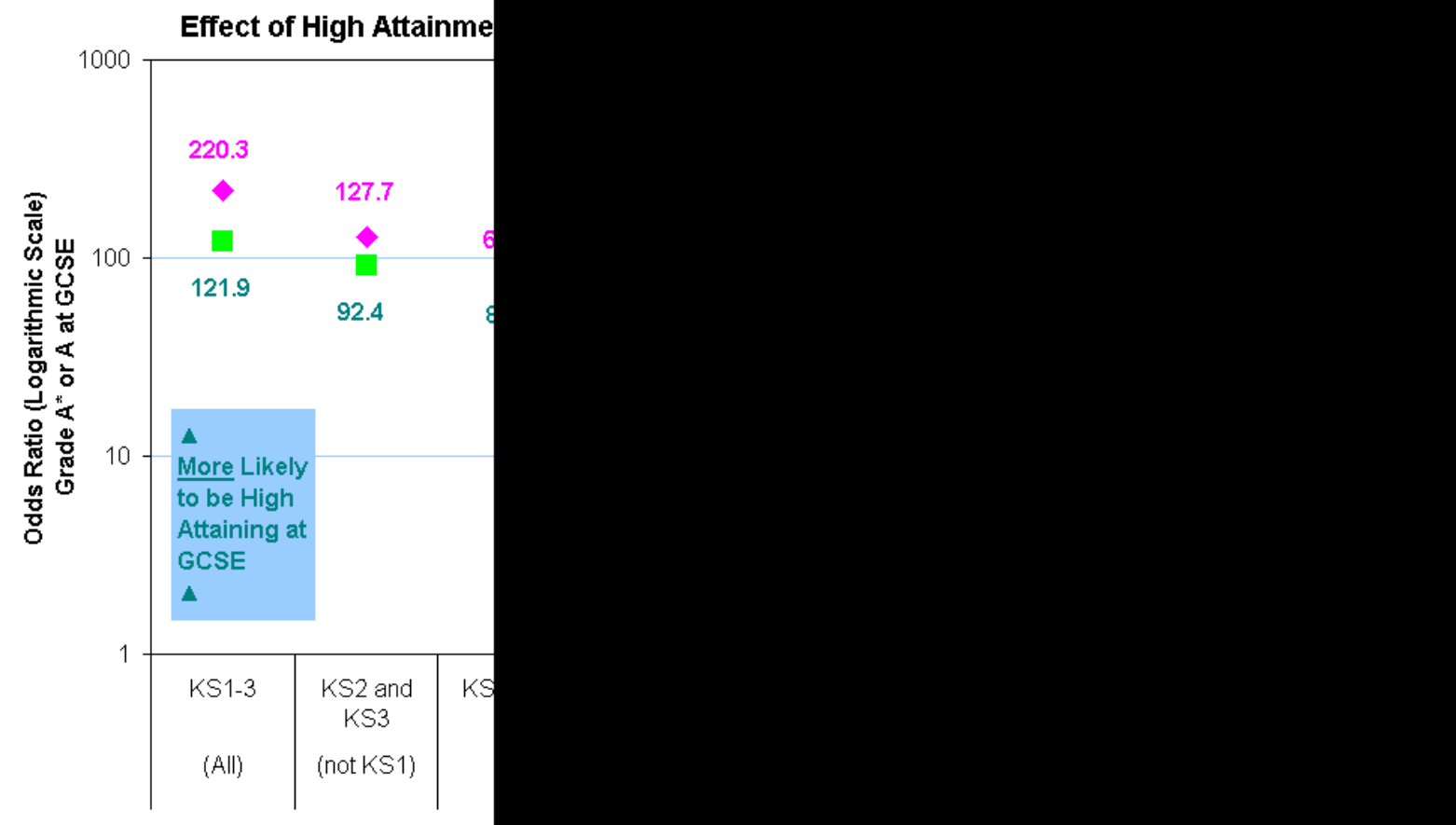

High Prior Attainment at... 


\section{Chapter 3. Pupil Characteristics and A Level Outcomes}

Chapter 3 explores the relationships between three key pupil characteristics and A level outcomes in maths, biology, chemistry and physics. Section 3.1 focuses in pupil gender, and reports on the participation and achievements of boys compared with girls. Section 3.2 then analyses the relationship between eligibility for free school meals and outcomes for maths and science A levels. Thirdly, section 3.3 examines the differences in entry and achievement associated with membership of different ethnic minority groups. Analyses in this chapter are restricted to pupils who attended maintained mainstream schools at the end of Key Stage 4.

\subsection{Gender and A level Maths and Sciences}

Section 3.1 examines the gender differences in A level entries and achievements for maths and the sciences. For each subject, the rates of entry and success at grades A or B amongst boys and girls in the cohort are examined; the third set of columns for each gender group then displays the percentage of entrants achieving grades $A$ or $\mathrm{B}$, to separate out the question of achievement from that of participation.

The following charts appear in pairs, with the first for each subject giving the rates for all pupils in the stated gender groups, and the second in each pair selecting just those pupils from within each group who had high prior attainment (at grades $A^{*}$ or $A$ ) for GCSE. Hence, the second chart for each subject focuses on those pupils with strong ability to continue studying the subject in question to A level. 
Figure 3.1a presents the rates of A level maths entry, and achievement of grades A or B for all pupils, by gender. In 2009 , boys were more likely to enter A level maths than girls (at $8 \%$ compared with $6 \%$ of the cohort respectively). As a percentage of the cohort, boys were also slightly more likely to achieve a grade A or B (5\% compared with 4\%), but this reflected the shortage in girls entering A level maths, rather than lower achievement, as $65 \%$ of female entrants achieved grade A or B compared with $63 \%$ of male entrants.

\section{Fig. 3.1a}

A level Maths Continuation and Achievement of Grades A and B All Pupils

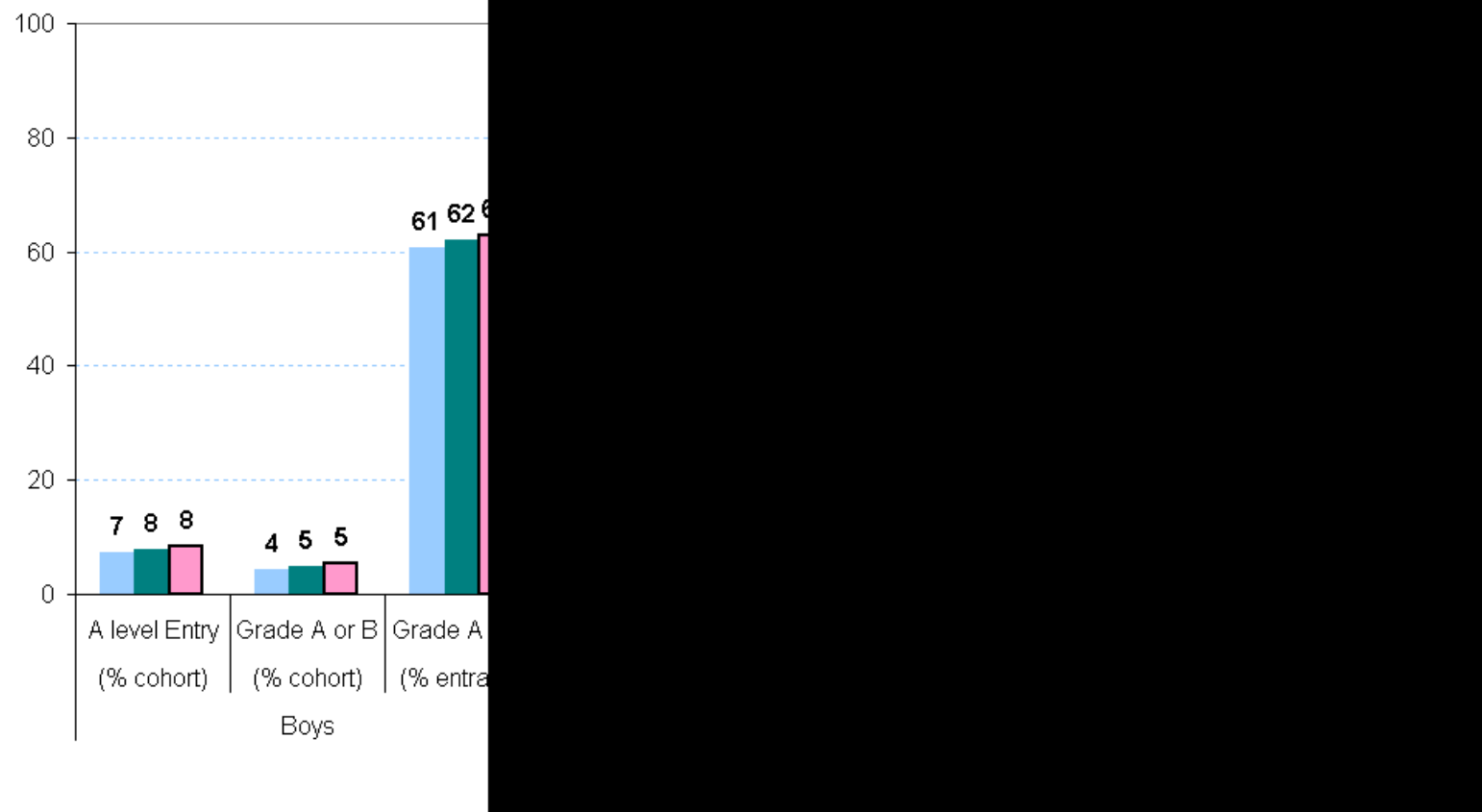


Focusing in on pupils who achieved grades $A^{*}$ or A in GCSE maths, figure 3.1b shows that high prior attaining boys are even more likely than their female counterparts to enter A level maths with $54 \%$ of boys choosing the subject compared with $39 \%$ of girls. High achieving boys are also more likely to enter and achieve grade A or B than high achieving girls, at $37 \%$ compared with $27 \%$. Perhaps as a result of the stronger selection operating amongst girls, if a high achieving girl entered A level maths, she was then slightly more likely than a high achieving male entrant to be awarded grade A or B (70\% compared with 69\%).

\section{Fig. 3.1b}

A level Maths Continuation and Achievement of Grades A and B Pupils with Grade $A^{*}$ or A at GCSE

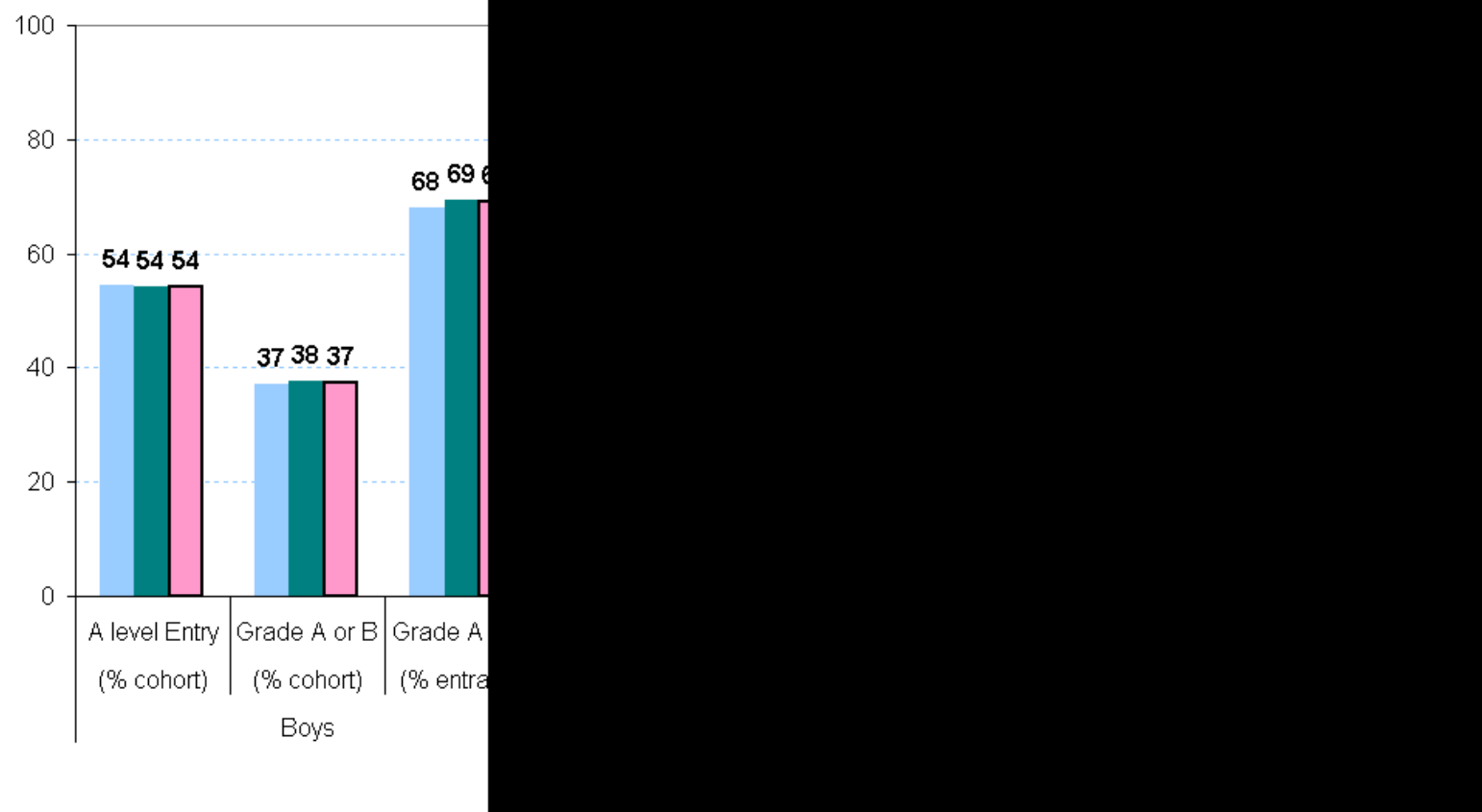


Moving on to biology, for all pupils, an opposite gender effect was evident, with girls being more likely to enter for A level (6\%) than boys (5\%). Girls were also more likely to enter and achieve a grade $A$ or $B$, at $3 \%$ compared with $2 \%$ of boys. Of those pupils who entered A level biology, girls had a further advantage in achievement, with $51 \%$ of entrants receiving grades A or B, compared with $47 \%$ of male entrants.

\section{Fig. 3.1c}

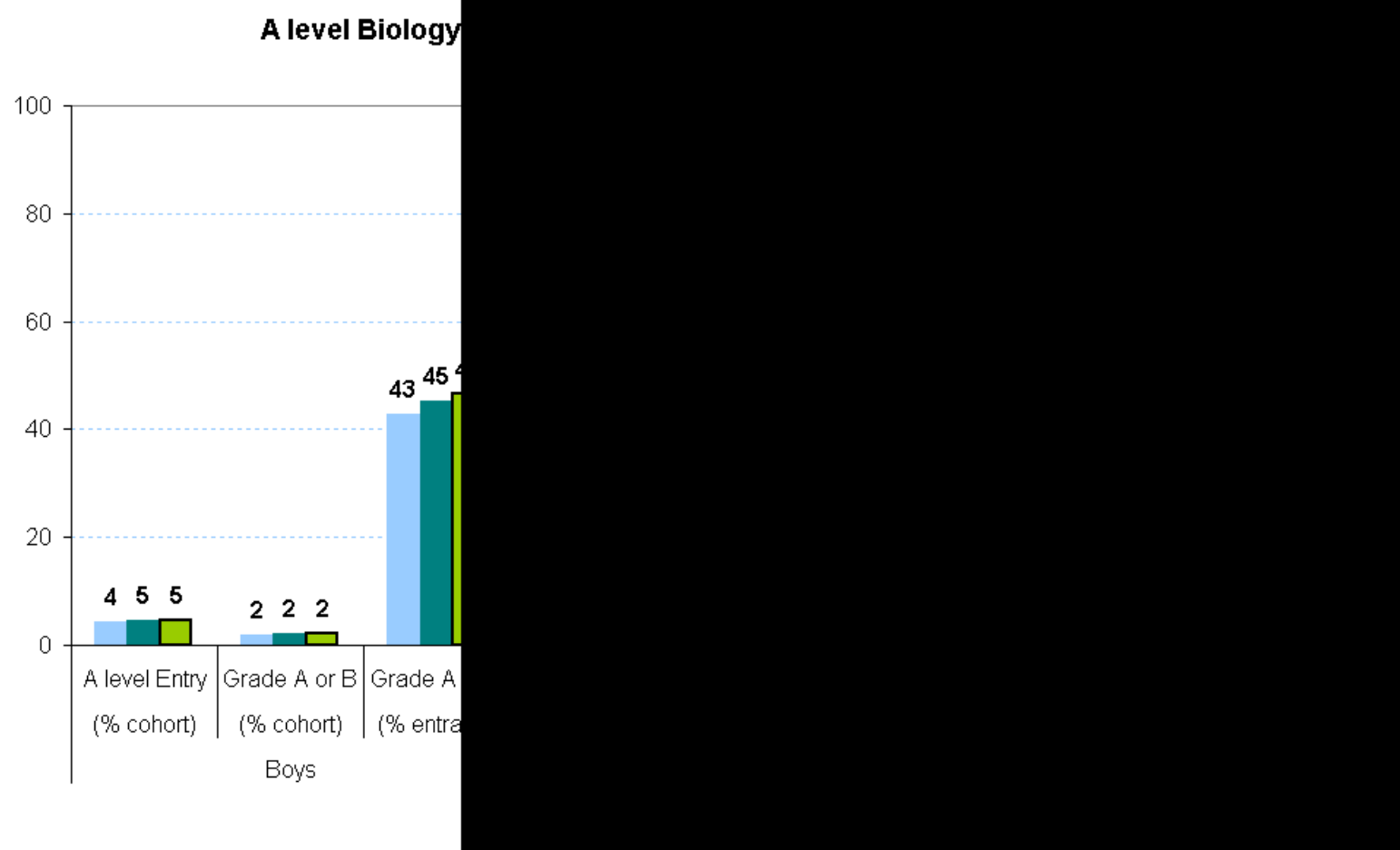


High prior achieving girls (with grades $A^{*}$ or A at GCSE biology or double science) had even stronger advantages over boys in both entry and achievement at $A$ level biology. $37 \%$ of high achieving girls entered A level biology compared with $27 \%$ of high achieving boys; $25 \%$ of the female cohort achieved grade $A$ or $B$, compared with $17 \%$ of high achieving boys. Of those high achievers who entered A level biology, $68 \%$ of the girls were awarded grade A or B, compared with $63 \%$ of the boys.

\section{Fig. 3.1d}

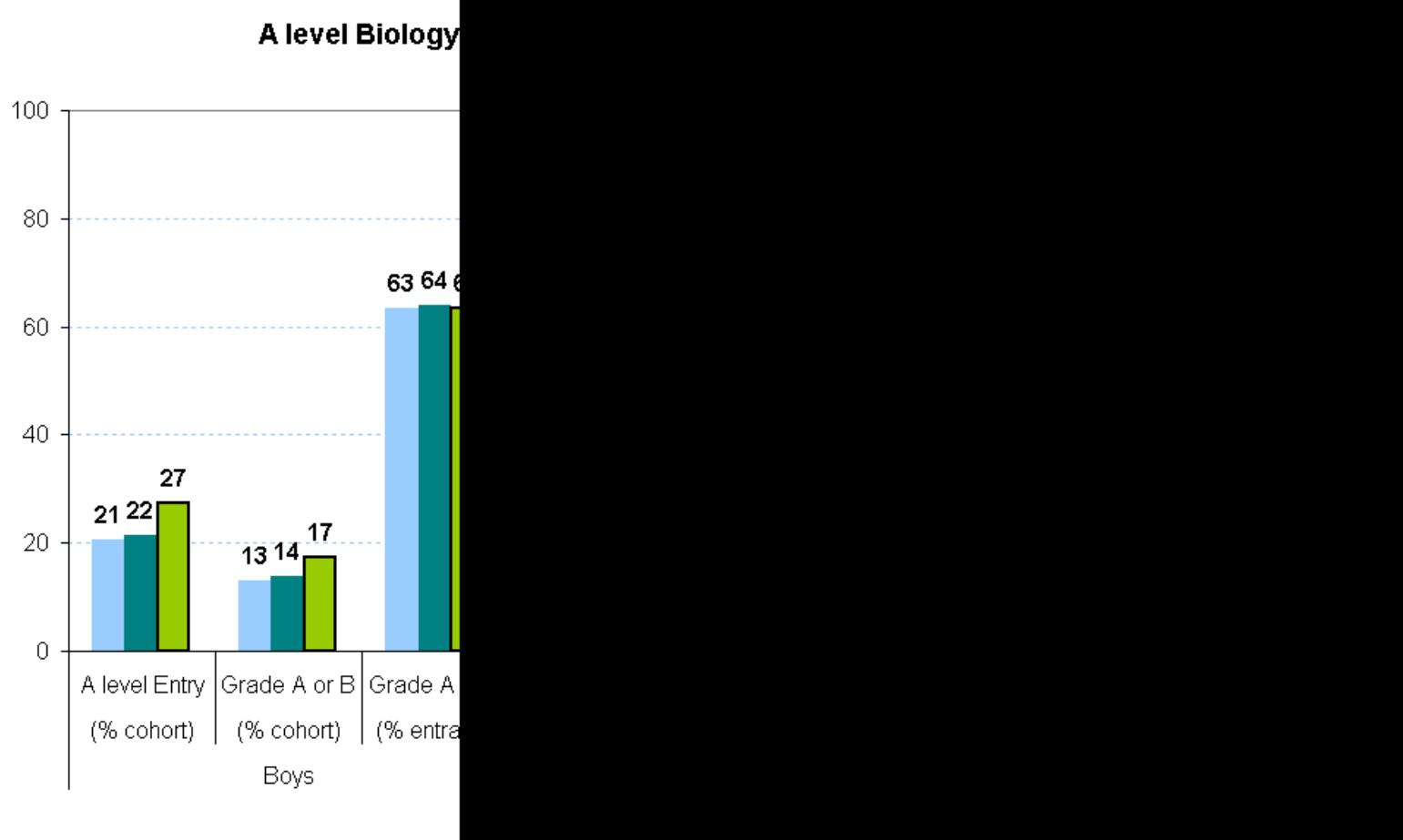


There were no differences between boys and girls in the rate of entry to A level chemistry (fig. 3.1e). However, girls who took this A level were more likely to achieve a grade $A$ or $B$, at $58 \%$ of entrants, compared with $55 \%$ of male entrants.

Fig. 3.1e

A level Chemistry Continuation and Achievement of Grades A and B All Pupils

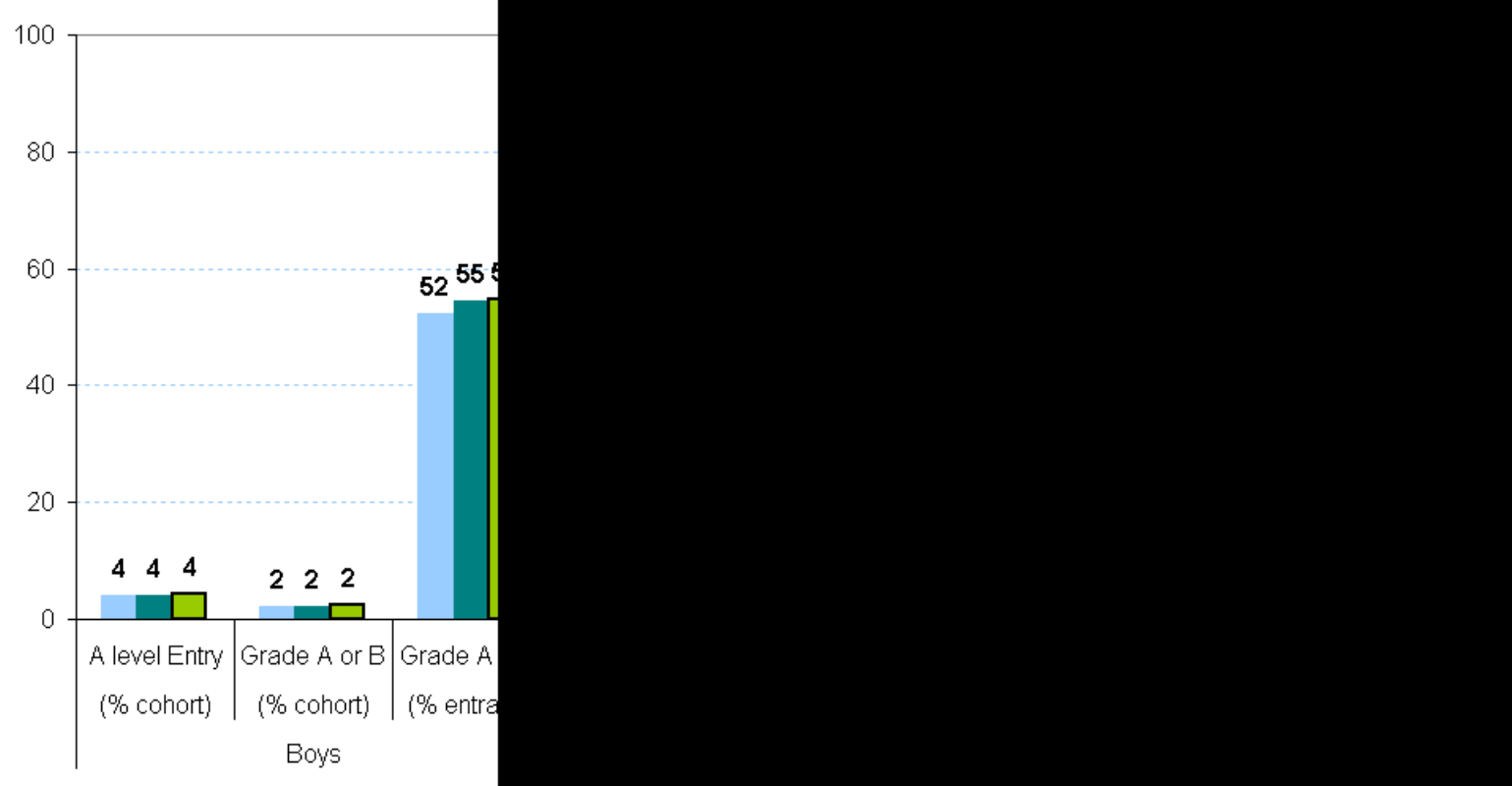

$2007 \square 2008 \square 2009$ 
Although there were no overall differences between boys and girls in the likelihood of entering A level chemistry, amongst those with high grades in chemistry or double science at GCSE, girls were less likely to take chemistry, at $28 \%$ of the high achieving group, compared with $30 \%$ of high achieving boys. This difference fed through into the likelihood of entering and achieving a grade A or B, which was $19 \%$ for high achieving girls, and $20 \%$ for high achieving boys. This was despite a higher achievement rate for girls with high prior attainment if they chose to take A level chemistry, with $69 \%$ gaining grades A or B, compared with $65 \%$ of high achieving male entrants.

\section{Fig. 3.1f}

A level Chemistry Continuation and Achievement of Grades A and B Pupils with Grade $A^{*}$ or A at GCSE

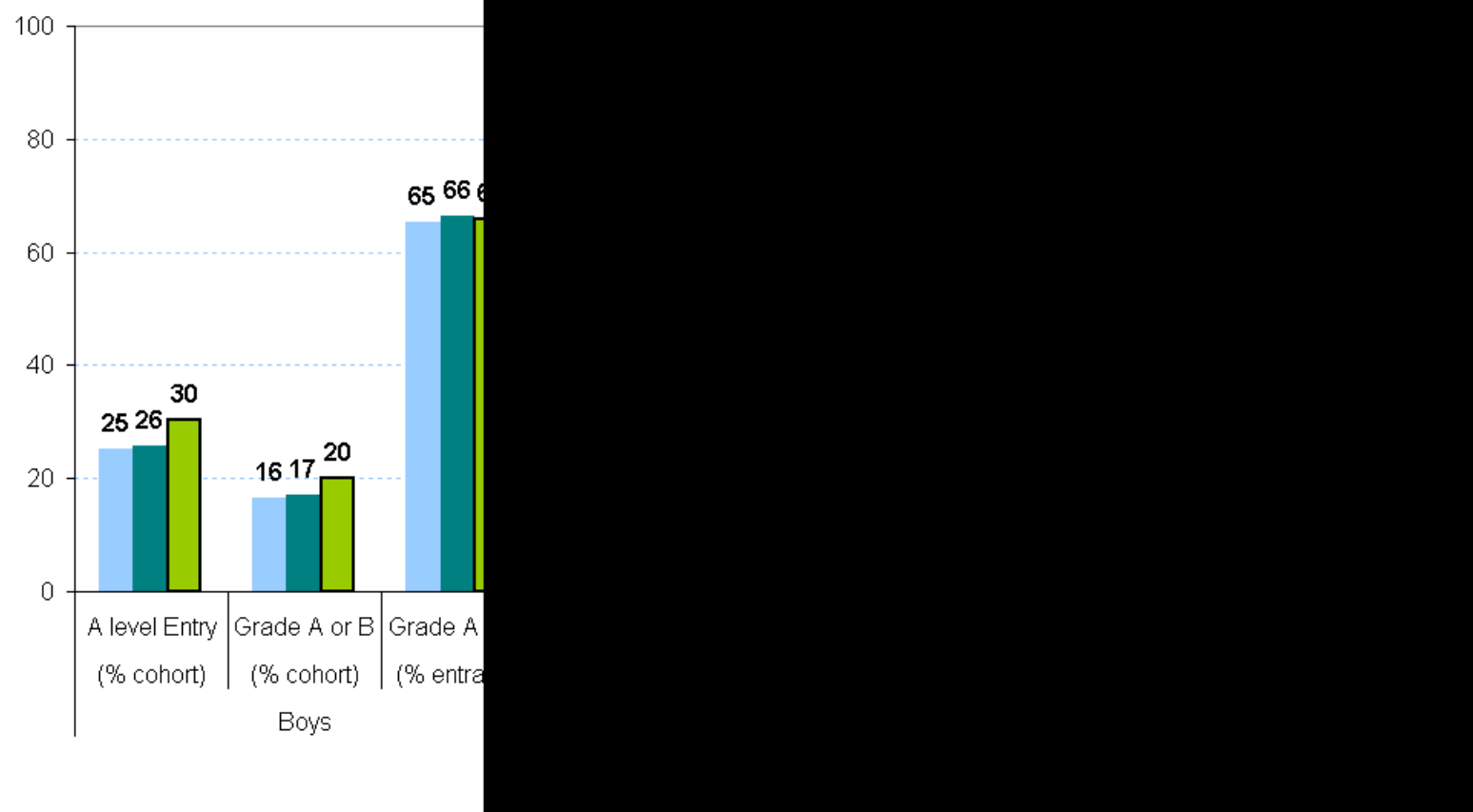


Figure 3.1g reveals that physics has the strongest gender effects of the maths and science A levels. Boys are five times as likely to enter A level physics as girls $(5 \%$ of boys compared with $1 \%$ of girls), and twice as likely to enter then achieve a grade $A$ or B ( $2 \%$ of boys compared with $1 \%$ of girls). These strong selection effects contribute to higher achievement by those girls who do enter physics A level (with $55 \%$ gaining grades $A$ or $B$ ) than for boys who enter (50\% of whom are awarded these grades).

\section{Fig. 3.1g}

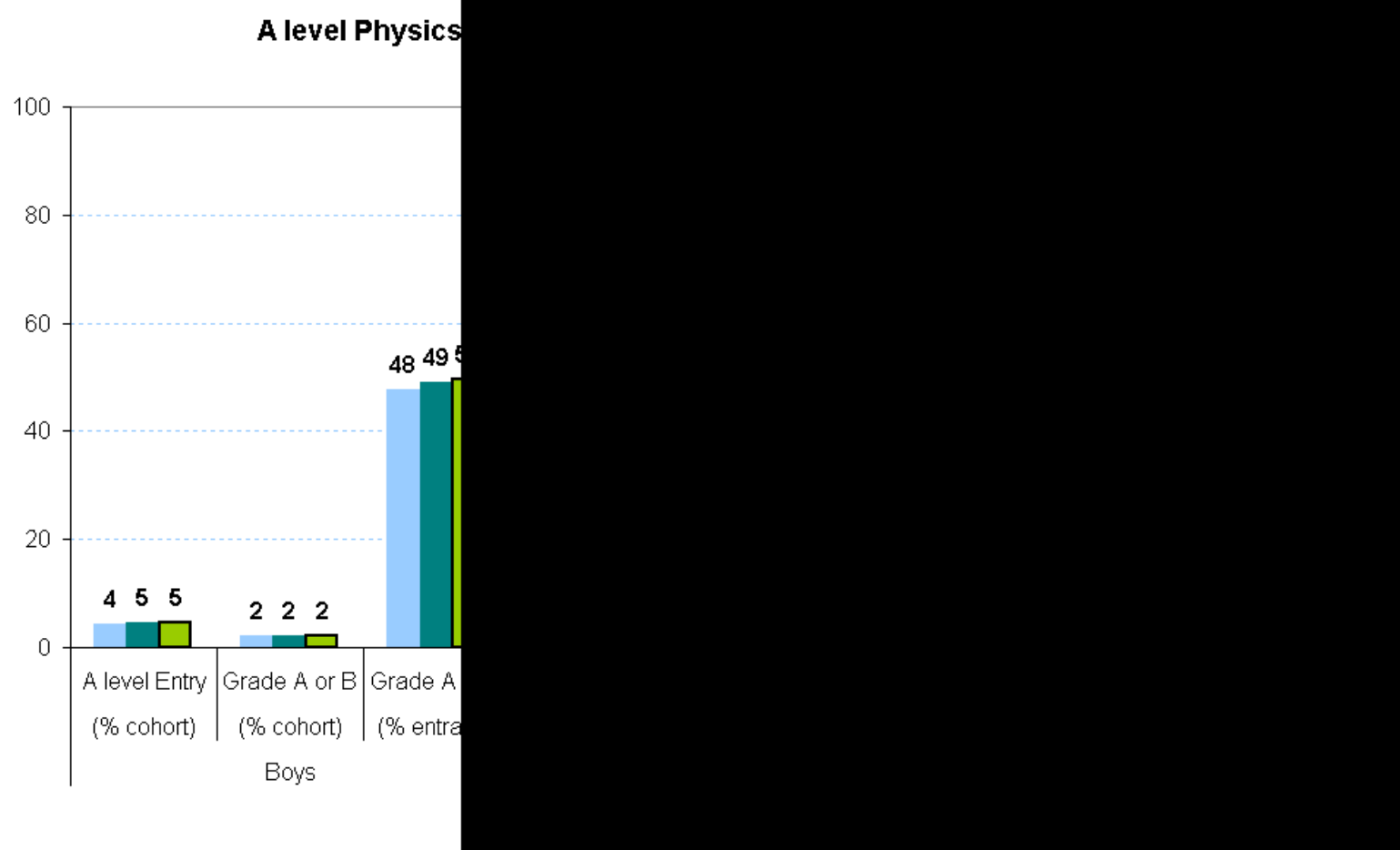


Strong gender effects also applied to high achieving pupils at GCSE physics or double science. Boys with grade $A^{*}$ or $A$ at GCSE were almost four times as likely to take A level physics as girls with the same prior attainment (31\% compared with $8 \%$ ). They were also over twice as likely to enter physics then achieve a grade A or $B$ (19\% compared with $8 \%$ ). Those high achieving girls who did enter A level physics were slightly more likely to subsequently achieve a grade A or B than their male counterparts, at $65 \%$ of girls compared with $63 \%$ of boys.

\section{Fig. 3.1h}

A level Physics Continuation and Achievement of Grades A and B Pupils with Grade $A *$ or A at GCSE

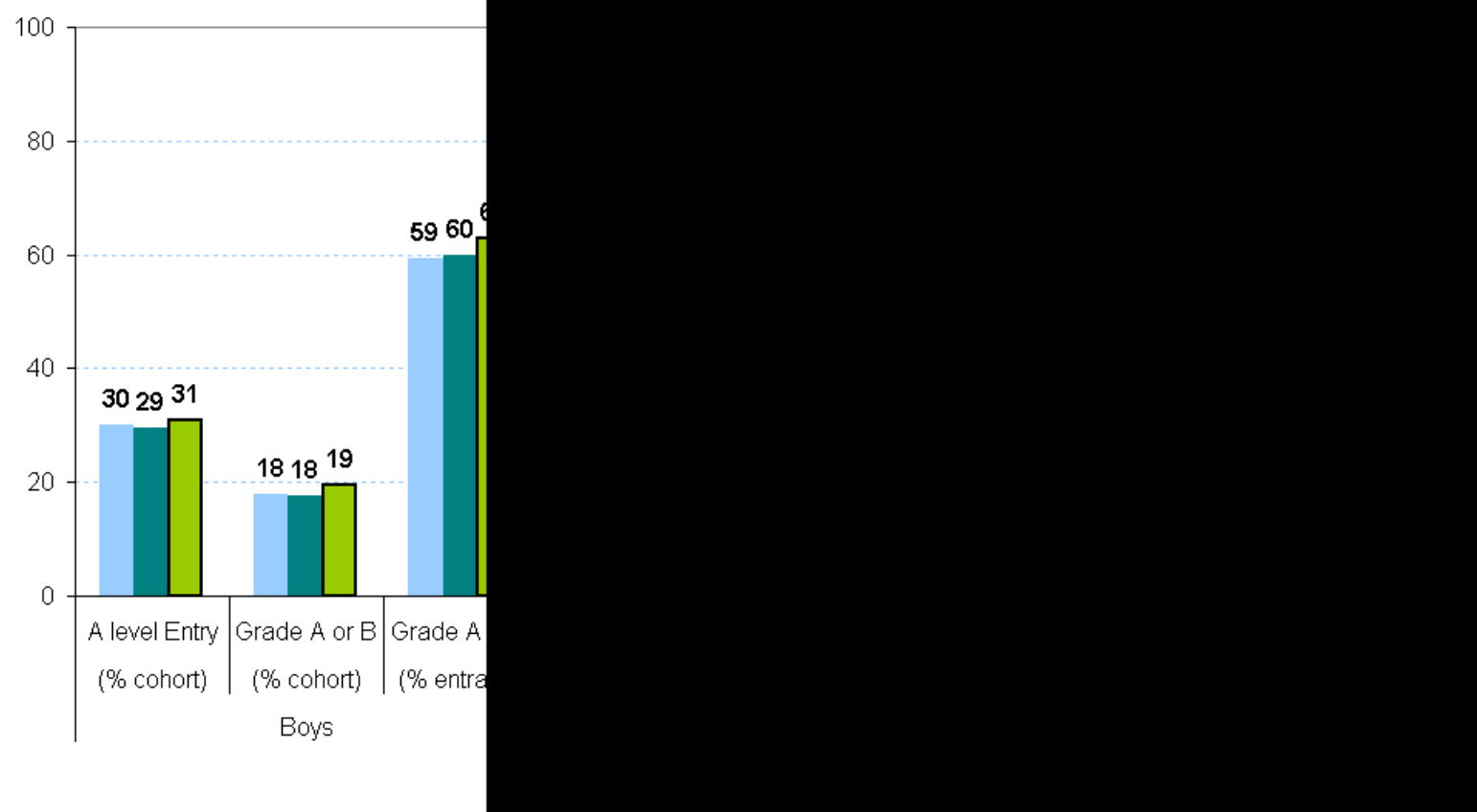


Figure 3.1h summarises the gender effects in A level maths and science subjects. Here, the effects are plotted as odds ratios (male probability / female probability) in order to standardise the effect sizes across the three A level outcome measures, four subjects, and two different prior attainment ranges. The pattern that is revealed confirms that physics has the strongest gender effects on A level entry, with boys having substantially larger odds of taking physics than girls, and reveals that this effect is actually stronger amongst high attaining pupils once the underlying attainment eligibility difference is taken into account by the standardised odds measure.

Female entrants had higher odds than boys of achieving top grades ( $A$ or $B$ ) at $A$ level in all four subjects. However, a given girl at Key Stage 4 was only more likely to end up with these grades in biology; and less likely to end up with them in maths, physics or chemistry, due to her lower propensity to take these A level subjects on. The lower odds of a given girl at KS4 to go on to take and succeed in A level chemistry only applied to girls with high prior attainment in that subject (or in double science), as across the full attainment range, the odds for girls and boys were similar. However, for maths, and particularly for physics, the odds advantage for boys persisted across the full ability range, but was slightly stronger amongst the subset of pupils with high prior attainment (and therefore the most suitability for A level).

\section{Fig. 3.1h}

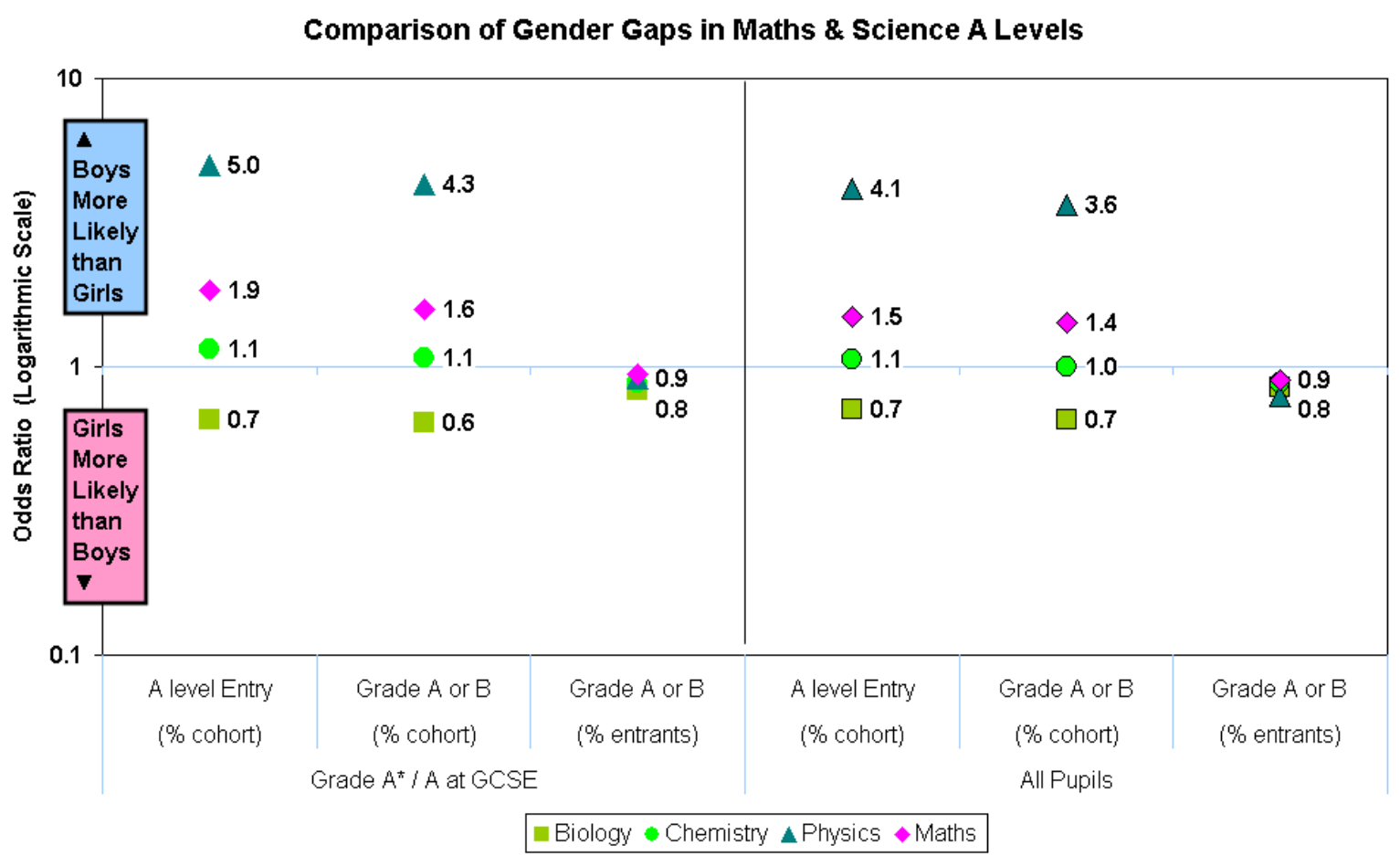

\subsection{Free School Meals and A level Maths and Sciences}

Section 3.2 examines A level participation and achievement gaps using free school meals (FSM) eligibility at Key Stage 4 as a proxy for deprivation. For maths and the sciences, the rates of entry and success at grades A or B in the cohort are examined for pupils with and without free school meal eligibility; the third set of columns for each group then displays the percentage of entrants achieving grades $A$ or $B$, to 
separate out the question of achievement from that of participation.

Figure 3.2a presents the rates of $A$ level maths entry, and achievement of grades $A$ or B for all pupils, by free school meal eligibility. In 2009, pupils eligible for free school meals were only one quarter as likely to enter A level maths than their noneligible peers (at $2 \%$ compared with $8 \%$ of the cohort respectively). As a percentage of the cohort, pupils with free school meals were also less likely to achieve a grade $A$ or B (1\% compared with $5 \%)$; this reflected both a shortage of pupils with free school meals entering A level maths, and an achievement gap for those who did, with 55\% of free school meal eligible entrants achieving grade A or B compared with $64 \%$ of non-FSM entrants.

\section{Fig. 3.2a}

A level Maths Continuation and Achievement of Grades A and B Al/ Pupils

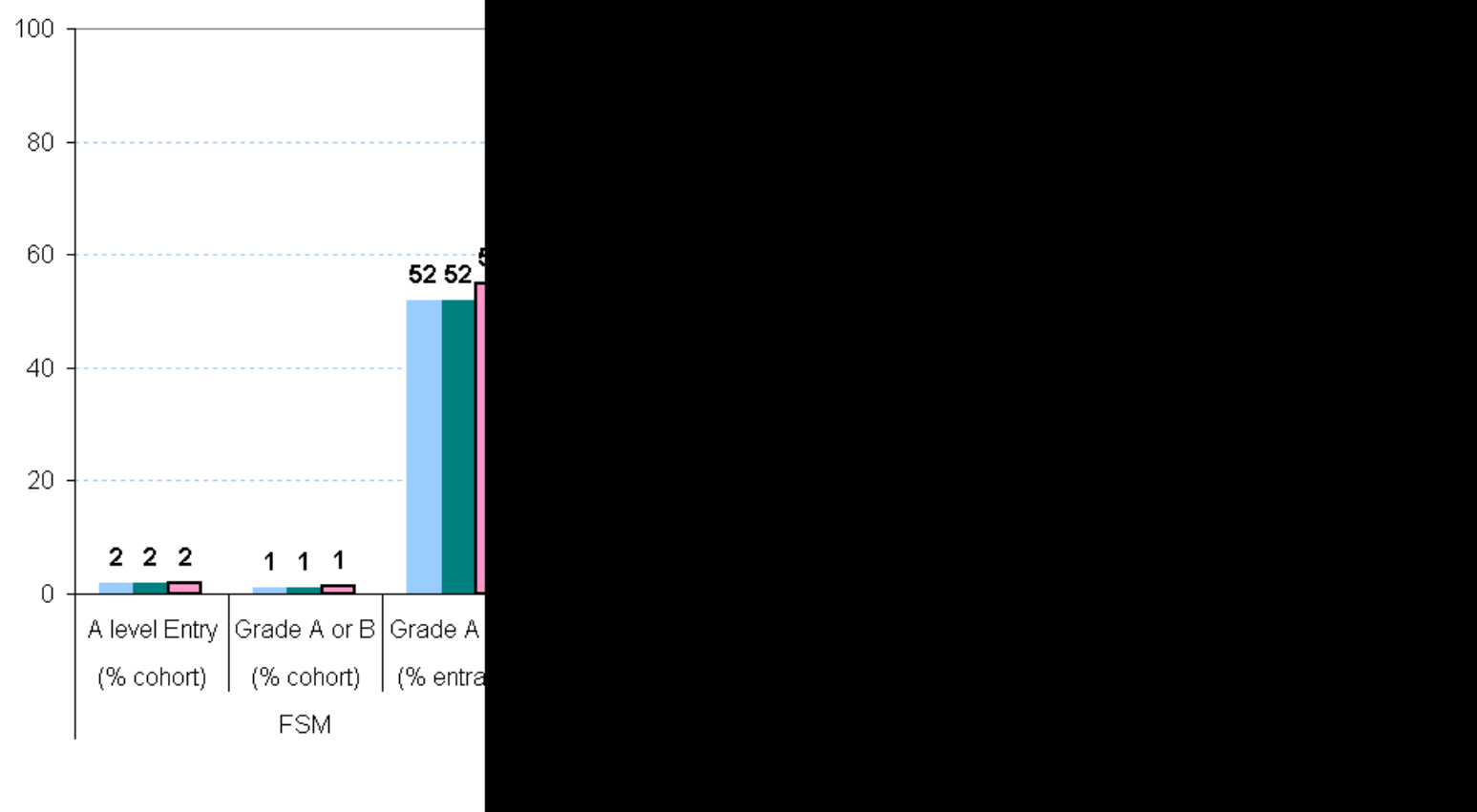


Focusing in on pupils who achieved grades $A^{*}$ or $A$ in GCSE maths, figure $3.2 b$ shows that pupils eligible for FSM, but who had achieved highly at GCSE, were actually slightly more likely to take A level maths than their high achieving noneligible counterparts (48\% compared with 47\%). This suggests that the deficit in maths entries amongst FSM pupils is entirely due to their lower probability of sufficiently high maths attainment at GCSE.

However, even amongst the high prior attaining group, pupils with free school meals were less likely to enter maths $A$ level then achieve a grade $A$ or $B$, at $30 \%$ compared with 33\% of non-FSM high attainers. This is due to a lower rate of achievement amongst FSM eligible maths entrants, $63 \%$ of whom gain A or B grades, compared with $70 \%$ of their non-FSM peers. Even when they have achieved highly at GCSE, deprivation exerts a downwards push on achievement of top grades at A level for pupils with free school meals.

\section{Fig. 3.2b}

A level Maths Continuation and Achievement of Grades A and B Pupils with Grade $A^{*}$ or $A$ at GCSE

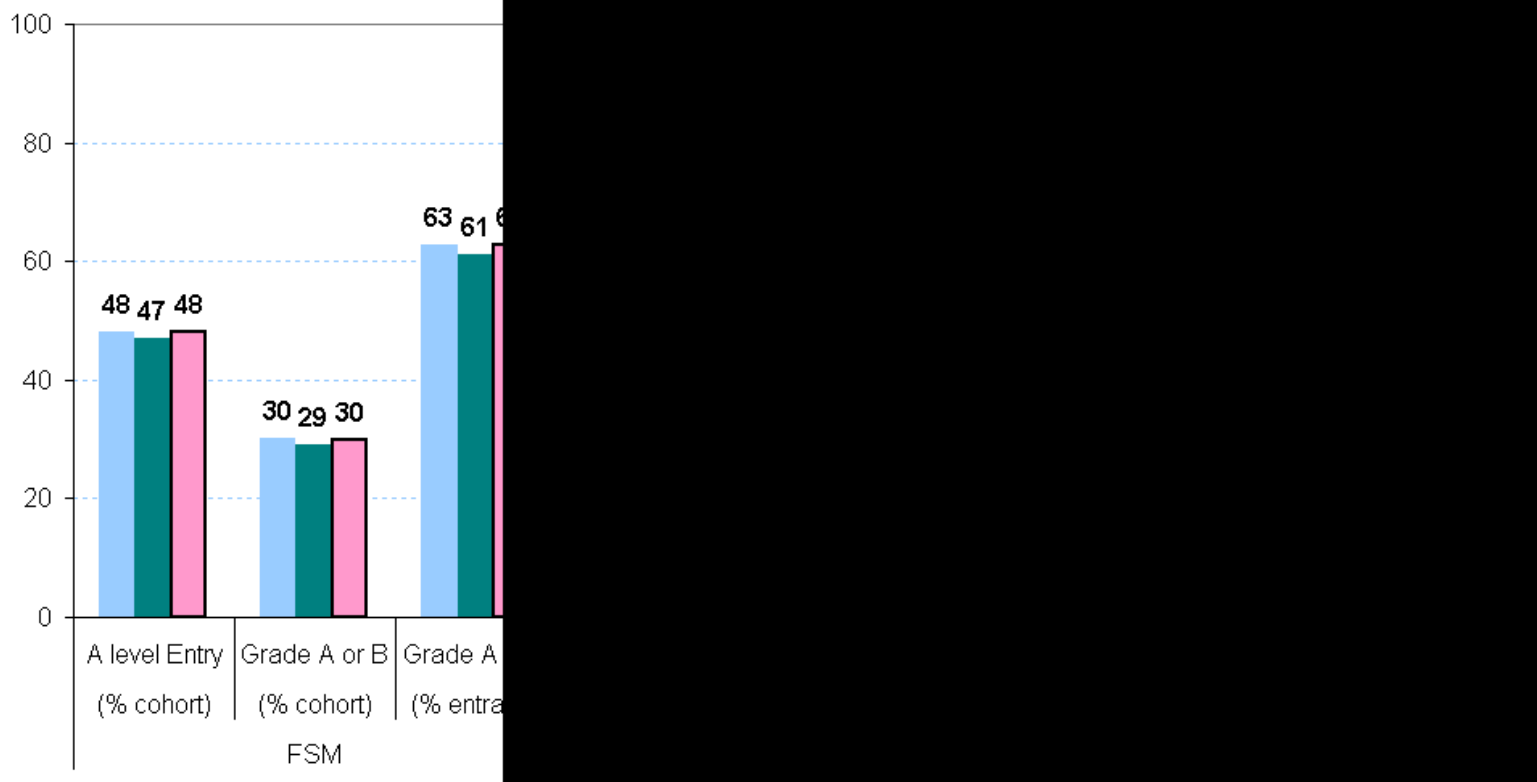

2007 a $2008 \mathbf{\square} 2009$ 
Moving to biology, pupils who were eligible for free school meals were again less likely to enter for A level, at $2 \%$ compared with $6 \%$ of non-eligible pupils (figure 3.2c). Pupils with FSM were only one third as likely to enter for biology then achieve a grade $\mathrm{A}$ or $\mathrm{B}$, at $1 \%$, compared with $3 \%$ of their non-FSM counterparts. The underlying achievement rate for pupils who entered $A$ level biology was also lower for those with free school meals, at $39 \%$ gaining grades A or B, compared with $50 \%$ of non-FSM pupils.

\section{Fig. 3.2c}

\section{A level Biology Continuation and Achievement of Grades A and B} All Pupils

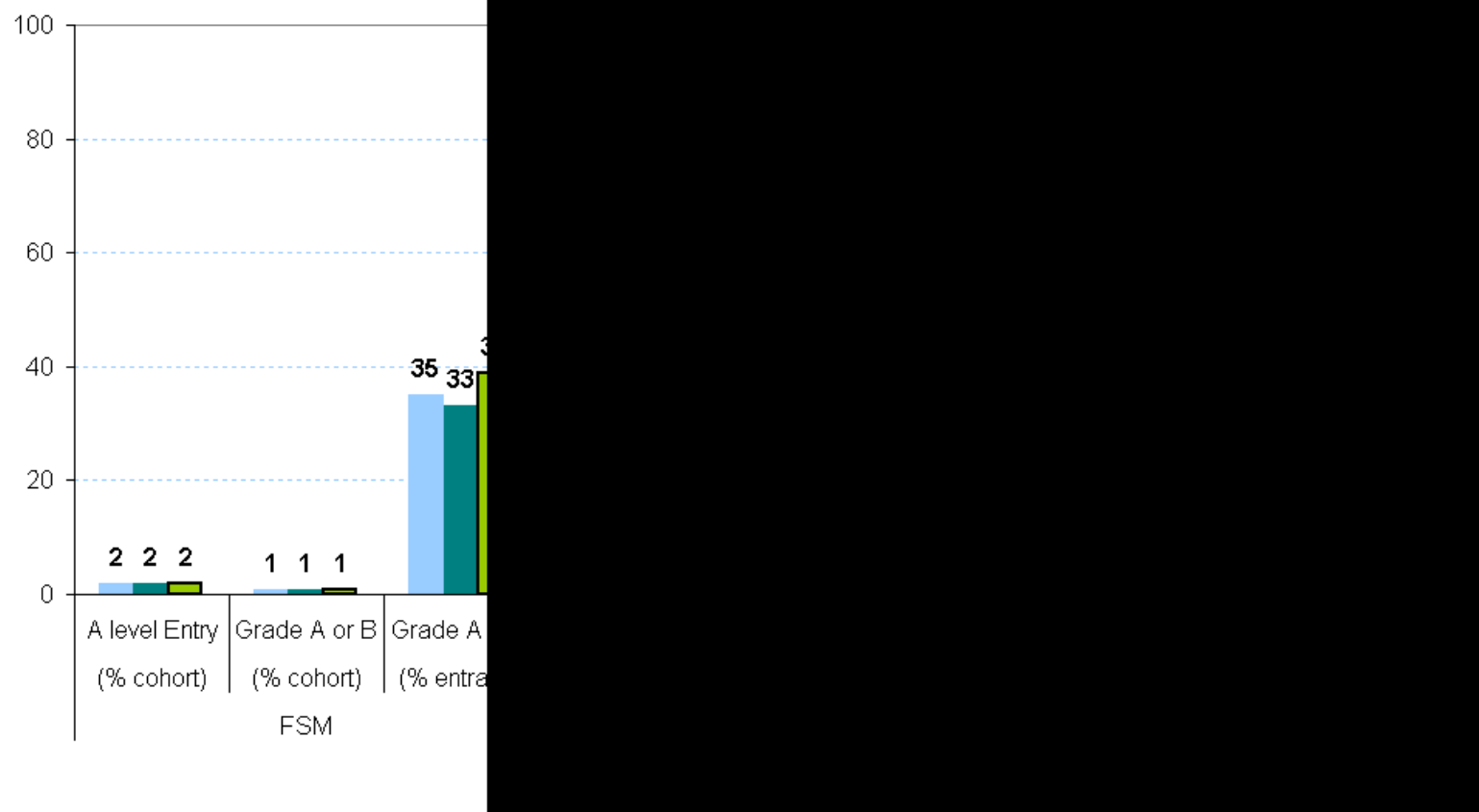


Figure $3.2 \mathrm{~d}$ reveals that those pupils with FSM who had achieved grades $A^{*}$ or $A$ in biology or double science at GCSE, were more likely to enter A level biology, but less likely to achieve a grade A or B, than their non-FSM high attaining peers. $37 \%$ of FSM pupils with high prior grades entered A level biology, compared with $32 \%$ of non-eligible pupils with equivalent prior grades. This advantage in entering biology results in high achieving FSM pupils having the same probability of entering then achieving top grades as their non-FSM counterparts (both 21\%); but the increased entry of the FSM group masks a lower underlying achievement rate for those who do take biology, at $57 \%$ of FSM entrants compared with $66 \%$ of non-FSM entrants gaining grades $\mathrm{A}$ or $\mathrm{B}$.

\section{Fig. 3.2d}

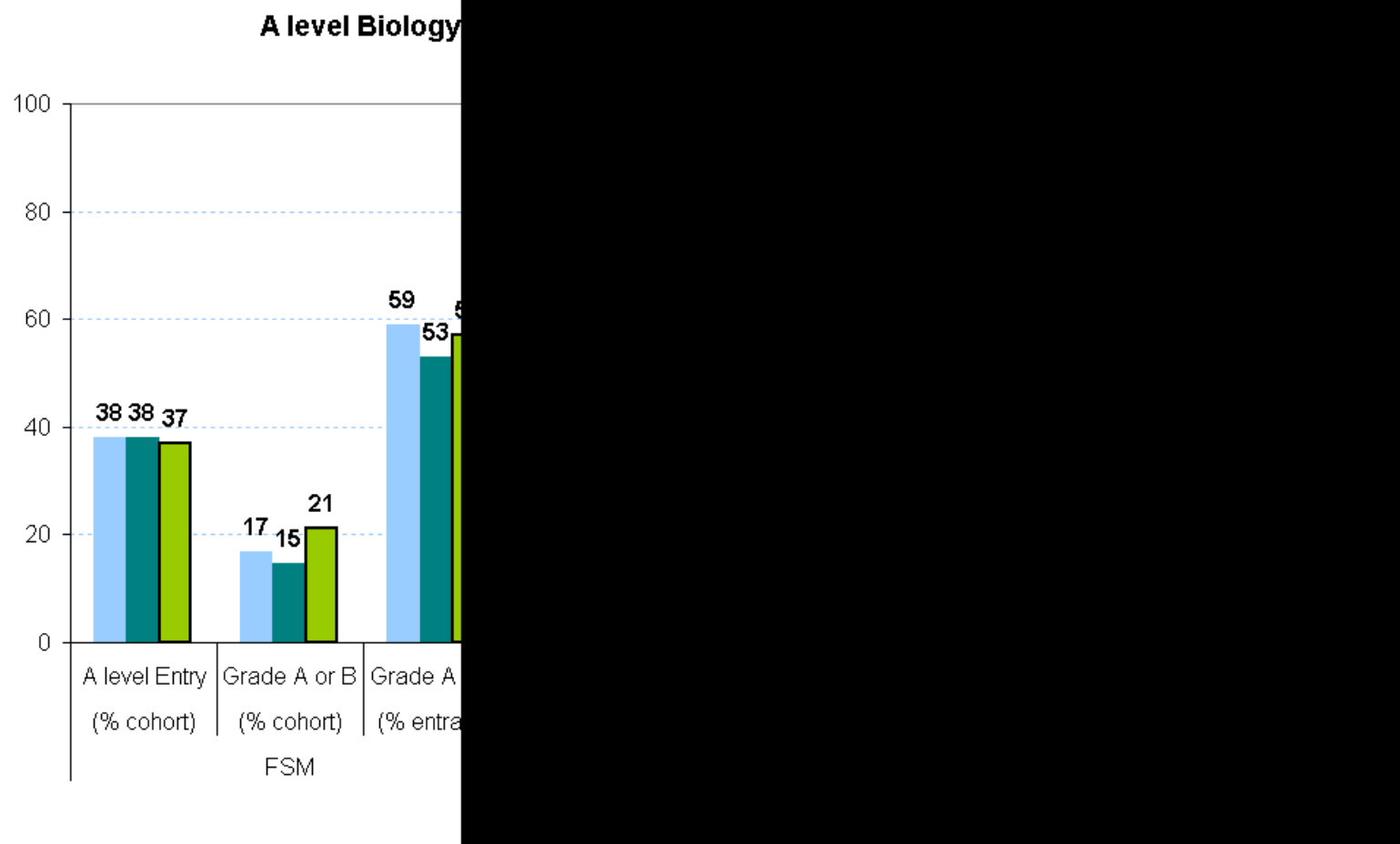


In figure 3.2e, the chemistry A level entry rate for pupils with free school meals stands at $2 \%$, compared with $5 \%$ of non-eligible pupils, reflecting the generally lower participation in A levels of the FSM group. Achievement of grades A or B is also lower for FSM pupils, at $1 \%$ of the group, or $44 \%$ of those who enter the A level; this compares with $3 \%$ of the non-FSM group entering then gaining top grades, or $57 \%$ of non-FSM entrants gaining top grades.

\section{Fig. 3.2e}

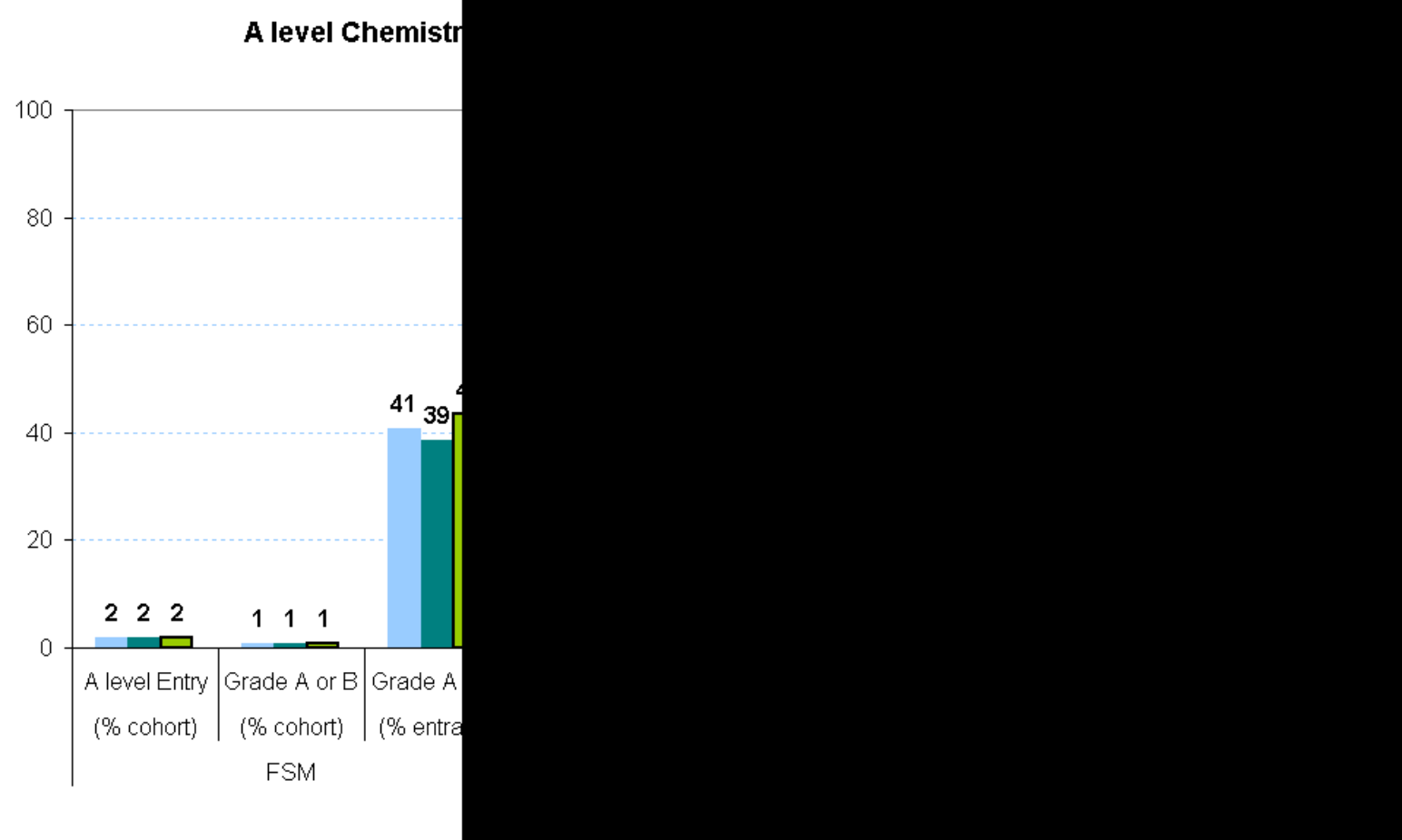


Figure $3.2 f$ mirrors the pattern seen amongst high achieving FSM pupils for A level maths and biology. The entry rate for chemistry exceeds that for non-FSM high achievers, at $36 \%$ compared with $29 \%$ of the respective groups. Achievement of grades A or B amongst those who take chemistry is lower for the deprived but high achieving group, at $58 \%$ compared with $68 \%$ of non-FSM high achieving entrants. Unlike maths or biology, the higher chemistry entry rate for the FSM high achievers outweighs their achievement disadvantage. This results in a higher proportion of all FSM pupils with high prior attainment in chemistry or double science ending up with A level chemistry grades A or B (21\%) than that of their non-FSM peers with equivalent prior attainment (at 19\%).

\section{Fig. $3.2 f$}

A level Chemistry Continuation and Achievement of Grades A and B Pupils with Grade $A^{*}$ or A at GCSE

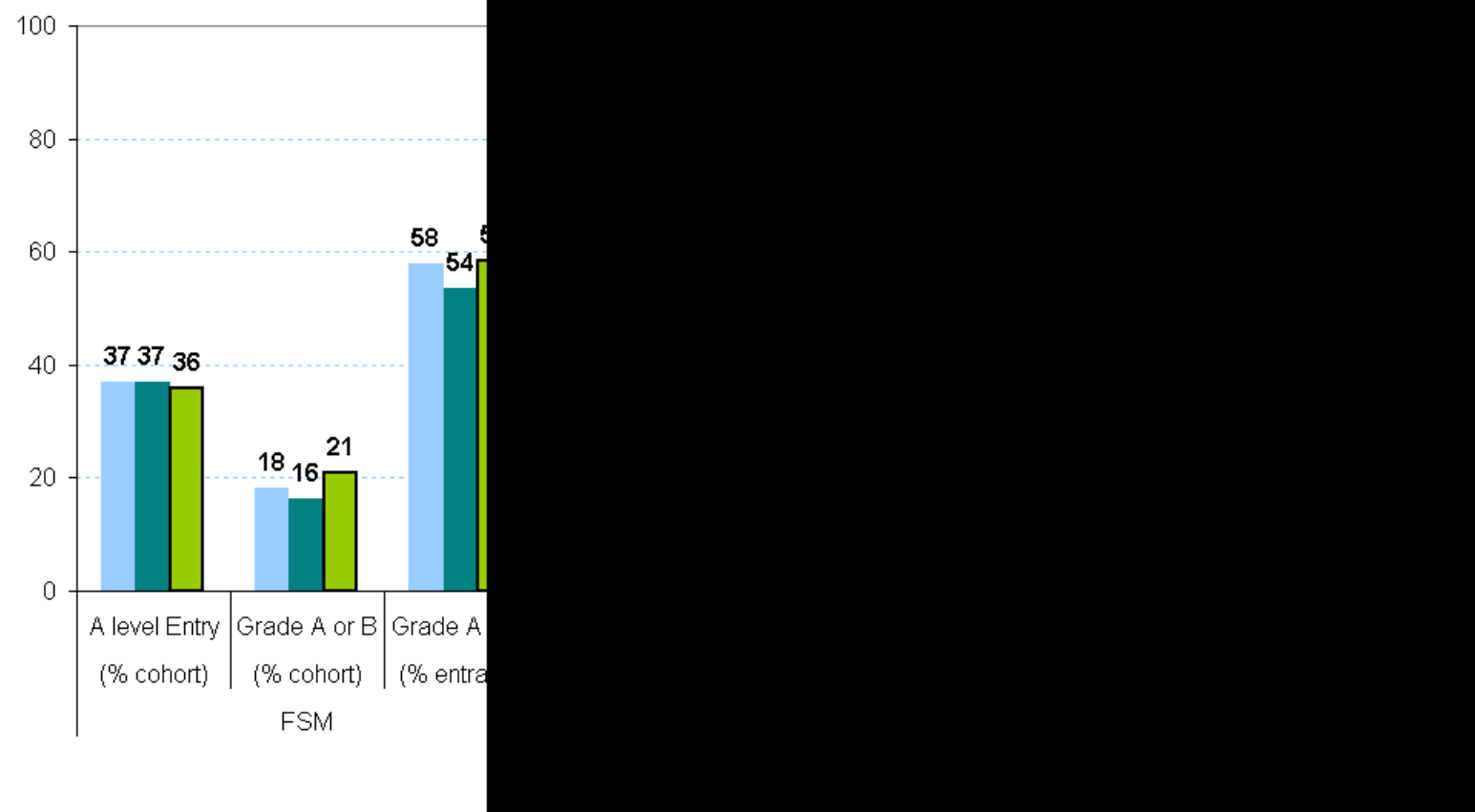


Turning to physics, figure $3.2 \mathrm{~g}$ echoes the patterns for the other maths and science subjects, with pupils eligible for free school meals again less likely to enter this A level, at $1 \%$ of the FSM group, compared with $3 \%$ of their non-FSM peers. Again this gap extends to achievement of top grades in physics A level, both as a proportion of the group and as a proportion of entrants. Less than 1\% of FSM pupils at Key Stage 4 go on to gain grades A or B at A level physics, compared with $2 \%$ of non-FSM pupils. Of those who do take physics, $34 \%$ of the FSM entrants gain top grades compared with $51 \%$ of non-eligible entrants.

\section{Fig. 3.2g}

A level Physics Continuation and Achievement of Grades A and B All Pupils

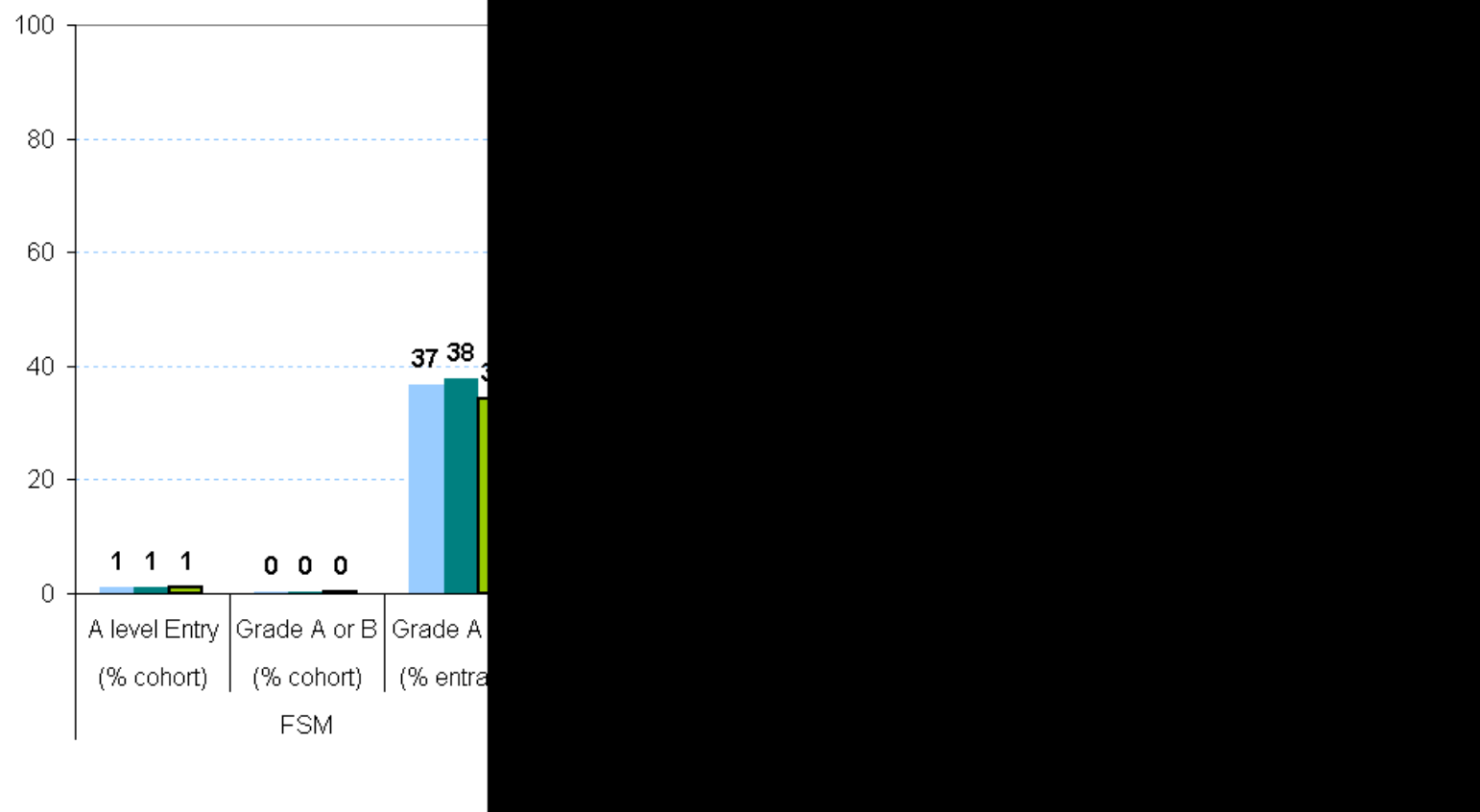


Physics A level has the most entrenched disadvantage gaps, with even those FSM pupils with prior attainment at grades $A^{*}$ or $A$ being less likely to take this $A$ level than their high achieving non-FSM peers (15\% compared with 19\%). Figure 3.2h shows that pupils with free school meals have a lower rate of achievement of top grades in A level physics than their counterparts, even if they have top $A^{*}$ or $A$ grades in physics or double science at GCSE. 7\% of FSM high achievers enter physics then achieve a grade $\mathrm{A}$ or $\mathrm{B}$, compared with $12 \%$ of non-FSM high achievers. Amongst those who did enter physics, the FSM high achievers at GCSE were less likely to be awarded top grades at A level, with $47 \%$ gaining grades A or B, compared with $64 \%$ of equivalent non-FSM entrants.

\section{Fig. 3.2h}

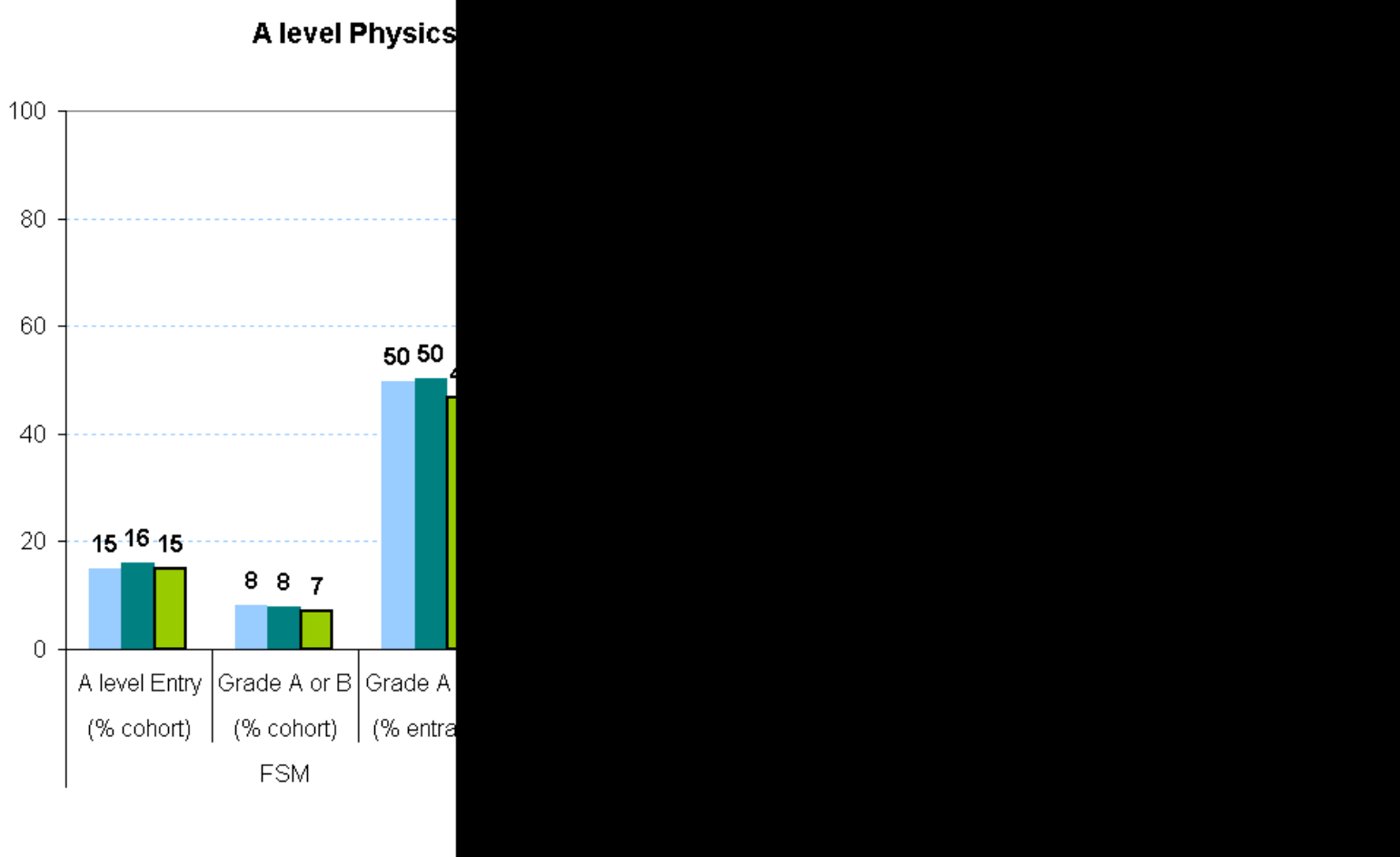

Figure 3.2i deploys odds ratios (FSM pupils / non-FSM pupils) to summarise the deprivation effects on A level maths and science outcomes. This enables meaningful comparisons to be made across subjects, across measures, and between the "all pupil" and "high prior attaining subset" analyses.

On the right-hand panel of the chart, all of the odds ratios have values of less than 1 , indicating that for the complete FSM and non-FSM groups (which have very different prior attainment bases), the FSM group is always less likely to enter maths and science A levels and to achieve high grades in those subjects at A level. Maths is the most affected of the subjects in terms of lower entry by pupils with FSM, but physics is more affected in terms of the proportion of pupils achieving top grades (whether as a percentage of the group, or as a percentage of entrants).

The left-hand panel plots the equivalent set of odds ratios for pupils with high prior attainment (of GCSE grades $A^{*}$ or $A$ ) within each subject. For this subset, who have the greatest suitability for A level, FSM pupils were more likely to take biology, chemistry or maths A levels than their peers, but less likely to take physics. They 
had similar odds to their non-FSM peers for entering biology, chemistry or maths then achieving a grade $A$ or $B$, but this was due to their greater propensity to take these A levels, and masks a lower achievement rate amongst entrants. For physics, the high prior attaining FSM pupils were still less likely than their peers with equivalent prior attainment to either enter or achieve a high grade at A level.

\section{Fig. 3.2i}

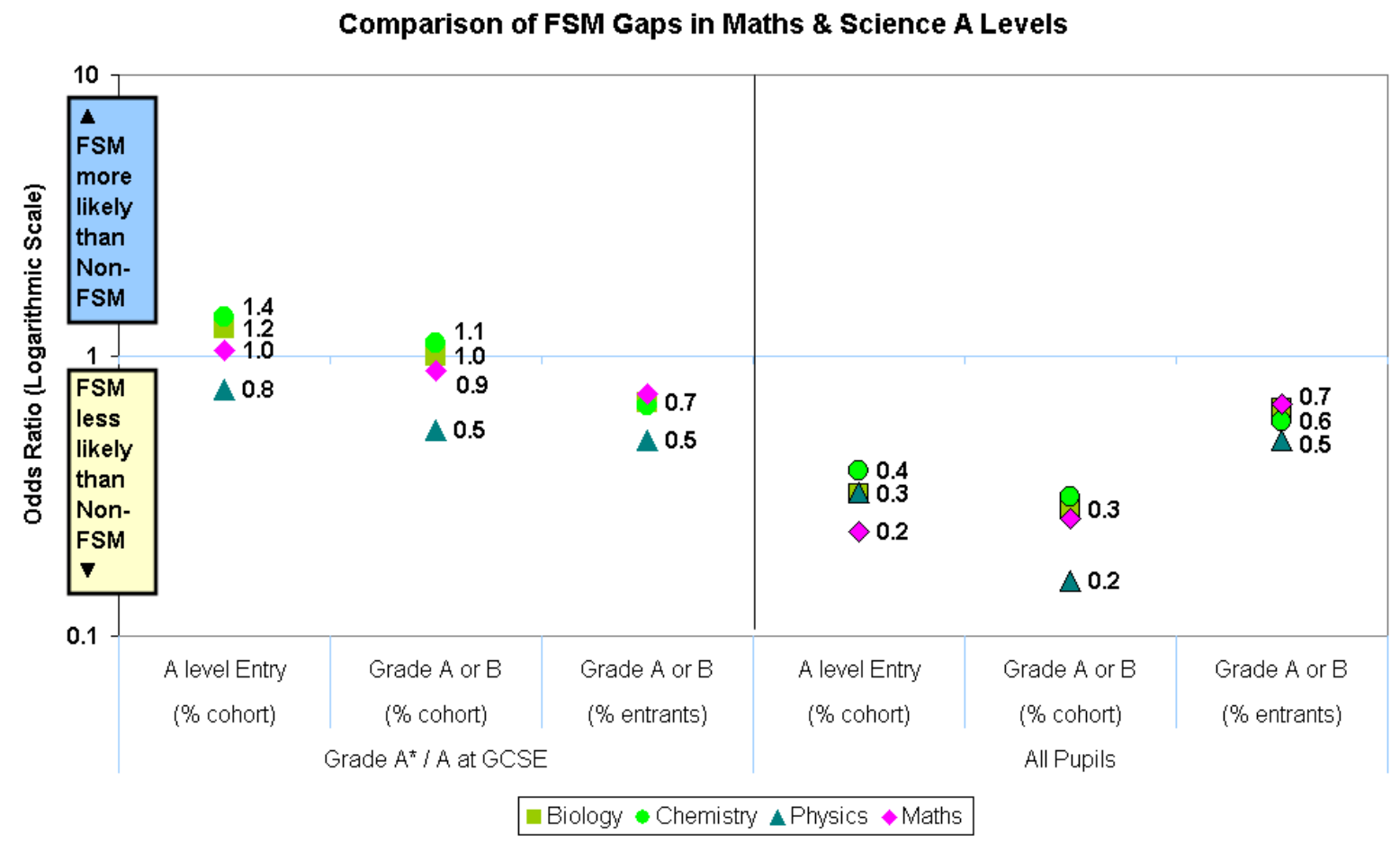

\subsection{Ethnicity and A level Maths and Sciences}

Section 3.3 examines A level participation and achievement gaps for different ethnic groups. For maths and the sciences, the rates of entry and success at grades A or B in the cohort are examined for each group, followed by the percentage of entrants achieving grades A or B in the third set of columns, to separate out the question of achievement from that of participation. 
Figure 3.3a presents the rates of A level maths entry, and achievement of grades $A$ or B for all pupils, by ethnic background. In 2009, Chinese, Indian, Black African, Bangladeshi and Pakistani pupils were more likely to enter A level maths than their White British peers. The probability of entering maths then achieving a grade $A$ or $B$ was also higher for the Chinese, Indian, Black African and Bangladeshi (but not Pakistani) groups. The only major ethnic group that was less likely to enter maths, or to go on to achieve a top grade, were Black Caribbean pupils.

However, amongst maths $A$ level entrants, the rate of success in gaining a grade $A$ or B, was only higher than White British pupils for the Chinese and Indian pupils.

The higher cohort incidence of these grades for Black African and Bangladeshi pupils was driven by higher entry rates rather than an achievement advantage.

Fig. 3.3a

A level Maths Continuation and Achievement of Grades A and B All Pupils

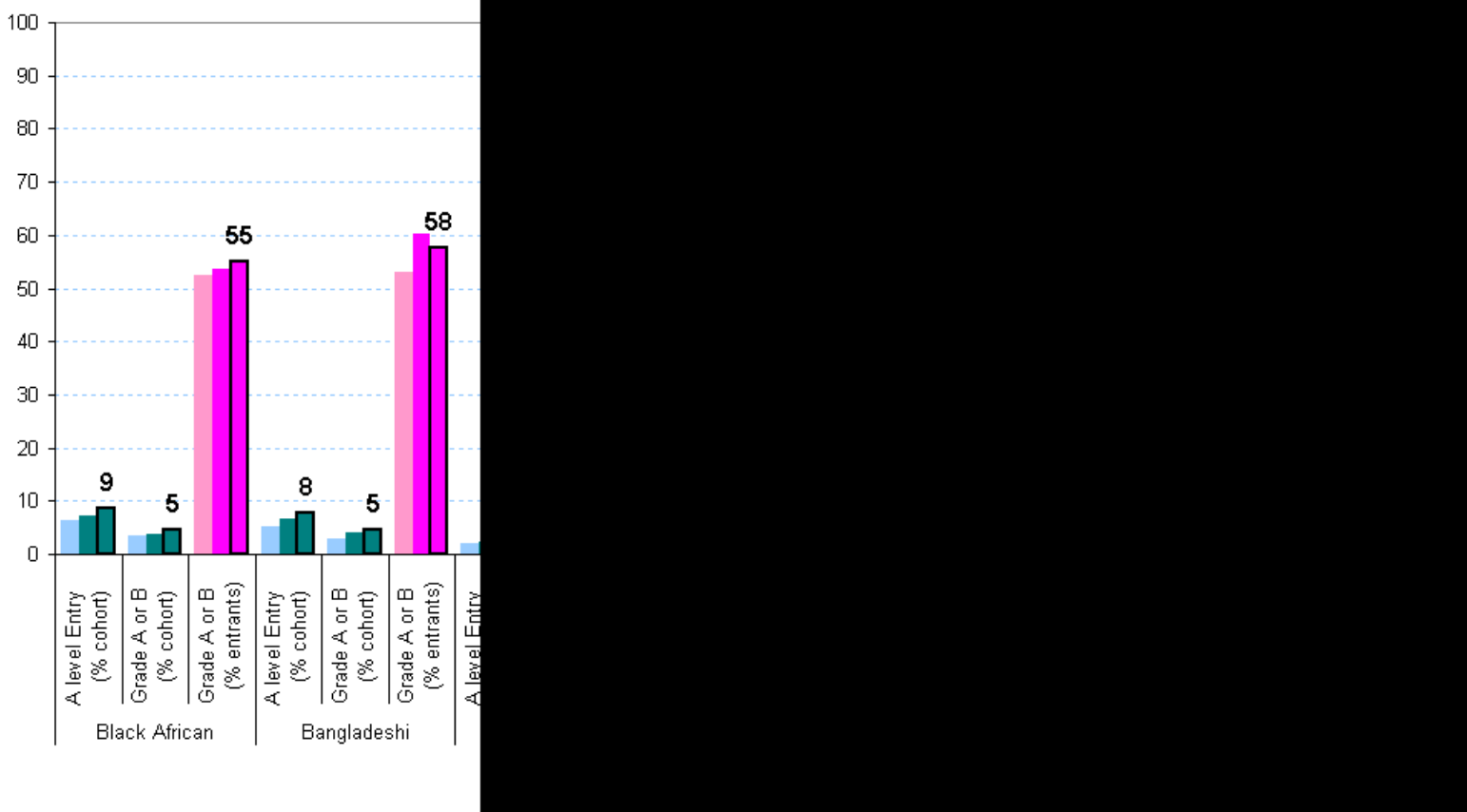

Narrowing the focus of the analysis to pupils who achieved grades $A^{*}$ or A in GCSE maths, figure 3.3b shows that the same ethnic groups (Chinese, Indian, Black African, Bangladeshi and Pakistani) had higher probabilities of their high achieving pupils taking A level maths as was the case for pupils of all prior achievement levels.

All of these groups, including Pakistani, also had higher rates of incidence for their high prior attaining pupils taking $A$ level maths then achieving a grade $A$ or $B$. Amongst those high achievers who entered maths, only the Chinese and Indian pupils had higher rates of grade A or B awards than White British pupils, with the higher cohort incidence of these grades for Black African, Bangladeshi and Pakistani pupils explained by their greater propensity to take A level maths.

The significance of the higher rates of A level maths entry and achievement by Chinese and Indian pupils can be seen from the finding that Chinese pupils with high GCSE maths grades are more than twice as likely to end up achieving a grade A or B 
in the subject at A level (62\% compared with $30 \%$ of White British peers); while Indian pupils with high GCSE maths grades have a 55\% incidence of $A$ level maths at grades A or B. Despite lower achievement profiles at A level maths, higher proportions of Black African (40\%), Bangladeshi and Pakistani (both 38\%) pupils with high GCSE maths grades eventually gain grades A or B at A level maths due to their increased rates of entry to the subject.

\section{Fig.3.3b}

\section{A level Maths Continuation and Achievement of Grades A and B} Pupils with Grade $A^{*}$ or $A$ at GCSE

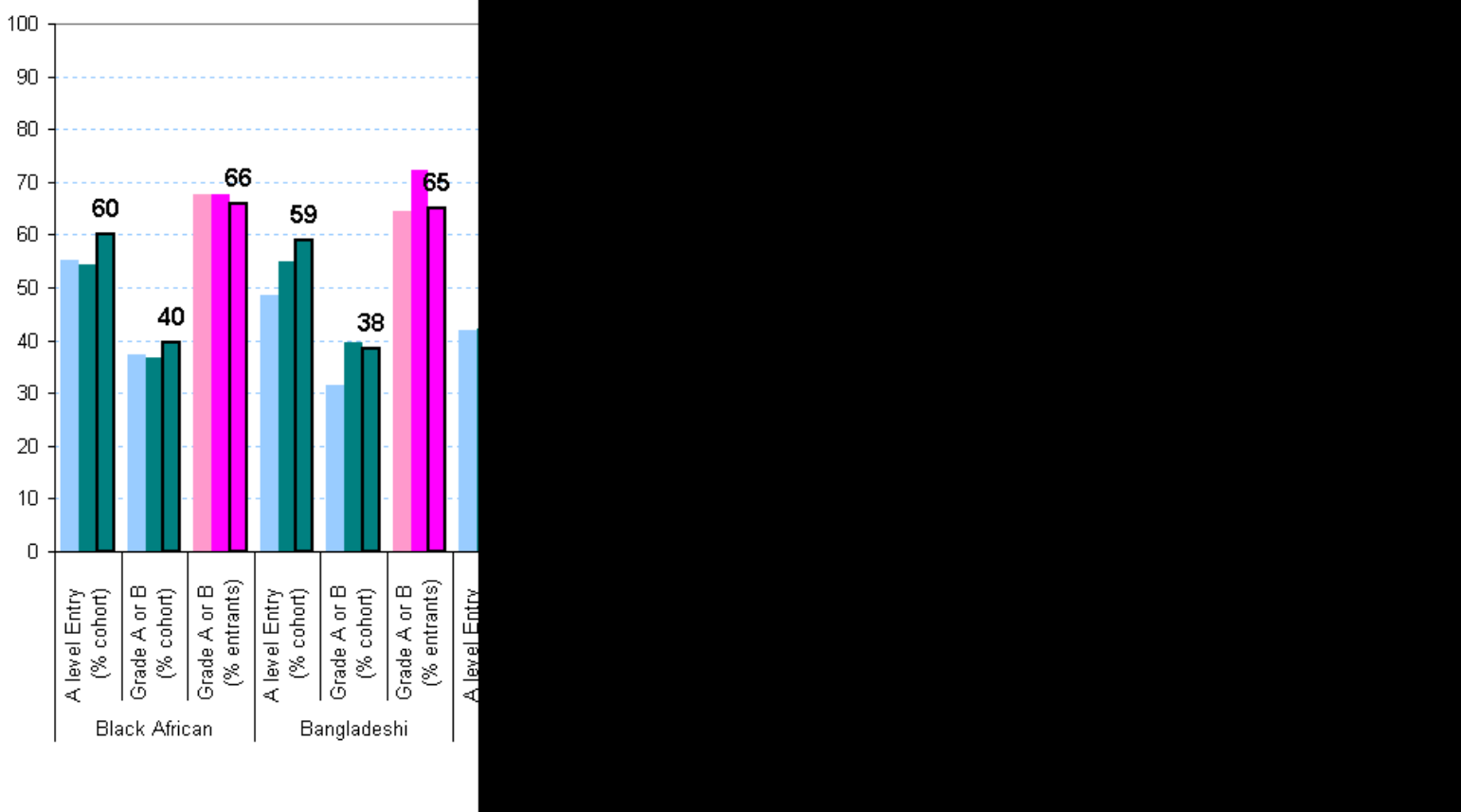

Figure 3.3c presents the rates of $A$ level biology entry, and achievement of grades $A$ or B for all pupils, by ethnic background. In 2009, the same ethnic groups as for maths (Chinese, Indian, Pakistani, Black African and Bangladeshi) were also more likely to enter A level biology than their White British peers.

The probability of entering biology then achieving a grade A or B was also higher for these groups, including Pakistani pupils. Again, the only major ethnic group that was less likely to enter biology, or to go on to achieve a top grade in the subject, were Black Caribbean pupils. 
However, amongst biology $\mathrm{A}$ level entrants, the rate of success in gaining a grade $\mathrm{A}$ or B, was only higher than White British pupils for the Chinese, Indian and Pakistani pupils. The higher cohort incidence of these grades for Black African and Bangladeshi pupils was driven by higher entry rates rather than an achievement advantage.

\section{Fig.3.3c}

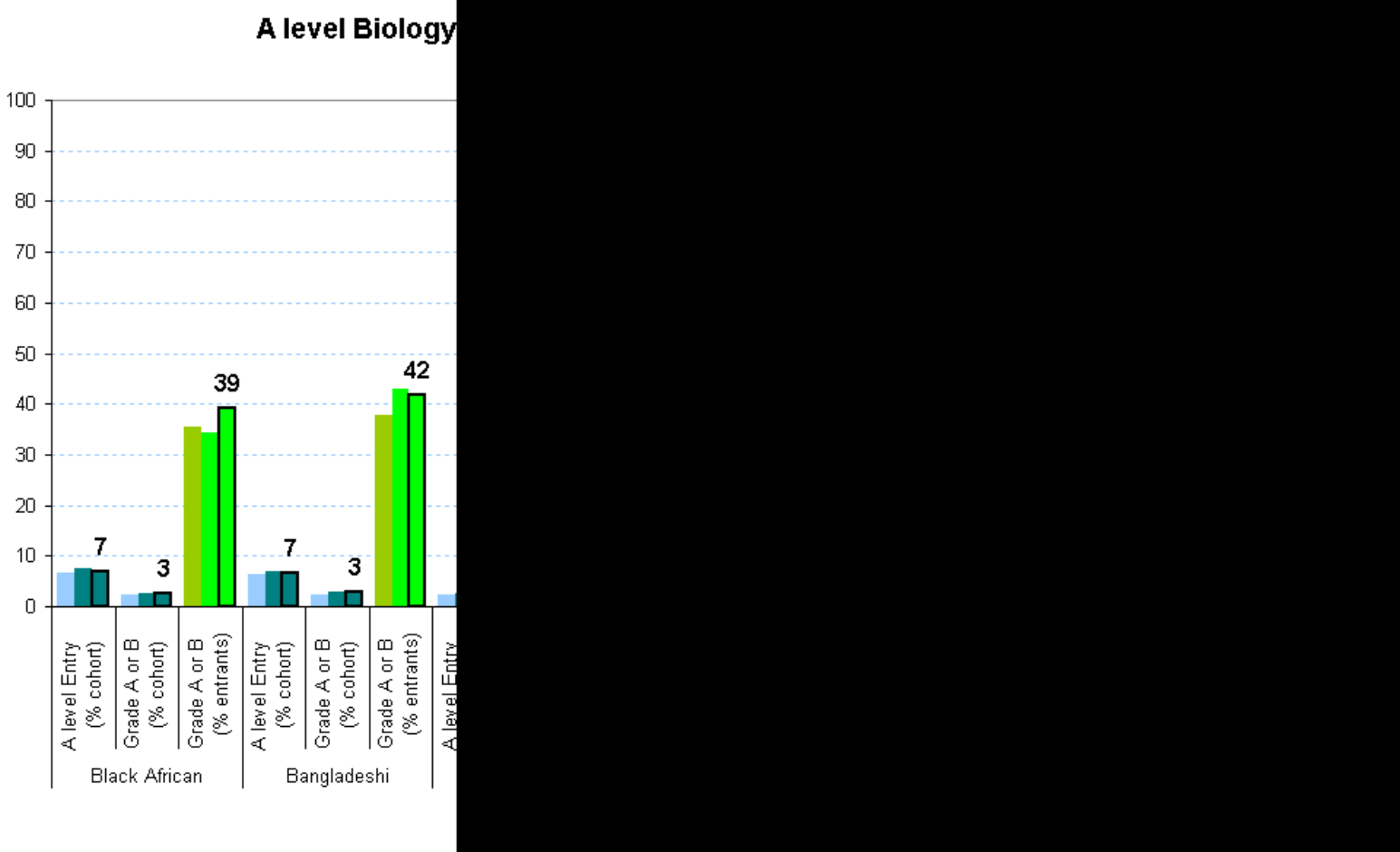

Narrowing the focus of the analysis to pupils who achieved grades $A^{*}$ or A in GCSE biology or double science, figure 3.3d shows that the same ethnic groups (Pakistani, Indian, Chinese, Black African and Bangladeshi) had higher probabilities of their high achieving pupils taking A level biology as was the case for pupils of all prior achievement levels. Black Caribbean pupils with high prior GCSE grades had the same probability of entering A level biology as their White British counterparts.

The same groups with raised entry levels for biology also had higher rates of incidence for their high prior attaining pupils taking $\mathrm{A}$ level biology then achieving a grade $A$ or $B$. However, amongst those high achievers who entered biology, only the Chinese, Indian and Pakistani pupils had higher rates of grade A or B awards than White British pupils, with the higher cohort incidence of these grades for Black African and Bangladeshi pupils explained by their greater propensity to take A level biology.

Pakistani pupils with high GCSE biology or double science grades are significantly more likely than their White British peers to end up achieving a grade A or B in the subject at A level (39\% compared with 19\%); followed by Indian pupils with high subject prior attainment who had a $36 \%$ incidence of $A$ level biology at grades A or B, and Chinese high prior attainers with $32 \%$ incidence. Despite lower achievement profiles at A level biology, higher proportions of Black African (25\%) and Bangladeshi 
(23\%) pupils with high subject prior attainment eventually gain grades A or B at A level biology due to their increased rates of entry to the subject.

Fig. 3.3d

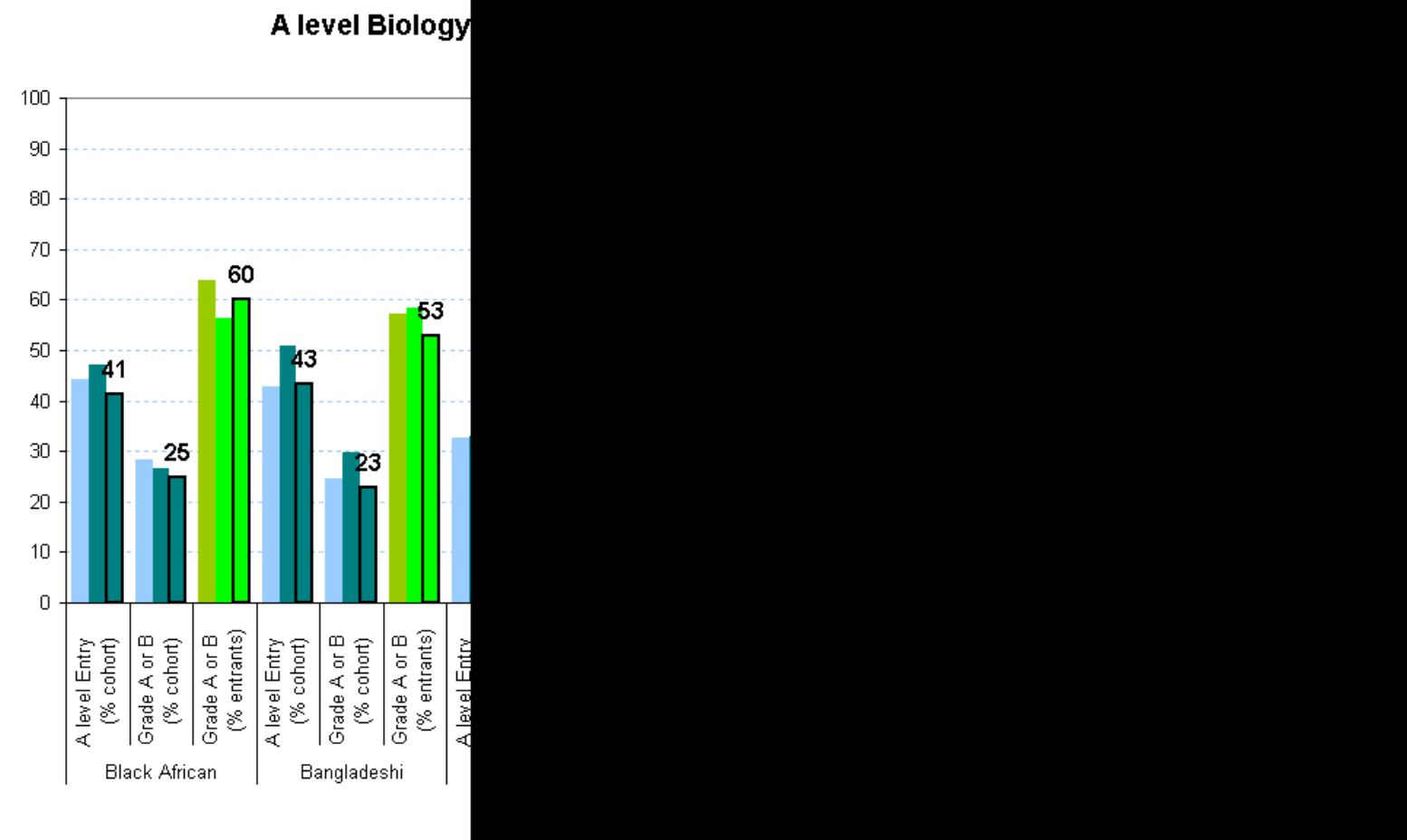

Figure 3.3e depicts the rates of A level chemistry entry, and achievement of grades A or B for all pupils, by ethnic background. Again for chemistry, the same ethnic groups had higher propensities to enter the subject at A level than White British pupils; these were the Chinese, Indian, Pakistani, Black African and Bangladeshi groups. The probability of entering chemistry then achieving a grade $A$ or $B$ was also higher for the same groups. The only major ethnic group that was less likely to enter chemistry, or to go on to achieve a top grade in the subject, were Black Caribbean pupils. 
However, amongst chemistry A level entrants, the rate of success in gaining a grade A or B, was only higher than White British pupils for the Chinese and Indian pupils. The higher cohort incidence of these grades for Pakistani, Black African and Bangladeshi pupils was driven by higher entry rates rather than an achievement advantage.

\section{Fig. 3.3e}

\section{A level Chemistry Continuation and Achievement of Grades A and B} All Pupils

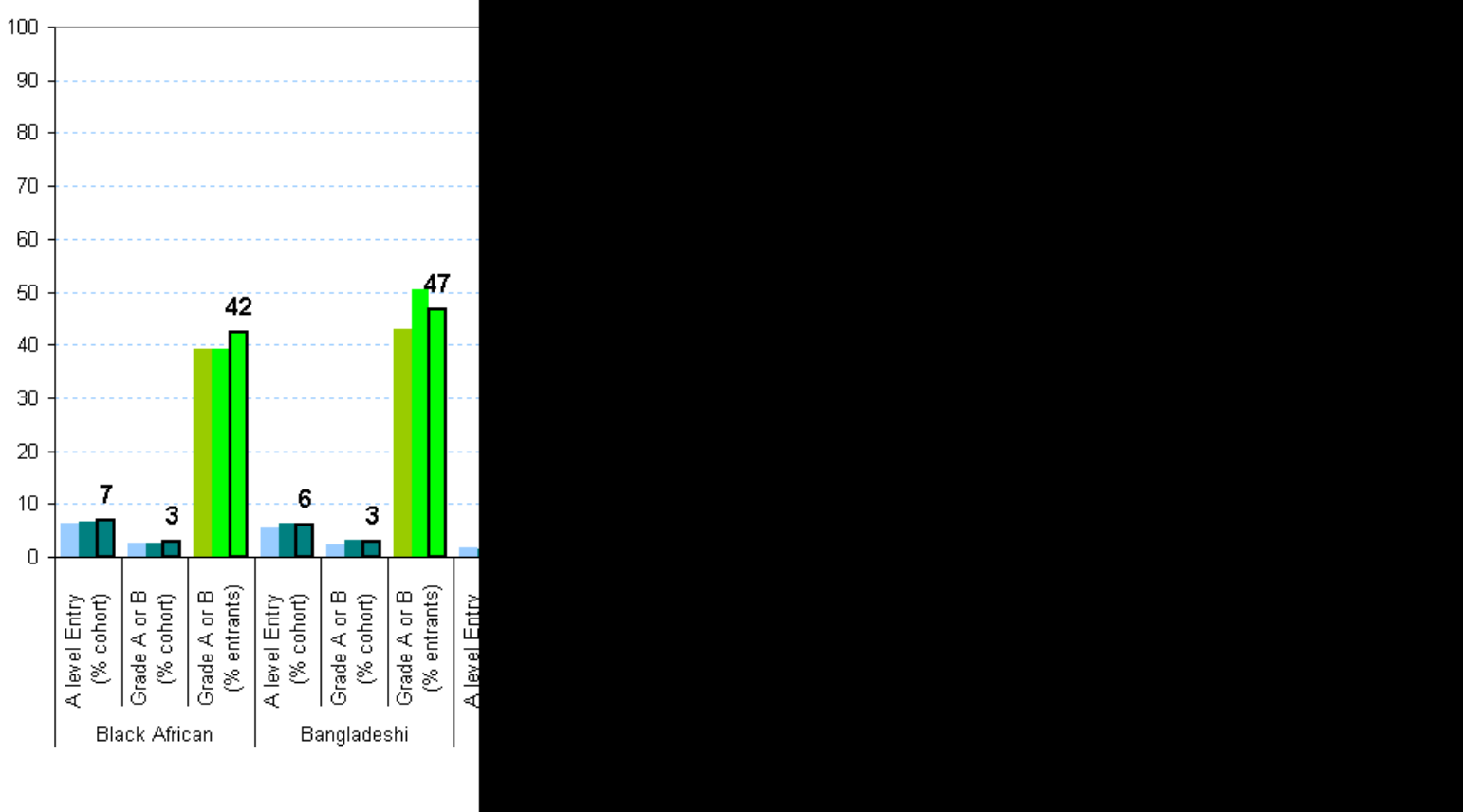

Selecting only those pupils who achieved grades $A^{*}$ or A in GCSE chemistry or double science to repeat the analysis, figure $3.3 f$ shows that the same ethnic groups (Pakistani, Indian, Chinese, Black African and Bangladeshi) had higher probabilities of their high achieving pupils taking A level chemistry as was the case for pupils of all prior achievement levels. Black Caribbean pupils with high prior GCSE grades had a lower probability of entering A level chemistry than their White British counterparts.

The same groups with raised entry levels for chemistry also had higher rates of incidence for their high prior attaining pupils taking A level chemistry then achieving a grade $A$ or $B$. However, amongst those high achievers who entered chemistry, only the Chinese and Indian pupils had higher rates of grade A or B awards than White British pupils, with the higher cohort incidence of these grades for Pakistani, Black African and Bangladeshi pupils explained by their greater propensity to take A level chemistry.

Chinese pupils with high GCSE chemistry or double science grades over twice as likely as their White British peers to end up achieving a grade A or B in the subject at A level (39\% compared with 17\%); followed by Indian and Pakistani pupils with high subject prior attainment who both had 37\% incidences of A level chemistry at grades A or B. Despite lower achievement profiles at A level chemistry, higher proportions of Black African (28\%) and Bangladeshi (24\%) pupils with high subject prior 
attainment go on to gain grades A or B at A level chemistry due to their increased rates of entry to the subject.

\section{Fig. 3.3f}

A level Chemistry Continuation and Achievement of Grades A and B Pupils with Grade $A^{*}$ or $A$ at GCSE

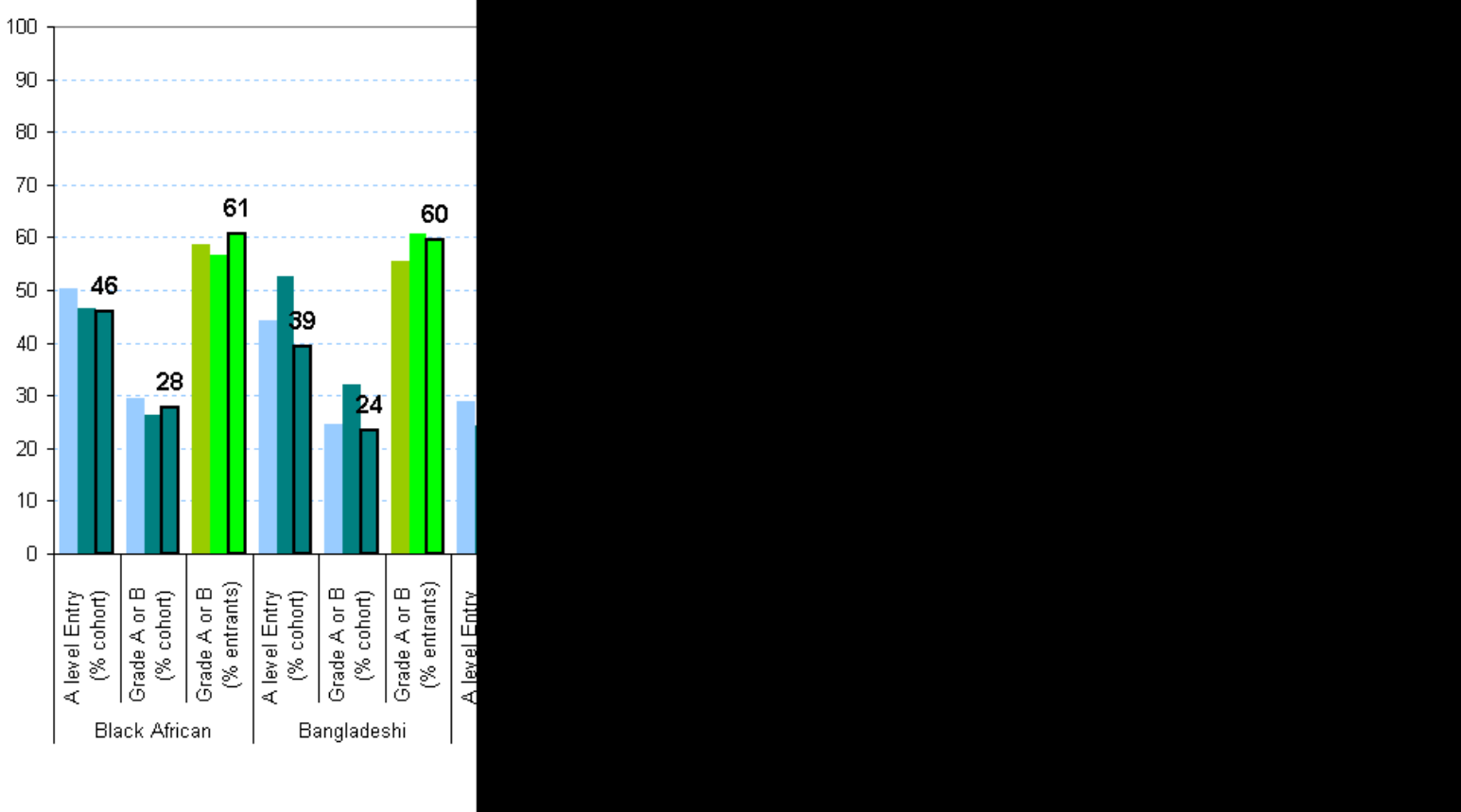


Figure $3.3 \mathrm{~g}$ presents the rates of A level physics entry, and achievement of grades $A$ or B for all pupils, by ethnic background. For physics, unlike the other maths and science subjects, only Chinese and Indian pupils had greater probabilities of taking the subject at $A$ level than White British pupils. The probability of entering chemistry then achieving a grade $A$ or $B$ was also higher for the same two groups. Pakistani, Bangladeshi, Black African and Black Caribbean pupils were less likely to enter chemistry, or to go on to achieve a top grade in the subject, than their White British peers.

For physics, only Chinese A level entrants were more likely to be awarded grade A or $B$ than White British entrants. Indian A level physics candidates had the same rate of achievement of top grades as White British entrants, whereas all other groups of entrants were less likely to gain Grades A or B.

\section{Fig. 3.3g}

A level Physics Continuation and Achievement of Grades A and B All Pupils

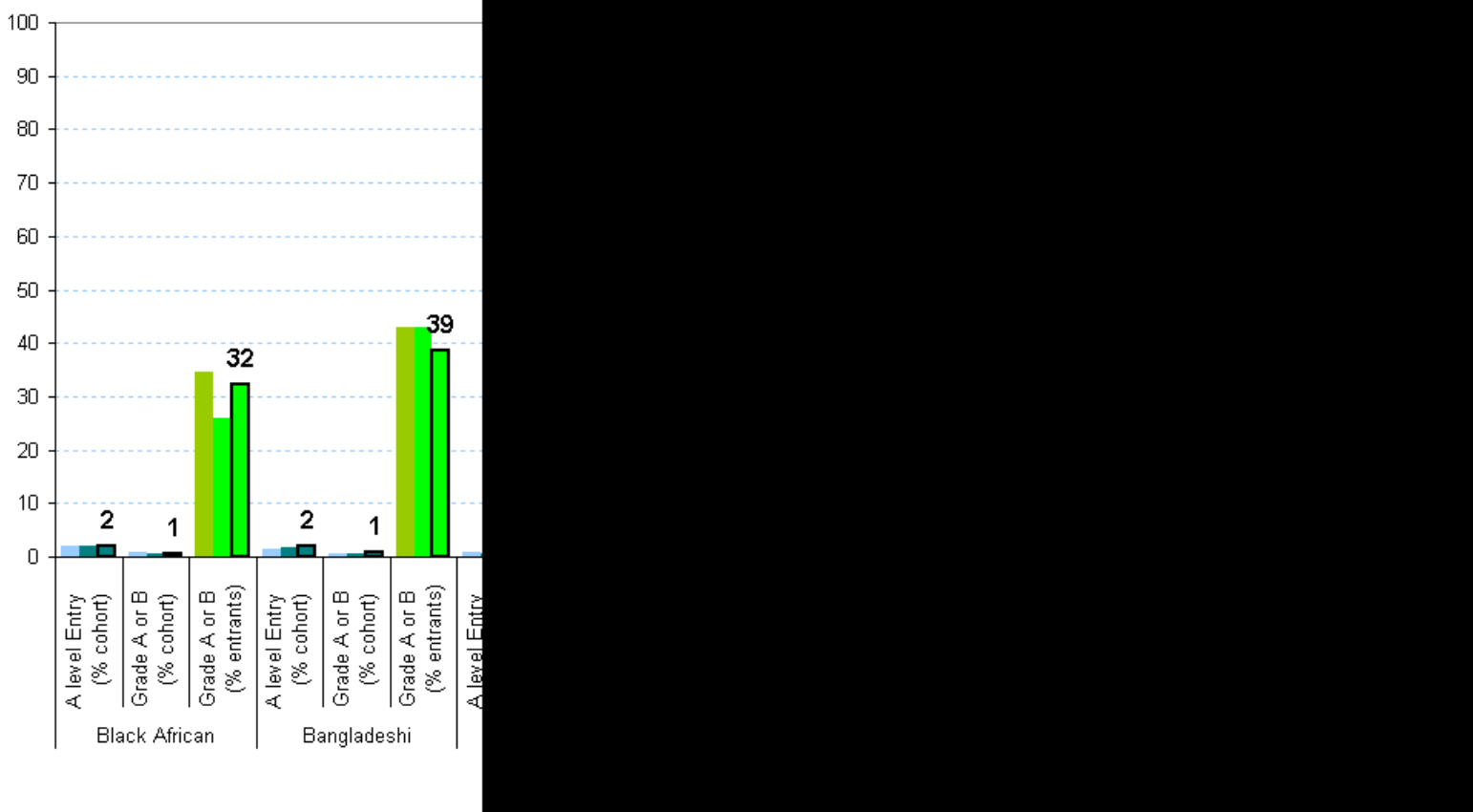

Narrowing the focus of the analysis to pupils who achieved grades $A^{*}$ or A in GCSE physics or double science, figure 3.3h shows that only the Chinese group had a higher probability of their pupils with grades $A^{*}$ or $A$ at GCSE taking A level physics than the White British equivalent high prior attaining group.

Chinese high achievers also had a higher rate of entering $A$ level physics then achieving a grade $A$ or $B$. However, due to higher underlying achievement of grades A or B by physics entrants than their White British peers, Indian high achieving pupils were equally as likely as White British high achievers to enter physics then gain a top grade, despite having a lower entry rate for the subject. All other ethnic groups had lower cohort incidence rates of physics grades A or B than White British pupils, for their high prior attaining subsets.

Chinese pupils with high GCSE physics or double science grades are over twice as 
likely overall as their White British peers to end up achieving a grade $A$ or $B$ in the subject at A level (34\% compared with 12\%); Indian pupils with equivalent GCSE grades equal their White British peers at $12 \%$. All other groups of high achievers have both lower entry and achievement rates for A level physics.

\section{Fig. $3.3 \mathrm{~h}$}

A level Physics Continuation and Achievement of Grades A and B Pupils with Grade $A^{*}$ or $A$ at GCSE

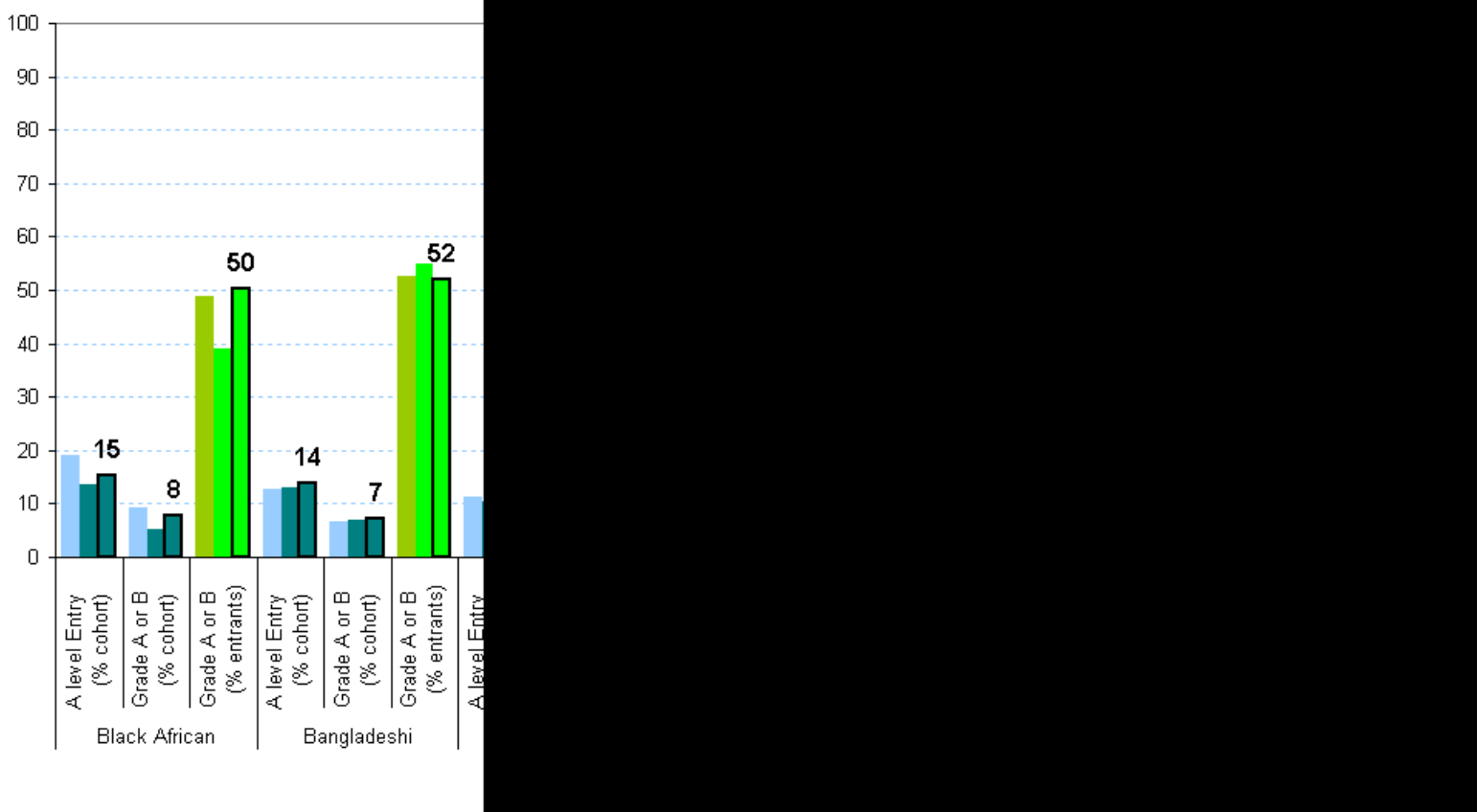

The charts in figures 3.3i-k deploy odds ratios (ethnic group / White British) to summarise the ethnicity effects on A level maths and science outcomes. This enables meaningful comparisons to be made across subjects, across measures, and between the "all pupil" and "high prior attaining subset" analyses.

The ethnicity effects follow roughly the same order from high entry / achievement to low entry / achievement in each of figures 3.3i to 3.3k. Chinese and Indian pupils have the highest odds of entry and achievement to maths and science A levels, followed by Pakistani, Black African and Bangladeshi pupils, with Black African pupils having the lowest odds for these outcomes. Where the White British majority fits in this order of propensity varies depending on the subject in question and whether the focus is on entry or achievement at A level.

Considering achievement of high grades by A level entrants, White British pupils fit in between Indian and Pakistani pupils (as the third ranked ethnic group), except in the case of physics, where they fit in after Chinese, but ahead of all other ethnic groups. By contrast, looking at A level entry effects, these are larger than the achievement effects, and all minority groups apart from Black Caribbean have stronger odds of taking A level maths and sciences than White British pupils, except in the case of physics, where only Chinese pupils are clearly ahead of the majority.

The odds ratios on achievement of grade A or B are generally similar for the high prior attaining subsets as for the complete prior attainment range, within each ethnic 
group. However for entry to A level, the effects were weaker, whether increases or decreases in odds, for the high prior attaining subsets (with the greatest suitability for A level study in these subjects), than across the complete ability range for each ethnic group.

\section{Fig. 3.3i}

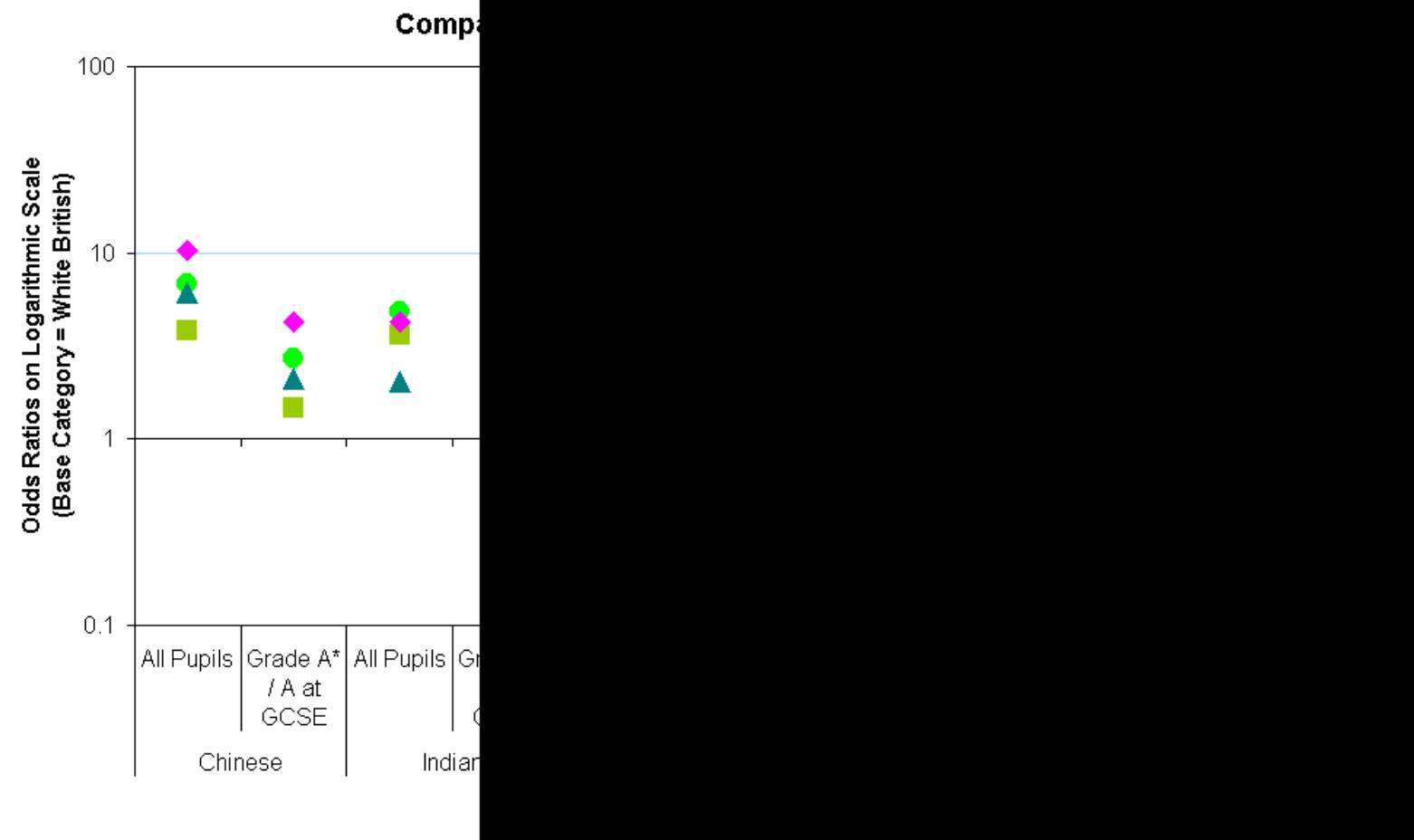

Fig. 3.3j

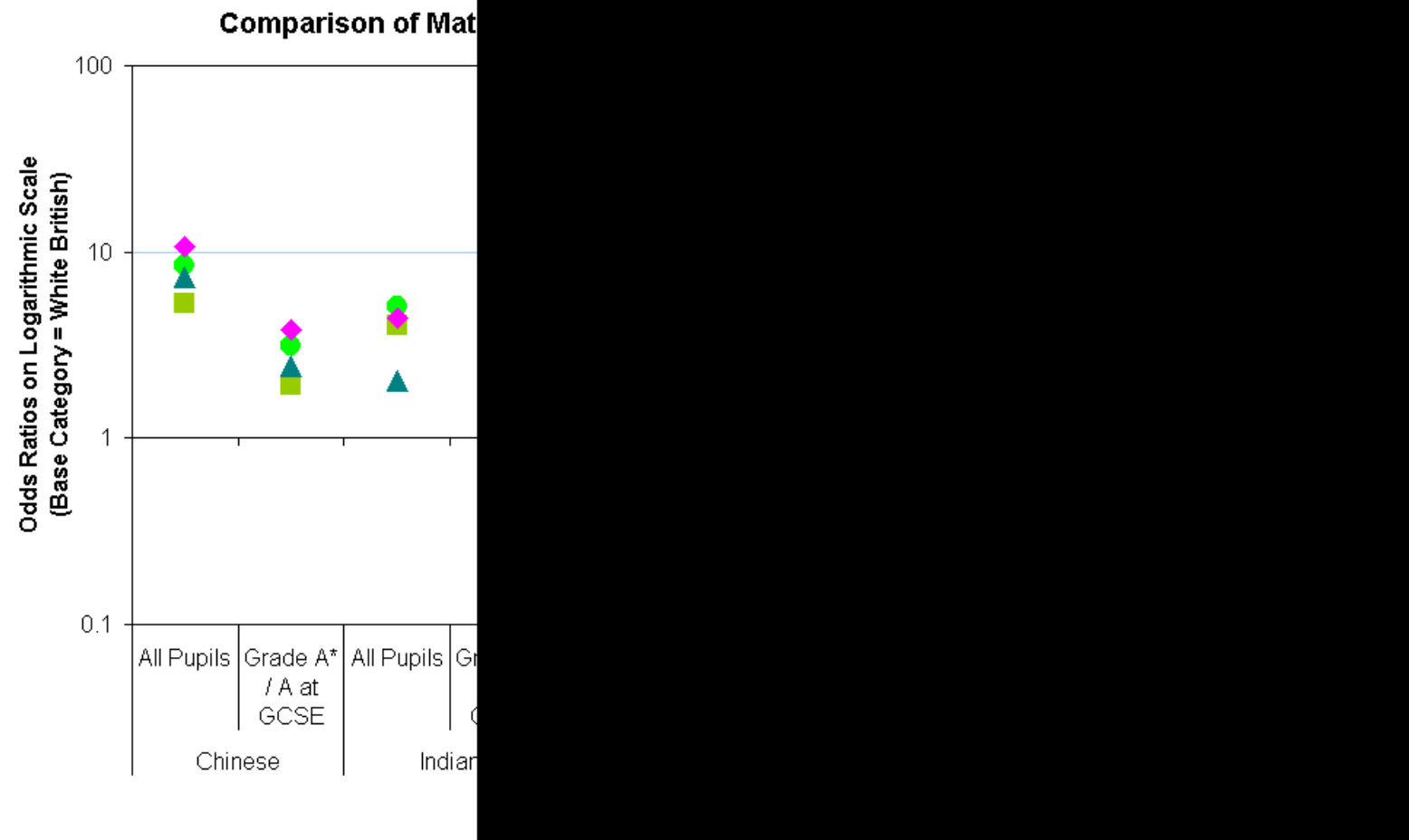




\section{Fig. 3.3k}

Comparison of Maths \& Science A Level Grade A/B (Proportion of Entrants)

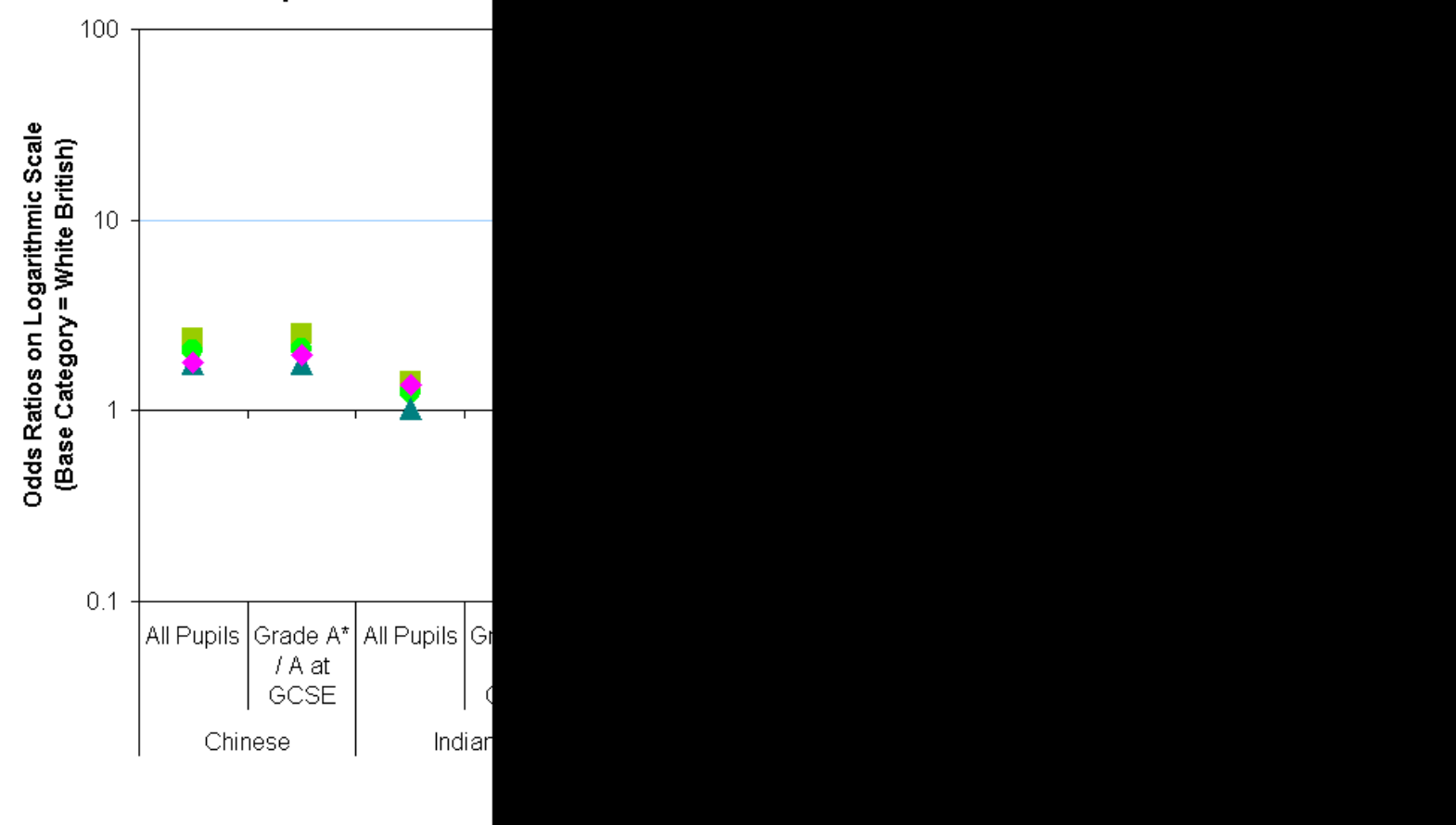




\section{Chapter 4. Provision: Education Settings \& Pathways}

Chapter 4 explores the influence of variations in the type of schools and A level providing institutions attended by young people, and in the most prevalent types of pre-A level qualification offered by schools in maths and science subjects. Section 4.1 begins by examining A level continuation and achievement for pupils attending different categories of Key Stage 4 schools. The focus then moves to GCSE science qualification routes in section 4.2, and the consequences of these options for subsequent biology, physics and chemistry A level outcomes; the link between different school types and the GCSE science routes offered is also covered. Section 4.3 then proceeds to analyse the continuation and achievement differences between alternative categories of Key Stage 5 institution. Finally, section 4.4 reports on the proportions of schools offering maths and science A levels for each Key Stage 5 institution type.

The analyses in this chapter cover all pupils in England with the relevant valid attainment records available from the National Pupil Database; this includes independent schools and FE sector institutions as well as maintained mainstream schools. Grammar schools, maths and computing specialist schools (MC), and science specialist schools (SS) are really subsets of the maintained mainstream (MM) group, but within the analyses here they are separated off from the rest of the MM sector as distinct groups. Where a school is both selective and specialist (MC or SS), they are counted as grammar schools and not as specialist schools. Special (SEN) schools do not appear in the analyses as a category because they account for only a tiny fraction of A level candidates, which is too small for meaningful analysis.

In sections 4.1 to 4.3 , figures are given separately for pupils with high prior subject attainment at GCSE, and for "all pupils" covering the complete range of prior attainment.

\subsection{GCSE School Types}

In this section, for each subject, rates of continuation to $A$ level, the proportion of $A$ level entries accounted for, and the rates of achieving grades $A$ or $B$, are presented for different types of Key Stage 4 school. The first chart for each subject reports the incidence of A level entries in that subject; the second chart takes the total number of A level entries in the subject and apportions it to the school types (placement); the third chart shows subsequent A level achievement, for all entrants and for high prior attaining entrants, by each GCSE school type.

Figure 4.1a shows that the highest rate of continuation to A level maths was for pupils who attended grammar schools for their GCSEs (34\%); independent school pupils are the next most likely to take maths A level, at 24\%. Eighteen percent of pupils at maths and computing specialist schools go on to take A level maths, compared with just $6 \%$ of other maintained mainstream schools.

These patterns are largely driven by prior attainment levels in GCSE maths for the various school types, as can be seen by examining the right-hand set of columns in the chart. Here, only pupils with the highest prior attainment in maths are included (those who achieved grade $A^{*}$ or A at GCSE), and the percentage point differences 
between school types become much smaller. For this subset of pupils, the highest continuation rate for A level maths is for maths and computing specialist schools, with over half $(53 \%)$ of their high attainers going on to take maths A level. This is followed closely by grammar schools with $50 \%$ maths continuation amongst high attainers in the subject, then the remaining maintained mainstream schools at $46 \%$, and finally the independent schools at $43 \%$.

Even within the high prior attaining subsets of pupils, the overall attainment profile of the pupils is likely to differ by school type, meaning that the school type itself may not be the factor driving the differences. For example, pupils in grammar and independent schools are likely to have stronger GCSE results across a range of subjects, giving them more options to choose from at A level. Also, whereas a grade A may be considered outstanding in many schools, it may be merely average at a selective or independent school, where an $A^{*}$, or even a high score within the $A^{*}$ range may be necessary to distinguish a pupil as one of the most able in the year group.

For these reasons, in Chapter 6 a range of factors including school types are modelled simultaneously, to identify their individual contributions to A level continuation in maths and science subjects. Nevertheless, it is still informative to examine the raw patterns of continuation and achievement in full, which this section proceeds to do.

\section{Fig. 4.1a}

Continuation to A level Maths by KS4 School Type

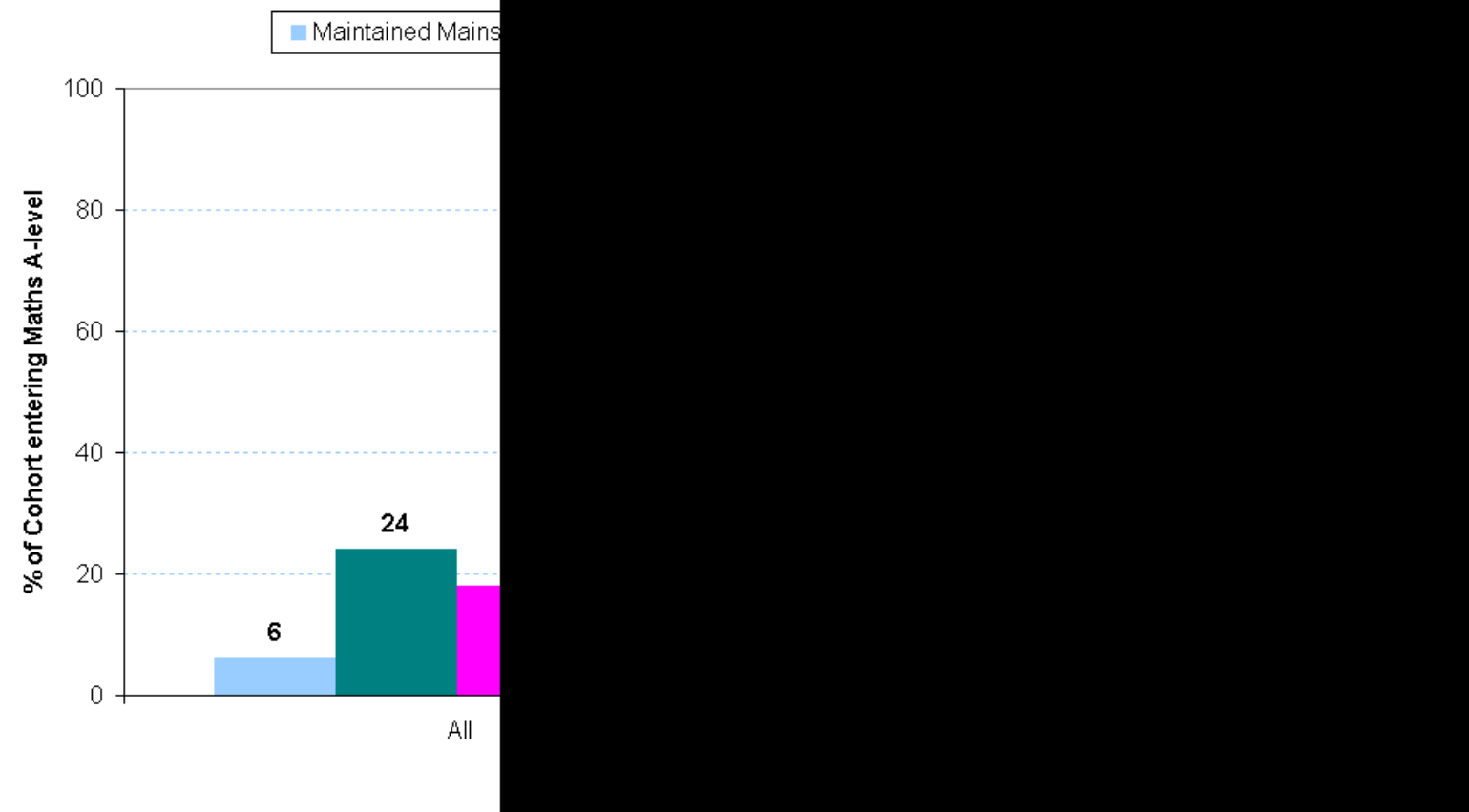

Figure 4.1b takes the same A level maths entries that were examined as percentages of the Key Stage 4 cohorts for each school type in figure $4.1 \mathrm{a}$, and looks at what percentage of the entries were for pupils who had attended each school type at GCSE. Despite having the lowest rate of continuation to A level maths, maintained mainstream schools nevertheless supply the majority (61\%) of A level 
maths entrants, because they dominate GCSE provision with the largest number of pupils passing through their doors. A further $21 \%$ attended independent schools at GCSE, 14\% were at grammar schools, and just 4\% came from maths and computing specialist schools.

Fig. 4.1b

Maths A level Entries in 2009 by Pupils from the 2007 KS4 Cohort

Grammar, $7,600,14 \%$

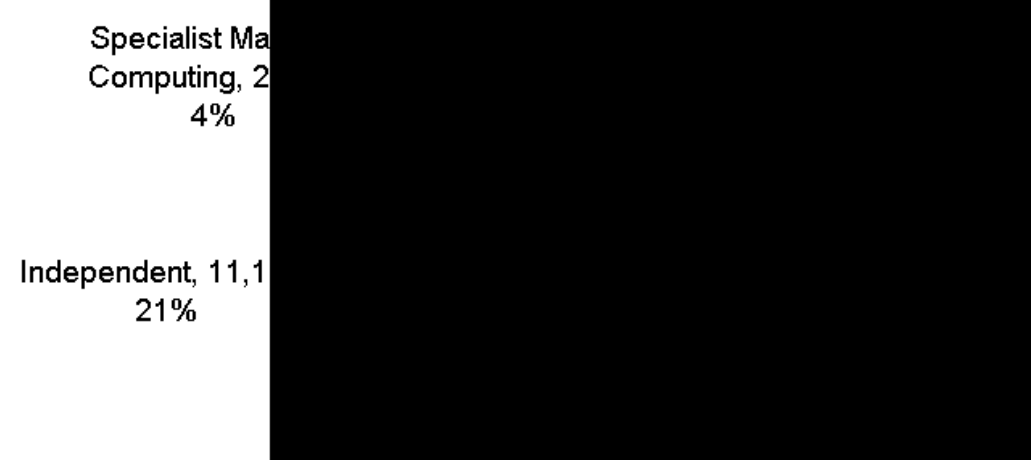


Figure 4.1c presents the achievement rates for top grades in A level maths by Key Stage 4 school type. Pupils who attended grammar or independent schools for their GCSEs were more likely to secure grades A or B at maths A level than their peers from other school types. For both maintained mainstream and maths and computing specialist schools, $61 \%$ of pupils who went on to take maths A level achieved either an A grade or a B grade; this compares with $82 \%$ of entrants from independent schools (at GCSE), and 78\% of entrants who came from grammar schools. The same pattern recurs amongst the subset of entrants with high prior attainment in maths (grades $A^{*}$ or A at GCSE), except with slightly smaller achievement deficits for pupils from maintained mainstream and specialist maths and computing schools.

\section{Fig. 4.1c}

Achievement in A level Maths by KS4 School Type

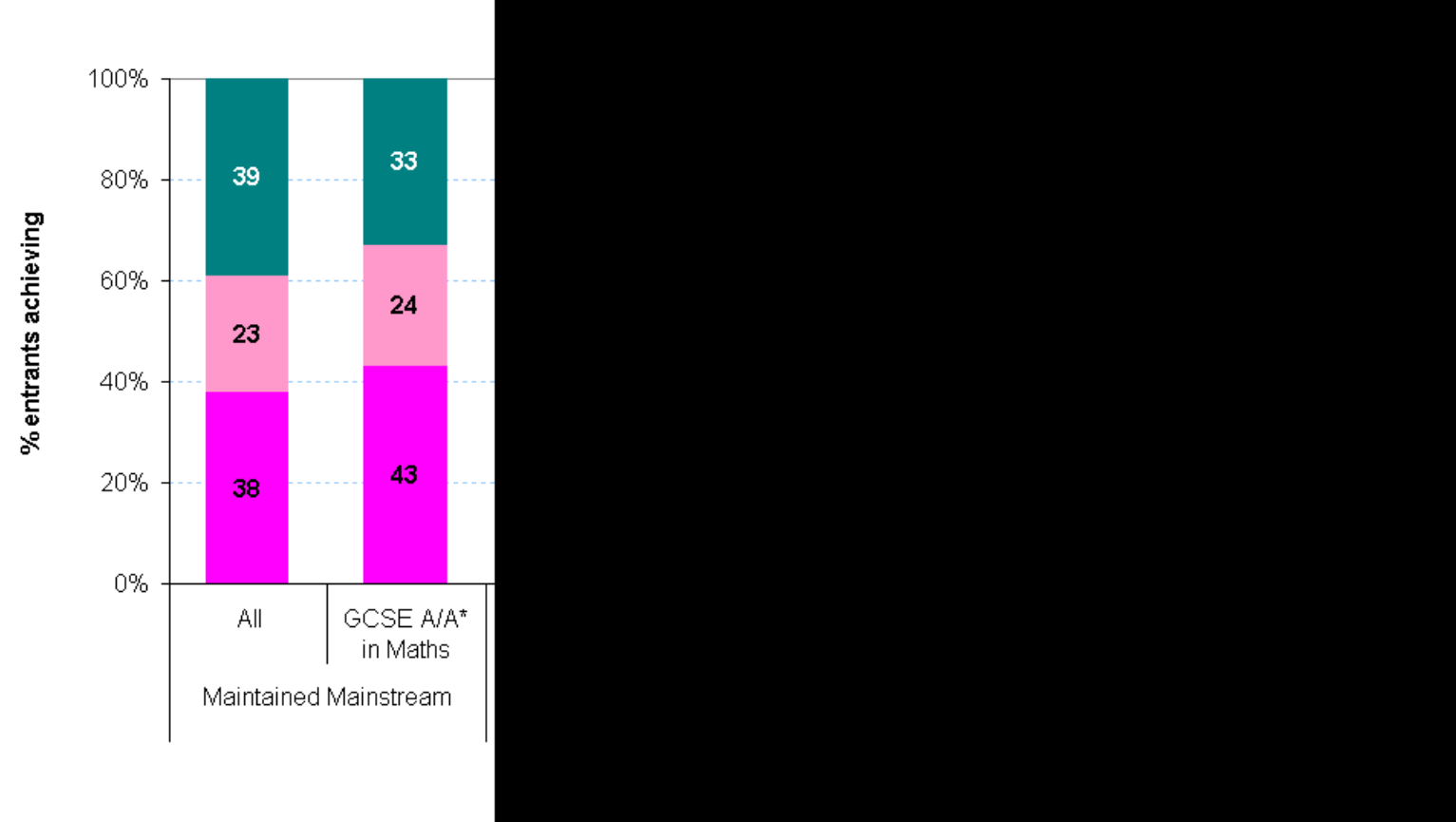

Figure 4.1d shows that the highest rate of continuation to A level biology was again for pupils who attended grammar schools for their GCSEs (21\%); independent school pupils are the next most likely to take biology A level, at 17\%. Seven percent of pupils at science specialist schools go on to take A level biology, just ahead of other maintained mainstream schools with $5 \%$ biology continuation.

The right-hand set of columns indicates that these patterns are again being driven by prior attainment differences between the school types. Only pupils with the highest prior attainment in biology or double science are included here (those who achieved grade $A^{*}$ or A at GCSE), and the percentage point differences between school types are a lot smaller.

For this subset of pupils, the school type with highest continuation rate for A level biology remains the grammar schools, with $35 \%$ of their high attainers going on to take biology at A level. This is followed closely by science specialist schools with $33 \%$ biology continuation amongst high attainers in the subject, and the other maintained mainstream schools with $32 \%$. Independent schools have $31 \%$ biology 
continuation amongst their high prior attainers in the subject.

Fig. 4.1d

\section{Continuation to A level Biology by KS4 School Type}

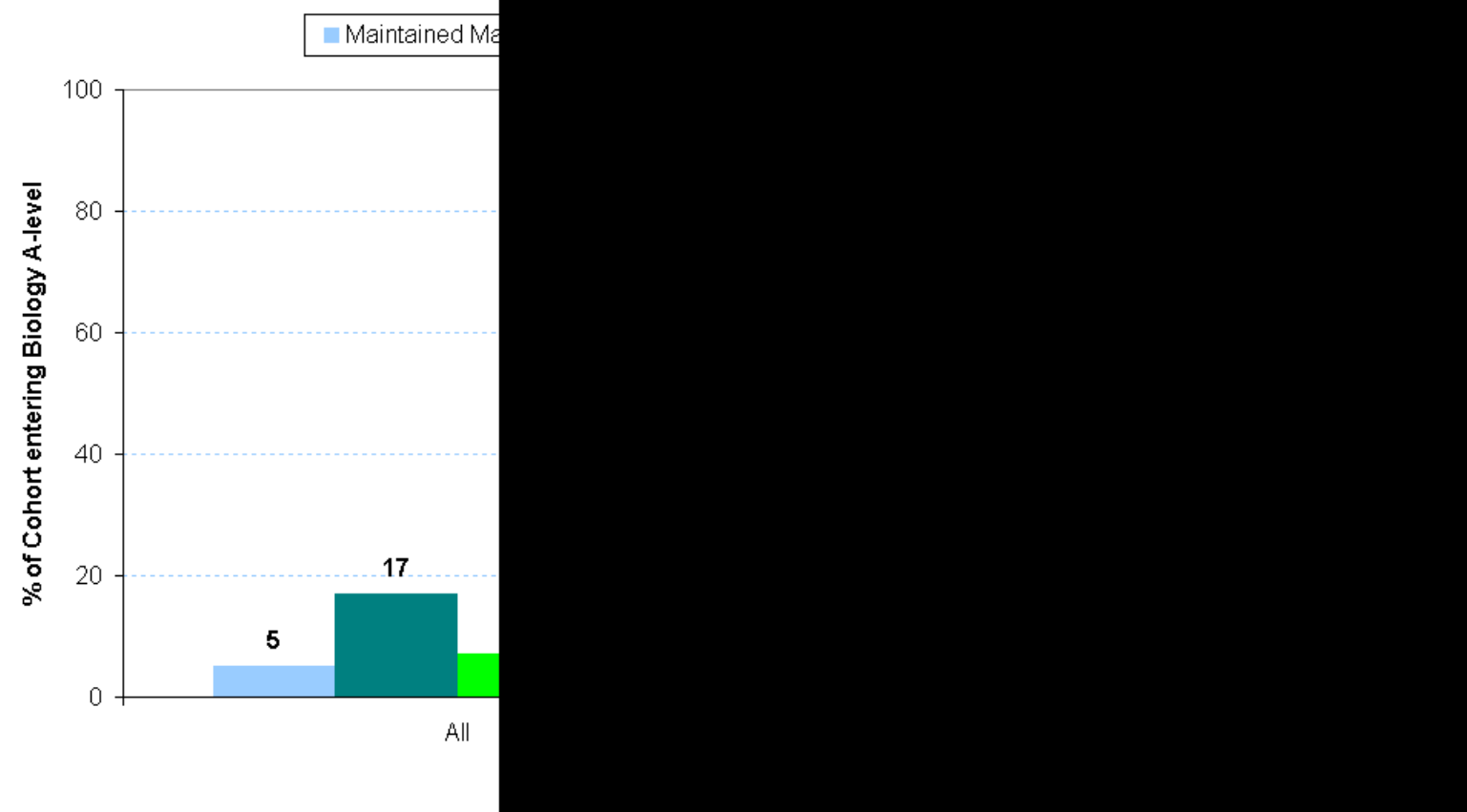


Figure 4.1e takes the same A level biology entries that were examined as percentages of the Key Stage 4 cohorts for each school type in figure $4.1 \mathrm{~d}$, and looks at what percentage of the entries were for pupils who had attended each school type at GCSE. Despite having the lowest rate of continuation to A level biology, maintained mainstream schools nevertheless supply the majority (59\%) of A level biology entrants, as they are responsible for the largest numbers of GCSE entrants. A further 20\% attended independent schools at GCSE, 12\% were at grammar schools, and the remaining 9\% came from science specialist schools.

Fig. 4.1e

Biology A level Entries in 2009 by Pupils from the 2007 KS4 Cohort

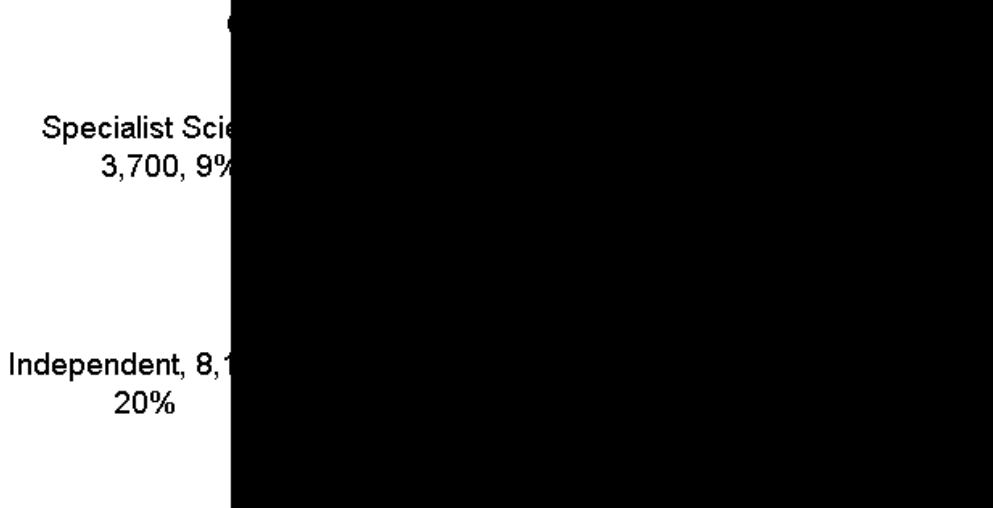


Figure $4.1 f$ presents the achievement rates for top grades in A level biology by Key Stage 4 school type. Biology entrants who attended independent schools or grammar schools for their GCSEs were more likely to secure grades A or B at biology $A$ level than their peers from other school types. For maintained mainstream schools, $45 \%$ of pupils who went on to take biology A level achieved either an $A$ grade or a B grade; this was slightly higher at $48 \%$ for biology entrants who came from science specialist schools at GCSE, $69 \%$ for those from selective grammar schools, and $71 \%$ for entrants who sat their GCSEs at independent schools. The same pattern recurs amongst the subset of entrants with high prior attainment in biology or double science (grades A* or A at GCSE), except with slightly smaller achievement deficits for pupils from maintained mainstream and science specialist schools.

\section{Fig. 4.1f}

\section{Achievement in A level Biology by KS4 School Type}

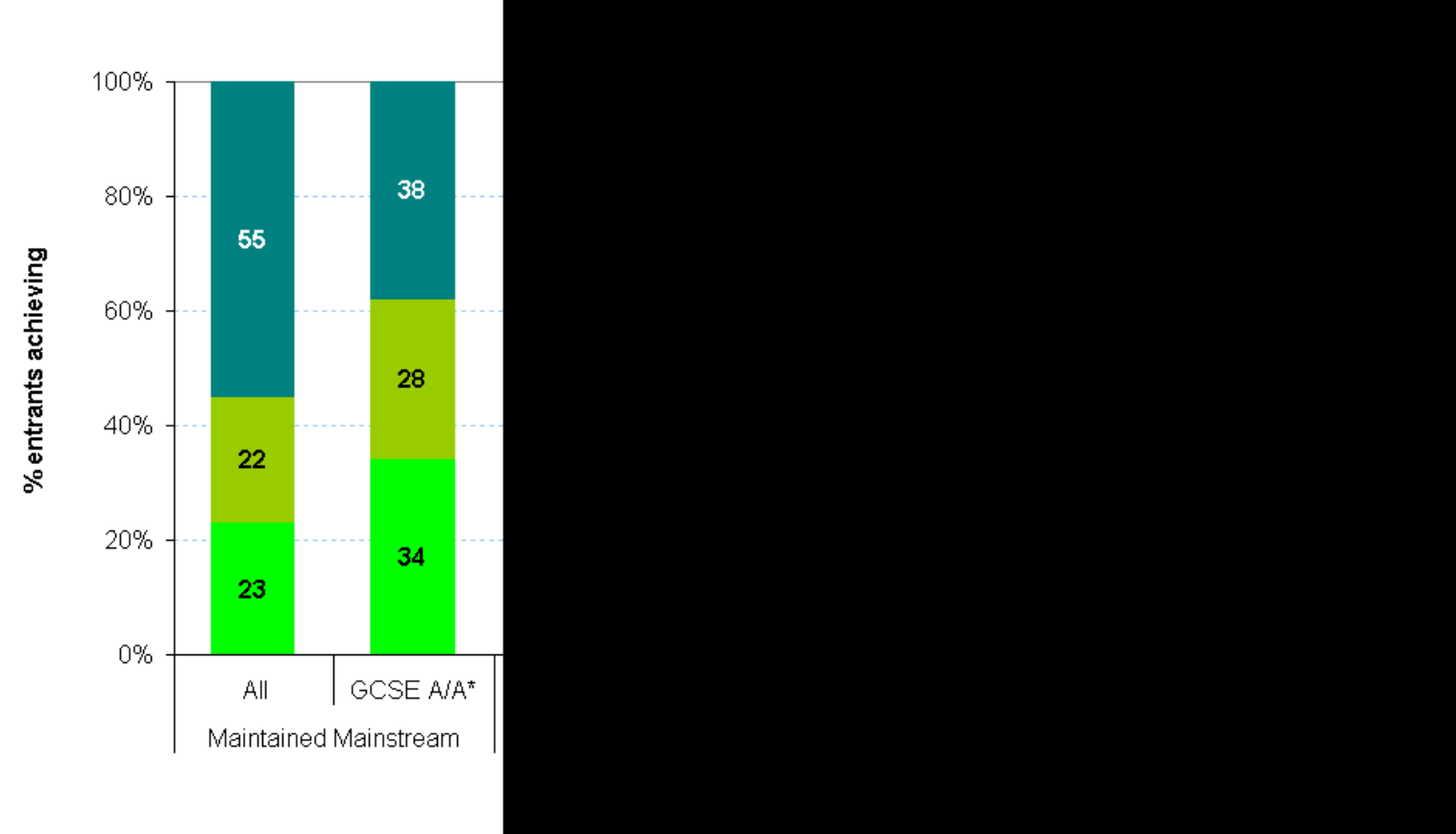

Figure 4.1g shows that the highest rate of continuation to A level chemistry, as with maths and biology, was for pupils who attended grammar schools for their GCSEs (18\%); independent school pupils are the next most likely to take chemistry A level, at $16 \%$. Chemistry continuation was considerably lower for pupils from science specialist schools at 5\%, and even less likely for those from the remaining maintained mainstream schools at $3 \%$.

The right-hand set of columns indicates that these patterns are again being driven by prior attainment differences between the school types. Only pupils with the highest prior attainment in chemistry or double science are included here (those who achieved grade $A^{*}$ or A at GCSE), and the percentage point differences between school types are much smaller.

For this subset of pupils, the school type with highest continuation rate for A level chemistry remains the grammar schools, with $33 \%$ of their high attainers going on to 
take chemistry at A level. This is followed closely by both science specialist schools and independent schools, with 30\% chemistry continuation amongst high attainers in the subject; the other maintained mainstream schools were a little further behind with $28 \%$ of their subject high attainers going on to take chemistry A level.

Fig. $4.1 \mathrm{~g}$

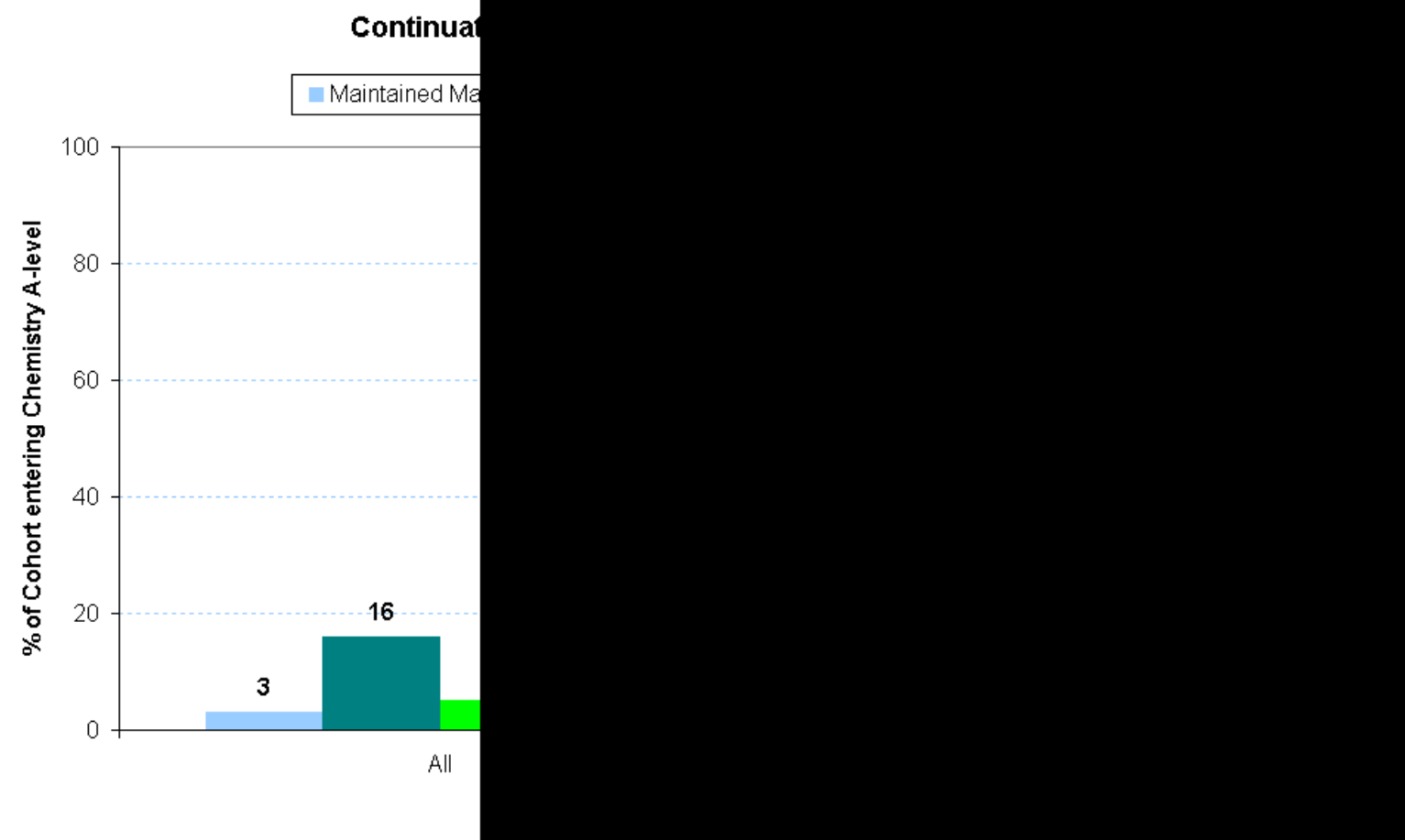


Figure 4.1h takes the same A level chemistry entries that were examined as percentages of the Key Stage 4 cohorts for each school type in figure $4.1 \mathrm{~g}$, and looks at what percentage of the entries were for pupils who had attended each school type at GCSE. Maintained mainstream schools supply more than half (55\%) of A level chemistry entrants even though they have the lowest continuation rate, because they are responsible for the largest numbers of GCSE entrants. A further $23 \%$ attended independent schools at GCSE, 13\% were at grammar schools, and the remaining 9\% came from science specialist schools.

\section{Fig. 4.1h}

Chemistry A level Entries in 2009 by Pupils from the 2007 KS4 Cohort

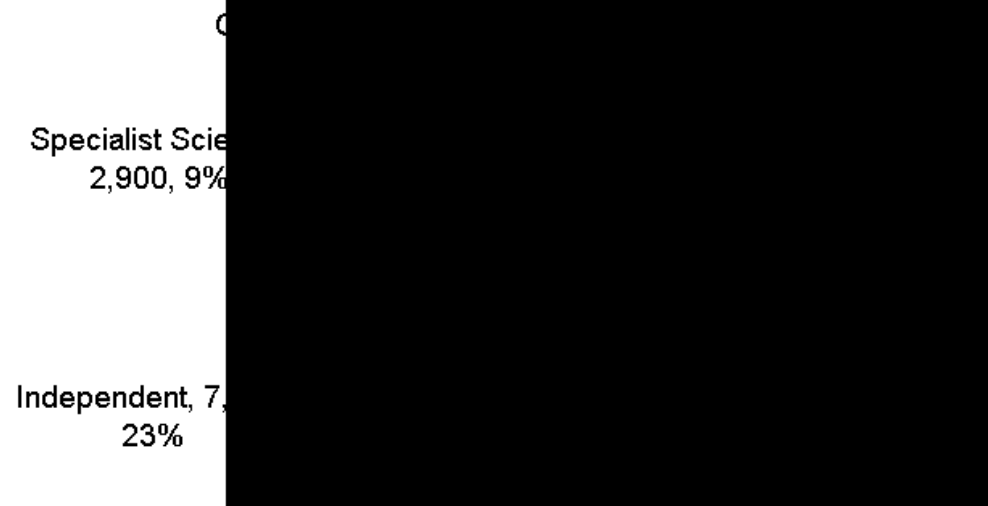


Figure 4.1i presents the achievement rates for top grades in A level chemistry by Key Stage 4 school type. Entrants who attended independent schools or grammar schools for their GCSEs were more likely to secure grades A or B at chemistry A level than their peers from other school types. For both science specialist schools and other maintained mainstream schools, $53 \%$ of pupils who went on to take chemistry A level achieved either an A grade or a B grade; achievement of top grades was stronger at $73 \%$ for chemistry entrants who came from grammar schools at GCSE, and highest for entrants from independent schools at $77 \%$.

The pattern was similar for chemistry entrants with high prior attainment in GCSE chemistry or double science (grades $A^{*}$ or A), except that the differences between school types were smaller, and achievement of top A level chemistry grades was a little higher for entrants from science specialist schools than for those who came from other maintained mainstream schools.

\section{Fig. 4.1i}

Achievement in A level Chemistry by KS4 School Type

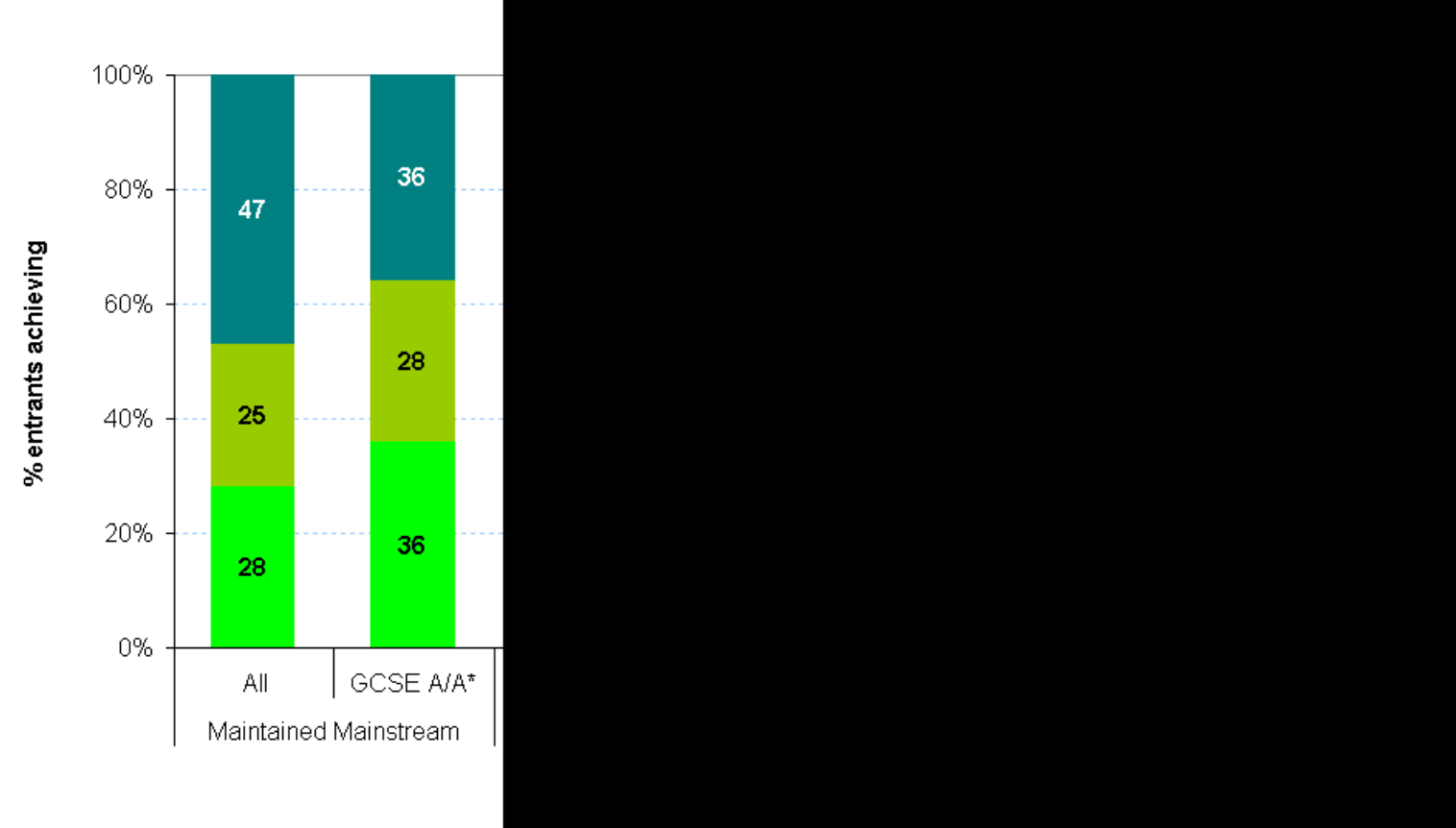


Figure 4.1j shows that the highest rate of continuation to A level physics, as with the other maths and science subjects, was for pupils who attended grammar schools for their GCSEs (13\%); independent school pupils are the next most likely to take physics A level, at $11 \%$. Chemistry continuation was considerably lower for pupils from science specialist schools at $4 \%$, and even less likely for those from the remaining maintained mainstream schools at $2 \%$.

The right-hand set of columns indicates that these patterns are again being driven by prior attainment differences between the school types. Only pupils with the highest prior attainment in physics or double science are included here (those who achieved grade $A^{*}$ or A at GCSE), and the percentage point differences between school types are much smaller.

For this subset of pupils, the school type with highest continuation rate for A level physics remains the grammar schools, with $22 \%$ of their high attainers going on to take chemistry at A level. This is followed closely by science specialist schools with $20 \%$ physics continuation amongst high attainers in the subject, then independent schools with $19 \%$ continuation, and maintained mainstream schools at $18 \%$ of high prior attainers in chemistry / double science.

\section{Fig. 4.1j}

Continuation from GCSE to A-level Physics by KS4 school type, Number of Pupils

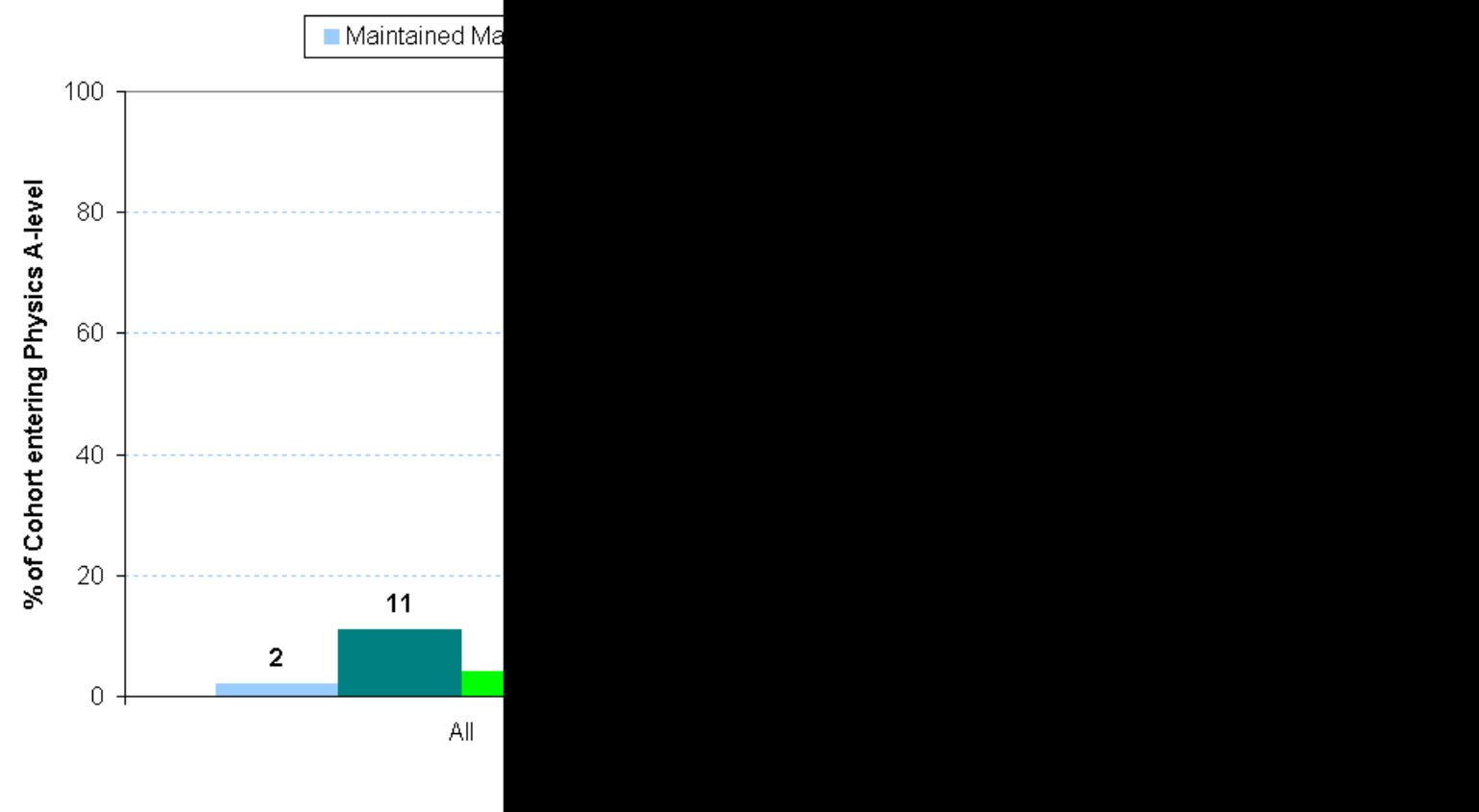


Figure 4.1k takes the same A level physics entries that were examined as percentages of the Key Stage 4 cohorts for each school type in figure $4.1 \mathrm{~g}$, and looks at what percentage of the entries were for pupils who had attended each school type at GCSE. As with maths, biology and chemistry, maintained mainstream schools supply more than half (56\%) of A level physics entrants even though they have the lowest continuation rate, because they are responsible for the largest numbers of GCSE entrants. A further 22\% attended independent schools at GCSE, $13 \%$ were at grammar schools, and the remaining $9 \%$ came from science specialist schools.

\section{Fig. 4.1k}

Physics A level Entries in 2009 by Pupils from the 2007 KS4 Cohort

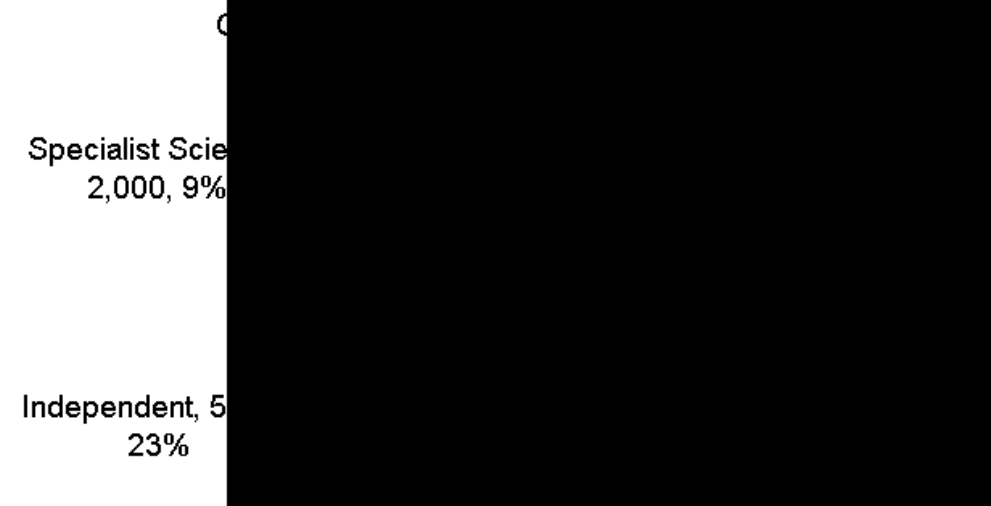


Figure 4.1I presents the achievement rates for top grades in A level physics by Key Stage 4 school type. Entrants who attended independent schools or grammar schools for their GCSEs were more likely to secure grades A or B at physics A level than their peers from other school types. For maintained mainstream schools, $47 \%$ of pupils who went on to take physics A level achieved either an A grade or a B grade; this was higher for entrants who came from science specialist schools at GCSE, $51 \%$ of whom achieved top grades. Achievement of grades A or B was stronger still at $67 \%$ for physics entrants who came from grammar schools at GCSE, and highest of all for entrants from independent schools at $76 \%$.

The pattern was the same for physics entrants with high prior attainment in GCSE physics or double science (grades $A^{*}$ or $A$ ), except with the smaller differences between school types that were also seen for the high prior attaining subsets in maths, biology and chemistry.

\section{Fig. 4.1I}

Achievement in A level Physics by KS4 School Type

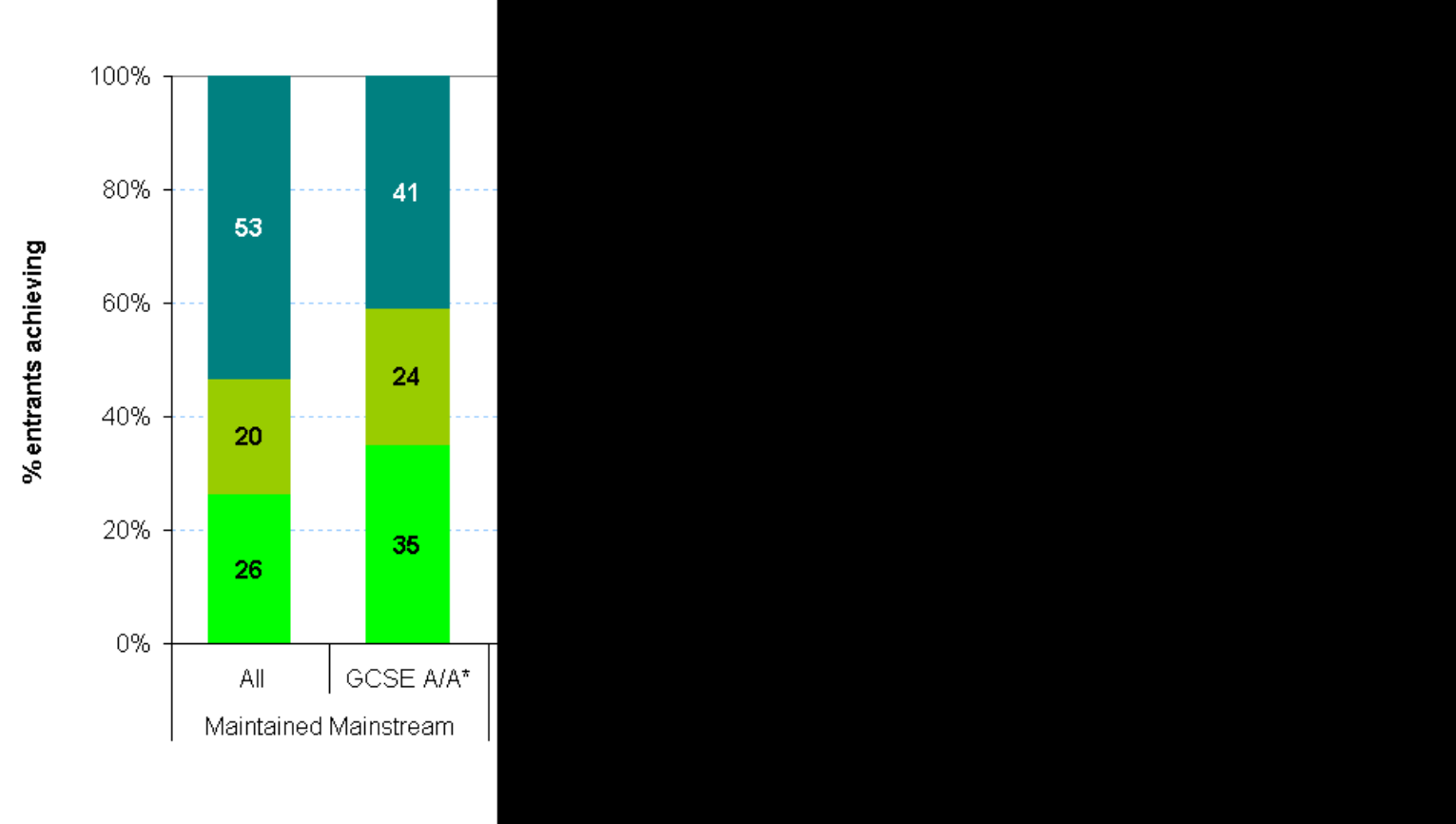

\subsection{GCSE Qualification Routes: The Separate Sciences Advantage}

In addition to the variation in science outcomes that exists between different types of Key Stage 4 school described in section 4.1 above, a further source of differences at this stage is associated with the type of GCSE science, or GCSE science route, taken by pupils. The two most prevalent Key Stage 4 science routes, up to 2007, were GCSE double science, and separate GCSE sciences in biology, chemistry and physics. From 2008, single and double science GCSEs were replaced by core and additional science GCSEs as the most common GCSE science route. Pupils taking core science receive one GCSE, equivalent to the old single science qualification; for most of these pupils this is then followed up with a second GCSE in additional science, equivalent to the old double science award. 
Figure 4.2a presents the 2009 uptake percentages for GCSE triple science (entering all three separate science GCSEs) for various groups of pupils. The level of uptake is not uniform across pupils with different characteristics, or attending different types of school at Key Stage 4. Those attending maintained mainstream schools that are not selective and not science specialist schools are less likely to take triple science, as are girls, pupils with FSM, and pupils of either White or Black ethnic backgrounds.

\section{Fig. $4.2 \mathrm{a}$}

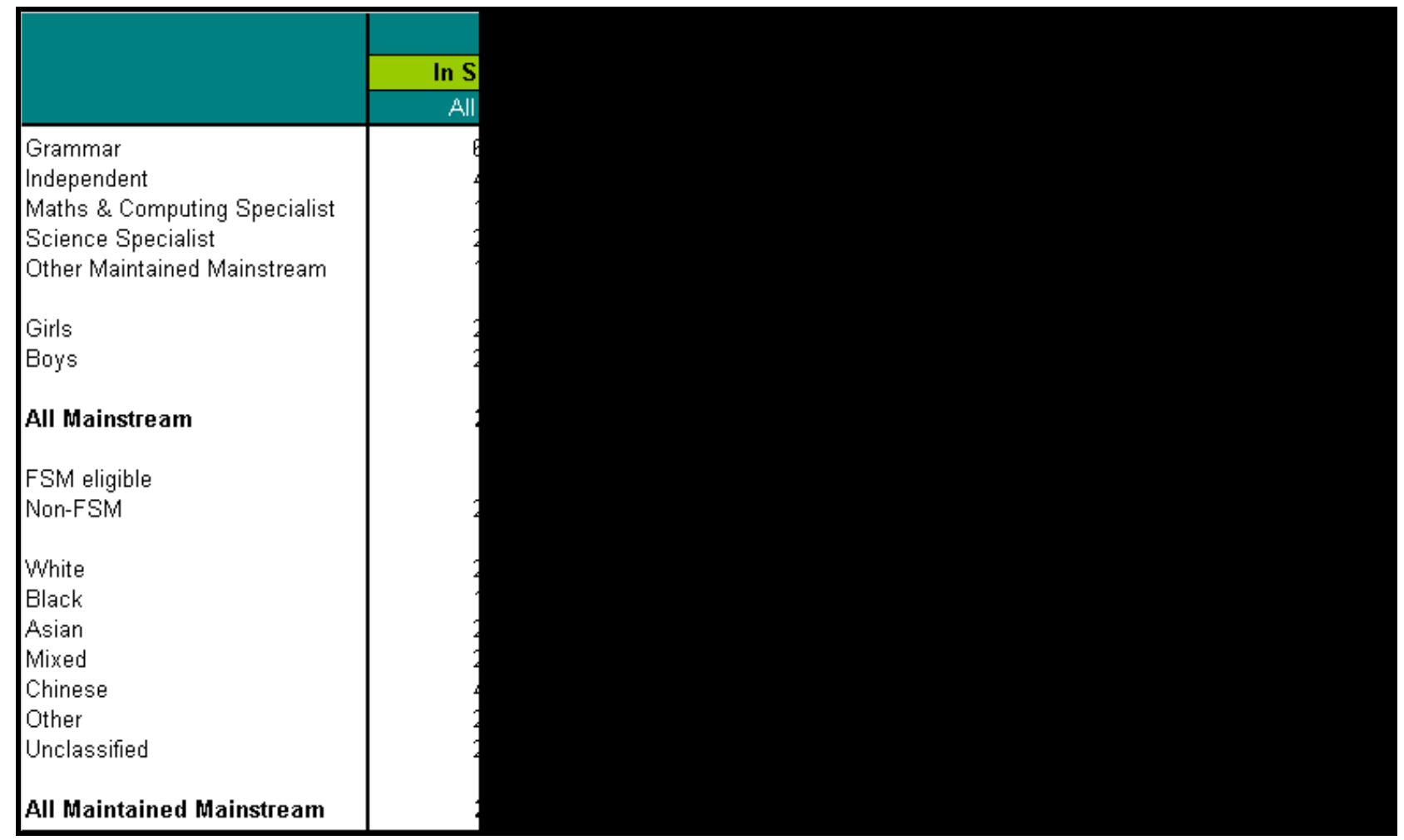

Pupils in the 2009 A level cohort predominantly sat their GCSEs in 2007, and will therefore have generally taken either GCSE double science or the three separate sciences. Figure $4.2 \mathrm{~b}$ depicts the A level continuation rates for this cohort for biology, chemistry and physics. It can be seen that for all three A level subjects, separate science GCSEs increased the chances of taking A level qualifications compared with double science GCSEs.

Pupils across the complete range of prior ability within the separate sciences group generally had similar rates of continuation to the high attaining subset from the double science group. It should be noted that separate science entrants have a higher distribution of prior attainment than double science entrants which will partly account for this (see section 1.6); Chapter 6 goes on to model these factors simultaneously to disentangle the separate effects.

Biology continuation stood at $24 \%$ for pupils who took biology GCSE compared with $5 \%$ for those who took double science GCSE; this was $23 \%$ for chemistry (separate GCSE) compared with $4 \%$ for double science entrants; for physics, the separate science GCSE entrants had 17\% continuation compared with $3 \%$ for double science 
entrants.

Fig. 4.2b

A level Continuation by GCSE Science Route and Prior Attainment (2009)

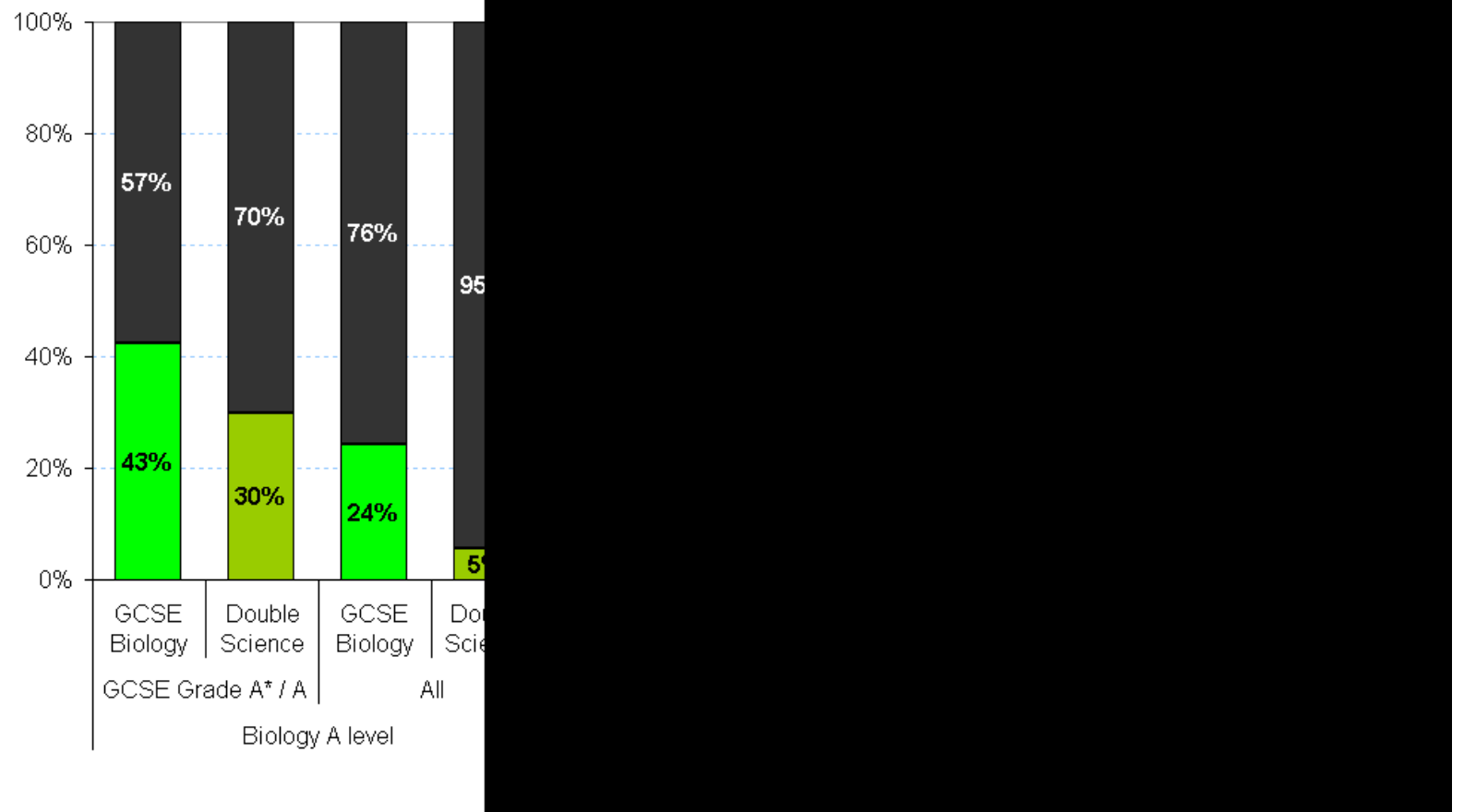

Considering A level science achievement and GCSE science routes, figure 4.2c presents the rates of achievement of grades $A$ and $B$ for entrants with separate science and double science GCSEs. A similar pattern of advantage for separate science GCSE holders appears for A level achievement in biology, chemistry and physics. In chemistry and physics, on average, all separate science entrants are more likely to continue to A level than the high attaining set for GCSE double science. 
For pupils with any GCSE grade, $60 \%$ of biology A level entrants who took a separate biology GCSE were awarded either an A grade or a B grade at A level, compared with $45 \%$ of entrants with GCSE double science. The rates of achievement for grades A or B in A level chemistry were $64 \%$ for separate chemistry GCSE holders, compared with $52 \%$ of double science GCSE holders. For physics $A$ level, $61 \%$ of entrants with a separate physics GCSE went on to gain grades A or B at A level, compared with $45 \%$ of entrants with double science GCSEs.

\section{Fig. 4.2c}

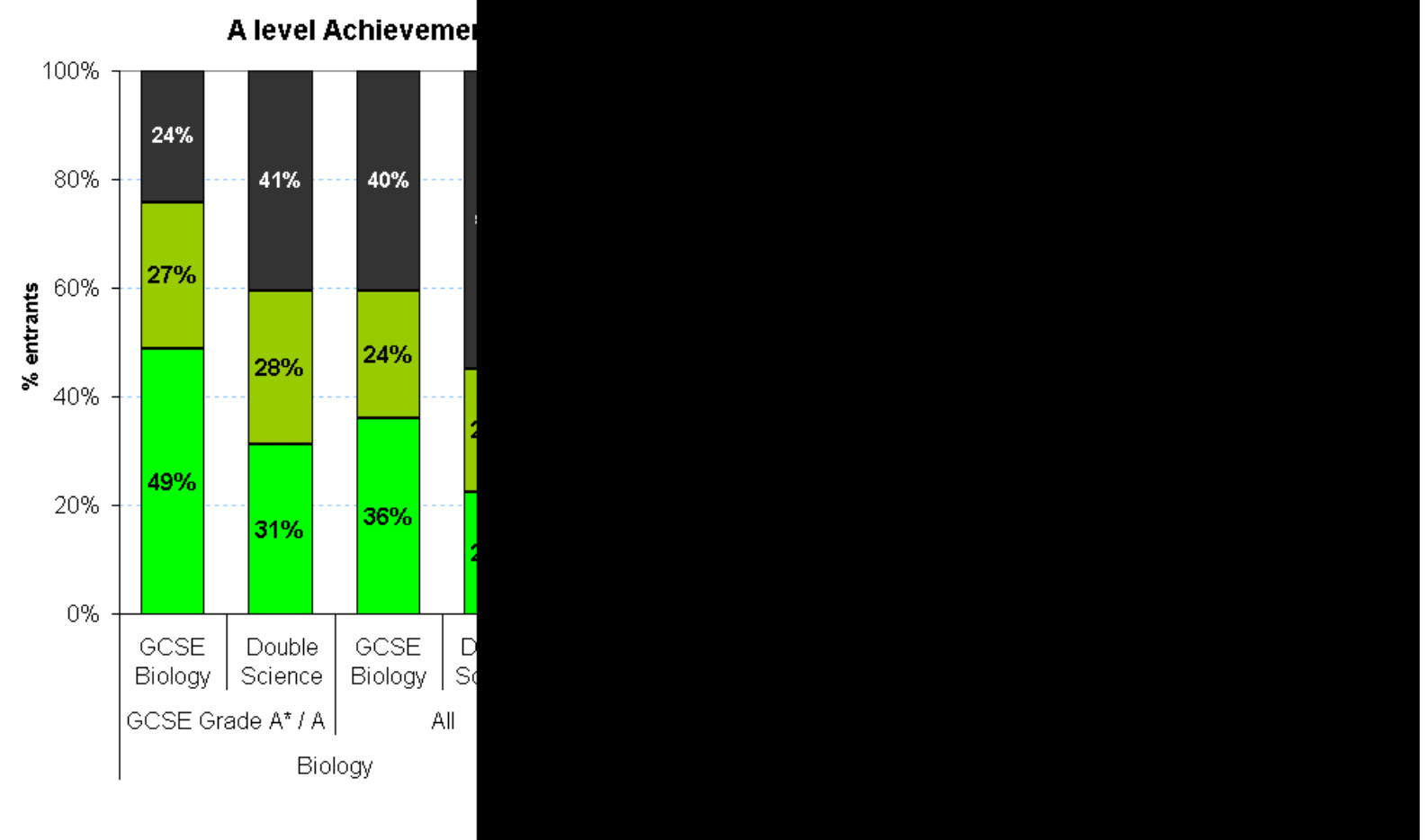

Because the three science subjects have different underlying levels of continuation and achievement, and to compare the high prior attainers with all pupils for each GCSE science route, figure $4.2 d$ uses odds ratios to standardise the percentage outcome measures across these groups. Odds ratios on continuation are similar to those on achieving top A level grades for those pupils with high prior GCSE grades ( $A^{*}$ or $\left.A\right)$, but much larger for the achievement measure than continuation when considering pupils across all grades at GCSE.

The diamond shaped green odds ratio points reveal that biology is the science subject whose continuation levels are least affected by the choice of GCSE science route (OR $=1.8$ for all pupils, and 6.0 for high attainers). The most affected subject for all pupils is chemistry, but the GCSE science route makes more difference to physics continuation amongst those pupils achieving high grades at GCSE, who are the most suited to A level studies. GCSE separate science entrants achieving grades $A^{*}$ or $A$ have 2.6 times the odds of double science entrants with equivalent grades for continuing to A level in chemistry (7.2 times the odds in the case of high GCSE attainers), and 2.7 times the odds of double science high attainers for taking A level physics (6.6 times the odds in the case of high GCSE attainers).

The square blue odds ratio points plot the relationship between GCSE science routes 
and achievement of grades A or B in A level science subjects. Here the differences between separate and double science GCSEs are slightly larger amongst those pupils with high GCSE grades than across the complete GCSE grade range. The odds ratios are broadly similar for all three subjects, and much more similar for high prior attainers compared with all pupils than was the case for A level uptake.

A level entrants across the GCSE grade range had 1.8 times the odds of achieving grade A or B in A level biology of an entrant with GCSE double science, if they took GCSE biology as a separate science instead (2.2 times the odds in the case of high GCSE attainers). This was slightly lower for A level chemistry where entrants with GCSE separate chemistry had 1.6 times the odds of those with GCSE double science of achieving a top A level grade (2.0 times the odds in the case of high GCSE attainers). GCSE science routes made most difference to achievement of grade A or B in A level physics, where the odds were 1.9 times those of entrants with GCSE double science for entrants with separate GCSE physics (or 2.1 times the odds in the case of GCSE high attainers).

\section{Fig.4.2d}

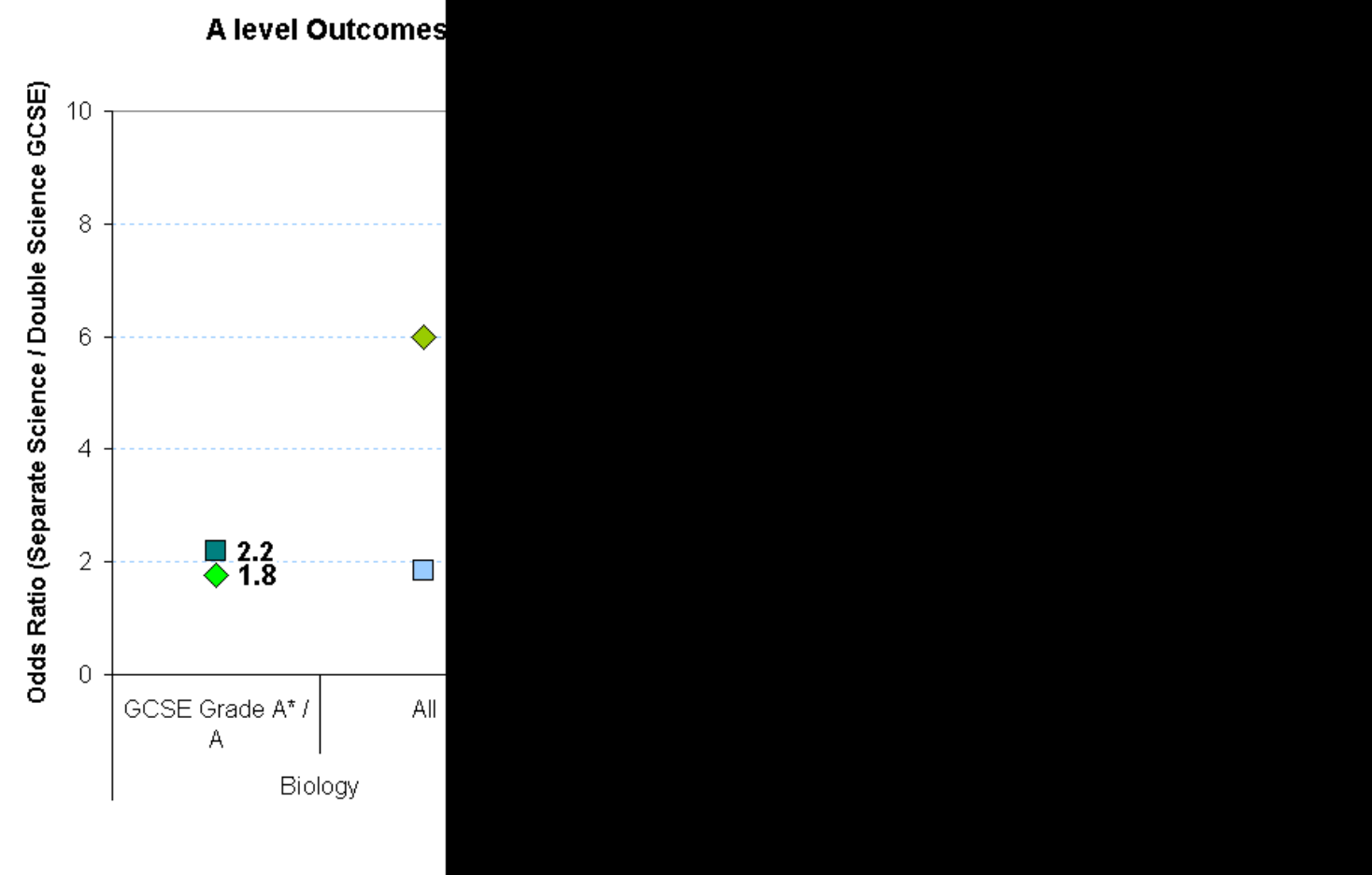

Figure 4.2e now considers the placement of A level science entrants and those achieving top grades across the different GCSE science routes that they came through from, and whether the pupil achieved a top GCSE grade in that route $\left(A^{*}\right.$ or A). Approximately half of $A$ level entries in each science subject are made by pupils who took double science at GCSE and achieved grades $A^{*}$ or $A$. Another fifth of biology entrants had achieved grades below A at GCSE double science, but closer to one tenth of chemistry and physics entrants were from this group.

Another fifth of biology entrants achieved top grades at GCSE separate biology, compared with nearer to one third of chemistry and physics entrants who held grades $A^{*}$ or $A$ in their respective separate science GCSEs. The remaining tenth of biology 
entrants held grades below A in separate biology at GCSE, with a slightly smaller fraction than this from the separate science at below A grade groups amongst $A$ level chemistry and physics entrants.

Separate science GCSE holders were more prominent amongst the group of A level sciences entrants who went on to achieve grades A or B at A level; they made up just over one third of biology grades $A$ or $B$, four tenths of chemistry grades $A$ or $B$, and almost half of physics grades $A$ or $B$. Entrants with grades below $A$ at the separate GCSE sciences made up only a tiny portion of these fractions. To summarise the analysis in another way, for the three sciences, $A$ level entrants with grades $A^{*}$ or $A$ at GCSE were overrepresented in the group of candidates who went on to achieve grades A or B at A level - but they were more overrepresented if their GCSE $A^{*}$ or A grade was in separate sciences at GCSE, than if it was in GCSE double science. 
Fig. 4.2e

A level Biology Entries

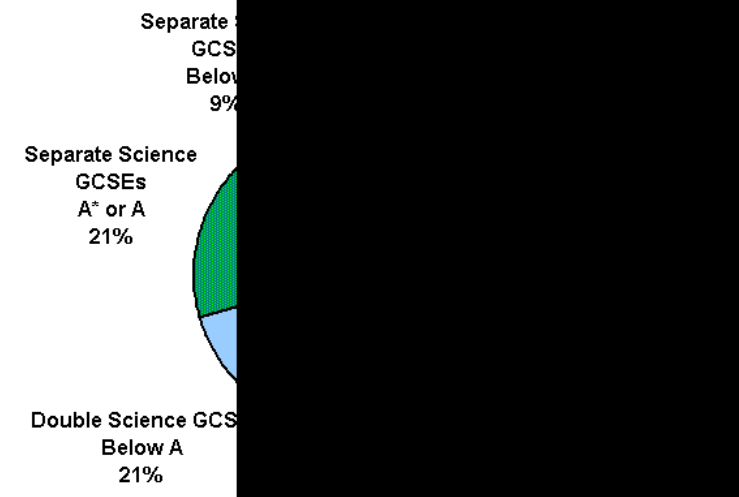

A level Biology Grade A/B

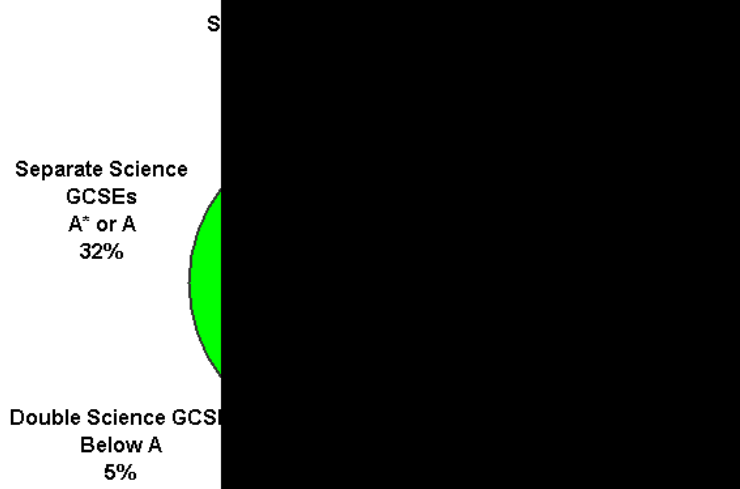

A level Chemistry Entries

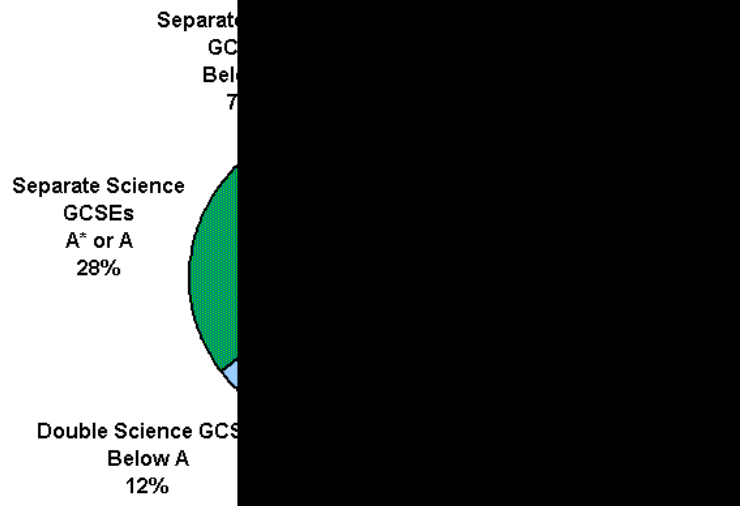

A level Chemistry Grade A/B

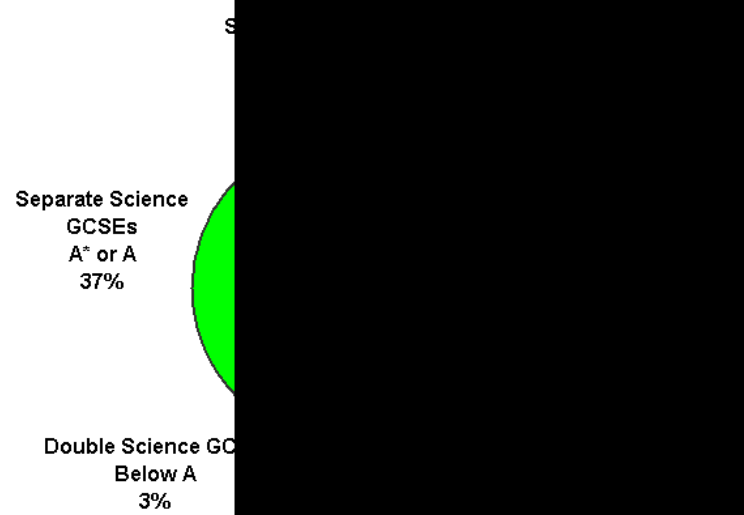

A level Physics Entries

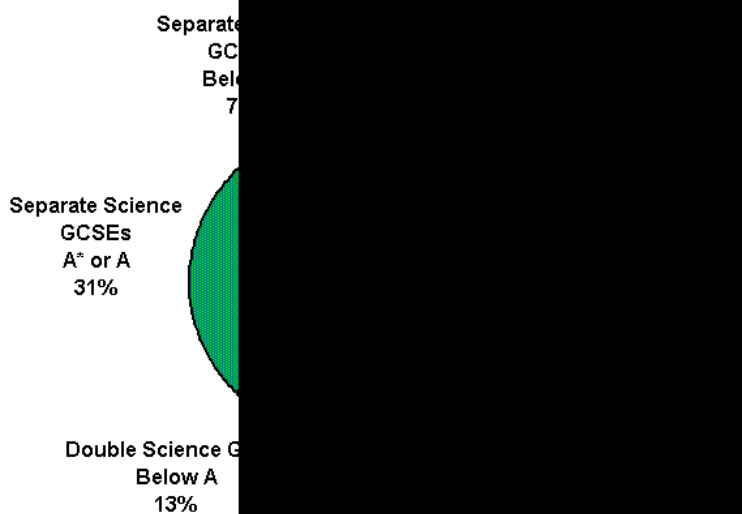

A level Physics Grade A/B

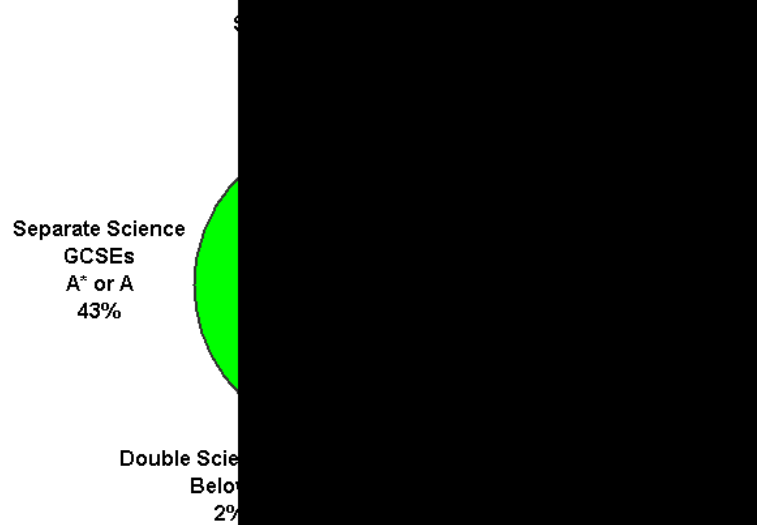

Moving forward to the 2010 A level results brings us into the first cohort who generally sat either core and additional GCSE science, or separate GCSE sciences in 2008 . Figure $4.2 \mathrm{f}$ repeats the analysis in figure $4.2 \mathrm{a}$, to test for whether the same 
relationships exist for core and additional science as were seen for double science.

As with double science A level continuation in 2009, pupils across the complete range of prior ability within the separate sciences group generally had similar rates of continuation to the high attaining subset from the double additional science group in 2010. It should be noted that separate science entrants have a higher distribution of prior attainment than additional science entrants which will partly account for this (see section 1.6); chapter 6 goes on to model these factors simultaneously to disentangle the separate effects.

Biology continuation stood at $25 \%$ for pupils who took biology GCSE compared with $7 \%$ for those who took double science GCSE; this was $24 \%$ for chemistry (separate GCSE) compared with $4 \%$ for double science entrants; for physics, the separate science GCSE entrants had $17 \%$ continuation compared with $3 \%$ for double science entrants. These were similar proportions to those seen in 2009 for the separate and double science comparison.

\section{Fig. 4.2f}

A level Continuation by GCSE Science Route and Prior Attainment (2010)

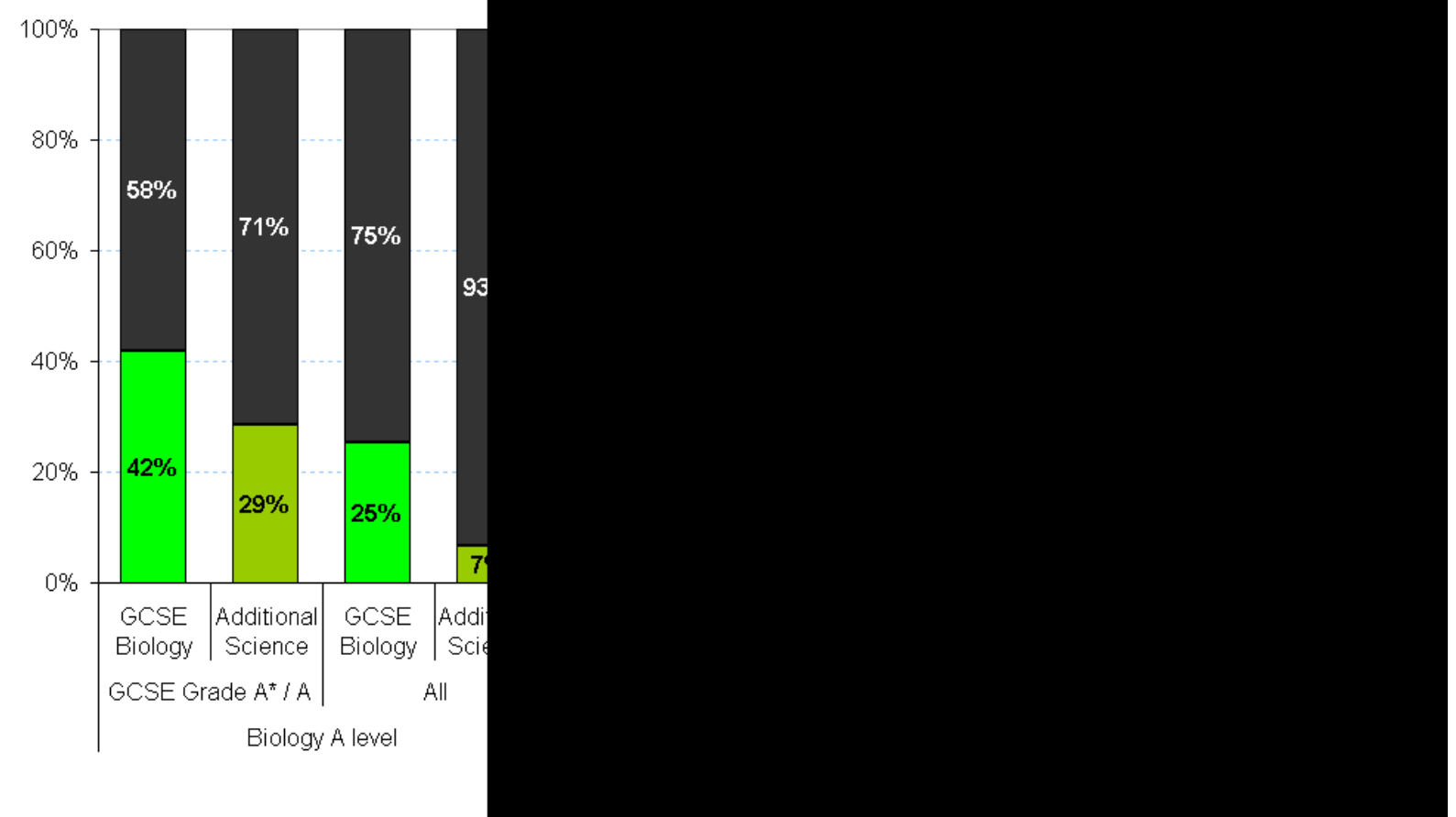

Moving on to A level achievement for core and additional GCSE takers, figure $4.2 \mathrm{~g}$ charts the equivalent percentages of entrants achieving grades $A$ and $B$ that appeared in figure $4.2 \mathrm{c}$ for double science entrants. This is with the addition of a new data series for $A^{*}$ grades which were introduced in 2010 , splitting the A grade category into higher and lower factions.

It's important to bear in mind, when comparing these relationships over time, that there is an ongoing shift in the proportion of GCSE entrants taking GCSE science subjects away from the combined GCSE science routes (double science or core and additional science), and towards the separate science GCSEs in biology, chemistry and physics. See section 1.5 where this is described in detail. The consequence for 
the analyses of achievement at A level by GCSE route of this compositional shift are that it is possible for achievement rates for both separate and combined science routes to decrease from year to year when in fact overall they may have increased for the two GCSE routes combined.

This is because the GCSE candidates with the strongest potential from the combined science group are moving into the bottom end of the range of potential for separate science GCSEs; with the top end of combined sciences denuded and the bottom end of separate sciences expanded, both groups are subject to downward pressure on their achievement measures. But because candidates are shifting from a group with lower achievement into a group with higher achievement, overall the combined rate may still have increased while the two component groups saw decreases.

Keeping this compositional shift in mind where the rates have dropped slightly since 2009 , figure $4.2 \mathrm{~g}$ presents the relationships between core and additional science and A level science achievements. A similar pattern of advantage for separate science GCSE holders appears for A level achievement in biology, chemistry and physics compared with additional science, as was the case comparing with double science for 2009. In all three subjects, on average, all separate science entrants are more likely to continue to A level than the high attaining set for GCSE double science.

For pupils with any GCSE grade, $59 \%$ of biology A level entrants who took a separate biology GCSE were awarded either grade $A^{*}-B$ at $A$ level, compared with $42 \%$ of entrants with GCSE additional science. The rates of achievement for grades $A *-B$ in A level chemistry were $63 \%$ for separate chemistry GCSE holders, compared with $51 \%$ of additional science GCSE holders. For physics A level, $60 \%$ of entrants with a separate physics GCSE went on to gain grades $A *-B$ at $A$ level, compared with $46 \%$ of entrants with additional science GCSEs.

\section{Fig. 4.2g}

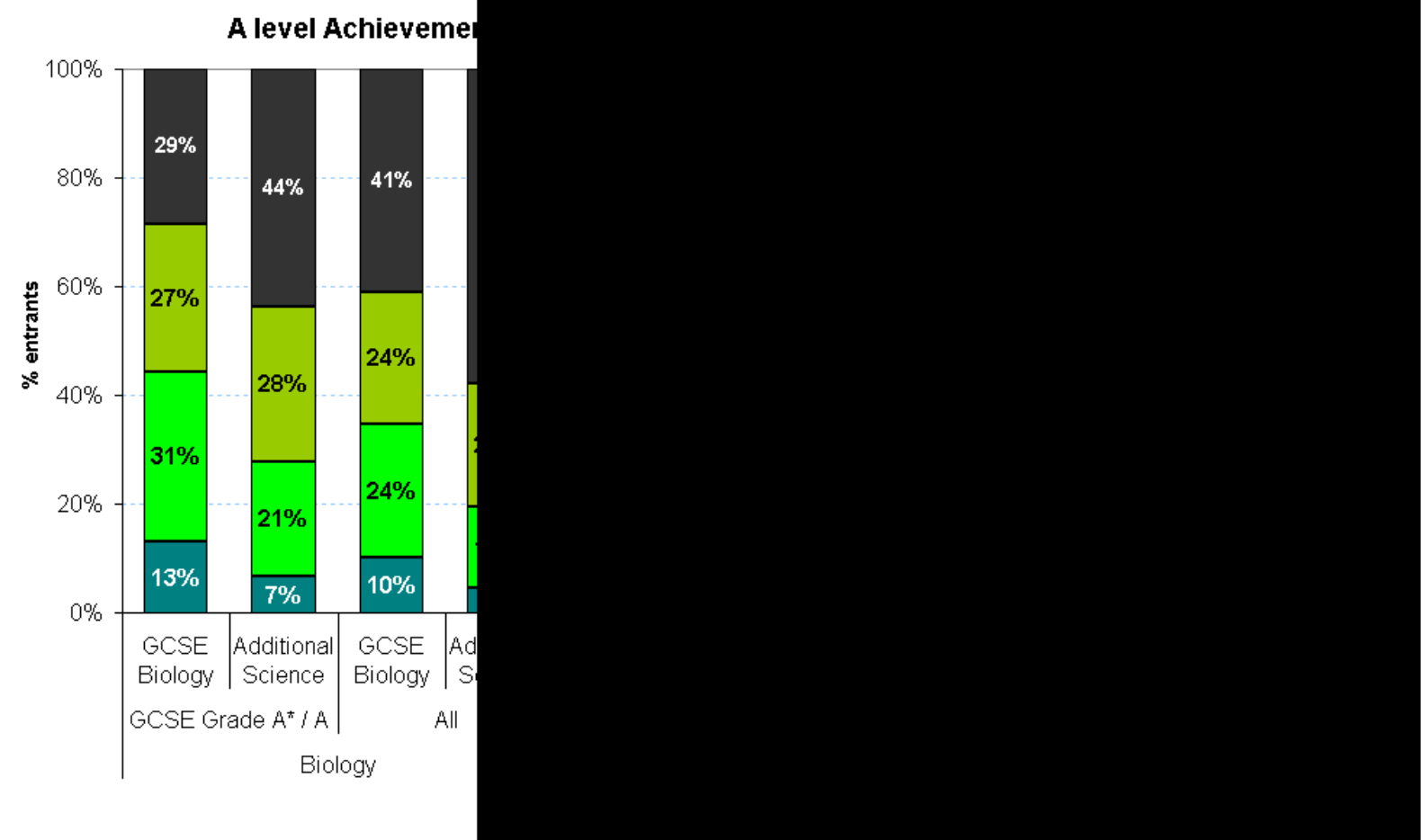


Figure $4.2 \mathrm{~h}$ uses odds ratios to standardise the percentage outcome measures from the previous two charts across the subjects and GCSE routes. The pattern is similar to that for the separate science compared with double science 2009 odds ratios in figure $4.2 \mathrm{~d}$, except for A level biology and chemistry continuation amongst pupils across the full grade range at GCSE. For biology, the ratio of odds on entering the $A$ level for entrants with separate biology at GCSE, compared with additional science at GCSE, is smaller than was the case when comparing separate biology with double science GCSEs. It has shrunk from 6.0 times the odds to 4.4 times the odds with additional science making less difference to biology continuation in 2010 than double science did in 2009. For chemistry continuation, the effect of additional science on 2010 A level entries is slightly larger than the effect of double science was on 2009 A level entries (the odds ratio has grown from 7.2 to 7.6).

The key finding, however, is that there remains an advantage to taking separate science GCSEs when it comes to A level continuation and achievement of top grades for all three science subjects since the introduction of core and additional science GCSEs in 2008.

\section{Fig. $4.2 \mathrm{~h}$}

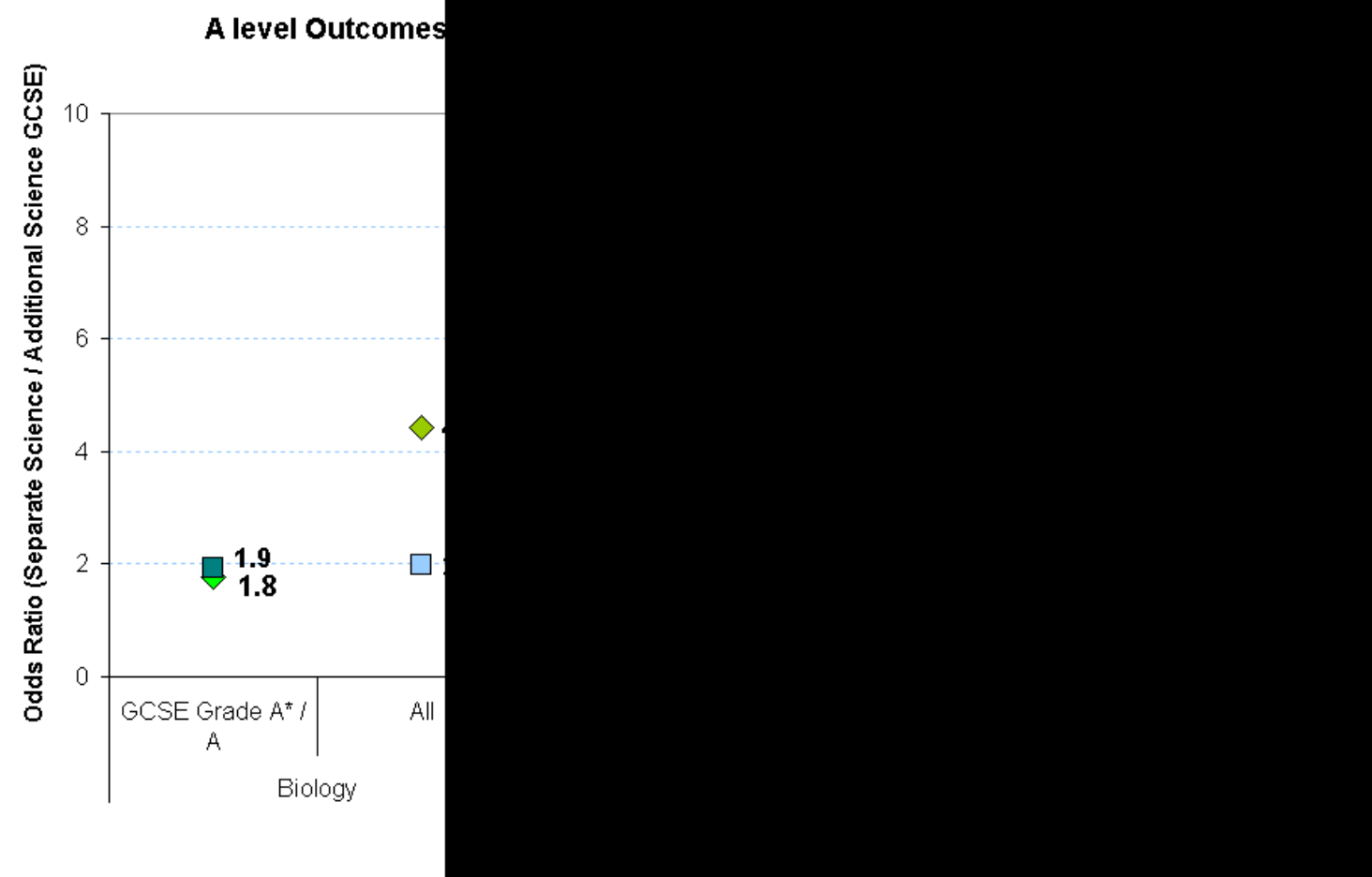


Returning to explore the shift in GCSE entries from core and additional GCSEs (and double science GCSEs before them) to greater popularity for separate science GCSEs, figure 4.2i presents the recent changes to the proportion of schools now offering separate science GCSEs, generally to their most able pupils but sometimes universally. Across all schools in England, the offer of separate sciences at GCSE has expanded from fewer than half of schools in 2008, to $52 \%$ in 2009 and $70 \%$ by 2010. Government policies have encouraged this expansion of separate or "triple" sciences over recent years in order to boost the supply of science candidates at $A$ level.

\section{Fig. 4.2i}

Increase in Schools Offering GCSE Triple Science, 2008-2010

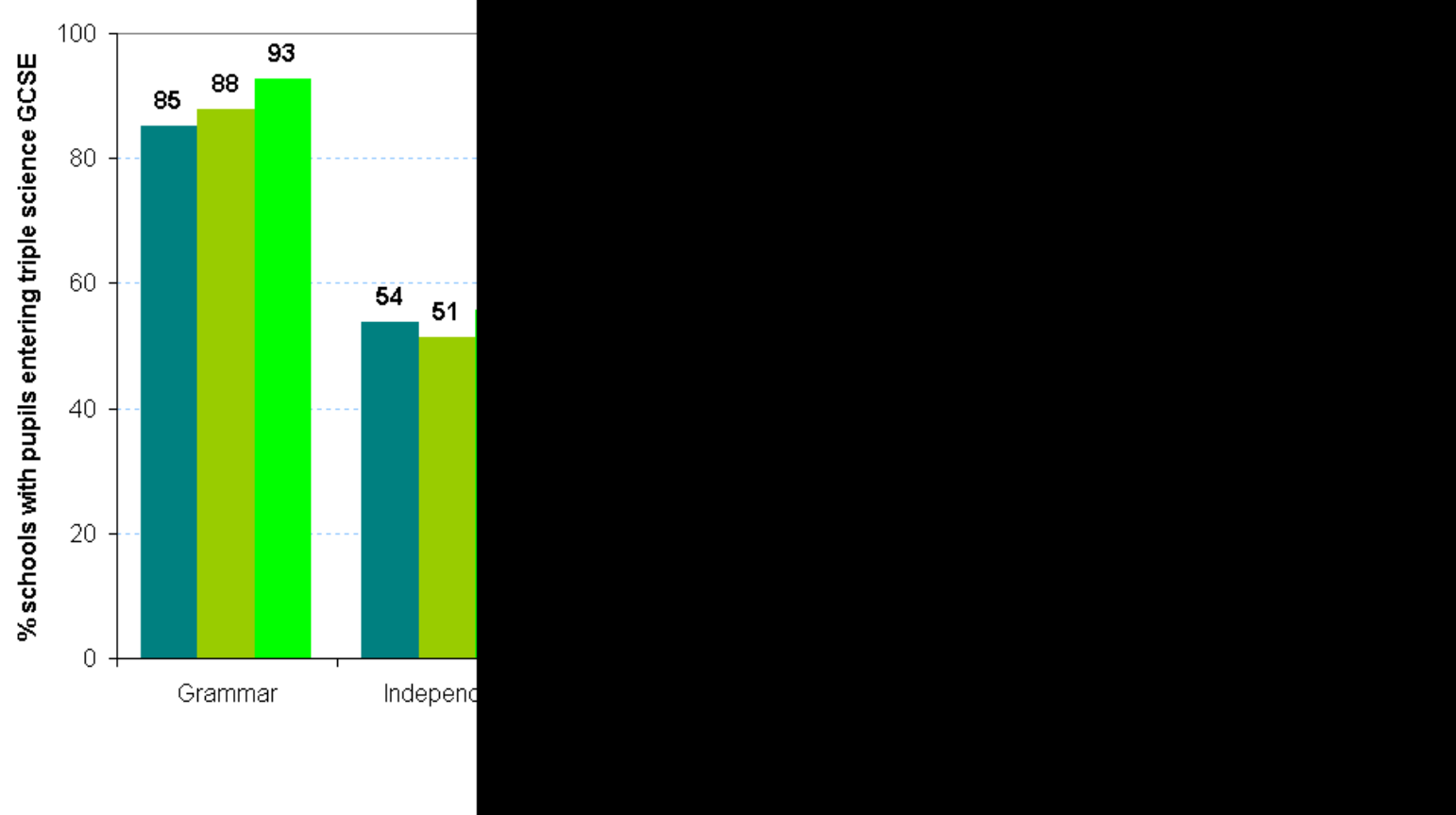

\subsection{A-level School \& Institution Types}

In this section, for each subject, the proportion of A level entries accounted for, and the rates of achieving grades $A$ or $B$, are presented for different types of Key Stage 5 schools and institutions. The first chart reports the incidence of top grades at A level for each of maths, biology, chemistry and physics; the second chart takes the total number of A level entries, and grades A or B, in each subject and apportions them to the Key Stage 5 school types where they were entered (placement).

Figure 4.3a depicts the higher A level achievement rates for all four maths and science subjects at independent and grammar schools. Sixth form colleges were ranked $3^{\text {rd }}$ or $4^{\text {th }}$ of the school types for all of the subjects and FE sector colleges had the lowest proportion of $A$ and $B$ grades in each case. The non-selective maintained mainstream school types had A level performance in the middle of the range of school types for each subject, with the order between maths and computing specialist, science specialist, and other maintained mainstream schools depending on the subject in question. The large differences in achievement between the 
institution types are significantly influenced by the different prior attainment levels of pupils attending each type of school. For simultaneous modelling of school types and prior attainment along with a range of other factors, please see chapter 6.

For maths, the rate of achievement of grades A or B was $85 \%$ at independent schools, $79 \%$ at grammar schools, $64 \%$ at science specialist schools, $61 \%$ at sixth form colleges, $60 \%$ at both maths and computing specialist schools and the remaining maintained mainstream schools, dropping to 56\% at FE sector colleges. Maths had the smallest range of percentage achievement rates between independent schools and FE sector colleges of the four maths and science subjects, but this was still considerable at 29 percentage points.

For biology A level, the rate of achievement of grades A or B was $74 \%$ at independent schools, $66 \%$ at grammar schools, $50 \%$ at sixth form colleges, $43 \%$ at science specialist schools, $42 \%$ at other maintained mainstream schools, $41 \%$ at maths and computing specialist schools, and 39\% at FE sector colleges. Biology had a range of percentage achievement rates of 35 percentage points between the top and bottom ranked institution types.

For chemistry A level, the rate of achievement of grades A or B was $79 \%$ at independent schools, $70 \%$ at grammar schools, $56 \%$ at sixth form colleges, $50 \%$ at maths and computing specialist schools, $49 \%$ at other maintained mainstream schools, $48 \%$ at science specialist schools, and $42 \%$ at FE sector colleges. Chemistry had the largest range of percentage achievement rates between independent schools and FE sector colleges, at 37 percentage points. 
For physics A level, the rate of achievement of grades A or B was $73 \%$ at independent schools, $64 \%$ at grammar schools, $50 \%$ at science specialist schools, $49 \%$ at sixth form colleges, $44 \%$ at the other maintained mainstream schools, $40 \%$ at maths and computing specialist schools, dropping to $39 \%$ at FE sector colleges. Physics had a range of percentage achievement rates of 34 percentage points between the top and bottom ranked institution types.

\section{Fig. 4.3a}

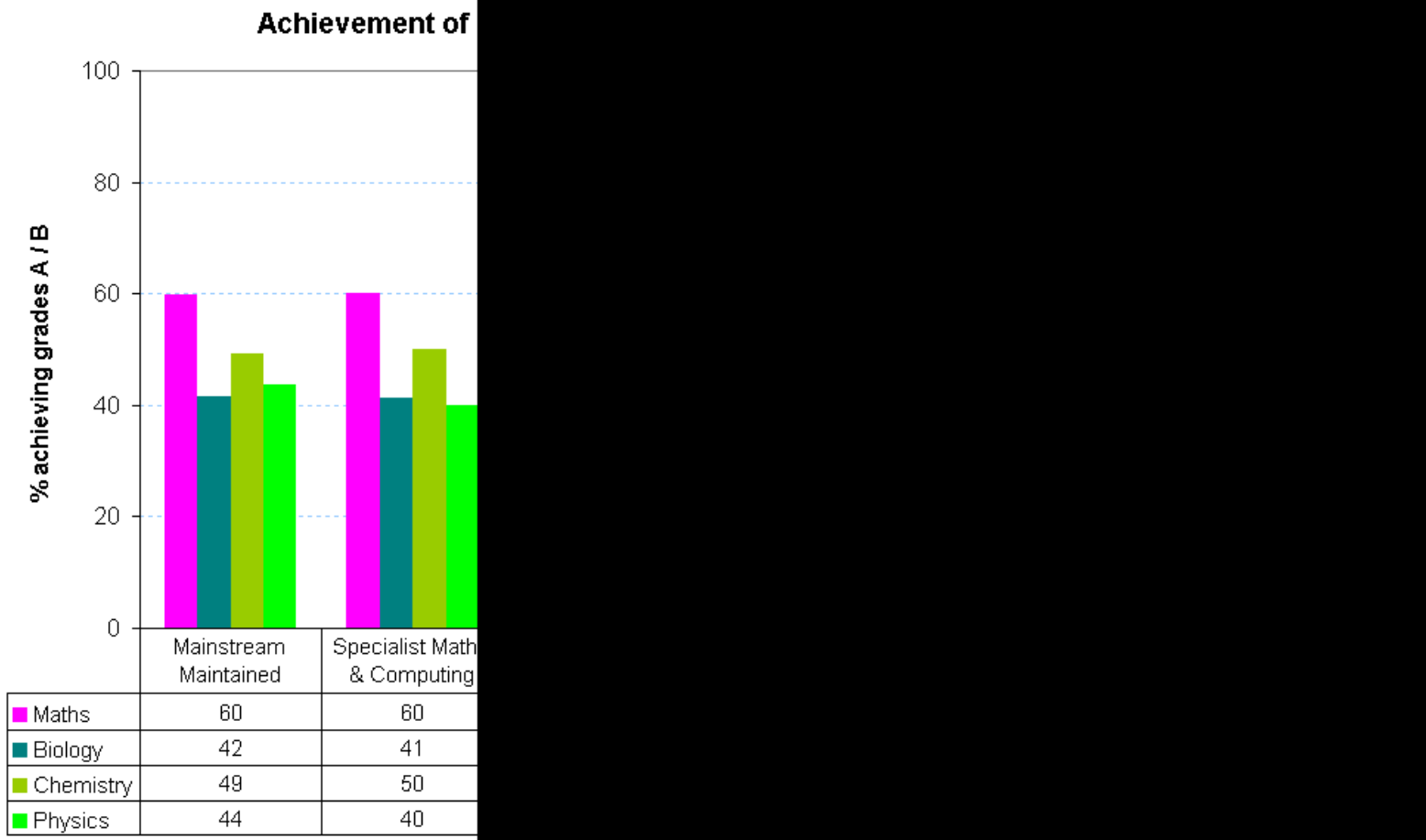

The charts in figure 4.3b show the placement of A level entries, and grades A or B, across the Key Stage 5 school and institution types from figure 4.3a above. Maintained mainstream schools make up the largest share, at between $32 \%$ and $36 \%$, of A level entries for each of maths, biology, chemistry and physics. Independent schools account for the next largest share at between 14\% and 17\% for each subject. Sixth form colleges and grammar schools follow next, each contributing similar shares of the maths and science A level entries, at between 13\% to $17 \%$ of the total entries. Specialist schools of either maths and computing, or science specialisms, make up the final $9 \%$ to $10 \%$ of entries for each subject.

Grammar schools and independent schools were overrepresented in the numbers of grades $A$ and $B$ for all four subjects. The independent schools were slightly less overrepresented in top maths grades than in top grades for biology, chemistry and physics. The disproportionate share of top A level grades commanded by grammar schools, was less extreme than for independent schools, but they were slightly more overrepresented for biology and chemistry than for maths and physics. The other school types were all underrepresented amongst the top A level grades for the four maths and science subjects. 
Fig. 4.3b

A level Maths Entries

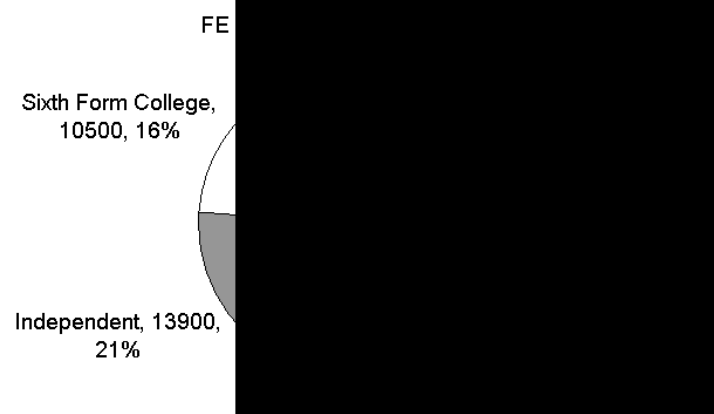

A level Maths Grades A / B

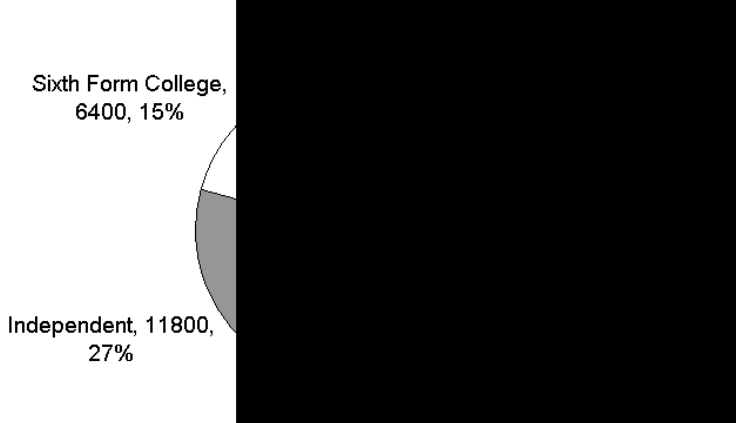

A level Biology Entries

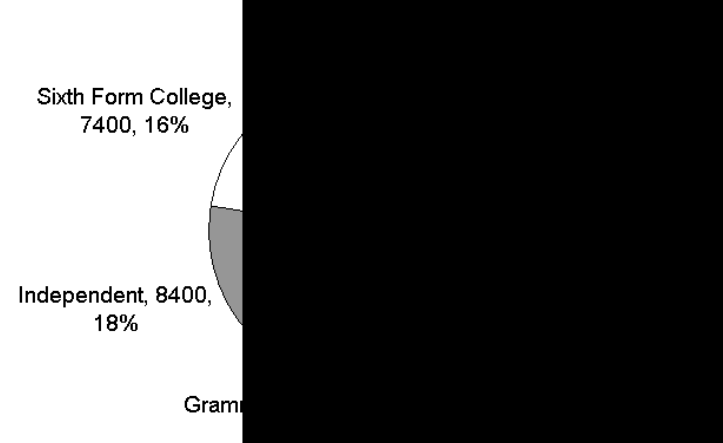

A level Biology Grades A / B

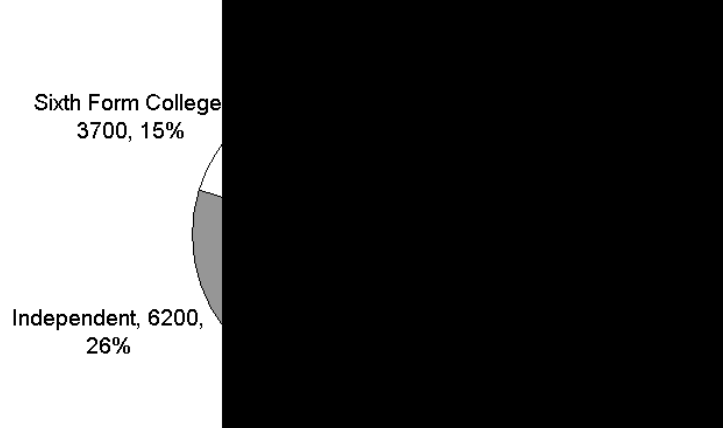

\section{A level Chemistry Entries}

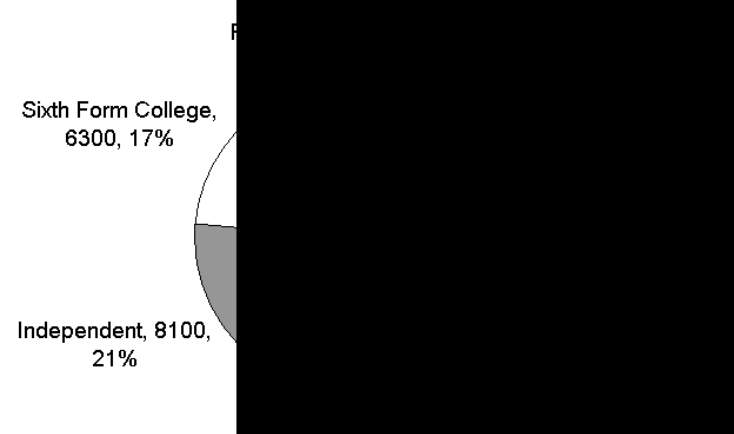

A level Chemistry Grades A / B

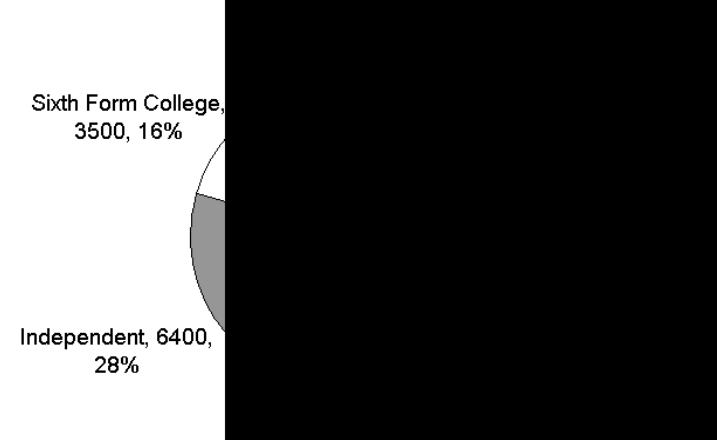

\section{A level Physics Entries}

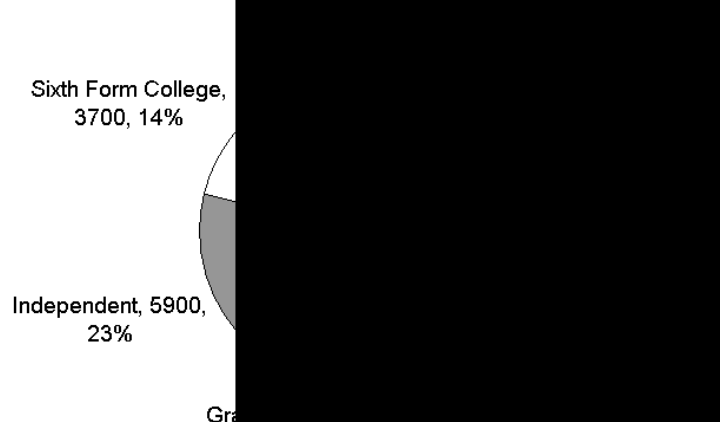

A level Physics Grades A / B

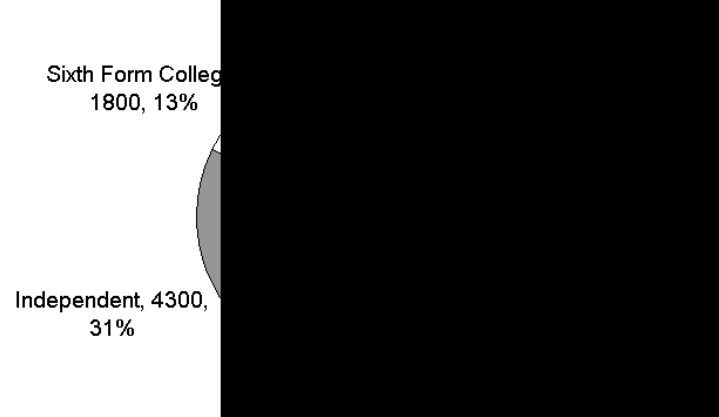


Further analyses of school types and A level achievement, combining the Key Stage 4 and Key Stage 5 institutions attended, are presented in annex B for reference.

\subsection{A-level Subjects Offered}

The different types of Key Stage 5 institution vary in the mix of qualifications offered. The table in figure $4.4 \mathrm{a}$ summarises some of the key differences in relation to maths and science A levels. For example, not all institutions at Key Stage 5 offer A levels at all, with just $78 \%$ of FE sector colleges offering one or more subject at A level. The average number of A level subjects on offer, for those schools and colleges offering at least one A level, varies between the school types. Sixth form colleges generally offer the widest range of subjects at $A$ level with an average of 38 subject options within their A level offer. Grammar schools offer the next largest range of A level subjects, with an average of 25 available per school; maintained mainstream schools (including specialist schools) and independent schools offer average A level ranges of approximately 20 subjects, as do FE sector colleges.

A level maths provision is widespread in all institution types apart from FE colleges, with over $85 \%$ availability; only $61 \%$ of FE colleges offer A level maths. A level biology and chemistry are offered at just $55 \%$ of FE colleges, but at over $80 \%$ of each of the other institution types. A level physics has the lowest availability of the four subjects at $78 \%$ or more of each institution type, except for FE colleges, where the rate of provision is $50 \%$.

All four subjects have near-universal availability at sixth form colleges and grammar schools. Virtually all science specialist schools offer maths, biology and chemistry at A level, with physics availability a little lower at $93 \%$ of these schools. Maintained mainstream, independent, and maths and computing specialist schools each have science availability at fewer than $90 \%$ of schools.

\section{Fig. 4.4a}

\begin{tabular}{|c|c|c|c|c|c|c|c|}
\hline & $\begin{array}{l}\text { Maintained } \\
\text { Mainstream }\end{array}$ & FE College & Independent & Grammar & $\begin{array}{c}\text { Science } \\
\text { Specialist }\end{array}$ & $\begin{array}{l}\text { Maths \& } \\
\text { Computing } \\
\text { Specialist } \\
\end{array}$ & $\begin{array}{c}\text { Sixth Form } \\
\text { College }\end{array}$ \\
\hline Number of KS5 Schools & 1390 & 270 & 610 & 160 & 160 & 150 & 90 \\
\hline Number Offering A levels & 1360 & 210 & 600 & 160 & 160 & 140 & 90 \\
\hline$\%$ Offering A levels & $98 \%$ & $78 \%$ & $97 \%$ & $100 \%$ & $100 \%$ & $98 \%$ & $99 \%$ \\
\hline $\begin{array}{l}\text { Mean Number of A level } \\
\text { Subjects Offered (by those } \\
\text { offering at least 1) }\end{array}$ & 20.0 & 19.7 & 19.5 & 25.4 & 22.1 & 20.6 & 38.3 \\
\hline Number Offering A level Maths & 1240 & 160 & 540 & 160 & 160 & 140 & 90 \\
\hline$\%$ Offering A level Maths & $89 \%$ & $61 \%$ & $88 \%$ & $100 \%$ & $97 \%$ & $92 \%$ & $99 \%$ \\
\hline Number Offering A level Biology & 1170 & 150 & 510 & 160 & 150 & 130 & 90 \\
\hline$\%$ Offering A level Biology & $84 \%$ & $55 \%$ & $83 \%$ & $99 \%$ & $96 \%$ & $88 \%$ & $99 \%$ \\
\hline Number Offering A level Chemistry & 1150 & 150 & 520 & 160 & 150 & 130 & 90 \\
\hline$\%$ Offering A level Chemistry & $82 \%$ & $55 \%$ & $85 \%$ & $99 \%$ & $96 \%$ & $86 \%$ & $99 \%$ \\
\hline Number Offering A level Physics & 1090 & 130 & 500 & 160 & 150 & 120 & 90 \\
\hline$\%$ Offering A level Physics & $78 \%$ & $50 \%$ & $81 \%$ & $99 \%$ & $93 \%$ & $82 \%$ & $99 \%$ \\
\hline
\end{tabular}




\section{Chapter 5: Choice \& Continuation}

The numbers of entries for maths and science A levels reported in Chapter 1 , and the rates of continuation presented in Chapter 2, amongst other factors such as prior attainment and pupil or school characteristics, are a function of the qualification and subject options chosen by individual young people, with a view to their future study and career ambitions. Chapter 5 examines some of the patterns of selection for $A$ level subjects in order to shed light on the processes of subject choice at Key Stage 5 , and how these influence the popularity of maths and science subjects.

\subsection{Competing for Entrants - Relative Subject Popularity Trends}

Section 5.1 begins by charting the relative popularity of maths, biology, chemistry and physics compared with other A level subjects. The table in figure 5.1 a shows the growth in entries across the range of A level subjects between 1996 and 2009.

Some subjects are grouped into categories to generate a manageable number of series.

\section{Fig. 5.1a}

\begin{tabular}{|c|c|c|c|c|c|c|c|c|c|c|c|c|c|c|c|c|c|}
\hline & & 1996 & 1997 & 1998 & 1999 & 2000 & 2001 & 2002 & 2003 & 2004 & 2005 & 2006 & 2007 & 2008 & 2009 & \begin{tabular}{|c|}
2009 \\
Entries \\
as a \\
Multiple \\
of 1996 \\
Entries \\
\end{tabular} & $\begin{array}{c}\% \\
\text { Change } \\
\text { in } \\
\text { Entries } \\
(1996- \\
2009) \\
\end{array}$ \\
\hline \multirow{5}{*}{ 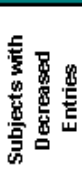 } & Modern Foreign Languages & 39,554 & 38,149 & 36,488 & 34,440 & 30,997 & 30,910 & 28,256 & 27,475 & 27,052 & 26,665 & 28,010 & 28,377 & 29,423 & 29,529 & 0.7 & $-25 \%$ \\
\hline & Geography & 37,162 & 37,979 & 38,832 & 37,054 & 33,017 & 33,106 & 31,067 & 31,179 & 29,906 & 28,383 & 28,283 & 27,494 & 27,846 & 28,450 & 0.8 & $-23 \%$ \\
\hline & General Studies & 62,020 & 71,742 & 78,806 & 84,261 & 87,834 & 90,979 & 58,521 & 56,897 & 57,190 & 58,674 & 59,653 & 57,674 & 57,086 & 52,484 & 0.8 & $-15 \%$ \\
\hline & Physics & 28,400 & 28,777 & 29,672 & 29,552 & 28,191 & 28,031 & 27,860 & 26,278 & 24,606 & 24,094 & 23,657 & 23,887 & 24,703 & 25,620 & 0.9 & $-10 \%$ \\
\hline & Other Science & 4,194 & 4,301 & 4,325 & 4,124 & 3,834 & 3,587 & 3,740 & 4,029 & 3,773 & 3,779 & 3,599 & 3,920 & 3,986 & 3,905 & 0.9 & $-7 \%$ \\
\hline \multirow{14}{*}{ 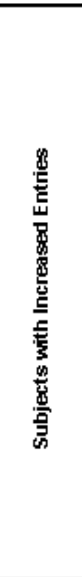 } & Chemistry & 34,677 & 36,429 & 37,103 & 35,831 & 35,290 & 33,871 & 32,324 & \begin{tabular}{|r|}
31,065 \\
\end{tabular} & 32,130 & 33,164 & 34,534 & 35,077 & 36,328 & 37,141 & 1.1 & $7 \%$ \\
\hline & Biology & 43,398 & 47,598 & 48,897 & 47,192 & 46,190 & 44,592 & 45,407 & 43,902 & 44,235 & 45,664 & 46,624 & 46,797 & 48,397 & 47,978 & 1.1 & $11 \%$ \\
\hline & English & 72,544 & 80,659 & 80,546 & 77,658 & 74,343 & 73,749 & 72,495 & 72,011 & 72,967 & 76,997 & 78,147 & 77,502 & 80,917 & 83,649 & 1.2 & $15 \%$ \\
\hline & History & 36,294 & 36,078 & 34,437 & 33,406 & 33,140 & 33,830 & 35,513 & 36,513 & 38,150 & 39,198 & 40,673 & 40,542 & 42,107 & 42,842 & 1.2 & $18 \%$ \\
\hline & Maths & 54,125 & 56,050 & 56,589 & 56,100 & 53,674 & 54,157 & 44,156 & 44,453 & 46,017 & 46,037 & 49,805 & 53,331 & 57,618 & 64,519 & 1.2 & $19 \%$ \\
\hline & $\begin{array}{l}\text { Accounting, Business, } \\
\text { Economics, Government, Law } \\
\text { Technology, IT, Home }\end{array}$ & 63,202 & 64,205 & 64,519 & 65,745 & 64,779 & 64,265 & 63,334 & 66,363 & 67,690 & 68,285 & 69,676 & 70,911 & 73,851 & 78,335 & 1.2 & $24 \%$ \\
\hline & Economics & 19,559 & 21,422 & 25,271 & 27,775 & 31,226 & 35,100 & 39,243 & 40,539 & 37,323 & 35,215 & 34,362 & 31,427 & 29,845 & 28,845 & 1.5 & $47 \%$ \\
\hline & Art, Music or Drama & 43,095 & 46,284 & 49,671 & 51,511 & 51,372 & 53,363 & 51,010 & 55,191 & 56,750 & 58,504 & 61,297 & 61,971 & 64,079 & 65,429 & 1.5 & $52 \%$ \\
\hline & Further Maths & 4,913 & 4,999 & 5,211 & 5,145 & 5,015 & 5,063 & 4,498 & 4,730 & 5,111 & 5,192 & 6,516 & 7,241 & 8,447 & 9,443 & 1.9 & $92 \%$ \\
\hline & $\begin{array}{l}\text { Psychology, Sociology or } \\
\text { Other Social }\end{array}$ & 41,201 & 46,217 & 46,050 & 46,780 & 46,072 & 47,128 & 53,706 & 60,898 & 67,505 & 72,251 & 75,720 & 76,399 & 78,106 & 79,659 & 1.9 & $93 \%$ \\
\hline & Physical Education & 9,042 & 11,696 & 13,680 & 14,740 & 15,853 & 16,779 & 16,655 & 18,763 & 19,234 & 19,759 & 21,533 & 21,527 & 22,344 & 21,609 & 2.4 & $139 \%$ \\
\hline & $\begin{array}{l}\text { Media or Other } \\
\text { Communications }\end{array}$ & 13,647 & 16,527 & 18,509 & 18,543 & 19,355 & 20,822 & 23,697 & 26,895 & 29,299 & 30,198 & 32,573 & 33,320 & 33,772 & 34,853 & 2.6 & $155 \%$ \\
\hline & Religious Studies & 6,728 & 6,956 & 6,740 & 5,044 & 7,161 & 7,484 & 8,607 & 10,173 & 11,725 & 13,856 & 14,900 & 15,593 & 16,842 & 17,488 & 2.6 & $160 \%$ \\
\hline & Total A Levels & $|620,164|$ & $|662,163|$ & $\mid 681,082$ & 680,048 & $\mid 672,362$ & $|681,553|$ & 645,033 & $|662,670|$ & $|675,924|$ & 691,371 & 715,203 & $\mid 718,756$ & $\mid 741,356$ & $|757,697|$ & 1.2 & $22 \%$ \\
\hline
\end{tabular}

Figure $5.1 \mathrm{~b}$ summarises the same data in bar chart format, showing the entry numbers in 1996, 2002 and 2009 for each subject or subject grouping. Most subjects have seen increases in entries over the time period resulting from the widening of access to Key Stage 5 studies, evident in the 22\% increase in the total number of $A$ levels entered. The largest increases were in religious studies and media or communications A levels, where entries in 2009 were 2.6 times their 1996 numbers. A level physical education has seen an increase in entries almost as large at 2.4 times the 1996 number by 2009. 
Only a small number of subjects have seen decreases in entries since 1996 . Entries in modern foreign languages have decreased by $25 \%$, followed by geography entries, which were down by $23 \%$ between 1996 and 2009. General studies and classics have also seen decreases in the number of entries per year. Focusing on maths and the sciences, despite the recovery in entries since 2006, physics numbers were still $10 \%$ down on 1996 by 2009 . As seen in chapter 1, the provisional 2010 data indicate that physics entries have now almost regained their 1996 level; however, they are still approximately 3,000 per year below their 1998 level. Other science A levels also fell between 1996 and 2010, by 7\%.

Chemistry and biology saw increased entries between 1996 and 2009, but these were the smallest increases over that period of all the subjects with increases, at $7 \%$ and $11 \%$ respectively. Maths has fared better, with a $19 \%$ increase in entry numbers over the same period, but still falls towards the bottom end of the increases by other subjects, and slightly below the total size of the increase in all A levels of $22 \%$.

\section{Fig. 5.1b}

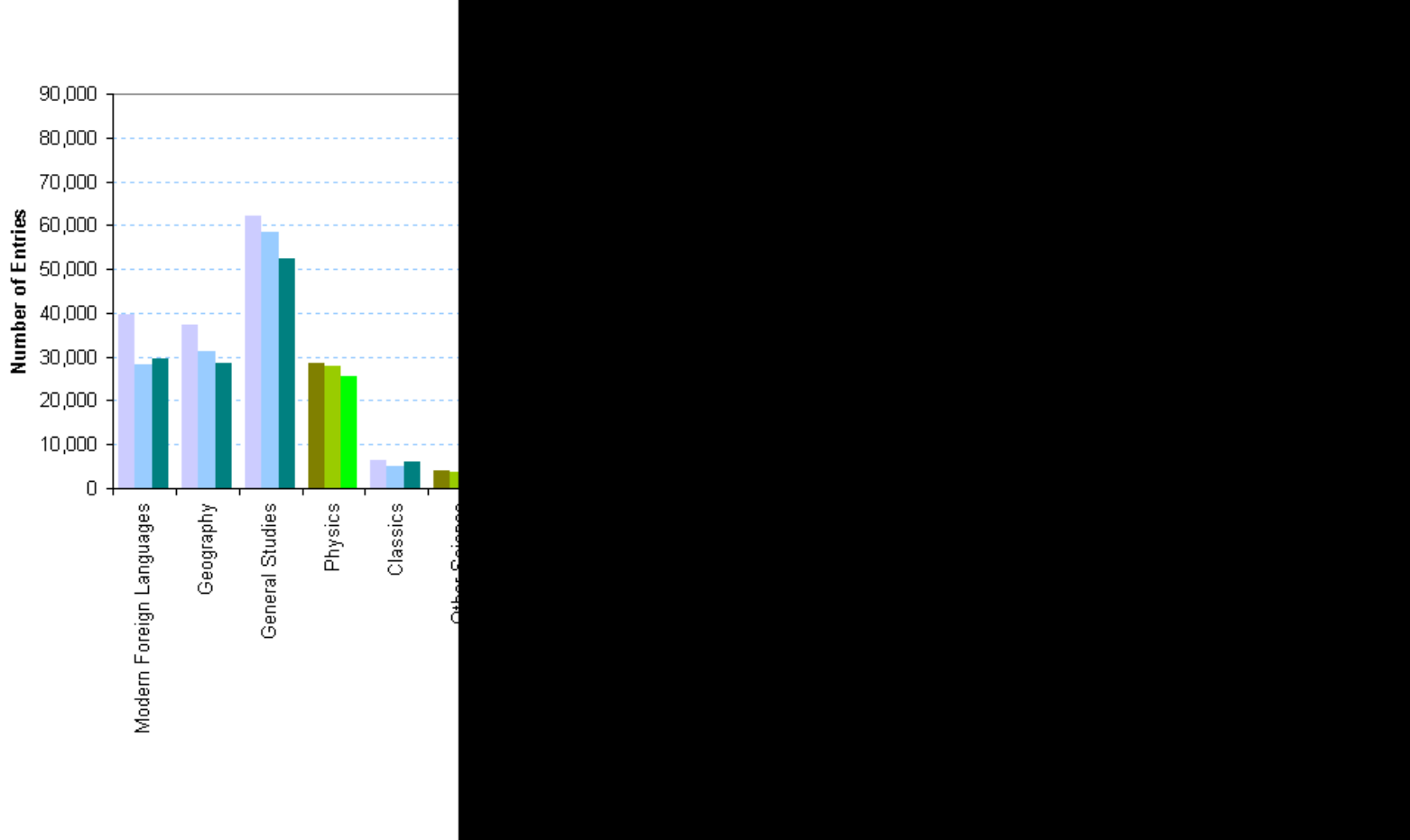


Figure 5.1c shows the relative increases in entries between subjects, with each subject's 1996 entry level set to a value of 1 .

Fig. 5.1c

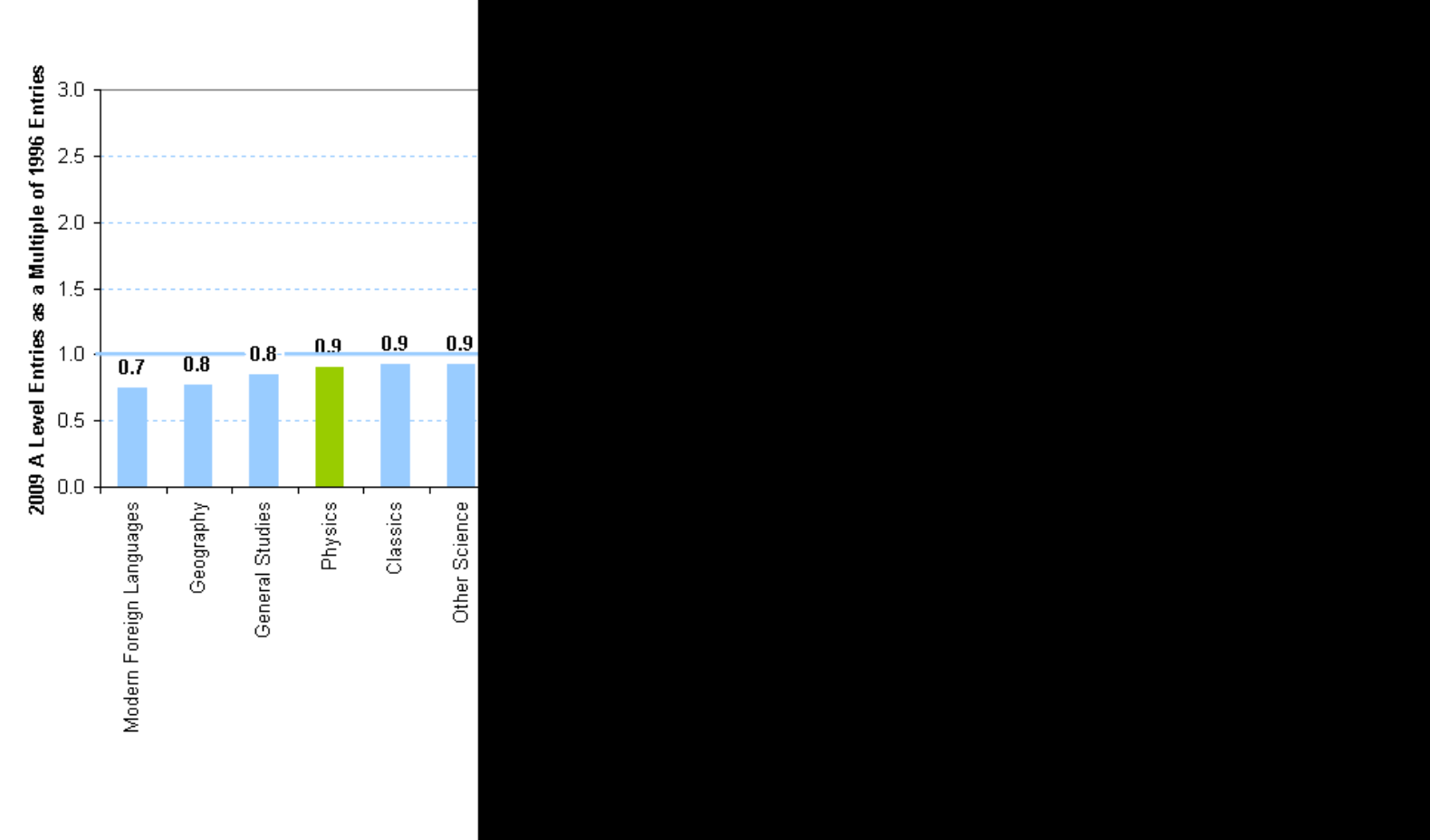




\subsection{Trend in the Number of Entries per Pupil}

Increases in individual subject entries have been influenced by the growth in Key Stage 5 qualifications as a whole. Figure 5.2a charts the numbers of additional entrants since 2003 , split by the number of A levels taken per candidate. Most of the increase has taken place within qualification types other than A levels, however there has also been a significant increase in the numbers of entrants taking 3 A levels. The numbers of candidates taking fewer than 3 or more than 3 A levels have remained fairly stable.

\section{Fig. 5.2a}

Trend in the Number of A Levels Per Entrant

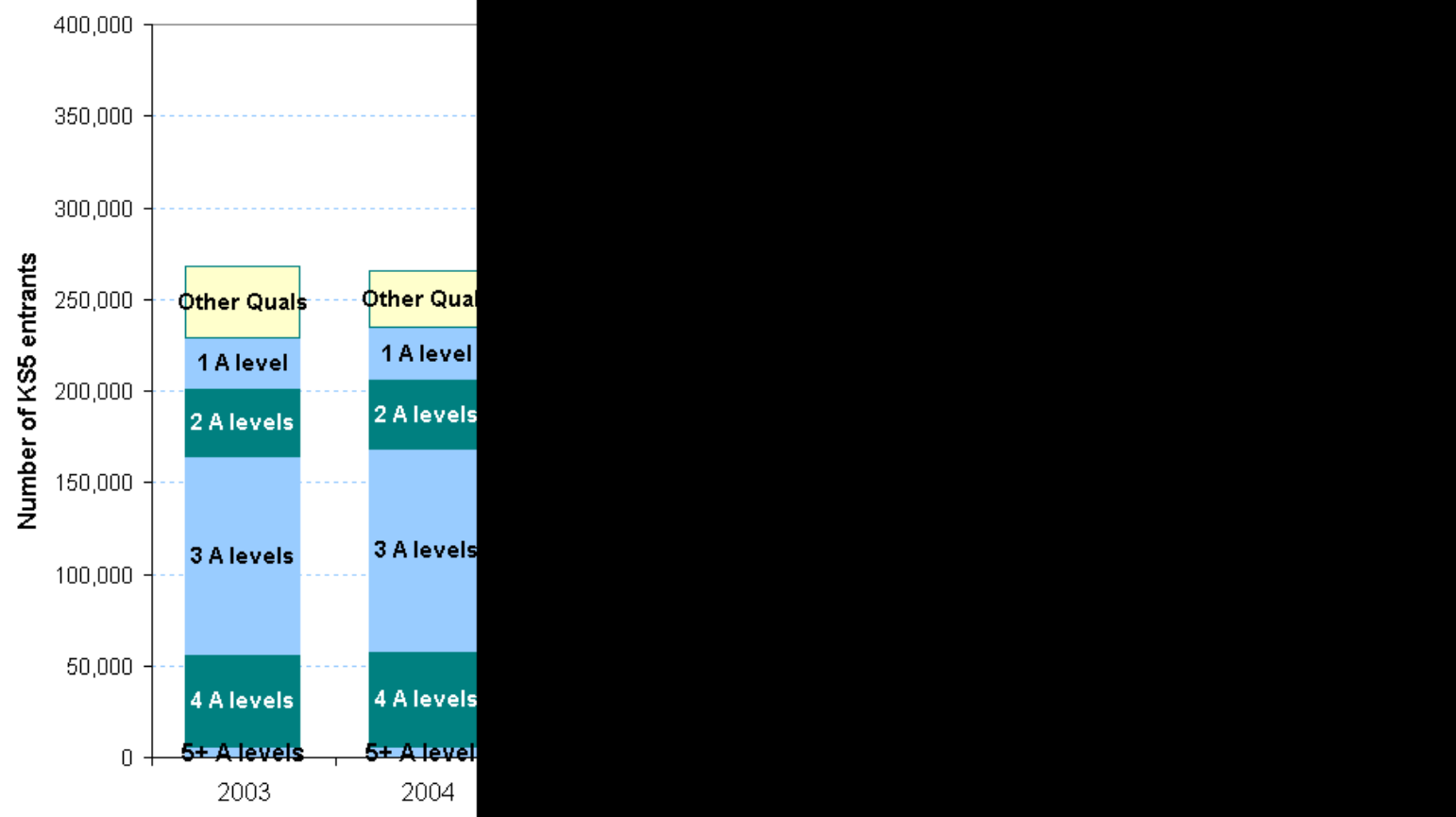


Figure $5.2 \mathrm{~b}$ charts the entry rates for maths and science A levels according to the number of $A$ levels taken by the candidate. The more subjects entered, the greater the likelihood that maths or science A levels will be amongst them. This effect of increased subjects entered on the probability of maths and science entries is stronger for maths than for the sciences, with biology benefiting the least from higher numbers of entries per candidate.

Entrants taking 3 subjects have slightly higher than average entry rates for maths and biology, but the same as the average for chemistry, and a lower than average physics rate (which is because almost half of physics entrants take 4 or more $A$ levels).

\section{Fig. 5.2b}

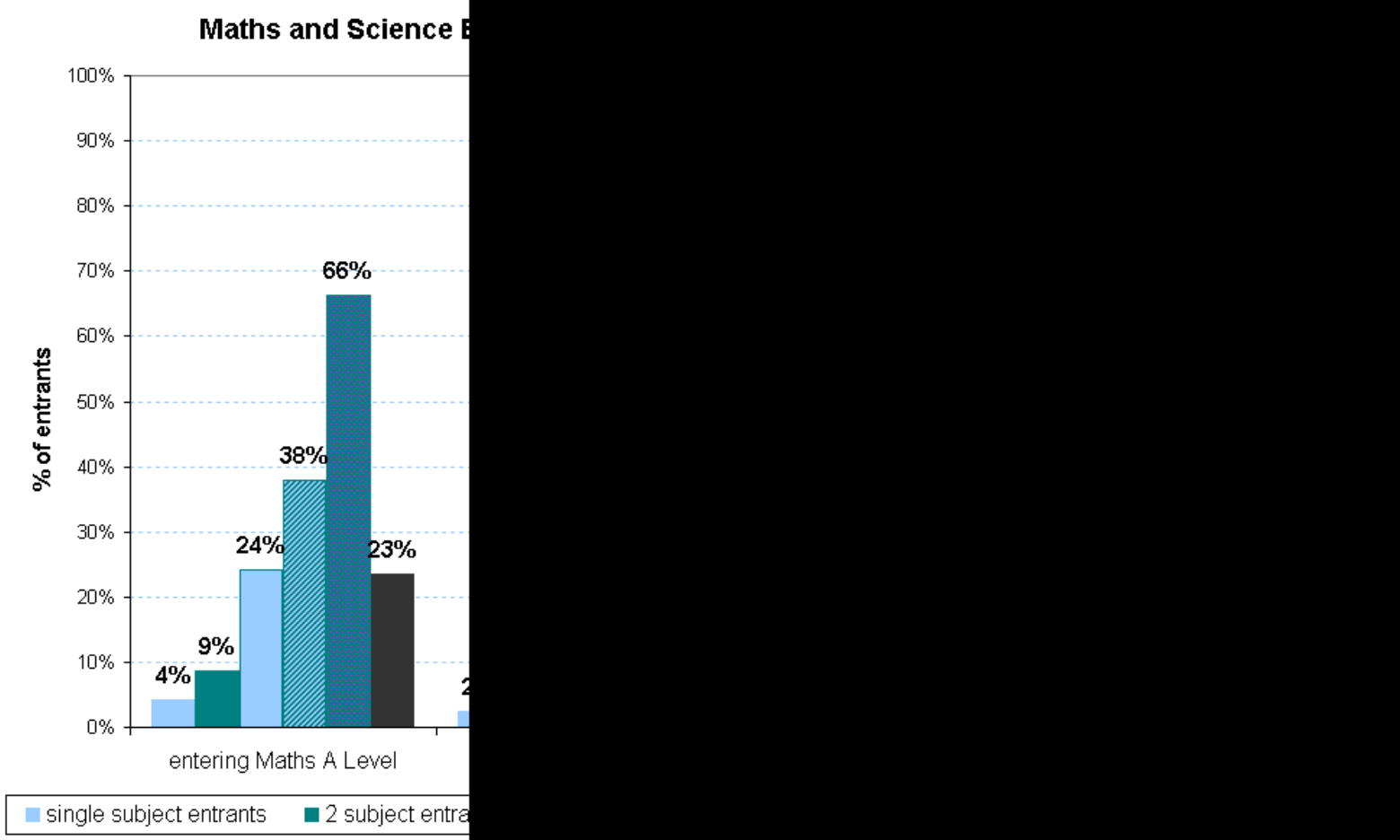

\subsection{Subject Combinations}

This section presents analyses of subjects most commonly combined with sciences and maths at A level, and of the number of A level candidates who sit more than one of the maths and science subjects. Figure 5.3a shows the numbers of A level entrants who sat one, two or three science subjects in 2009. The dark blue righthand columns then show how many of these entrants also combined their science entries with maths A level. Of the 75 thousand entrants with at least one science subject at A level, $57 \%$ sat just one science; $38 \%$ sat two sciences, and just $5 \%$ entered three sciences.

Entrants who combined maths with science A levels, made up almost half (48\%) of the entrants with one science, or $27 \%$ of the total science entrants. Fifty-nine percent of the entrants with two sciences also sat maths; they made up $23 \%$ of the total science entrants. Almost half $(48 \%)$ of the entrants with three sciences also sat 
maths A level; they made up $2 \%$ of the total science entrants.

As a proportion of the 65 thousand maths A level entrants in 2009, over half (60\%) took at least one science in combination with maths. This breaks down into $31 \%$ who sat one science with maths, $26 \%$ who took two sciences plus maths, and $3 \%$ who took three sciences as well as maths.

\section{Fig. 5.3a}

Number of A Level Entrants with Multiple Science Entries, 2009

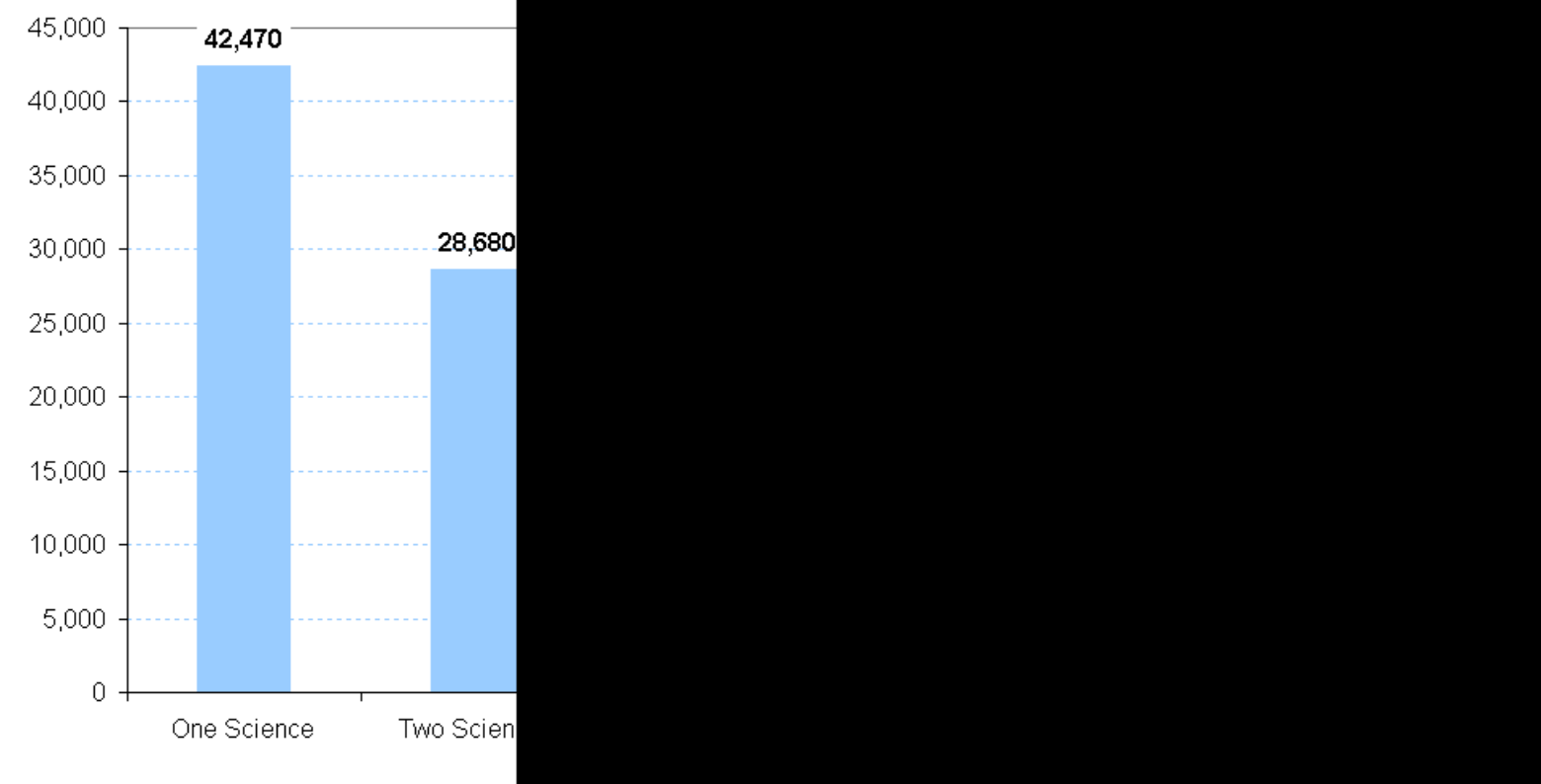

Figures $5.3 \mathrm{~b}$ to $5.3 \mathrm{e}$ give the most commonly combined A level subjects with each of maths, biology, chemistry and physics. Subjects which were taken by $5 \%$ or more of the maths / science entrants are included in each case. The number of entrants who took the subject as their sole A level entry is also given for reference. The top three subjects combined with maths were chemistry (22 thousand entrants), physics (20 thousand entrants) and biology (17 thousand entrants). For biology, the top three combined subjects were chemistry (24 thousand entrants), maths (17 thousand entrants) and general studies (10 thousand entrants); physics was ranked sixth with 5 thousand entrants taking this combination.

The top three subjects combined with chemistry were biology (24 thousand entrants), maths (22 thousand entrants) and physics (10 thousand entrants). For physics, the most commonly combined three subjects were maths (20 thousand entrants), chemistry (10 thousand entrants) and further maths (6 thousand entrants); biology was ranked fifth with 5 thousand entrants taking this combination.

General studies, further maths, history and geography were combined with all four maths and science subjects by at least $5 \%$ of their entrants. Other subjects that cropped up more than once as common combinations were social psychology, economics, and English literature. 
Fig. $5.3 b$

A Level Subjects Taken in Combination with Maths, 2009

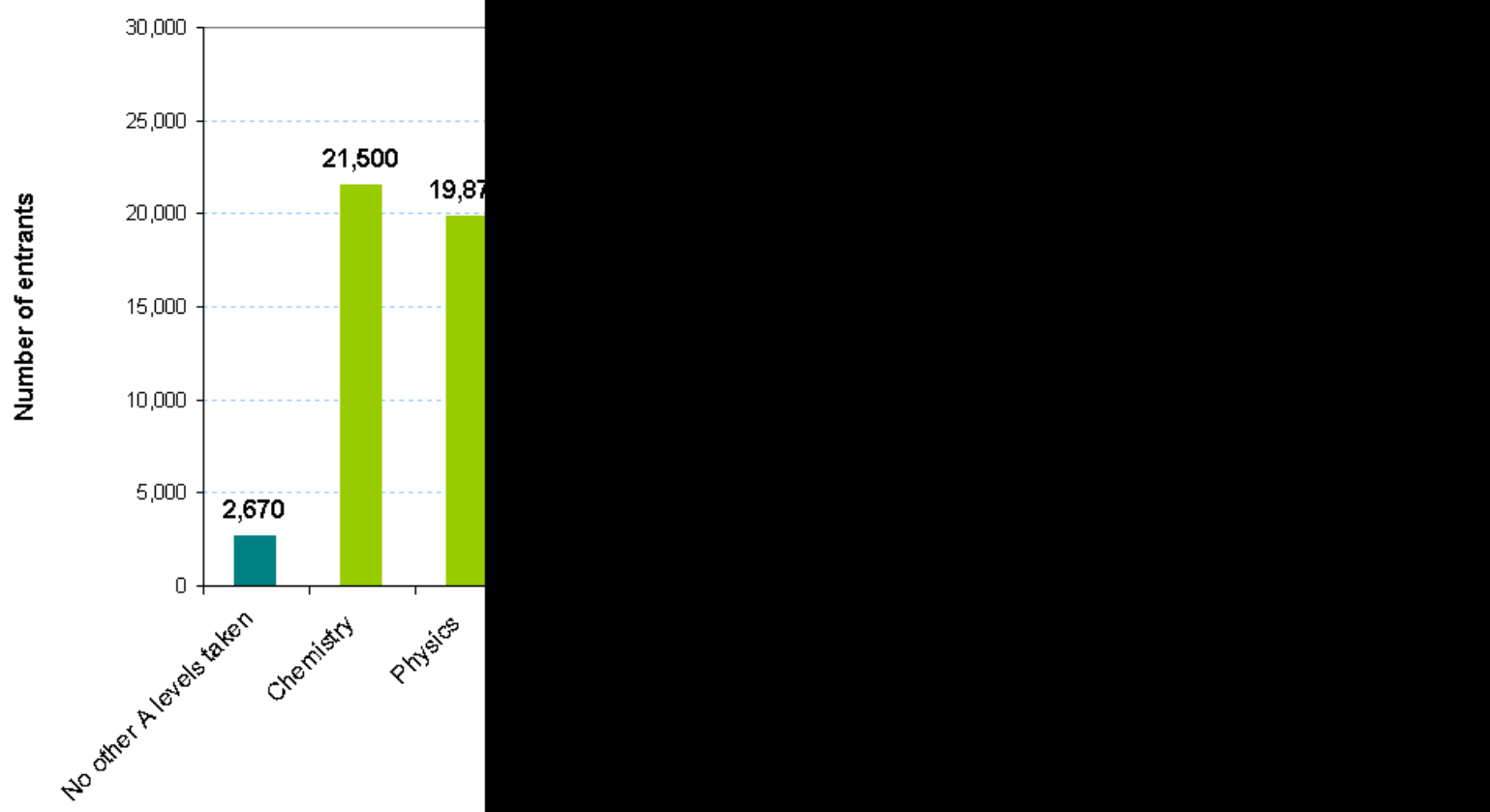

Fig. 5.3c

A Level Subjects Taken in Combination with Biology, 2009

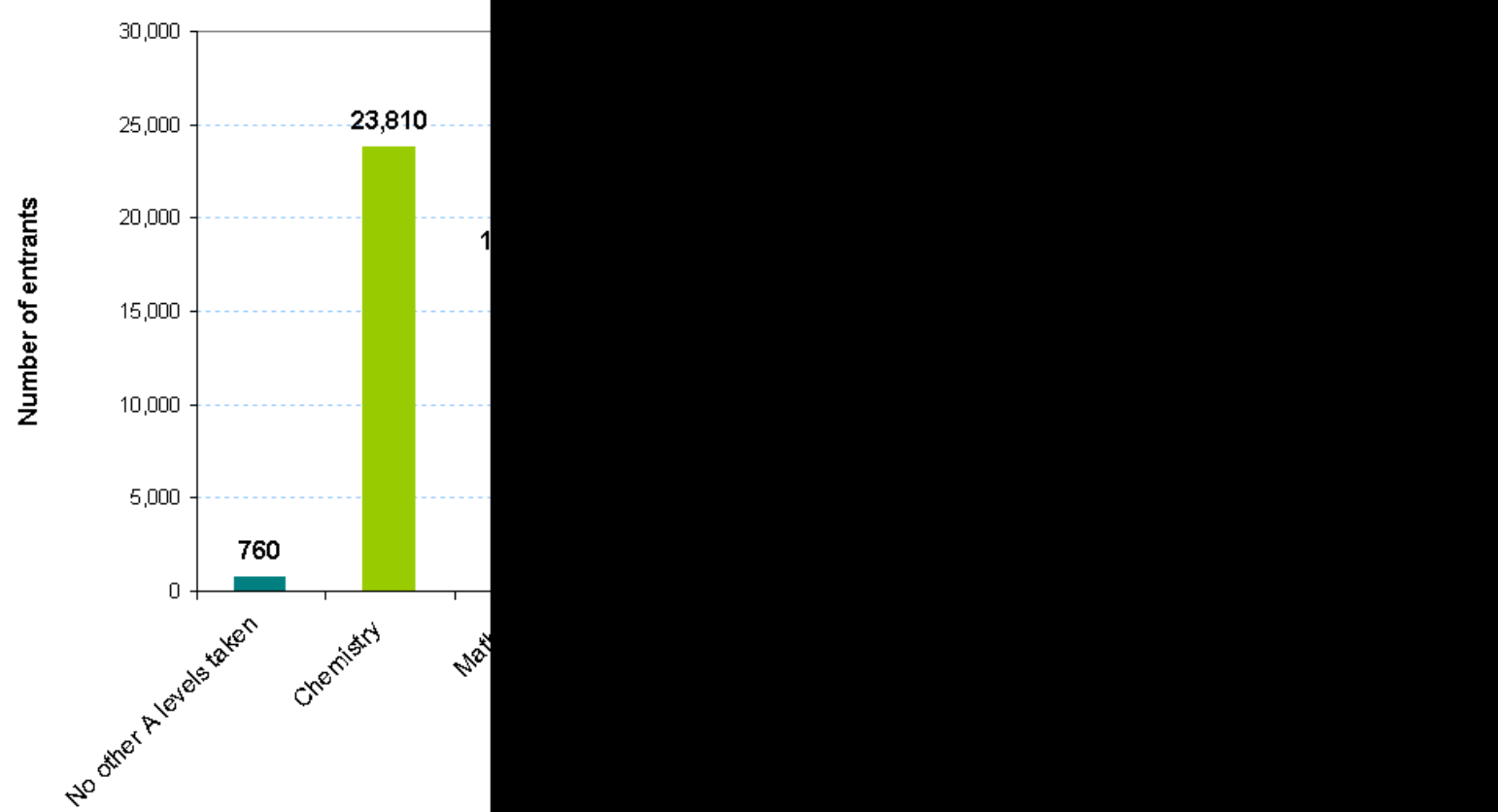


Fig. 5.3d

A Level Subjects Taken in Combination with Chemistry, 2009

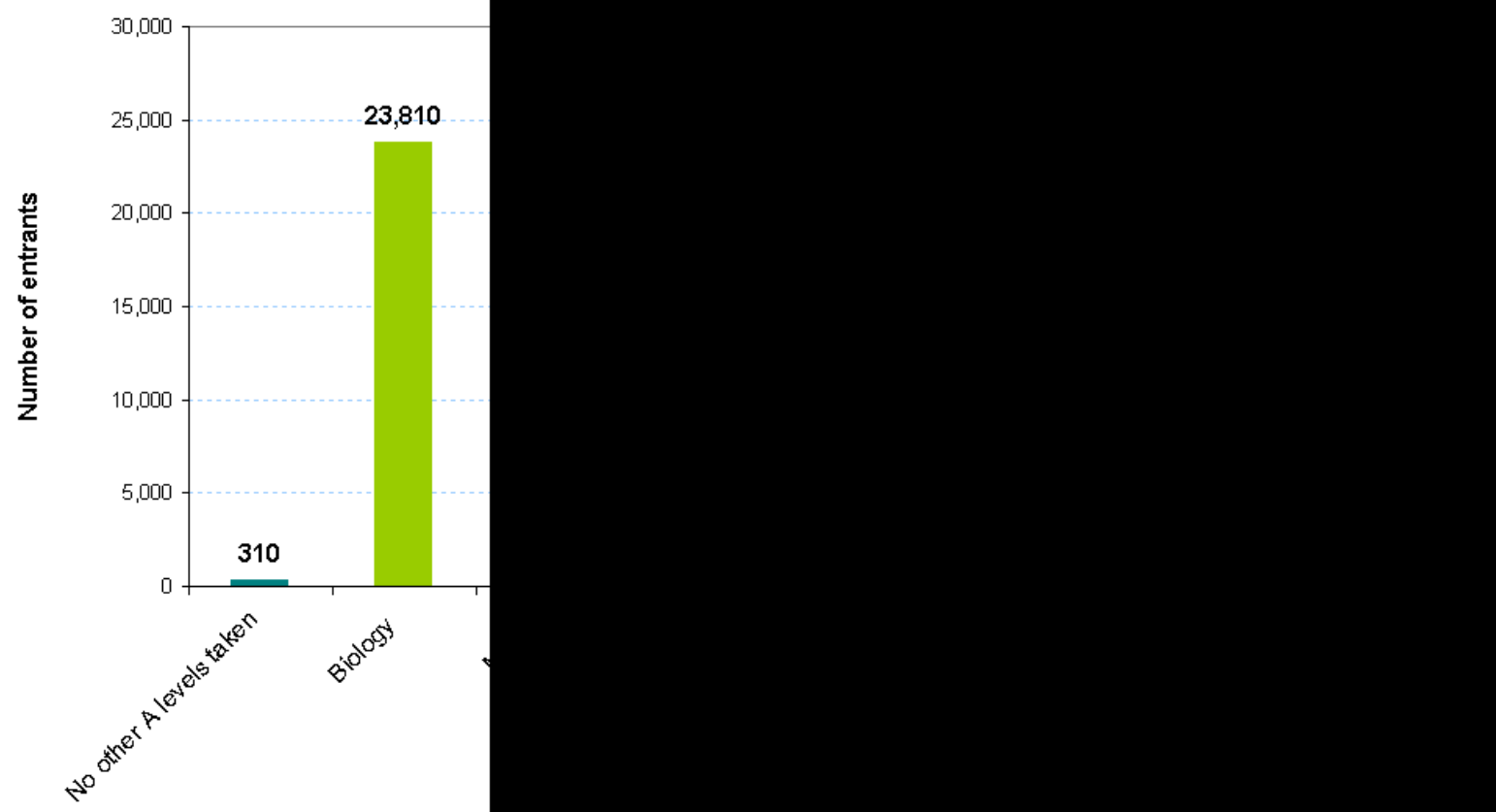

Fig. 5.3e

A Level Subjects Taken in Combination with Physics, 2009

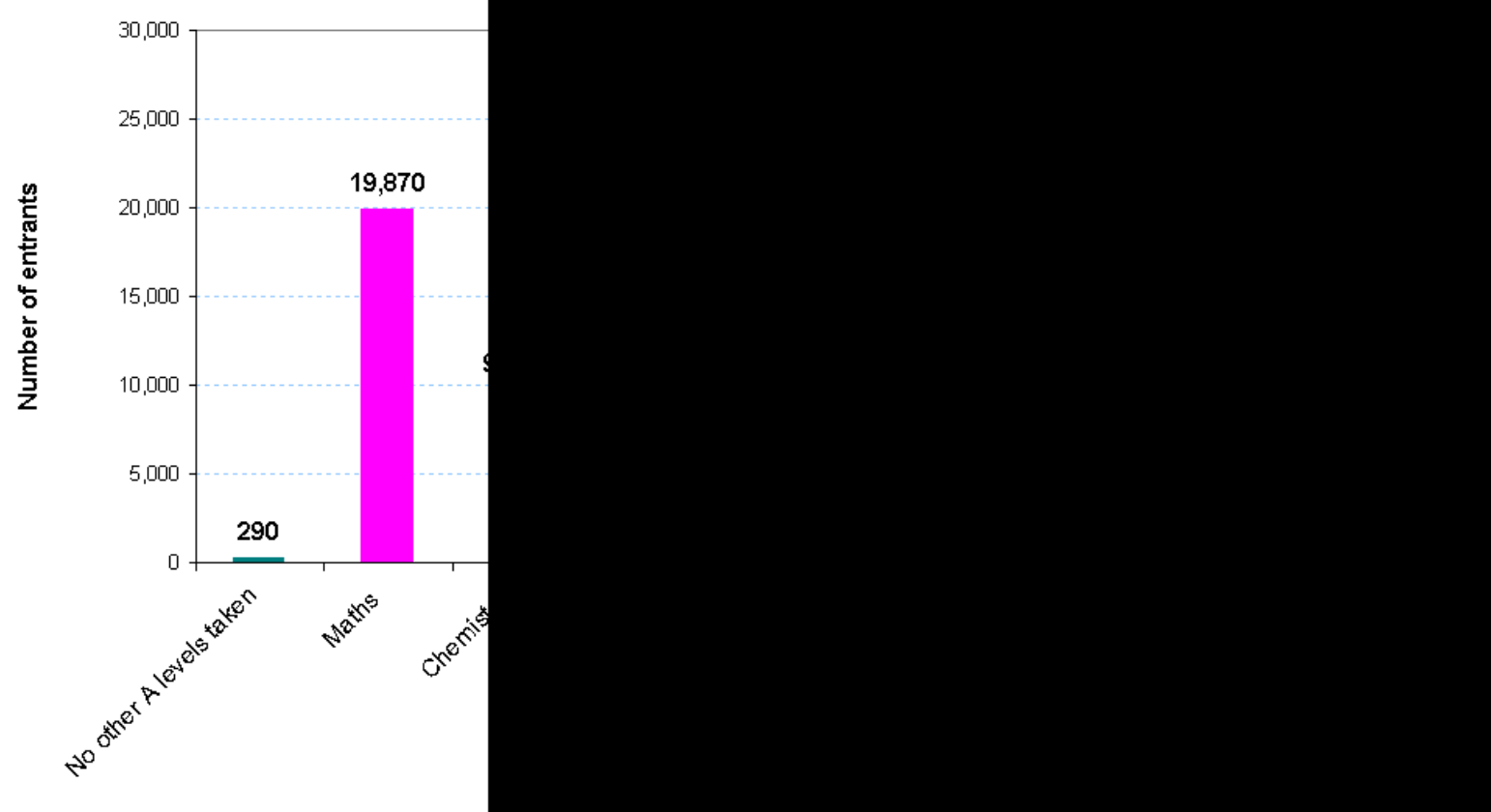

5.4 How Hard is it to Achieve a Top Grade in Maths and Science?

Subject options at A level may also be influenced by the individual entrant's perception of how likely they are to achieve a good grade in each subject that they are considering. Chapter 2 has demonstrated the key impact of prior attainment in 
determining the chances of securing a grade A or B at A level, which feeds into the entrant's decision process. However, whether based in perception alone, or also in objective reality, maths in particular is sometimes believed to be a relatively difficult subject $^{5}$. One way of assessing whether there is any truth in this belief is to analyse the rates of success in securing top grades by the most able entrants for each subject. This analysis is undertaken in figure 5.4a for maths, biology, chemistry and physics, plus a selection of other subjects.

By holding constant the prior attainment for each subject at grades $A^{*}$ or $A$, some indication of how challenging the selected $A$ levels are can be deduced. On the right of the chart appear the subjects for which the fewest entrants with high prior attainment were able to secure a grade A or B at A level. These can be characterised as relatively more difficult. Of the selected subjects, physics and biology appear at the more difficult end of the scale, with $63 \%$ and $66 \%$ of high prior attaining entrants managing to secure a top grade. By contrast, English and maths appear at the less difficult end of the scale, with $74 \%$ and $70 \%$ of high prior attainers respectively gaining grades $A$ or $B$. Chemistry appears in the middle of the scale with $67 \%$ of high prior attainers achieving top grades. This analysis may not capture the full sense of what people mean when they discuss the difficulty of different subjects, but does address the objective probabilities of success controlling for prior attainment in the subject.

\section{Fig. 5.4a}

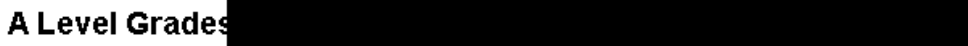

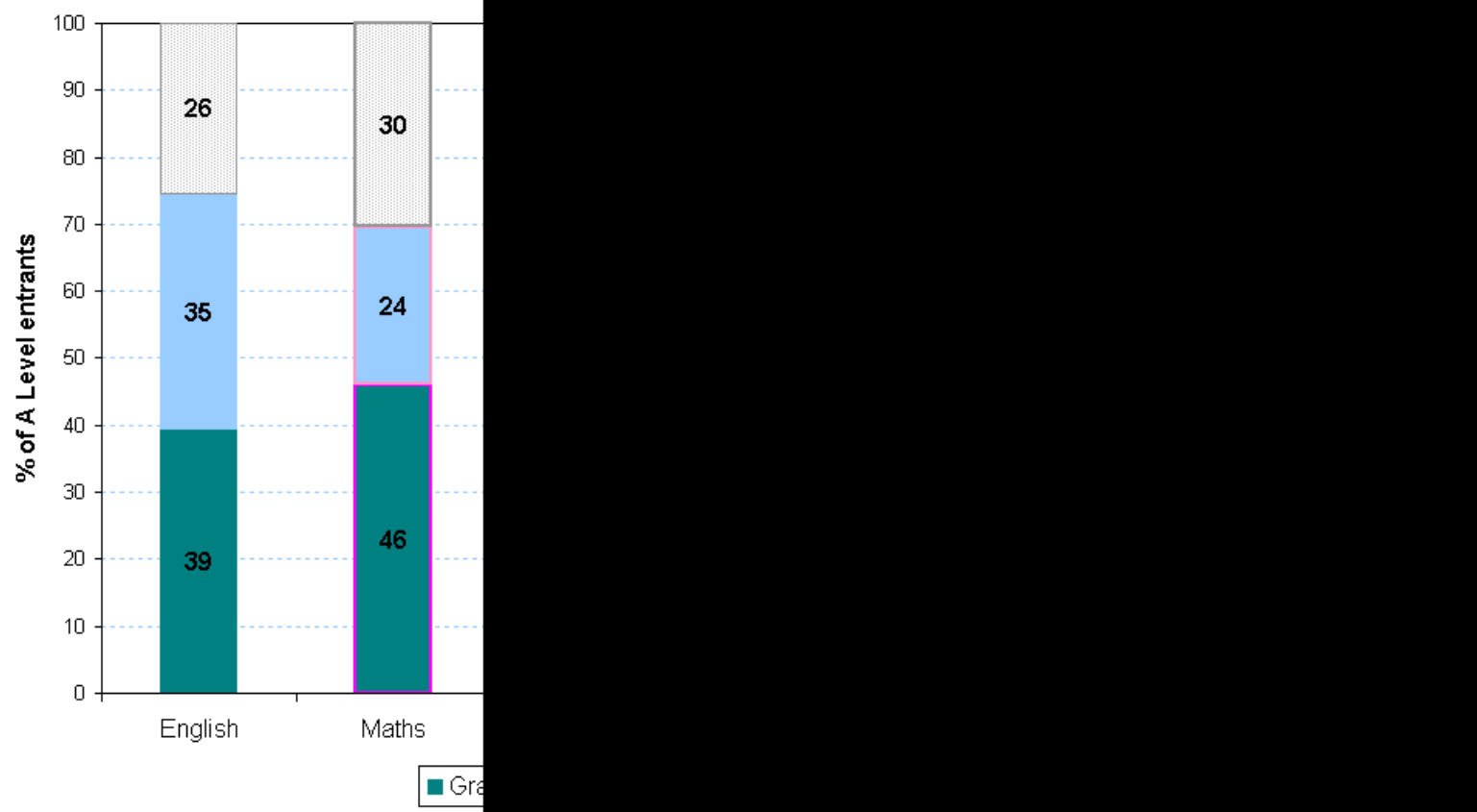

\footnotetext{
${ }^{5}$ Qualifications and Curriculum Authority, (2007) Evaluation of participation in GCE Mathematics. QCA.
} 


\subsection{Asymmetric Point Scores}

Continuing with the theme of subject difficulty, but taking an individual approach rather than a general one, this section investigates the proportions of pupils whose GCSE results suggest that they find maths or science subjects relatively easy or difficult. A factor which might influence subject choice, related to prior attainment in the subject in question, is the relative strength of a candidate's ability in that subject compared with their complete range of prior attainment across subjects. To illustrate the logic of this proposition, if a young person is good at maths, and not very good at other subjects, we might expect them to be more likely to choose to continue studying maths than another young person who is equally good at maths, but also attained strongly in nine other subjects at GCSE. This proposition is tested in Chapter 6 , where a factor for relative subject strength is entered in the models to predict A level entry and achievement in maths and the sciences (see figure $6.5 \mathrm{f}$ in particular). In this section, the distributions of GCSE grades achieved for selected subjects, relative to each entrant's average GCSE grade, are presented to illustrate which subjects entrants are most likely to have asymmetric (or unusual) scores in.

Figure 5.5a plots the percentage frequencies for achieving a GCSE grade that is a certain number of grades higher or lower than the entrant's average grade across all the subjects they took. The bar labelled "- 1 grade" includes all cases where the subject grade was between zero and one grade lower than the pupil's average grade, and is the most common outcome for all subjects. The bar labelled "-2 grades" contains the pupils whose subject grade was more than one grade and up to two grades lower than their average grade, and so forth. All the darker shaded bars on the right of the "- 1 grade" bar are therefore made up of cases where the pupil has a relative strength in that subject; bars to the left of the "-1 grade" bar can be considered to be cases where the pupil has a relative weakness.

The separate sciences are selective in the sense that they tend to attract higher achieving entrants, and history and Modern Foreign Languages are selective in the sense that they are not compulsory subjects, whereas maths and English GCSEs are taken by virtually all pupils. This may influence the distributions. Keeping this in mind and considering the seven subjects in figure $5.5 \mathrm{a}$, maths, the three separate sciences, and English are skewed towards relative subject strength for a greater proportion of pupils than relative weakness. This is strongest for the sciences, where the percentages of pupils with relative strength outnumber the percentages with relative weakness by 11 to 13 percentage points. For maths, the distribution is less skewed, with relatively strong pupils outnumbering relatively weak pupils by 2 percentage points; for English, the strong outnumber the weak by 11 percentage points, on a similar scale to the sciences despite the subject being compulsory at GCSE.

History and Modern Foreign Language entrants are skewed towards relative subject weakness, rather than relative subject strength, despite these subjects being optional at GCSE. For History, the percentage of pupils with relative subject weakness outnumbers the percentage with relative strength by 11 percentage points; for MFL, this is strikingly more pronounced at 25 percentage points. Looking back to the subject entry trends in section 5.1, this relative subject weakness in MFL coincides with a comparatively small and shrinking number of A level entries, but for history, 
the opposite is the case as A level entries are growing. English (including English Literature) is the most popular A level subject and entries have grown in recent years, coinciding with the skew towards relative subject strength.

The A level sciences have fared differently from one another with physics entries remaining fragile while biology entries are strong, and chemistry entries are recovering well. The fact that all separate GCSE sciences have relative subject strengths is probably linked to the selective nature of the separate sciences, but the order of strength in A level entries is still reflected in the slight differences in the size of the relative subject strengths at GCSE.

Maths A level entries are strong and growing, having recovered from decreases in the last decade, but the relative subject strength at GCSE is only slight compared with English, which has similar growth in A levels. Across the subjects included in the analysis, there is some coincidence between A level growth and distributions of GCSE results that skew towards relative subject strength rather than weakness, but the pattern is imperfect, reflecting the many other factors that influence subject choice at A level.

\section{Fig. 5.5a}
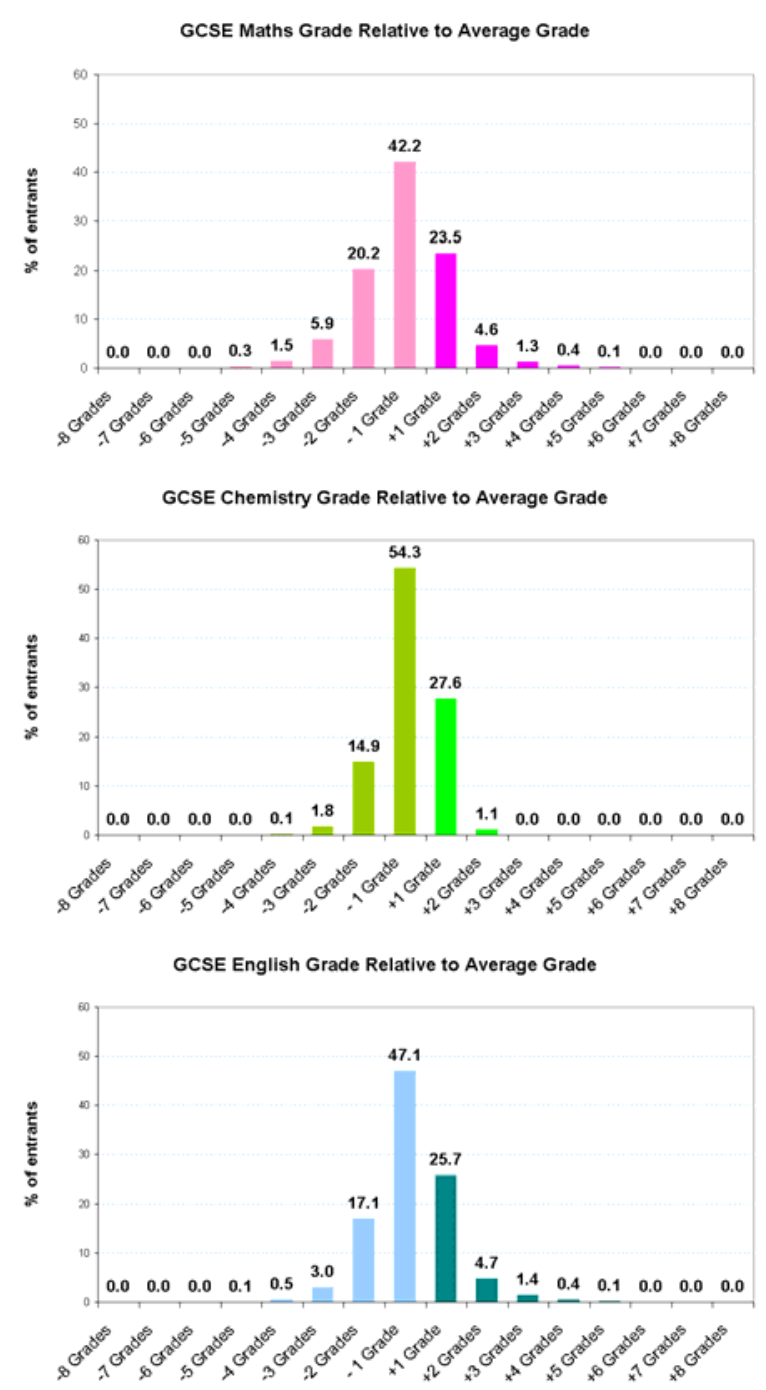
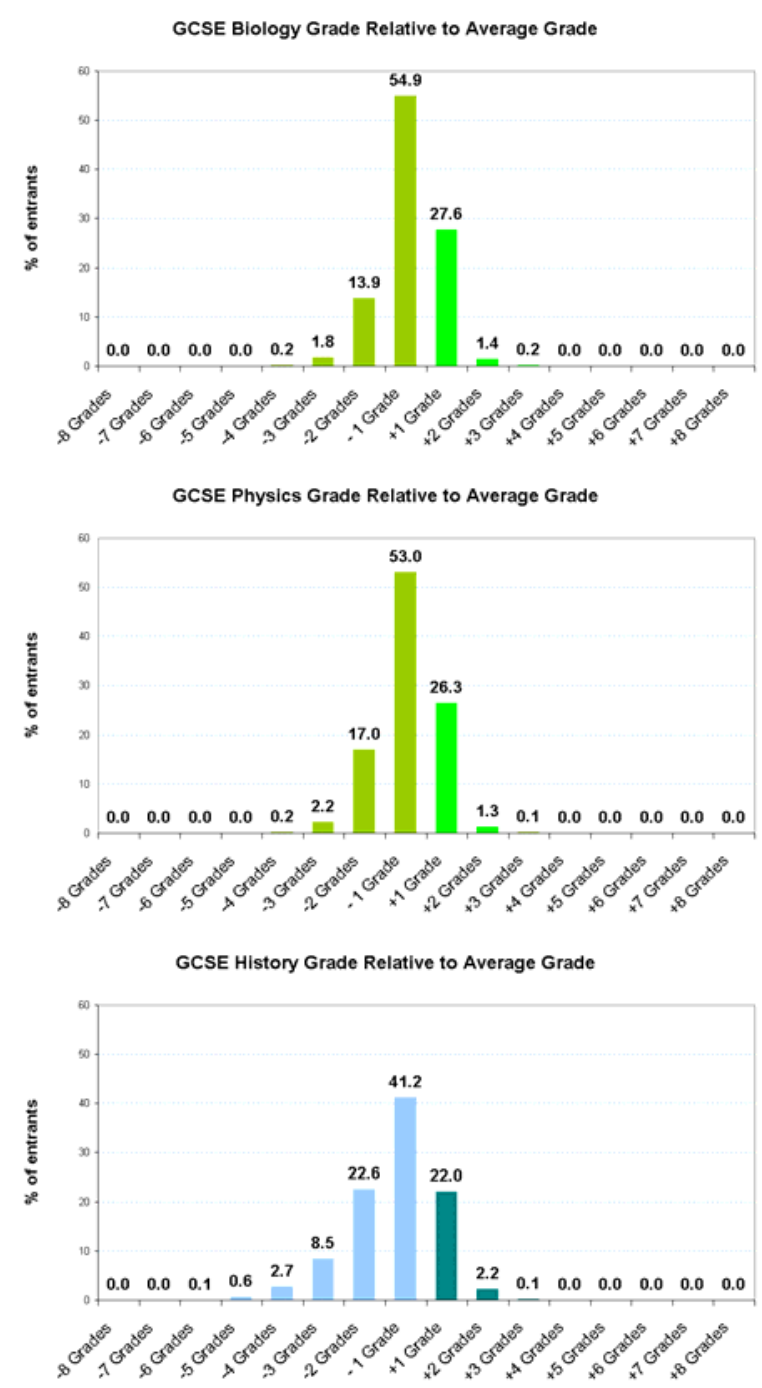


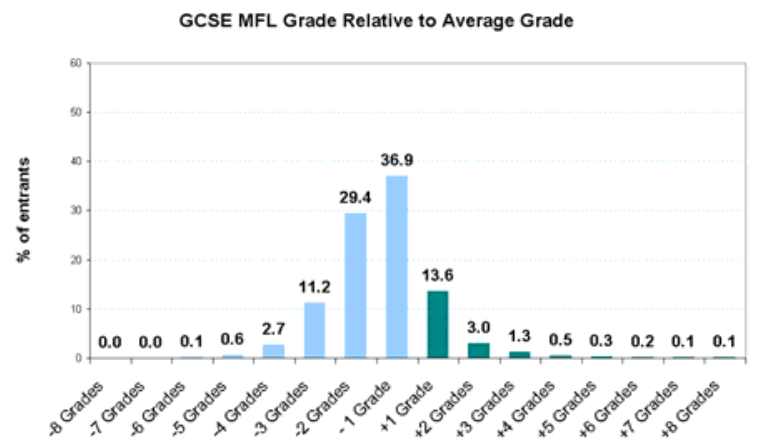




\section{Chapter 6. Modelling Maths \& Science Uptake \& Attainment}

\subsection{Introduction to the Models}

This chapter will examine the factors associated with uptake and achievement of high grades in A level maths, biology, chemistry and physics. Logistic regression modelling is used as an analytic framework to identify and control for a range of relevant factors from administrative and survey data. The National Pupil Database is used for the basic models, which encompass pupils' attainment histories, individual characteristics and characteristics of the schools they have attended. The models are scoped to cover pupils who attended a maintained mainstream school in England during their Key Stage 4 GCSE exam year. Three variants of the models are presented for each of the maths and science subjects, which have varying scopes in order to focus in on different aspects of the selection of pupils into these A levels, and into the high attaining subset within A level maths and science subjects. A 95\% confidence level is applied to factors from the main administrative data models.

Additionally, the twelve administrative models have been extended using survey data from the Longitudinal Study of Young People in England (LSYPE) to test a wider range of factors. The administrative model results are presented for 2008 and 2009 A level cohorts, with the discussion focusing on the 2009 results. The LSYPE-based model extensions use the 2008 A level cohort, which coincides with the timing of the cohort sampled for the survey. Factors from the survey data are tested at both the $90 \%$ and $95 \%$ confidence levels.

\section{The Model Variants}

For each subject, two models of A level entry and one model of A level achievement are reported in sections 6.2 to 6.4 .

The first of the entry models, referred to as "KS4 MM Pupils" includes the whole of the Key Stage 4 cohort from maintained mainstream schools in 2006/07, who went on to form the potential A level cohort of 2008/09. This model variant focuses on the complete decision process for continuing in education post-16, choosing to study via the A level route, and selecting a maths or science subject. It therefore presents factors which weed pupils out of further study altogether, as well as those who simply choose other subjects or qualification types at Key Stage 5.

The second entry model variant, "KS4 MM with GCSE $A{ }^{*}-B, 1+A$ level entries", narrows the field of pupils down to those who obtained grade $B$ or higher in the relevant subject at GCSE (maths or science, including double science as well as separate science entries), and to those who continued in education post-16 and entered at least one A level. In this way, issues of insufficient prior attainment and the choice between further education and work are reduced within the model, and it focuses mainly on A level subject choice. This variant therefore views maths and science subjects in the context of competition with other possible A level options.

During model development, a third entry model variant which limited the pupils in the model to those entering at least one A level, but did not filter for prior attainment at GCSE was also tested, but produced results which were very similar to the second entry model variant presented, and so is not reported separately here. 
The model variant for A level achievement, "KS4 MM and 1+ A level entries, Grade $A / B$ ", models success at grade B or higher at A level in the relevant subject, and is scoped to include pupils from the maintained mainstream GCSE cohort who went on to enter at least one A level (with no restriction on prior attainment at GCSE). This variant therefore focuses on the selection of pupils from the A level cohort, who chose to study maths or science, and achieved a high grade in that subject. Elements of both subject choice and attainment are modelled using the same set of factors that were tested for predicting A level entry.

Figure 6.1a presents the range of factors included in the set of twelve models described above. The factors are listed on the left-hand side of the table and their respective reference categories in each model are described on the right. The main difference between the maths and science models is the inclusion of factors capturing the GCSE science route taken in the latter. Factors are entered for having sat separate sciences at GCSE, for whether the KS4 school attended offered both separate and double science options, and an interaction term for the coincidence of both of these factors.

For brevity, pupils attending schools which offered both separate and double science at GCSE are characterised by the GCSE route factor labelled "choice". However, it should be born in mind when interpreting the results that the models are unable to distinguish how the selection of pupils into separate sciences (rather than double science) takes place within these schools - it may be that there is no actual choice made by the pupil; it is possible that schools determine which pupils are suitable candidates for separate science GCSEs. 
Fig. 6.1a

\begin{tabular}{|c|c|c|c|c|c|c|c|c|c|c|c|c|c|}
\hline & \multirow{3}{*}{ Factors in the Models } & \multicolumn{8}{|c|}{ A Level Entry Mlodels } & \multirow{2}{*}{\multicolumn{4}{|c|}{$\begin{array}{l}\text { A level Achievement Mlodels (grade A or B } \\
\text { s KS4 MHA and 1+ A level entries }\end{array}$}} \\
\hline & & \multicolumn{4}{|c|}{ KS4 MMH pupils } & \multicolumn{4}{|c|}{ KS4 MHI with GCSE $A^{*}-B, 1+$ A level entries } & & & & \\
\hline & & Maths & Biology & Chemistry & Physics & Maths & Biology & Chemistry & Physics & Maths & Biology & Chemistry & Physics \\
\hline \multirow{5}{*}{ 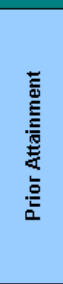 } & \begin{tabular}{|l} 
GCSE Grade A* \\
GCSE Grade A
\end{tabular} & \multirow{2}{*}{\multicolumn{4}{|c|}{ GCSE grade C-G or unclassified }} & \multirow{2}{*}{\multicolumn{4}{|c|}{ GCSE grade B }} & \multirow{2}{*}{\multicolumn{4}{|c|}{ GCSE grade C-G or unclassified }} \\
\hline & GCSE Grade B & & & & & & & & & & & & \\
\hline & $\begin{array}{l}\text { Maths GCSE Grade > average } \\
\text { grade across subjects entered }\end{array}$ & $\begin{array}{c}\text { Subject } \\
\text { Average } \\
>=\text { Maths }\end{array}$ & $\begin{array}{c}\text { Subject } \\
\text { Average } \\
>=\text { Biology } \\
\text { / Science }\end{array}$ & \begin{tabular}{|c|} 
Subject \\
Average \\
$>=$ \\
Chemistry \\
/ Science \\
\end{tabular} & $\begin{array}{c}\text { Subject } \\
\text { Average } \\
>= \\
\text { Physics / } \\
\text { Science }\end{array}$ & $\begin{array}{l}\text { Subject } \\
\text { Average } \\
>=\text { Maths }\end{array}$ & $\begin{array}{c}\text { Subject } \\
\text { Average } \\
>=\text { Biology } \\
\text { / Science }\end{array}$ & $\begin{array}{c}\text { Subject } \\
\text { Average } \\
>= \\
\text { Chemistry } \\
\text { / Science }\end{array}$ & \begin{tabular}{|c|} 
Subject \\
Average \\
$>=$ \\
Physics / \\
Science \\
\end{tabular} & $\begin{array}{c}\text { Subject } \\
\text { Average } \\
>=\text { Maths }\end{array}$ & $\begin{array}{c}\text { Subject } \\
\text { Average } \\
>=\text { Biology } \\
\text { / Science }\end{array}$ & $\begin{array}{c}\text { Subject } \\
\text { Average } \\
>= \\
\text { Chemistry } \\
\text { / Science }\end{array}$ & $\begin{array}{c}\text { Subject } \\
\text { Average } \\
>= \\
\text { Physics / } \\
\text { Science }\end{array}$ \\
\hline & KS3 Level 7+ & L5 or & \multirow{2}{*}{\multicolumn{3}{|c|}{ L5 or below in Science }} & & \multirow{2}{*}{\multicolumn{3}{|c|}{ L5 or below in Science }} & & \multirow{2}{*}{\multicolumn{3}{|c|}{ L5 or below in Science }} \\
\hline & KS3 Level 6 & $\begin{array}{l}\text { below in } \\
\text { Maths }\end{array}$ & & & & $\begin{array}{l}\text { below in } \\
\text { Maths }\end{array}$ & & & & $\begin{array}{l}\text { below in } \\
\text { Maths }\end{array}$ & & & \\
\hline \multirow[b]{2}{*}{ 岂 苛 } & Separate Science at GCSE & \multirow[b]{2}{*}{$\begin{array}{c}\text { Factor not } \\
\text { entered }\end{array} \mid$} & \multirow{2}{*}{\multicolumn{3}{|c|}{$\begin{array}{l}\text { Double or Vocational Science } \\
\text { without Choice (school has no } \\
\text { separate science entries) }\end{array}$}} & & \multirow{2}{*}{\multicolumn{3}{|c|}{$\begin{array}{l}\text { Double or Vocational Science } \\
\text { without Choice (school has no } \\
\text { separate science entries) }\end{array}$}} & & & & \\
\hline & $\begin{array}{l}\text { Choice } \\
\text { (Separate + Double Science) } \\
\text { Separate Science with Choice }\end{array}$ & & & & & $\begin{array}{c}\text { Factor not } \\
\text { entered }\end{array}$ & & & & $\begin{array}{c}\text { Factor not } \\
\text { entered }\end{array}$ & without 0 & hoice (scho & entries) \\
\hline & Boys & & & & & & & & & & & & \\
\hline & Free School Meals & & & & & & & & & & & & \\
\hline & Irish & & & & & & & & & & & & \\
\hline & Other White & & & & & & & & & & & & \\
\hline & White \& Black Caribbean & & & & & & & & & & & & \\
\hline & White \& Black African & & & & & & & & & & & & \\
\hline & White \& Asian & & & & & & & & & & & & \\
\hline$\stackrel{9}{=}$ & Other Mixed & & & & & & & & & & & & \\
\hline$\underline{\underline{m}}$ & Indian & & & & & & & & & & & & \\
\hline$\overline{\mathrm{g}} \mathrm{u}$ & Pakistani & & & & White Britis & sh Girls, not & eligible for $F$ & SM, no Spe & ecial Educati & ational Needs & & & \\
\hline 苋 & Bangladeshi & & & & & & & & & & & & \\
\hline 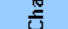 & Other Asian & & & & & & & & & & & & \\
\hline & Black Caribbean & & & & & & & & & & & & \\
\hline & Black African & & & & & & & & & & & & \\
\hline & Other Black & & & & & & & & & & & & \\
\hline & Chinese & & & & & & & & & & & & \\
\hline & Other Ethnic Background & & & & & & & & & & & & \\
\hline & SEN Statement or Action Plus & & & & & & & & & & & & \\
\hline & SEN School Action & & & & & & & & & & & & \\
\hline$\overline{\mathrm{g}}$ & $\begin{array}{l}\text { Maths \& Computing [/ Science] } \\
\text { Specialist }\end{array}$ & & & & & & & & & & & & \\
\hline 흠 & \begin{tabular}{|l|} 
Grammar \\
\end{tabular} & Mixed, no & n-selective & school, no si & sixth form, & & Mixed no & on-selective & school no s & sixth form $n$ & ot Science & specialist & \\
\hline 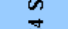 & Girls School & not $\mathrm{M}$ & aths and $\mathrm{Cot}_{0}$ & mputing spe & ecialist & & Mixed, no & on-selective & schood, no s & Sixth form, $\mathrm{n}$ & lot Sclence & speclalist & \\
\hline 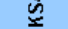 & Boys School & & & & & & & & & & & & \\
\hline & Sixth Form & & & & & & & & & & & & \\
\hline 政 & Entered 4 A levels & & Factor no & t entered & & & & & pils with 1-3 & 3 A level entri & & & \\
\hline$x$ & Entered 5+ A levels & & & & & & & & & & & & \\
\hline & $\begin{array}{l}\text { School Offers 16-30 A level } \\
\text { Subjects }\end{array}$ & & & & & & & & & & & & \\
\hline & $\begin{array}{l}\text { School Offers } 31+\text { A level } \\
\text { Subjects }\end{array}$ & & & & & & & & & & & & \\
\hline $\bar{\Xi}$ & 11-20 Maths [/Biology / & & & & & & & & & & & & \\
\hline$\frac{E}{\breve{U}}$ & Chemistry / Physics] Entries & & Factorno & it entered & & Pupils at $s$ & schools offeri & ing $1-15$ sub & jects at A le & level, and wit & th $0-10$ entri & es for Maths & $s[B i o l o g y /$ \\
\hline$\underset{n}{0}$ & $\begin{array}{l}\text { 21-30 Maths [/Biology / } \\
\text { Chemistry / Physics] Entries }\end{array}$ & & Factor no & & & & & & emistry / $\mathrm{Pr}$ & Physics] A le & & & \\
\hline & 31-40 Maths [/Biology / & & & & & & & & & & & & \\
\hline & Chemistry / Physics] Entries & & & & & & & & & & & & \\
\hline & $\begin{array}{l}\text { 41+ Maths [/Biology / } \\
\text { Chemistry / Physics] Entries }\end{array}$ & & & & & & & & & & & & \\
\hline & Traveller of Irish Heritage & & & & & & & & & & & & \\
\hline 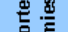 & Gypsy/Romany & Entered to & all models & to avoid cont & tamination & of the referer & nce categoris & es by unusu & Jal cases. $T$ & Traveller of $\mathrm{Ir}$ & rish Heritage & and Gypsy & / Romany \\
\hline 훙 & Unclassified ethnicity & ethnic cate & egories wer & too small to & 0 produc & ffects signific & cantly differe & nt from Whi & ite British; U & Unclassified & hnicity, ur & 10wn KS3 & levels and \\
\hline 岂 & KS3 level unknown & & & & & & 46 & 10 & licy interest. & & & & \\
\hline & SEN status unknown & & & & & & & & & & & & \\
\hline
\end{tabular}

\section{Data Source for the Model Extensions}

LSYPE is a panel study, managed by DfE, which involves over 15,000 young people. They and their families have been interviewed annually since 2004, at which point they were aged 13 or 14, making them the cohort of pupils who reached the end of KS4 in 2006. The analysis in this report, (with the exception of the receipt of Education Maintenance Allowance at age 17), is based on questions asked in that first wave of the study when the young people were half way through secondary school.

LSYPE collects a very broad range of information, including details about parental background as well as the attitudes, behaviours and aspirations of the young person and their parent. This data has been matched to the National Pupil Database (NPD), which contains pupil level attainment as well as both pupil and school characteristics, 
such as ethnicity, free school meals (FSM) eligibility, information about Special Education Needs (SEN) and school level prior attainment.

The two datasets together allow comparison of the relative importance of the different types of factors in determining maths and science A level outcomes. Factors can be weighed against one another, for example, how subject preferences at 14 compare with gender, how family composition compares with a pupil's ethnicity, and how a pupil's locus of control compares with their prior attainment.

After the twelve models, including their survey data extended versions, have been illustrated in sections 6.2 to 6.4, the importance of the factors across subjects and model variants is compared in section 6.5. Here, examples of how the odds effects presented for the models translate into the probability of entering A level maths or science, or of achieving grade $A$ or $B$, are described. Further factors which were tested but found to be unimportant in predicting maths and science A level outcomes are also listed.

\subsection{A level Entry for KS4 MM Pupils}

As described in the previous section, the A level entry model for the complete Key Stage 4 cohort from maintained mainstream schools includes the wider decisions faced by pupils post-16 of whether to continue their education, which qualification route to follow, and which subjects to study.

Beginning with maths in figure 6.2a, the following charts illustrate the odds effects of the model factors on entry to A level for each of the four maths and science subjects. The value labels give the odds factors for 2009, while the lighter coloured bars depict the 2008 effects for comparison purposes. The bars representing the odds effects appear on a logarithmic scale. This is in order to balance the influence of factors with negative effects (odds of less than 1) against those of factors with positive effects (odds of greater than 1). Charted on a logarithmic scale, negative factors appear with the same bar length as positive factors of equivalent importance.

Where the characteristic has a factor of more than one, a pupil displaying this characteristic is more likely to continue to maths A level, all other things being equal. For example, boys in the cohort have a factor of 2.2, meaning the odds of entering $A$ level maths are $120 \%$ higher than those for girls in the cohort. All effects for which a bar appears in the chart were statistically significant at the 5\% level.

Prior attainment at GCSE has the largest influence on whether the pupils will continue to A level maths. The odds of a pupil with grade A at GCSE maths are approximately four hundred times the odds for a pupil who achieved grade $C$ or below at GCSE. The individual factors can be multiplied together to give an overall effect for pupils with a given combination of characteristics. Section 6.5 below explores some combinations of characteristics across the models, and translates these odds effects into probabilities by combining them with the background level of probability for e.g. entering A level maths.

From figure 6.2a, it can be seen that the higher a pupil's prior attainment in maths, the greater their odds of entering for A level. Key Stage 3 maths attainment has an 
independent effect on A level entry over and above high grades at GCSE, with pupils who achieved level 7 or higher having four times the odds of continuation of those with level 5 or below at KS3, all other things (including GCSE grade) being equal. Boys and certain ethnic groups were also more likely to enter A level maths, with pupils of Indian, Other Asian or Chinese ethnic backgrounds having odds of continuation around four times higher than those of White British pupils. There were no school types with large odds effects on A level maths entry after prior attainment and the other factors were controlled. 
Fig. 6.2a

\section{Who Enters A Level Maths? \\ (all pupils in the maintained mainstream KS4 cohort)}

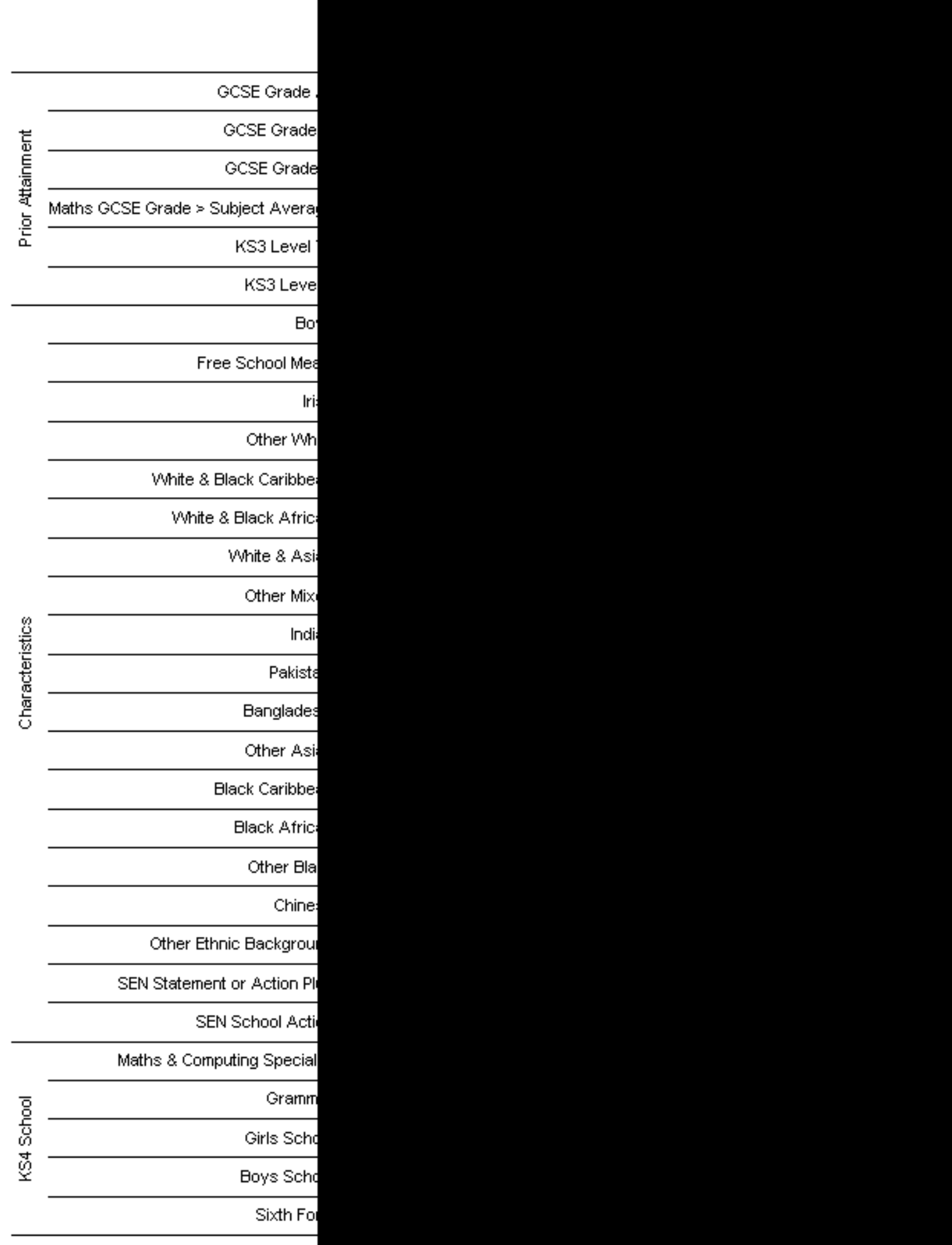


Figures $6.2 \mathrm{~b}-\mathrm{d}$ display the odds effects for the same set of factors on entry of KS4 pupils to A level in biology, chemistry and physics. Prior attainment in the science subject in question (or in GCSE double science) remains the most critical factor in driving uptake of science A levels as was the case for maths. In addition to withinsubject prior attainment, small increases in the odds of continuation are seen for pupils where the science in question was one of their stronger GCSE subjects. This factor is entered into the model flagging where the GCSE science grade was higher than the pupil's average GCSE grade, indicating relative strength in that subject.

Pupils who sat separate science GCSEs in schools where this was the only route on offer had twice the odds of going on to enter A level biology as pupils who sat double science GCSE in schools where that was the only route offered. Multiplying the additional effects of attending a school where both routes were offered into this reveals that the presence of both science routes results in odds of continuation that are 2.2 times higher for those who take separate sciences. The odds of continuation are lower for pupils who sat double science in schools where both routes were offered, at $80 \%$ of the odds of pupils in schools where double science was the only route taught.

The odds effects resulting from the GCSE science route variations for A level physics entry were the same size as those for biology. However, the separate science effects were stronger for chemistry, where pupils in schools only offering separate sciences had odds 2.5 times as large as pupils in schools where only double science was taught. Choosing or being selected for separate science within a school offering both routes resulted in odds that were 3 times as large, whereas choosing or being selected for double science gave odds that were only $70 \%$ as large.

The odds for A level entry by boys were only $60 \%$ of those for girls in biology, but $20 \%$ higher than the odds for girls in chemistry and 6 times as large as the odds for girls in physics.

The ethnic groups with the highest odds of entering A level sciences were Pakistani, Other Asian and Indian for biology; Pakistani, Other Asian and Black African for chemistry; and (with a smaller odds effect) Chinese in the case of Physics. Pupils with Special Educational Needs were slightly less likely to continue to A levels in biology or chemistry, but slightly more likely to enter A levels in maths or physics after their prior attainment had been controlled for, e.g. comparing a pupil with no SEN and GCSE grade B with a pupil at with needs at School Action level, but also with a GCSE grade B. Attending a Key Stage 4 school with a sixth form had small but consistent positive effects on the odds of continuing to maths or science A levels. 
Fig. 6.2b

\section{Who Enters A Level Biology? (all pupils in the maintained mainstream KS4 cohort)}

Effect on Odds (Logarithmic Scale)

0.1

1

10

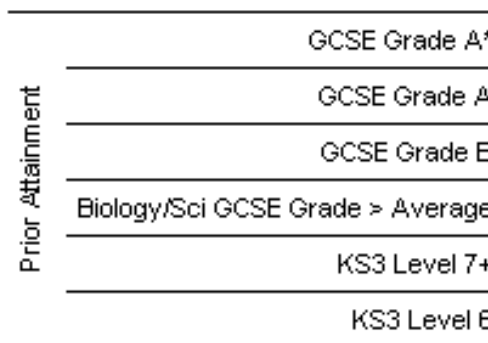

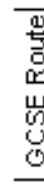

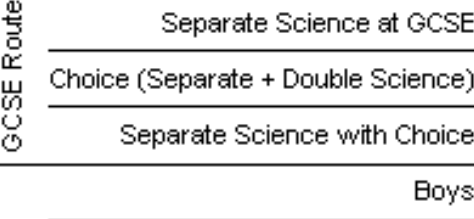

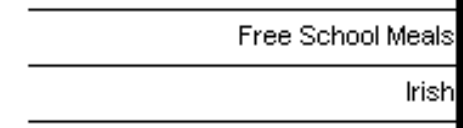

Other White

White \& Black Caribbean

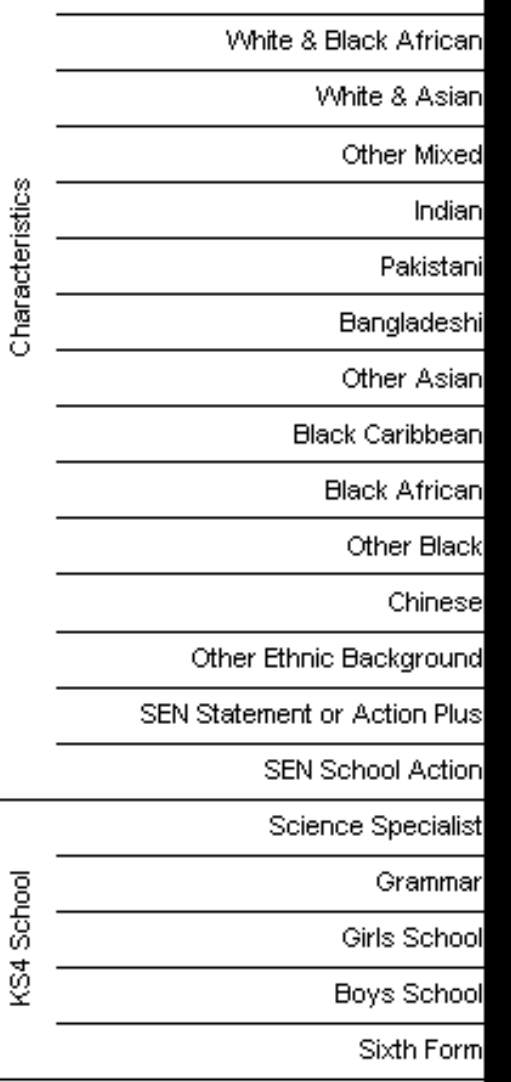

口 $2009 \square 2008$

$\leftarrow$ Less Likely ... More Likely $\rightarrow$ 
Fig. 6.2c

\section{Who Enters A Level Chemistry? (all pupils in the maintained mainstream KS4 cohort)}

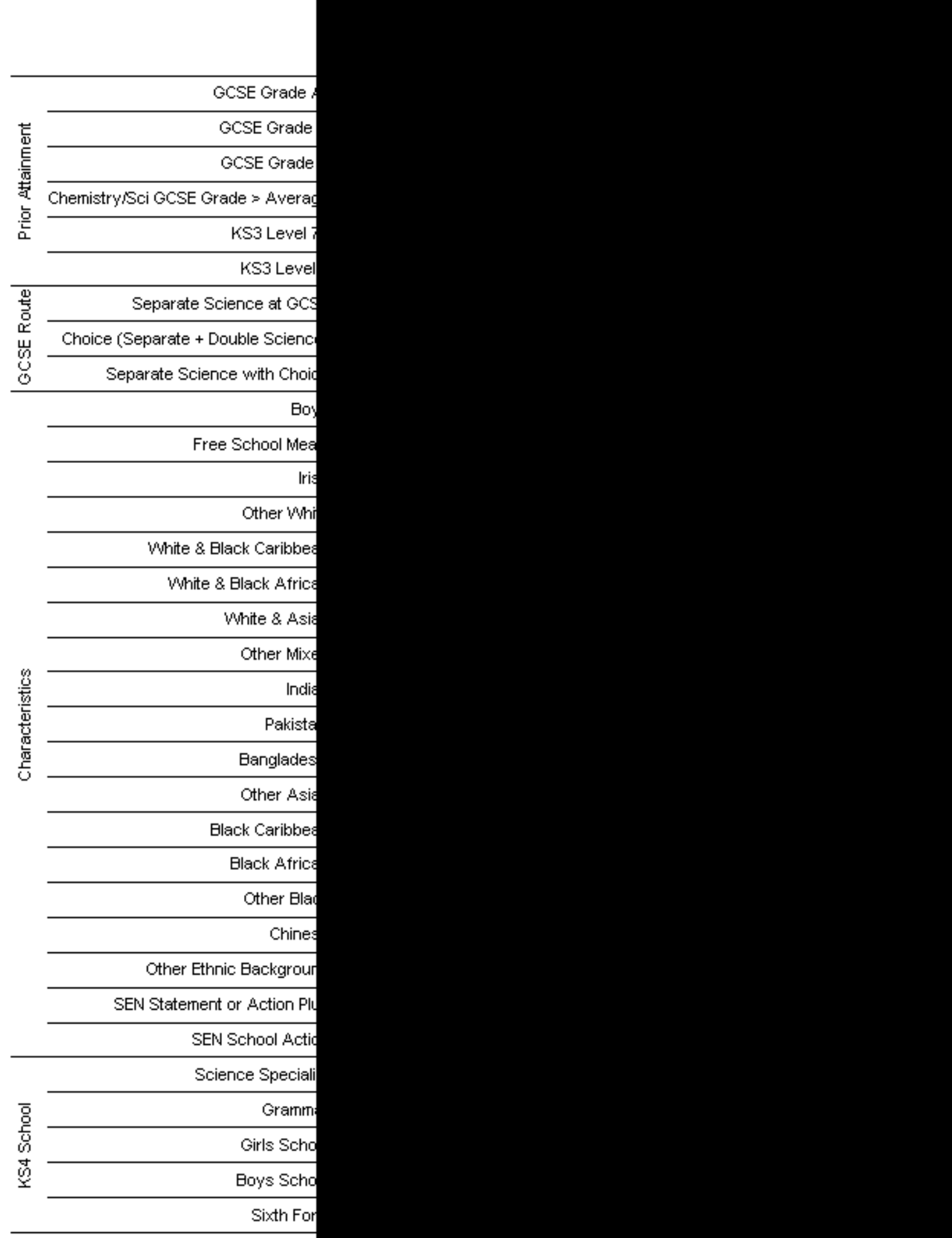


Fig. 6.2d

\section{Who Enters A Level Physics? \\ (all pupils in the maintained mainstream KS4 cohort)}

Effect on Odds (Logarithmic Scale)

0.1

1

10

100

1000

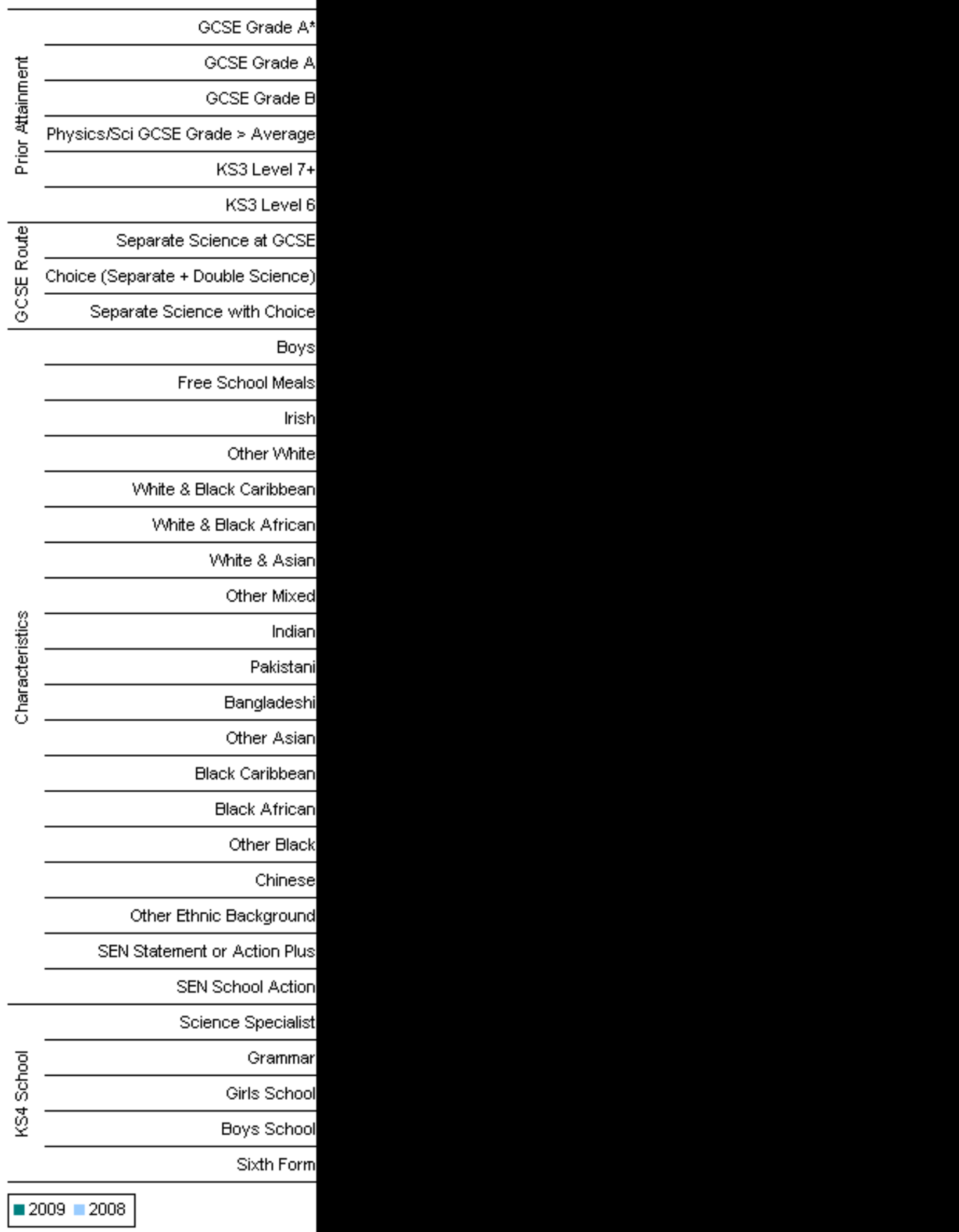


The Key Stage 4 to A level entry models are further explored using data from LSYPE to test for the significance of additional factors derived from this richer sampled survey source. In the charts in figures $6.2 \mathrm{e}-\mathrm{h}$, the dark blue bars represent prior attainment within subject, the strongest factors from the administrative data models. These are shown in the charts to enable comparisons of scale with the new model factors. The remaining factors from the administrative data models were still included and controlled for in the new LSYPE versions of the models, but are not displayed in the charts in order to make space for the new factors.

The pale (pink/green) bars represent new factors which were not significant within the model in question; the bright (pink/green) bars, displayed with odds effect value labels, are factors which were significant to at least $p=0.1$ ( $90 \%$ confidence intervals); the error bar "whiskers" depict 95\% confidence intervals $(p=0.05)$. The less stringent $90 \%$ significance level is allowed in the interpretation of the LSYPE model versions in order to pick up effects which are weaker in some model variants, possibly due to the smaller sample size, which ranges from 3,000 to 15,000 depending on the model scope. Further details of the LSYPE factors tested, and those rejected, from the extended models appear in section 6.5.

Enjoyment of science and maths at age 14 were amongst the strongest new factors in the model for A level entry, with enjoyment of science increasing the odds of continuation for all four subjects to 2 - 3 times those of pupils who had not enjoyed science earlier in their secondary education. "Liking maths" also raised the odds of A level entry for maths, chemistry and physics by between $40 \%$ and $140 \%$ compared with pupils who had not enjoyed maths at that stage, but was not linked to A level biology entry.

Pupils who perceived themselves to be hard workers at school at 14 , and those who had an internal locus of control, were also more likely to take A level maths and sciences after completing their GCSEs. Pupils who reported "working as hard as I can" had odds of A level maths, biology and chemistry entry that were $40 \%$ to $100 \%$ larger than pupils who did not identify themselves as hard workers. Pupils who agreed with the statement "if someone is not a success in life, it is usually their own fault" had odds of continuation in chemistry and physics that were $60 \%$ and $40 \%$ (respectively) higher than those who did not agree with the statement.

Family demographic factors were significant in A level continuation for some science and maths subjects. For maths and chemistry, pupils who lived in areas of low deprivation at age 14 had odds of entry that were $30-40 \%$ higher than those of pupils who lived in more deprived areas. There was no deprivation effect for biology or physics, or for any of the other model variants, however. Pupils living with two married or cohabiting adults at 14 were also more likely to enter A level chemistry, and to enter biology A level. The odds of continuation for pupils living in these household structures were $30 \%$ higher for biology and $60 \%$ higher for chemistry, than the odds of entering these A levels for pupils who lived with lone parents or in other types of household.

Family behaviours were also related to A level entry in biology. Pupils who reported that their family ate together on most nights or every night at age 14 had odds of entry raised by $30 \%$ compared with pupils whose family ate together less frequently 
or not at all. Similarly, pupils whose parents reported attending parents evening at school had odds of biology entry that were $50 \%$ higher than pupils whose parents did not report attending these events. These factors were not related to continuation in any of the other sciences or maths. Pupils who reported attending private tuition in school subjects outside of school hours at age 14 had raised odds of entering A level chemistry, $30 \%$ higher than the odds for pupils who had private tuition less frequently or never. Again, this was the only maths or science subject that was statistically linked to this factor.

In addition to the effects of enjoyment of maths and science, there were some significant effects of pupils' belief in their own ability in these subjects at age 14 . These effects persisted after controlling for actual attainment in these subjects. For example, the odds of a KS4 pupil going on to enter A level biology were $60-90 \%$ higher for those who reported that they were "good at maths" or "good at science" earlier in their secondary education, compared with those who did not believe they were good at these subjects. Pupils who reported being "good at science" at age 14 were less likely to enter A level maths, however, after controlling for Key Stage attainment in maths. 
Fig. 6.2e

\section{Who Enters A Level Maths? Additional Factors (maintained mainstream KS4 cohort in LSYPE)}

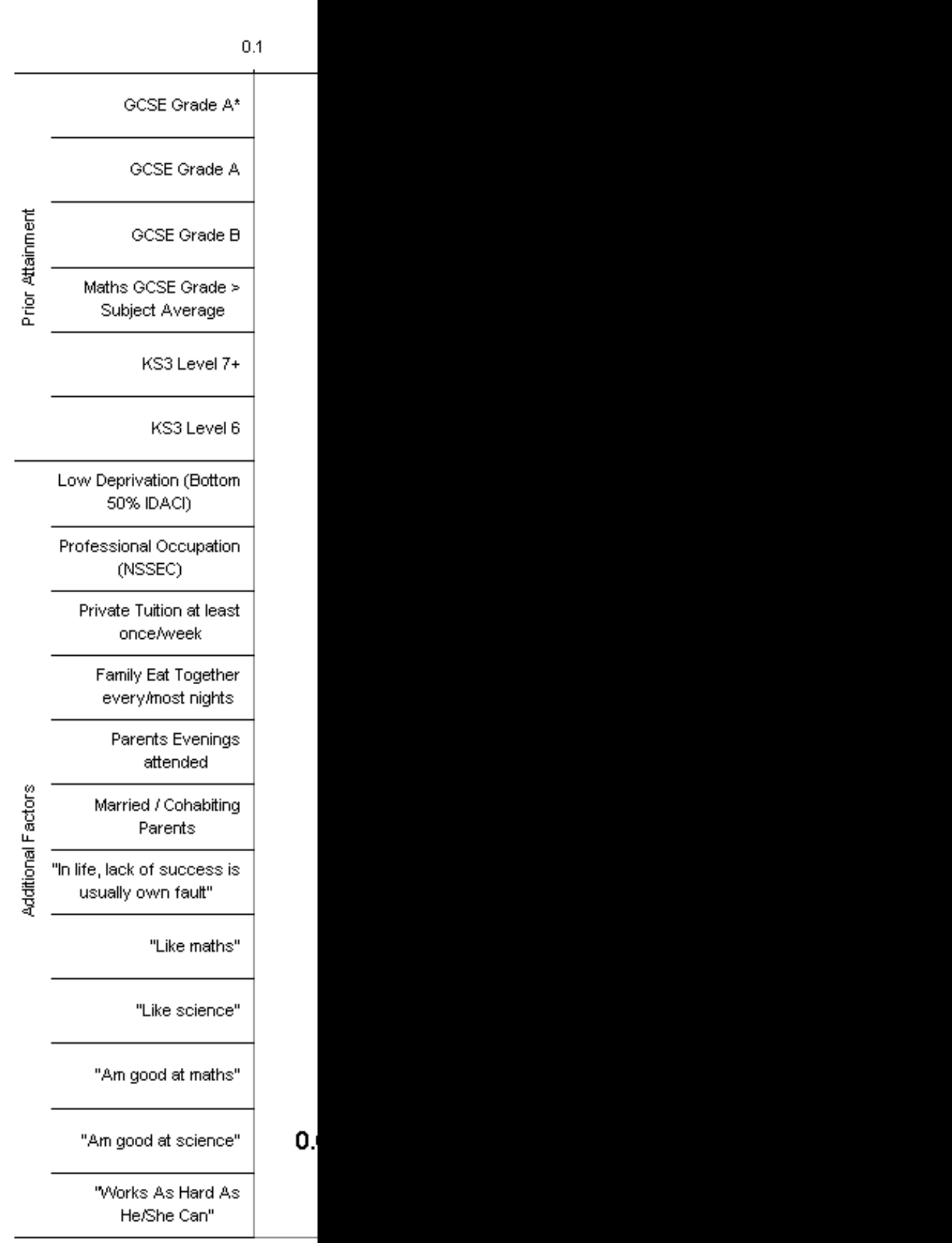

$\leftarrow$ Less Likely ... More Likely $\rightarrow$ 
Fig. 6.2f

\section{Who Enters A Level Biology? Additional Factors (maintained mainstream KS4 cohort in LSYPE)}

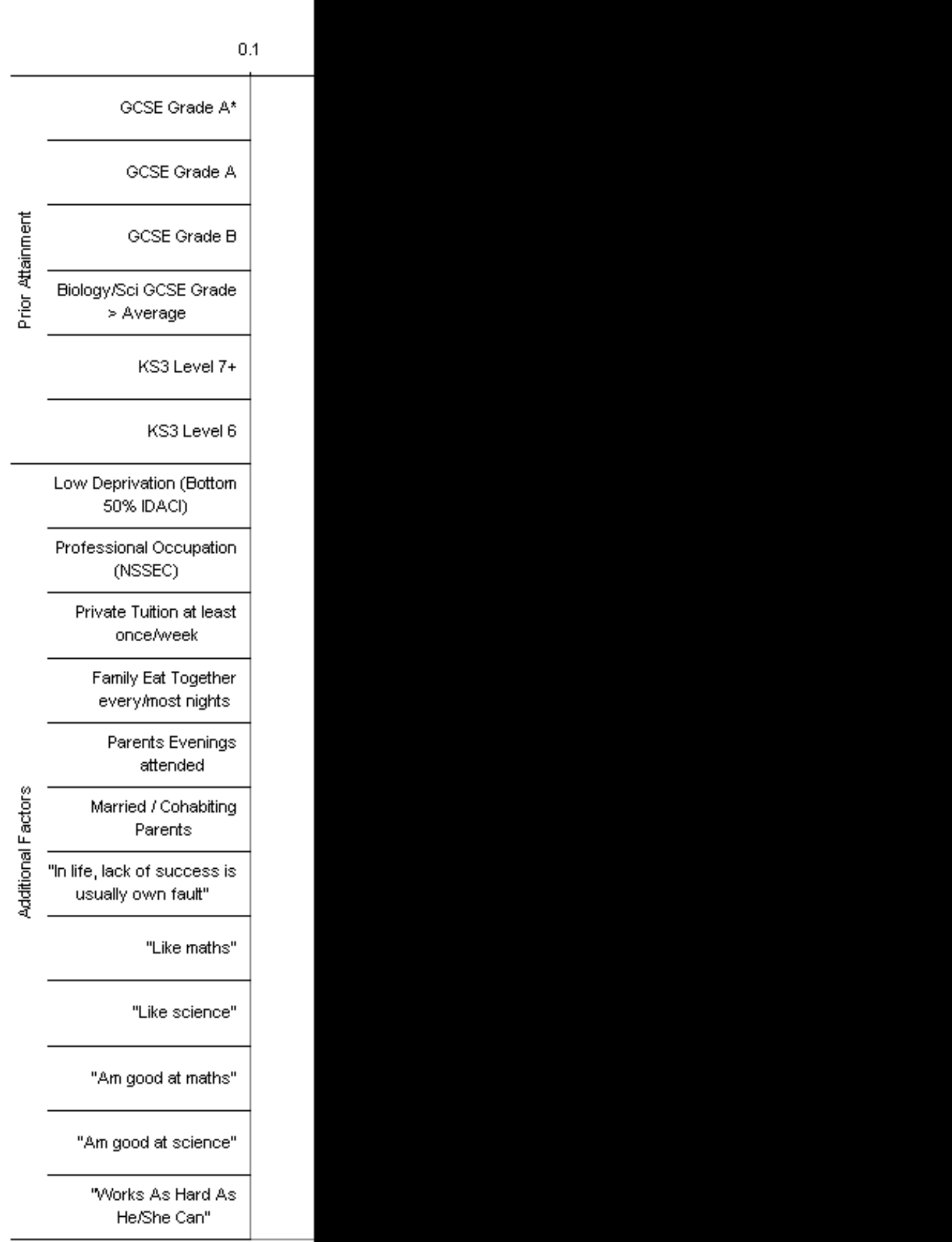

$\leftarrow$ Less Likely ... More Likely $\rightarrow$ 
Fig. $6.2 \mathrm{~g}$

Who Enters A Level Chemistry? Additional Factors (maintained mainstream KS4 cohort in LSYPE)

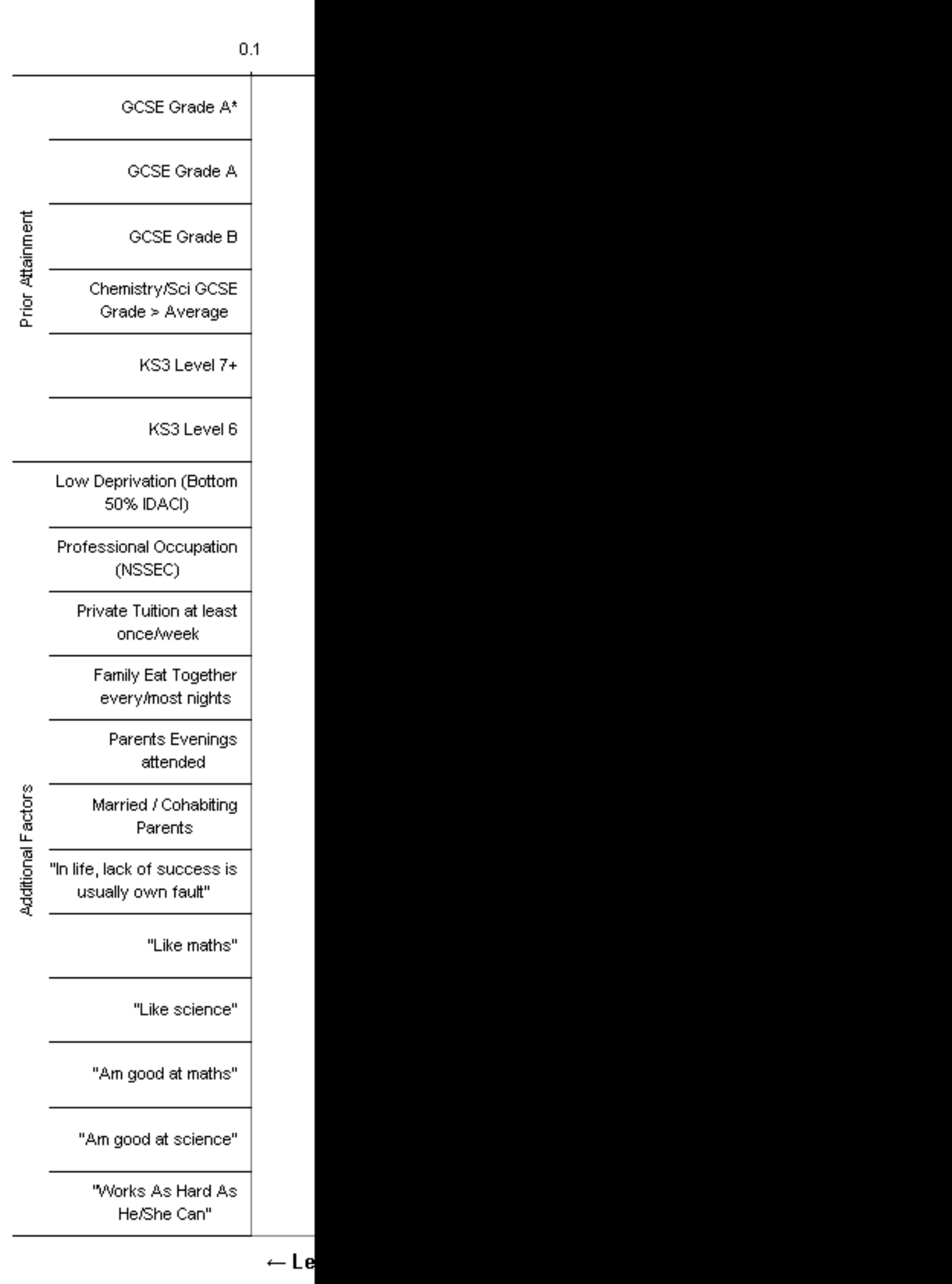


Fig. 6.2h

\section{Who Enters A Level Physics? Additional Factors (maintained mainstream KS4 cohort in LSYPE)}

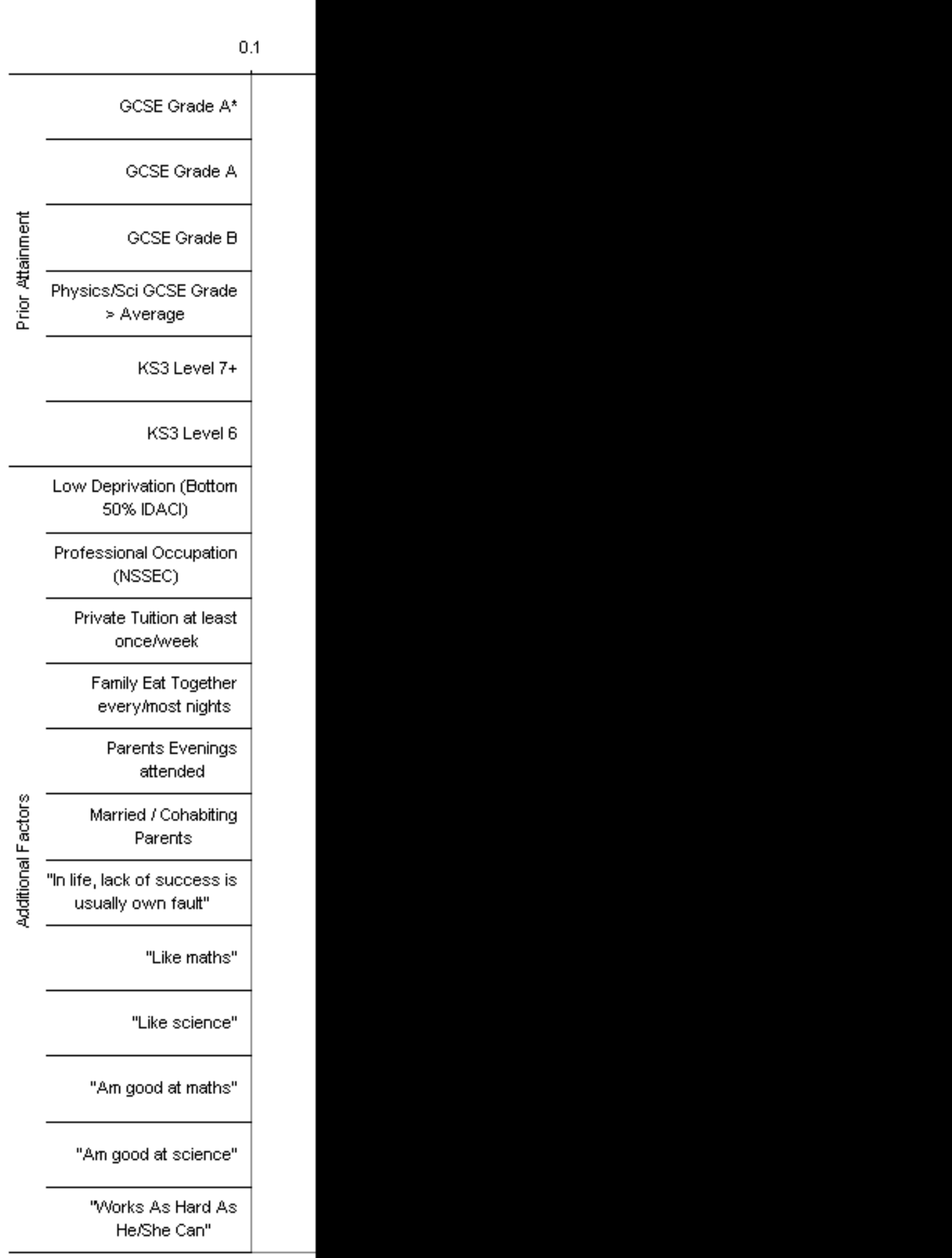

$\leftarrow$ Less Likely ... More Likely $\rightarrow$ 


\subsection{Subject Choice in A level Entry}

The second entry model variant narrows the field of pupils down to those who obtained grade B or higher in the relevant subject at GCSE (maths or science, including double science as well as separate science entries), and to those who continued in education post-16 and entered at least one A level, thereby focusing mainly on A level subject choice. Figures $6.3 a-d$ present factors affecting the choice to enter maths and science subjects rather than other possible A level options.

Now that the reference case for GCSE prior attainment has shifted up to become pupils with grade $B$ in the subject, the odds effects on $A$ level entry for grade $A^{*}$ or grade A GCSE achievement have shortened considerably. Nevertheless, prior attainment at grade $A^{*}$ remains the strongest factor for maths or chemistry entry and the second strongest factor for biology and physics, behind Pakistani ethnicity and male gender respectively. Key Stage 3 prior attainment within subject remained a significant influence over and above subsequent GCSE performance.

Relative strength in that subject at GCSE increases the odds of continuing to A level for all four maths and science subjects after limiting the analysis to high prior attainers who go on to enter at least one A level. The strongest effect of relative subject strength was for physics, where pupils with GCSE physics or double science grades higher than their own individual grade average had odds $90 \%$ higher than pupils whose physics / science GCSE performance was equal to or lower than their average. Gender retained its importance in increasing the odds of maths, chemistry and physics A level choice for boys compared with girls, but decreasing the odds for boys of entering A level biology.

The odds effects for different GCSE science routes remained very similar to those seen in section 6.2, before the scope of the models was narrowed to focus down on subject choice. Pupils who sat separate science GCSEs in schools where this was the only route on offer had twice the odds of going on to enter A levels in biology, chemistry or physics compared with pupils in schools where only double science was offered. Choosing or being selected for separate science within a school offering both routes resulted in odds that were two to three times as large, whereas choosing or being selected for double science gave odds that were only $70 \%-80 \%$ as large.

The ethnic groups described in section 6.2 continued to have raised odds of $A$ level entry for all four subjects when prior attainment and continuing to A level studies in at least one subject were controlled in the model specifications. The order of the effects was similar to the previous models, with 4-5 times the odds of White British pupils for Indian, Other Asian and Chinese pupils in maths; 3-4 times the odds of White British pupils for Pakistani, Other Asian and Indian pupils in biology; 5-6 times the odds of White British pupils for Pakistani, Other Asian and Black African pupils in chemistry; and twice the odds of White British pupils for Chinese pupils in physics.

Looking at the two categories of Special Educational Needs entered in the models, shifting the focus towards subject choice by those pupils who have entered at least one A level (which would include only the very top attaining pupils for the groups of pupils with SEN), results in slightly larger increases in odds for pupils with SEN entering A level maths and physics. The odds of entering A level biology go from 
being lower for pupils with SEN, to being no different from the odds for pupils without SEN. There is also a shift away from decreases in odds and towards raised odds, for pupils with SEN compared with other pupils, in the case of physics A level entry.

Pupils who entered 4 A levels had odds of choosing A level maths that were $20 \%$ higher than the odds of pupils who entered 3 or fewer A level subjects. For biology, this was a smaller increase at 10\% greater odds for those entering $4 \mathrm{~A}$ levels, but for chemistry and physics A level choice, the increases in odds associated with taking 4 A levels were larger at $30 \%$ and $40 \%$ respectively.

The number of other pupils at the KS5 institution attended sitting maths or science A levels was also associated with the odds of continuing in these subjects. For example, the odds of entering A level biology were $90 \%$ higher for pupils where the biology A level cohort was 41 pupils or larger, than for pupils attending institutions where 10 or fewer pupils sat biology A level in that year. Similar but slightly smaller effects were seen for A level subject cohort size on chemistry, physics and maths $A$ level entry. 
Fig. 6.3a

Who Enters A Level Maths?

(KS4 MM pupils with GCSE grade $A^{*} / A$ / B, at least one A level entry)

Effect on Odds (Logarithmic Scale)

0.1

1

10

100

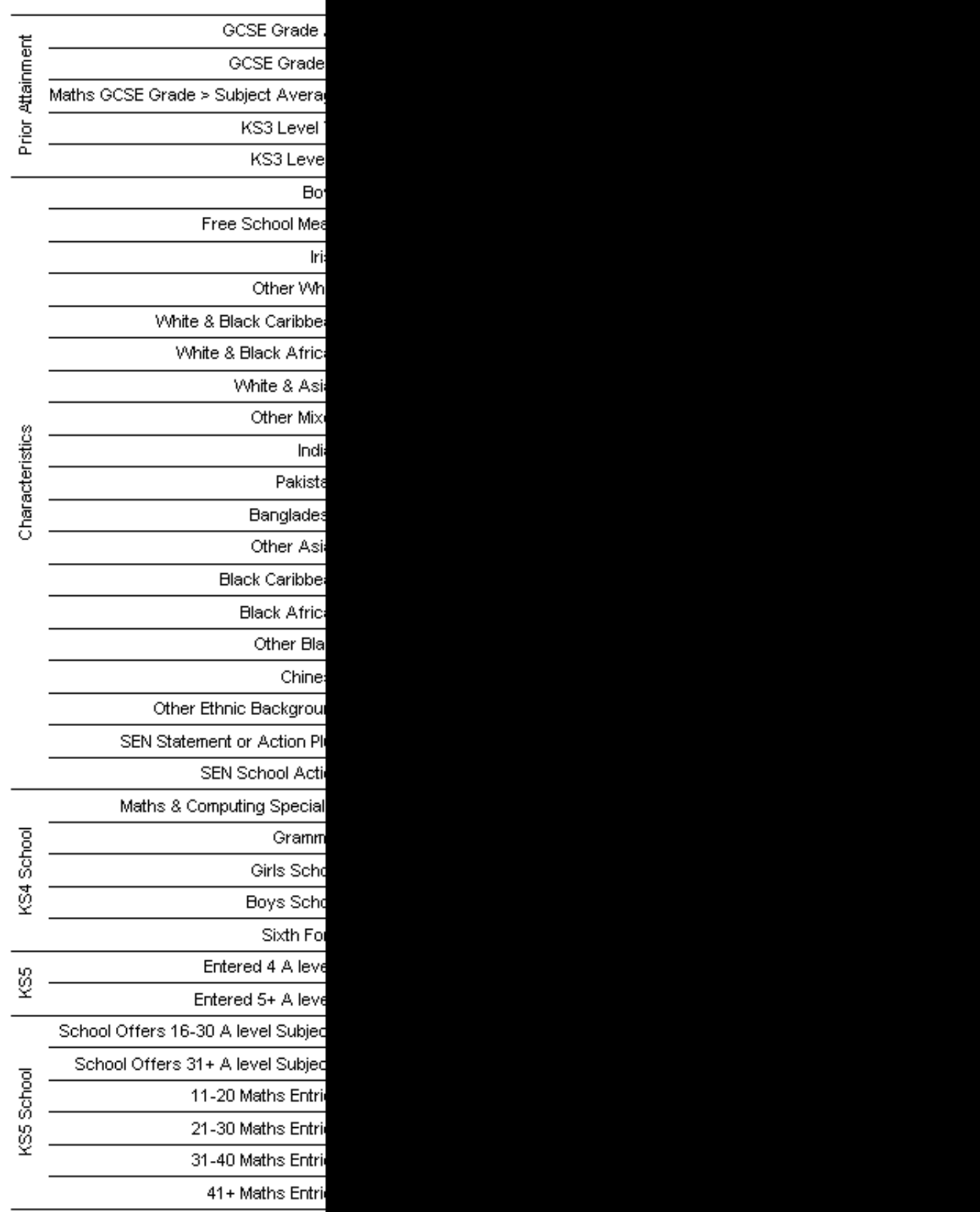

$\square 2009 \square 2008$

$\leftarrow$ Less Likely ... More Likely $\rightarrow$ 
Fig. 6.3b

\section{Who Enters A Level Biology? \\ (KS4 MM pupils with GCSE grade $A^{*} / A$ / $B$, at least one $A$ level entry)}

Effect on Odds (Logarithmic Scale)

0.1

1

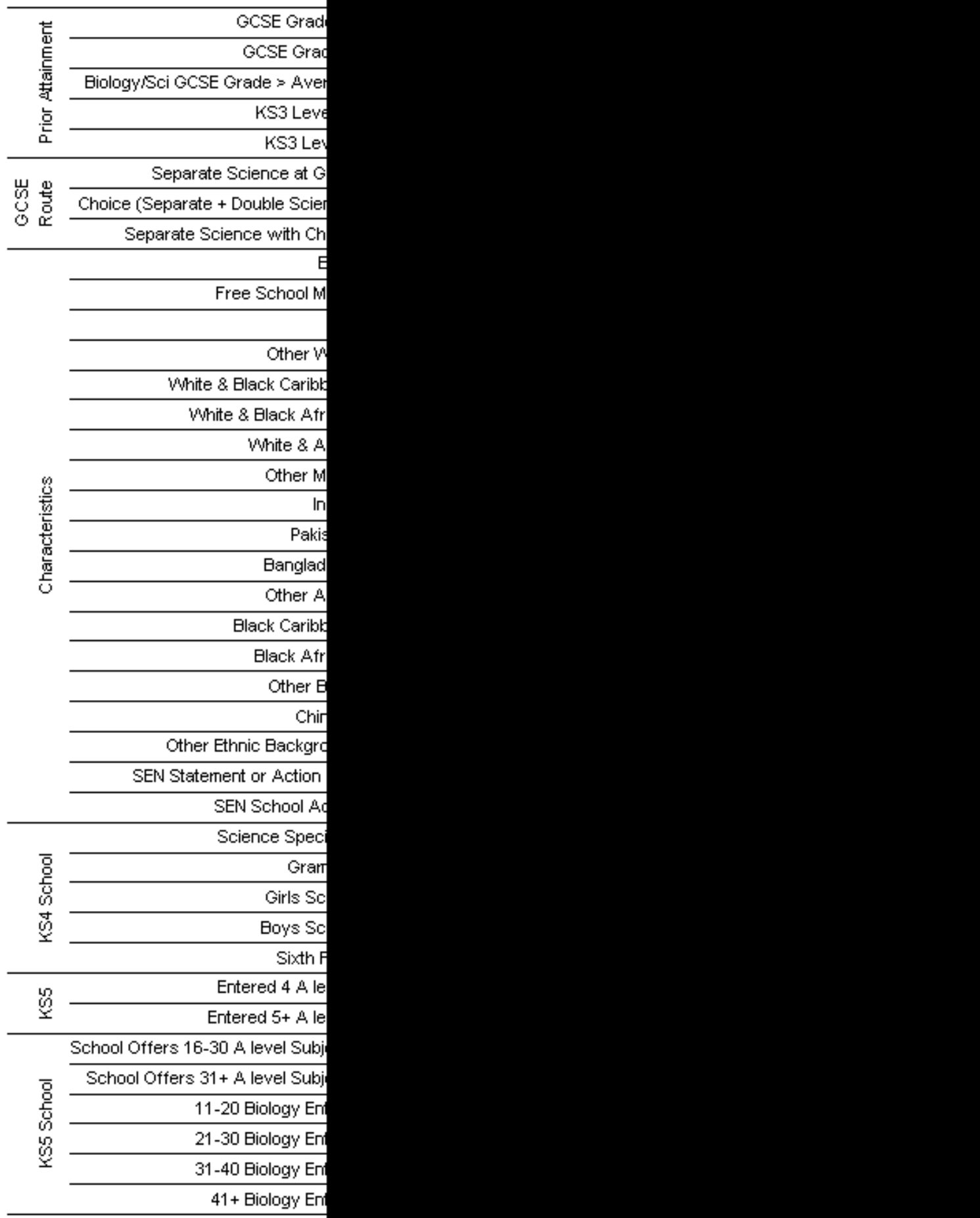


Fig. 6.3c

\section{Who Enters A Level Chemistry? \\ (KS4 MM pupils with GCSE grade $A^{*} / A / B$, at least one A level entry)}

Effect on Odds (Logarithmic Scale)

0.1

1

10

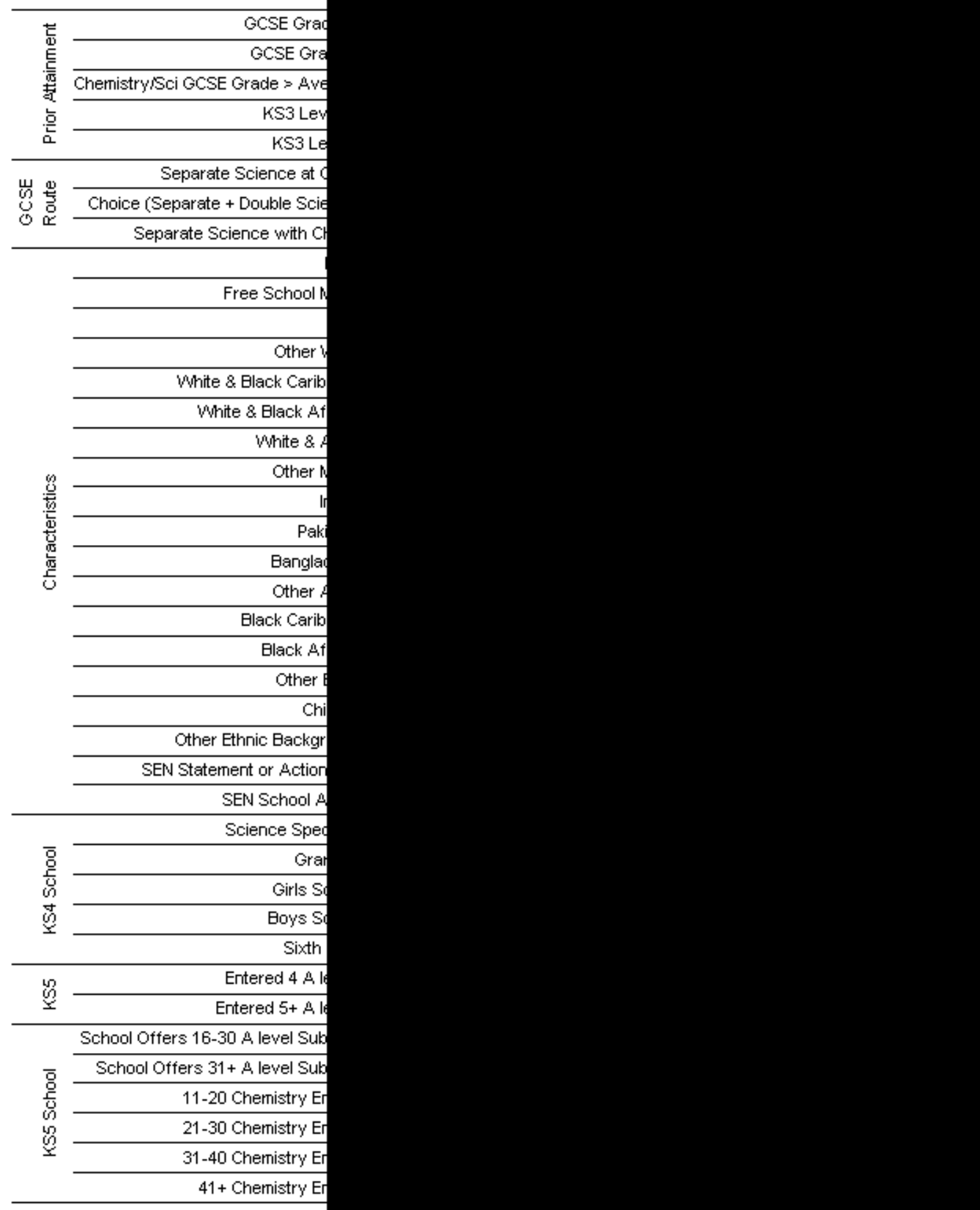

$\square 2009 \approx 2008$

$\leftarrow$ Less Likely ... More Likely $\rightarrow$ 
Fig. 6.3d

Who Enters A Level Physics?

(KS4 MM pupils with GCSE grade $A^{*} / A / B$, at least one $A$ level entry)

0.1

Effect on Odds (Logarithmic Scale)

1

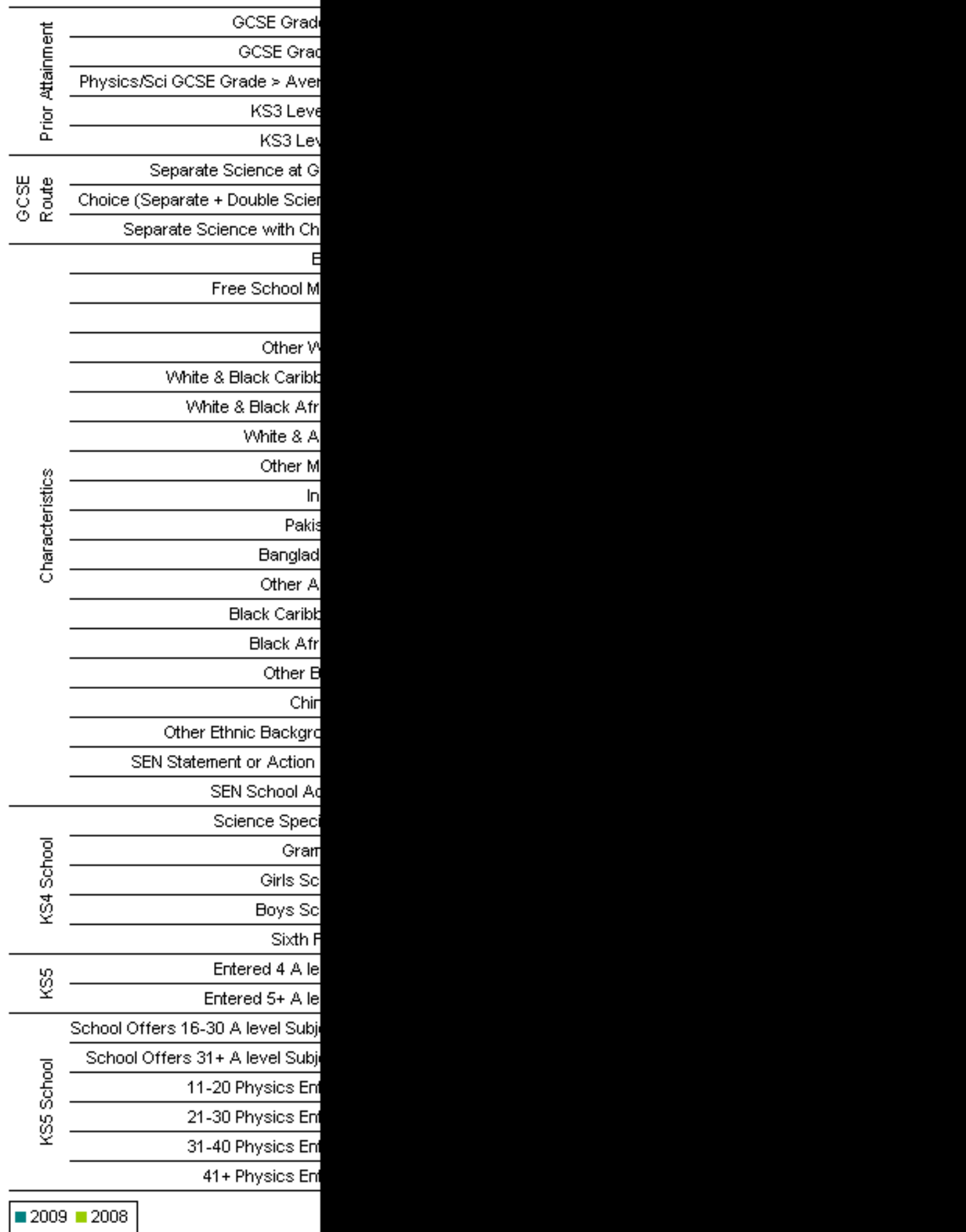


The entry models focused on subject choice described above are now explored using data from LSYPE to test for the significance of additional factors. Figures 6.3e-h present the extra factors in the extended subject choice model; inclusion on the charts is based on the factor being found to be significant (to the $10 \%$ level) in at least one of the full set of twelve entry and achievement models.

As with the wider, full cohort entry models described in section 6.2 above, enjoyment of science and maths at age 14 were still amongst the strongest new factors in the model for A level entry when the scope was narrowed to pupils who had high prior attainment in maths / science and had gone on to enter at least one A level. Enjoyment of science increased the odds of continuation for all four subjects to 2 - 3 times those of pupils who had not enjoyed science earlier in their secondary education. "Liking maths" also raised the odds of A level entry for maths, chemistry and physics by between $40 \%$ and $170 \%$ compared with pupils who had not enjoyed maths at that stage, but was not linked to A level biology entry.

The odds effects for working hard at school persist for maths ${ }^{6}$ and chemistry entry, but are no longer present for biology when focusing on subject choice. Pupils who reported "working as hard as I can" at age 14 had odds of A level maths continuation that were $40 \%$ higher, and A level chemistry entry that were $70 \%$ higher, than the odds for pupils who did not identify themselves as hard workers. Pupils with internal locus of control, who agreed with the statement "if someone is not a success in life, it is usually their own fault" had odds of continuation in physics and chemistry that were $30 \%-60 \%$ higher than the odds for those pupils who did not agree with the statement.

When focusing on subject choice at A level, living in an area of low deprivation at age 14 , and living with two cohabiting or married adults at this age, were both only significantly related to chemistry A level continuation ${ }^{6}$. The maths and biology effects disappeared when the model scope was limited to pupils with high prior subject attainment who entered at least one $A$ level. The odds effect for low area deprivation on chemistry continuation was an increase in odds of $30 \%$ compared with pupils living in areas of higher deprivation. For pupils with married or cohabiting adults in the household, the odds were raised by $50 \%$ compared with those for pupils who lived in other household types.

Pupils who reported that their family ate together on most nights or every night at age 14 had odds of A level choice raised by $30 \%$ for both biology and physics, compared with pupils whose family ate together less frequently or not at all. Having parents who attended parents evenings at school continued to raise the odds of A level biology subject choice by $50 \%$ compared with pupils whose parents did not report attending these events. Again, this factor was not related to continuation in any of the other sciences or maths. Pupils who reported attending private tuition in school subjects outside of school hours at age 14 had raised odds of entering A level chemistry, but not for the other sciences or maths, at $40 \%$ higher than the odds for pupils who had private tuition less frequently or never.

In the subject choice model variant, there were significant effects of pupils' belief in

\footnotetext{
${ }^{6}$ Significant only at the $90 \%$ level.
} 
their own ability in maths and science as reported at age 14. These effects persisted after controlling for actual prior attainment in these subjects. For example, the odds of a KS4 pupil going on to enter A level biology were $60-90 \%$ higher for those who reported that they were "good at maths" or "good at science" earlier in their secondary education, compared with those who did not believe they were good at these subjects. Pupils who reported being "good at science" at age 14 were less likely to enter A level maths, but those who reported being "good at maths" had odds of maths continuation that were $110 \%$ higher than the odds for pupils who did not say they were good at maths. 
Fig. 6.3e

\section{Who Enters A Level Maths? Additional Factors \\ (KS4 MM LSYPE with GCSE grade $A^{*} / A$ / B, at least one A level entry)}

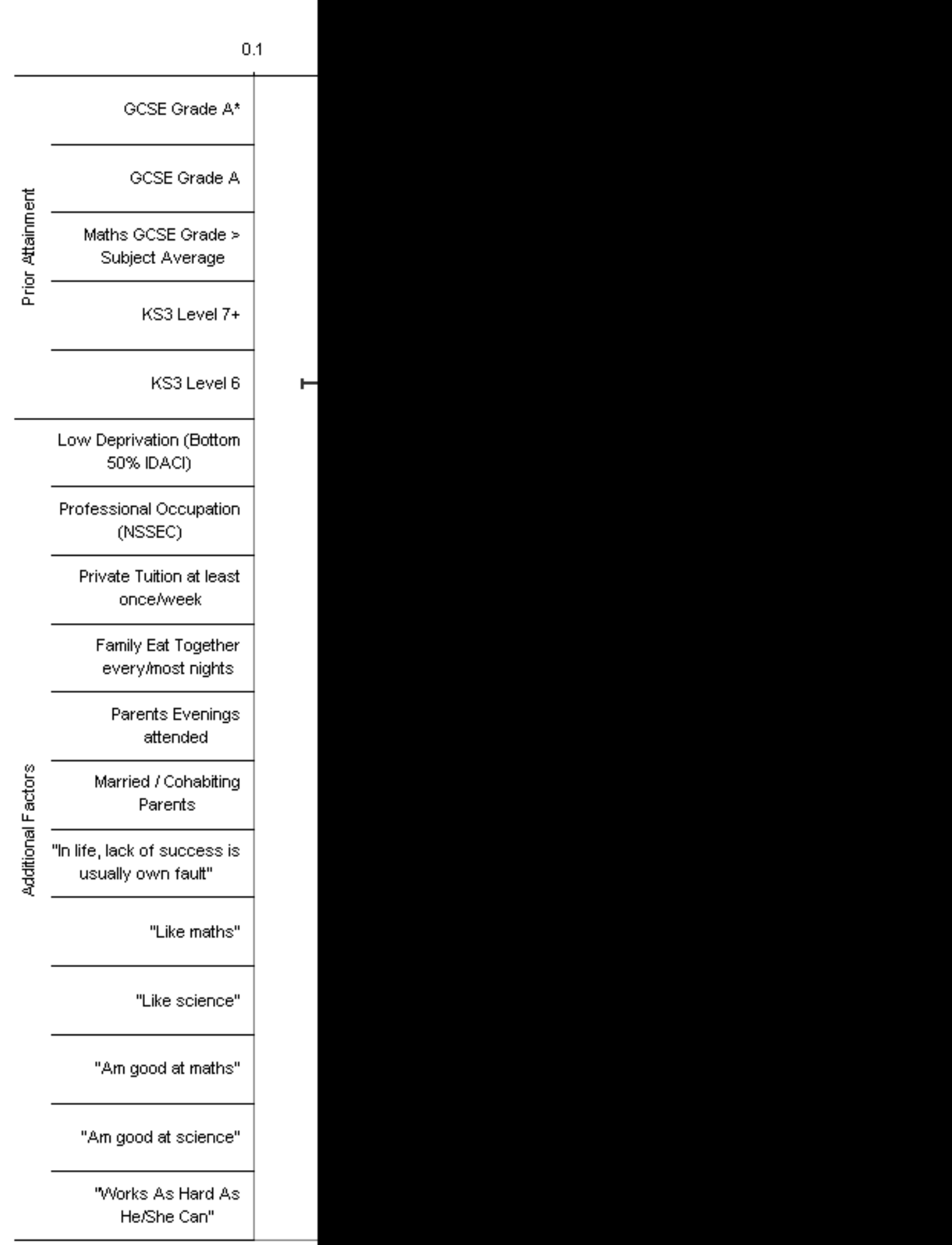

$\leftarrow$ Less Likely... More Likely $\rightarrow$ 
Fig. 6.3f

Who Enters A Level Biology? Additional Factors
(KS4 MM LSYPE with GCSE grade $A^{*} / A$ / $B$, at least one $A$ level entry)

Effect on Odds (Logarithmic Scale)

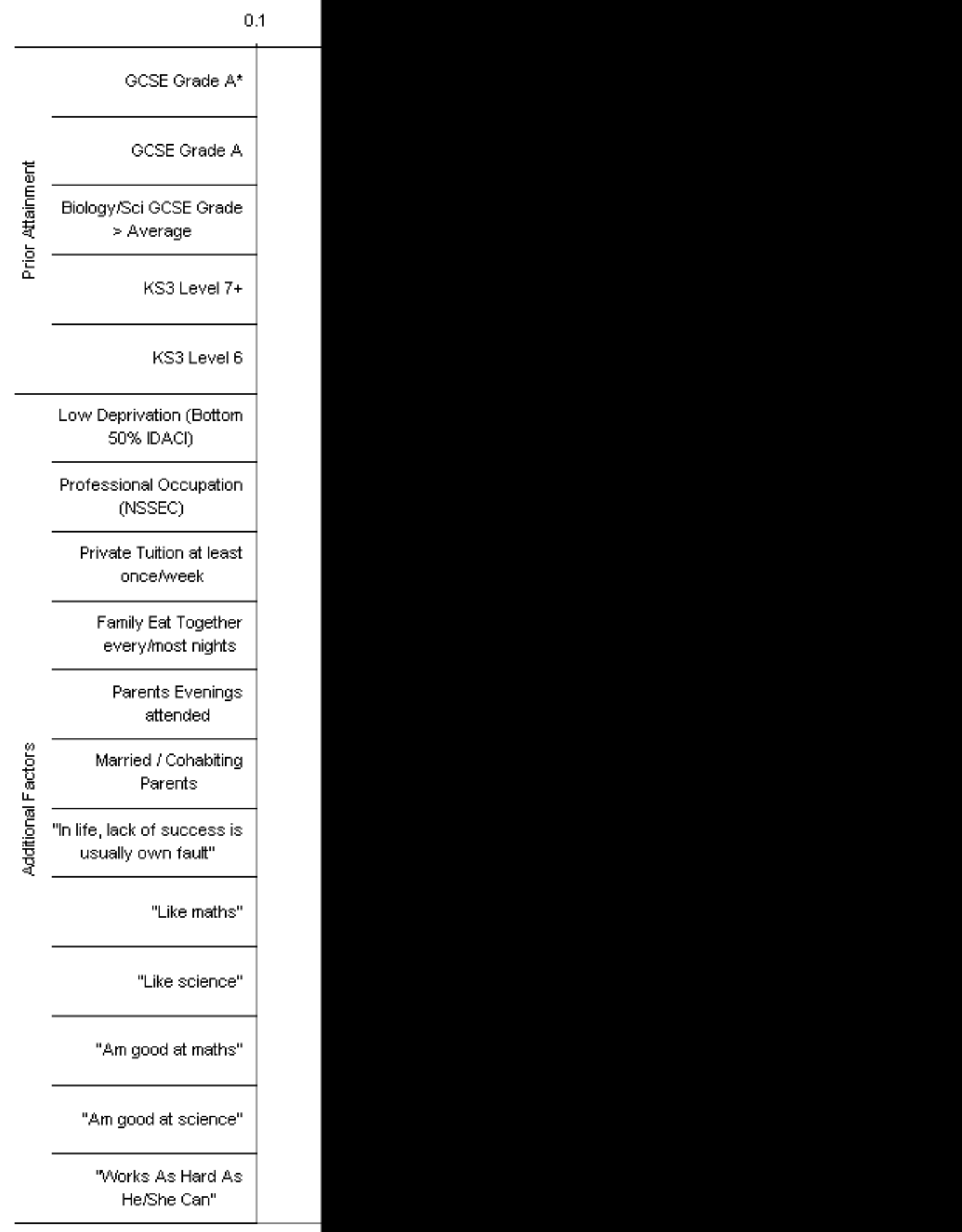

$\leftarrow$ Less Likely ... More Likely $\rightarrow$ 
Fig. $6.3 \mathrm{~g}$

Who Enters A Level Chemistry? Additional Factors

(KS4 MM LSYPE with GCSE grade $A^{*} / A$ / $B$, at least one $A$ level entry)

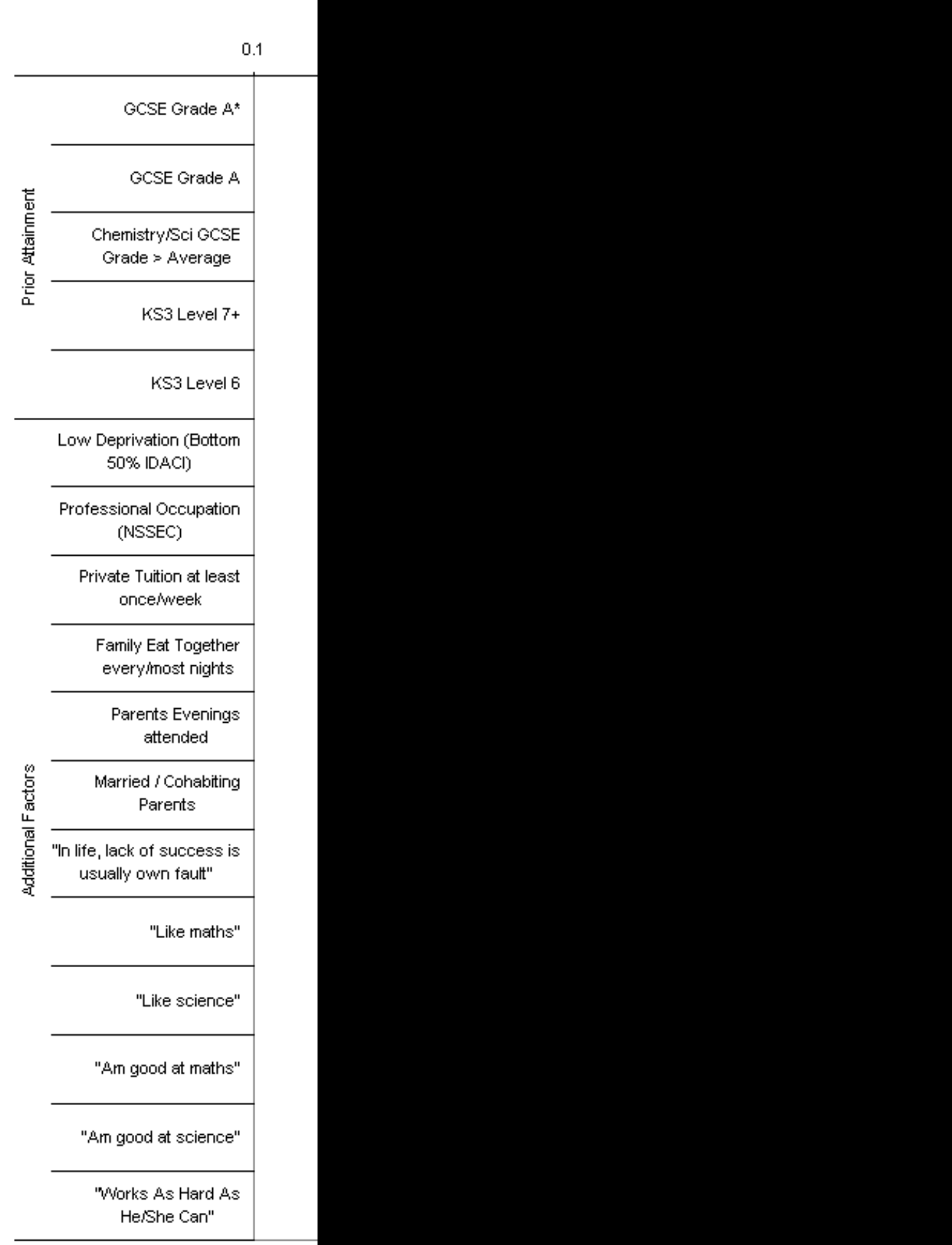

$\leftarrow$ Less Likely ... More Likely $\rightarrow$ 
Fig. 6.3h

Who Enters A Level Physics? Additional Factors
(KS4 MM LSYPE with GCSE grade $A^{*} / A$ / $B$, at least one A level entry)

Effect on Odds (Logarithmic Scale)

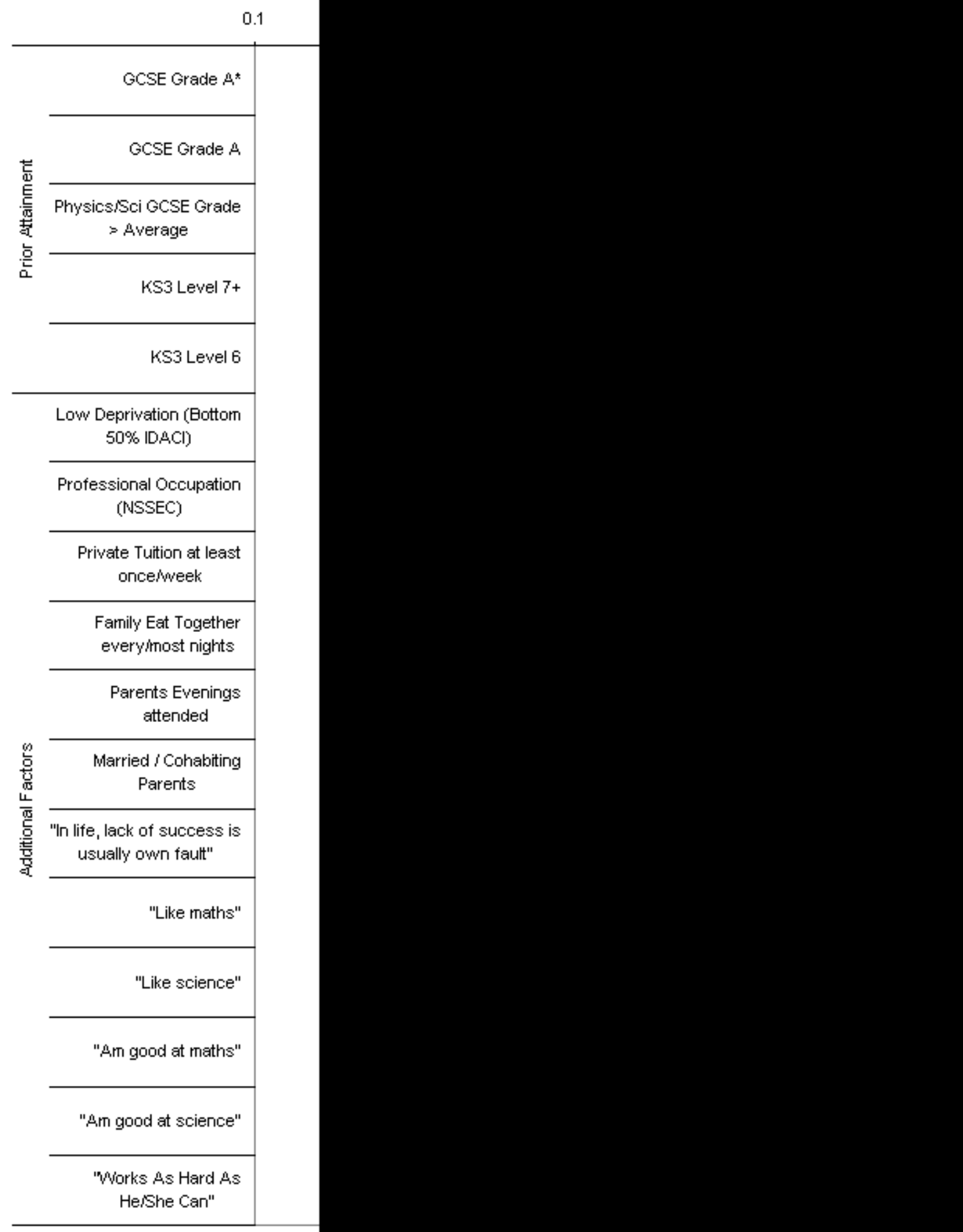

$\leftarrow$ Less Likely ... More Likely $\rightarrow$ 


\subsection{A level Achievement}

The model variant for success at grade B or higher at A level is scoped to include pupils from the maintained mainstream GCSE cohort who went on to enter at least one A level (with no restriction on prior attainment at GCSE). The following models therefore focus on the selection of pupils from the A level cohort who chose to study maths or science and achieved a high grade in that subject. Figures 6.4a-d illustrate the odds effects on achievement for the set of factors from the previous models.

Allowing the prior attainment to vary again, this time across the complete range for pupils who entered at least one A level, results in odds effects for the within-subject GCSE grade that are almost as high for maths achievement at $A$ level as they were for maths A level entry from the complete KS4 cohort. For the three science subjects, the increases in odds associated with high prior GCSE attainment are actually larger for achieving grade A or B at A level than they were for entering the A level, even though the models in this section are selective of pupils with high enough prior attainment at least to enter an A level in one subject. Across the maths and science subjects, for achievement of a high grade at A level the odds effects are in the order of around ten times the odds for prior attainment at grade B, 100 times the odds for prior attainment at grade A, and over 100 times the odds for prior attainment at grade $A^{*}$. These multiples are compared with the odds for pupils who attained at grade $\mathrm{C}$ or below in the relevant GCSE.

The influence of Key Stage 3 within-subject prior attainment, above that of GCSE prior attainment, was smaller but still significant for all four subjects when modelling the odds of high grade achievement rather than A level entry. For maths, the odds of achieving a grade $A$ or $B$ at $A$ level were $60 \%$ higher for pupils who achieved a level $7+$ at KS3 than for pupils with level 5 or below. For chemistry, the odds were $30 \%$ higher for pupils with science level 7+ at KS3, but for biology they were $80 \%$ higher and for physics, $120 \%$ higher than for pupils with level 5 or below.

In maths, biology and chemistry (but not physics), the odds of achieving grade A or B at $A$ level were decreased for pupils where this subject was stronger than their average grade at GCSE. This is because each pupil's prior GCSE grade in that subject is controlled for in the model, meaning that relative strength of the subject grade also reflects relative weakness of the pupil's grade average. Within the comparison group of pupils with subject grades equal to or lower than their grade average across all subjects, are found pupils who achieved $A^{*}$ in every subject they took, who are stronger candidates at $A$ level than those who got $A^{*}$ in maths or science, but $B$ or $C$ grades in other subjects.

The odds effects for different GCSE science routes were similar to those seen in sections 6.2 and 6.3, looking at A level entry. Pupils who sat separate science GCSEs in schools where this was the only route on offer had twice the odds of going on to achieve grade A or B in A level biology, chemistry or physics compared with pupils in schools where only double science was offered. Choosing or being selected for separate science within a school offering both routes resulted in odds that were two to three times as large, whereas choosing or being selected for double science gave odds that were only $70 \%-80 \%$ as large. For A level physics, the combination of separate sciences and having chosen or being selected for this route 
resulted in odds that were raised by slightly more for achievement of grade A or B than they were for entering A level physics.

The pattern of odds effects by pupil gender followed that for A level entry with similar sized effects in the same direction. Boys had raised odds of achieving grade A or B in maths, physics and chemistry (120\% higher than the odds for girls in maths, $10 \%$ higher in chemistry, and $470 \%$ higher in physics). For biology, the odds of achieving grade A or B at A level were only $60 \%$ as large as the odds for girls.

The ethnic minority groups who had raised odds of entering A levels in maths and science subjects also have raised odds of achieving grades $A$ or $B$ at $A$ level in those subjects, compared with White British pupils. For A level maths achievement, the odds effects for Indian, Other Asian and Chinese pupils are factors of around 4 times the odds for White British pupils. For biology, the odds effects for Pakistani, Other Asian and Indian pupils are in the order of 3 to 4; for chemistry, the odds effects for Pakistani, Other Asian and Black African pupils are in the order of 3 to 5; for physics, the odds for Chinese pupils are twice the odds for White British pupils.

Pupils with Special Educational Needs at School Action have raised odds of achieving grades $A$ or $B$ in maths or physics compared with their prior attainment peers who do not have SEN. Although pupils with SEN are overall much less likely to achieve at A level, for the minority who do attain sufficiently at GCSE to continue to A level, their odds of achievement in maths and physics are better than those of their counterparts without SEN.

The odds effects on achievement of grades A or B at A level are slightly stronger than those for A level entry for the model factors related to the number of A levels entered by the pupil, and the number of pupils entering that $A$ level in their school or FE college. The odds of achievement are raised by $40 \%$ for maths, $20 \%$ for biology, $50 \%$ for chemistry, and $70 \%$ for physics for those pupils who enter four A levels, compared with those who enter three or fewer subjects. The odds of attaining grades A or B at A level, for all four maths and science subjects, are about twice as large for pupils attending an institution with a large cohort of pupils sitting that subject (41 or more in that year), as for pupils at schools or colleges with ten or fewer entries in that subject. 
Fig. 6.4a

\section{Who Achieves Grade A or B in A level Maths? (KS4 MM pupils with at least one A level entry)}

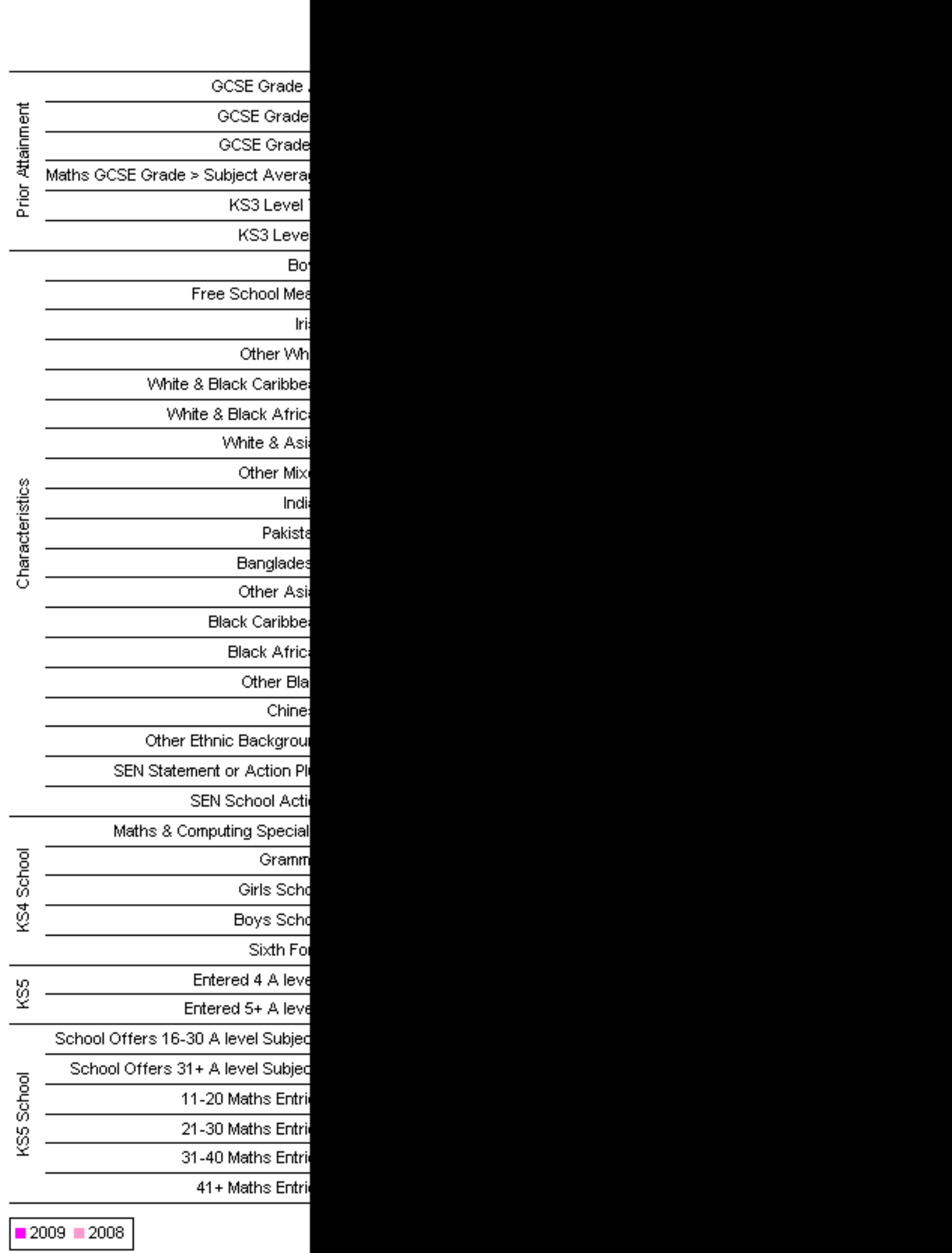


Fig. 6.4b

Who Achieves Grade A or B in A level Biology? (KS4 MM pupils with at least one $A$ level entry)

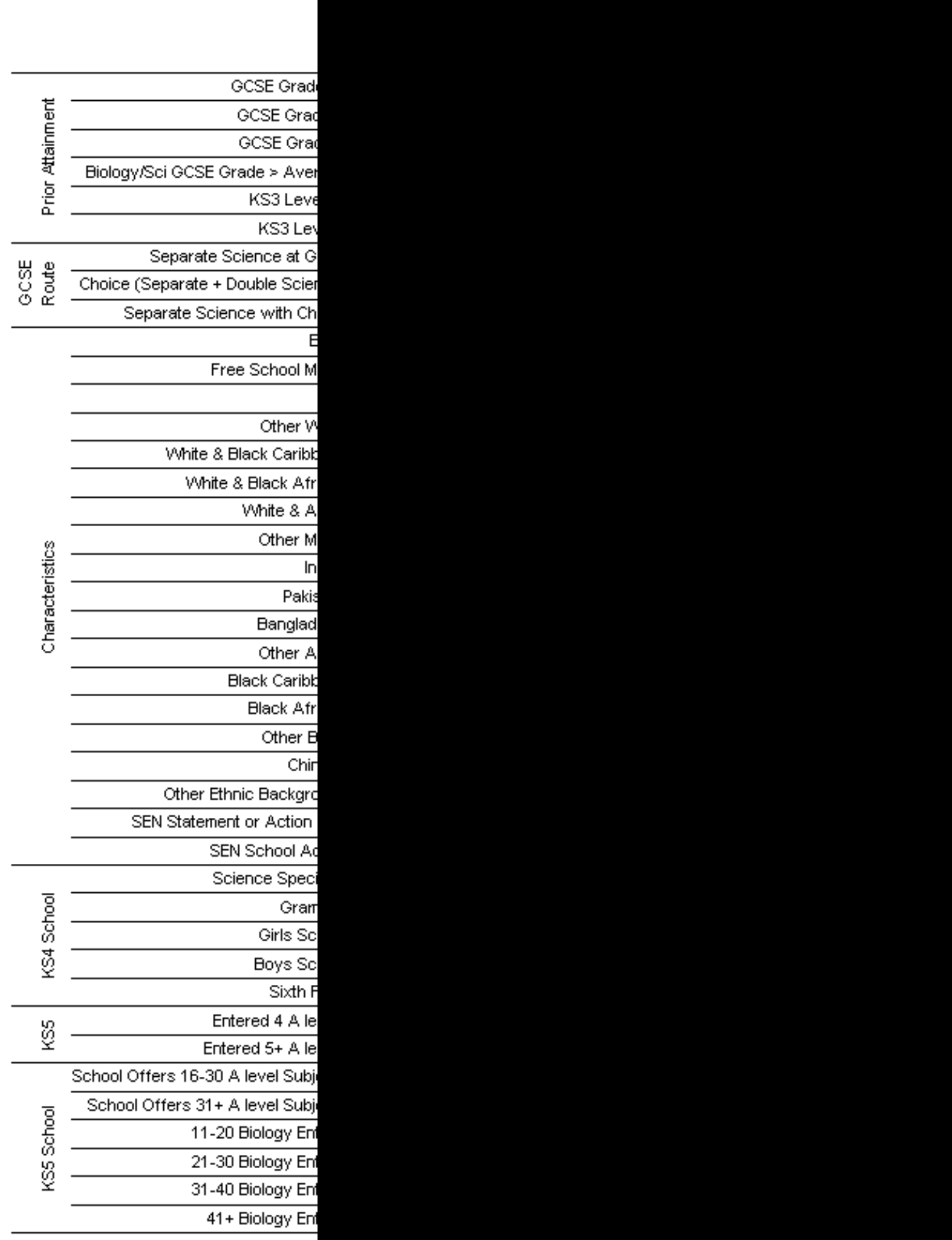


Fig. 6.4c

\section{Who Achieves Grade A or B in A level Chemistry? (KS4 MM pupils with at least one A level entry)}

\begin{tabular}{|c|c|c|c|c|c|}
\hline & & & Effect on Odds (Lo & ale) & \\
\hline & & 0.1 & 1 & 100 & 100 \\
\hline & GCSE Grade $A^{*}$ & & & & 73.4 \\
\hline$\stackrel{\underset{\mathbb{W}}{E}}{\mathrm{Z}}$ & GCSE Grade A & & & 92.6 & \\
\hline 彭 & GCSE Grade $\mathrm{B}$ & & & & \\
\hline 曹 & Chemistry/Sci GCSE Grade > Average & 0.8 & & & \\
\hline 흔 & KS3 Level 7+ & & 1.3 & & \\
\hline & KS3 Level 6 & & & & \\
\hline & Separate Science at GCSE & & 2.3 & & \\
\hline 恣 & Choice (Separate + Double Science) & 0.7 & & & \\
\hline & Separate Science with Choice & & 1.8 & & \\
\hline & Boys & & 1.1 & & \\
\hline & Free School Meals & & 1.2 & & \\
\hline & Irish & & & & \\
\hline & Other White & & 1.2 & & \\
\hline & White 8 Black Caribbean & 0.7 & & & \\
\hline & White \& Black African & & & & \\
\hline & White \& Asian & & 1.3 & & \\
\hline & Other Mixed & & 1.3 & & \\
\hline 总 & Indian & & 3.3 & & \\
\hline$\stackrel{\bar{s}}{\mathrm{~s}}$ & Pakistani & & 4.7 & & \\
\hline 产 & Bangladeshi & & 2.3 & & \\
\hline$\overleftarrow{J}$ & Other Asian & & 3.6 & & \\
\hline & Black Caribbean & & & & \\
\hline & Black African & & 3.4 & & \\
\hline & Other Black & & 2.4 & & \\
\hline & Chinese & & 2.3 & & \\
\hline & Other Ethnic Background & & 3.2 & & \\
\hline & SEN Statement or Action Plus & & & & \\
\hline & SEN School Action & & & & \\
\hline & Science Specialist & 0.9 & & & \\
\hline$\overline{\mathrm{g}}$ & Grammar & 0.7 & & & \\
\hline 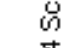 & Girls School & & & & \\
\hline 苞 & Boys School & & & & \\
\hline & Sixth Form & & 1.1 & & \\
\hline 少 & Entered $4 \mathrm{~A}$ levels & & 1.5 & & \\
\hline$Y$ & Entered 5+ A levels & & 2.1 & & \\
\hline & School Offers 16-30 A level Subjects & & & & \\
\hline $\bar{D}$ & School Offers 31+ A level Subjects & 0.5 & & & \\
\hline$\frac{9}{0}$ & 11-20 Chernistry Entries & & 1.4 & & \\
\hline in & 21-30 Chernistry Entries & & 1.7 & & \\
\hline$\underline{Y}$ & 31-40 Chernistry Entries & & 1.8 & & \\
\hline & $41+$ Chernistry Entries & & 2.2 & & \\
\hline
\end{tabular}


Fig. 6.4d

\section{Who Achieves Grade A or B in A level Physics? (KS4 MM pupils with at least one A level entry)}

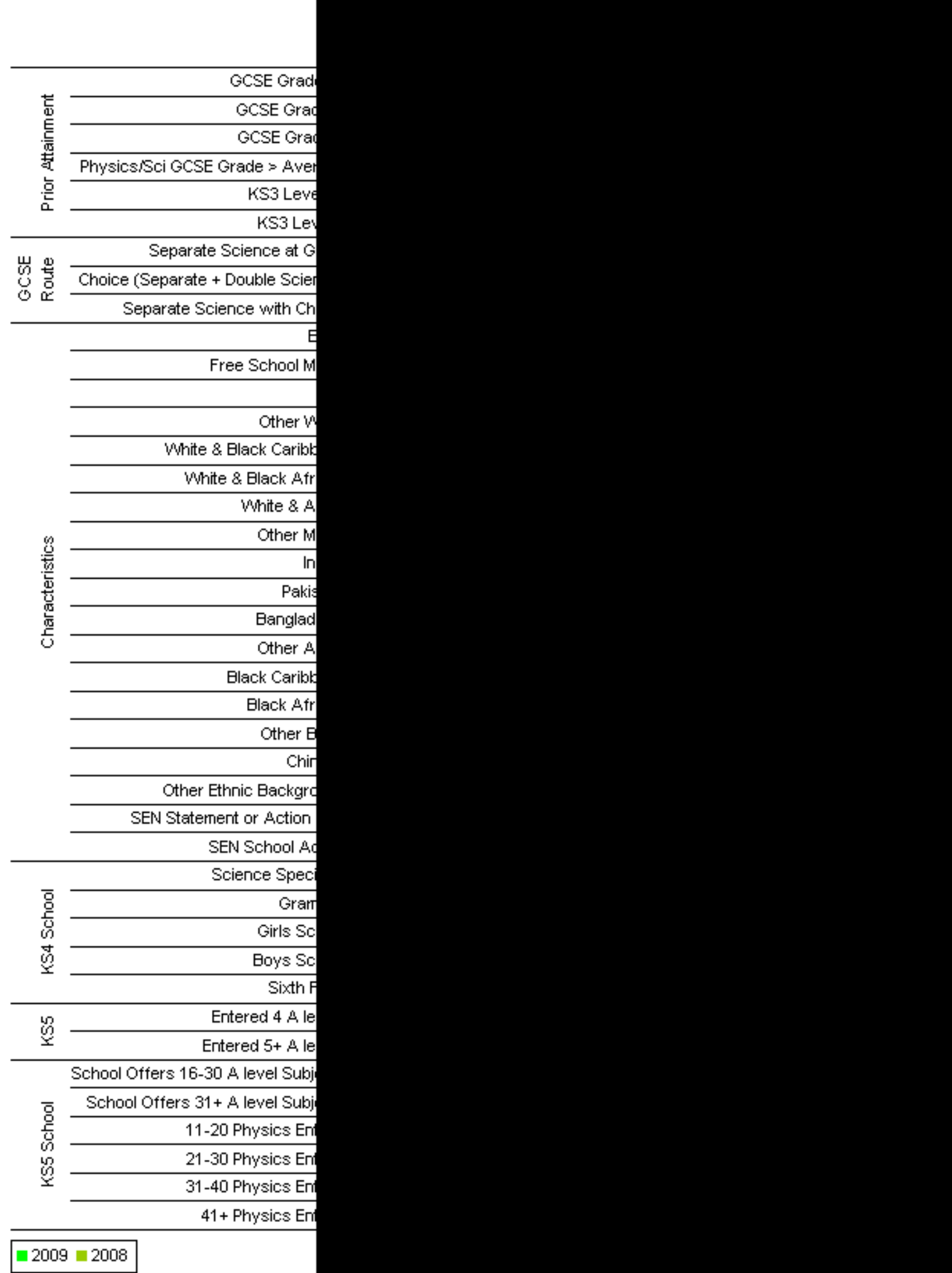


The administrative data models of achievement at $A$ level in maths and science subjects were further explored, using data from LSYPE to test for the significance of additional factors. Figures $6.4 \mathrm{e}-\mathrm{h}$ present the extra factors in the extended versions of the A level grade A or B achievement models.

Just as was the case in the A level entry model variants, subject enjoyment was the most prevalent additional effect in the achievement models. For pupils who reported liking science at age 14, the odds of achieving high grades at A level in maths and the sciences were raised to between 2 and 3 times the odds for pupils who did not say they enjoyed science. For pupils who said they liked maths at age 14, the odds of achieving grade A or B in maths, chemistry or physics (but not biology) were also 2 to 3 times the size of the odds for pupils who didn't report liking maths at the younger age.

The odds effects for pupils whose family eat together most nights or every night were stronger for achievement of high grades at A level than for choosing to enter science subjects. The effect was significant for biology only when looking at entry from the whole Key Stage 4 cohort, for both biology and physics when focusing on those pupils with high prior attainment who continued to A level in at least one subject, and for all three sciences when testing the effects on A level achievement. The odds of gaining grade $\mathrm{A}$ or B were $60 \%$ higher in biology A level, $50 \%$ higher in physics, and $40 \%$ higher in chemistry, compared with the odds for pupils whose family did not eat together this frequently.

Pupils who believed they were good at maths at age 14 also had raised odds of achieving grades A or B in A level maths (three times the odds) or A level biology (twice the odds), compared with pupils who did not identify themselves as good at maths. Interestingly, perceiving oneself to be good at science at 14 was not significant in any of the A level achievement models, although it was linked to raised odds of subject entry for maths and biology.

The A level chemistry extended achievement model contained significant effects for pupils who lived in areas of low deprivation and those who had a parent with a professional occupation, both with odds that were 50\% greater, for achieving grades A or B, than the odds for pupils who lived in more deprived areas, or did not have a parent with a professional job. Neither of the other sciences had significant odds effects on these factors, nor did maths. Similarly, having a parent who attended parents evenings raised the odds for achievement in biology, just as it had raised the odds for biology A level entry, but again this was the only one of the four subjects associated with this factor.

Factors that were associated with A level maths or sciences entries, but which were not related to achievement of high grades in any of these subjects were living with married or cohabiting adults, having an internal locus of control, reporting working as hard as one can, and receiving private tuition outside of school lessons. 
Fig. 6.4e

\section{Grade A or B in A level Maths: Additional Factors \\ (KS4 MM pupils in LSYPE with at least one A level entry)}

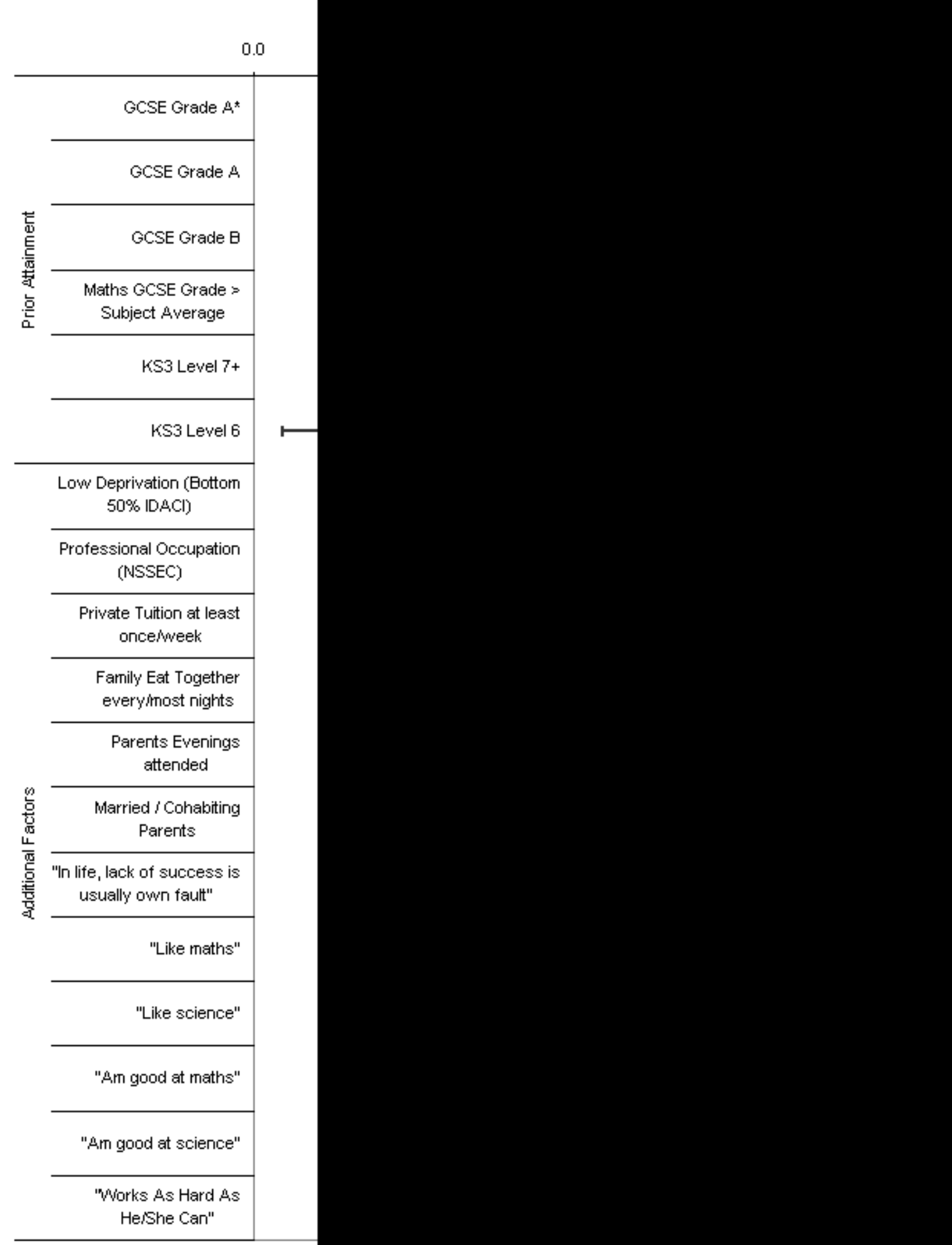

$\leftarrow$ Less Likely ... More Likely $\rightarrow$ 
Fig. $6.4 f$

Grade A or B in A level Biology: Additional Factors

(KS4 MM pupils in LSYPE with at least one A level entry)

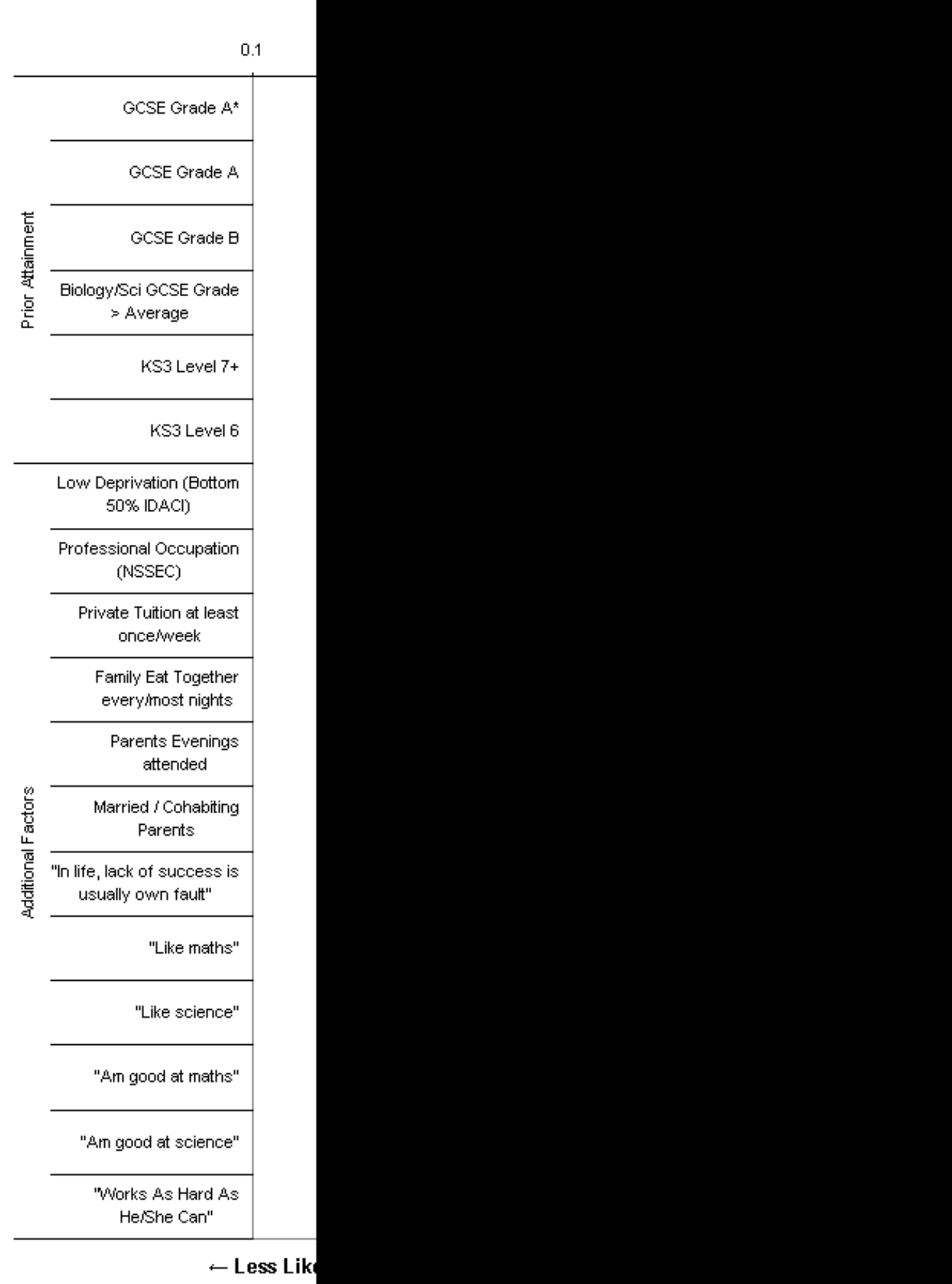


Fig. $6.4 \mathrm{~g}$

\section{Grade A or B in A level Chemistry: Additional Factors (KS4 MM pupils in LSYPE with at least one A level entry)}

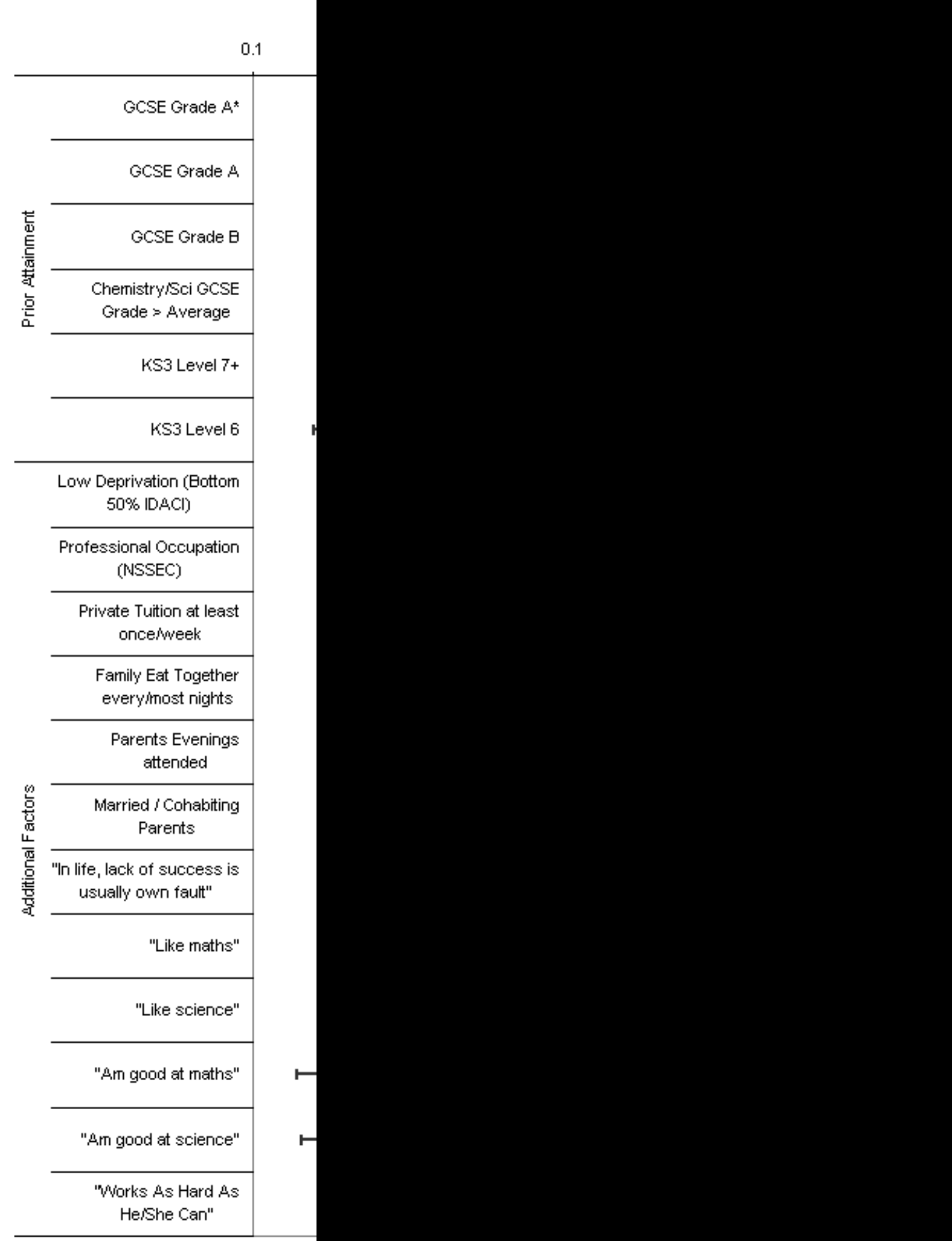

$\leftarrow$ Less Likely ... More Likely $\rightarrow$ 
Fig. 6.4h

\section{Grade A or B in A level Physics: Additional Factors (KS4 MM pupils in LSYPE with at least one A level entry)}

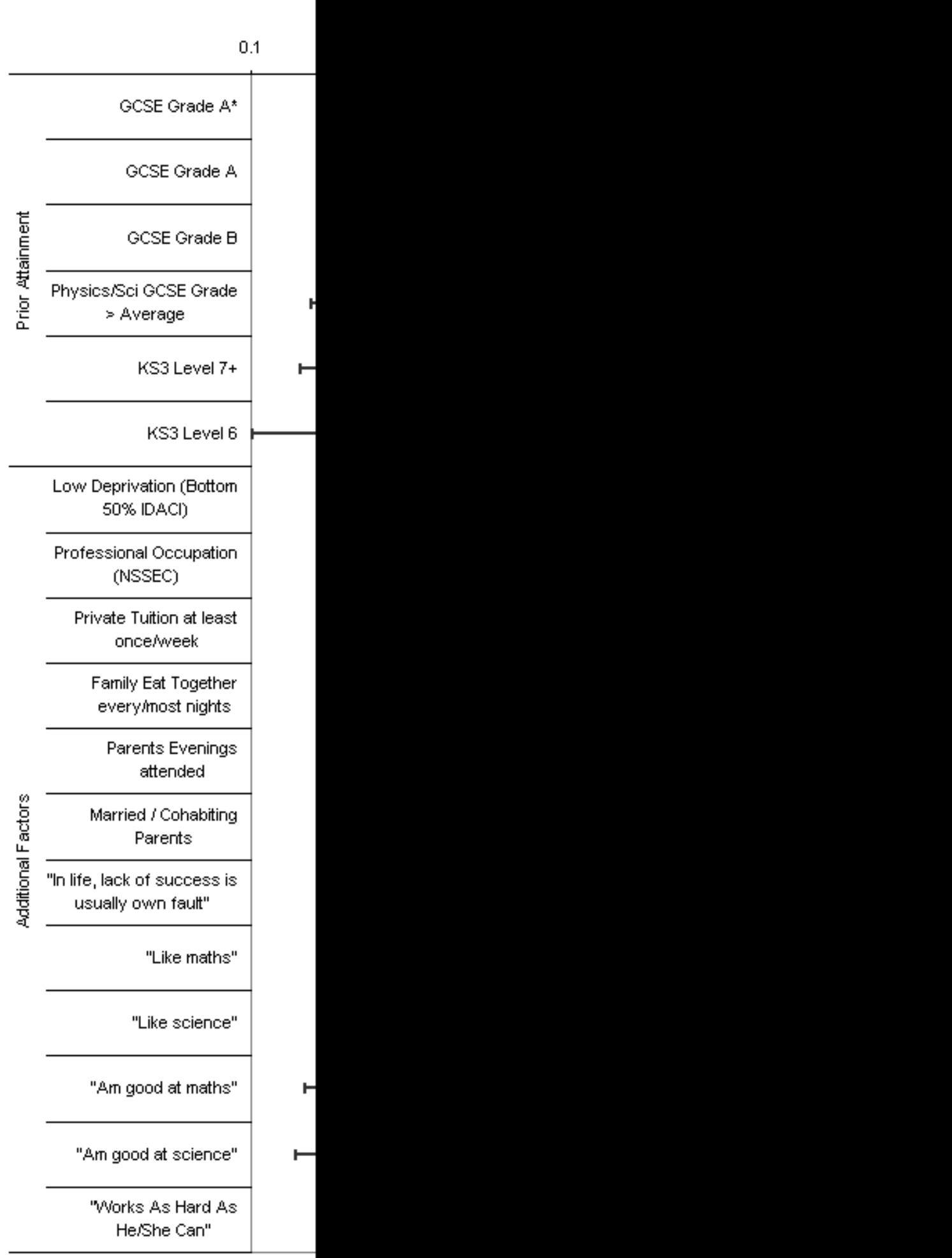

$\leftarrow$ Less Likely ... More Likely $\rightarrow$ 


\subsection{Comparing and Summarising the Model Findings}

In this section, the effects described in sections 6.2 to 6.4 , which were there organised according to the A level outcome in question, are summarised across the three model variants and four subjects. Figure 6.5 a presents a summary of the significant odds effects from the pupil level factors in the administrative data versions of the models. Figure $6.5 \mathrm{~b}$ then presents the school level factors in the administrative models, and $6.5 \mathrm{~h}$ gives an equivalent summary of the additional survey data sourced factors in the extended models. These summaries are intended to facilitate a review of some of the key factors and their effects across the range of models presented above.

\section{Fig. 6.5a}

\begin{tabular}{|c|c|c|c|c|c|c|c|c|c|c|c|c|}
\hline \multirow{3}{*}{$\begin{array}{l}\text { A level Outcome } \\
\text { Model Scope }\end{array}$} & \multicolumn{3}{|c|}{ Maths } & \multicolumn{3}{|c|}{ Biology } & \multicolumn{3}{|c|}{ Chemistry } & \multicolumn{3}{|c|}{ Physics } \\
\hline & \multicolumn{2}{|c|}{ Maths Entry } & \multirow{2}{*}{$\begin{array}{l}\text { Grade A/B } \\
\mid \begin{array}{c}\mathrm{KS} 4 \mathrm{MM} \\
1+\mathrm{A} \text { level }\end{array}\end{array}$} & \multicolumn{2}{|c|}{ Biology Entry } & \multirow{2}{*}{$\begin{array}{l}\text { Grade A/B } \\
\mid \begin{array}{l}\mathrm{KS} 4 \mathrm{MM} \\
1+\mathrm{A} \text { level }\end{array} \\
\end{array}$} & \multicolumn{2}{|c|}{ Chemistry Entry } & \multirow{2}{*}{$\begin{array}{l}\text { Grade } \mathbf{A} / \mathbf{B} \\
\mid \begin{array}{l}\mathrm{KS} 4 \mathrm{MM} \\
1+\mathrm{A} \text { level }\end{array}\end{array}$} & \multicolumn{2}{|c|}{ Physics Entry } & \multirow{2}{*}{$\begin{array}{l}\text { Grade A/ } \\
\begin{array}{|l}\mathrm{KS} 4 \mathrm{MM} \\
1+\mathrm{A} \text { level }\end{array}\end{array}$} \\
\hline & KS4 MM & \begin{tabular}{|c} 
KS4 MM \\
GCSE A*-B \\
$1+$ A level
\end{tabular} & & KS4 MM & $\begin{array}{c}\text { KS4 MM } \\
\text { GCSE A*-B } \\
1+\mathrm{A} \text { level }\end{array}$ & & KS4 MM & $\mid \begin{array}{c}\text { KS4 MM } \\
\text { GCSE A*-B } \\
1+\text { A level }\end{array}$ & & KS4 MM & \begin{tabular}{|c} 
KS4 MM \\
GCSE A*-B \\
$1+$ A level
\end{tabular} & \\
\hline \multirow{2}{*}{$\begin{array}{l}\text { Individual Level Factors... } \\
\text { GCSE Grade } A^{*}\end{array}$} & \multicolumn{12}{|c|}{2009 Effects on Odds (all reported effects are significant at the $5 \%$ level) } \\
\hline & 2775.7 & 38.9 & 2154.4 & 78.3 & 2.9 & 379.7 & 251.5 & 6.2 & 473.4 & 134.1 & 3.5 & 249.0 \\
\hline GCSE Grade A & 445.9 & 7.5 & 223.9 & 46.3 & 2.0 & 105.7 & 91.9 & 2.8 & 92.6 & 63.7 & 2.1 & 61.1 \\
\hline GCSE Grade B & 44.1 & & 15.8 & 17.7 & & 16.6 & 25.0 & & 12.9 & 21.1 & & 8.6 \\
\hline Grade > Subject Average & 0.9 & 1.3 & 0.7 & 1.1 & 1.5 & 0.7 & 1.2 & 1.6 & 0.8 & 1.3 & 1.9 & \\
\hline Key Stage 3 Level 7+ & 4.4 & 2.4 & 1.6 & 2.3 & 1.3 & 1.8 & 1.9 & 1.3 & 1.3 & 3.8 & 2.2 & 2.2 \\
\hline Key Stage 3 Level 6 & 2.2 & & & 2.2 & 1.3 & 1.4 & 1.4 & & & 2.0 & 1.2 & \\
\hline Separate Science & & & & 2.0 & 1.8 & 2.4 & 2.5 & 2.1 & 2.3 & 2.0 & 2.0 & 2.0 \\
\hline Choice (Separate / Double) & & & & 0.8 & 0.8 & 0.8 & 0.7 & 0.7 & 0.7 & 0.8 & 0.8 & 0.7 \\
\hline Separate * Choice & & & & 1.4 & 1.5 & & 1.7 & 1.9 & 1.8 & 1.4 & 1.4 & 1.7 \\
\hline Boys & 2.2 & 2.5 & 2.2 & 0.6 & 0.7 & 0.6 & 1.2 & 1.2 & 1.1 & 5.9 & 6.3 & 5.7 \\
\hline Free School Meals & & 1.1 & & 0.9 & 1.1 & & 1.1 & 1.3 & 1.2 & 0.8 & & \\
\hline \multicolumn{13}{|l|}{ Irish } \\
\hline Other White & 1.3 & 1.3 & 1.5 & & & & 1.2 & 1.2 & 1.2 & 1.2 & 1.1 & 1.2 \\
\hline White \& Black Caribbean & & & & & & & & & 0.7 & 0.7 & 0.7 & \\
\hline White \& Black African & 1.9 & 2.1 & & & 1.4 & 1.5 & 1.8 & 1.9 & & & & \\
\hline White \& Asian & 1.6 & 1.7 & 1.7 & 1.3 & 1.2 & 1.3 & 1.4 & 1.4 & 1.3 & 1.4 & 1.4 & \\
\hline Other Mixed & 1.3 & 1.4 & 1.6 & 1.3 & 1.3 & 1.3 & 1.4 & 1.4 & 1.3 & & & \\
\hline Indian & 4.1 & 4.2 & 3.8 & 2.7 & 2.3 & 2.5 & 3.8 & 3.4 & 3.3 & 1.2 & & \\
\hline Pakistani & 2.5 & 2.6 & 2.1 & 4.0 & 3.8 & 3.8 & 6.0 & 5.6 & 4.7 & & 0.8 & 0.8 \\
\hline Bangladeshi & 2.4 & 2.5 & 2.0 & 2.0 & 1.8 & 1.6 & 3.1 & 2.7 & 2.3 & & & \\
\hline Other Asian & 4.1 & 4.6 & 3.8 & 3.0 & 2.6 & 2.5 & 4.7 & 4.3 & 3.6 & 1.4 & 1.3 & \\
\hline Black Caribbean & 1.2 & 1.4 & & & & & 1.3 & 1.3 & & 0.7 & 0.7 & 0.5 \\
\hline Black African & 3.2 & 3.4 & 2.7 & 2.6 & 2.2 & 2.3 & 4.5 & 4.0 & 3.4 & 1.2 & & \\
\hline Other Black & 1.8 & 2.2 & 1.7 & 1.5 & 1.7 & 1.6 & 2.3 & 2.6 & 2.4 & & & \\
\hline Chinese & 4.6 & 4.9 & 3.9 & 1.4 & 1.2 & 1.4 & 2.5 & 2.1 & 2.3 & 2.5 & 2.1 & 2.2 \\
\hline Other Ethnic Background & 3.1 & 3.6 & 2.8 & 2.2 & 2.0 & 2.1 & 3.6 & 3.3 & 3.2 & & & \\
\hline SEN Statement / Action+ & & 1.2 & 1.3 & 0.6 & & & 0.8 & & & & 1.4 & \\
\hline SEN School Action & 1.1 & 1.5 & 1.4 & 0.7 & & 0.8 & 0.9 & 1.1 & & 1.2 & 1.6 & 1.5 \\
\hline
\end{tabular}

\section{Prior Attainment}

Prior attainment within subject has the strongest consistent effects on both A level entry and achievement of top grades. The effects of achieving a grade A within subject at GCSE are largest for maths, followed by chemistry, then physics, then biology; the odds effects range from 400 down to 50 times the odds for a pupil with grade $\mathrm{C}$ or below within subject for A level entry from the KS4 cohort; and from 7 down to 2 times the odds for a pupil with grade $C$ or below for choosing the maths or science subjects from amongst $A$ level alternatives. For achievement of grade A or B at A level, prior attainment at grade $A$ is also strongest for maths, followed by biology, then chemistry, then physics; the odds effects range from 200 down to 60 times the odds for a pupil with grade $\mathrm{C}$ or below, from amongst the $\mathrm{A}$ level cohort.

Achievement of level 7+ at Key Stage 3 within subject has independent positive effects on the odds of both entry and achievement at A level, above and beyond those for GCSE prior attainment. For A level entry and subject choice, the effects are largest for maths, followed by physics, then biology, then chemistry, ranging from 
4.4 down to 1.3 times the odds for a pupil with KS3 level 5 or below within subject. The order of effects is different for achievement of grades A or B in the maths and science subjects from amongst the A level cohort; level 7+ makes most difference to physics A level, then biology, then maths, then chemistry, with the odds effects ranging from 2.2 down to 1.3 .

\section{Relative Subject Strength}

The effects of the relative subject strength model factor, an indicator that the pupil's maths or science GCSE grade is higher than their average grade across all GCSES, are generally positive on A level entry, with relative subject strength associated with increased odds of entering the maths or science subject in question at A level. These positive effects on A level entry are generally stronger in the subject choice model (limited to pupils with strong prior attainment who took at least one a level), than in the entry model for the complete Key Stage 4 cohort. Maths is the exception to this, beginning with decreased odds of A level entry for pupils from the KS4 cohort with relative subject strength in maths; the effect is reversed for the subject choice model, resulting in a positive association with choosing maths $A$ level as was the case for biology, physics and chemistry.

For the models of achievement of grades A or B in maths, biology and chemistry, relative strength in these subjects at GCSE was associated with lower odds of achievement at $A$ level. The effect was not statistically significant for physics $A$ level achievement. These reduced chances of achieving top grades at $A$ level for pupils with GCSE grades higher than their subject averages in maths, biology and chemistry reflect the influence of overall GCSE attainment on A level results; an entrant with relative subject strength has other weaker subjects by definition, and a lower overall point score. Figure $6.5 \mathrm{f}$ and discussion (below) gives a worked example translating the odds effects into probabilities.

\section{GCSE Science Route}

The GCSE science route taken by a pupil was significantly predictive of entering $A$ level and of achieving grade A or B at A level for all three sciences. These A level outcomes are most likely for pupils who chose or were selected for separate science GCSEs at a school offering both separate and double science GCSEs, followed by pupils at schools where only separate science GCSEs were offered. Pupils who entered double science GCSEs were less likely to enter or achieve top grades in A level sciences, with those selected for or choosing double science at a school where both routes were offered being even less likely to enter or achieve than those pupils at schools where only double science was offered. The odds effects found for GCSE science routes on A level entry were slightly larger for chemistry; those for achieving grade A or B at A level were slightly larger for physics. Figure $6.5 e$ and discussion (below) gives a worked example translating the odds effects into probabilities.

Although significant odds effects have been found for the GCSE science route taken, it should be borne in mind that this factor is likely to be correlated with other individual or school characteristics, some of which may not be captured in the model, but may affect the pupil's propensity to enter A level sciences and achieve well. In other words, the pupils who choose separate sciences are likely to be different from those who choose double science, and pupils who attend schools where only separate sciences are offered are likely to be different from those who attend schools 
where double science is offered. It is therefore possible that the odds effects on this factor overestimate the true effect to some unknown degree.

\section{Gender}

The odds of entering A level physics are 6 times higher for boys than for girls; this is mirrored in the odds of achieving grade A or B in physics a level, with the odds for boys again being approximately six times those for girls of achieving these top grades. The next strongest gender effects were for biology, where boys had lower odds than girls, at $60 \%$ of the odds for girls of both entering A level biology, and of achieving grade $A$ or $B$ at $A$ level biology. For maths, boys had just over twice the odds of girls, both for entering the A level, and for achieving grades A or B in it. Chemistry had the smallest gender effects, with the odds of entering the A level being $20 \%$ higher for boys than for girls, and the odds of achieving a grade A or B being just $10 \%$ higher for boys than for girls.

\section{Ethnicity}

Almost all ethnic minority groups had higher odds than White British pupils of entering A levels in maths, biology or chemistry, and of achieving grades A or B in these A levels. The strongest effects were for Asian and Chinese pupils, but Black African and Other Black pupils also had smaller raised odds in these subjects. Black Caribbean pupils had slightly raised odds of entering maths or chemistry A levels, but not of achieving top grades in them. Ethnicity effects in physics A level were much less prevalent, with only Chinese and Other White groups having raised odds of both entering and achieving grades A or B. Pakistani and Black Caribbean pupils had lower odds of entering and achieving in A level physics than the White British reference category.

\section{Special Educational Needs}

Pupils with SEN were slightly less likely to continue to A levels in biology or chemistry, but slightly more likely to enter A levels in maths or physics after their prior attainment had been controlled for, e.g. comparing a pupil with no SEN and GCSE grade B with a pupil at with needs at School Action level, but also with a GCSE grade $B$. When considering subject choice by those pupils who have entered at least one $A$ level (which would include only the very top attaining pupils for the groups of pupils with SEN), the odds increases for pupils with SEN on entering A level maths or physics were slightly larger. The odds of entering A level biology go from being lower for pupils with SEN, to being no different from the odds for pupils without SEN. There is also a shift away from decreases in odds and towards raised odds, for pupils with SEN in the case of physics A level entry.

Pupils with Special Educational Needs at School Action have raised odds of achieving grades $A$ or $B$ in maths or physics compared with their prior attainment peers who do not have SEN. Although pupils with SEN are overall much less likely to achieve at A level, for the minority who do attain sufficiently at GCSE to continue to A level, their odds of achievement in maths and physics are better than those of their counterparts without SEN.

\section{Free School Meals}

Eligibility for free school meals, a proxy measure for deprivation, had some fairly small and inconsistent effects on entry to maths and science A levels. Although 
pupils with FSM have smaller absolute probabilities for these outcomes, this appears to be absorbed by other factors in the model, with the prior attainment measures being the likeliest candidates for this. Once prior attainment and the other model factors are controlled for, pupils with FSM have slightly raised odds of choosing A levels in maths, biology and chemistry; these were odds increases of $10-30 \%$. The negative effects of FSM can be traced in slightly reduced odds of entering A levels in physics and biology across the complete KS4 cohort - these are likely to reflect lower odds of entering any A levels (not just physics and biology) for pupils who are eligible for free school meals.

Figure 6.5b now summarises the school level effects in the administrative data models for A level entry and achievement in maths and science subjects.

Fig. $6.5 \mathrm{~b}$

\begin{tabular}{|c|c|c|c|c|c|c|c|c|c|c|c|c|}
\hline \multirow{3}{*}{$\begin{array}{l}\text { A level Outcome } \\
\text { Model Scope }\end{array}$} & \multicolumn{3}{|c|}{ Maths } & \multicolumn{3}{|c|}{ Biology } & \multicolumn{3}{|c|}{ Chemistry } & \multicolumn{3}{|c|}{ Physics } \\
\hline & \multicolumn{2}{|c|}{ Maths Entry } & \multirow{2}{*}{$\begin{array}{l}\text { Grade A/B } \\
\text { KS4 MM } \\
1+\mathrm{A} \text { level }\end{array}$} & \multicolumn{2}{|c|}{ Biology Entry } & \multirow{2}{*}{$\begin{array}{l}\text { Grade A/B } \\
\mid \begin{array}{l}\mathrm{KS} 4 \mathrm{MM} \\
1+\mathrm{A} \text { level }\end{array}\end{array}$} & \multicolumn{2}{|c|}{ Chemistry Entry } & \multirow{2}{*}{$\begin{array}{l}\text { Grade A/B } \\
\mid \begin{array}{c}\mathrm{KS} 4 \mathrm{MM} \\
1+\mathrm{A} \text { level }\end{array}\end{array}$} & \multicolumn{2}{|c|}{ Physics Entry } & \multirow{2}{*}{$\begin{array}{l}\text { Grade A/B } \\
\text { KS4 MM } \\
1+\text { A level }\end{array}$} \\
\hline & KS4 MM & \begin{tabular}{|c|} 
KS4 MM \\
GCSE A*-B \\
$1+$ A level \\
\end{tabular} & & KS4 MM & \begin{tabular}{|c} 
KS4 MM \\
GCSE A*-B \\
$1+\mathrm{A}$ level \\
\end{tabular} & & KS4 MM & \begin{tabular}{|c|} 
KS4 MM \\
GCSE A*-B \\
$1+$ A level \\
\end{tabular} & & KS4 MM & \begin{tabular}{|c|} 
KS4 MM \\
GCSE A*-B \\
$1+$ A level \\
\end{tabular} & \\
\hline School Level Factors... & \multicolumn{12}{|c|}{2009 Effects on Odds (all reported effects are significant at the $5 \%$ level) } \\
\hline KS4 Specialist School & 1.0 & 1.1 & & 0.9 & 0.9 & 0.9 & 0.9 & 0.9 & 0.9 & & & \\
\hline KS4 Grammar & 0.8 & 0.6 & 0.8 & & 0.8 & 0.8 & 0.9 & 0.7 & 0.7 & & 0.7 & 0.7 \\
\hline KS4 Girls School & & & & & 0.9 & 0.9 & & & & 1.5 & 1.8 & 1.9 \\
\hline KS4 Boys School & 0.9 & 0.8 & 0.8 & 0.9 & 0.9 & & 0.7 & 0.7 & 0.7 & 0.7 & 0.6 & 0.7 \\
\hline KS4 has Sixth Form & 1.1 & & 1.1 & 1.2 & & & 1.1 & & 1.1 & 1.3 & 1.2 & 1.1 \\
\hline Entered 4 A Levels & & 1.2 & 1.4 & & 1.1 & 1.2 & & 1.3 & 1.5 & & 1.4 & 1.7 \\
\hline Entered 5+ A Levels & & 2.0 & 2.3 & & & & & 1.7 & 2.1 & & 2.4 & 3.4 \\
\hline KS5 Offers 16-30 A Levels & & 0.6 & 0.7 & & 0.7 & 0.7 & & 0.7 & 0.6 & & 0.7 & 0.7 \\
\hline KS5 Offers 31+ A Levels & & 0.5 & 0.8 & & 0.5 & 0.5 & & 0.5 & 0.5 & & 0.5 & 0.4 \\
\hline 11-20 Entrants (in subject) & & 1.3 & 1.3 & & 1.5 & 1.6 & & 1.3 & 1.4 & & 1.5 & 1.5 \\
\hline 21.30 Entrants (in subject) & & 1.3 & 1.4 & & 1.8 & 1.9 & & 1.6 & 1.7 & & 1.7 & 1.7 \\
\hline 3140 Entrants (in subject) & & 1.4 & 1.5 & & 1.8 & 2.1 & & 1.7 & 1.8 & & 1.4 & 1.6 \\
\hline 41+ Entrants (in subject) & & 1.5 & 1.7 & & 1.9 & 2.3 & & 1.8 & 2.2 & & 1.5 & 1.8 \\
\hline
\end{tabular}

\section{Key Stage 4 School Types}

Pupils who attended KS4 schools with sixth forms had slightly raised odds of entering A levels in maths or science subjects from amongst the complete Key Stage 4 cohort, but these disappeared in the subject choice model, indicating that the effect was on A level continuation in general rather than specific to maths and science subjects. The odds of achieving grades A or B at A level were also raised slightly for maths, chemistry and physics, for pupils who had attended schools with sixth forms at GCSE.

There were no positive and significant effects of attending a specialist (maths and computing / science specialist) school at GCSE after the other model factors were controlled for, suggesting that the raw effects seen in Chapter 5 are likely to operate mainly via increased prior attainment, which is already captured in the models. Probably for similar reasons, grammar schools had decreased odds of A level entry and achievement in the maths and science subjects after controlling for prior attainment. Girls' schools at GCSE had some positive effects on physics entry and achievement, whereas boys' schools were associated with lower odds of entry for all four subjects and achievement for all except biology. Figure $6.5 \mathrm{~g}$ and discussion (below) gives a worked example translating the odds effects into probabilities.

\section{Number of A levels Entered}

Entering four or more resulted in increased odds of entering each of the four maths and science subjects (as would be likely to be the case); it is also associated with 
raised odds of achieving grades $A$ or $B$ in these subjects. This is after prior attainment within subject is controlled for in the models, but may reflect some uncaptured elements of total GCSE attainment, such as having successfully entered larger numbers of GCSEs.

\section{Number of A levels Offered at Key Stage 5 Institution Attended}

Attending a Key Stage 5 institution which offers larger numbers of subjects at A level is associated with lower odds of choosing maths, biology, chemistry or physics, (unsurprisingly), but is also linked to lower odds of achieving grades A or B in these subjects should they be entered.

\section{Number of Entrants in Subject at Key Stage 5 Institution Attended}

Attending an A level institution where there is a large cohort of pupils studying that subject is associated with raised odds of both entering, and achieving top grades in, maths, biology, chemistry and physics A levels.

An alternative way of understanding the odds effects discussed above and summarised in figures $6.5 \mathrm{a}$ and $6.5 \mathrm{~b}$ is to convert them into probabilities for entry and achievement in the maths and science A levels. The background odds of these events are combined with the reported odds effects in order to illustrate a range of scenarios for A level physics entry in figure 6.5c; this example uses the complete Key Stage 4 cohort entry model, encompassing the decision to continue study post-16 and opt for A level qualifications, as well as to choose physics. 


\section{Fig. 6.5c}

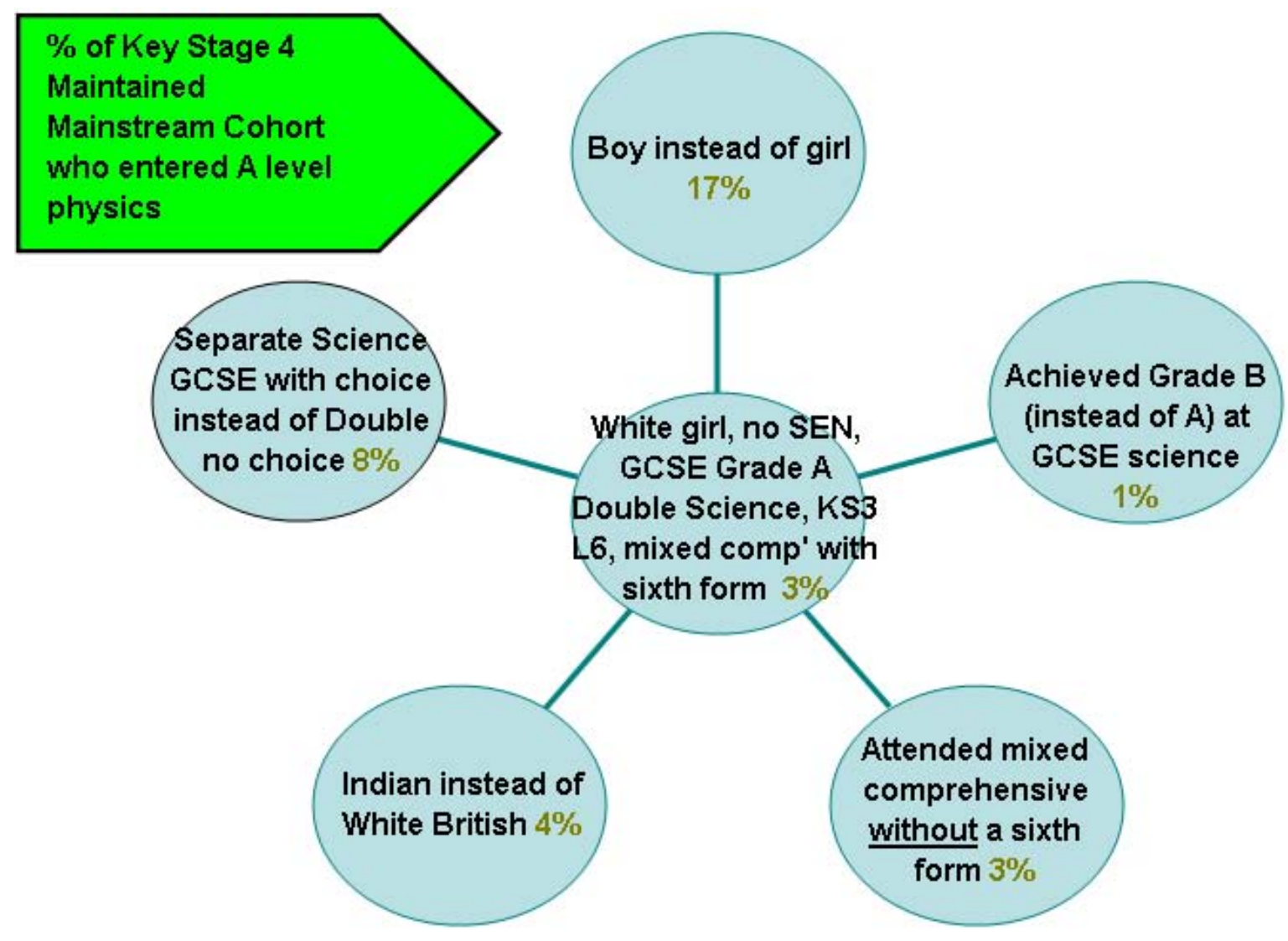

The example begins with a base case (appearing in the centre of the web diagram) of a White British girl, without any special educational needs, who attended a Mixed comprehensive school with a sixth form at GCSE, and achieved a grade AA in double science, following on from level 6 in science at Key Stage 3 . Her probability of entering A level physics is 3\%. Each arm of the web diagram then alters one characteristics from the base case to illustrate the effects of the different characteristics on this probability. 
If instead of being a girl, the pupil was a boy, but with all other factors remaining as they were in the base case, the probability of entering physics A level is much higher at $17 \%$. If the pupil was still a girl, but achieved grade BB at GCSE double science instead of $A A$, her probability of entering $A$ level physics would then be just $1 \%$. If she still had grade AA at GCSE, but attended a mixed comprehensive school without a sixth form for GCSEs, her probability would still be 3\% (this factor is too small on the small underlying probability of entering physics for a girl to make a visible difference). If the pupil was Indian instead of White British, her probability of entering A level physics would be increased to $4 \%$. If she were still White British, but had achieved an A grade in a separate physics GCSE, at a school offering both separate and double sciences, instead of her original double science GCSE at a school only offering this route, her probability would then be $8 \%$. 
Figure $6.5 d$ repeats the probability analysis for achievement of grades A or B in A level physics. The girl in the base case has a probability of entering physics and then gaining a top grade of just $1 \%$. If she were a boy instead of a girl, all other factors being equal, her probability of success would be $8 \%$. If she had achieved grade BB instead of AA at GCSE double science, her probability would then be less than $1 \%$. If she were Indian instead of White British, but with grade AA at GCSE, her probability of achieving grades $A$ or $B$ at $A$ level physics would be $2 \%$. If she were still White British, but held A separate science GCSE in physics at grade A, at a school offering both separate and doubles sciences, instead of her original double science grade AA at a school offering only this route, her probability would then be $3 \%$.

\section{Fig. 6.5d}

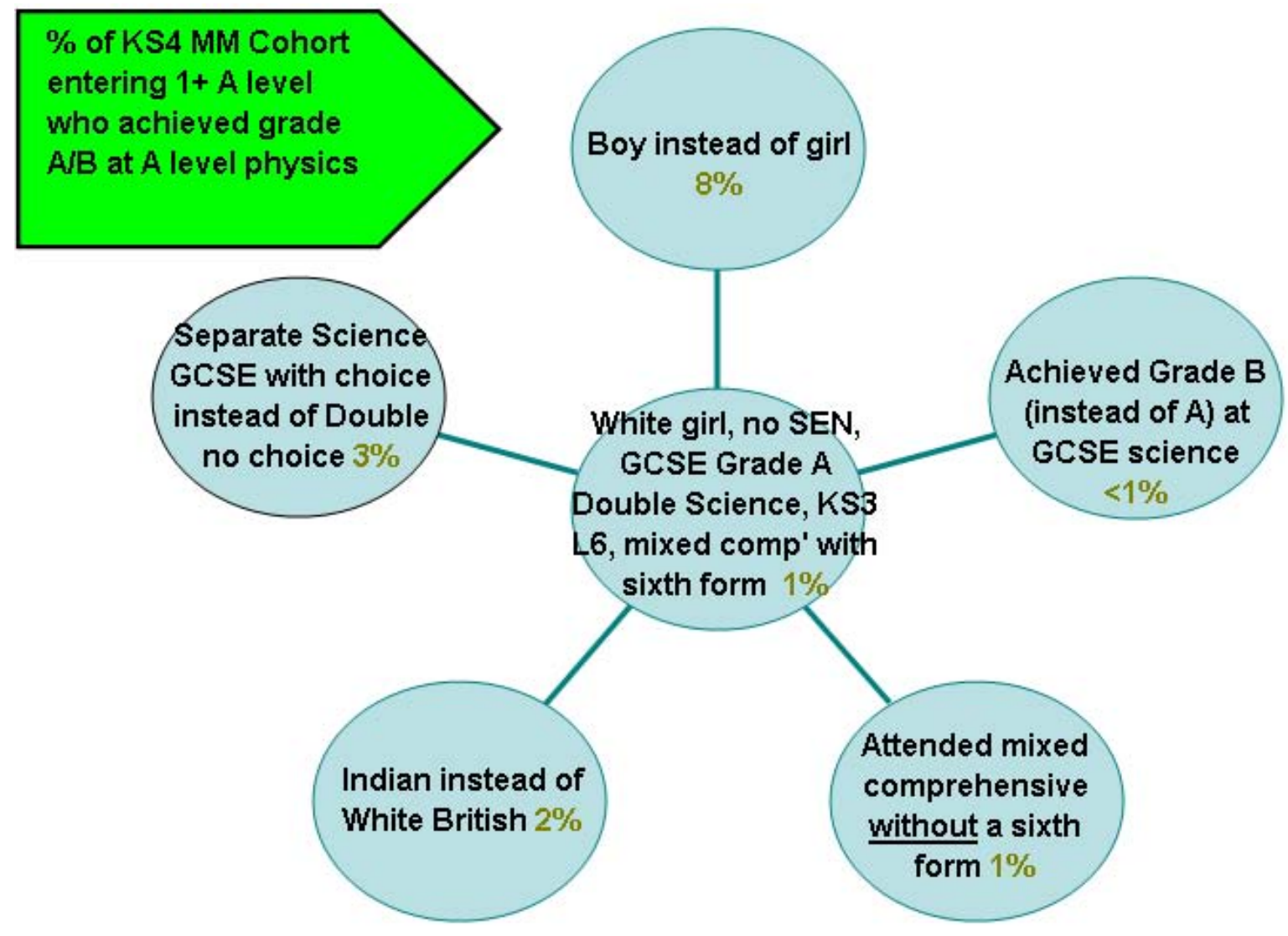

Probability web diagrams for maths, biology and chemistry A levels are included at Annex $\mathrm{C}$ for reference.

Probability analyses generated from the logistic odds models can also be used to examine more detailed sets of scenarios, such as the full exposition of GCSE science routes appearing in figure 6.5e. A base case is selected around which the probabilities for the various scenarios are fitted; in this analysis the base case is a White British girl with within subject prior attainment at grade A (or AA for the double science scenarios); she also has level 6 at Key Stage 3 within subject. 
With one small exception for biology achievement, for all three sciences and across both entry / subject choice models and the achievement model, science A level outcomes are better for pupils who took GCSE separate sciences than those who took GCSE double science. Within these routes, outcomes are better for separate science entrants if they were selected for or chose separate sciences at a school offering both routes; but they are better for double science entrants if they were not selected for and did not choose double science, but rather attended a school where double science was the only route offered. The differences in the probabilities of entering and achieving high grades in A level sciences, between separate sciences and double science, are larger than the differences associated with whether both routes were offered at the school attended.

\section{Fig. $6.5 \mathrm{e}$}

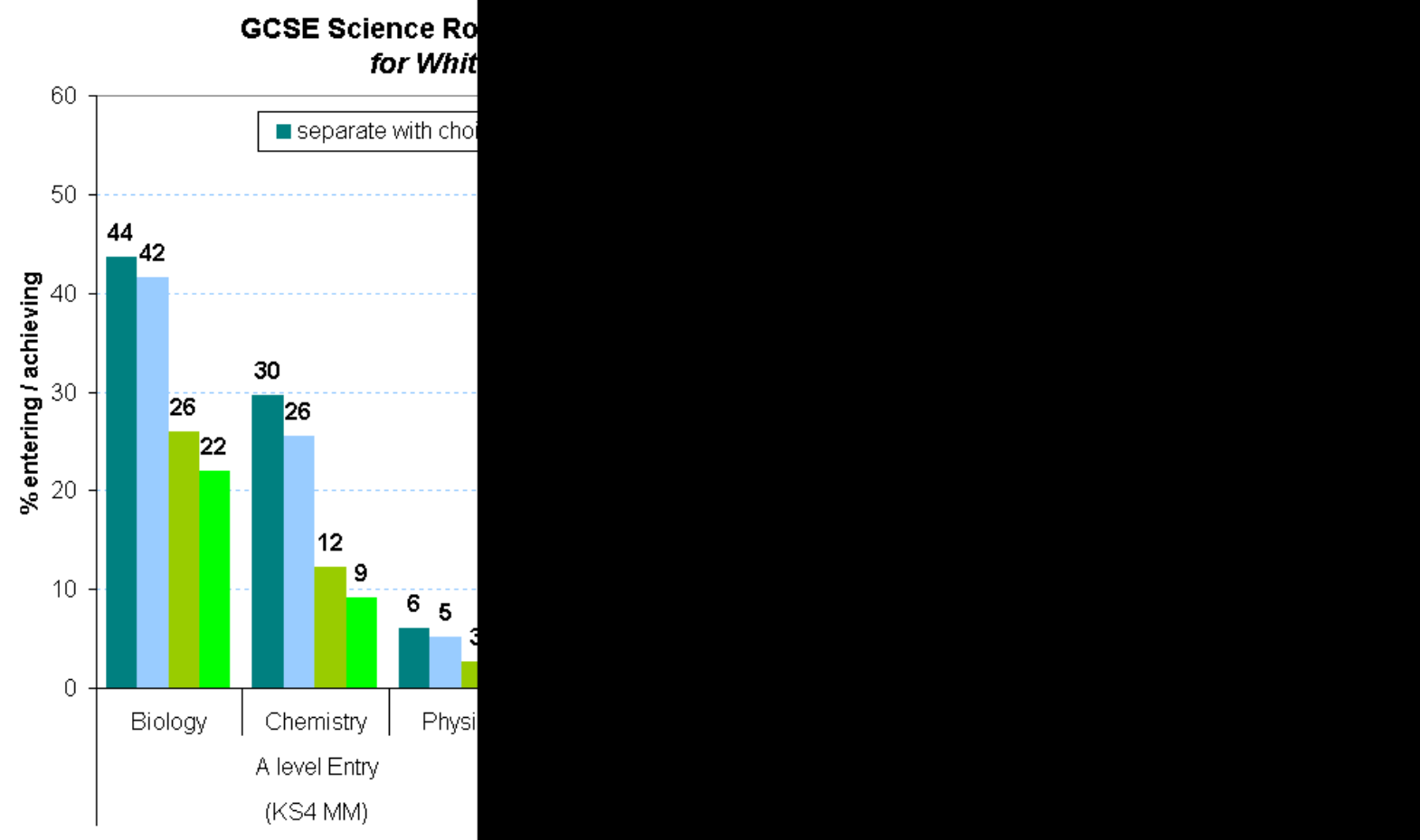


Using the base case of a White British girl with level 6 in science at Key Stage 3, figure $6.5 f$ applies a probability analysis to the odds effects from the science models for different levels of absolute and relative prior attainment. For subject entry at $A$ level, the probability is almost always higher for a pupil with GCSE grade A* within subject than for a pupil with grade $A$, and is always higher for a pupil with grade $A$ than a pupil with grade B. Within grades, pupils have a greater chance of A level continuation if their grade within subject is higher than their average grade across all their GCSEs.

For biology and physics, in the A level subject choice models which limit their scope to pupils with high prior attainment (at B or better) and who have continued to $A$ levels in at least one subject, pupils with GCSE grade A within subject for whom that grade A was above their average grade actually had slightly higher probabilities of continuing to A level than pupils with GCSE grade $A^{*}$ within subject, but for whom $A^{*}$ was their average grade across all their GCSEs.

For achievement of grades A or B at A level, the relative subject strength pattern is also reversed, with each extra grade bringing a higher probability of securing grades A or B in the A level sciences, but with a within subject GCSE grade greater than the pupil's average GCSE grade resulting in a lower probability of achieving a top grade at A level.

\section{Fig. $6.5 f$}

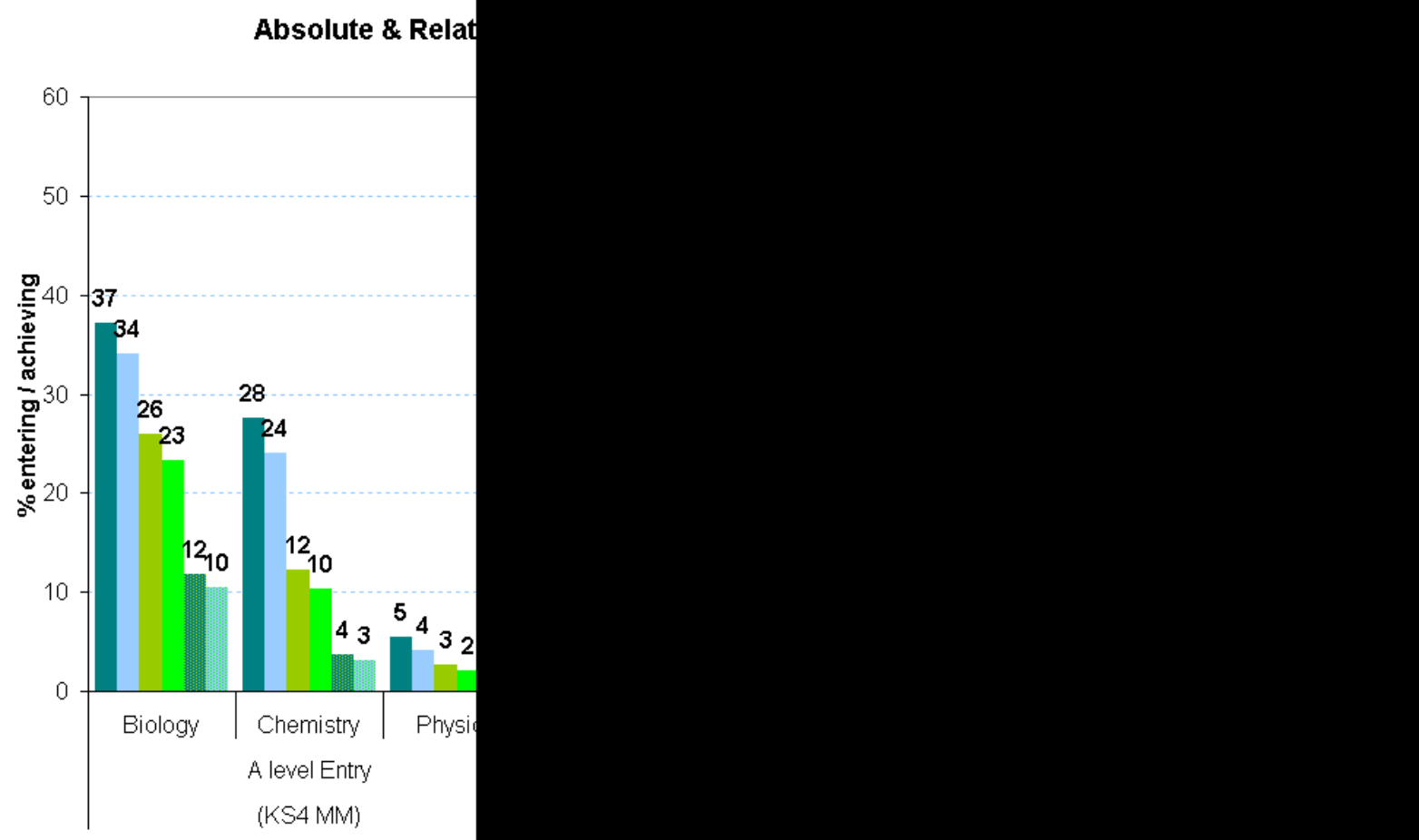

This time using the maths models for the example, figure $6.5 \mathrm{~g}$ translated the odds effects for school type and gender into probabilities of entry and achievement at $A$ level maths. The base case is a White British pupil with level 6 in Key Stage 3 maths and grade $A$ in GCSE maths. The probabilities of entering A level maths are noticeably lower for girls than for boys at all Key Stage 4 school types; the probability of entering maths and going on to achieve a grade $A$ at $A$ level is also lower for girls 
than for boys across the board, though with smaller percentage gaps than was the case for A level entry. Entering A level maths was more likely for pupils from comprehensive schools than those from grammar schools once prior attainment was controlled for, and higher for those comprehensive schools with sixth forms than those without, for both boys and girls.

Pupils from single sex schools also had higher probabilities of going on to take $A$ level maths than those from mixed gender schools. However, this effect largely operated through greater probabilities of continuing to A level in any subject (rather than maths in particular), and when considering only those pupils with high prior attainment who took at least one A level, it was pupils who came from mixed gender schools who were more likely to choose maths (with the exception of boys from grammar schools).

After controlling for prior attainment, pupils from comprehensive schools were also more likely to achieve grades A or B at A level maths than those who had attended grammar schools at Key Stage 4. Boys were again more likely to achieve this outcome than girls for every school type. Single sex schools at GCSE had the advantage over mixed schools for both boys and girls in comprehensive schools, when it came to gaining top grades in A level maths. GCSE schools with sixth forms again produced pupils who were a little more likely to succeed in this outcome than schools without sixth forms.

\section{Fig. $6.5 \mathrm{~g}$}

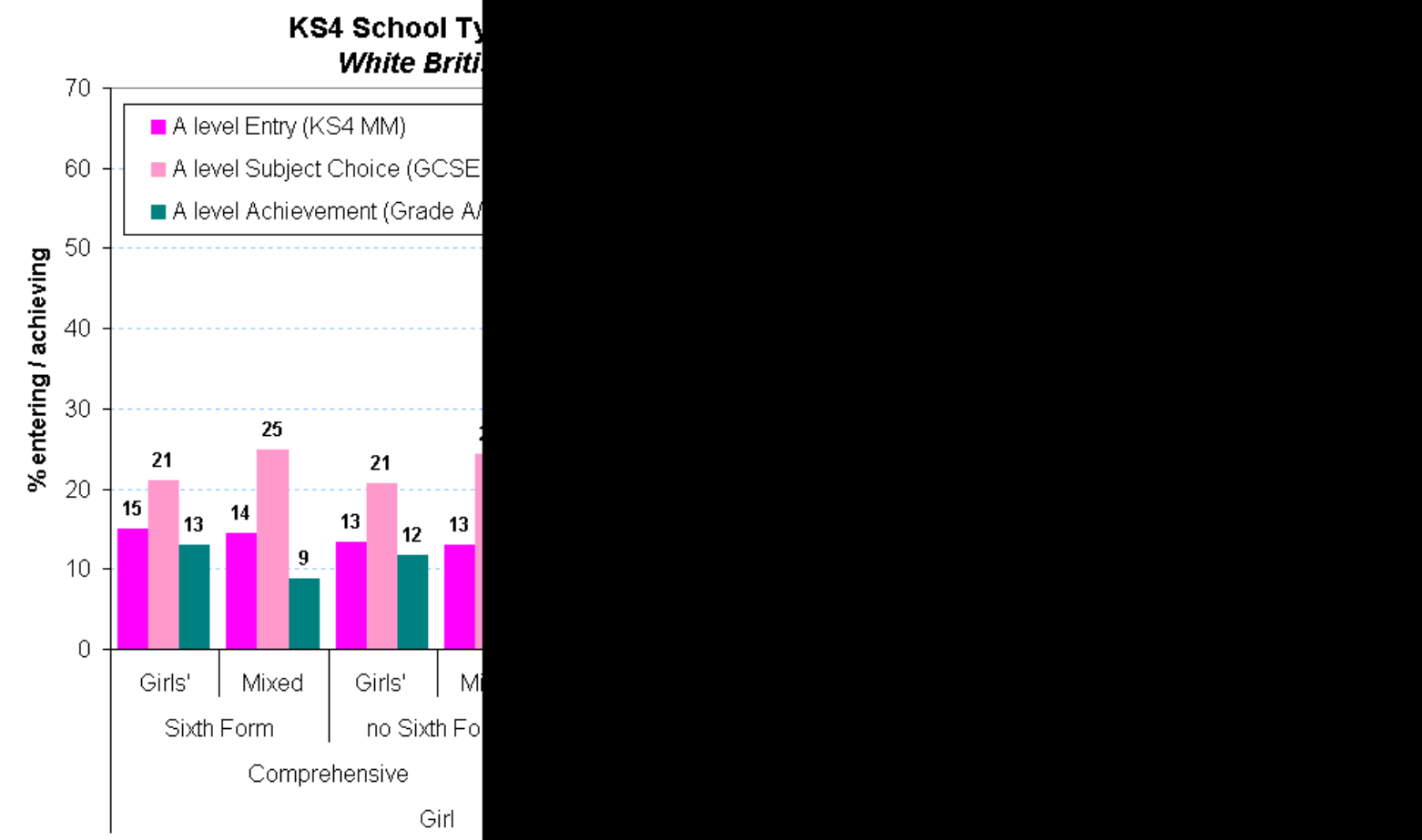

Proceeding to the additional model factors added to the extended models using LSYPE survey data, figure $6.5 \mathrm{~h}$ provides a summary of the odds effects for these wider factors in maths and science A level entry and achievement. 


\section{Subject Enjoyment}

Liking maths and science at age 14 were the most consistently important factors from the LSYPE survey data in predicting subsequent A level entry and achievement. Liking maths increased the odds of entering $A$ level and achieving grades $A$ or $B$ for all of the subjects except biology. Liking science increased the odds on both outcomes for maths as well as biology, chemistry and physics.

Using the logarithmic scale applied in the charts in sections 6.2 to 6.4 , liking the subject at age 14 is approximately one fifth as important as having a grade $A$ at GCSE in predicting A level entry for maths, biology and chemistry, a little less so for physics. Restricting the comparisons to pupils with a minimum of grade B at GCSE within subject, who entered at least one A level (focusing in on subject choice), the importance of liking the subject at age 14 is even greater, at around one third as important as a GCSE grade A for maths, almost as important as a grade A for biology and chemistry, and more important than a grade A for choosing physics.

\section{Belief in Subject Ability}

For A level maths, thinking that they were good at maths at age 14 also gives higher odds of choosing the subject at A level and of achieving a grade A or B at A level; this is above and beyond the effects of actual prior attainment at KS3 and GCSE, and the effects of liking the subject or otherwise. Thinking they were good at science at the age of 14 similarly increased the odds of entering $A$ level biology and of then achieving grades $\mathrm{A}$ or $\mathrm{B}$. However, this belief in ability at an earlier age was not significantly associated with better A level outcomes for chemistry or physics after actual prior attainment was controlled for.

\section{Working as Hard as Possible}

After controlling for KS3 and GCSE attainment, pupils who stated that they worked as hard as they could at school at age 14 had raised odds of continuing to A level and choosing maths as one of their subjects; they also had greater odds of entering biology and chemistry A levels, but not of entering physics. This factor was not associated with achieving higher grades at A level however.

\section{Locus of Control}

Pupils who said at age 14 that they believed a lack of success in life is usually one's own fault had increased odds of taking A levels and choosing chemistry or physics. Maths and biology entry were not significantly associated with this attitude, however, and it did not raise the odds of actually achieving high grades in maths and science $A$ levels.

\section{Eating Together as a Family}

Pupils who reported that they ate together with their family most nights or every night at age 14 had increased odds of choosing biology or physics A levels, and of achieving grades A or B in biology, chemistry and physics. This family behaviour was not significantly associated with maths A level outcomes, however.

\section{Living with Married or Cohabiting Parents}

Pupils who lived with married or cohabiting parents at age 14 were more likely to enter biology or chemistry at A level (but not maths or physics), but this seems to operate through a greater probability of taking any A levels rather than subject 
choice, with no effect for biology amongst those pupils with high prior attainment who took at least one A level, and a smaller effect for chemistry for this group.

\section{Parents Evenings and Private Tuition}

There were isolated effects in biology only for pupils whose parents attended parents' evenings or similar events during Key Stage 3, and for A level entry in chemistry only for pupils who received private tuition at least once a week at age 14. It is not clear why these factors were significant for specific sciences but not more generally.

\section{IDACI Deprivation}

Living in an area of lower deprivation (bottom 50\%) also had some isolated effects in particular models, with this group of pupils having raised odds of entering A level maths and chemistry (but not subject choice from amongst the high prior attaining $A$ level cohort in maths). They also had higher odds of achieving grades $A$ or $B$ in $A$ level chemistry. Again, it is not clear why chemistry outcomes are associated with this factor but biology and physics outcomes are not.

\section{Fig. 6.5h}

\begin{tabular}{|c|c|c|c|c|c|c|c|c|c|c|c|c|}
\hline \multirow{3}{*}{$\begin{array}{l}\text { A level Outcome } \\
\text { Model Scope }\end{array}$} & \multicolumn{3}{|c|}{ Maths } & \multicolumn{3}{|c|}{ Biology } & \multicolumn{3}{|c|}{ Chemistry } & \multicolumn{3}{|c|}{ Physics } \\
\hline & \multicolumn{2}{|c|}{ Maths Entry } & \multirow{2}{*}{$\begin{array}{c}\text { Grade A/B } \\
\text { KS4 MM } \\
1+\mathrm{A} \text { level }\end{array}$} & \multicolumn{2}{|c|}{ Biology Entry } & \multirow{2}{*}{ 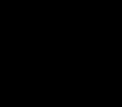 } & \multicolumn{2}{|c|}{ Chemistry Entry } & \multirow{2}{*}{$\begin{array}{l}\text { Grade A/B } \\
\qquad S S 4 \text { MM } \\
1+\mathrm{A} \text { level }\end{array}$} & \multicolumn{2}{|c|}{ Physics Entry } & \multirow{2}{*}{$\begin{array}{l}\text { Grade A/B } \\
\text { KS4 MM } \\
1+\mathrm{A} \text { level }\end{array}$} \\
\hline & KS4 MM & $\begin{array}{c}\text { KS4 MM } \\
\text { GCSE } A^{*}-\mathrm{B} \\
1+\mathrm{A} \text { level } \\
\end{array}$ & & KS4 MM & $\begin{array}{c}\text { KS4 MM } \\
\text { GCSE } A^{*}-\mathrm{B} \\
1+\mathrm{A} \text { level } \\
\end{array}$ & & KS4 MM & $\begin{array}{l}\text { KS4 MM } \\
\text { GCSE A*-E } \\
1+\text { A level }\end{array}$ & & KS4 MM & $\begin{array}{l}\text { KS4 MM } \\
\text { GCSE A*-E } \\
1+\text { A level }\end{array}$ & \\
\hline & \multicolumn{12}{|c|}{ Effects on Odds (- significant at the $5 \%$ level; * significant at the $10 \%$ level) } \\
\hline $\begin{array}{l}\text { Low Deprivation } \\
\text { (Bottom } 50 \% \text { IDACl) }\end{array}$ & $\begin{array}{c}1.3^{* *} \\
\text { (increase) }\end{array}$ & & & & & & $\begin{array}{c}1.4^{* *} \\
\text { (increase) }\end{array}$ & \begin{tabular}{|c|c|}
$1.3^{*}$ \\
(increase)
\end{tabular} & $\mid \begin{array}{c}1.5^{*} \\
\text { (increase) }\end{array}$ & & & \\
\hline $\begin{array}{l}\text { Professional Occupation } \\
\text { (NSSEC) }\end{array}$ & & & & & & & & & \begin{tabular}{|c|}
$1.5^{*}$ \\
(increase)
\end{tabular} & & & \\
\hline $\begin{array}{l}\text { Private Tuition } \\
\text { at least oncelweek }\end{array}$ & & & & & & & $\begin{array}{c}1.3^{ \pm *} \\
\text { (increase) }\end{array}$ & $\begin{array}{c}1.4^{*} \\
\text { (increase) }\end{array}$ & & & & \\
\hline $\begin{array}{l}\text { Family Eat Together } \\
\text { everyimost nights }\end{array}$ & & & & $\begin{array}{c}1.3^{* *} \\
\text { (increase) }\end{array}$ & \begin{tabular}{|c|}
$1.3^{* *}$ \\
(increase)
\end{tabular} & $\begin{array}{c}1.6^{* *} \\
\text { (increase) }\end{array}$ & & & $\begin{array}{c}1.4^{*} \\
\text { (increase) }\end{array}$ & & $\begin{array}{c}1.3^{*} \\
\text { (increase) }\end{array}$ & $\begin{array}{c}1.5^{*} \\
\text { (increase) }\end{array}$ \\
\hline $\begin{array}{l}\text { Parents Evenings } \\
\text { attended }\end{array}$ & & & & $\begin{array}{c}1.5^{\text {t* }} \\
\text { (increase) }\end{array}$ & \begin{tabular}{|c|}
$1.5^{* \pm}$ \\
(increase)
\end{tabular} & $\begin{array}{c}1.8^{*} \\
\text { (increase) }\end{array}$ & & & & & & \\
\hline $\begin{array}{l}\text { Married / Cohabiting } \\
\text { Parents }\end{array}$ & & & & $\begin{array}{c}1.3^{\text {t* }} \\
\text { (increase) }\end{array}$ & & & $\begin{array}{c}1.6^{\text {t*t }} \\
\text { (increase) }\end{array}$ & $\begin{array}{c}1.5^{*} \\
\text { (increase) }\end{array}$ & & & & \\
\hline $\begin{array}{l}\text { "In life, lack of success } \\
\text { is usually own fault" }\end{array}$ & & & & & & & $\begin{array}{c}1.6^{\text {t* }} \\
\text { (increase) }\end{array}$ & $\begin{array}{c}1.6^{\text {t* }} \\
\text { (increase) }\end{array}$ & & $\begin{array}{c}1.4^{\text {** }} \\
\text { (increase) }\end{array}$ & $\begin{array}{c}1.3^{*} \\
\text { (increase) }\end{array}$ & \\
\hline "Like maths" & $\begin{array}{l}2.4^{\text {** }} \\
\text { (increase) }\end{array}$ & $\begin{array}{c}2.7^{* *} \\
\text { (increase) }\end{array}$ & $\begin{array}{c}3.2^{\text {** }} \\
\text { (increase) }\end{array}$ & & & & $\begin{array}{c}1.6^{* *} \\
\text { (increase) }\end{array}$ & $\begin{array}{c}1.6^{\text {t* }} \\
\text { (increase) }\end{array}$ & $\begin{array}{c}1.6^{* *} \\
\text { (increase) }\end{array}$ & $\begin{array}{c}1.4^{* *} \\
\text { (increase) }\end{array}$ & $\begin{array}{c}1.4^{*} \\
\text { (increase) }\end{array}$ & $\begin{array}{c}1.6^{*} \\
\text { (increase) }\end{array}$ \\
\hline "Like science" & $\begin{array}{l}2.1^{* \pm} \\
\text { (increase) }\end{array}$ & $\begin{array}{c}2.3^{* *} \\
\text { (increase) }\end{array}$ & $\begin{array}{l}2.1^{* *} \\
\text { (increase) }\end{array}$ & $\begin{array}{c}1.9^{* *} \\
\text { (increase) }\end{array}$ & $\begin{array}{c}2.1^{* *} \\
\text { (increase) }\end{array}$ & $\begin{array}{c}1.6^{*} \\
\text { (increase) }\end{array}$ & $\begin{array}{c}2.6^{\text {t* }} \\
\text { (increase) }\end{array}$ & $\begin{array}{c}2.7^{* *} \\
\text { (increase) }\end{array}$ & \begin{tabular}{|c|}
$2.7^{* * *}$ \\
(increase)
\end{tabular} & $\begin{array}{l}2.6^{\text {t* }} \\
\text { (increase) }\end{array}$ & $\begin{array}{c}2.5^{\text {t* }} \\
\text { (increase) }\end{array}$ & $\begin{array}{c}2.5^{*} \\
\text { (increase) }\end{array}$ \\
\hline "Am good at maths" & & \begin{tabular}{|c|}
$2.1^{*}$ \\
(increase)
\end{tabular} & \begin{tabular}{|c|}
$3.1^{*}$ \\
(increase)
\end{tabular} & $\begin{array}{c}1.6^{\star} \\
\text { (increase) }\end{array}$ & \begin{tabular}{|c|}
$1.6^{ \pm}$ \\
(increase)
\end{tabular} & \begin{tabular}{|c|}
$2.0^{*}$ \\
(increase)
\end{tabular} & & & & & & \\
\hline "Am good at science" & $\begin{array}{l}0.6^{\text {t* }} \\
\text { decrease }\end{array}$ & $\begin{array}{c}0.6^{* *} \\
\text { (decrease) }\end{array}$ & & $\begin{array}{c}1.9^{* *} \\
\text { (increase) }\end{array}$ & $\begin{array}{c}1.9^{* *} \\
\text { (increase) }\end{array}$ & & & & & & & \\
\hline $\begin{array}{l}\text { "Works As Hard } \\
\text { As HeiShe Can" }\end{array}$ & $\begin{array}{c}1.4^{*} \\
\text { (increase) }\end{array}$ & $\begin{array}{c}1.4^{*} \\
\text { (increase) }\end{array}$ & & $\begin{array}{c}1.4^{*} \\
\text { (increase) }\end{array}$ & & & $\begin{array}{c}2.0^{* \pm} \\
\text { (increase) }\end{array}$ & $\begin{array}{c}1.7^{* \pm} \\
\text { (increase) }\end{array}$ & & & & \\
\hline
\end{tabular}


To conclude, figure 6.5i presents the factors from the LSYPE survey data that were tested in the A level maths and science models, but were not important for either A level entry and subject choice or for achievement of high grades. Amongst these factors which were not associated with the outcomes of interest were pupil aspirations to stay on in full time education post-16 (measured at age 14), parental employment status, receipt of an Education Maintenance Allowance, and the pupil's perception of their school's reputation. Factors which were re-tested in different categorisations as they came closer to significance, but which ultimately were dropped as they were still not significant, included household income and highest parental qualification.

\section{Fig. 6.5d}

\begin{tabular}{|c|c|c|c|}
\hline \multicolumn{4}{|c|}{ Additional (LSYPE) Factors that Made no Difference to Maths and Science A Level Outcomes } \\
\hline \multicolumn{4}{|c|}{ Rejected and removed from the models (neither significant nor near-significant) } \\
\hline Factor & Age & Categories Tested in Early Models & \\
\hline & & Omitted reference category appears in grey & \\
\hline Post-16 Aspirations of Pupil & 14 & $\begin{array}{l}\text { Stay on in full-time education (including those who plan to } \\
\text { leave but return later) / Leave full-time education / Not sure / } \\
\text { Missing or not stated aspirations. }\end{array}$ & \\
\hline Parental Aspirations for Pupil & 14 & $\begin{array}{l}\text { Stay on in full-time education (including return later) / Leave full- } \\
\text { time education / Not sure / Missing or not stated aspirations. }\end{array}$ & \\
\hline Discussing School & 14 & $\begin{array}{l}\text { Parent talks to child about school at least once a week / Once } \\
\text { or twice a week or at least once a term / Less often than once } \\
\text { a term / Never / Don't know or missing. }\end{array}$ & \\
\hline Parental Employment Status & 14 & $\begin{array}{l}\text { At least one parent works / Couple, neither works / Lone } \\
\text { parent, doesn't work. }\end{array}$ & \\
\hline Birth Order & 14 & $\begin{array}{l}\text { Only child / Older of two children / Younger of two children / } \\
\text { Oldest of } 3+\text { children / Youngest of } 3+\text { children / In the middle } \\
\text { of } 3+\text { children / Unclear or missing birth order. }\end{array}$ & \\
\hline Bullying (Victim of & 14 & $\begin{array}{l}\text { Pupil reported having been bullied / Parent reported / Both } \\
\text { reported / Neither reported / missing. }\end{array}$ & \\
\hline School Free School Meals Rate & 14 & $\begin{array}{l}1.5 \% / 5-9 \% / 9-13 \% / 13-21 \% / 21-35 \% / 35-50 \% / 50 \%+/ \\
\text { missing. }\end{array}$ & \\
\hline School Reputation Perception & 14 & $\begin{array}{l}\text { Pupil feels people think their school is a good school/ Does } \\
\text { not feel people think their school is a good school/ } \\
\text { Missing or not stated. }\end{array}$ & \\
\hline Education Maintenance Allowance & 17 & $\begin{array}{l}\text { Receives EMA / Does not receive EMA / Applied for EMA, } \\
\text { awaiting outcome / Unknown. }\end{array}$ & \\
\hline \multicolumn{4}{|c|}{ Retained in the models but still failed to reach significance when retested using simpler categorisations } \\
\hline Factor & Age & Categories Tested in Early Models & Categories Tested in Revised Models \\
\hline Household Income & 14 & $\begin{array}{l}\text { Refused or missing income / Don't know income / Less than } \\
£ 5,200 / £ 5,200-£ 10,400 / £ 10,400-£ 15,600 / £ 15,600 \text { - } \\
£ 20,800 / £ 20,800-£ 26,000 / £ 26,000-£ 31,200 / £ 31,200 \text { - } \\
£ 36,400 / \text { More than } £ 36,400 \text {. }\end{array}$ & $\begin{array}{l}\text { More than } £ 32,100 / £ 32,100 \text { or less / Refused or missing } \\
\text { incomve / Don't know income. }\end{array}$ \\
\hline Highest Parental Qualification & 14 & $\begin{array}{l}\text { No qualifications held / Degree / Higher Education / A Level / } \\
\text { GCSE } A^{*}-C \text { / Level } 1 \text { or below or other qualifications / No } \\
\text { information given. }\end{array}$ & $\begin{array}{l}\text { Degree or Higher Education / Lower than Higher Education / } \\
\text { No informations given. }\end{array}$ \\
\hline Supplementary Classes & 14 & $\begin{array}{l}\text { Pupil attends supplementary classes (additional subjects) } 3 \text { or } \\
4 \text { times a week over the last } 12 \text { months / Less than } 3 \text { but more } \\
\text { than } 1 \text { times a week over the last } 12 \text { months / Once a week or } \\
\text { less over the last } 12 \text { months / Never / Missing info. }\end{array}$ & $\begin{array}{l}\text { More than once a week / Once a week or less (including } \\
\text { never) / Missing info. }\end{array}$ \\
\hline
\end{tabular}




\section{Annex A: Numbers of Pupils Progressing to A level}

\section{GCSE}

Progression of GCSE Maths Cohorts to A-level Maths for 2007-2009

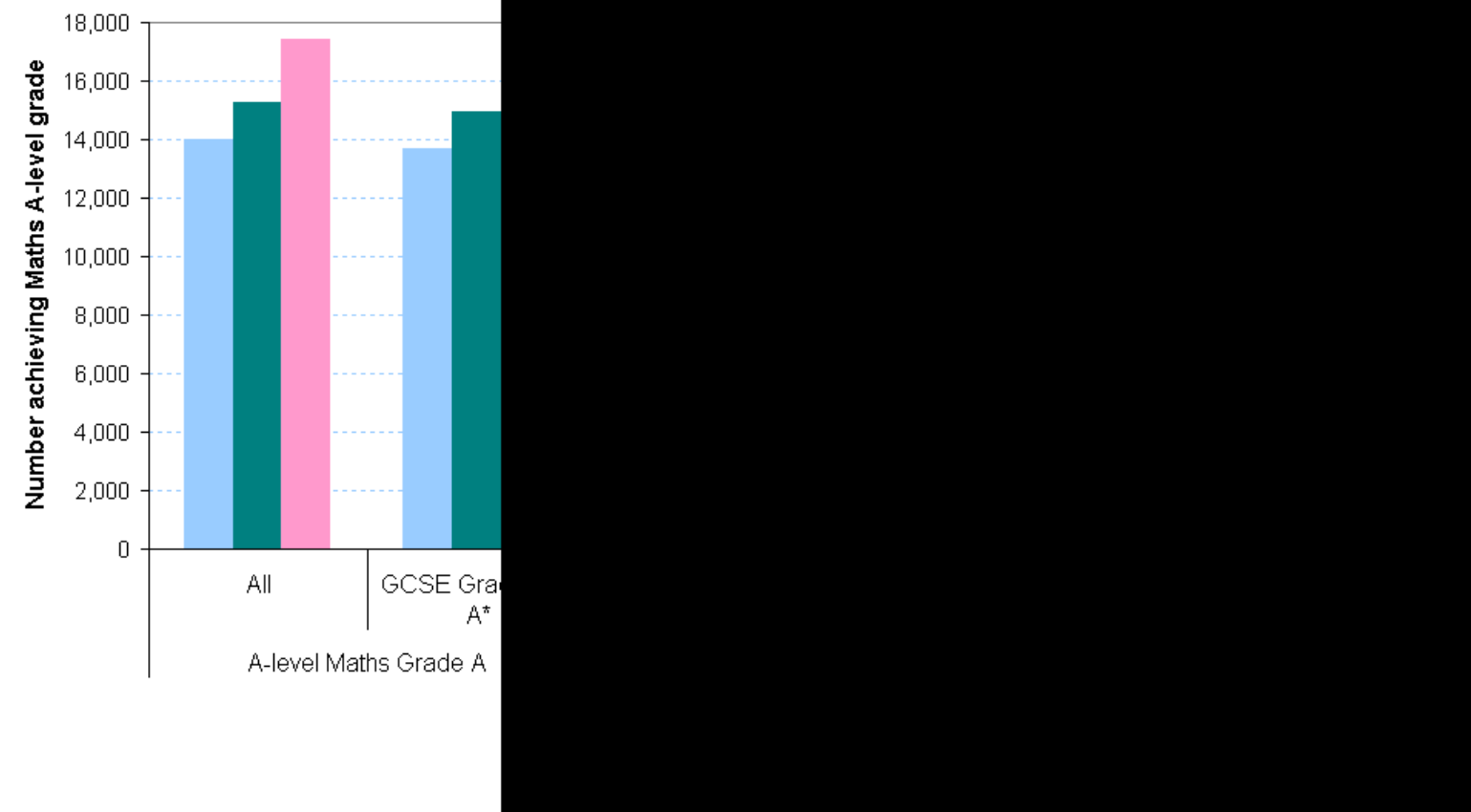

Progression of GCSE Science Cohorts to A-level Biology for 2007-2009

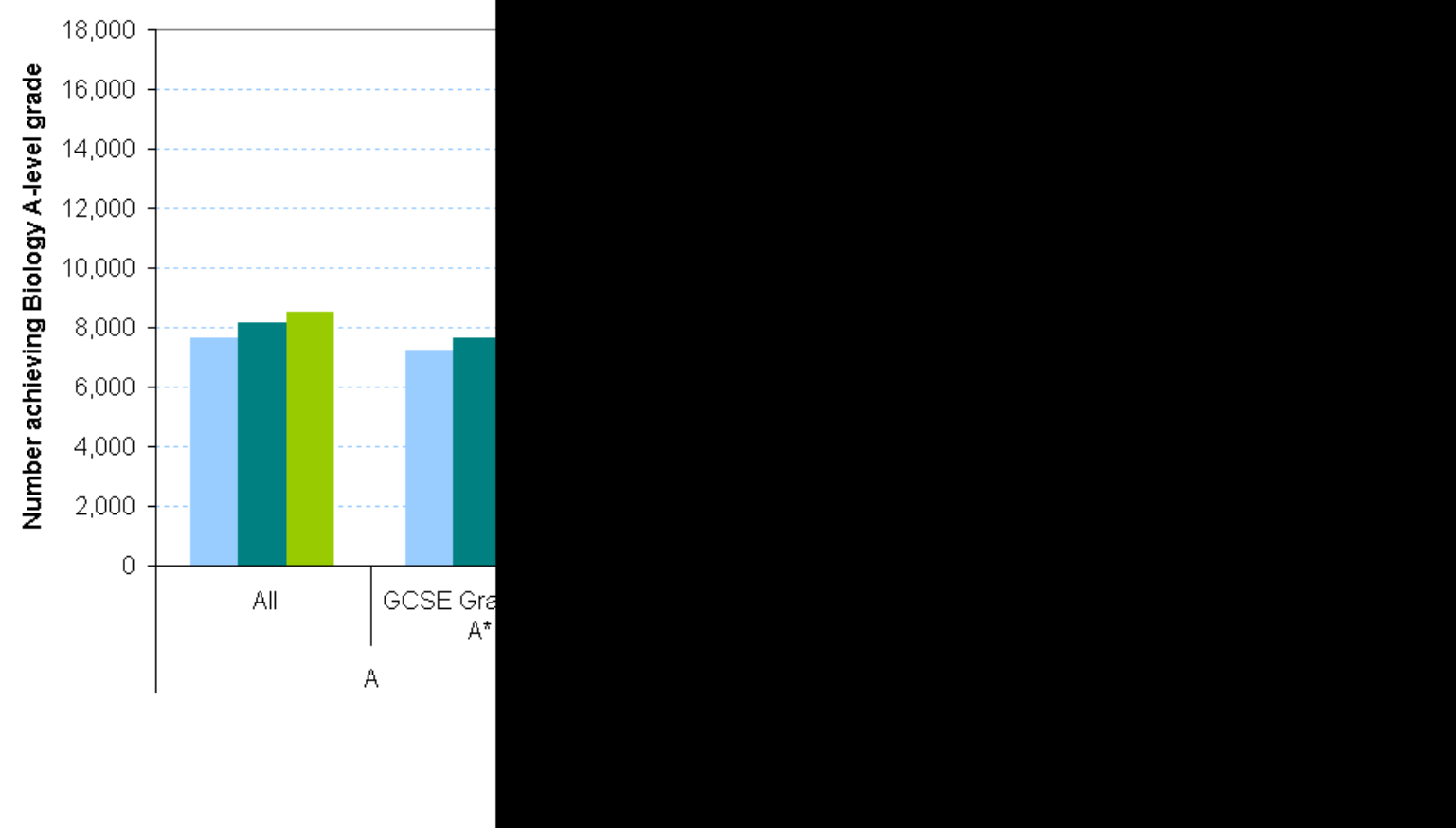




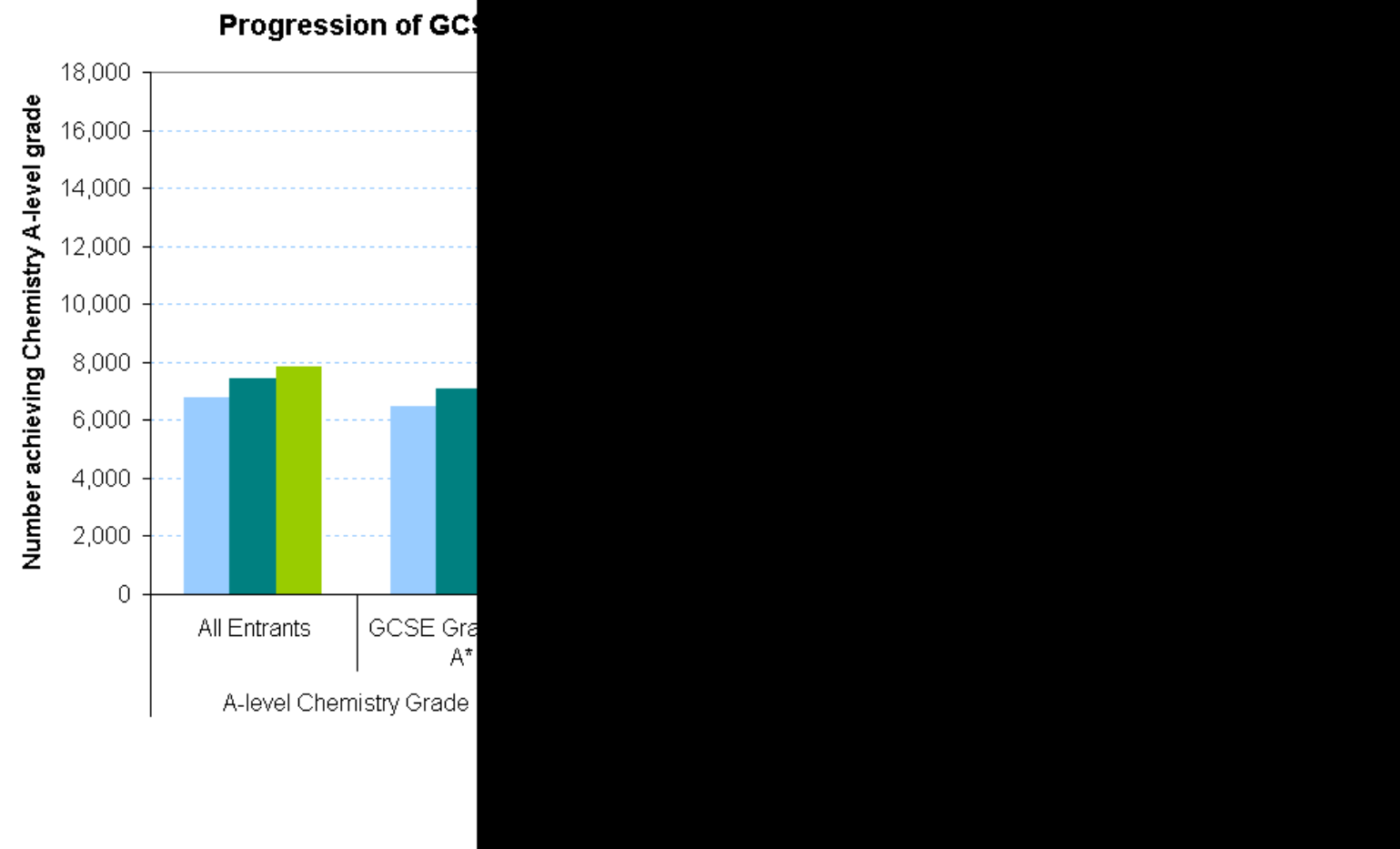

Progression of GCSE Science Cohorts to A-level Physics for 2007-2009

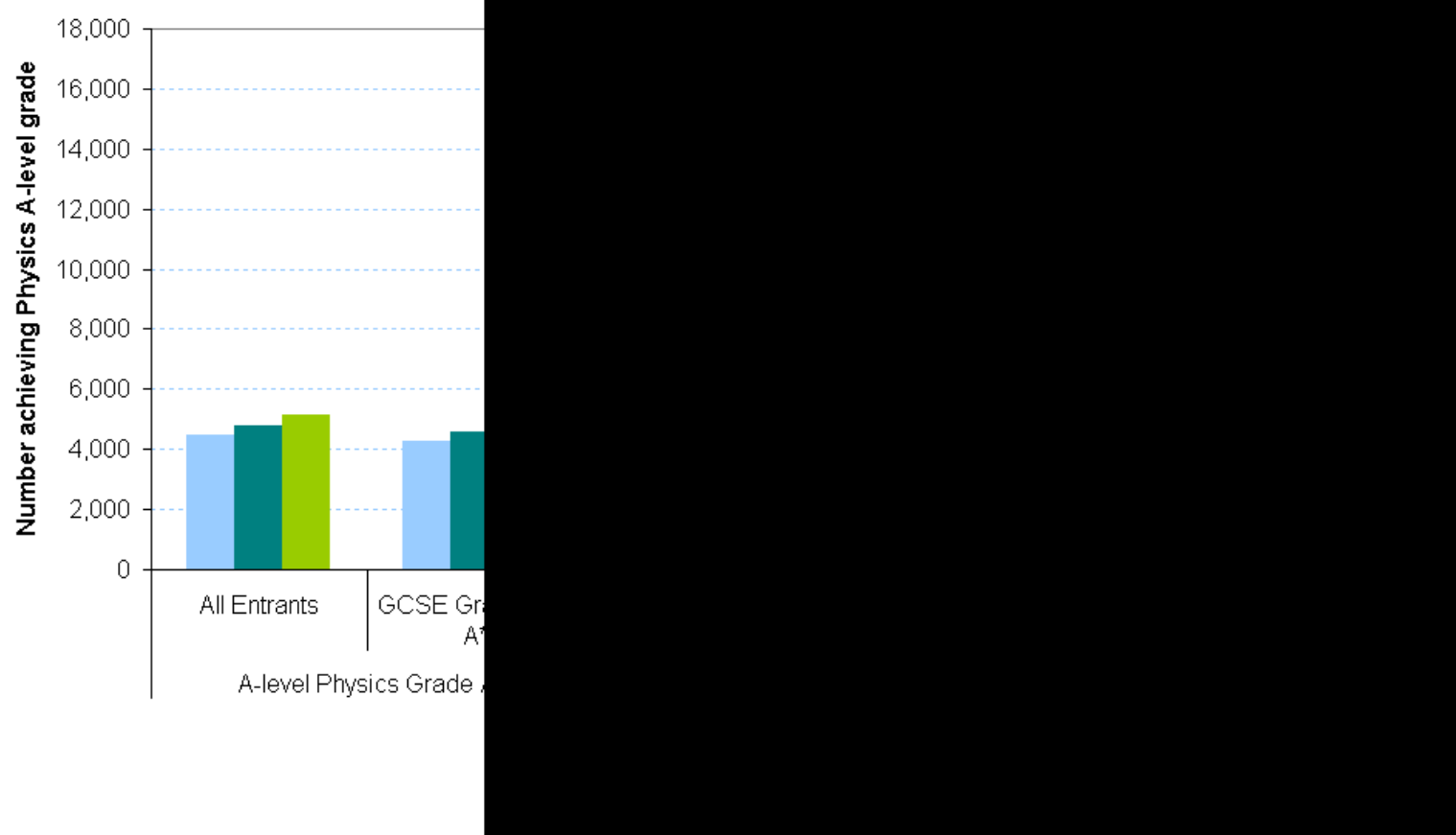




\section{Key Stage 3}

Progression of KS3 Maths Cohorts to A-level Maths for 2007-2009

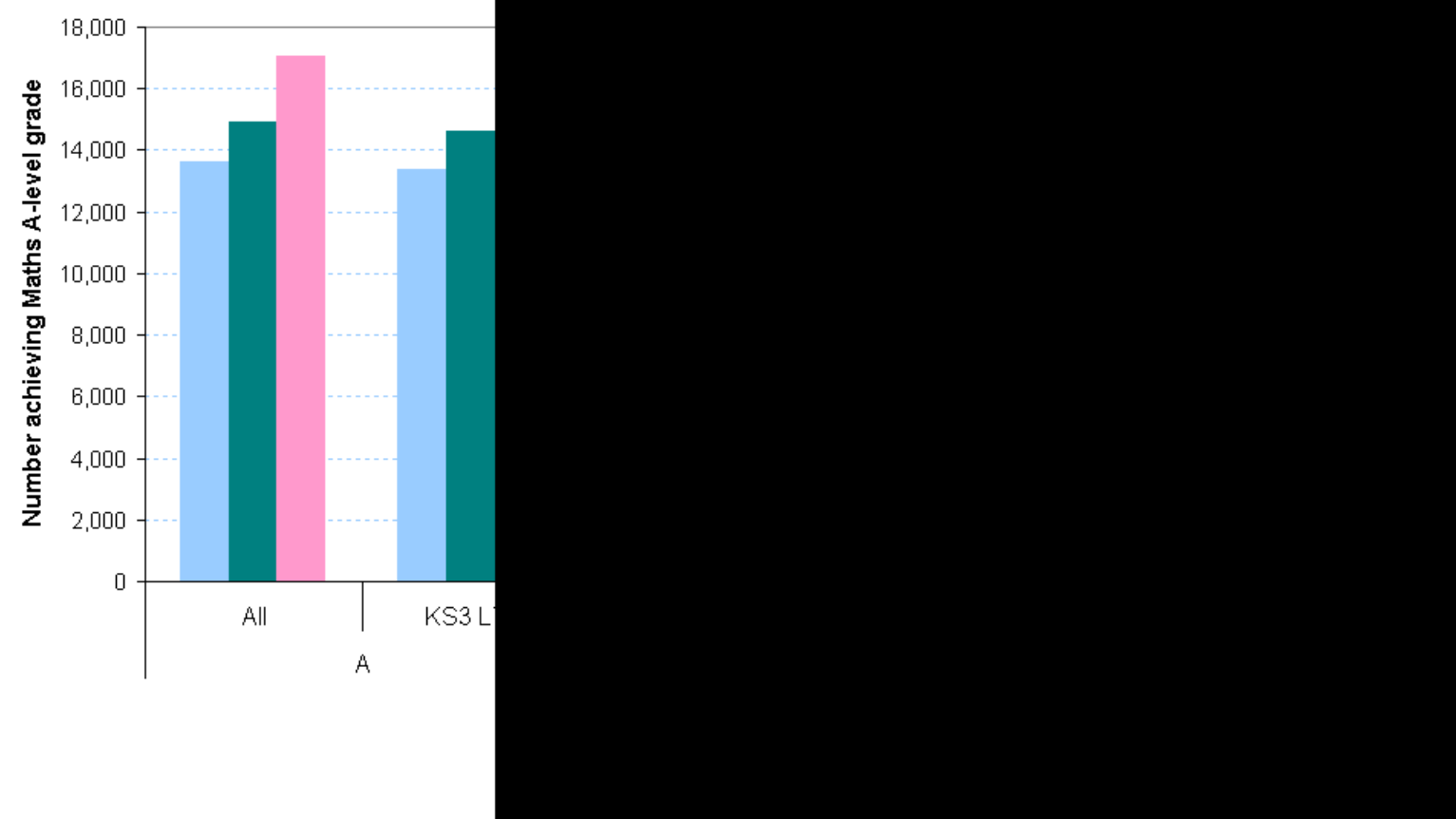

Progression of KS3 Science Cohorts to A-level Biology for 2007-2009

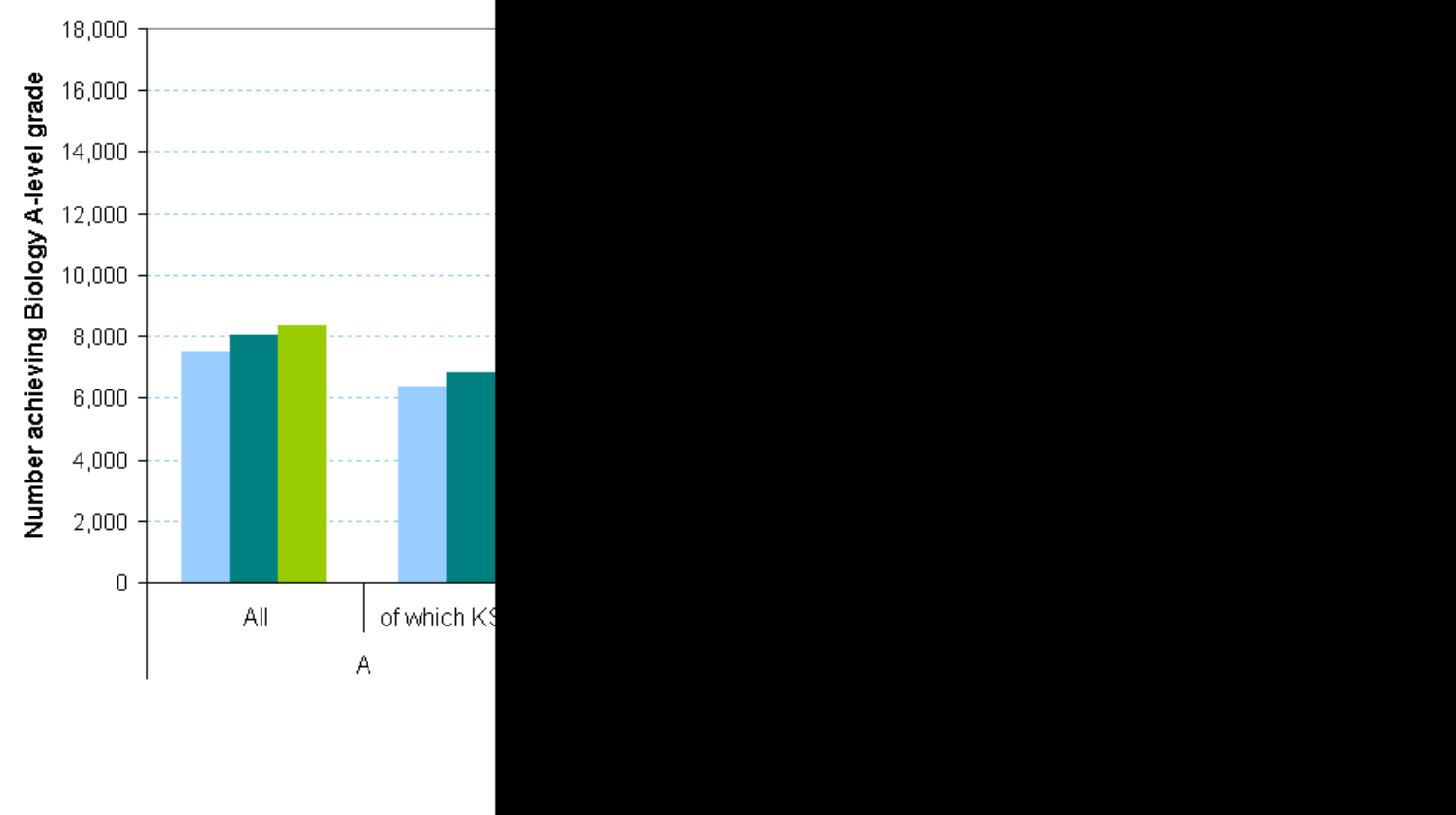


Progression of KS3 Science Cohorts to A-level Chemistry for 2007-2009

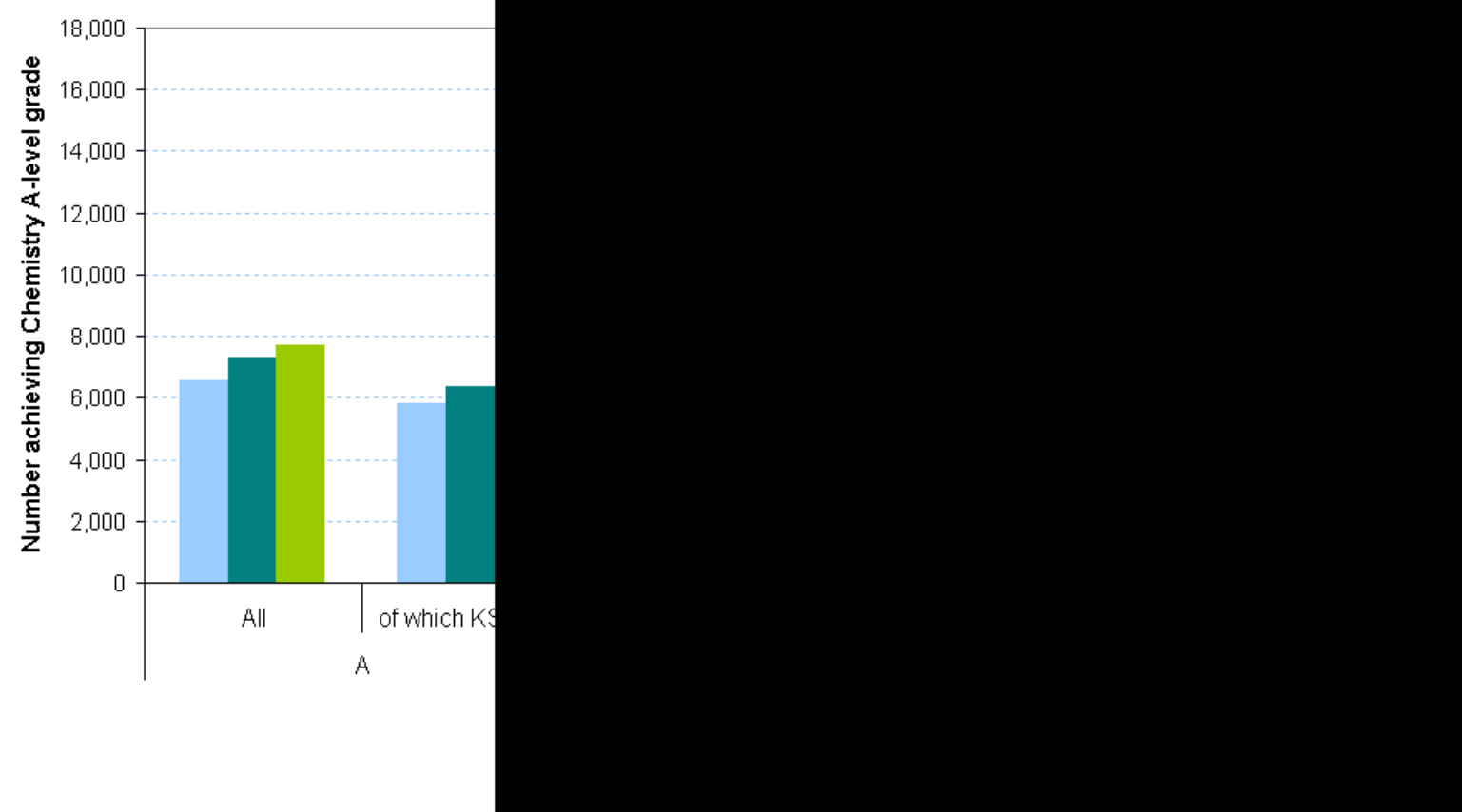

Progression of KS3 Science Cohorts to A-level Physics for 2007-2009

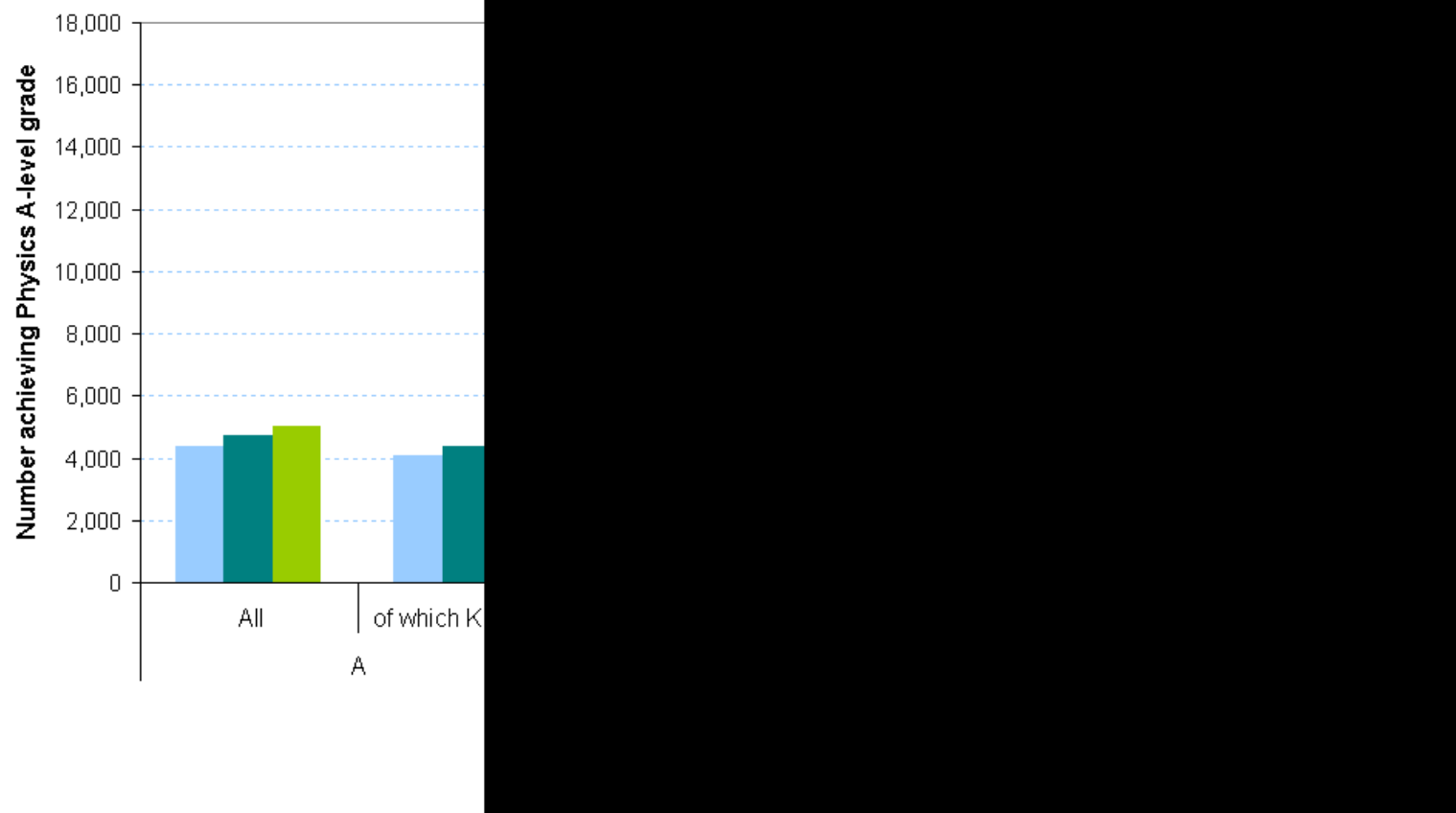




\section{Key Stage 2}

Progression of KS2 Maths Cohorts to A-level Maths for 2007-2009

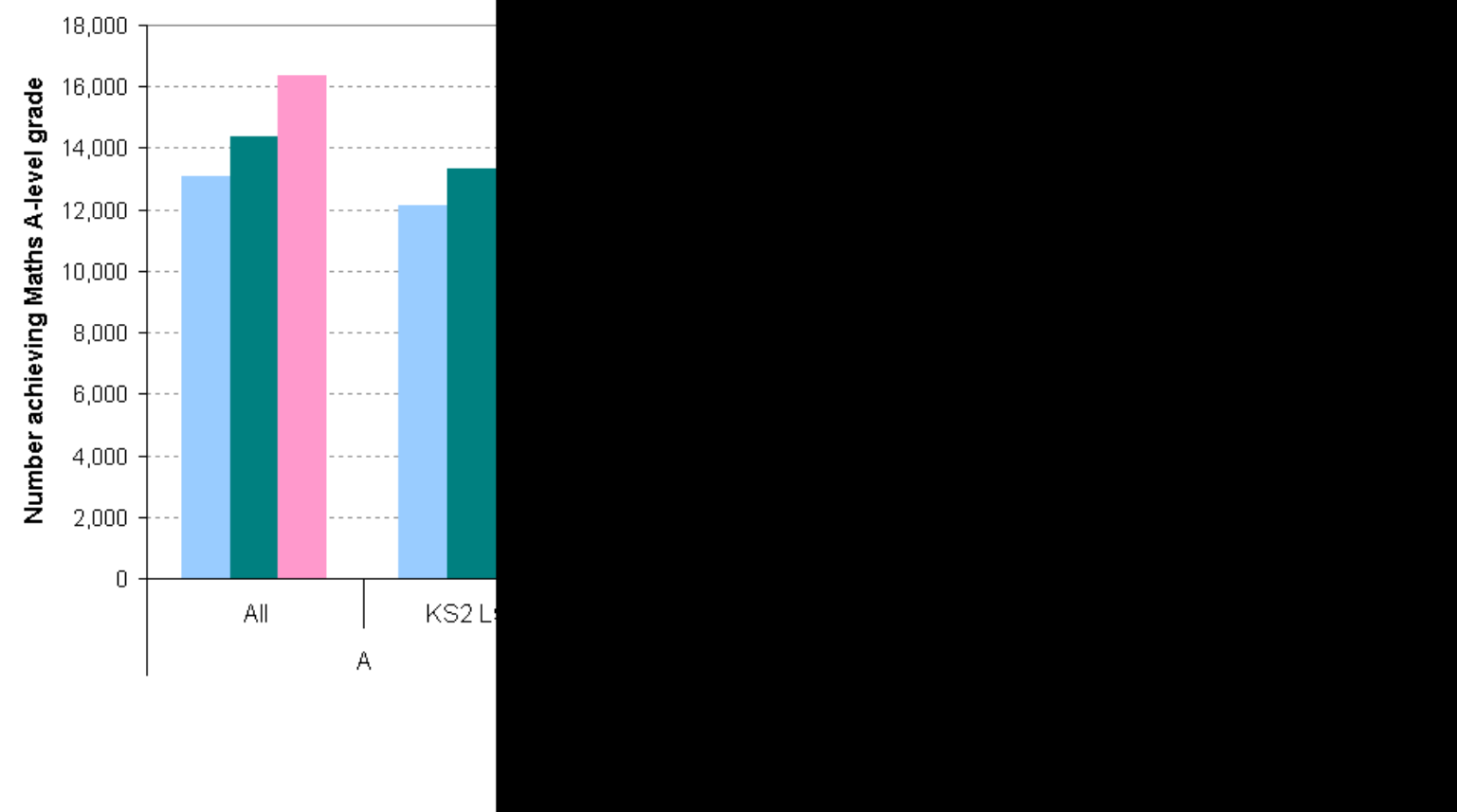

Progression of KS2 Science Cohorts to A-level Biology for 2007-2009

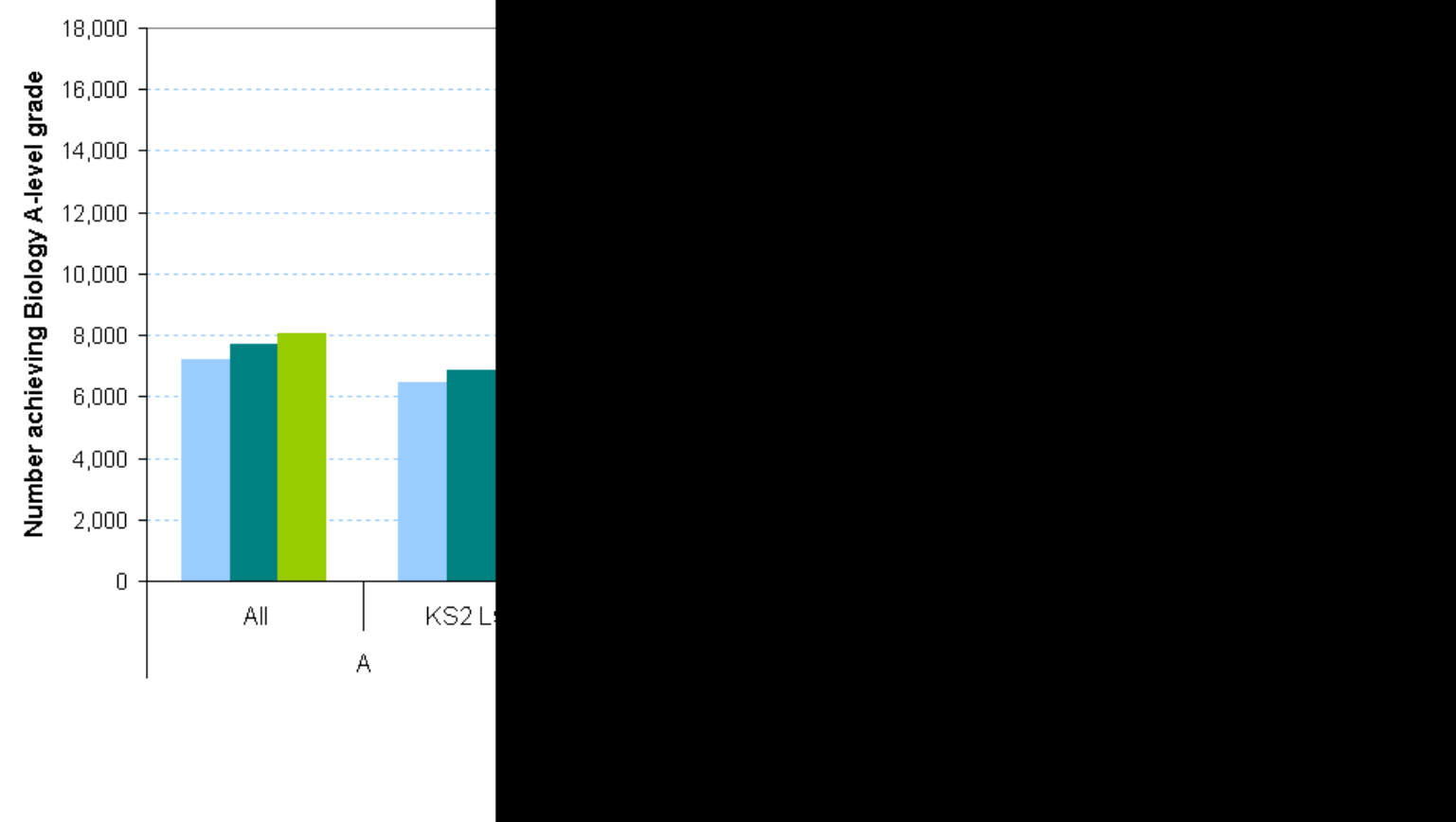


Progression of KS2 Science Cohorts to A-level Chemistry for 2007-2009

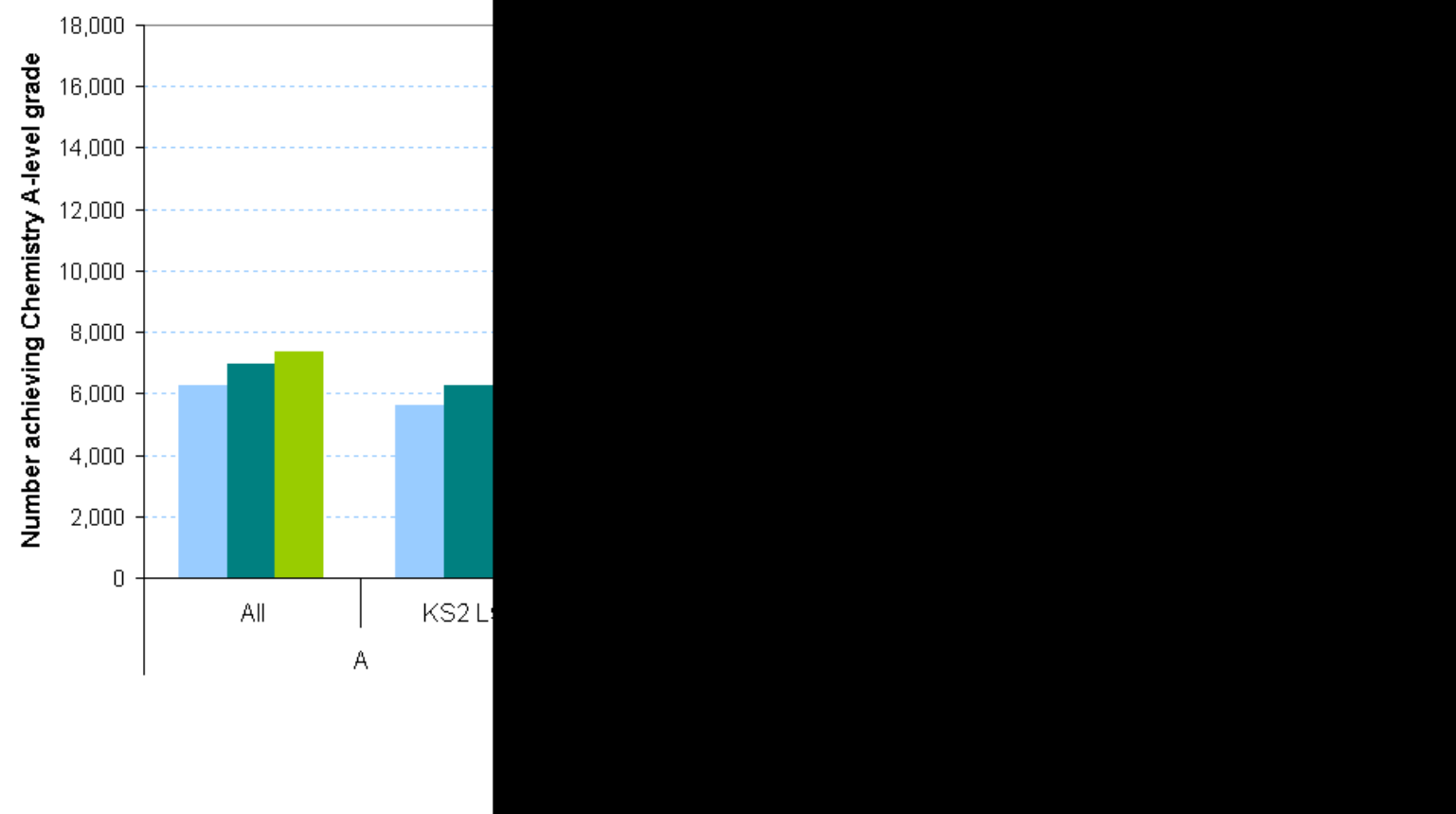

Progression of KS2 Science Cohorts to A-level Physics for 2007-2009

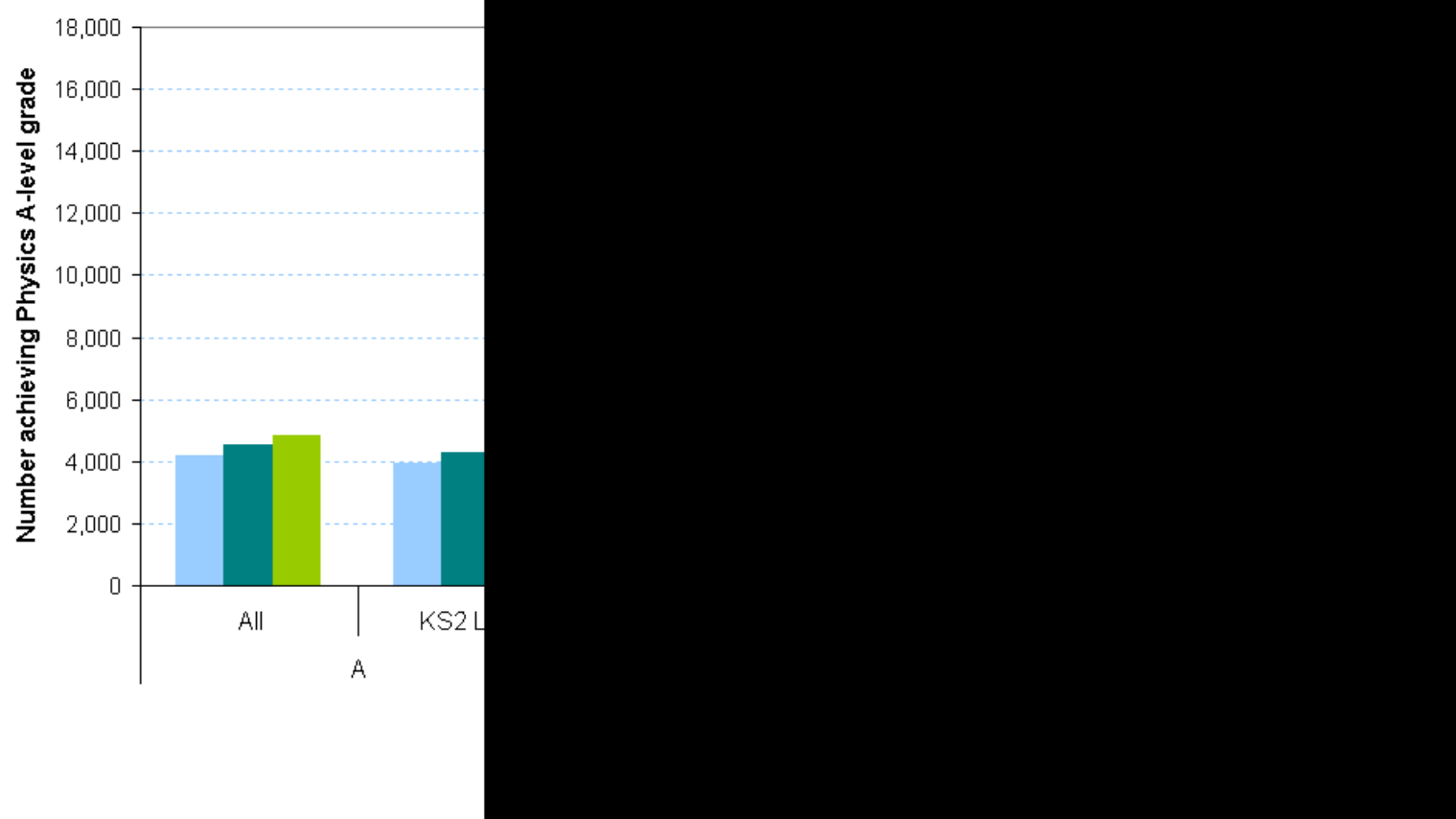




\section{Annex B: School Type Pathways and A level Achievement}
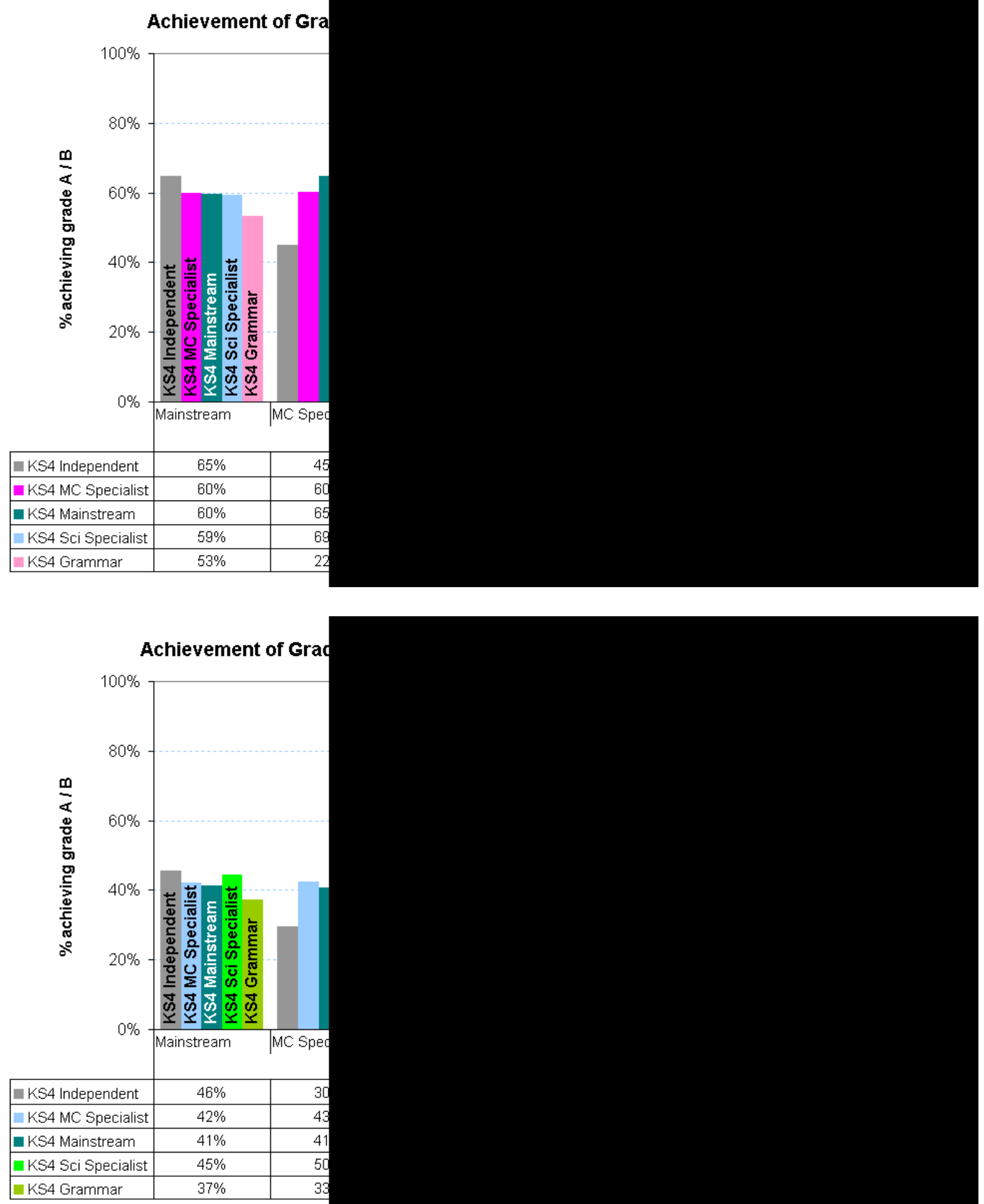
Achievement of Grades A / B in A level Chemistry by KS4 and KS5 School Type

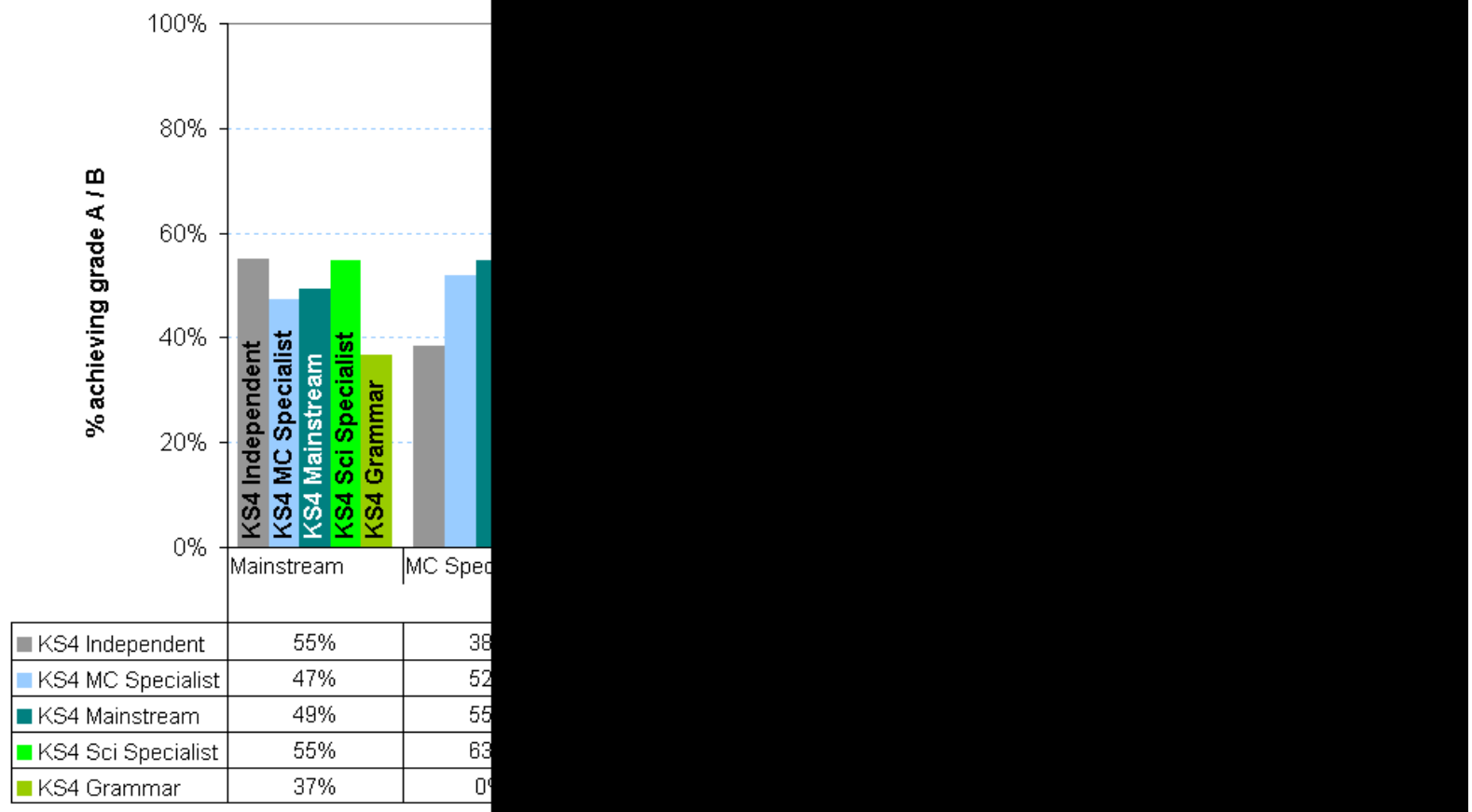

Achievement of Grades A / B in A level Physics by KS4 and KS5 School Type

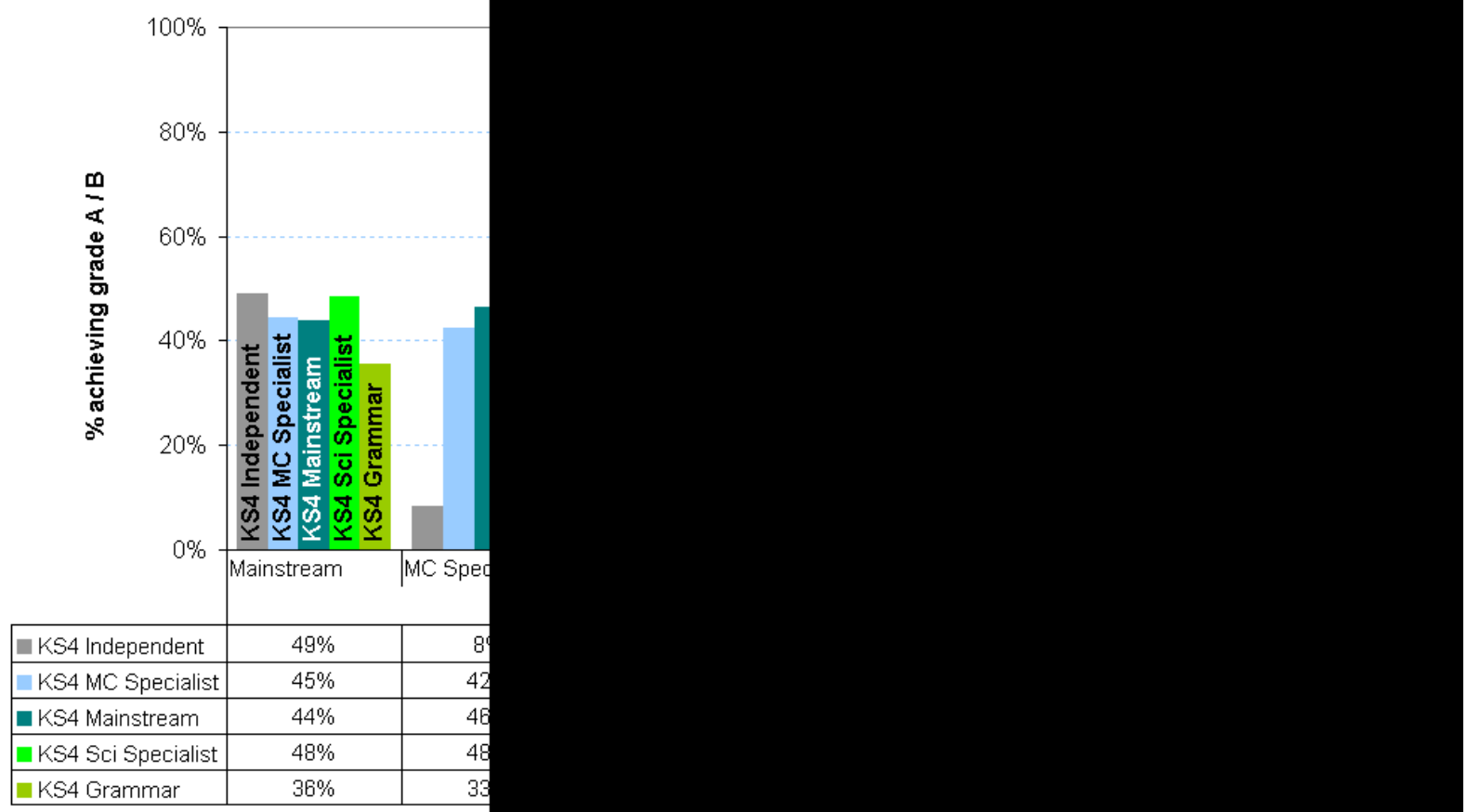




\section{Annex C: Model Effects Translated to Probabilities for Maths, Biology \& Chemistry}

\section{$\%$ of Key Stage 4 Maintained Mainstream Cohort who entered A level maths}

$\%$ of KS4 MM Cohort entering 1+ A level who achieved grade $A / B$ at $A$ level maths
Boy instead of girl $27 \%$

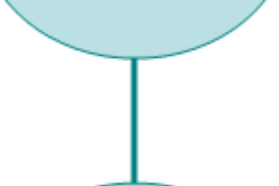

White girl, no SEN, GCSE Grade A, KS3 L6, mixed comprehensive with sixth form

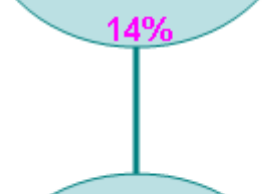

Attended mixed comprehensive without a sixth form $13 \%$
Achieved Grade B (instead of A) at GCSE maths

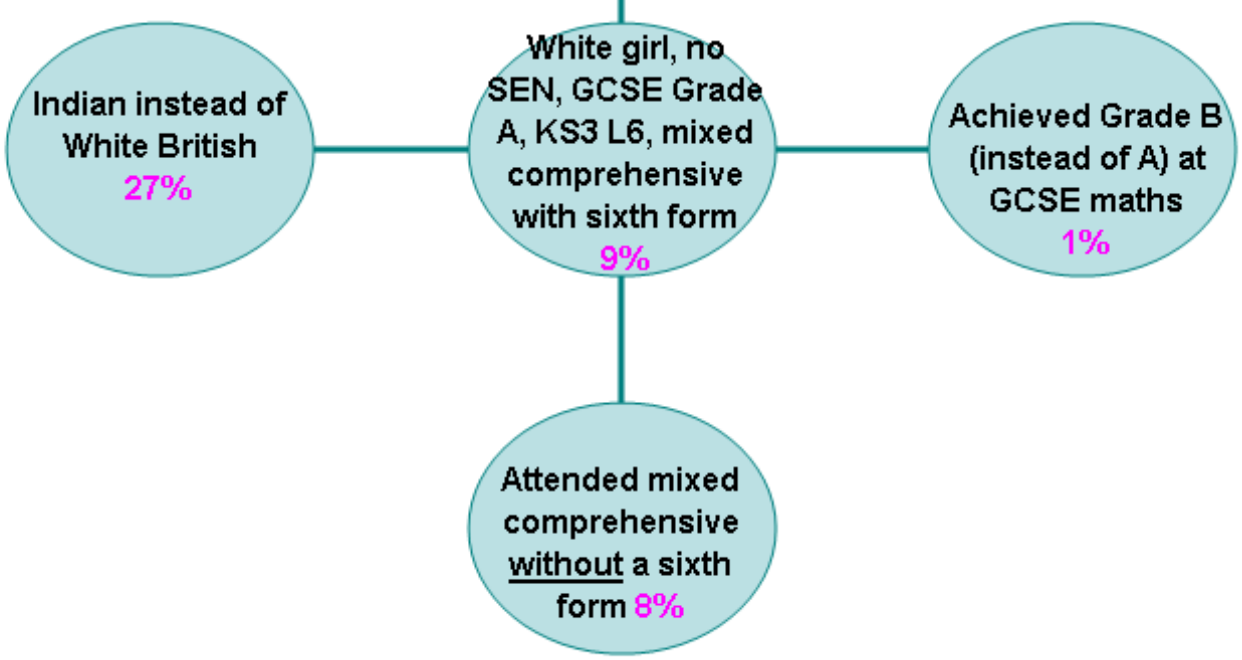


$\%$ of Key Stage 4

Maintained

Mainstream Cohort

who entered A level

biology
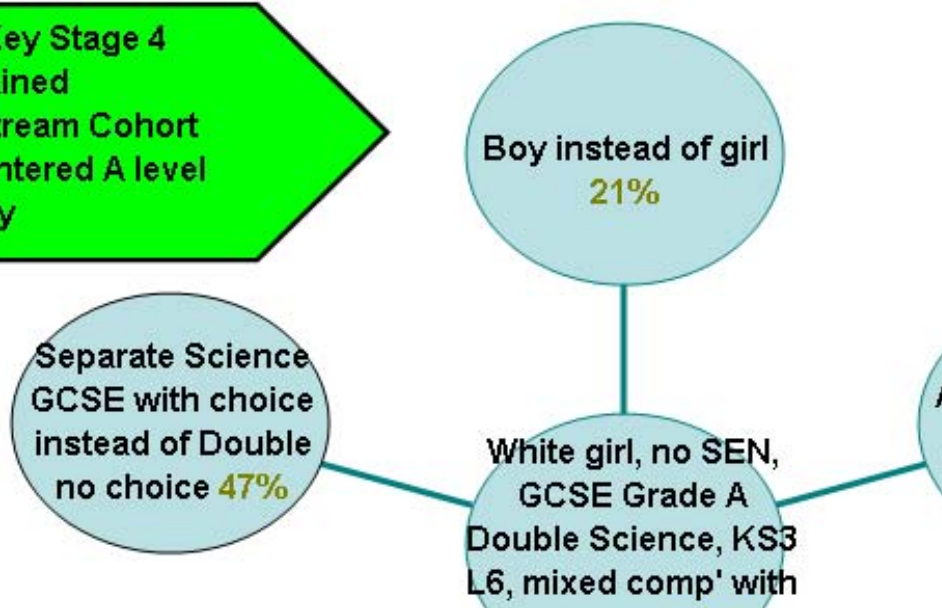

Achieved Grade B (instead of A) at GCSE science $14 \%$

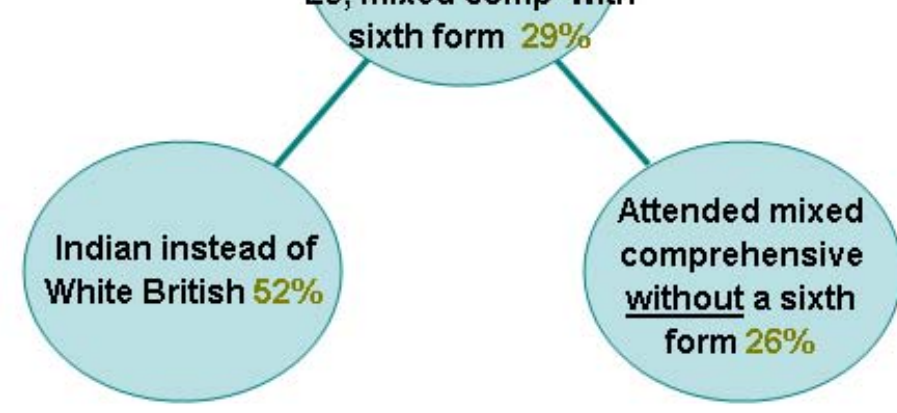

$\%$ of KS4 MM Cohort entering 1+ A level who achieved grade A/B at A level biology

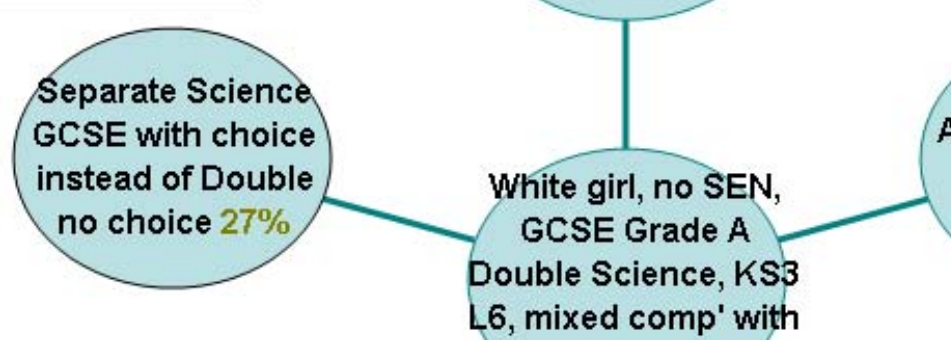

Boy instead of girl $10 \%$

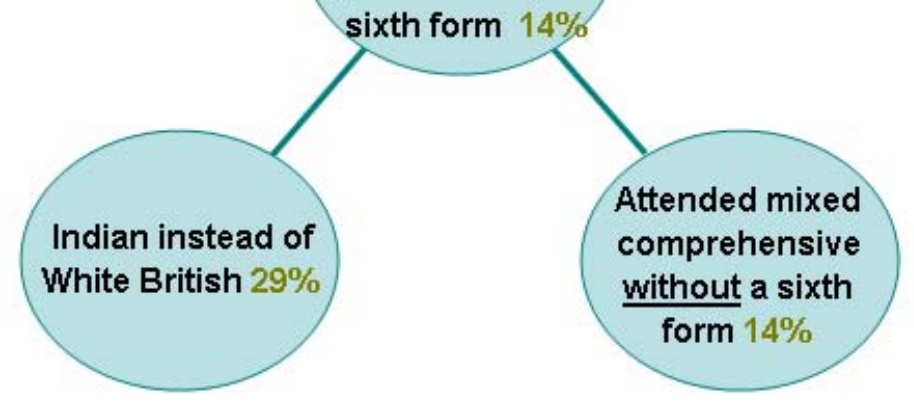


$\%$ of Key Stage 4

Maintained

Mainstream Cohort

who entered A level

chemistry
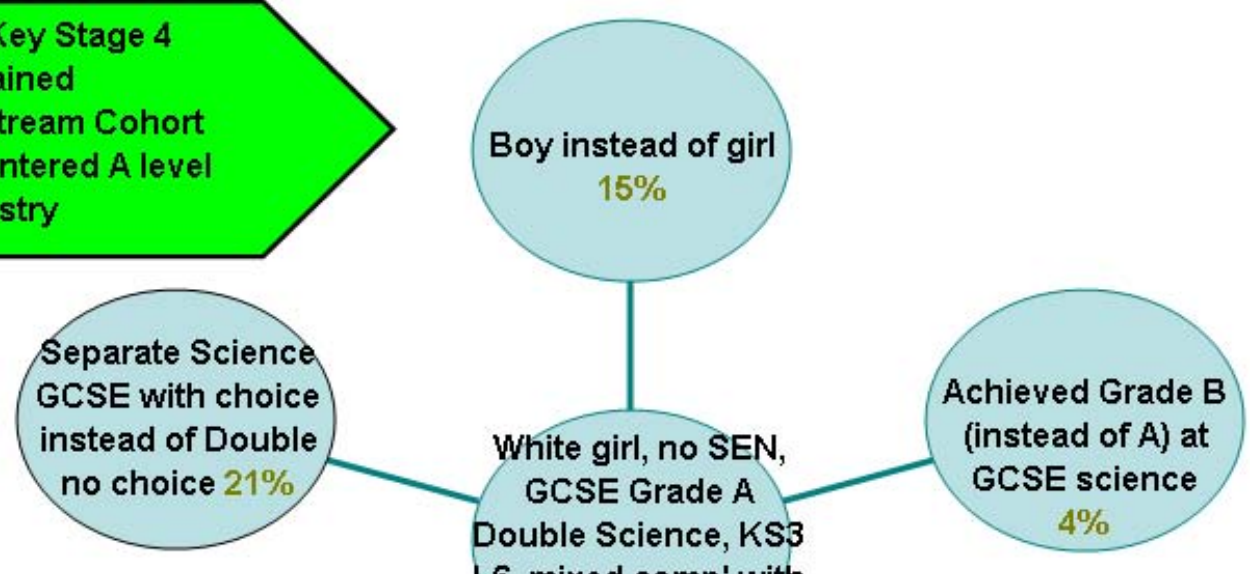

L6, mixed comp' with

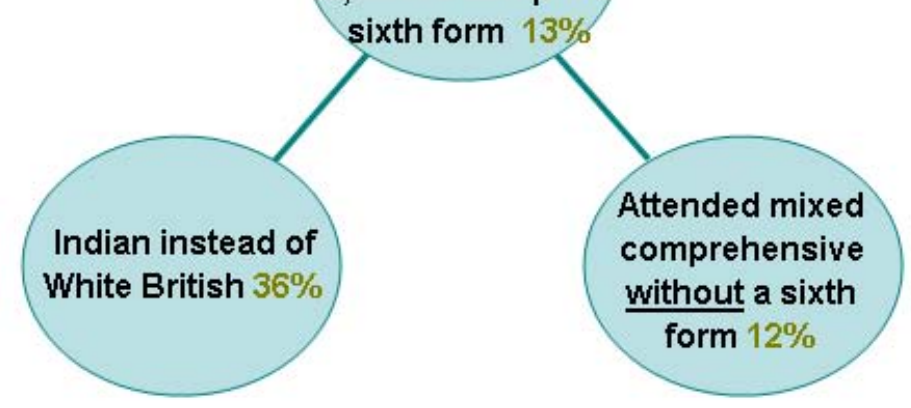

$\%$ of KS4 MM Cohort entering 1+A level who achieved grade AlB at $A$ level chemistry

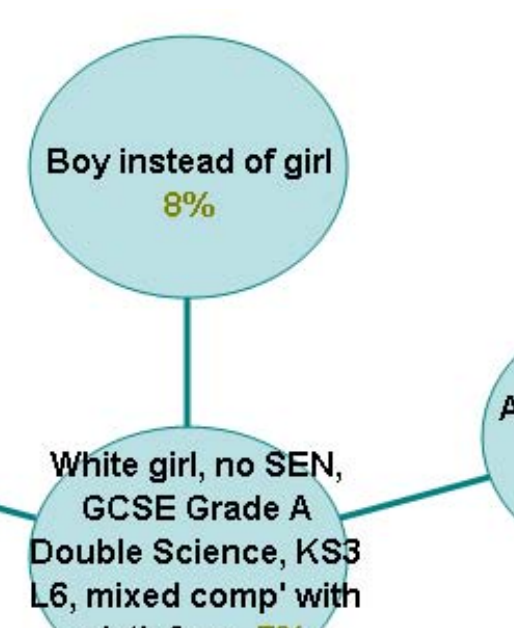

Achieved Grade B (instead of A) at GCSE science

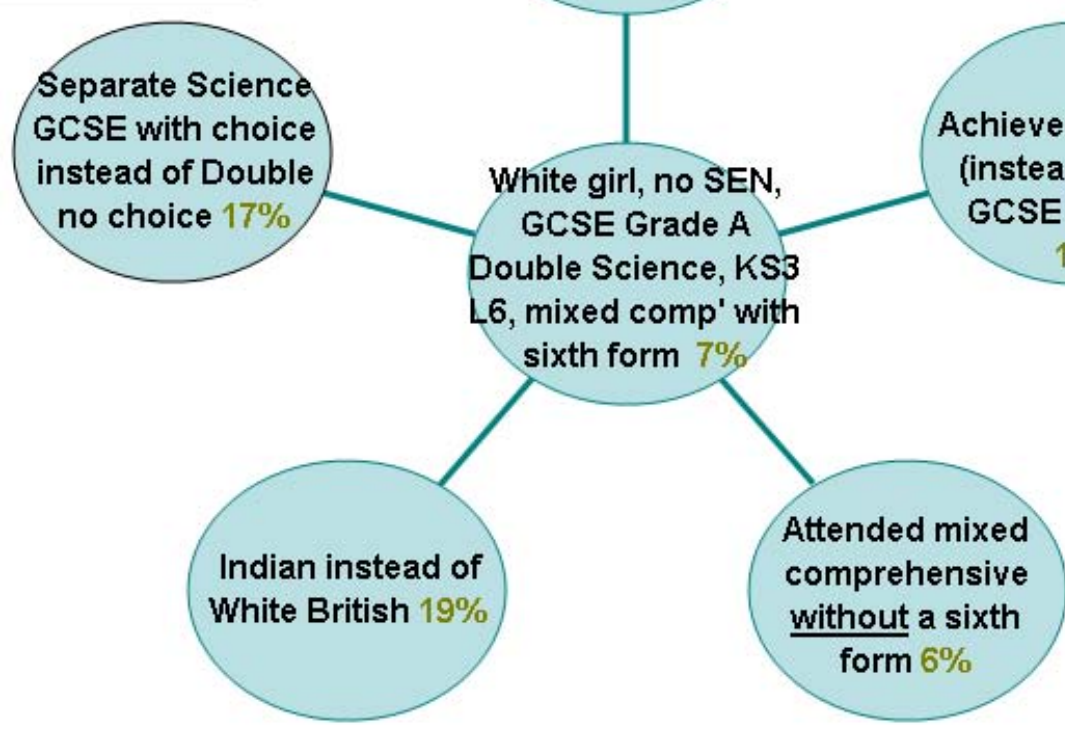


Annex D: Local Authority Maths Science Indicators at KS4 (2010)

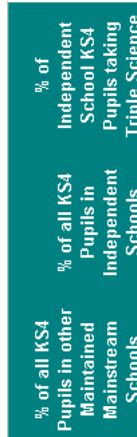

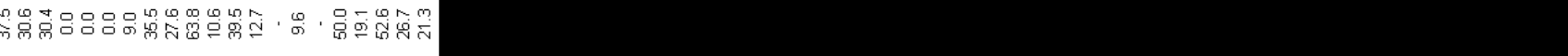

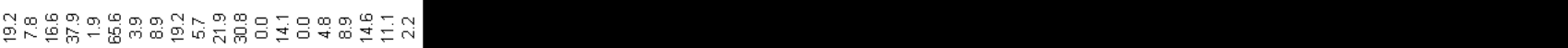

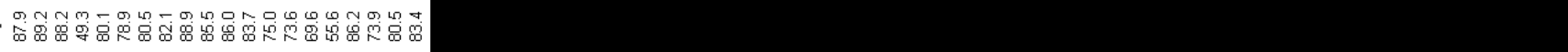

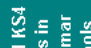

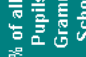

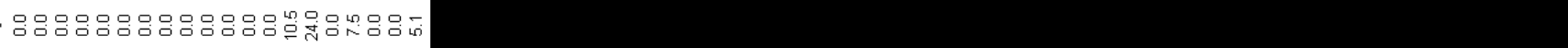

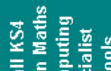

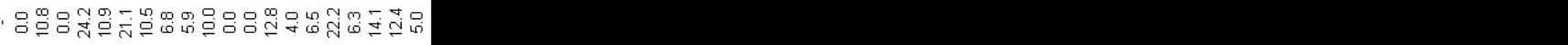

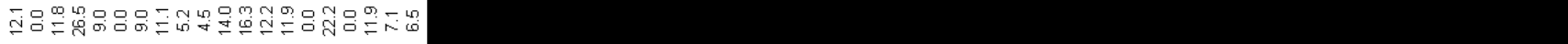

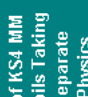

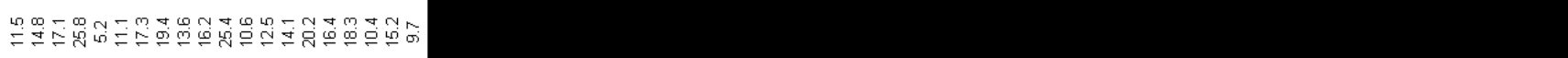

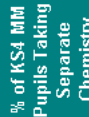

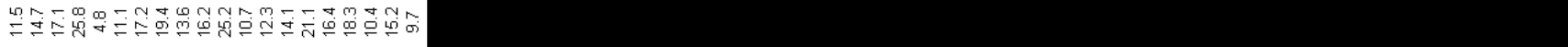

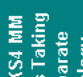

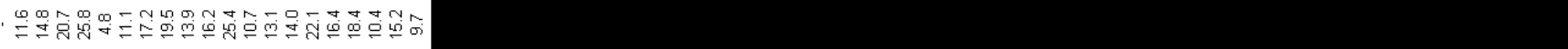

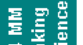

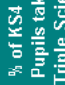

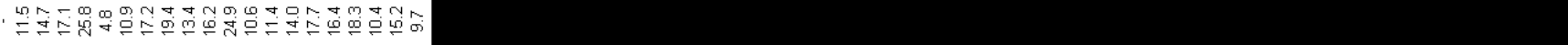

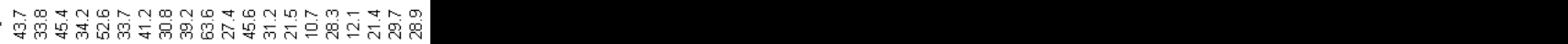

$\bar{s}$

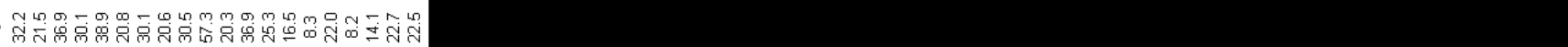

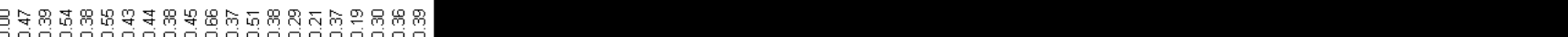
高言弯

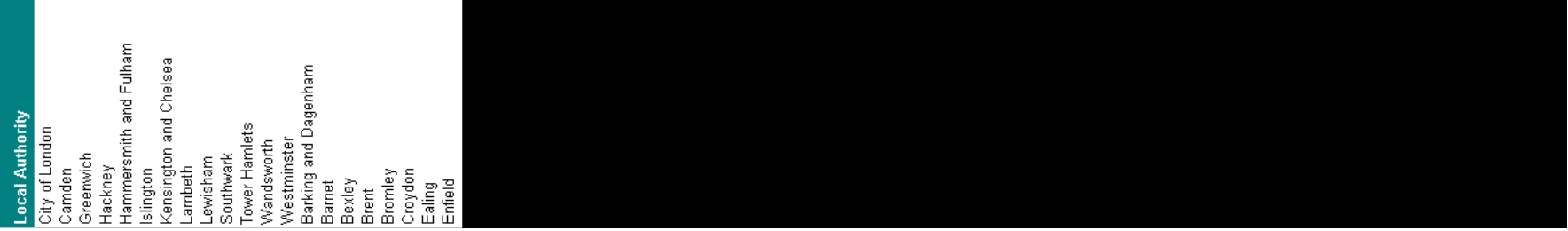


Annex D: Local Authority Maths Science Indicators at KS4 (2010) ...continued

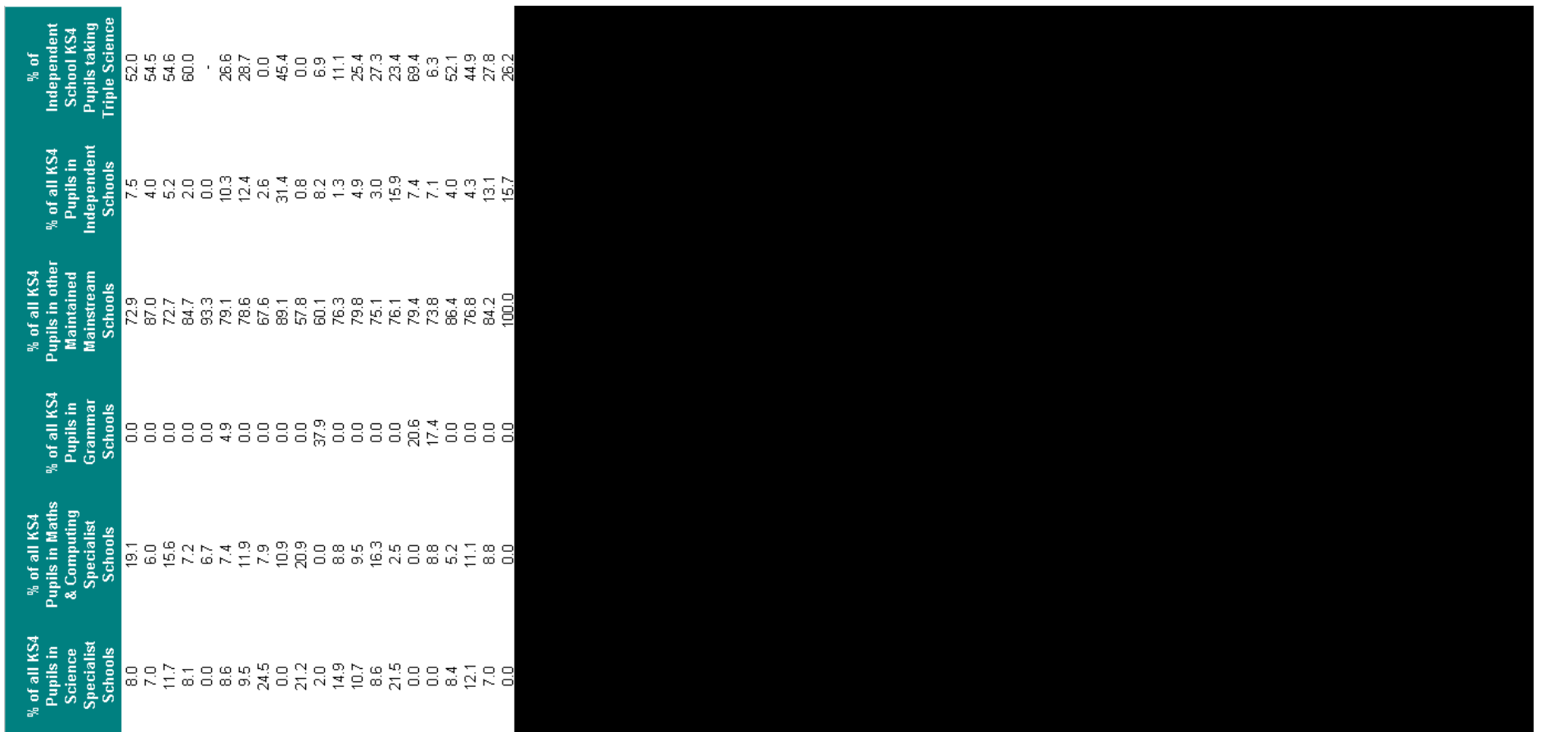

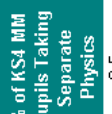

管

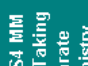

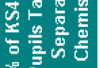
等

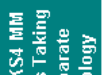
等 몰

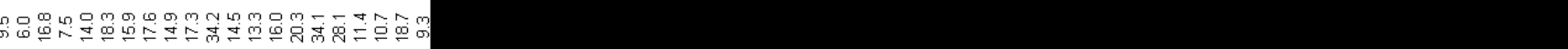

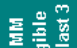

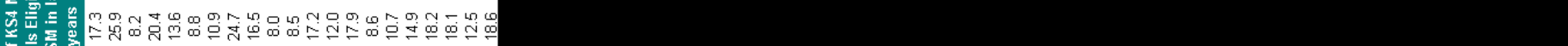

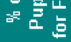

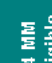

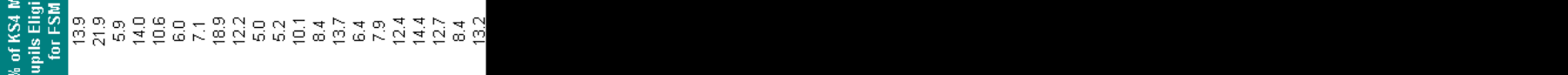

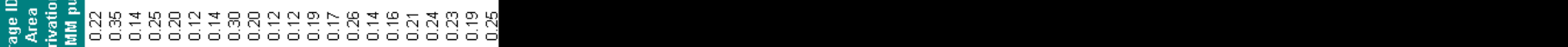

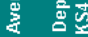
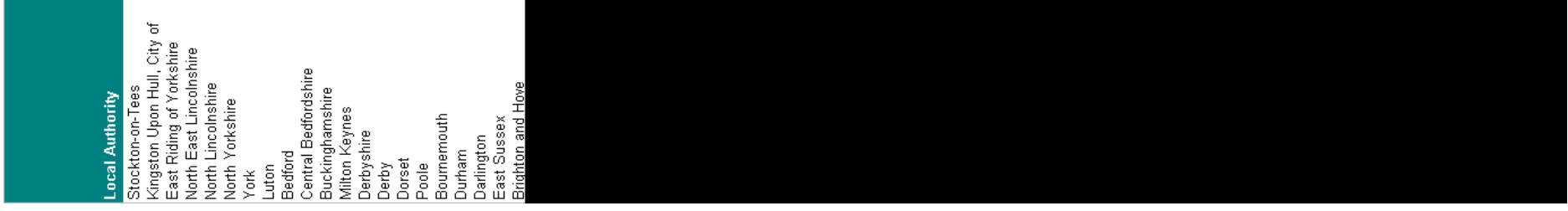
Annex D: Local Authority Maths Science Indicators at KS5 (2010)

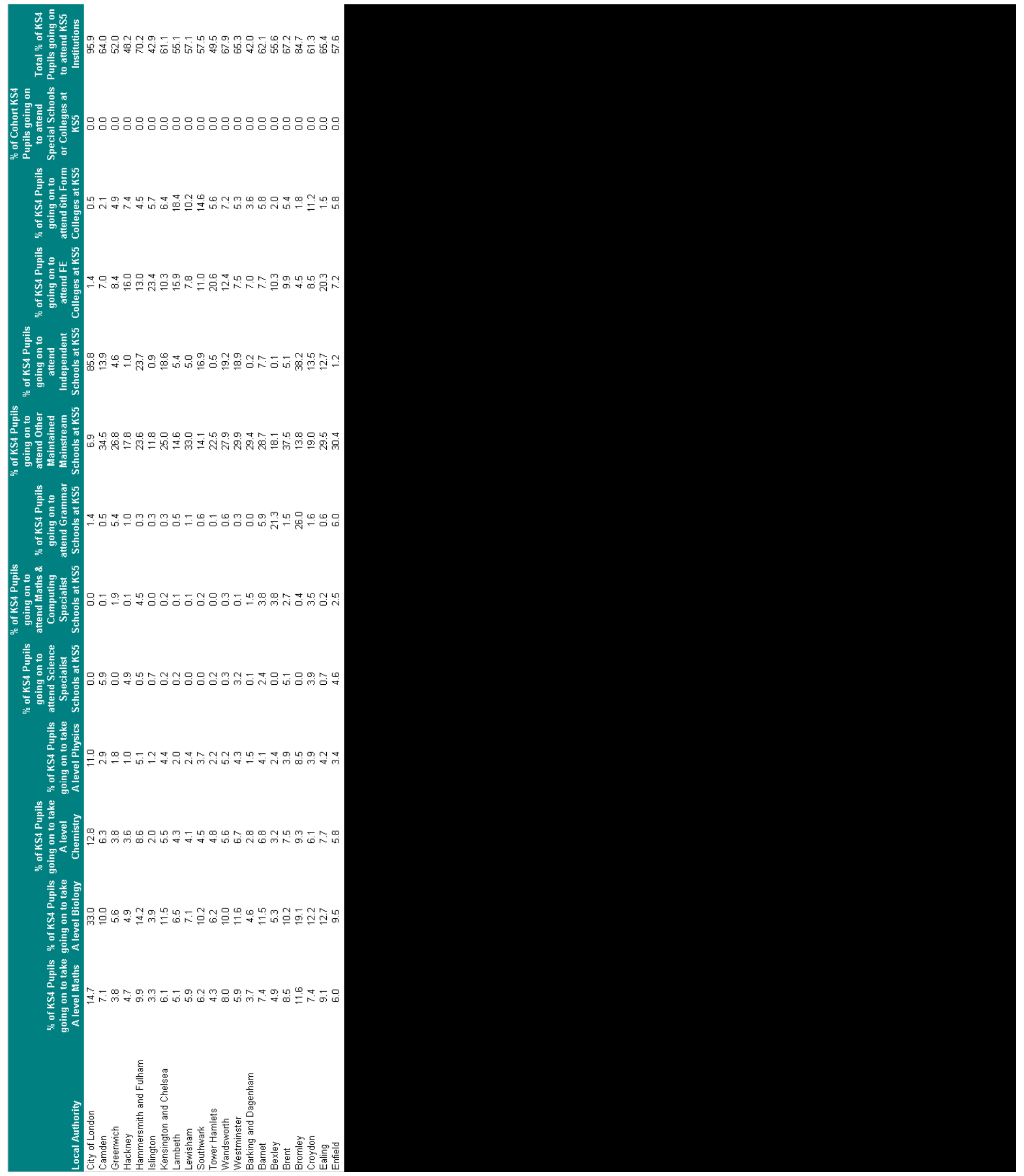


Annex D: Local Authority Maths Science Indicators at KS5 (2010) ... continued

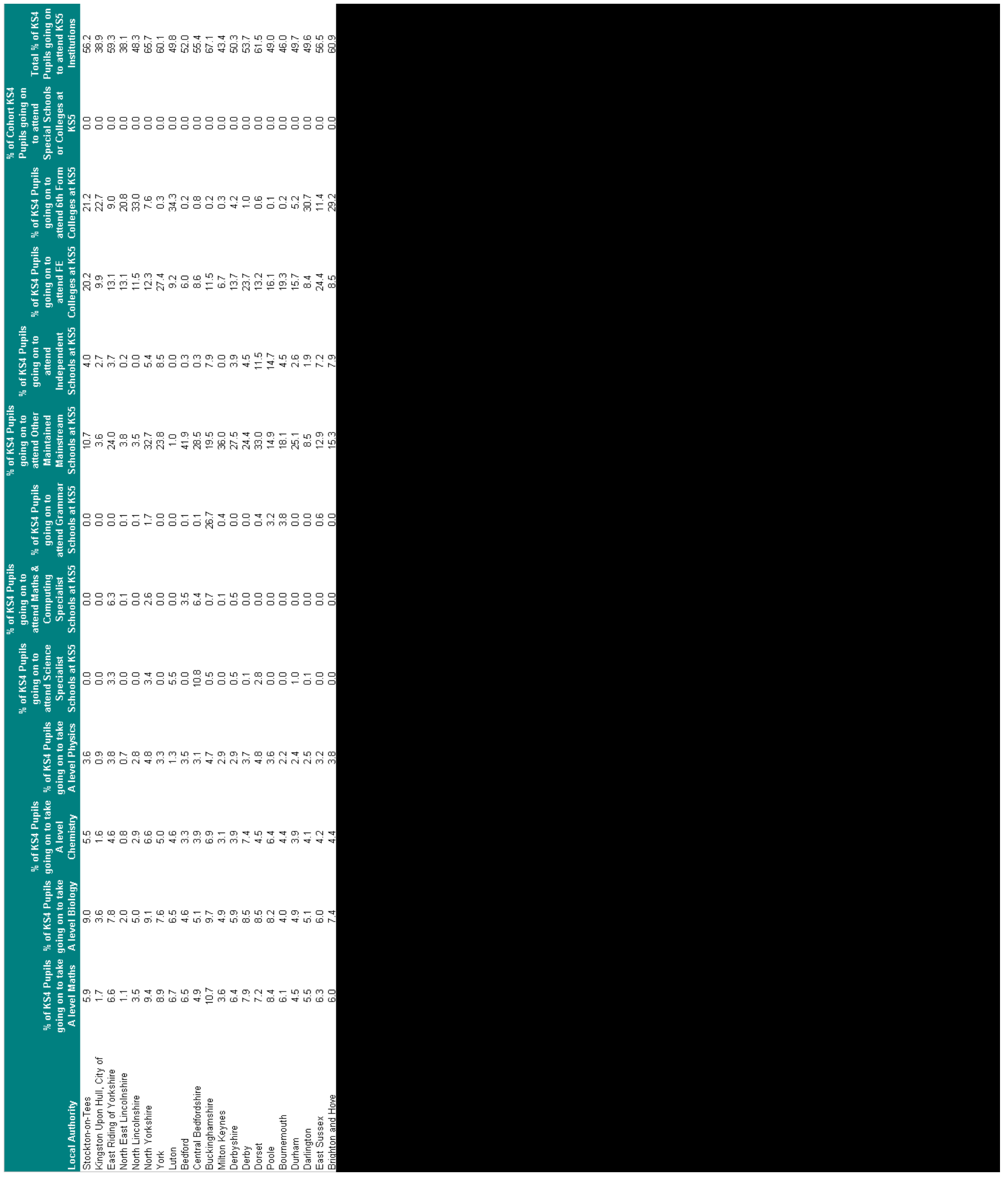


Ref: DFE-RR079

ISBN: 978-1-84775-850-7

(c) Department for Education

January 2011 